Gabriele Somaggio

\title{
Start mit Hindernissen
}

Eine theoretische und empirische Analyse der Ursachen von Arbeitslosigkeit nach der dualen Berufsausbildung 


\section{Gabriele Somaggio}

\section{Start mit Hindernissen}

Die duale Berufsausbildung gilt als Garant für einen reibungslosen Einstieg ins Erwerbsleben. Aber nicht bei allen Absolventen einer dualen Berufsausbildung gelingt dieser Einstieg erfolgreich. Bereits zu Beginn des Berufslebens sind Unterschiede $\mathrm{zu}$ erkennen, die den weiteren Erwerbsverlauf beeinflussen. Deshalb ist es notwendig, die Ursachen der Arbeitslosigkeit nach der dualen Berufsausbildung zu identifizieren. Daran setzt diese Arbeit an. Der Fokus der theoretischen und empirischen Untersuchung liegt auf der Bestimmung der Wahrscheinlichkeit des Eintritts in Arbeitslosigkeit. Grundlage bildet hier die unterschiedliche Ausbildungsbereitschaft der Betriebe. Die Einflussfaktoren auf die Wahrscheinlichkeit und auf die Länge der Arbeitslosigkeit werden ökonometrisch überprüft.

Gabriele Somaggio studierte Wirtschaftswissenschaften an der Universität Hohenheim. Zwischen 2005 und 2009 war sie Wissenschaftliche Mitarbeiterin am Lehrstuhl für Wirtschaftswissenschaft an der Universität Koblenz-Landau. Ab Mitte 2009 ist sie Mitarbeiterin am IAB in Saarbrücken. 
Start mit Hindernissen

Gabriele Somaggio - 978-3-631-74988-3

Downloaded from PubFactory at 01/11/2019 09:32:31AM

via free access 


\section{Sozialökonomische Schriften}

Herausgegeben von

Bert Rürup und Werner Sesselmeier

Band 39

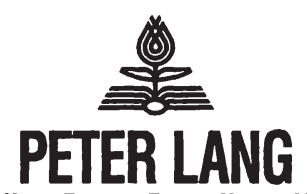

Frankturt am Main - Berlin - Bern - Bruxelles · New York - Oxłord - Wien

Gabriele Somaggio - 978-3-631-74988-3

Downloaded from PubFactory at 01/11/2019 09:32:31AM

via free access 


\section{Gabriele Somaggio}

\section{Start mit Hindernissen}

Eine theoretische und empirische Analyse der Ursachen von Arbeitslosigkeit nach der dualen Berufsausbildung

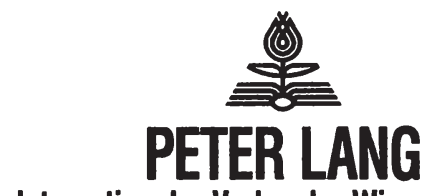

Internationaler Verlag der Wissenschaften

Gabriele Somaggio - 978-3-631-74988-3

Downloaded from PubFactory at 01/11/2019 09:32:31AM 
Bibliografische Information der Deutschen Nationalbibliothek Die Deutsche Nationalbibliothek verzeichnet diese Publikation in der Deutschen Nationalbibliografie; detaillierte bibliografische Daten sind im Internet über <http://www.d-nb.de> abrufbar.

Open Access: The online version of this publication is published on www.peterlang.com and www.econstor.eu under the international Creative Commons License CC-BY 4.0. Learn more on how you can use and share this work: http://creativecommons.org/licenses/ by/4.0.

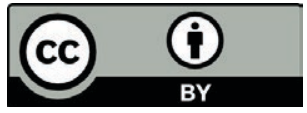

This book is available Open Access thanks to the kind support of ZBW - Leibniz-Informationszentrum Wirtschaft.

Zugl.: Koblenz-Landau, Univ., Diss., 2009

Die Dissertation wurde vom Fachbereich 6 der Universität Koblenz-Landau zur Erlangung des akademischen Grades einer Doktorin der Staatswissenschaften am 16.02.2009 angenommen.

Gedruckt auf alterungsbeständigem, säurefreiem Papier.

ISSN 0172-1747

ISBN 978-3-631-58728-7

ISBN 978-3-631-74988-3 (eBook)

(C) Peter Lang GmbH

Internationaler Verlag der Wissenschaften

Frankfurt am Main 2009

Alle Rechte vorbehalten.

Das Werk einschließlich aller seiner Teile ist urheberrechtlich geschützt. Jede Verwertung außerhalb der engen Grenzen des

Urheberrechtsgesetzes ist ohne Zustimmung des Verlages

unzulässig und strafbar. Das gilt insbesondere für

Vervielfältigungen, Übersetzungen, Mikroverfilmungen und die Einspeicherung und Verarbeitung in elektronischen Systemen. 


\section{Vorwort}

Die vorliegende Arbeit ist im Rahmen meiner Lehrstuhltätigkeit am Institut für Sozialwissenschaften - Abteilung Wirtschaftswissenschaften an der Universität Koblenz-Landau in Landau entstanden. Hierfür möchte mich bei Frau Prof. Dr. Renate Neubäumer herzlich bedanken, die mir die Gelegenheit gab, diese Arbeit zu erstellen und für günstige Rahmenbedingungen sorgte. Ebenso danke ich Herrn Prof. Dr. Werner Sesselmeier, der das Zweitgutachten übernommen hat und der mich während meiner Doktorandenzeit ebenfalls sehr unterstützt und durch die Organisation eines Doktorandenseminars mit der Universität Göttingen immer wieder für zeitlichen Druck - der sich auf das Vorankommen positiv auswirkte - gesorgt hat. Außerdem haben mir beide während meiner Schlussphase viel Freiraum zugestanden.

Für die Unterstützung und die unkomplizierte Kooperation und Beratung danke ich dem Forschungsdatenzentrum des Instituts für Arbeit- und Berufsforschung der Bundesagentur für Arbeit, insbesondere Nils Drews, Dr. Peter Jacobebbinghaus und Alexandra Schmucker. Ebenso möchte ich Dr. Hans Dietrich für die anregenden Diskussionen danken.

Mein Dank gilt ebenso meinen Lehrstuhlkollegen Dr. Ulrich Steinmetz, Marion Kleiber, Matthias Balzer, Kathrin Engelhard und Amrei Schommers, Dr. Iris Brandenburger und schließlich meiner Mit-Doktorandin Aysel Yollu-Tok für ihr offenes Ohr und die hilfreichen Gespräche. Schließlich möchte meinen Eltern für die lebenslange Unterstützung und Elke Nettelbeck-Fehling danken.

Einen bedeutenden Teil zur Entstehung dieser Dissertation hat Sven Wydra beigetragen. Ohne sein ständiges Motivieren und Aufrichten wäre diese Arbeit nicht fertig gestellt worden. Dafür möchte ich ihm besonders danken. 
Gabriele Somaggio - 978-3-631-74988-3

Downloaded from PubFactory at 01/11/2019 09:32:31AM

via free access 
Inhaltsverzeichnis

Abbildungsverzeichnis .............................................................................................11

Tabellenverzeichnis ..............................................................................................................13

$1 \quad$ Einleitung ............................................................................................................15

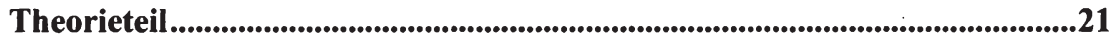

2 Einfluss der Institutionen auf den Übergang vom Schulsystem ins

Erwerbsleben ...........................................................................................................................21

2.1 Struktur der dualen Berufsausbildung .......................................................21

2.2 Fakten des dualen Berufsbildungssystems ..............................................25

2.3 Übergang von der dualen Berufsausbildung in das Erwerbsleben ............31

3 Theoretische Erklärungsansätze der Ausbildungsbereitschaft..............39

3.1 Duale Berufsausbildung aus humankapitaltheoretischer Sicht.................39

3.1.1 Humankapitaltheorie als Grundlage für Humankapital-

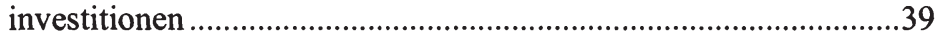

3.1.2 Duale Berufsausbildung als allgemeine Humankapitalinvestition .42

3.2 Mikroökonomische Erklärungsansätze und der Einfluss auf das

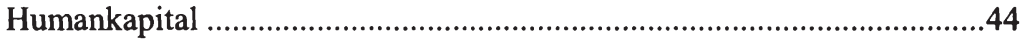

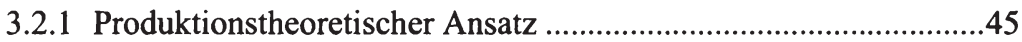

3.2.2 Investitionstheoretische Ansätze...................................................48

3.2.2.1 Grundmodell investitionstheoretischer Ansätze .................49

3.2.2.2 Bestimmungsgründe von Investitionen in allgemeines

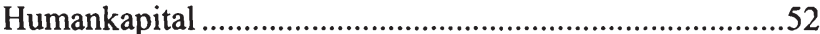

3.2.2.2.1 Informationsasymmetrien und Ausbildung ..........53

3.2.2.2.2 Allgemeines und spezifisches Humankapital als Komplementärgüter .............................................55

3.2.2.3 Institutionelle Rahmenbedingungen der Ausbildung ........59

3.2.2.3.1 Existenz von Gewerkschaften..............................59

3.2.2.3.2 Unvollkommene Produktmärkte...........................62

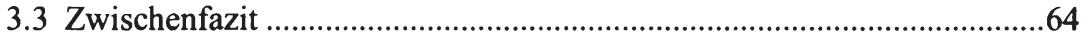


4 Bedeutung der unterschiedlichen Ausbildungsbereitschaft für den

Verbleib nach dem Abschluss ............................................................................67

4.1 Mikroökonomische Determinanten und der Übergang in den

Arbeitsmarkt

4.1.1 Produktionstheoretischer Ansatz und Verbleib nach der

Ausbildung.

4.1.2 Investitionstheoretische Ansätze und Verbleib nach der

Ausbildung

4.2 Makroökonomische Determinanten und der Übergang in den

Arbeitsmarkt

4.2.1 Institutionelle Rahmenbedingungen an der zweiten Schwelle .......72

4.2.2 Wirtschaftliche Rahmenbedingungen bei Abschluss der

Ausbildung .74

4.3 Mobilität seitens der Ausbildungsabsolventen..........................................77

4.4 Heterogene Humankapitalstruktur der Ausbildungsabsolventen...............78

5 Erklärungsansätze für Arbeitslosigkeit nach der Ausbildung und deren Dauer ................................................................................................................81

5.1 Situation der Ausbildungsabsolventen auf dem Gesamtarbeitsmarkt.......81

5.2 Theoretische Grundlagen für Arbeitslosigkeit nach der dualen Berufsausbildung und deren Dauer.

5.2.1 Suchtheoretische Ansätze ...

5.2.1.1 Arbeitgeberseite: Adverse Selektion bei der Stellenbesetzung...

5.2.1.2 Arbeitnehmerseite: Individuelles Suchverhalten

5.2.1.3 Erweiterungen des Suchmodells um humankapitaltheoretische Aspekte

5.2.2 Insider-Outsider-Theorie.

5.3 Implikationen der heterogenen Humankapitalstruktur der Absolventen

für die Arbeitslosigkeit .95

5.3.1 Bedeutung von Informationsasymmetrien. 
5.3.2 Einfluss eines veränderten Humankapitalbedarfs der Betriebe ......99

5.4 Auswirkungen einer Arbeitslosigkeit auf weiteres Erwerbsleben .101

6 Zusammenfassung der theoretisch abgeleiteten Ergebnisse und Hypothesenbildung .105

Empirieteil

7 Einflussfaktoren auf die Arbeitslosigkeit nach der dualen

Berufsausbildung

7.1 Empirische Umsetzung zur Messung des Humankapitals am Übergang nach der Ausbildung............................................................................... 113

7.1.1 Bedeutung der individuellen Merkmale.....................................115

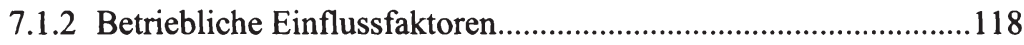

7.1.3 Wirtschaftliche und demografische Einflussgrößen......................123

7.2 Überblick über die Wirkungszusammenhänge .......................................128

7.3 Beschreibung der Datengrundlage ..........................................................131

8 Ökonometrische Verfahren und empirische Evidenz ...........................139

8.1 Bestimmungsgründe für den Verbleib nach der dualen

Berufsausbildung

8.1.1 Methodisches Vorgehen zur Bestimmung des Arbeitsmarkteintritts 140

8.1.2 Empirische Evidenz des Arbeitsmarkteintritts ............................ 144

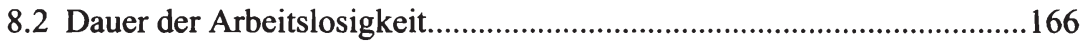

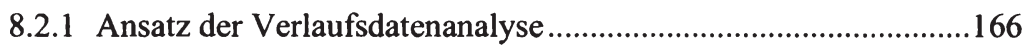

8.2.2 Einflussfaktoren auf die Dauer der Arbeitslosigkeit....................168

8.3 Bedeutung der Arbeitslosigkeit für die Beschäftigungsdauer zu Beginn

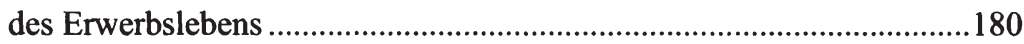

8.3.1 Beschreibung der Methode ........................................................180

8.3.2 Empirische Evidenz des Einflusses der Arbeitslosigkeit...............185 


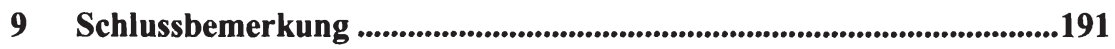

Literaturverzeichnis ................................................................................................201

Anhang .......................................................................................................................................223 


\section{Abbildungsverzeichnis}

Abbildung 1: Ausbildungsquoten zwischen 1999 und 2006 (in \%)..................26

Abbildung 2: Abstimmungsebenen im Zwei-Schwellen-Konzept......................33

Abbildung 3: Veränderung der Beschäftigtenzahl 2006 und

Auszubildendenzahl in ausgewählten Ausbildungsbereichen

2003 (in \%)

Abbildung 4: Lohnkompression und Humankapitalinvestition

Abbildung 5: Vermittlung von komplementärem spezifischem und allgemeinem Humankapital

Abbildung 6: Zusammenhang zwischen Ausbildungsbereitschaft und

Humankapital

Abbildung 7: Zusammenhang zwischen Ausbildungsbereitschaft und

Übergang ins Erwerbsleben.

Abbildung 8: Einflussfaktoren auf Höhe und Struktur des Humankapitals.....114

Abbildung 9: Darstellung der Verbleibsalternativen nach der dualen

Berufsausbildung.

Abbildung 10: Verteilung der Verbleibsalternativen der Abschlusskohorten

2000-2003

Abbildung 11: Verbleib nach Schulabschluss für die Abschlusskohorten

2000-2003 (in \%)

Abbildung 12: Abgang aus Arbeitslosigkeit der Abschlusskohorten 2000-

2003 nach Schulabschluss - Schätzung eines Kaplan-Meier-

Modells

Abbildung 13: Abgang aus der Arbeitslosigkeit der Abschlusskohorten 1996-

1999 nach Schulabschluss - Schätzung eines Kaplan-Meier-

Modells 169 
Abbildung 14: Abgang aus der Arbeitslosigkeit der Abschlusskohorten 19921995 nach Schulabschluss - Schätzung eines Kaplan-MeierModells 170

Abbildung 15: Abgang aus der Arbeitslosigkeit der Abschlusskohorten 19751991 nach Schulabschluss - Schätzung eines Kaplan-MeierModells .235 Abbildung 16: Abgang aus der Arbeitslosigkeit nach Ausbildungsbereichen Schätzung eines Kaplan-Meier-Modells 236 


\section{Tabellenverzeichnis}

Tabelle 1: Übernahmequoten nach Wirtschaftsbereichen zwischen 2002 und 2006 (in \%) 28

Tabelle 2: Arbeitslosenquote der 15- bis 29-jährigen nach Wirtschaftsbereichen 2006 (in \%) .................................................................30

Tabelle 3: Auswirkungen des Humankapitals auf die zweite Schwelle........99

Tabelle 4: Übersicht über Wirkungszusammenhänge zwischen Einflussfaktoren auf das Humankapital und dem Verbleib nach der Ausbildung

Tabelle 5: Arbeitslosenquote nach Schulabschluss für die Ausbildungsabsolventen zwischen 1975-2003 (in \%)

Tabelle 6: Verbleib nach Abschlussbereichen für die Abschlusskohorten 2000-2003 (in \%)

Tabelle 7: Einflussfaktoren auf den Verbleib nach der dualen Berufsausbildung für die Abschlusskohorten 2000-2003 -Schätzungen eines multi-nomialen Logitmodells.

Tabelle 8: Einflussfaktoren auf den Verbleib nach der dualen Berufsausbildung für die Abschlusskohorten 1996-1999 - Schätzungen eines multi-nomialen Logitmodells.

Tabelle 9: Einflussfaktoren auf den Verbleib nach der dualen Berufsausbildung für die Abschlusskohorten 1992-1995 - Schätzungen eines multi-nomialen Logitmodells.

Tabelle 10: Einflussfaktoren auf die Abgangsrate aus Arbeitslosigkeit Schätzungen eines Cox-Modells für die Abschlusskohorten zwischen 1992 und 2003 (Hazardratios)

Tabelle 11: Average Treatment Effect der Arbeitslosigkeit auf die Beschäftigungsdauer im ersten Berufsjahr - nach Schulbildung (in Tagen) 
Tabelle 12: Average Treatment Effect der Arbeitslosigkeit auf die Beschäftigungsdauer im ersten Berufsjahr - nach Ausbildungsbereichen (in Tagen)

Tabelle 13: Zuordnung der staatlich anerkannten Ausbildungsberufe zu den Ausbildungsbereichen

Tabelle 14: Häufigkeitsverteilung der Ausbildungsabsolventen an der zweiten Schwelle (1975 und 2003 in \%)

Tabelle 15: Eintritt in den Arbeitsmarkt nach der dualen Berufsausbildung für die Abschlusskohorten 1975-1980 - multinomiales Logitmodell .228

Tabelle 16: Eintritt in den Arbeitsmarkt nach der dualen Berufsausbildung für die Abschlusskohorten 1981-1986 - multinomiales Logitmodell 230

Tabelle 17: Eintritt in den Arbeitsmarkt nach der dualen Berufsausbildung für die Abschlusskohorten 1987-1991 - multinomiales Logitmodell .232

Tabelle 18: Einflussfaktoren auf die Abgangsrate aus Arbeitslosigkeit Schätzungen eines Cox-Modells für die Abschlusskohorten zwischen 1975 und 1991 (Hazardratios) 


\section{Einleitung}

Die duale Berufsausbildung gilt in Deutschland als Garant für einen reibungslosen Einstieg der Jugendlichen in das Erwerbsleben. Im internationalen Vergleich führte dies auch lange Zeit zu unterdurchschnittlichen Arbeitslosenquoten innerhalb der Gruppe der jungen Erwachsenen zwischen 20 und 25 Jahren (u. a. Büchtemann et al. 1993: 512), weshalb im Ausland das duale Ausbildungssystem als Vorbild angesehen wird, weil dessen institutionelle Strukturen die „smooth transitions“ zu begünstigen scheinen (Saar et al. 2008; Steinmann 2000; Blossfeld/Stockmann 1999). Die Besonderheit des dualen Bildungssystems liegt einerseits darin, dass gesetzlich keine Mindestanforderungen an die Qualifikationen zur Erlangung eines Berufsabschlusses gestellt werden. Auch Jugendlichen ohne Schulabschluss wird ermöglicht, sich über die duale Berufsausbildung beruflich zu qualifizieren und so den Einstieg in den Arbeitsmarkt zu schaffen. Andererseits kennzeichnet die Verknüpfung zwischen allgemeinem, in der Berufsschule, und spezifischem, im Betrieb, vermitteltem $\mathrm{Hu}-$ mankapital die Verberuflichung sowie Standardisierung des Ausbildungssystems. Dies bildet zugleich die Grundlage eines erfolgreichen Übergangs zwischen Berufsbildungssystem und Erwerbsleben, der so genannten zweiten Schwelle (Müller/Shavit 1998). Denn über die betriebliche Komponente können aktuelle Anforderungen, die der Arbeitsmarkt an die Fachkräfte stellt, vermittelt werden. Die Berufsschule ergänzt die betriebliche Ausbildung um die allgemeine Komponente. Daher ist es den Unternehmen, mehr als in anderen Berufsbildungssystemen, wie der Universität oder der schulischen Berufsausbildung, möglich, selbst Einfluss auf die Qualifikationen der Jugendlichen zu nehmen und gleichermaßen die Ausbildung eines Jugendlichen und dessen Arbeitsmarktschancen zu beeinflussen. Somit wird innerhalb der Ausbildung arbeitsplatz- sowie arbeitsmarktnahes Humankapital vermittelt. Deshalb haben die Arbeitgeber ihrerseits ein Interesse an der Durchführung der dualen Berufsausbildung, was im Zusammenhang eines bevorstehenden Fachkräftemangels auch notwendig ist. Vor diesem Hintergrund hat die Diskussion um die duale Berufsausbildung in den letzten Jahren wieder mehr an Bedeutung gewonnen. Insbesondere der Lehrstellenmangel und die Vorschläge, Anreize für Unternehmen zu schaffen bzw. ihnen Sanktionen, bspw. über die Ausbildungsplatzabgabe, aufzuerlegen, rückten in den Mittelpunkt der Debatte. Aussagen wie „Besser eine schlechte Ausbildung als keine Ausbildung“" oder „Ausbildung vor Übernahme" (Beicht/Berger 2003; Bispinck et al. 2002) werden zwar gefordert, aber diese Forderungen sind wirkungslos, wenn die ausgebildeten jungen Fachkräfte nach der Ausbildung nicht die Möglichkeit bekommen, ihr Humankapital (adäquat) einzusetzen. Am ehesten wird dies durch eine Übernahme oder doch zumindest durch eine Erwerbstätigkeit im erlernten Beruf gewährleistet.

In jüngerer Zeit garantieren diese Vorteile für Jugendliche gegenüber ihrer Altersklasse in anderen Ländern jedoch keine schnellere und erfolgreichere Integration in den Arbeitsmarkt mehr (OECD 2008). Bereits seit den 1990er Jah- 
ren ist die Übernahmequote nach der dualen Berufsausbildung rückläufig. Gleichzeitig nimmt der Anteil der Ausbildungsabsolventen, die in Arbeitslosigkeit eintreten, zu (OECD 2008; Rothe/Tinter 2007). Von Arbeitslosigkeit betroffen sind junge Erwachsene insbesondere dann, wenn sich die gesamtwirtschaftliche Situation unvorteilhaft für die Arbeitsnachfrage entwickelt (Riphahn 2002: 131f.). 2005 betrug die Arbeitslosenrate für Ausbildungsabsolventen nach der dualen Berufsausbildung 36\% (BMBF 2007: 206). Dies hat zur Folge, dass sich die Arbeitslosenquote der 15- bis 25-jährigen Personen dem EU-Durchschnitt annähert. Bereits im Zeitraum von 1998 bis 2004 hat die Arbeitslosenquote der zwischen 15- und 34-jährigen mit dualer Berufsausbildung um vier Prozentpunkte zugenommen (Reinberg/Hummel 2005). Beispielsweise gelingt nur $80 \%$ unter den Absolventen mit Hauptschulabschluss ein erfolgreicher Start ins Erwerbsleben. Aber auch zwischen den einzelnen Berufsgruppen sind unterschiedliche Arbeitslosenquoten zu erkennen. Absolventen in handwerklichen Sektoren sind eher und häufiger von Arbeitslosigkeit betroffen als Ausbildungsabsolventen in Verwaltungs- und Dienstleistungsbereichen. Schließlich kann davon ausgegangen werden, dass die Arbeitslosigkeit mittlerweile einen Teil der Erwerbsbiografie von Jugendlichen und jungen Erwachsenen darstellt und teilweise die Übergänge an den beiden Schwellen determiniert (Dundler/Müller 2006; Dietrich 2001a). Infolgedessen gewinnt der Übergang an der zweiten Schwelle zunehmend an Bedeutung. Eine Erweiterung der Diskussion um die Situation nach der dualen Berufsausbildung wird erforderlich.

Indes konzentriert sich der Forschungsschwerpunkt vorwiegend auf die mangelnde Ausbildungsbereitschaft und den daraus resultierenden Lehrstellenmangel sowie die Arbeitslosigkeit vor Eintritt in die Berufsausbildung und die Wirkungen der eingesetzten arbeitsmarktpolitischen Maßnahmen, welche auf die Unterstützung der Jugendlichen zur Aufnahme einer Ausbildung abzielen (Dietrich/Gerner 2008; Niederalt 2004). Auch Studien (u. a. Konietzka 2002; Konietzka/Seibert 2001; Büchel/Neubäumer 2001; Neubäumer 1999; Stegmann/Kraft 1983; Stegmann/Kraft 1982), welche die zweite Schwelle untersuchen, legen den Fokus nicht auf den Eintritt in Arbeitslosigkeit, sondern vielmehr auf die Bestimmungsgründe für die Abweichung des durch die duale Berufsausbildung vorgegebenen strukturierten Übergangs. Weitere Studien (Lauterbach/Sacher 2001; Sackmann 2001; Fuchs/Schettkat 2000, Fürstenberg 2000; Mayer 2000; Blossfeld 1990) bescheinigen ebenfalls eine Prekarität bzw. eine Erosion des Übergangssystems an der zweiten Schwelle. Auch Buchholz (2008) untersucht die zunehmende Flexibilisierung beim Eintritt in den Arbeitsmarkt. Darüber hinaus existieren Untersuchungen, die insbesondere die Art der Abgänge aus Arbeitslosigkeit und deren Einflussfaktoren analysieren (Dietrich/Kleinert 2006 und 2005). Zudem gibt der bisherige Forschungsstand vorwiegend Aufschluss über die Folgewirkungen eines weniger erfolgreichen Übergangs von der dualen Berufsausbildung in den Arbeitsmarkt sowie einer Arbeitslosigkeitsperiode zu Beginn des Erwerbslebens. In diesem Kontext werden 
häufig individuelle und soziale Merkmale als Einflussvariablen herangezogen, während betriebliche Eigenschaften vornehmlich der Erklärung der unterschiedlichen Ausbildungsbereitschaft dienen. Jedoch beeinflussen diese betrieblichen Variablen im Zusammenhang mit individuellen Faktoren aber auch den Eintritt in das Erwerbsleben und determinieren infolgedessen den Eintritt in Arbeitslosigkeit mit. Diesen Gründen bzw. Ursachen für den Eintritt in die Arbeitslosigkeit nach der dualen Berufsausbildung wird bisher wenig Beachtung geschenkt. Aber nur durch die Bestimmung der Ursachen für einen weniger erfolgreichen Eintritt können Handlungsempfehlungen abgeleitet werden, welche auf die Situation junger Erwachsener einwirken und so die Arbeitsmarktsituation dieser Gruppe gegenüber Arbeitskräften mit Berufserfahrung verbessern. Daher steht im Mittelpunkt der vorliegenden Arbeit die theoretische und empirische Identifikation der Bestimmungsgründe für den Eintritt in die Arbeitslosigkeit.

Hierfür liegt der Fokus auf der betrieblichen Ebene, da die Merkmale der Ausbildungsbetriebe zu Unterschieden der erworbenen Fähigkeiten innerhalb des betrachteten formalen Abschlusses der dualen Berufsausbildung führen. Ausgangspunkt ist die Kostenstruktur der Ausbildungsbetriebe, welche die Anteile der Humankapitalarten innerhalb der dualen Berufsausbildung determinieren. So ergänzen und erweitern die betrieblichen Rahmenbedingungen, die während der Ausbildung vorherrschen, das Humankapital auf unterschiedliche Weise. Dies hat Auswirkung auf das Beschäftigungsinteresse über die Ausbildung hinaus. Zudem beeinflussen individuelle Merkmale und gesamtwirtschaftliche Voraussetzungen die Art der Ausbildung. Über diesen Wirkungskanal wird wiederum der Verbleib nach der dualen Berufsausbildung und damit auch der Eintritt in die Arbeitslosigkeit bestimmt.

Ziel der Arbeit ist es somit, die betrieblichen, individuellen und gesamtwirtschaftlichen Bestimmungsgründe für das Arbeitslosigkeitsrisiko nach der dualen Berufsausbildung herauszuarbeiten und abzuleiten, wobei die betriebliche Ausbildungsbereitschaft die Erklärungsgrundlage für die unterschiedliche Vermittlung von Qualifikationen und folglich für den Übergang ins Erwerbsleben, insbesondere für die Arbeitslosigkeit nach Abschluss der Ausbildung, bildet. Daraus ergibt sich die Fragestellung, inwieweit sich diese unterschiedliche Ausbildungsbereitschaft auf den Übergang von der dualen Berufsausbildung ins Erwerbsleben auswirkt. Zur Beantwortung müssen zunächst deren Gründe identifiziert und dann untersucht werden, ob diese den Übergang nach Abschluss der Ausbildung beeinflussen. Hierbei wird der Fokus auf die arbeitslosen Ausbildungsabsolventen gelegt. Drei Aspekte stehen dabei im Mittelpunkt: Im ersten Schritt werden betriebliche und individuelle Einflussfaktoren analysiert, die den Eintritt bestimmen. In einem zweiten Schritt werden dieselben Einflussfaktoren zur Erklärung der Verbleibsdauer in Arbeitslosigkeit herangezogen. Im dritten Schritt wird untersucht, inwieweit sich die erste Arbeitslosigkeit auf das weitere Erwerbsleben im ersten Berufsjahr auswirkt und infolgedessen die Periode in Erwerbstätigkeit beeinflusst. Aus den Erklärungsansätzen für die betriebliche 
Ausbildungsbereitschaft in Verbindung mit den individuellen Faktoren werden Implikationen für den Übergang von der dualen Berufsausbildung ins Erwerbsleben abgeleitet. Um diese zu fundieren, werden die Theorien zur Erklärung der Ausbildung mit den Arbeitslosigkeitstheorien verknüpft, wodurch sich dann die Gründe für den Eintritt in Arbeitslosigkeit und deren Dauer herausarbeiten lassen.

Der Aufbau der Arbeit gestaltet sich wie folgt: Der theoretische Teil beginnt in Kapitel zwei mit einer Beschreibung der institutionellen Besonderheiten der dualen Berufsausbildung und des Übergangs von der dualen Berufsausbildung in den Arbeitsmarkt. Bereits an dieser Stelle wird deutlich, dass allgemeines, betriebsspezifisches, aber vor allem berufsspezifisches Humankapital vermittelt wird. Diese Grundlagen stellen darüber hinaus die Verbindung zwischen den theoretischen Modellen und dem Spezialfall der dualen Berufsausbildung dar. In Kapitel drei wird das theoretische Modell zur Humankapitalbildung aufgezeigt und um Ansätze erweitert, welche sich als Erklärung für die Ausbildungsbereitschaft innerhalb des dualen Berufsbildungssystems besser eignen. Mikroökonomische aber auch institutionelle Gründe beeinflussen die betriebliche Motivation für eine Ausbildung und darüber hinaus die Zusammensetzung der einzelnen Humankapitalkomponenten. Diese determinieren wiederum die Ausbildungsqualität und die Höhe des Bildungsstandes. Daraus resultiert die unterschiedliche Ausbildungsbereitschaft, die Implikationen für den Übergang von der dualen Berufsausbildung in das Erwerbsleben gibt. In Kapitel vier wird daher konkretisiert, welche Bedeutung diese unterschiedliche Ausbildungsbereitschaft auf die betriebliche Entscheidung hat, Ausbildungsabsolventen unbefristet, befristet oder überhaupt nicht weiterzubeschäftigen. Im Mittelpunkt steht das formal vermittelte Humankapital, das um die informellen Fähigkeiten erweitert wird. Die Zusammensetzung der verschiedenen Humankapitalkomponenten spielt eine entscheidende Rolle für den Verbleib nach der Ausbildung. Der Einfluss der Betriebe am Übergang ins Erwerbsleben wird um die individuelle Sichtweise ergänzt, indem beschrieben wird, was Ausbildungsabsolventen dazu bewegt, ihren Ausbildungsbetrieb zu verlassen. Schließlich betrachtet Kapitel fünf die Situation der nicht weiterbeschäftigten Ausbildungsabsolventen im Kontext des gesamten Arbeitsmarktes. Besonderheiten auf dem Arbeitsmarkt werden für arbeitslose Ausbildungsabsolventen verdeutlicht, die sich durch die Rahmenbedingungen des Bildungssystems und des Arbeitsmarktes kennzeichnen. Gegenüber älteren erwerblosen Arbeitnehmern weisen die arbeitslosen jungen Erwachsenen nur wenig Berufserfahrung auf, da sie meist direkt aus dem Ausbildungssystem in die Arbeitslosigkeit eintreten. So bestimmen die vermittelten Humankapitalkomponenten, welche sich je nach Ausbildung unterschiedlich zusammensetzen, die Wahrscheinlichkeit, in Arbeitslosigkeit einzutreten, sowie deren Dauer. Die Erklärung der Arbeitslosigkeit ist durch die Verknüpfung der Ausbildungs- mit den Arbeitslosigkeitstheorien möglich. Kapitel sechs fasst die the- 
oretischen Erkenntnisse zusammen und leitet die zu überprüfenden Hypothesen ab.

Diese Hypothesen werden im empirischen Teil überprüft. Da das Humankapital empirisch nur eingeschränkt erfasst werden kann, befasst sich das Kapitel sieben vor allem mit der konkreten Umsetzung des Humankapitals in Variablen, mit denen die Hypothesen überprüft werden können. Bereits vorhandene Studien dienen als Grundlage. Eine zentrale Rolle spielen hierbei die betrieblichen Rahmenbedingungen während der Ausbildung. Diese bestimmen insbesondere die Qualifikationen innerhalb der dualen Berufsausbildung, welche wiederum den Verbleib an der zweiten Schwelle beeinflussen. Da aber ebenfalls individuelle und wirtschaftliche Rahmenbedingungen auf den Übergang in das Erwerbsleben wirken, werden diese im Zusammenhang mit den betrieblichen Rahmenbedingungen untersucht. Gegenstand der ökonometrischen Schätzungen bilden daher die Determinanten, welche die Ausbildung und deren Wirkungen auf die Arbeitslosigkeit nach der dualen Berufsausbildung bestimmen. Abschließend erfolgt eine Beschreibung der IAB-Beschäftigtenstichprobe Version 1975-2004, welche als Datengrundlage dient. Kapitel acht überprüft die Hypothesen empirisch, indem die Einflussfaktoren auf das Arbeitslosigkeitsrisiko sowie auf die Arbeitslosigkeitsdauer in getrennten Schätzungen analysiert werden. Um Implikationen für den weiteren Erwerbsverlauf zu erhalten, wird der Einfluss der Arbeitslosigkeit direkt nach der dualen Berufsausbildung auf die Beschäftigungsdauer innerhalb des ersten Jahres nach Abschluss der betrieblichen Ausbildung ermittelt. Die Arbeit schließt in Kapitel neun mit einer Zusammenfassung und einem Ausblick über die Möglichkeiten und Empfehlungen zur Verbesserung der Situation am Übergang von der Ausbildung in das Erwerbsleben. 
Gabriele Somaggio - 978-3-631-74988-3

Downloaded from PubFactory at 01/11/2019 09:32:31AM

via free access 


\section{Theorieteil}

\section{Einfluss der Institutionen auf den Übergang vom Schulsystem ins Er- werbsleben}

\subsection{Struktur der dualen Berufsausbildung}

Das deutsche Bildungssystem wird entscheidend von der Institution der dualen Berufsausbildung geprägt, die eine effiziente Methode zur Bildung von beruflicher Qualifikation darstellt (Hofer/Pichelmann 1995: 327). Im Jahr 2007 erlernten $66,2 \%$ eines Schulabsolventenjahrganges innerhalb des dualen Berufsbildungssystems einen Ausbildungsberuf (BMBF 2008: 10). Somit bildet es den Hauptpfeiler der Berufsausbildung innerhalb des deutschen Bildungssystems. Ferner weisen Absolventen dieses Systems im internationalen Vergleich eine eher geringe Arbeitslosigkeit auf. Dies wird durch die institutionellen Rahmenbedingungen, innerhalb derer die Organisation der dualen Berufsausbildung stattfindet, begünstigt (Steinmann 2000: 164; Werwatz 2002: 280; McGinnity et al. 2005: 359), wobei insbesondere der Verbindung zwischen den Bildungssystemen und dem Arbeitsmarkt eine bedeutende Rolle zukommt (Steinmann 2000: 164). Deutlich wird dies bei der genaueren Betrachtung der Bezeichnung ,dual', welche das Zusammenwirken der zwei Lernorte Betrieb und (Berufs-)Schule beschreibt. Die erste Säule des dualen Berufsbildungssystems bildet der betriebliche Ausbildungsteil. Dieser ist zugleich die Voraussetzung für das Bestehen des Systems. Das Berufsbildungsgesetz von 1969 (BBiG), das 2005 novelliert wurde und nunmehr als Berufsbildungsreformgesetz bezeichnet wird (kurz: BerBiRefG), bildet die privat- rechtliche Grundlage für die betriebliche Ausbildung. ${ }^{1}$ Ausbildungen im handwerklichen Bereich werden gemäß $\S 3$ Abs. 3 BerBiRefG durch die Handwerksordnung (HwO) geregelt, die als Ergänzung des Berufsbildungsreformgesetzes zu verstehen ist. ${ }^{2}$ Das Berufsbildungsgesetz, wie auch die Handwerksordnung, legt dem Ausbildungsverhältnis einen Ausbildungsvertrag zwischen dem Auszubildenden und dem Ausbildungsbetrieb gemäß $§ 10$ BerBiRefG (bzw. $\S 28 \mathrm{HwO}$ ) zugrunde. Der Ausbildungsbetrieb hat „soweit sich aus seinem Wesen und Zweck und aus diesem Gesetz nichts anderes ergibt, die für den Arbeitsvertrag geltenden Rechtsvorschriften und Rechts-

\footnotetext{
${ }^{1}$ Das novellierte Bcrufsbildungsreformgesetz von 2005 stärkt insbesondere den Lernort Berufsschule. Notwendig wurde dies im Zuge der zunchmenden Lehrstellenkrise. Aufgrund des Mangels an betricblichen Ausbildungsplätzen wird das duale System seinem Anspruch nicht mehr gerecht, den Jugendlichen cine zeiteffiziente Integration bereits während der Ausbildung in den Arbeitsmarkt zu gewährleisten (Lorenz et al. 2005: 167).

${ }^{2}$ Dies bedeutet, dass bspw. Regelungen zur Struktur der Ausbildung (insbesondere $§ 5$ BerBiRefG), zur Eignung von Ausbildungsstätten und Regelungen zur Überwachung der Berufsausbildung durch die zuständigen Stellen und deren Berufsbildungsausschüsse keine Anwendung im Handwerksbereich finden. Folglich gilt für diese Fragen der zweite Teil der Handwerksordnung. Im Folgenden wird dic duale Berufsausbildung nur nach dem Berufsbildungsreformgesetz beschricben, da die Handwerksordnung im Großen und Ganzen dieselben Anforderungen an die Ausbildungsbetriebe stellt.
} 
grundsätze anzuwenden" ( $\$ 10$ BerBiRefG). Da eine Ausbildung erst mit einer vertraglichen Regelung auf privat-rechtlicher Basis zustande kommt, wählen Ausbildungsbetriebe die Schulabgänger aus, die den betrieblichen Anforderungen gerecht werden. So wird der Zugang zur dualen Berufsausbildung kontrolliert und durch den Markt geregelt (Hillmert 2007: 87; Konietzka 2007: 278). Nach Hillmert (2004) erfolgt die Besetzung der Ausbildungsplätze unter Konkurrenzbedingungen, da deren Anzahl begrenzt ist. Dies bringt zugleich eine Ungleichverteilung der Chancen zur Besetzung der Ausbildungsplätze mit sich.

Die Vermittlung von vergleichbaren betrieblichen Ausbildungsinhalten für Auszubildende derselben Berufe garantieren Ausbildungsordnungen (Neubäumer/Somaggio 2006: 692; Neubäumer 2002: 304), welche die Grundlage für eine betriebliche Ausbildung bilden. Dadurch erfolgt die Wissensvermittlung innerhalb des Betriebes nach gewissen Mindeststandards. Inhalt der Ausbildungsordnungen sind gemäß $\S 5$ Abs. 1 BerBiRefG die „Bezeichnung des Ausbildungsberufes“, „die Ausbildungsdauer“, ,die Fertigkeiten und Kenntnisse, die Gegenstand der Berufsausbildung sind“, „der Ausbildungsrahmenplan“ und ,die Prüfungsanforderungen". Die gezielte Vermittlung von berufsspezifischem Wissen wird zudem durch die Ausbildungsordnungen gefördert (Konietzka 2007: 278). Dadurch wird bundesweit eine einheitliche, staatlich anerkannte Berufsausbildung sichergestellt (Neubäumer 2002: 304). Dies erlaubt eine effiziente Allokation von ausgebildeten Arbeitskräften einerseits durch Betriebswechsel nach Abschluss der Berufsausbildung. Andererseits gewährleistet die Struktur der Gewerkschaften in Deutschland Flexibilität der ausgebildeten Jugendlichen innerhalb des Betriebes, aber auch zwischen den Betrieben. Grund hierfür ist die Abgrenzung der Arbeitsplätze nach Qualifikationsanforderungen. Dadurch können die Fachkräfte an mehreren sich unterscheidenden Stellen eingesetzt werden.

Da die Ausbildungsordnungen indirekt auch hohe Fachkenntnisse von Seiten der Ausbildungsbetriebe verlangen, ist eine Ausbildungsberechtigung gemäß $\S 28$ BerBiRefG zu erwerben. Danach kann nur einstellen, wer ,persönlich geeignet ist" und ausbilden, wer ,persönlich und fachlich geeignet ist" ( $\S 28 \mathrm{Abs}$. 3 und $29 \mathrm{BerBiRefG}$ ). Diese Überprüfung der Betriebe zur Eignung für eine Ausbildung ist notwendig, um die Vermittlung betrieblichen Wissens nach gewissen Mindeststandards sowie eine Vergleichbarkeit der Berufsabschlüsse zu gewährleisten. Aufgrund der Beteiligung unterschiedlichster Betriebe bzw. Unternehmen kann dennoch nicht verhindert werden, dass durch die Verberuflichung eine starke Differenzierung der Abschlüsse nach anerkannten Ausbildungsberufen besteht (Müller/Shavit 1998). Darüber hinaus geht ebenfalls eine Differenzierung innerhalb der Ausbildungsberufe wegen der betrieblichen Komponente der dualen Berufsausbildung einher, weil betriebsspezifische Faktoren das informelle Wissen auf unterschiedliche Weise fördern.

Schließlich werden, um die betriebliche Berufsausbildung zu garantieren, Institutionen eingesetzt, die die Durchführung regeln und kontrollieren. $\mathrm{Zu}$ diesen 
Institutionen gehören insbesondere die Industrie- und Handelskammern, die Handwerkskammern sowie die Ärzte- und die Rechtsanwaltskammer, die so genannten „zuständigen Stellen“ (Neubäumer/Somaggio 2006: 692). Ihre Zuständigkeitsbereiche sind im $\S 71$ BerBiRefG verankert. Neben der Kontrolle und Regelung überprüfen die Kammern, inwieweit die Ausbildungsstätten geeignet sind und den Anforderungen der $\S \S 22$ und 96 BerBiRefG gerecht werden. Schließlich nehmen sie Abschlussprüfungen ab und üben eine beratende Funktion gegenüber den Betrieben, aber auch gegenüber den Auszubildenden gemäß §76 BerBiRefG aus (Neubäumer 2002: 304). Insgesamt haben in Westdeutschland knapp $60 \%$ aller Betriebe eine Ausbildungsberechtigung, erfüllen demnach die gesetzlichen Anforderungen gemäß BerBiRefG, jedoch beteiligen sich nur $53 \%$ aller ausbildungsberechtigten Betriebe an der dualen Berufsausbildung (BMBF 2008: 304).

Die zweite Säule der dualen Berufsausbildung bildet der Besuch der Berufsschule. Dieser ist für die meisten der Schulabgänger noch obligatorisch, da in Deutschland eine Schulpflicht bis zum 18. Lebensjahr gilt. Die schulische bzw. allgemein bildende Komponente der dualen Berufsausbildung findet in den Schulgesetzen der Länder eine rechtliche Grundlage, wodurch die Zuständigkeit der einzelnen Bundesländer zum Ausdruck kommt. Die Verantwortlichkeiten der Berufsschulen sind demnach, wie die der allgemein bildenden Schulen, föderal organisiert. Die Details der Lehrpläne differieren daher von Bundesland zu Bundesland. Um jedoch die Standardisierung des Abschlusses der dualen Berufsausbildung zu garantieren, koordiniert der Bund die Aufgaben und die schulischen Lehrinhalte. So gibt der Bund einen Rahmenlehrplan vor, innerhalb dessen die einzelnen Länder das zu vermittelnde Wissen konkretisieren. Dabei werden die berufstheoretischen und berufspraktischen Inhalte festgelegt sowie das bei den Auszubildenden bereits vorhandene allgemein bildende Wissen erweitert (Neubäumer 2002: 305). Die Schulbehörde eines jeden Bundeslandes ist für die zu vermittelnden Kenntnisse und Fähigkeiten verantwortlich. Entsprechend dieser Aufteilung ist der schulische Teil der dualen Berufsausbildung den öffentlich-rechtlichen Institutionen zugeordnet. So übernehmen die Regierungspräsidenten, die Schulaufsicht oder die Schulämter die Beratung und die Überwachung der Berufsschulen. ${ }^{3}$

${ }^{3}$ Gemäß $\$ 77$ Abs. 1 BerBiRefG „errichtet dic zuständige Stclle einen Berufsbildungsausschuss“, welchem „sechs Bcauftragte der Arbcitgeber, sechs Beauftragte der Arbeitnehmer und sechs Lehrkräfte an berufsbildenden Schulen“ angehören. Der Berufsbildungsausschuss ist mit dem Beschluss der zu crlassenden Rechtsvorschriften betraut. Die Lehrkräfte haben allerdings nur cine beratende Funktion. Des Weiteren werden die Ausbildungsbetriebe und deren Ausbildungsstätten durch den Berufsbildungsausschuss auf ihre Eignung sowic dic Einhaltung der Ausbildungsbestimmungen hin geprüft. $\mathrm{Zu}$ den weiteren Aufgaben gehört auch die Förderung der Berufsausbildung. Dazu dienen Instrumente wie dic Beratung der Firmen und der Auszubildenden. Die Zuständigkeiten finden ihre rechtliche Grundlage im $\S 79$ BerBiRefG. Darüber hinaus führen die Berufsbildungsausschüsse dic Prüfungsordnungen durch. Aufgrund der Zusammensetzung dieses Ausschusses wird eine (Selbst-)Kontrolle der 
Aufgrund der Beteiligung von unterschiedlichen Akteuren an der dualen Berufsausbildung ist eine Standardisierung erforderlich, unterschiedliche Arten und Qualitäten von Humanakkumulation vergleichbar zu machen. Daher ist eine enge Zusammenarbeit zwischen Betrieb und Berufsschule notwendig (Niederalt 2004: 23; Neubäumer 2002: 303), damit deren Ziel, nach dem ,die Berufsausbildung die für die Ausübung einer qualifizierten beruflichen Tätigkeit in einer sich wandelnden Arbeitswelt notwendigen beruflichen Fertigkeiten, Kenntnisse und Fähigkeiten (berufliche Handlungsfähigkeit) in einem geordneten Ausbildungsgang zu vermitteln hat" ( $\$ 1$ BerBiRefG), gewährleistet wird. Sie hat ferner „den Erwerb der erforderlichen Berufserfahrungen zu ermöglichen“ ( $\$ 1$ Abs. 3 BerBiRefG). Das eigenverantwortliche Zusammenwirken der beiden Institutionen der dualen Berufsausbildung wird durch das Bundesinstitut für Berufsbildung (BiBB) unterstützend koordiniert. Gegründet wurde es mit dem Berufsbildungsgesetz im Jahr 1969. Das BiBB ist vor allem mit der Anpassung der Ausbildungsordnungen an sich wandelnde Anforderungen des Arbeitsleben beauftragt sowie der Entwicklung von Ausbildungsordnungen für neue Berufe (Neubäumer 2002: 304). Damit wird garantiert, dass die Lehrinhalte den sich durch den technischen Fortschritt und durch die Tertialisierung verändernden Gegebenheiten gerecht werden. Dies erfordert aber zugleich auch, dass die Ausbildungsordnungen der Ausbildungsberufe in immer kürzeren Abständen überarbeitet bzw. erneuert werden (müssen). So werden neue Ausbildungsberufe geschaffen und andere zusammengefasst, um einer umfassenderen Ausbildung Rechnung tragen zu können, welche durch einen sich in Richtung Tertialisierung wandelnden Arbeitsmarkt erforderlich wird (Grünewald/Moraal 2001: 12). Gewährleistet wird dies durch eine enge Zusammenarbeit des BiBB mit Vertretern der Arbeitgeber, der Arbeitnehmer sowie den Berufsschullehrern (Neubäumer 2002: 304). ${ }^{4}$ Trotz dieser regelmäßigen Überarbeitungen gilt das System der dualen Berufsausbildung bei Kritikern aufgrund des hohen Koordinationsaufwandes als inflexibel, weil sich die Anforderungen in der Arbeitswelt in immer kürzer werdenden Abständen ändern.

Entsprechend der Verantwortlichkeiten ist die Finanzierung der dualen Berufsausbildung auf die beteiligten Akteure aufgeteilt. Hierbei trägt der Ausbildungsbetrieb die Kosten des betrieblichen Teils. Darunter fallen unter anderem Personalkosten der haupt- aber auch nebenberuflichen Ausbilder sowie Kosten für Materialen. In den letzten Jahren nahm die Bedeutung der überbetrieblichen Lehrwerkstätten als weitere Lernorte zu. Diese sind dadurch gekennzeichnet, dass sie meist außerhalb des Ausbildungsbetriebes angesiedelt sind und für Auszubildende unterschiedlicher Unternehmen zur Verfügung stehen. Die Kosten

Betriebe untereinander gewährleistet sowie ein Wissenstransfer zwischen den Betrieben zugclassen (Neubäumer/Somaggio 2006: 692).

${ }^{4}$ Vertreter der Arbeitgeber können unter anderem Ausbildungsleiter bei großen Unternehmen und Mitarbeiter von Forschungsinstituten sein. Zu den Arbeitnehmervertretern sind Betriebräte, Mitarbeiter wissenschaftlicher Institute der Gewerkschaften zu zählen. 
dieser Lehrwerkstätten werden auf die beteiligten Unternehmen umgelegt. Darüber hinaus gibt es aber auch Labore und Räume als innerbetriebliche Lehrwerkstätten. Letztere sind vor allem in Großbetrieben vorzufinden, deren Arbeitsabläufe zu komplex sind und zudem an teuren Maschinen durchgeführt werden, sodass Auszubildende an kleineren ausrangierten Maschinen üben können (Neubäumer 1997: 11). Diese Art von Lernorten wird ebenfalls von den jeweiligen Ausbildungsbetrieben finanziert. Den Großteil der betrieblichen Ausbildungskosten machen aber die Ausbildungsvergütungen der Jugendlichen aus, die im Rahmen eines Flächentarifvertrags bzw. von tariflichen Vereinbarungen durch die Gewerkschaften ausgehandelt werden. Mit den Ausbildungsvergütungen erhalten die Auszubildenden aber eine Entlohnung, welche zum einen unterhalb ihrer Produktivität liegt. Zum anderen liegt sie auch unter dem Lohn, den sie bei einer ungelernten Tätigkeit bekommen würden (siehe auch Kap. 3.2.2.1). Dadurch beteiligen sich die Auszubildenden selbst ebenfalls an der Finanzierung der Ausbildung. Schließlich obliegt die Finanzierung der schulischen Berufsausbildung dem Bund und den Ländern. Das nächste Kapitel belegt die Beteiligung der dualen Berufsausbildung sowie den Übergang in Erwerbsleben mit deskriptiven Daten, um die Bedeutung dieses Teils des Bildungssystems hervorzuheben.

\subsection{Fakten des dualen Berufsbildungssystems}

Das duale Berufsausbildungssystem bietet für den größten Anteil eines Schulabsolventenjahrgangs die Möglichkeit, einen Beruf zu erlernen. Im Folgenden sollen die Bedeutung der dualen Berufsausbildung beschrieben und mögliche $\mathrm{Zu}-$ sammenhänge zwischen Ausbildungszeit und dem Arbeitsmarkteintritt aufgezeigt werden. Denn trotz des großen Beitrags der dualen Berufsausbildung für die Integration junger Erwachsener in das Erwerbsleben verläuft diese für einen beträchtlichen Anteil weniger erfolgreich. Hierbei wird als erfolgreich definiert, wenn einerseits die Ausbildungsabsolventen in ihrem Ausbildungsbetrieb verbleiben können bzw. sie andererseits den erlernten Beruf ausüben können. Hierbei findet die horizontale Abstimmungsebene Berücksichtigung, die in Abschnitt 2.3. beschrieben wird. Brüche, in Form von Arbeitslosigkeit, direkt am Übergang nach der dualen Berufsausbildung haben Auswirkungen auf das spätere Erwerbsleben (BMBF 2007; Mroz/Savage 2006; Giesecke/Groß 2003). Hinweise hierfür liefert bereits die Betrachtung der Ausbildungsphase. Während dieser Zeit ist aufgrund der unterschiedlichen Gründe für die Ausbildungsbereitschaft durch den Ausbildungsbetrieb eine Tendenz zu erkennen, ob ein Ausbildungsabsolvent ohne Hürden in das Arbeitsleben startet. Eine hohe Ausbildungsbereitschaft lässt einerseits auf einen hohen Bedarf an zukünftigen Fachkräften schließen. Andererseits ist sie im Allgemeinen Kennzeichen für die Steigerung des gesamtwirtschaftlichen Bildungsstandes. Als Indikator wird die 
Ausbildungsquote ${ }^{5}$ herangezogen, deren gesamtwirtschaftlicher Durchschnitt in Abbildung 1 dargestellt ist.

Abbildung 1: Ausbildungsquoten zwischen 1999 und 2006 (in \%)

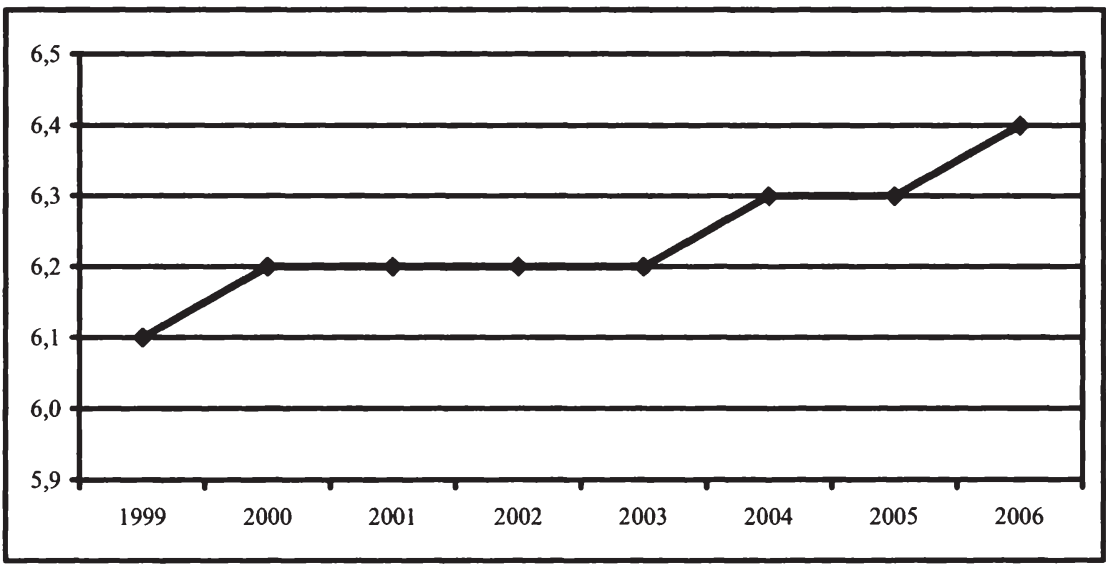

Quelle: BMBF (2003-2008)

Der Anteil der Auszubildenden an allen Beschäftigten betrug zwischen 2003 und 2006 6\% und blieb nahezu konstant. Ein Vergleich der einzelnen Ausbildungsbereiche mit der gesamtwirtschaftlichen Ausbildungsquote deutet auf die unterschiedliche Ausbildungsbereitschaft hin: Überdurchschnittliche Ausbildungsquoten weisen insbesondere die Wirtschaftsbereiche Land und Forstwirtschaft, Bauhaupt- bzw. Ausbaugewerbe, Kfz-, Groß- und Einzelhandel, insbesondere Reparatur, das Gaststättengewerbe und haushaltsnahe bzw. personenbezogene Dienstleistungen auf. So übersteigen die Quoten den Durchschnitt um bis zu sechs Prozentpunkte, im Groß- und Einzelhandel bzw. in den haushaltsnahen Dienstleistungen sogar um bis zu 10 Prozentpunkte. Dieser hohe Anteil an Auszubildenden kann zum einen auf die Tradition der dualen Berufsausbildung in einem Teil der Bereiche (vor allem in den handwerklichen Bereichen) zurückgeführt werden (Greinert 1998) und zum anderen ökonomische Gründe haben, welche in Kapitel 3.1.1 ausführlich beschrieben werden. Andere Bereiche, die innerhalb des dualen Berufsbildungssystems anerkannte Ausbildungsberufe beinhalten, bilden unterdurchschnittlich aus. Hierbei sind vor allem unternehmensnahe Dienstleistungen, Softwareentwicklung, -beratung, Hardwareberatung zu nennen, deren Quote zwei Prozentpunkte unter der Gesamtausbildungsquote liegt. In diesen Bereichen sind häufig junge Unternehmen vertreten, die sich erst im Markt etablieren müssen. Zudem hat die duale Berufsausbildung in

\footnotetext{
${ }^{5}$ Die Ausbildungsquote berechnet sich aus dem Verhältnis der Auszubildenden zu allen Beschäftigten in einem Betrieb.
} 
dieser Sparte wenig Tradition. Schließlich erfordern die Tätigkeitsbereiche in diesen Wirtschaftsbereichen hoch qualifizierte Arbeitskräfte, welche sich durch einen Hochschulabschluss auszeichnen.

Studien (u. a. Konietzka 2007; Büchel/Neubäumer 2001; Neubäumer 1999) untersuchten die individuellen Arbeitsmarktchancen Jugendlicher und stellten fest, dass der Einstieg ins Erwerbsleben von der vorangegangenen Ausbildung abhängt. Aufgrund der betrieblichen Beteiligung der Unternehmen und letztlich ihrer Auswahl der Absolventen werden die Schulabsolventen bereits vor der Ausbildung gefiltert (Konietzka 2007: 284). Die Erlernung eines Ausbildungsberufes hängt damit vom formalen Schulabschluss ab. Bei der Betrachtung der am meisten belegten Ausbildungsberufe, differenziert nach Schulabschluss, ist bereits eine Selektion festzustellen.

Insgesamt stellen Personen mit Realschulabschluss die größte Gruppe der Auszubildenden innerhalb des dualen Berufsausbildungssystems mit $40 \%$ aller, die 2005 eine Ausbildung begonnen haben, dar, gefolgt von Personen mit Hauptschulabschluss (knapp 31\%). Die Beteiligung von Abiturienten lag bei einem Anteil von ungefähr 18\% aller Auszubildenden im Jahr 2005. Die Besetzung der Ausbildungsberufe variiert zwischen den Auszubildenden mit unterschiedlichen Schulabschlüssen. Jugendliche ohne Schulabschluss bilden die Minderheit der Auszubildenden im dualen Berufsbildungssystem. Sie erlernen meist einen handwerklichen Ausbildungsberuf. Ungefähr 8\% der Auszubildenden, die den Beruf des Malers erlernen, sind Auszubildende mit Hauptschulabschluss; von allen Auszubildenden im Malerberuf belief sich der Anteil auf knapp 63\%. Aber auch in anderen Berufen, wie Metallbauer, Anlagenmechaniker, sowie in Dienstleistungsberufen mit einfachen Tätigkeiten, wie Fachverkäufer/in im Lebensmittelhandwerk, Verkäufer/in und Friseure bilden Auszubildende mit Hauptschulabschluss die Mehrheit aller Auszubildenden.

Auszubildende mit Realschulabschluss erlernen vor allem den Beruf des/der Kaufmanns/-frau im Einzelhandel (6\%), verglichen mit Abgängern anderer Schularten machen sie aber nur ungefähr 38\% aller Auszubildenden des Berufes aus. Auszubildende mit Realschulabschluss sind vor allem in den technischmedizinischen Ausbildungsberufen vertreten. Bspw. besitzen $64 \%$ der Jugendlichen, die den Beruf des/der medizinischen Fachangestellten und $62 \%$ der Jugendlichen in der Ausbildung zum/zur zahnmedizinischen Fachangestellten einen Realschulabschluss. 54\% der Auszubildenden zum Industriemechaniker verfügen über eine mittlere Reife. Auszubildende mit Realschulabschluss besetzen auch zunehmend Ausbildungsstellen in kaufmännischen Tätigkeiten, innerhalb derer sie in Konkurrenz mit den Abiturienten stehen. 61\% der Auszubildenden, die den Ausbildungsberuf zum/zur Bankangestellten erlernen, sind Abiturienten. Über die Hälfte der Auszubildenden im Ausbildungsberuf zum/zur Versicherungskaufmann/-frau (56\%) sowie zum/zur Steuerfachangestellten (57\%) repräsentieren Abiturienten. Ebenso verfügt ein Großteil der Auszubildenden zum/ 
zur Fachinformatiker/-in (47\%) und zum/zur Industriekaufmann/-frau (46\%) über Abitur (BMBF 2008).

Der Verbleib der Ausbildungsabsolventen in den einzelnen Betrieben wird anhand der Übernahmequote der Wirtschaftszweige genauer analysiert. Das Bundesinstitut für berufliche Bildung (BiBB) und das Bundesministerium für Bildung und Forschung (BMBF) veröffentlichen regelmäßig Daten von Übernahmequoten, die nach Betriebsgröße und Branchen aufgegliedert sind (Granato/Dorau 2004; BMBF 2007). Dabei ist die Übernahme auf Betriebsebene definiert. Die Berechnung dieses Indikators wird auf der Grundlage des Betriebspanels durchgeführt. Hier werden die Betriebe befragt, welchen Anteil ihrer Ausbildungsabsolventen sie übernehmen. Dieser Anteil setzt sich aus dem Verhältnis der im Ausbildungsbetrieb verbliebenen Absolventen an allen Ausbildungsabsolventen zusammen. Jedoch kann anhand der gestellten Frage nicht zwischen befristeten und unbefristeten Übernahmen unterschieden werden. Demnach sind in der Übernahmequote auch Ausbildungsabsolventen enthalten, die auf Grundlage tariflicher Regelungen für einen bestimmten Zeitraum übernommen wurden (BMBF 2007: 206). Die Übernahmequoten differieren zwischen den Ausbildungsbranchen zum Teil erheblich, wie folgende Übersicht zeigt:

Tabelle 1: Übernahmequoten nach Wirtschaftsbereichen zwischen 2002 und 2006 (in \%)

\begin{tabular}{|l|l|l|l|l|l|}
\hline & $\mathbf{2 0 0 2}$ & $\mathbf{2 0 0 3}$ & $\mathbf{2 0 0 4}$ & $\mathbf{2 0 0 5}$ & $\mathbf{2 0 0 6}$ \\
\hline Land-Forstwirtschaft, Fischerei & 14,8 & 45,9 & 36,2 & 36,0 & 30,5 \\
\hline Bergbau, Energie Wasserversorgung & 72,0 & 60,4 & 75,7 & 76,3 & 74,4 \\
\hline Nahrungs- und Genussmittel & 58,3 & 59,7 & 66,5 & 46,8 & 50,3 \\
\hline Verbrauchsgüter & 60,9 & 55,0 & 65,5 & 59,7 & 67,2 \\
\hline Produktionsgüter & 72,5 & 68,0 & 70,0 & 67,9 & 74,3 \\
\hline Investitions- und Gebrauchsgüter & 80,0 & 79,0 & 76,1 & 72,9 & 76,4 \\
\hline Baugewerbe & 56,3 & 54,2 & 50,1 & 51,7 & 56,6 \\
\hline Handel, Instandhaltung und Reparatur & 56,4 & 52,0 & 53,6 & 56,1 & 59,1 \\
\hline Verkehr und Nachrichtenübermittlung & 63,9 & 69,4 & 49,7 & 60,1 & 61,7 \\
\hline Kredit- und Versicherungsgewerbe & 81,4 & 74,5 & 73,7 & 77,4 & 80,7 \\
\hline Gastgewerbe & 28,3 & 32,6 & 31,1 & 36,6 & 38,1 \\
\hline Gesundheits-, Veterinär- und Sozialwesen & 44,3 & 48,5 & 34,0 & 36,6 & 37,8 \\
\hline Dienstleistungen überwiegend für Unternehmen & 46,6 & 62,9 & 57,4 & 63,5 & 57,7 \\
\hline sonst. Dienstleistungen insb. für Unternehmen & 58,7 & 42,8 & 60,2 & 58,8 & 63,3 \\
\hline andere Dienstleistungen & 52,9 & 56,3 & 53,5 & 49,4 & 59,0 \\
\hline Organisationen ohne Erwerbscharakter/Staat & 65,6 & 65,2 & 54,2 & 56,3 & 58,6 \\
\hline Gesamt & 57,0 & 56,7 & 53,8 & 55,0 & 57,0 \\
\hline
\end{tabular}

Quelle: BMBF (2003-2008)

Während der betrachteten Jahre ist festzustellen, dass die durchschnittliche Übernahmequote in Westdeutschland ungefähr 57\% beträgt. Allerdings ist 2004 die Quote dramatisch gesunken als Folge der Rezession von 2003. Nach Bellmann et al. (2006) hängen Übernahmequoten stark von der aktuellen wirtschaft- 
lichen Situation und der betrieblichen Auftragslage ab. Die Unternehmen reagierten wohl auf die schlechte Wirtschaftslage, die 2003 und 2004 einsetzte. Der Anteil der übernommenen Ausbildungsabsolventen stieg 2005 wieder an. Hier ist eine parallele Entwicklung der konjunkturellen Lage und der Übernahmequote zu erkennen, sodass die gesamtwirtschaftliche Lage einen Einfluss auf die Übernahmeentscheidung der Ausbildungsbetriebe vermuten lässt. Wird nun die Verteilung über die Besetzung der Ausbildungsberufe mit der Übernahmequote betrachtet, ist zu erkennen, dass in den Bereichen Land- und Forstwirtschaft, Gastgewerbe sowie Gesundheits-, Veterinär- und Sozialwesen die Übernahmequoten sehr gering sind. Darüber hinaus weist das „Bauhauptgewerbe“ unterdurchschnittliche Übernahmequoten auf. In diesen Bereich sind insbesondere handwerkliche Berufe, wie Maler und Lackierer oder Metallbauer, zu subsumieren. Allerdings liegt die Übernahmequote nicht allzu weit von der gesamtwirtschaftlichen Übernahmerate entfernt, was auf saisonale Bedingungen zurückzuführen ist. Denn das Ausbildungsjahr endet in der Regel im (Früh-)Sommer, wenn die handwerklichen Berufe ausreichende Aufträge haben und eine Weiterbeschäftigung über diese Jahreszeit erforderlich ist. In diesen beschriebenen Bereichen erlernen meist Auszubildende mit geringerer Schulbildung einen Beruf. Zudem sind sie stark konjunkturabhängig. Den hohen Übernahmequoten in den Sektoren Energiewirtschaft, Kredit- und Versicherungsgewerbe, Verkehr und Nachrichtenübermittlung stehen jedoch im Gegensatz zu der Branche der sonstigen Dienstleistungen (insbesondere für Unternehmen) geringe Ausbildungsquoten gegenüber, sodass von diesen hohen Übernahmequoten relativ wenige Ausbildungsabsolventen profitieren. In den anderen Dienstleistungen dagegen werden viele junge Erwachsene ausgebildet, wodurch die geringe Übernahmequote eine hohe Betroffenheit auslöst (Fischer et al. 2008: 68).

Eine entscheidende Rolle bei der Übernahmeentscheidung spielen zudem tarifliche Regelungen. Zur Förderung der Ausbildung wurden vor allem ab Mitte der 1990er Jahre als Reaktion auf die sich verschlechternde Situation auf dem Ausbildungsstellenmarkt sowie auf dem Arbeitsmarkt für junge Arbeitnehmer Übernahmeregelungen vereinbart (Bispinck et al. 2002: 213). Diese stellen aber häufig Selbstverpflichtungen der Unternehmen dar, auf die sich die Ausbildungsabsolventen nicht berufen können, weil sie unverbindlich sind. Dennoch ist ein Zusammenhang zwischen den gewerkschaftlich stark organisierten Wirtschaftsbereichen und der Übernahmequote zu erkennen. In stark organisierten Bereichen, wie Bergbau, Energie, Wasserversorgung, Produktionsgüter, Investitions- und Gebrauchsgüter sowie Kredit- und Versicherungsgewerbe liegen die Übernahmequoten mit über 70\% über dem Durchschnitt. In diesen Wirtschaftsbereichen sind aber auch Ausbildungsberufe enthalten, die meist von Jugendlichen mit höherem Schulabschluss ausgeübt werden, sodass die höheren Übernahmequoten ebenfalls durch ein höheres Ausbildungsniveau begründet werden können (Acemoglu/Pischke 1998). 
Tabelle 2: Arbeitslosenquote ${ }^{6}$ der 15- bis 29 -jährigen nach Wirtschaftsbereichen 2006 (in \%)

\begin{tabular}{|l|c|}
\hline & $\mathbf{2 0 0 6}$ \\
\hline Land- und Forstwirtschaft, Fischerei & 16,5 \\
\hline Bergbau & 12,6 \\
\hline verarbeitendes Gewerbe & 20,0 \\
\hline Energie, Gas und Wasserversorgung & 16,6 \\
\hline Baugewerbe & 21,7 \\
\hline Handel & 23,6 \\
\hline Gastgewerbe & 34,5 \\
\hline Verkehr und Nachrichtenubermittlung & 16,4 \\
\hline Kredit- und Versicherungsgewerbe & 18,8 \\
\hline Immobilien und produktionsnahe Dienstleistungen & 21,1 \\
\hline offentliche Dienstleistung, Verwaltung & 18,8 \\
\hline Gesundheits-, Veterinär- und Sozialwesen & 22,3 \\
\hline andere personenbezogene Dienstleistungen & 20,0 \\
\hline Gesamt & 20,9 \\
\hline
\end{tabular}

Quelle: EC (2007)

Tabelle 2 zeigt den Anteil der erwerbstätigen Jugendlichen zwischen 15 und 29 Jahren an allen Beschäftigten zwischen 15 und 64 Jahren in Deutschland nach Wirtschaftsbereichen. Der europäische Durchschnitt dieses Indikators liegt bei 22,2\%. Daher ist diese Altersgruppe in Deutschland unterdurchschnittlich beschäftigt, was teilweise auf die institutionellen Rahmenbedingungen innerhalb des gesamten Berufsbildungssystems (Übergang in und Austritt aus dem Berufsbildungssystem) zurückgeführt werden kann. Vor allem Jugendliche zwischen 15 und 19 Jahren befinden sich noch im Schulsystem, sind damit keinem Wirtschaftsbereich zuzuordnen und daher nicht erfasst. Die Beschäftigtenquote differiert zwischen den einzelnen Wirtschaftsbereichen zum Teil erheblich: Im europäischen Vergleich überdurchschnittlich vertreten ist die Altersgruppe im Bau-, Gastgewerbe oder Handel tätig. Dabei handelt es sich vor allem um Wirtschaftsbereiche, die stark konjunkturell beeinflusst werden. Ebenso finden sich in diesen Bereichen Ferien- bzw. Nebenjobs, welche insbesondere von Jugendlichen oder jungen Erwachsenen während der Ferien oder neben dem Studium ausgeübt werden. Zudem schwankt die Arbeitsnachfrage saisonal. Von Konstanz geprägte Wirtschaftsbereiche weisen dagegen unterdurchschnittliche $\mathrm{Be}$ schäftigtenquoten auf. Es sind vor allem Verkehr und Nachrichtenübermittlung sowie Energie und Wasserversorgung zu nennen. Sie benötigen gut ausgebildete Fachkräfte, deren Ausbildungsdauer sich entsprechend verlängert. Entsprechend ist der Anteil der jungen Erwachsenen gering. Darüber hinaus machen letztere

\footnotetext{
${ }^{6}$ Die Arbeitslosigkeitsquoten von EUROSTAT werden nach der ILO-Definition berechnet, die sich von der Ermittlung der Arbeitslosen durch die Bundesagentur für Arbeit unterscheidet.
} 
gegenüber den ersteren Wirtschaftsbereichen einen geringeren Anteil an der gesamten Beschäftigung aus.

Aus diesen Deskriptionen ist zu erkennen, dass zu Beginn der dualen Berufsausbildung bereits ein Filterungs- und Selektionsprozess durch die Betriebe stattfindet, der sich an der zweiten Schwelle weiter auswirkt. So werden Schulabgänger aufgrund ihrer potenziellen Eignung zum Erlernen des Ausbildungsberufes ausgewählt und eingestellt (Konietzka 2007: 281$)^{7}$, weil die duale Berufsausbildung einen Eintritt in den Arbeitsmarkt bereits vor Abschluss einer Berufsausbildung erfordert. Die Informationsgewinnung über die Fähigkeit eines Auszubildenden (Screening) erfolgt daher, anders als bei der schulischen Berufsausbildung, während der Ausbildungsphase (Konietzka 2007: 281). Inwieweit die Jugendlichen nach dem Abschluss ihrer dualen Berufsausbildung in anderen Berufen unterkommen oder arbeitslos werden, wird durch die Rahmenbedingungen an dem Übergang vom Berufsbildungssystem ins Erwerbsleben strukturiert.

\section{3 Übergang von der dualen Berufsausbildung in das Erwerbsleben}

Die Art des Eintrittes in den Arbeitsmarkt spielt eine bedeutende Rolle für die spätere Erwerbskarriere, das heißt, die Art der ersten Beschäftigung entscheidet über den zukünftigen Berufsverlauf (Bender/Dietrich 2001). ${ }^{8}$ Die Interaktion des betrieblichen und des schulischen Ausbildungsteils mit den institutionellen Rahmenbedingungen bildet eine wichtige Voraussetzung des dualen Berufsausbildungssystems für den erfolgreichen Übergang aus dem Bildungssystem in das Erwerbsleben (siehe auch Kap. 2.1). Weil es formal keine Zulassungsvoraussetzungen für das Absolvieren einer dualen Berufsausbildung gibt, steht auch schwächeren Schülern der Weg für den Erwerb einer Berufsausbildung offen. ${ }^{9}$ Die Ausbildungsbeteiligung der Wirtschaft gewährleistet jedem Jugendlichen eine frühzeitige Integration in den Arbeitsmarkt. Durch den betrieblichen Teil

\footnotetext{
${ }^{7}$ Es ist aber auch zu erkennen, dass sich die Berufswünsche der Schulabgänger an ihren Chancen, die durch den Arbeitsmarkt vorgegeben werden, sowie an den eigenen Fähigkeiten und an den regionalen Arbeitsmarktgegebenheiten orientieren und sich entsprechend anpassen sowie die Selektion in Ausbildungsberufe verstärken (Evans/Heinz 1994; Heinz et al. 1987; Beck et al. 1979). Dies wirkt sich auf den Verbleib nach der dualen Berufsausbildung aus.

${ }^{8}$ Bender und Dietrich (2001) untersuchten dic Einmündungsprozesse der Geburtskohorten von 1964 und 1971. Der Einsticg in das Erwerbsleben war für einen größeren Anteil des älteren Jahrgangs weniger erfolgreich als für den jüngeren Jahrgang, was sich auf das spätere Erwerbsleben auswirkte. Zurückzuführen war dies insbesondere auf das niedrigere Bildungsniveau, auf die schlechten demografischen und konjunkturellen Rahmenbedingungen der ältcren Kohorte.

9 Jedoch waren in den letzten Jahren gerade Jugendliche ohne Schulabschluss von dem Lehrstellenmangel betroffen. Um Ausbildungsreife zu erlangen und somit dic Chancen auf einen Ausbildungsplatz zu erhöhen, gibt es zahlreiche berufsvorbereitende Maßnahmen, deren Ziele es sind, Einblicke in verschiedene Berufe zu geben, schulische Lücken zu schließen und somit den beruflichen Einstieg zu erleichtern.
} 
der Ausbildung haben Unternehmen die Möglichkeit, einerseits den Auszubildenden über drei Jahre kennen zu lernen. Andererseits können sie das Wissen vermitteln, das im Betrieb bzw. zur Ausübung der nachgefragten Tätigkeitsbereiche notwendig ist. Insgesamt kann durch die Mitwirkung der Betriebe an der dualen Berufsausbildung der Übergang nach der dualen Berufsausbildung in das Erwerbsleben theoretisch erleichtert und somit als Brücke verstanden werden. Durch die strukturierende Wirkung der Institutionen gelingt den betrieblichen Ausbildungsabsolventen die erste Platzierung im Arbeitsmarkt besser als anderen, die bspw. eine schulische Ausbildung oder ein Hochschulstudium absolvieren (Dietrich/Abraham 2005: 74). Allmendinger spricht hierbei von einer Standardisierung des Übergangs, welche die Vereinheitlichung und die Anerkennung der Lehrinhalte innerhalb einer Volkswirtschaft beschreibt (Allmendinger 1989: 46) und damit auch bei Verlassen des Ausbildungsbetriebes den Einsatz in anderen Unternehmen ermöglicht. ${ }^{10}$ Allerdings verläuft der Eintritt in den Arbeitsmarkt im Sinne von Erwerbstätigkeit bzw. ausbildungsadäquater Erwerbstätigkeit innerhalb der Gruppe der Ausbildungsabsolventen nicht immer erfolgreich.

Bei dem Übergang von der Schule in das Erwerbsleben spielen das Bildungssystem und der Arbeitsmarkt eine entscheidende Rolle (Müller 2005; Kerckhoff 2000). Von Mertens und Parmentier (1982) geben eine Grundlage zur Erklärung der Übergänge und deren Problematik. In ihrem Konzept unterstellen sie zwei Schwellen innerhalb des Arbeitslebens, die zwischen dem Übergang vom allgemein bildenden Schulsystem ins Berufsbildungssystem (erste Schwelle) und von dem Berufsbildungssystem in den Arbeitsmarkt (zweite Schwelle) unterscheiden. Somit rückt der Verlauf der Übergänge unter Berücksichtigung des Bildungssystems in den Mittelpunkt der Betrachtung. Diese Festlegung entspricht nach Mertens und Parmentier (1982) der Standardbiografie der Akteure. ${ }^{11}$ Andere Wechsel, wie bspw. die Aufnahme eines Studiums nach Abschluss einer dualen Berufsausbildung oder ein Arbeitsplatzwechsel, spielen sich innerhalb der genannten Systeme ab und werden daher nicht in diese Betrachtung der Übergänge aufgenommen. Die Übergänge an der ersten bzw. der zweiten Schwelle sind in unterschiedliche Abstimmungsebenen zu unterteilen, in denen sich die Systeme des Bildungs- bzw. des Berufsanfängermarktes einander angleichen müssen.

${ }^{10}$ Ein weiteres Merkmal, welches bei der Verbindung des Bildungssystems mit dem Arbeitsmarkt berücksichtigt werden muss, ist dic Stratifizierung. Stratifizierung bedeutet dic Durchlässigkeit zwischen den einzelnen Bildungssystemen. Innerhalb des dualen Berufsbildungssystem, in dem ein Ausbildungsverhältnis zwischen Betrieb und Auszubildendem zustande kommt, ist es notwendig, dass Betrieben relevante Informationen über Ausbildungsstellenbewerber zur Verfügung gestellt werden, damit die Durchlässigkeit von allgemein bildendem System und Berufsbildungssystem gewährleistet wird (Blossfeld et al. 2005: 10).

11 Andere, wie bspw. Mayer (2001) unterscheiden über bis zu sieben verschiedene Arten von Übergängen, welche die zwei Hauptübergänge noch einmal untergliedern. Letztlich können aber die entscheidenden Übergänge im Erwerbsleben auf die beschriebenen Schwellen reduziert werden, da die weiteren Übergänge als Ergänzungen zu interpretieren sind. 
Von besonderer Relevanz für diese Arbeit ist jedoch die Betrachtung des Übergangs an der zweiten Schwelle. Daher wird im Folgenden nur die Problematik des Übergangs von den Berufsbildungssystemen in die Erwerbstätigkeit betrachtet. Das Zwei-Schwellen-Konzept ordnet den Übergängen jeweils vier Abstimmungsebenen zu. Es ist für die erste und zweite Schwelle gleichermaßen anzuwenden. Hier sollen sie hauptsächlich die Problematik des Übergangs von der dualen Berufsausbildung auf den ersten Arbeitsplatz nach Abschluss konkretisieren:

\section{Abbildung 2: Abstimmungsebenen im Zwei-Schwellen-Konzept}

\begin{tabular}{|l|l|}
\hline Abstimmungsebene & Bedeutung \\
\hline global & Übereinstimmung zwischen Angebot und Nachfrage \\
\hline vertikal & Übereinstimmung zwischen Niveau der Abgänge und Niveau der Zugänge \\
\hline horizontal & Übereinstimmung der Fachrichtungsstrukturen mit den Berufswünschen \\
\hline qualitativ & $\begin{array}{l}\text { Übereinstimmung zwischen Inhalt im Bildungswesen und Anforderungen } \\
\text { Berufsleben }\end{array}$ \\
\hline
\end{tabular}

Quelle: Mertens/Parmentier (1982); eigene Darstellung

Die erste Ebene betrifft die globale Abstimmung. Sie gibt lediglich an, ob die Zahl der Absolventen mit der Zahl der angebotenen Stellen übereinstimmt (Mertens/Parmentier 1982: 360f). Auf dieser Stufe der Übergangsbetrachtung wird nur auf quantitative Indikatoren eingegangen. Eine Unterscheidung zwischen der Art der dualen Berufsausbildung bzw. der Art der Berufsausbildung im Allgemeinen und der Art der angebotenen Stellen wird vorerst nicht gemacht. Bei einem Angebotsüberschuss ergibt sich aus dieser Ebene die Arbeitslosenquote der Absolventenjahrgänge. Wenn die Zahl der Absolventen eines Abschlussjahrgangs der Anzahl der Stellen, die für Berufsanfänger direkt nach der dualen Berufsausbildung zur Verfügung gehalten werden, entspricht, kann die zweite Abstimmungsebene betrachtet werden. Sie behandelt ebenfalls die Problematik aus quantitativer Sicht, differenziert aber nun nach dem Niveau der Berufsausbildung und dem Qualifikationsniveau der angebotenen Arbeitsplätze. Mertens und Parmentier (1982) kennzeichnen die vertikale Abstimmungsebene durch die quantitative Übereinstimmung zwischen Absolventen und vorhandenen bzw. neu geschaffenen Arbeitsplätzen, welche jeweils die Anforderungen dieses Abschlusses weder über- noch unterschreiten. Bspw. kann in einer Abschlusskohorte der Anteil der nachgefragten Hochschulabsolventen nicht mit dem Angebot der tatsächlich von der Hochschule abgegangenen Absolventen deckungsgleich sein. Liegt ein Überschussangebot an Absolventen der dualen Berufsausbildung bei gleichzeitiger Überschussnachfrage nach Hochschulabsolventen vor, können die offenen Stellen für Hochschulabsolventen nicht durch Ausbildungsabsolventen besetzt werden. Hier liegt somit ein vertikales Abstimmungsproblem vor. Diese Abstimmungsproblematik lässt sich aber auch innerhalb der Gruppe der betrieblichen Ausbildungsabsolventen übertragen, weil Jugendliche nach Abschluss einer dualen Berufsausbildung in bestimmten Sektoren oder Be- 
rufen als Hilfsarbeiter, und somit unterhalb ihrer Qualifikation, tätig sein können. ${ }^{12}$ Demnach erfolgt in diesen Fällen keine vertikale Abstimmung, da für diese Anstellung prinzipiell keine Berufsausbildung erforderlich ist. Daher liegen die Anforderungen des Erwerbsberufes unter den Fähigkeiten, die die Jugendlichen während der Ausbildung erworben haben. Dies kann aber bei der Betrachtung nur eines formalen Bildungsabschlusses nicht von der horizontalen $\mathrm{Ab}$ stimmungsebene losgelöst werden. Diese berücksichtigt die Fachrichtungsstrukturen. Hierbei kommt die qualitative Komponente „Berufswünsche“ zum Ausdruck. Die Anzahl von Zugängen aus dem Berufsbildungssystem kann mit dem Angebot von Arbeitsplätzen auf den vorangegangenen Ebenen übereinstimmen. Dennoch ist es möglich, dass die Berufswünsche der Ausbildungsabsolventen nicht mit den angebotenen Arbeitsplätzen übereinstimmen. Unstimmigkeiten auf der horizontalen Abstimmungsebene treten in den letzten Jahren häufig auf. Dies wird bei der Betrachtung der Veränderungsraten der Zahl der Auszubildenden und der Zahl der in diesem Wirtschaftsbereich Beschäftigten deutlich. Entsprechend wird als möglicher Indikator für die Nachfrage die Veränderung der Beschäftigten eines Wirtschaftsbereiches mit der Veränderung der Anzahl der Auszubildenden drei Jahre zuvor verglichen. Denn der Verbleib nach der dualen Berufsausbildung hängt von der Nachfrage nach Fachkräften zu dem entsprechenden Zeitpunkt ab. Die folgende Abbildung gibt einen Überblick über ausgewählte Wirtschaftsbereiche, in denen sich die Veränderungen innerhalb von drei Jahren gegenläufig entwickelt haben und somit mögliche Anhaltspunkte für die Abstimmungsproblematik auf der horizontalen Ebene liefern.

Es ist zu erkennen, dass die Bereiche Bergbau, Gewinnung von Erdöl, -gas, Steinen, Erden, Nahrungsmittelgewerbe, Fahrzeugbau und Einzelhandel ein Wachstum der Anzahl an Auszubildenden im Jahr 2004 verzeichnen konnten, die Zahl der Beschäftigten zum Zeitpunkt des Abschlusses des betrachteten Jahrgangs aber gegenüber dem Vorjahr zurückging. Aus diesen Zahlen kann man zwar nicht schließen, dass die betrachteten Auszubildenden nach der dualen Berufsausbildung nicht übernommen werden, ebenso wenig, dass sie arbeitslos werden. Jedoch kann davon ausgegangen werden, dass bei gleich bleibender Entwicklung dieser Größen in dem jeweiligen Wirtschaftsbereich ausgebildete Fachkräfte eine geringere Wahrscheinlichkeit haben, in ihrem erlernten Beruf eine Anstellung zu finden. In den anderen dargestellten Bereichen war die gegenläufige Entwicklung in diesem Zeitraum umgekehrt. Das heißt, die Verände-

\footnotetext{
12 Nach Sengenberger (1987) sind die Ausbildungen auf verschiedene Segmente aufgeteilt, die verschiedene Mobilitätsprozesse und -möglichkeiten implizieren. Das externe Segment eröffnet den ausgebildeten Fachkräften die meisten Alternativen der beruflichen Mobilität, wobei dies in manchen Fällen in dic Ausübung einer angelernten Tätigkeit mündet, dic in der Regel nicht ausbildungsadäquat ist. Dagegen eröffnet das interne Segment dem Ausbildungsabsolventen Mobilitätsmöglichkeiten innerhalb eines Unternehmens, was eine hohe Beschäftigungssicherheit zur Folge hat. Schließlich ist das berufspezifische Segment von einer hohen Flexibilität innerhalb der jeweiligen Berufsgruppe gekennzeichnet. Charakteristisch ist dies für die Handwerksberufe.
} 

denzahl in ausgewählten Ausbildungsbereichen 2003 (in \%)

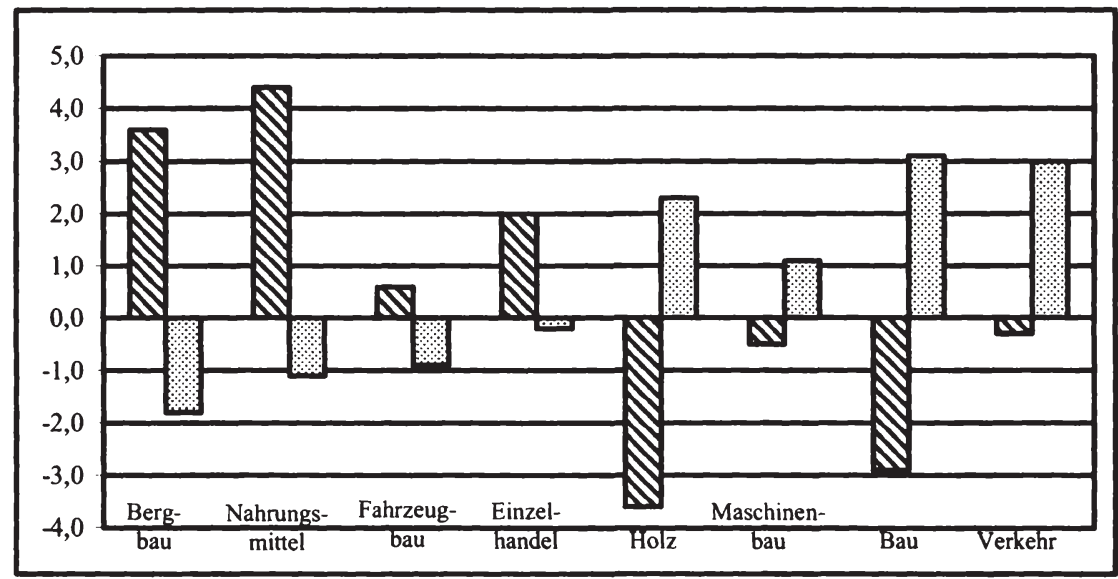

Quelle: BMBF (2003-2007)

DIIIUW = Veränderung der Auszubildenden 2003

₹ = Veränderung der Beschäftigten 2006

rung der Anzahl der Auszubildenden ist negativ, während die Veränderungen in der Beschäftigtenzahl positiv verliefen. Dies bedeutet, dass die Bereiche die Fachkräfte extern beschaffen. Bspw. werden zunehmend Fachkräfte aus dem Ausland beschäftigt, die unter Umständen günstiger sind als die ausgebildeten Fachkräfte. Dies ist für das Bauhauptgewerbe zu vermuten, wo ausländische Unternehmen bzw. Fachkräfte zunehmend die Arbeitsaufträge übernehmen. Allerdings können die Anforderungen der Tätigkeiten in den entsprechenden Wirtschaftsbereichen gestiegen sein, sodass die Unternehmen verstärkt Hochschulabsolventen nachfragen. Vorstellbar ist dies im Fahrzeugbau.

Schließlich hebt sich die qualitative Abstimmungsebene von den anderen Ebenen ab, weil sie mehr die Kongruenzschwierigkeiten zwischen dem Berufsbildungssystem und den Anforderungen des Arbeitslebens anspricht. ${ }^{13}$ Nach einer Studie von Szydlik (1996) gibt es wenig Übereinstimmung zwischen den während der Ausbildung erworbenen Qualifikationen und am Arbeitsplatz erforderlichen Fähigkeiten. Die inadäquate Stellenbesetzung, sofern sie eine Überqualifikation bedeutet, hat aber (langfristig) keine negativen Auswirkungen auf die Volkswirtschaft, weil dies zugleich auch ein hohes Ausbildungsniveau impli-

${ }^{13}$ Die Problematik an der crsten Schwelle ist durch die mangelnde Ausbildungsreife gegeben (Anger et al. 2006; Eberhard 2006). Grundlegende inhaltliche Anforderungen des Berufslebens sind nicht über grundlegende Kenntnisse, wic Sprachverständnis, mathematische Grundrechenarten, was die Unternehmen hindert, Lehrstellen zu besetzen. 
ziert. Zudem nehmen die Diskrepanzen zwischen Ausbildung und Arbeitsmarktanforderung im Erwerbsverlauf mit zunehmender Berufserfahrung ab. Kritik wird aber an den Ausbildungsordnungen geübt, die sich nicht in gleichem Tempo wie der technische Fortschritt verändern, sodass es deswegen zu einer Fehlinvestition in berufliches Wissen kommt (Bellmann/Janik 2007; Niederalt 2004).

Trotz der institutionellen Rahmenbedingungen, die innerhalb der dualen Berufsausbildung gelten, treten Abstimmungsproblematiken auf, weil Ausbildungsbetriebe in ihrem Ausbildungskalkül die Interaktion zwischen Berufsbildungs- und Beschäftigungssystem nicht bedenken. Somit üben sie großen Einfluss auf den Übergang aus. Das Zwei-Schwellen-System von Parmentier und Mertens (1982) verdeutlicht, dass der reibungslose Einstieg ins Erwerbsleben ebenfalls von Marktmechanismen abhängt (Dietrich/Abraham 2005: 72). Denn der Eintritt erfolgt bei Abschluss der Ausbildung, gleich, ob sich der Markt im Gleichgewicht befindet oder nicht. Saisonale, demografische sowie konjunkturelle Faktoren beeinflussen den Grad der Abstimmungsproblematiken, deren Stärke von der Besetzung eines Absolventenjahrgangs abhängt (Rees 1986: 616, siehe auch Kap. 4.2). Die konjunkturellen Faktoren wirken bereits an der ersten Schwelle, weil hier die Jugendlichen einen Ausbildungsplatz finden müssen. Sie wirken jedoch auch an der zweiten Schwelle, weil die Übernahmeentscheidung abhängig von der wirtschaftlichen Situation getroffen wird. Nicht übernommene Ausbildungsabsolventen sind an der zweiten Schwelle erneut mit den Marktmechanismen konfrontiert, während übernommene junge Erwachsene von den konjunkturellen Veränderungen unberührt bleiben. Zudem wird von einem heterogenen Arbeitsangebot ausgegangen, das sich zum einem auf das formal erworbene Humankapital und zum anderen auf das informell erworbene Wissen, wie z.B. Berufserfahrung, aufteilt. Dies hat zur Folge, dass Berufsanfänger auf dem Arbeitsmarkt mit älteren Arbeitskräften konkurrieren müssen, die bereits unter Umständen jahrelange Berufserfahrung aufweisen (Dietrich/Abraham 2005: 72f.; siehe auch Kap. 5.1). Darüber hinaus kann sich der Mangel an individuellen Fähigkeiten, die bereits vor Beginn der dualen Berufsausbildung vorhanden waren, und derentwegen die Selektion in einen Ausbildungsberuf mit weniger günstigen Beschäftigungsaussichten erfolgt ist, auf den Verbleib nach der dualen Berufsausbildung auswirken (Hanushek/Wößmann 2006; Brunello et al. 2004). Daneben können aber auch betriebliche Gegebenheiten während der Ausbildungszeit Gründe für einen weniger erfolgreichen Übergang in das Erwerbsleben sein. Dies führt dazu, dass die Ausbildungsabsolventen erneut einen Arbeitsplatz suchen müssen.

Die Abstimmungsebenen und infolgedessen der Übergang in das Erwerbsleben werden neben den gesamtwirtschaftlichen Voraussetzungen vor allem von betrieblichen Rahmenbedingungen bestimmt. Im Folgenden wird daher der Fokus auf das Ausbildungsverhalten und die Abgangsraten aus Sicht der Betriebe gelegt. Dabei werden Gemeinsamkeiten und Unterschiede zwischen den einzel- 
nen Ausbildungsberufen herausgearbeitet und mögliche Zusammenhänge über den Verbleib nach Abschluss der dualen Berufsausbildung aufgezeigt. Als Erklärungsgrundlage werden mikroökonomische Modelle herangezogen. 
Gabriele Somaggio - 978-3-631-74988-3

Downloaded from PubFactory at 01/11/2019 09:32:31AM

via free access 


\section{Theoretische Erklärungsansätze der Ausbildungsbereitschaft}

Im folgenden Kapitel werden nun die verschiedenen Erklärungsansätze zur betrieblichen Ausbildungsbereitschaft dargestellt. Dies dient als Grundlage zur Erklärung des Zusammenhangs zwischen der ersten und der zweiten Schwelle, denn Unternehmen sind bei unterschiedlicher formaler Schulbildung bereit, in unterschiedlicher Höhe Humankapitalinvestitionen vorzunehmen (Brunello et al. 2004). Daraus resultiert eine Ungleichverteilung der Chancen der Schulabsolventen hinsichtlich der Aufnahme von Ausbildungen, welche durch die SchoolTracking-Ansätze (u. a. Mühlenweg 2007; Hanuschek/Wößmann 2006; Figlio/ Page 2002) erklärt werden. Diese stellen den Zusammenhang zwischen dem Schulabschluss und dem Erlernen eines Ausbildungsberufes dar, denn aufgrund des dreigliedrigen Schulsystems wird der Bildungspfad der Jugendlichen, den diese nur schwer verlassen können, bereits frühzeitig vorgezeichnet. ${ }^{14}$ Infolgedessen haben Schüler mit guten Noten bzw. mit einem höheren formalen Schulabschluss einen Vorteil gegenüber den Schülern mit niedrigerem formalem Abschluss, einen Ausbildungsplatz mit guten Beschäftigungsaussichten zu erhalten. Dies bestimmt sich durch die unterschiedliche Ausbildungsbereitschaft der Unternehmen, woraus Schwerpunkte hinsichtlich der Vermittlung von unterschiedlichen Arten von Wissen und Fähigkeiten resultieren. Die Wirkungen der Ausbildungsbereitschaft auf die Höhe des Bildungsstandes, die sich aus diesem $\mathrm{Zu}$ sammenhang ergeben, werden im folgenden Kapitel herausgearbeitet.

\subsection{Duale Berufsausbildung aus humankapitaltheoretischer Sicht}

\subsubsection{Humankapitaltheorie als Grundlage für Humankapitalinvestitionen}

Die Humankapitaltheorie von Becker (1962) beschreibt die Ausbildungsentscheidungen der Unternehmen. Wie bei der Abwägung einer Investition in Sachkapital wird auch die Entscheidung für eine Humankapitalinvestition von deren Kosten und deren daraus resultierenden Erträgen beeinflusst. Je mehr in Humankapital investiert wird, desto produktiver werden Fachkräfte und entsprechend höher sind die Erträge, aber desto teurer ist auch die Ausbildung. Relevante Größe für die Ausbildungsentscheidung ist zunächst der Lohn, den ein Auszubildender während seiner Zeit im Ausbildungsbetrieb bekommt. Demgegenüber steht der produktive Einsatz der Auszubildenden, da die Ausbildung innerhalb des Betriebes erfolgt und die Arbeitgeber dadurch den Auszubildenden im Produktionsprozess einsetzen können. Somit erfolgt unter der Annahme vollkommenen Wettbewerbs die Entlohnung der Auszubildenden durch die Un-

\footnotetext{
${ }^{14}$ Erfolgt erst einmal die Zuteilung in die verschiedenen Typen der weiterführenden Schulen, ist danach ein Wechsel zwischen diesen Typen nur schwer möglich.
} 
ternehmen in Höhe der Grenzproduktivität ${ }^{15}$ (Becker 1962: 11). Da eine Ausbildungsphase meist mehrere Perioden andauert, im Falle der dualen Berufsausbildung in der Regel drei Jahre, sind Unternehmen nur dann bereit, in Ausbildung zu investieren, wenn die Bildungserträge während der Ausbildungszeit mindestens den Bildungsinvestitionen entsprechen. Zudem ist eine über die Ausbildungszeit hinausgehende Beschäftigungsdauer zu berücksichtigen. Daher muss die Summe der Löhne $(W)$ der Summe der bewerteten Grenzprodukte $(M P)$, den Wertgrenzprodukten, entsprechen und demnach $M P_{t}=W$, gelten. Dabei gibt $t$ die Anzahl der Ausbildungsperioden an. Neben der Entlohnung fallen noch weitere Kostenarten, wie bspw. Material- oder Personalkosten der Ausbilder, an, die in die Investitionsentscheidung einbezogen werden. Daher spiegelt folgende allgemeine Gleichgewichtsbedingung die Humankapitalinvestition wider (Becker 1962: 11f.):

$$
\sum_{t=0}^{n-1} \frac{R_{t}}{(1+i)^{t+1}}=\sum_{t=0}^{n-1} \frac{E_{t}}{(1+i)^{t+1}}
$$

wobei $R_{t}$ die Einnahmen bzw. Erträge und $E_{t}$ die Aufwendungen über die gesamte Ausbildungszeit $t$ darstellen. Die Ausbildungsentscheidung wird von den Unternehmen zu Beginn der Ausbildung über den gesamten Zeitraum festgelegt. Grundlage bildet der Barwert der Erträge und Kosten, die zum Zinssatz $i$ abdiskontiert werden. Der Zinssatz beläuft sich auf die Höhe einer alternativen Anlagemöglichkeit des Geldes auf dem Markt, sowohl für das Unternehmen als auch für die Arbeitskraft.

Da vollkommene Mobilität auf Seiten der Auszubildenden bzw. der Ausbildungsabsolventen herrscht, spielt die Art des Humankapitals als Begründung für die betriebliche Ausbildungsbereitschaft eine entscheidende Rolle. Denn im Gegensatz zu Sachkapital ist das Humankapital an das einzelne Individuum gebunden (Hofmann 2001). Bei Verlassen des Betriebes steht das angeeignete Wissen diesem nicht mehr zur Verfügung. Entsprechend wird der Ausbildungsbetrieb keine weiteren (Bildungs-)Erträge realisieren können. Daher ist es nach Becker (1962) notwendig, dass Unternehmen möglichst während der Ausbildungsphase die Humankapitalinvestitionen amortisieren. Andernfalls sind sie nicht bereit, die Bildung zu finanzieren. Die Mobilität sowie die Realisierung von Bildungserträgen hängen von der Art des vermittelten Humankapitals ab. Die Humankapitaltheorie nimmt daher die Existenz von allgemeinem und (betriebs-) spezifischem Humankapital an. Diese Unterscheidung ist in einem Betrieb sowohl für die Aufteilung der Finanzierung während der als auch für die Beschäftigungsdauer über die Ausbildung hinaus von Bedeutung.

${ }^{15}$ Die Entlohnung $(W)$, die aus Sicht der Unternehmen die Ausgaben darstellt, muss dem Grenzprodukt $(M P)$ und somit den Erträgen entsprechen. Die formale Bedingung lautet daher $M P=W$. 
Für die Eigenschaft der unterschiedlichen Humankapitalarten gilt: „General training is useful in many firms in addition to the firms providing it" (Becker 1962: 12). Bei einem Betriebswechsel bleibt diese Art von Humankapital erhalten und erhöht in anderen Unternehmen ebenfalls die Produktivität (Becker 1962: 13). Demnach ist allgemeines Wissen marktfähig. Wegen der Annahme des vollkommenen Wettbewerbs und der vollkommenen Mobilität müssen die Ausbildungsbetriebe nach der Ausbildung für den Marktlohn aufkommen. Denn wenn sie einen geringeren Lohn als den Marktlohn bezahlen würden, um Erträge realisieren zu können, würden die ausgebildeten Fachkräfte verstärkt das Unternehmen verlassen. So wirkt sich die Entlohnung nach Marktlohn negativ auf die Wahrscheinlichkeit einer Realisation von Bildungserträgen für die Betriebe aus. Folglich ziehen die Ausgebildeten Bildungserträge auf sich, weil sie jederzeit zum Marktlohn wechseln können, ohne einen Produktivitätsverlust zu erleiden. Der Ausbildungsbetrieb kann die getätigten Bildungsaufwendungen daher nicht ausgleichen und kommt somit auch nicht für die Kosten in allgemeines Humankapital auf (Becker 1962: 13). Demgegenüber steht das (betriebs-)spezifische Wissen. Darunter versteht man , [...]training that increases productivity more in firms providing it [...]. Completely specific training can be defined as training that has no effect on the productivity of trainees that would be useful in other firms" (Becker 1962: 17). In diesem Fall verlieren die ausgebildeten Fachkräfte bei einem Betriebswechsel das (betriebs-)spezifische Humankapital, da dieses lediglich in dem Betrieb angewendet werden kann, in dem dieses Wissen erlernt wird. In anderen Unternehmen findet das (betriebs-)spezifische Humankapital dagegen keine Anwendung. Dadurch wird automatisch die Mobilität auf Seiten der ausgebildeten Fachkräfte eingeschränkt (Blundell et al. 1999: 11). Die Wahrscheinlichkeit, dass sich Bildungserträge nach der Ausbildung für die Firmen realisieren lassen, ist bei der Durchführung einer (betriebs-)spezifischer ausgerichteten Ausbildung größer als bei der Vermittlung von allgemeinem Humankapital. Dies bedeutet eine höhere Rentabilität einer Ausbildung dieser Art. Unternehmen sind bereit, in (betriebs-)spezifisches Humankapital zu investieren, weil aufgrund der Existenz eines Nachfragemonopols nach der Art des Humankapitals Bildungserträge resultieren (Leuven/Oosterbeek 2001: 342). ${ }^{16}$ Beide Seiten profitieren von der (betriebs-)spezifischen Humankapitalinvestition, weil die Unternehmen einerseits die Ausbildungsabsolventen langfristig im Unternehmen halten können. Dadurch werden die Kosten der Ausbildung amortisiert. Gleichzeitig bedeutet dies, dass die Ausbildungsabsolventen andererseits keinen Anreiz haben zu wechseln, weil sie dadurch Lohneinbußen hinnehmen

${ }^{16}$ Oosterbeek et al. (2007) haben Experimente mit unterschiedlichen Rahmenbedingungen (vollkommener Markt versus ein Markt mit Transaktionskosten) durchgeführt, um zu erfahren, ob die Finanzierung in betriebsspezifisches Humankapital immer bei den Unternehmen liegt. Die Theorie wurde bei einem vollkommenen Markt bestätigt. Sobald Transaktionskosten auftreten, finanzieren sowohl die Betriebe als auch Auszubildenden in betriebsspezifisches Humankapital. 
müssten. Dementsprechend werden auch die Bildungserträge unter den Akteuren geteilt (Leuven/Oosterbeek 2001: 342). Schließlich profitieren die Ausbildungsabsolventen von dem Lohn, den sie für ihre (betriebs-)spezifische Ausbildung bei einem Verbleib im Betrieb erhalten.

Aufgrund der Eigenschaften der Humankapitalarten und der Annahmen über deren Finanzierung entstehen innerhalb des humankapitaltheoretischen Ansatzes Bildungserträge erst nach der Ausbildung. Allein der Zeitpunkt der Amortisation der Kosten unterscheidet sich für Betriebe. Dabei ergeben sich Unterschiede bei der Ausbildungsbereitschaft, denn die Folgen der unterschiedlichen Humankapitalarten auf die Realisierung der Bildungserträge setzen verschiedene Anreize. Ist die Ausbildung auf die Vermittlung von allgemeinem Humankapital ausgelegt, werden nach Becker (1962) Ausbildungsbetriebe wenig Anreiz haben, in diese Art von Wissen zu investieren, vorausgesetzt die Kosten werden bereits während der Ausbildungszeit durch die Erträge gedeckt. Andernfalls sind die Auszubildenden bereit, für die Aneignung bzw. die Vermittlung von allgemeinem Humankapital selbst aufzukommen, weil sie später die Bildungserträge in Form höherer Löhne in anderen Betrieben realisieren wollen (Becker 1962: 13). Bei einem Wechsel der Fachkräfte nach der Ausbildung haben die Unternehmen dadurch keine finanziellen Verluste. Dagegen werden bei einer Vermittlung von (betriebs-)spezifischem Humankapital die Unternehmen bereit sein, dieses zu finanzieren, da wiederum die Auszubildenden keinen Anreiz haben, den Betrieb zu wechseln. Fachkräfte, die ihren Betrieb dennoch wechseln, erhalten von dem neuen Unternehmen dann lediglich den Marktlohn, der unter dem Lohn liegt, den der Ausbildungsbetrieb bezahlt. Der Marktlohn entspricht ihrer Produktivität, die sich durch das nach einem Wechsel immer noch einsetzbare (allgemeine) Humankapital ergibt. Bei der Annahme einer ausschließlichen Vermittlung von (betriebs-)spezifischem Humankapital ist diese Produktivität dann null. Zusammenfassend lässt sich sagen, dass in allgemeines Humankapital investiert wird, sobald die Auszubildenden diese Investition selbst finanzieren.

\subsubsection{Duale Berufsausbildung als allgemeine Humankapitalinvestition}

Um die Funktionsweise der dualen Berufsausbildung theoretisch begründen zu können, müssen aber die Annahmen des humankapitaltheoretischen Modells modifiziert werden. Die duale Berufsausbildung wird als ein Mischmodell charakterisiert, weil spezifisches und allgemeines Humankapital vermittelt wird. Zudem müssen die von Becker eingeführten Humankapitalarten im Kontext der dualen Berufsausbildung um die berufsspezifische Humankapitalkomponente, welche das Hauptmerkmal dieser Art von Berufsausbildung darstellt, ergänzt werden. Dabei ist zu beachten, dass berufsspezifisches Wissen mehr die Eigenschaften des allgemeinen als des betriebsspezifischen Humankapitals besitzt. So kann berufsspezifisches Wissen nach einem Betriebswechsel uneingeschränkt eingesetzt werden, sofern der Beruf beibehalten wird. Diese Art von Wissen verfällt demnach nicht. In diesem Fall profitieren andere Unternehmen, die sich 
nicht an der Ausbildung beteiligen, die aber die gleichen beruflichen Qualifikationen benötigen wie die Ausbildungsbetriebe in gleichem Maße wie von dem allgemeinen Humankapital. Berufsspezifisches Humankapital hat dieselbe Eigenschaft wie allgemeines Humankapital und bildet somit eine wichtige Erklärungsgrundlage für die Funktionsweise des dualen Berufsausbildungssystems (Politaev/Robinson 2008). Zudem führen staatlich anerkannte Abschlüsse der dualen Berufsausbildung, die durch die Kammern abgenommen werden und der parallele Besuch der Berufsschule zu einer weiteren Verallgemeinerung des Wissens der dualen Berufsausbildung (Acemoglu/Pischke 1999b; 1999a; Schettkat 1992), wodurch für Ausbildungsabsolventen Betriebswechsel erleichtert werden. Die formalen Bildungskomponenten der dualen Berufsausbildung sind transferierbar (Stevens 1996). Aus diesem Grunde sehen auch Clark und Fahr (2001) sowie Harhoff und Kane (1997) die duale Berufsausbildung als eine Investition in allgemeines Humankapital an. Wird die Argumentation von Lazear (2004) betrachtet, gibt es kein (betriebs-)spezifisches Humankapital, da jede Art von Wissen ohne größere Einschränkungen angewendet werden kann. Betriebsspezifisch ist dann die Zusammensetzung der verschiedenen (allgemeinen) Fähigkeiten, die nach ihrer Bedeutung bei der Ausübung der Tätigkeit jeweils unterschiedlich gewichtet werden. ${ }^{17}$ So sind Unternehmen eher bereit, in Ausbildung $\mathrm{zu}$ investieren, wenn die Kombination einzelner Fähigkeiten sehr betriebsspezifisch ist und nur in wenigen Bereichen Anwendung findet (BackesGellner/Mure 2008: 4). Trotz der Ausrichtung der dualen Berufsausbildung als eher allgemeine Investition muss weiterhin zwischen allgemeinem und betriebsspezifischem Humankapital differenziert werden, um die verschiedenen Bildungsniveaus und deren Einflussfaktoren identifizieren zu können. Damit sind nach der Theorie von Becker Ausbildungsbetriebe nicht gewillt, Lehrstellen im Rahmen der dualen Berufsausbildung anzubieten, weil die Auszubildenden nach Erwerb ihres Berufsabschlusses den Ausbildungsbetrieb verlassen können, ohne bei einem anderen Arbeitgeber der gleichen Berufsgruppe größere Einkommenseinbußen erleiden zu müssen (Pigou 1912). Darüber hinaus weisen nach einer Kostenstudie der dualen Berufsausbildung von Beicht et al. (2004) 96\% der Ausbildungsbetriebe Nettokosten nach der dualen Berufsausbildung auf, ${ }^{18}$

${ }^{17}$ Bspw. benötigen Bürokaufleute mehr analytische Fähigkeiten als handwerkliches Geschick, wohingegen bei Tischlern der Schwerpunkt umgekehrt ausgerichtet ist. Entsprechend werden die beiden Fähigkeiten in den genannten Berufen unterschiedlich bewertet.

${ }^{18}$ Hierbei ist zwischen den unterschiedlichen Kostenbegriffen zu unterscheiden. Die Sachverständigenkommission („Edding Kommission 1974) unterscheidet dabei zwischen zwei Kostenarten: Vollkosten und Teilkosten. Der Berechnung liegt der Ansatz der Vollkosten einer Berufsausbildung zugrunde, der in wissenschaftlichen Arbeiten meist zitiert wird. Vollkosten erfassen alle Leistungen, die im gesamten Prozess einer beruflichen Qualifizierung innerhalb des Betriebes anfallen. Es müssen daher alle eingesetzten Personen- und Sachmittel dem Auszubildenden (anteilig) zugerechnet werden; es werden somit auch Kosten berücksichtigt, die durch den Produktionsprozess in jedem Falle, also auch ohne Ausbildungsbeteiligung entstehen (Beicht et al. 2004: 21f.). Demnach fließen auch die nicht entscheidungsrelevanten Kos- 
was den Anreiz für eine betriebliche Ausbildungsbereitschaft weiter beeinträchtigen sollte. Dennoch sind Unternehmen bereit, in Humankapital im Rahmen der dualen Berufsausbildung zu investieren (Booth/Bryan 2002; Acemoglu/Pischke 1999a, 1999b, 1998; Autor 2001; Loewenstein/Spletzer 1999/1998; Katz/ Ziderman 1990). Um die Ausbildungsbereitschaft für die duale Berufsausbildung erklären zu können, muss nach unterschiedlichen Zeithorizonten differenziert werden, die von den Humankapitalarten bestimmt werden. Diese Differenzierung erlaubt es somit, den Verbleib an der zweiten Schwelle und somit freiwilligen und vor allem die unfreiwillige Abgangsrate (Nichtübernahme), die bei Becker nicht existiert, zu verdeutlichen. Im Folgenden werden diese Ansätze genauer beschrieben.

\subsection{Mikroökonomische Erklärungsansätze und der Einfluss auf das Hu- mankapital}

Aufgrund der begrenzten Anwendung des humankapitaltheoretischen Ansatzes, der vor allem auf den amerikanischen Arbeitsmarkt und entsprechend auf das On-the-Job-Training angewendet wird, muss das Modell um zwei unterschiedliche Zeithorizonte erweitert werden, welche sich durch die Kosten-NutzenStruktur bestimmen. Unternehmen bilden einerseits dann aus, wenn die Kosten bereits während der Ausbildung amortisiert werden. Eine Beschäftigung über die Ausbildung hinaus ist nicht notwendig bzw. vorgesehen. In einem Lagerhaltungsmodell ist diese Situation durch geringe Fehlmengenkosten, welche die vorgehaltenen Potenziale innerhalb der Fachkräfte reduzieren, gekennzeichnet. In diesem Fall ist die Ausbildung eher auf ein kurzfristiges Arbeitsverhältnis angelegt. Andererseits bilden Unternehmen auch bei Nettokosten aus. Die Motivation ist daher ein langfristiges Beschäftigungsinteresse, um den zukünftigen Fachkräftebedarf zu decken. Entsprechend sind die Kosten der Vorratshaltung gemäß einem Lagerhaltungsmodell niedrig (Backes-Gellner 2008: 7).

In diesem Fall sind für die Erklärung der Motivation der betrieblichen Ausbildungsbereitschaft vor allem institutionelle Rahmenbedingungen zu berücksichtigen. Aus diesen jeweiligen Planungshorizonten lassen sich Implikationen für die Art und die Höhe des Humankapitals, das im Kontext der institutionellen Rahmenbedingungen vermittelt wird, ableiten. Zur Erklärung der unterschiedlichen Ausbildungsbereitschaft muss der Zeithorizont, der im humankapitaltheo-

ten, wie Personalkosten nebenberuflicher Ausbilder in den Vollkostenansatz ein. Auf Vollkostenbasis entscheidet sich ein Betrieb, ob er ausbildet oder nicht. Dagegen berücksichtigt der Teilkostenansatz die Ausbildungskosten, die zusätzlich bei der Entscheidung für eine Ausbildung anfallen, also entscheidungsrelevant sind (Mohrenweiser/Backes-Gellner 2008). Bspw. fallen hierunter die Ausbildungsvergütungen, die Personalkosten externer Ausbilder etc. (Beicht et al. 2004: 25). Bruttokosten sind alle Aufwendungen und Kosten, die im Zuge einer dualen Berufsausbildung anfallen. Werden die Erträge, die aus der dualen Berufsausbildung im Laufe der Ausbildungsjahre entstehen, abgezogen, ergeben sich die Nettokosten. Die Nettokosten geben demnach die tatsächlich verbleibenden Ausbildungskosten wieder. 
retischen Grundmodell gilt, in eine Ausbildungs- und in eine Beschäftigungsphase unterteilt werden. Daraus leitet sich der Fokus auf die Ausbildungsphase $\mathrm{ab}$, da bei Beendigung der Ausbildung das Ausbildungsverhältnis zwischen Betrieb und Ausbildungsabsolvent automatisch endet. Die Kosten-Nutzen-Struktur zu diesem Zeitpunkt bildet die Entscheidungsgrundlage für eine weitere Beschäftigungsperiode.

\subsubsection{Produktionstheoretischer Ansatz}

Aus der humankapitaltheoretischen Theorie geht hervor, dass betriebliche Investitionen in allgemeines Humankapital nur dann stattfinden, wenn während der Beschäftigung im Ausbildungsbetrieb positive Erträge entstehen (Zwick 2007: 4; Fougère/Schwerdt 2002: 318; Neubäumer 1999: 67; Lindley 1975: 4ff.). Im produktionstheoretischen Ansatz beschränkt sich die Betrachtung auf den Ausbildungszeitraum. Unternehmen sind bereit, in allgemeines Humankapital zu investieren, weil die Erträge bereits während der Ausbildung die Kosten übersteigen. Dies ist nur möglich, weil der Auszubildende direkt am Arbeitsplatz lernt und so auf fast denselben Produktionsbeitrag kommt wie ein bereits fertig ausgebildeter Mitarbeiter (Brunello/Medio 2001; Lindley 1975). So trägt in diesem Fall der Jugendliche während seiner Ausbildung selbst einen großen Anteil zur laufenden Produktion bei. Dadurch können die Ausbildungsbetriebe die Löhne der un- bzw. angelernten Arbeitskräfte an die Löhne der Auszubildenden (nach unten) angleichen. Diese Idee hat Lindley (1975) in seinem Modell der Ausbildungsbereitschaft aufgegriffen, welche auf die Kosten-Nutzen-Struktur der Ausbildungsbetriebe angewendet wurde. Dem produktionstheoretischen Ansatz liegt eine Cobb-Douglas-Produktionsfunktion zugrunde, welche die Beziehung zwischen den Auszubildenden, den Fachkräften und den ungelernten Arbeitern darstellt (Lindley 1975: 4f). Dabei können die Fachkräfte nur von den Auszubildenden ersetzt werden und umgekehrt (Lindley 1975: 3). Die Unternehmen bestimmen die Höhe des Outputs, die sie erreichen wollen. Ausgehend davon werden der Kapitalstock sowie die Struktur der Arbeitskräfte festgelegt, sodass die Kosten der Unternehmung minimiert werden (Lindley 1975: 5). In Zusammenhang mit der Kapitalausstattung, die bestimmte Qualifikationsniveaus erfordert, kann schließlich der Bedarf an Auszubildenden abgeleitet werden (Lindley 1975: 4ff.; Neubäumer 1999: 36). Hierbei werden unterschiedliche Produktivitäten der einzelnen Arbeitsgruppen angenommen, die unter Berücksichtigung der entsprechenden Lohnhöhe die Zusammensetzung der Arbeitskräfte bestimmen (Franz et al. 2000: 59). ${ }^{19}$ Demnach hängt die Nachfrage nach Auszubildenden von dem aktuellen Arbeitsbedarf, der durch die Kapitalausstattung gegeben ist, ab.

${ }^{19}$ Es wird von der Existenz von Arbeitsplätzen ausgegangen, die entweder ungelernte oder ausgebildete Arbeitskräfte erfordern. 
Der produktive Einsatz der Auszubildenden lässt die Wahrscheinlichkeit steigen, dass die Ausbildungskosten $\left(E_{t}\right)$ während der Ausbildungsphase amortisiert werden und der Barwert ${ }^{20}$ der Erträge $\left(R_{t}\right)$ über dem der Ausbildungskosten liegt. Die Bedingung, die sich aus Gleichung (2) ergibt, lautet in diesem Fall

(2) $\sum_{t=0}^{n-1} \frac{R_{t}}{(1+i)^{t+1}} \geq \sum_{t=0}^{n-1} \frac{E_{t}}{(1+i)^{t+1}}$.

Die Erträge entstehen aufgrund des direkten Einsatzes der Auszubildenden in den Produktionsprozess. Daraus resultiert ein hoher Produktionsbeitrag, weswegen prinzipiell nicht von einer Ausbildung gesprochen werden kann, da über das am Arbeitsplatz eingesetzte Wissen hinaus kein zusätzliches Humankapital vermittelt wird (Kempf 1985: 66; Merilees 1983: 4ff.). Folglich beschränkt sich das Humankapitalniveau der Ausbildungsabsolventen auf Komponenten, die direkt am Arbeitsplatz entstehen. Das Humankapitalniveau geht daher nicht über das Wissen des Arbeitsprozesses hinaus und ist entsprechend gering. Hierbei handelt es sich um berufspezifisches Wissen, das in Betrieben mit denselben Tätigkeitsbereichen nahezu uneingeschränkt eingesetzt werden kann.

Das Angebot für einen Ausbildungsplatz liegt in diesem Fall in einem kurzfristigen Planungshorizont begründet, da keine Überlegungen zum zukünftigen Fachkräftebedarf angestellt werden (Niederalt 2004: 84). Dies entspricht auch der Idee Lindleys, der (zunächst) die gegenwärtige Produktion in Unabhängigkeit von den zukünftigen Produktionserwartungen sieht. Für das Unternehmen ist es daher nicht von großer Bedeutung, über den Ausbildungshorizont hinaus zu planen.

Folglich hängen die Einstellungen von Auszubildenden nach Lindley (1975) nicht von dem künftigen Fachkräftebedarf ab, sondern von einem Mangel an Ausbildung im Allgemeinen. Die Motivation der Betriebe ergibt sich aus der Ausbildung von Fachkräften für den jeweiligen Industriesektor. Allerdings weichen Ausbildungsbetriebe nur dann von ihren optimalen Einstellungsquoten ab, wenn die Löhne im Verhältnis zu dem Mangel an Fachkräften unterproportional steigen (Lindley 1975: 9). Darüber hinaus stellen die Unternehmen ebenfalls mehr Auszubildende ein, wenn eine geringere gesamtwirtschaftliche Arbeitslosenquote vorherrscht bzw. stellen weniger ein, wenn die Arbeitslosigkeit hoch ist, da sie in letzterem Fall auf mehr ausgebildete Fachkräfte zurückgreifen können (Lindley 1975: 10). Hier wird die Abhängigkeit von der Konjunktur deutlich. Nach Lindley (1975) ist es in Bezug auf die Einstellung von Auszubildenden notwendig, die verschiedenen Produktionsbeiträge der einzelnen Gruppen, das heißt, die unterschiedliche Produktivität von Auszubildenden und qualifizierten Arbeitnehmern zu berücksichtigen. Ebenso beeinflussen der Abgang der Auszubildenden nach der Ausbildung und die Fluktuation der Fachkräfte die Einstellung von Schulabsolventen (Lindley 1975: 19).

${ }^{20}$ Die Berechnung des Barwertes erfolgt analog zu der Vorgehensweise in Kap. 3.1. 
Die Motivation der Betriebe, nach dem produktionstheoretischen Ansatz auszubilden, ist innerhalb des dualen Berufsausbildungssystems nur schwer vorstellbar. Dies liegt insbesondere daran, dass der Bildungsprozess für die Auszubildenden, gleich welcher Branche bzw. gleich welchen Ausbildungsberufes, innerhalb der Arbeitsprozesse abläuft und Bildung als ein Kuppelprodukt der Produktionsprozesse zu verstehen ist. Vielmehr schreiben die Ausbildungsordnungen Mindeststandards für das zu erreichende formale Bildungsniveau vor, sodass der Ausbildungsinhalt über das am Arbeitsplatz benötigte Humankapital hinausgeht. Entsprechend beschränkt sich die Bildung auf die vorgegebenen Standards. Ferner verbringen Auszubildende einen Teil ihrer Ausbildung in der Berufsschule. Daher können Betriebe, die im Rahmen der dualen Berufsausbildung ausbilden, nicht in dem Maße Produktionserträge realisieren wie durch den produktionstheoretischen Ansatz beschrieben wird.

Dennoch weisen auch Ausbildungsbetriebe innerhalb der dualen Berufsausbildung Nettoausbildungserträge auf und bilden entsprechend nach dem produktionstheoretischen Ansatz aus (Mohrenweiser/Zwick 2008; Zwick 2007; Fougère/Schwerdt 2002). Nach Mohrenweiser und Backes-Gellner (2008) verfolgen ungefähr 18,5\% der Ausbildungsbetriebe die Substitutionsstrategie. Der unmittelbare produktive Einsatz der Auszubildenden zu Beginn ihrer Ausbildungszeit wird ermöglicht, weil die Tätigkeitsprofile nur geringe Anforderungen an den Bildungsstand stellen. Deshalb reicht ein niedriger Humankapitalstand aus. Da es sich meist um Ausbildungsbetriebe handelt, die in der Regel standardisierte Produkte in ebenso standardisierten Prozessen herstellen (Mohrenweiser/Backes-Gellner 2008), kann das erlernte Wissen als berufsspezifisch bezeichnet werden. Somit wird der berufs- und der sehr geringe Anteil an betriebsspezifischem Humankapital aufgrund der arbeitsplatznahen Ausbildung sowie der Möglichkeit, Leerzeiten während der Produktion als Einarbeitungszeit zu nutzen, vermittelt (Neubäumer 1997; 1999). Betriebe mit standardisierten Prozessen erzielen zum Teil während einer Ausbildungsphase Nettoerträge (Niederalt 2004: 84). Der Auszubildende nimmt hierbei die Rolle einer zusätzlichen Arbeitskraft bzw. Hilfskraft ein. Die Auszubildenden sind nach dem produktionstheoretischen Ansatz als günstiger Ersatz für andere qualifizierte Arbeitskräfte zu sehen (Zwick 2007: 195; Fougère/Schwerdt 2002: 318; Franz et al. 2000: 59; Neubäumer 1999: 36). Aufgrund der niedrigen bzw. negativen Nettokosten, die sich aus der Differenz zwischen den Bruttokosten und den Erträgen aus der Ausbildung heraus ergeben, stellen die Unternehmen die Auszubildenden kurzfristig ein. Dies führt wiederum dazu, dass Ausbildungsabsolventen nur über das notwendige Humankapital verfügen, welches für den Tätigkeitsbereich erforderlich ist. Dies kann unter Umständen sehr gering sein, weil auch hier positive Externalitäten durch die Ausbildung entstehen. 
Viele Ausbildungsbetriebe weisen jedoch am Ende der Ausbildungsphase Nettokosten auf (Beicht et al. 2004; Bardeleben et al. 1995). ${ }^{21}$ Nun muss die Frage beantwortet werden, warum diese Betriebe dennoch bereit sind, sich an der (dualen) Berufsausbildung zu beteiligen.

\subsubsection{Investitionstheoretische Ansätze}

Den Betrieben entstehen insbesondere Kosten durch Ausbildungsvergütungen, die Löhne der hauptberuflichen Ausbilder sowie Materialkosten. Ferner findet in vielen Betrieben die Ausbildung zunehmend in (außer-)betrieblichen Lehrwerkstätten statt. Während dieser Zeit leisten die Auszubildenden keinen Beitrag zur Produktion (Bellmann/Neubäumer 2001). Darüber hinaus benötigen Unternehmen mit hohem technologischem Fortschritt qualifizierte Arbeitskräfte, welche die Maschinen bedienen können. Um diese zu erhalten, sind sie bereit, hohe Kosten für die Ausbildung aufzuwenden (Bartel/Sichermann 1998). Aber damit die Ausbildung auch tatsächlich einen Investitionscharakter aufweist, muss sichergestellt werden, dass die Bildungserträge von den Ausbildungsbetrieben realisiert werden können (Niederalt 2004: 86). Daher werden theoretisch nur die Unternehmen ausbilden, die eine Übernahme planen. Die Bedingung der Kosten-Ertragssituation nach der dualen Berufsausbildung nach Becker (1962) kehrt sich gegenüber dem produktionstheoretischen Ansatz entsprechend um. Unternehmen mit Nettoausbildungskosten sind direkt nach Beendigung der Ausbildung der folgenden Situation gegenüber gestellt:

$$
\sum_{t=0}^{n-1} \frac{R_{t}}{(1+i)^{t+1}}<\sum_{t=0}^{n-1} \frac{E_{t}}{(1+i)^{t+1}}
$$

Voraussetzung für die Ausbildungsbereitschaft ist auch in dieser Situation die Möglichkeit, Bildungserträge zu realisieren. Da die Kosten die Erträge nach der Ausbildungsphase übersteigen, werden diese (Kosten), im Vergleich zum produktionstheoretischen Ansatz, erst nach der Ausbildung bei einer Übernahme und Weiterbeschäftigung der Ausbildungsabsolventen amortisiert. Daher bilden Unternehmen aus, weil sie an einem längerfristigen Beschäftigungsverhältnis interessiert sind. Der zukünftige Fachkräftebedarf lässt sich durch technologischen Fortschritt innerhalb des Ausbildungsbetriebs begründen. Eine Ausbildung ist notwendig, da entsprechende Arbeitskräfte benötigt werden und durch die technologisierten Prozesse hohe Einarbeitungskosten für extern rekrutierte Fachkräfte entstehen. In diesem Fall ist eine Ausbildung kostengünstiger (Niederalt 2004: 85; Großmann/Meyer 2002: 68; Lehne 1991: 50; Kempf 1985: 61). Um die Ausbildungsabsolventen nach Beendigung der Ausbildung im Ausbil-

${ }^{21}$ Studien (u. a. Dionisius et al. 2008) zeigen, dass in Deutschland mehr Ausbildungsbetriebe Nettokosten nach der dualen Berufsausbildung aufweisen als in der Schweiz. Zurückzuführen ist dies vor allem auf die institutionellen Rahmenbedingungen, die sich zwischen den beiden Ländern unterscheiden. 
dungsbetrieb halten zu können, müssen Rahmenbedingungen geschaffen werden, welche die freiwillige Mobilität einschränken. ${ }^{22}$ Nur so kann die betriebliche Ausbildungsbereitschaft der Unternehmen gewährleistet werden.

\subsubsection{Grundmodell investitionstheoretischer Ansätze}

Um die Ausbildungsbereitschaft bei Nettoausbildungskosten, wie es größtenteils auf die duale Berufsausbildung zutrifft, zu ermöglichen, müssen die Annahmen eines vollkommenen Arbeitsmarktes fallen gelassen werden. Um die Ausbildungsbereitschaft der Unternehmen bei allgemeinem Humankapital dennoch zu garantieren, gehen Acemoglu und Pischke (1999a; 1999b; 1998) davon aus, dass Unvollkommenheiten auf dem Arbeitsmarkt und institutionelle Rahmenbedingungen zunehmende Lohnunterschiede zwischen qualifizierten und unqualifizierten Arbeitskräften verhindern, weil die Löhne der qualifizierten Arbeitskräfte nach unten verzerrt sind und somit Lohnkompression vorliegt. Das heißt, die Entlohnung liegt unter der Produktivität (Beckmann 2002b: 369f.), wodurch den Ausbildungsbetrieben im Falle einer Weiterbeschäftigung Bildungserträge entstehen.

Hierbei wird die Humankapitaltheorie von Becker (1962) modifiziert, damit allgemeine Humankapitalinvestitionen auch bei betrieblicher Finanzierung getätigt werden. Erklärungsgrundlage bildet das Modell von Acemoglu und Pischke $(1999 \mathrm{a} ; 1999 \mathrm{~b} ; 1998)$ Darin hängt der Lohn $\omega(\tau)$ direkt vom Bildungsstand einer Person $a b .^{23} \mathrm{Da}$ lediglich allgemeines Humankapital betrachtet wird, beschreibt $\tau$ somit das Niveau der allgemeinen Bildung, welche den Produktionsbeitrag einer qualifizierten Arbeitskraft in Höhe von $f(\tau)$ determiniert. Die Gleichgewichtsbedingung lautet bei vollkommenem Wettbewerb $\omega(\tau)=f(\tau)$. Der Lohn $\omega(\tau)$ gilt für alle Unternehmen, weshalb er gleichzeitig den Marktlohn repräsentiert. ${ }^{24}$ Dies ermöglicht jedoch qualifizierten Arbeitskräften weiterhin ihren Ausbildungsbetrieb zu verlassen, da sie in einem anderen Unternehmen in gleicher Höhe entlohnt werden. Erleichtert werden Betriebswechsel weiterhin durch das Fehlen von Mobilitätskosten. Wie in der Humankapitaltheorie werden auch in dem Modell von Acemoglu und Pischke (1999a; 1999b; 1998) die Unternehmen zunächst bei Marktvollkommenheit nicht bereit sein, in allgemeines Humankapital zu investieren, da durch die Ausbildung positive Externalitäten entstehen (Acemoglu/Pischke 1999b: $552 \mathrm{ff}$ ), welche nicht internalisiert werden können. Das heißt, andere Unternehmen, die sich nicht an der Ausbildung beteiligen, profitieren ebenso von den qualifizierten Arbeitskräften, weil diese auch

\footnotetext{
${ }^{22}$ Ein Anreiz für Ausbildungsabsolventen zu wechseln, liegt immer dann vor, wenn sie die Optionen auf dem externen Arbeitsmarkt besser als im Ausbildungsbetrieb einschätzen.

${ }^{23}$ Bei Becker wird der Humankapitalstand auf Unternehmensebene betrachtet, wenn in betriebsspezifisches Humankapital investiert wird bzw. auf gesamtwirtschaftlicher Ebene, sofern eine Investition in allgemeines Humankapital erfolgt (Pütz 2003: 70ff.).

${ }^{24} \mathrm{Da}$ nur Auszubildende betrachtet werden spiegelt $\omega$ deren Lohn, die Ausbildungsvergütungen wider.
} 
in nicht ausbildenden Betrieben die Produktivität erhöhen. Daher liegt die Finanzierung auch hier bei den Auszubildenden, welche entweder in Form eines Bildungsbeitrages oder in Form eines im Vergleich zu einer ungelernten Arbeitskraft niedrigeren Lohnes (Acemoglu/Pischke 1999a: 113) erfolgt. Hierbei müssen aber jeweils perfekte Kreditmärkte angenommen werden (Acemoglu/Pischke 1999a: 117f). ${ }^{25}$

Um dem Aspekt eines längerfristigen Beschäftigungshorizontes gerecht $\mathrm{zu}$ werden, wird davon ausgegangen, dass die Arbeitskräfte unterhalb ihrer Produktivität entlohnt werden und somit $\omega(\tau)<f(\tau)$ gilt. Die Differenz zwischen Produktionsbeitrag und Entlohnung beschreibt die Lohnkompression (Acemoglu/Pischke 1999a, 1999b, 1998). Formal lässt sich diese Bedingung zu Beginn einer Ausbildung $(\tau=0)$ wie folgt darstellen:

(4) $\Delta^{\prime}(0)=f^{\prime}(0)-\omega^{\prime}(0)$,

wobei $\Delta^{\prime}(\tau)$ die Lohnkompression in Form einer Differenz zwischen Produktionsbeitrag $f^{\prime}(\tau)$ und Entlohnung $\omega^{\prime}(\tau)$, jeweils in Abhängigkeit der angeeigneten Bildung $(\tau)$, darstellt. Booth und Zoega (2001) bezeichnen dies als absolute Lohnkompression. Darunter wird der absolute Unterschied zwischen der Veränderung der Entlohnung und der Veränderung der Produktivität bei steigendem Bildungsstand verstanden (Booth/Zoega 2001: 2).

Wie Abbildung 4 zeigt, erhöhen sich die Bildungserträge und damit die Produktivität bei zunehmendem Bildungsstand. Daher gilt $\omega^{\prime}(\tau)<f^{\prime}(\tau)$ als (hinreichende) Bedingung für die Ausbildungsbereitschaft (Acemoglu/Pischke 1999a: 120; 1999b: 547). Dies bedeutet, dass der Lohn bei zunehmendem Bildungsstand weniger stark steigt als die Produktivität. Wegen der in $\tau$ steigenden Lohnfunktion treten nun ebenfalls Unterschiede in den Erträgen von qualifizierten und nicht qualifizierten Arbeitnehmern im Gegensatz zu einem perfekten Markt auf, das heißt, aufgrund der zunehmenden Lohnkompression (Lohndifferentials) realisieren die Betriebe höhere Bildungserträge, je mehr sie in Bildung investieren. Der optimale Bildungsstand $\tau *$ ist dann erreicht, wenn die Grenzkosten $c^{\prime}(\tau)$ dem Grenzprodukt $f^{\prime}(\tau)$ entsprechen. Dies ist dann gegeben, wenn die Differenz $(f(\tau)-\Delta)$ und $c(\tau)$ maximiert wird.

\footnotetext{
${ }^{25}$ In einem perfekten Kreditmarkt gibt es keine Beschränkungen bei der Kreditaufnahme. Hier gewähren Banken ein Darlehen ohne Sicherheiten. Dies ist Voraussetzung, da Humankapital an das Individuum gebunden und somit nicht veräußerbar ist, das heißt, das Humankapital als Sicherheit, wie bspw. eine Hypothek auf Immobilien zu belangen.
} 


\section{Abbildung 4: Lohnkompression und Humankapitalinvestition}

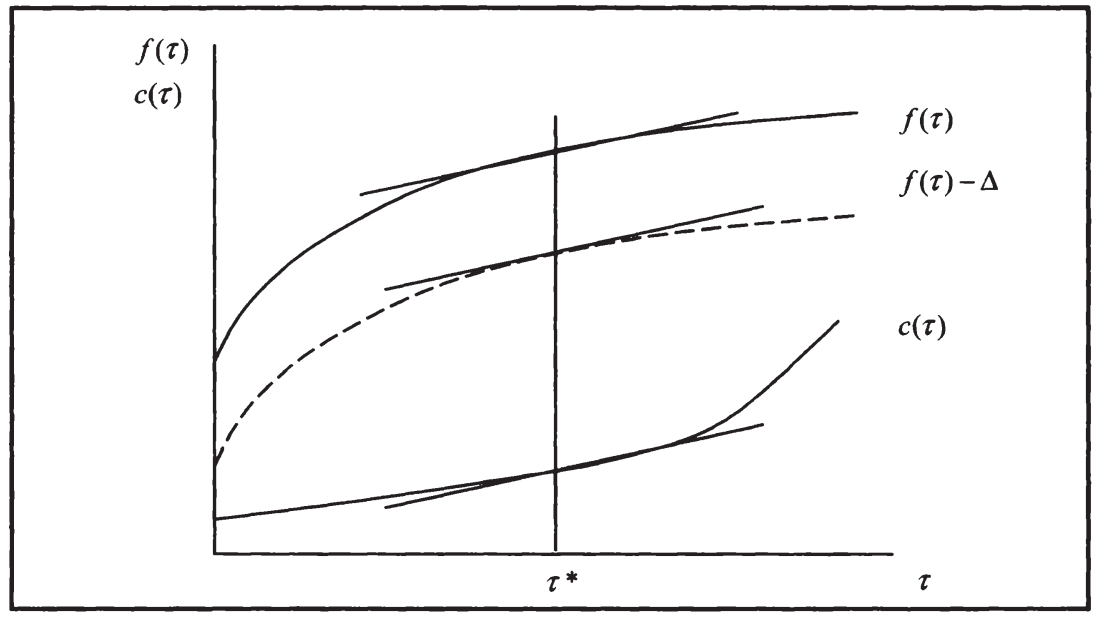

Quelle: Acemoglu/Pischke (1999a)

Dagegen beschreibt die relative Lohnkompression $p(\tau)$ das Verhältnis zwischen Produktionsbeitrag und Entlohnung $P / W$. Dies bedeutet, dass das Verhältnis, und somit der Unterschied, der betrachteten Determinanten mit zunehmendem Bildungsstand steigt, das heißt, dass eine Zunahme der Bildung den Produktionsbeitrag im Vergleich zur Entlohnung überproportional ansteigen lässt (Acemoglu/Pischke 1999a: 120ff., 1999b: 548; Booth/Zoega 2001: 3). So kann sich zwar die absolute Differenz zwischen Produktivität und Entlohnung erhöhen, das Verhältnis, also die relative Lohnkompression zwischen den beiden Größen aber konstant bleiben. ${ }^{26}$

Lohnkompression existiert auf dem gesamten Arbeitsmarkt, weshalb die Absolventen auch in einem anderen Betrieb unterhalb ihrer Produktivität ent-lohnt werden. Daher gilt aufgrund des unvollkommenen Arbeitsmarkts mit vollkommenem Wettbewerb $v(\tau)<f(\tau)$. Dies bedeutet, dass auch für Unternehmen auf dem externen Arbeitmarkt für nicht selbst ausgebildete Personen ein Marktlohn $v(\tau)$ gilt, der unterhalb der Produktivität $f(\tau)$ des Arbeitnehmers zu gegebenem Bildungsniveau ( $\tau$ ) liegt (Acemoglu/Pischke 1999b: 547). Dies schränkt zwar den Anreiz, den Ausbildungsbetrieb zu verlassen, ein, reicht aber zur Vermeidung von Mobilität nicht aus. Infolgedessen muss weiterhin gewährleistet sein, dass der innerhalb des Ausbildungsbetriebes geltende Lohn $\omega(\tau)$ den

${ }^{26}$ Die relative Lohnkompression entspricht dem allgemeinen Verständnis der Lohnstauchung, welche die Einkommensverteilung bestimmt (Katz/Murphy 1991; Beckmann 2002b; Booth/Zoega 2001) 
Marktlohn $v(\tau)$ übersteigt, wobei $\omega(\tau)>v(\tau)$ gilt. ${ }^{27}$ Mobilitätskosten fallen nur an, weil Auszubildende bei einem Wechsel eine geringere Entlohnung als im Ausbildungsbetrieb erhalten. ${ }^{28}$ Zusätzliche Mobilitätskosten existieren nicht. Aufgrund des Ausbildungsverhaltens der Betriebe steigt das gesamtwirtschaftliche Bildungsniveau und infolgedessen der Marktlohn. Bei steigendem Marktlohn und gegebenem Niveau an Ausbildung und entsprechender betrieblicher Entlohnung wird aber der Anreiz für einen Betriebswechsel wieder steigen. Damit die Mobilität möglichst gering bleibt und Bildungserträge künftig entstehen, muss weiter in Bildung investiert werden. So setzt sich ein Prozess in Gang, der zu einem immer höheren Bildungsniveau führt.

Die Durchsetzung von absoluter Lohnkompression veranlasst die Ausbildungsfirmen, in Bildung zu investieren. Durch die Entlohnung der Ausbildungsabsolventen unterhalb der Produktivität entstehen Bildungserträge, welche mit zunehmendem Bildungsstand steigen. Unternehmen, denen Nettokosten entstehen, werden mehr als die vorgegebenen Mindestanforderungen, die in den Ausbildungsverordnungen verankert sind, vermitteln. Denn nur so kann das Potenzial einer Lohnkompression ausgeschöpft werden. Daher werden die Ausbildungsabsolventen einen hohen Bildungsstand vorweisen, der sich aus formellen Humankapitalanforderungen und informellen Fähigkeiten zusammensetzt. Diese Art der Humankapitalvermittlung wird durch weitere existierende Marktunvollkommenheiten verursacht, die gleichzeitig zur Lohnkompression beitragen.

\subsubsection{Bestimmungsgründe von Investitionen in allgemeines Human- kapital}

Um betriebliche Investitionen in allgemeines Humankapital zu gewährleisten, gilt es, die Mobilität der Ausbildungsabsolventen zu reduzieren, denn höhere betriebliche Humankapitalinvestitionen führen mit hoher Wahrscheinlichkeit nach Abschluss der dualen Berufsausbildung zu einem freiwilligen Betriebswechsel (Adnett et al. 2003: 49ff.; Hashimoto 1981: 478). Nach Hashimoto (1981) besteht eine Möglichkeit zur Reduzierung der Mobilität aber darin, die Finanzierung der Ausbildung auf die beiden Akteure aufzuteilen. Infolge einer Aufteilung der Finanzierung entstehen dem Auszubildenden bei Wechsel Austrittskosten in Form eines Produktivitätsverlustes. Weiterhin lässt sich Lohnkompression deswegen durchsetzen, weil die neoklassischen Annahmen eines vollkommenen Marktes aufgegeben werden (Booth/Zoega 2001; Acemoglu/

\footnotetext{
${ }^{27}$ Der Marktlohn spiegelt Unsicherheiten der Unternehmen bei der Einstellung von Arbeitskräften hinsichtlich der tatsächlichen Produktivität wider. Existiert, nach Hashimoto (1981), eine relativ hohe Unsicherheit auf dem externen Arbeitsmarkt, können Ausbildungsbetriebe relativ niedrige Löhne durchsetzen, da Ausbildungsabsolventen schlechte Optionen außerhalb des Unternehmens haben. Die Lohnkompression ist hoch und führt entsprechend zu hohen Bildungserträgen.

${ }^{28}$ Die Mobilitätskosten entsprechen demnach der Differenz zwischen dem Lohn des Ausbildungsbetriebes $\omega(\tau)$ und dem Marktlohn $v(\tau)$.
} 
Pischke 1999a; 1999b; 1998). Gekennzeichnet ist dieser vor allem von Informationsasymmetrien, durch deren Existenz Ausbildungsabsolventen nicht ihre tatsächliche Produktivität gegenüber anderen Unternehmen signalisieren können. Daneben ist es den Ausbildungsabsolventen aber auch teilweise nicht möglich, ihr im Ausbildungsbetrieb erhaltenes Wissen vollständig einzusetzen, weil neben allgemeinem auch betriebsspezifisches Humankapital vermittelt wird. Infolgedessen trägt dies ebenfalls zur Reduzierung der Mobilität bei.

\subsection{Informationsasymmetrien und Ausbildung}

Merkmal eines Marktes mit asymmetrischen Informationen ist, dass ein Akteur mehr Informationen hinsichtlich eines Verhaltens hat als ein anderer Akteur. Diese zusätzliche Information kann bzw. möchte dieser seinem Gegenüber nicht mitteilen. Bspw. verfügt der Ausbildungsbetrieb über einen Informationsvorsprung gegenüber anderen Nichtausbildungsbetrieben, den er während der dualen Berufsausbildung erzielt. Informationsasymmetrien entstehen vor allem durch die Existenz von unbeobachtbaren Faktoren. Dabei handelt es sich bspw. um zu Beginn der Ausbildung vorhandene informelle Fähigkeiten. Derartiges Humankapital ist angeboren, wird aber auch durch entsprechende Sozialisation erworben (Schweri 2006: 69). Daneben kann die individuelle Produktivität der Auszubildenden von Unternehmen auf dem externen Arbeitsmarkt nicht beobachtet werden, da sie auf diesem noch nicht aktiv waren. Aufgrund der mangelnden Kenntnis über die tatsächlichen Fähigkeiten der Auszubildenden bietet das Unternehmen für jeden Arbeitnehmer dieselben Ausbildungsinhalte an (Acemoglu/Pischke 1998). ${ }^{29}$ Dabei spielt bei der Anwendung und Umsetzung des formellen Humankapitals informelles Wissen eine entscheidende Rolle. Der Produktionsbeitrag nach der Ausbildung ergibt sich daher aus dem informellen Humankapital $\eta$, das die Begabungen widerspiegelt und somit zunächst nicht beobachtbar ist, sowie aus dem formellen Humankapital, das nun in der Funktion $\alpha(\tau)$ dargestellt ist. Entsprechend wird der Produktionsbeitrag von $f=\alpha(\tau) \eta$ bestimmt. Der ausgezahlte Lohn $\omega(\tau, \eta)$ hängt ebenfalls von den formellen und informellen Fähigkeiten der Ausgebildeten ab. Hierbei weisen Individuen mit geringen (informellen) Fähigkeiten einen Wert von null auf, wobei $\eta=0$ gilt; für Individuen mit hohen (informellen) Fähigkeiten gilt dagegen $\eta=1$ (Acemoglu/Pischke 1998). Innerhalb der Ausbildungsphase werden Informationsasymmetrien abgebaut. Arbeitgeber sind danach in der Lage, die Fähigkeiten (Begabungen) der Ausbildungsabsolventen einzuordnen und entsprechend $\mathrm{zu}$ entlohnen. Unter der Annahme, dass die Ausbildungsbetriebe die vollständige Verhandlungsmacht haben, bekommen Arbeitnehmer mit geringen Fähigkeiten einen Lohn von $\omega(\tau, \eta=0)=0$; Arbeitnehmer, die bessere Fähigkeiten aufweisen,

${ }^{29}$ Dies bedeutet, dass keine Unterschiede bei der formalen Bildung bestehen. Darüber hinaus haben die neu eingestellten Arbeitnehmer während der ersten Periode eine Produktivität von null. 
erhalten einen Lohn mindestens in Höhe von $\omega(\tau, \eta=1)=v(\tau)$ (Acemoglu/Pischke 1999b: 557). Der Informationsvorsprung verursacht ein Nachfragemonopol für den Ausbildungsbetrieb, weil nur das Ausbildungsunternehmen die tatsächlichen Fähigkeiten der Ausbildungsabsolventen, die zum Teil über die Ausbildungsinhalte der Ausbildungsordnung hinausgehen, kennt. ${ }^{30}$ Bei einem Betriebswechsel können Ausbildungsabsolventen, wegen der Annahme existierender Informationsasymmetrien, ihr Wissen und ihre Fähigkeiten den Unternehmen auf dem externen Arbeitsmarkt nicht ausreichend signalisieren. Dies führt bei einem Betriebswechsel zu einer Verschlechterung der Position, sodass Fachkräfte einen geringen Anreiz, haben zu einem anderen Unternehmen zu gehen. Entsprechend der verschlechterten Position auf dem externen Arbeitsmarkt ist es dem auszubildenden Betrieb möglich, einen Lohn unterhalb der Produktivität durchzusetzen, welcher gleichzeitig den Marktlohn übersteigt. Für die Bedingung der Lohnkompression gilt nun $v(\tau)<\omega(\tau, \eta=1)<f=\alpha(\tau) \eta$ (Acemoglu/Pischke 1999b: 557). Durch den Verbleib kann der Ausbildungsbetrieb Erträge aus der Ausbildung erzielen und hat somit den Anreiz, weiter in $\mathrm{Hu}-$ mankapital des Arbeitnehmers zu investieren (Smits/Stromback 2001; Acemoglu/Pischke 1998).

Trotz der hohen Standardisierung existieren innerhalb der dualen Berufsausbildung Informationsasymmetrien, weil sich unterschiedliche Betriebe beteiligen. Diesen wird bei der Vermittlung von Humankapital über die Ausbildungsordnungen hinaus ein gewisser Spielraum zugestanden. Für das Absolvieren einer Ausbildung werden Qualifikationsanforderungen in unterschiedlicher Höhe gestellt. Vor diesem Hintergrund werden Schulabsolventen bereits an der ersten Schwelle ausgewählt, denn neben den formellen Anforderungen müssen auch die betrieblichen Anforderungen am Ende der Ausbildung erreicht werden. Da diese bei Ausbildungsbetrieben mit langfristigem Zeithorizont höher liegen als bei denjenigen mit kurzfristigem Zeithorizont, stellen erstere Betriebe die formal besseren Schulabsolventen ein. Um die bestehenden Informationsasymmetrien $\mathrm{zu}$ reduzieren, wird davon ausgegangen, dass bei besseren Schulabsolventen auch die unbeobachtbaren Fähigkeiten positiv ausgeprägt sind. Infolgedessen steigen die Chancen auf eine betriebliche Ausbildung in entsprechenden Betrieben bei höherer Bildung, weil nach Rosen (1976) fähigere Auszubildende nied-

${ }^{30}$ Während der Ausbildungsphase ist das Unternehmen zwar in der Lage, Informationsasymmetrien abzubauen, aber das Problem der Adversen Selektion ist damit keineswegs behoben. Dem entgegen wirken die Unternehmen, indem sie einen Effizienzlohn festlegen, der für die Auszubildenden bzw. Ausbildungsabsolventen einen Anreiz bietet, sich weiterhin anzustrengen. Um auch in diesem Fall eine Lohnkompression zu erreichen, wird angenommen, dass die Höhe des Effizienzlohnes unabhängig von den Fähigkeiten ist. In diesem Fall ist nur die interne Lohnstruktur verzerrt, was zur Ausbildungsbereitschaft führt. In den bisherigen Überlegungen von Acemoglu/Pischke (1999a, 1999b, 1998) veranlasst jedoch die Verzerrung der externen Lohnstruktur die Unternehmen, in allgemeines Humankapital zu investieren. Dadurch resultiert entsprechend eine komprimierte interne Lohnstruktur (Acemoglu/Pischke 1999b: 561f.). 
rigere Kosten für Schul- bzw. Ausbildung für eine Arbeitsstelle mit gegebenen Anforderungen verursachen. Gegenüber unbeobachtbaren Faktoren besteht jedoch Unsicherheit, weswegen die Ausbildung zunächst auf einem befristeten Vertrag basiert, der zu Beginn Transaktionskosten verursacht und nach Cappelli (2002) daher die Einstellung besserer Schulabsolventen fördert. So ist die duale Berufsausbildung als Informationsgewinnungsprozess zu betrachten, weil der Betrieb anhand des formalen Bildungsstandes die Auszubildenden einstellt, jedoch über die unbeobachtbaren Merkmale erst während der Beschäftigung Kenntnis erlangt (Autor 2001; Clark 2001; Katz/Ziderman 1990; Franz/Soskice 1995). Daher müssen die unbeobachtbaren bzw. informellen Fähigkeiten, wie Zuverlässigkeit, Auffassungsgabe etc., ebenfalls berücksichtigt werden. Hierbei werden diese Fähigkeiten weiterhin durch das Niveau an Fähigkeiten, das innerhalb des Ausbildungsbetriebes herrscht, determiniert. Nach Asplund (2004) sowie Blundell et al. (1999) bildet bspw. ein produktives Arbeitsumfeld damit einen weiteren Anreiz für die Ausbildungsbereitschaft. Begründet liegt dies im Zusammenhang zwischen der Produktivität eines einzelnen Arbeitnehmers und der Arbeitsproduktivität der Belegschaft. Verfügt das Unternehmen bereits über ein hohes Produktivitätsniveau und somit über eine hohe Arbeitsqualität, hat das Unternehmen einen Anreiz, Arbeitskräfte auszubilden, weil die Auszubildenden von dem Wissen der Fachkräfte profitieren und lernen können (Brunello 2001: 3). Dadurch erreichen die Auszubildenden in dem Ausbildungsbetrieb eine höhere Produktivität, begründet durch eine höhere Qualifikation innerhalb der Belegschaft gegenüber anderen Unternehmen mit weniger guten ausgebildeten Fachkräften (Asplund 2004: 50; Booth/Zoega 2000: 11). Das Unternehmen erzielt dadurch höhere Bildungserträge, was dazu führt, dass die Entlohnung des Auszubildenden bei einem Betriebswechsel weit unterhalb seiner Produktivität liegt. Ein Großteil der Produktivität und damit auch des Humankapitals, welches zu einem gewissen Teil auf die Belegschaft zurückgeführt werden kann, ist für außen stehende Unternehmen nicht beobachtbar. Aufgrund dieser Informationsasymmetrien sind die Ausbildungsbetriebe bereit, über die Mindestanforderungen hinaus auszubilden, weil Absolventen ihre Arbeitsmarktposition bei einem freiwilligen Wechsel verschlechtern.

\subsection{Allgemeines und spezifisches Humankapital als Komplementär- güter}

Wegen der Organisation der dualen Berufsausbildung ist es unerlässlich, die simultane Vermittlung von allgemeinem bzw. berufsspezifischem und betriebsspezifischem Humankapital zu betrachten. Die allgemeinen und berufsspezifischen Kenntnisse werden durch den betrieblichen Teil dieser Ausbildung, in dem eine Vermittlung von betriebsspezifischem Wissen stattfindet, ergänzt. Daher fördern der Aufbau und die Struktur der betrieblichen Arbeitsprozesse sowie die Bedienung von Maschinen die Vermittlung der betriebsspezifischen Humankapitalkomponenten aufgrund der Integration in den Produktionsprozess (Rei- 
chenbach 2001: 164; Neubäumer 1999: 69f.; Soskice 1994: 45). Bei der Analyse der dualen Berufsausbildung kann daher die Vermittlung von allgemeinem und betriebsspezifischem Humankapital nicht getrennt betrachtet werden. Die Existenz von betriebsspezifischem Humankapital in den Ausbildungsinhalten fördert die Bereitschaft der Unternehmen, in betriebliche Ausbildung zu investieren. Diese Art Wissensvermittlung unterliegt dann mehr dem Zufallsprinzip und kann die Informationsasymmetrien gegenüber anderen Unternehmen verstärken, weil sie über die festgelegten Ausbildungsinhalte hinausgeht und es dem Betrieb obliegt, welche Art und welche Höhe er an zusätzlichem Wissen an die Auszubildenden weitergibt. Zudem kann der Ausbildungsbetrieb ex ante festlegen, wie viel betriebsspezifisches Wissen angeeignet werden soll. In beiden Fällen wird die betriebliche Investition in Humankapital positiv beeinflusst. Aufgrund der Ausbildungsordnungen, die den Betrieben Mindestqualifikationen vorgeben und dafür sorgen, dass der duale Berufsabschluss allgemein anerkannt wird, ist der Anteil der betriebsspezifischen Komponente nach oben begrenzt. Unter diesen Rahmenbedingungen muss der Produktionsbeitrag schließlich um die betriebsspezifische Komponente erweitert werden. Der Output kann durch

$$
f(s, \tau, \theta)=f^{s}(s ; \theta)+f^{\tau}(\tau ; \theta)
$$

beschrieben werden (Kessler/Lülfesmann 2002: 5). Daraus ergibt sich, dass die Produktivität am Ende der Ausbildung, $f$, vom betriebsspezifischen, $(s)$, und vom allgemeinen Humankapital, $(\tau)$, abhängt. Darüber hinaus beeinflusst der Parameter $\theta$ den Output, der Auskunft bspw. über einen Nachfrageschock gibt. Dieser Parameter ist nicht zu Beginn der Ausbildung, sondern erst nach einer gewissen Zeit innerhalb der Ausbildungsphase bekannt. Findet nun keine allgemeine Ausbildung statt, bestimmt sich die Produktivität nach der Ausbildung lediglich aus der Produktivität, die aus der betriebsspezifischen Ausbildung $f^{s}$ heraus resultiert und umgekehrt (Kessler/Lülfesmann 2002: 6). In diesem Fall sind die beiden Humankapitalkomponenten als Substitute zu betrachten.

Für die Beendigung des Beschäftigungsverhältnisses bedeutet dies, dass auch dem Unternehmen der Anteil des betriebsspezifischen Humankapitals verloren geht. Entsprechend erhält der Ausbildungsabsolvent bei einem Betriebswechsel einen Lohn, welcher der Höhe seiner Produktivität, resultierend aus dem allgemeinen bzw. dem formalen Wissensstand, entspricht (Kessler/Lülfesmann 2002: 8). Somit haben die Auszubildenden einen Anreiz, im Unternehmen zu verbleiben, da ihnen sowohl ein Teil der Erträge aus allgemeinem und betriebsspezifischem Humankapital zusteht. Dasselbe gilt für das Unternehmen, das nun auch von allgemeinem Humankapital profitiert. ${ }^{31}$ So werden auch Anreize für Auszu-

${ }^{31}$ Durch die gemeinsame Vermittlung der beiden Humankapitalkomponenten erhöhen sich aber auch die Kosten der Investition um den Anteil für die betriebsspezifische Bildung. Die Ausbildungskosten $c(\tau, s)$ eines Betriebes hängen nun von der allgemeinen Bildung $(\tau)$ und von der betriebsspezifischen Bildung $(s)$ ab. 
bildende selbst gesetzt, in betriebsspezifisches Humankapital zu investieren (Balmaceda 2005: 117).

Die gemeinsame Vermittlung der Humankapitalarten reicht aber für die Komplementaritätseigenschaft nicht aus. Vielmehr muss ein Zusammenhang zwischen den beiden Arten bestehen. So liegt Komplementarität zwischen allgemeinem und betriebsspezifischem Humankapital vor, wenn mit zunehmendem allgemeinem Humankapitalstock bei gegebenem betriebsspezifischem Humankapitalbestand die Produktivität stärker als der Marktlohn steigt. Dies entspricht nach Acemoglu und Pischke (1999a, 1999b, 1998) der relativen Lohnkompression, weil ein hohes allgemeines Humankapitalniveau die Aneignung betriebsspezifischen Wissens wiederum positiv beeinflusst. Umgekehrt weisen Steigerungen des betriebsspezifischen Humankapitals bei gegebenem allgemeinem Humankapitalstock ebenfalls steigende, aber abnehmende Erträge auf (siehe Abbildung 5).

Abbildung 5: Vermittlung von komplementärem spezifischem und allgemeinem Humankapital

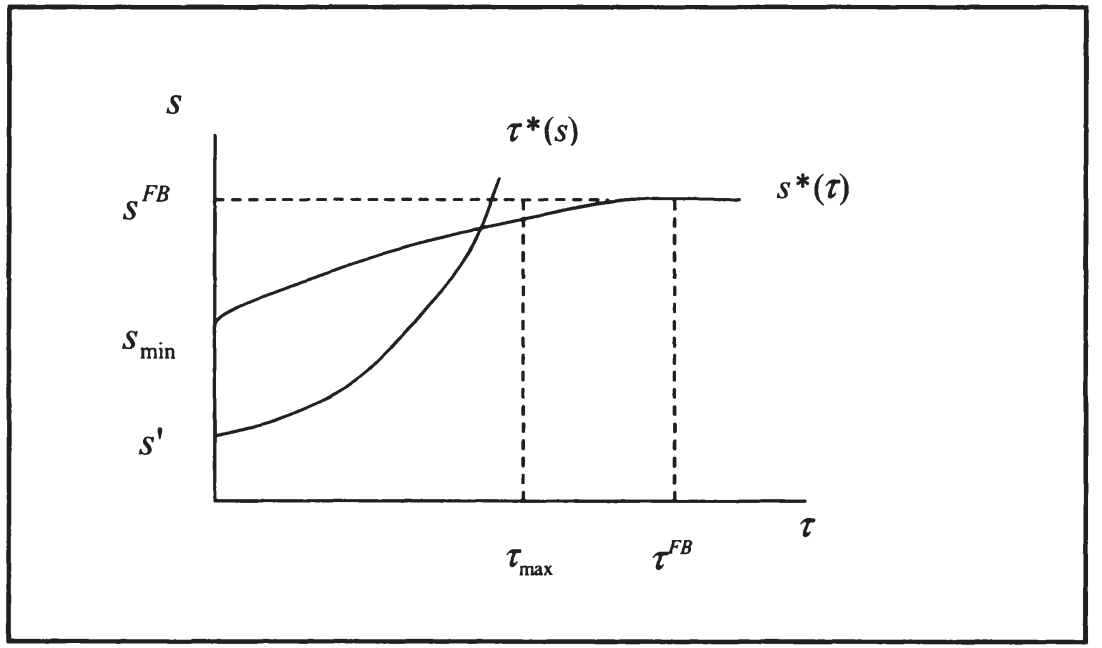

Quelle: Kessler/Lülfesmann (2002)

Infolgedessen genießen in der Regel die Auszubildenden, die über ein hohes Humankapital verfügen, eine weitere und auch höhere Ausbildung als Personen mit geringerem Bildungsstand, weil eine Vermittlung von betriebsspezifischem Humankapital erfolgen kann. Bei Vorliegen von Komplementarität zwischen allgemeinem und betriebsspezifischem Humankapital steigen die Bildungserträge und die Bereitschaft der Betriebe für eine allgemeine Ausbildung mit dem Anteil der Vermittlung von betriebsspezifischem Humankapital (Acemoglu/Pischke 1999b: 560). Diese Bereitschaft kann durchaus auch von der $\mathrm{Zu}$ - 
sammensetzung des physischen Kapitals abhängen. Kapitalintensive Unternehmen legen Wert auf gut ausgebildete Fachkräfte. Schließlich wirkt sich die Art der Ausbildungsinhalte auf die Fluktuation aus. Aufgrund der Komplementarität von betriebsspezifischem und allgemeinem Humankapital sinkt die Fluktuationsrate bei steigendem allgemeinem Humankapital (Acemoglu/Pischke 1999b: 560), weil aufgrund der Komplementarität auch der betriebsspezifische Bildungsanteil wächst. Jedoch beeinflusst aber auch eine exogen vorgegebene Fluktuationsrate die Bildungsinvestition: Ist diese hoch, wird Ausbildungsabsolventen mehr betriebsspezifisches Humankapital vermittelt, wodurch das Verlassen des Betriebes weniger attraktiv erscheint. So ermöglicht auch die Komplementarität der beiden Humankapitalarten eine Entlohnung unterhalb der tatsächlichen Produktivität.

Zugleich verhindert gerade die gemeinsame Vermittlung von allgemeinem Wissen bei gegebenem betriebsspezifischem Humankapital das Problem des Hold-up ${ }^{32}$ in Form einer Unterinvestition in Bildung (Balmaceda 2005; Williamson 1975), da die Unternehmen durch die Investition in allgemeines Wissen Bildungserträge realisieren können (Leuven 2005; Kessler/Lülfesmann 2002; Blinder/Krüger 1996). Die Unterinvestition in Humankapital wird wegen des negativen Zusammenhangs zwischen der Fluktuationsrate und der Höhe der Bildung (bei Komplementarität von betriebsspezifischem und allgemeinem Humankapital) vermindert. Um den Hold-up zu verhindern, müssen am Ende der Ausbildung Vertragsverhandlungen stattfinden. Dabei haben sowohl die Ausbildungsbetriebe als auch die Ausbildungsabsolventen verschiedene Alternativen, sich auf dem externen Arbeitsmarkt umzusehen. Entsprechend hängt die Verhandlungsmacht von der Zusammensetzung des Humankapitalbestandes ab. Je höher die betriebsspezifische Humankapitalkomponente, desto schwächer ist die Verhandlungsposition der Unternehmen, da sie die Bildungserträge nicht vollständig realisieren können, sofern die Ausbildungsabsolventen wechseln (Leuven 2005: 99). In diesem Fall investiert das Unternehmen in allgemeines Humankapital (Kessler/Lülfesmann 2002: 11). Darüber hinaus steigt das Interesse an einer eigenen Ausbildung, wenn aufgrund der verwendeten Technologien bzw. aufgrund der Komplexität der Arbeitsprozesse die externe Rekrutierung von Fachkräften teurer ist. In diesem Fall wird die Besetzung der Stellen aufwendiger und die Einarbeitungszeit verlängert sich. Daher ist eine Ausbildung günstiger (Genda et al. 2001).

Für die Ausbildungsbetriebe ist es daher möglich, die Erträge der verbleibenden Nettoinvestitionen der Ausbildung zu realisieren. Deshalb ist ein langfristi-

\footnotetext{
${ }^{32}$ Hold-up entsteht bei Unsicherheit nach Vertragsabschluss. Der Ausbildungsbetrieb (Agent) verpflichtet sich, dem Auszubildenden (Prinzipal) Rahmenbedingungen zu schaffen, die eine ordentliche Ausbildung gewährleisten. Wenn diese nicht vorherrschen, erfolgt eine Vertragsauflösung von Seiten des Auszubildenden. Durch die Vermittlung des betriebsspezifischen zusätzlich zum allgemeinen Humankapital kann dem Hold-up (der überraschenden Vertragsaufkündigung) entgegengewirkt werden.
} 
ges Beschäftigungsinteresse umso größer, je mehr der Anteil des betriebsspezifischen Humankapitals an der Ausbildung ausmacht. Dies erfordert zugleich aber auch einen höheren allgemeinen Bildungsstand. Dadurch verstärkt sich die Bildung von internen Arbeitsmärkten (Sengenberger 1987). ${ }^{33}$ Innerhalb diesen herrscht ein höheres Ausbildungsgleichgewicht, was zu einer niedrigeren Abgangsrate aus dem Ausbildungsbetrieb führt (Acemoglu/Pischke 1998).

Die Notwendigkeit, dass trotz dieser Komplementarität zwischen Humankapitalarten dennoch in allgemeine Bildung investiert wird, muss durch die Existenz von entsprechenden Institutionen ergänzt werden (Kessler/Lülfesmann 2002: 22), die im System der dualen Berufsausbildung durch Gewerkschaften, Industrie- und Handelskammern sowie Handwerkskammern, aber auch durch die lange Tradition der betrieblichen Ausbildung gegeben sind.

\subsubsection{Institutionelle Rahmenbedingungen der Ausbildung}

Die duale Berufsausbildung in Deutschland funktioniert vor allem deswegen, weil verschiedene öffentlich-rechtliche und privat-rechtliche Institutionen den Inhalt der verschiedenen Ausbildungsberufe mitgestalten und koordinieren. Somit wird der Berufsausbildung ein reglementierter Rahmen vorgegeben (Schweri 2006: 51f.). Hierbei bestimmen neben den Informationsasymmetrien auch die Gewerkschaften die Höhe der Ausbildungsintensität. Zudem beeinflusst die Position des Betriebes auf dem Gütermarkt die Art der Ausbildungsbereitschaft.

\subsection{Existenz von Gewerkschaften}

Die Gewerkschaften tragen zur Gewährleistung der Ausbildungsbereitschaft in allgemeines Humankapital bei. Sie entscheiden in ihren jährlichen Verhandlungen auf regionaler sowie sektoraler Ebene über die Höhe der Ausbildungsvergütungen und nehmen dadurch Einfluss auf die Lohnstruktur der Unternehmen (Fitzenberger/Kohn 2005: 1; Dustmann/Schönberg 2004: 3).$^{34}$ Dies impliziert, dass Gewerkschaften u.a. die Höhe der Ausbildungskosten bestimmen, jedoch nicht direkt die Bildungsintensität bzw. die Ausbildungsinhalte aushandeln (Bispinck et al. 2002). Die Löhne der Auszubildenden machen ungefähr die

${ }^{33}$ Interne Arbeitsmärkte sind von einem hohen Qualifikationsniveau der Mitarbeiter gekennzeichnet. Entsprechend liegt eine hohe Beschäftigungssicherheit vor. Mobilität ist hauptsächlich innerhalb des internen Segments vorhanden, nicht aber zwischen internem und externem bzw. berufsspezifischem Segment. Interne Arbeitsmärkte finden sich vor allen in großen Unternehmen. Hier ist insbesondere die Kernbelegschaft diesem Teilbereich zuzuordnen (Sengenberger 1987; Doeringer/Piore 1971).

${ }^{34}$ Dabei werden für die Arbeitnehmer, die unterschiedlichen Berufsgruppen angehören und auch unterschiedliche Bildungsniveaus aufweisen, die jeweiligen Löhne ausgehandelt. Prinzipiell gelten die Tariflöhne ausschließlich für Arbeitnehmer, die Mitglied einer Gewerkschaft sind. Jedoch gibt es in der Praxis keinen Unterschied. Gehört das Unternehmen einem Arbeitgeberverband an, gelten die Tariflöhne auch für Nichtgewerkschaftsmitglieder (Fitzenberger/Kohn 2005; Dustmann/Schönberg 2004). 
Hälfte der gesamten Ausbildungskosten aus (Beicht et al. 2004), weswegen die Rolle der Gewerkschaften im Rahmen der dualen Berufsausbildung nicht vernachlässigt werden darf. Denn die gewerkschaftlich gebundenen Ausbildungsbetriebe sind verpflichtet, mindestens die tariflichen Ausbildungsvergütungen $\mathrm{zu}$ bezahlen. Diese übernehmen dadurch die Funktion von Mindestlöhnen (Dustmann/Schönberg 2004; 2002). Entsprechend werden bei der Lohnverhandlung die Ausbildungsvergütungen $\omega$ zunächst unabhängig vom Bildungsniveau ausgehandelt (Acemoglu/Pischke 1999b: 565). Unter der Annahme, dass Unternehmen nur bei vorhandener Lohnkompression bereit sind auszubilden, liegt hier ein hoher Anreiz vor, in Bildung zu investieren. Da die Löhne durch die Gewerkschaften teilweise exogen vorgegeben werden, verbleibt als beeinflussbare Größe für die Betriebe die Produktivität, um die Differenz zwischen den beiden Größen zu maximieren. Dies erfolgt durch höhere betriebliche Bildungsinvestitionen. Die Entlohnung steigt weniger stark als die Produktivität, weil nur der Lohn nach unten bindend ist, Erhöhungen aber freiwilliger Natur sind. Dadurch nimmt die Verzerrung der Lohnstruktur mit zunehmendem Bildungsstand zu (Beckmann 2002a: 190), weil die besser ausgebildeten Arbeitnehmer zwar einen höheren Lohn erhalten, dieser aber unter ihrer wahren Produktivität liegt und mit dieser nicht proportional steigt. Die Lohnkompression ist somit durch den geltenden Tariflohn im unteren Bereich kaum bzw. gar nicht vorhanden (Howell/Hübler 2005: 8), nimmt aber zu, je mehr in Bildung investiert wird, weil die Entlohnung weniger stark steigt.

Die Organisation der dualen Berufsausbildung gibt Mindestanforderungen der Auszubildenden hinsichtlich des Humankapitalniveaus vor, was wiederum ein hohes Bildungsniveau impliziert. Entsprechend tendieren diese Unternehmen dazu, über die Mindeststandards hinaus in Bildung zu investieren. Denn durch die höheren Bildungsinvestitionen kann die Differenz zwischen der Produktivität und den tariflich ausgehandelten Ausbildungsvergütungen vergrößert werden. Daher wird die Ausbildung in Unternehmen mit Tarifbindung höher sein als in Ausbildungsbetrieben ohne tarifliche Bindung, die Absprachen mit der betrieblichen Arbeitnehmervertretung oder den Arbeitnehmern selbst treffen (Dustmann/Schönberg 2004). Infolgedessen werden Gewerkschaften über die Lohnverhandlungen den Bildungsstand der Ausbildungsbetriebe beeinflussen und aufgrund der Externalitäten positiv zum gesamtwirtschaftlichen Bildungsniveau beitragen.

Weil nicht alle Ausbildungsbetriebe an tariflich vereinbarte Regelungen gebunden sind, gelten daher unterschiedliche Lohnniveaus. Dies schränkt die Mobilität der Ausbildungsabsolventen ein. Ausbildungsabsolventen werden bei einem freiwilligen Verlassen in jedem Fall Lohneinbußen hinnehmen müssen, die zunehmen, wenn sie ihren gewerkschaftlich gebundenen Ausbildungsbetrieb verlassen und $\mathrm{zu}$ einem nicht tarifgebundenen Unternehmen wechseln. Nicht tarifgebundene Unternehmen entlohnen die Fachkräfte nach dem Marktlohn, der einerseits unterhalb der tatsächlichen Produktivität und andererseits unter den 
tariflich ausgehandelten Löhnen liegt. Durch diese Lohndifferenzen zwischen den Unternehmen kann auch das Poaching ${ }^{35}$ verhindert werden. Durch die Existenz von Gewerkschaften werden somit Rahmenbedingungen geschaffen, die zum einen den Bildungsstand beeinflussen und zum anderen die Mobilität nach der dualen Berufsausbildung verringern und entsprechende Voraussetzungen für die Realisation von Bildungserträgen für die Betriebe schaffen (Acemoglu/Pischke 1999b: 566; Booth/Chatterji 1998: 340). Trotz der institutionellen Rahmenbedingungen, die durch Arbeitnehmerverbände gesetzt werden, und der besseren Entlohnung im Ausbildungsbetrieb, welche die Mobilität auf Seiten der Ausbildungsabsolventen reduziert, zeigen Studien, dass Ausbildungsabsolventen ihren Ausbildungsbetrieb häufig nach einer bestimmten Zeit verlassen. So gehen nach Franz und Zimmermann (1999) 55\% der Ausbildungsabsolventen bereits nach spätestens zwei Jahren aus dem Ausbildungsbetrieb. Betrachtet man Unternehmen mit über 1000 Beschäftigten, bei denen von einem hohen Organisationsgrad auszugehen ist, kann eine höhere Verbleibsquote nach zwei Jahren festgestellt werden, die sich auf knapp über $60 \%$ der Ausbildungsabsolventen beläuft. Nach Harhoff und Kane (1997) müssen die Ausbildungskosten auf jeden Fall zu dem Zeitpunkt des Verlassens des Ausbildungsbetriebes gedeckt sein, da sich ansonsten eine Humankapitalinvestition für die Unternehmen nicht lohnen würde. ${ }^{36}$ Grundsätzlich widerspricht dies nicht den Annahmen des humankapitaltheoretischen Ansatzes.

Zusammenfassend kann gesagt werden, dass Gewerkschaften einen direkten Einfluss auf die Ausbildung und die Höhe des Bildungsstandes ausüben. Da der durch die Gewerkschaften ausgehandelte Lohn, der im Betrieb gilt, in Anlehnung an die Gewerkschaftsmodelle den Marktlohn übersteigt (Gerlach/Stephan 2005: 6), wird der Anreiz der Ausbildungsabsolventen, den Betrieb zu verlassen, reduziert. Daher sind an diese Löhne gebundene Unternehmen auch bereit, mehr in Humankapital zu investieren als nicht tariflich gebundene Unternehmen. $\mathrm{Zu}$ dem steigt die Ausbildungsqualität, weil Unternehmen in erster Linie wegen des eigenen Fachkräftebedarfs ausbilden. So führt die Rigidität der stark koordinierten (Teil-)Märkte zu einer engeren Bindung zwischen Arbeitgebern und Arbeitnehmern (Blossfeld et al. 2005: 13). Dies ist Voraussetzung für eine Investition in allgemeines Humankapital. Bei tariflich gebundenen Unternehmen handelt es sich meist um Großunternehmen und auch um mittelständische Betriebe, die ebenfalls in den Hochqualitätssegmenten der Produktmärkte vertreten sind. Daher sind weiterhin die institutionellen Rahmenbedingungen auf dem Gütermarkt zu berücksichtigen.

\footnotetext{
${ }^{35}$ Unternehmen, die sich nicht an der Ausbildung beteiligen, werben gut ausgebildete Fachkräfte bzw. Ausbildungsabsolventen von Ausbildungsunternehmen ab (Stevens 2001; Alewell/Richter 1998).

${ }^{36}$ Entgegen Becker (1962) und Acemoglu/Pischke (1999a; 1999b; 1998), die einen unendlichen Zeithorizont annehmen, gehen Harhoff und Kane (1997) von einem begrenzten Zeitraum aus.
} 


\subsection{Unvollkommene Produktmärkte}

Durch die Beteiligung der Betriebe an der dualen Berufsausbildung darf die Analyse nicht auf den Arbeitsmarkt und die entsprechenden Regulierungen reduziert werden. Vielmehr ist die Ausbildungsbereitschaft mit den Rahmenbedingungen auf dem Gütermarkt verknüpft (Gersbach/Schmutzler 2001). Da sich die Ausbildungsbetriebe in unterschiedlichen Wettbewerbsbedingungen bzw. positionen hinsichtlich der produzierten Güter befinden, ist die Ausbildung eigener Fachkräfte von Vorteil. Der Betrieb bleibt damit konkurrenzfähig und spart durch die externe Rekrutierung entstehende Einarbeitungskosten ein. Hierzu benötigen sie langfristig gut ausgebildete Fachkräfte, die sowohl hohes allgemeines als auch betriebsspezifisches Humankapital aufweisen (Soskice 1994: 37). Betriebsspezifisches Humankapital ist vor allem aufgrund der hergestellten Produkte und den daraus notwendigen Arbeitsprozessen erforderlich. Als Begründung für die Investitionen in allgemeines Humankapital wird daher neben einem unvollkommenen Arbeitsmarkt ein unvollkommener Gütermarkt angenommen. Dieser ist durch verschiedene Größen der Teilproduktmärkte und durch unterschiedliche Produktdifferenzierungen gekennzeichnet (Gersbach/Schmutzler 2006: 4). Ausbildungsbetriebe können in zweierlei Hinsicht von der Ausbildung profitieren: Erstens steigert jeder weitere Auszubildende, der im Betrieb verbleibt, die betriebliche Produktivität. ${ }^{37}$ Zweitens sinken die Löhne bei zunehmender Ausbildungsbeteiligung, weil das Angebot an ausgebildeten Fachkräften steigt. Entsprechend sinkt die Wettbewerbsfähigkeit der Konkurrenzunternehmen und infolgedessen verschlechtert sich deren Stellung auf dem Gütermarkt (Gersbach/Schmutzler 2006). Deshalb ist nur eine geringe Anzahl an Ausbildungsbetrieben auf dem Markt, die in Konkurrenz zueinander stehen. Steigt die Anzahl der Unternehmen in dem jeweiligen Sektor, sinkt die Ausbildungsbereitschaft. Jedoch nimmt bei einer zu geringen Anzahl an ausbildenden Betrieben die Bereitschaft aufgrund der abnehmenden Bildungserträge ebenfalls ab. Dies liegt darin begründet, dass bei zunehmenden Einstellungen von Ausbildungsabsolventen geringere Bildungserträge erzielt werden, das heißt, ein Ausbildungsbetrieb allein würde immer negative Effekte aus der Ausbildung ziehen, da maximale Bildungserträge innerhalb einer Industrie Gleichverteilung der ausgebildeten Fachkräfte voraussetzen (Gersbach/Schmutzler 2006: 3).

Kritisch am Erklärungsansatz für die Ausbildungsbereitschaft durch die Situation auf den Produktmärkten bleibt, dass lediglich auf die Intensität der Ausbildung eingegangen wird. Um aber den Verbleib an der zweiten Schwelle erörtern zu können, ist die Berücksichtigung der Qualität sowie die Art des vermittelten Humankapitals erforderlich. Schließlich spielt die Differenzierung der verschie-

${ }^{37}$ Gleichzeitig steigt auch die Produktivität der in Konkurrenz zum Ausbildungsbetrieb stehenden Unternehmen, was wiederum als negativer Effekt hinsichtlich der betrieblichen Ausbildungsbereitschaft zu interpretieren ist. In dieselbe (negative) Richtung weist der durch die Zunahme der Ausbildung angestiegene Lohn (Gersbach/Schmutzler 2001: 4). 
denen Produkte innerhalb der Teilgütermärkte hinsichtlich der Ausbildungsbereitschaft eine Rolle. Für Unternehmen mit Standardprodukten sind bei der Herstellung geringere betriebsspezifische sowie allgemeine Kenntnisse erforderlich als für Unternehmen, die Produkte oder Dienstleistungen im Hochpreissegment herstellen und entsprechend auch einen hohen Anspruch an die Qualität stellen. Unternehmen für Qualitätsprodukte sind somit freiwillig bereit, in Ausbildung zu investieren, da sie entsprechende Fachkräfte zukünftig benötigen, um weiterhin in der Marktnische bestehen zu können. Deshalb benötigen die Ausbildungsbetriebe Fachkräfte, die die entsprechende Unternehmensphilosophie, die unter betriebsspezifisches Humankapital zu subsumieren ist, verinnerlichen. Dies geschieht während der Ausbildung am effizientesten. Darüber hinaus werden in dem entsprechenden Marktbereich in der Regel die neuesten Technologien eingesetzt, die technologie- und in diesem Sinne auch betriebsspezifisches Wissen erfordern. ${ }^{38}$ Schließlich gewährleistet, wie in Kapitel 2.1 beschrieben, die duale Berufsausbildung den flexiblen Einsatz von Arbeitskräften, sobald Nachfrageverschiebungen auf dem Gütermarkt auftreten. Dadurch treten Spillover-Effekte ausgehend vom Gütermarkt auf den Ausbildungsmarkt auf, welche durch Institutionen auf den Gütermärkten entstehen. Teilgütermärkte, die aufgrund des Wettbewerbs schnelle Veränderungen im Produktionsprozess durchleben, wie technologische Produkte oder auch Qualitätsprodukte, benötigen gut qualifizierte Arbeitskräfte. Der Aspekt der Komplementarität zwischen betriebsspezifischem und allgemeinem Humankapital ist daher von besonderer Bedeutung. Nach Estevez-Abe et al. (2000) sind in einem hoch spezialisierten Gütermarkt, wie es der deutsche ist, die Anreize besonders hoch, bereits während der Schulzeit in allgemeines Humankapital zu investieren. So kann während der Ausbildung einfacher das (technologie-) spezifische Wissen erlernt bzw. vermittelt werden und die Ausbildungsabsolventen flexibel innerhalb des Betriebes eingesetzt werden (Soskice 1994: 38). Damit wird der Zugang zu wettbewerbsfähigeren Jobs gewährleistet, welcher ein hohes durchschnittliches allgemeines Humankapital voraussetzt. Dadurch kann aber wiederum auch spezifisches $\mathrm{Hu}-$ mankapital, das im Kontext der Betrachtung des Gütermarktes vor allem güterspezifisches Wissen einschließt, vermittelt werden. Infolgedessen wird die Ausbildungsbereitschaft der Unternehmen gefördert, wobei in Nischen mit hohem Wettbewerbsdruck mehr in Ausbildung investiert wird als auf dem Markt für Standardprodukte. ${ }^{39}$ Folglich weisen Ausbildungsabsolventen aus ersteren Un-

${ }^{38}$ Auf dem Gütermarkt sind auch verstärkt ausländische Firmen vertreten, die neue Technologien auf den Arbeitsmarkt etablieren. Dazu bedarf es entsprechend qualifizierter Fachkräfte, die auf eine sich ändernde Nachfrage und Produktion der in der Regel wenig standardisierten Produkte flexibel reagieren müssen. Junge Erwachsene, die in ausländischen Firmen ausgebildet werden, verfügen daher über einen hohen Bildungsstand (Görg et al. 2007).

${ }^{39}$ Dies verdeutlicht auch der Ansatz des Lagerhaltungsmodells. Der Markt für Standardprodukte ist durch eine Massenproduktionsstrategie gekennzeichnet, die keine umfassenden Qualifikationen benötigt, weil sich die erforderlichen Fähigkeiten durch „Learning by doing“ aneignen lassen. Dagegen sind Unternehmen mit einem Produktregime „flexibler Spezialisie- 
ternehmen einen höheren Bildungsstand und eine höhere Ausbildungsqualität auf als Ausbildungsabsolventen aus letzteren Betrieben.

\subsection{Zwischenfazit}

Es wurde deutlich, dass die unterschiedlichen Gründe für eine betriebliche Ausbildungsbereitschaft die Humankapitalkapitalakkumulation auf unterschiedliche Weise beeinflussen. Da die duale Berufsausbildung vorwiegend aus allgemeinem bzw. berufspezifischem Humankapital besteht, das innerhalb der gesamten Volkswirtschaft bzw. innerhalb eines Industriezweiges uneingeschränkt eingesetzt werden kann, gilt es zu klären, warum die Betriebe dennoch freiwillig bereit sind, sich an dieser Art der Berufsausbildung zu beteiligen. Als Erklärungsansätze dienen Erweiterungen des humankapitaltheoretischen Ansatzes von Becker (1962). Je nach getroffenen Annahmen differieren Ausbildungsbetriebe in der Verfolgung ihrer Ziele, die zu unterschiedlichen Bildungsniveaus und -arten führen. Zudem müssen die theoretischen Ansätze um die berufsspezifische Humankapitalkomponente ergänzt werden, die in den Grundmodellen keine Berücksichtigung findet. Abbildung 6 fasst dies zusammen.

Dabei weisen Betriebe, welche nach dem produktionstheoretischen Ansatz ausbilden, einen kurzen Zeithorizont auf, bei dem Nettoerträge oder zumindest geringe Nettokosten entstehen. Der Stand des Humankapitals ist niedrig und setzt sich vor allem aus allgemeinem bzw. berufsspezifischem Wissen zusammen. Dagegen ist der Zeithorizont bei Betrieben mit investitionstheoretischem Hintergrund langfristig, was sich in hohen Ausbildungskosten niederschlägt. Aufgrund dessen verfügen Ausbildungsabsolventen über einen hohen Bildungsstand, der aus betriebsspezifischen und allgemeinen sowie berufsspezifischen Humankapitalkomponenten besteht. Dabei liegt die allgemeine Bildung auf einem höheren Niveau als bei Ausbildungsabsolventen, die nach dem produktionstheoretischen Ansatz ausgebildet werden. Dementsprechend sind die Bildungsinvestitionen am Ende der Ausbildung noch nicht amortisiert. Die Ausbildungsbereitschaft ist in diesem Fall geprägt von der Unsicherheit der Betriebe hinsichtlich des Verbleibs der ausgebildeten Fachkräfte. Daher gilt es, die Mobilität auf Seiten der Ausbildungsabsolventen einzuschränken und so die betriebliche Ausbildungsbereitschaft zu erhöhen (Bellmann/Janik 2007: 208) ${ }^{40}$ Denn nur

rung" auf qualifizierte Fachkräfte angewiesen, weil hier eine strikte Aufgabentrennung aufgehoben und dementsprechend ein breites Wissen notwendig ist (Backes-Gellner 2008: 7f.).

${ }^{40}$ Nach Bellmann und Janik (2007) investieren Betriebe nicht in Ausbildung, da sie mit vielen Unsicherheiten, welche die Situation an der zweiten Schwelle betreffen, konfrontiert sind. Diese beziehen Veränderungen in der Arbeitsnachfrage und in der Nachfrage nach Fähigkeiten, die der Arbeits- bzw. Gütermarkt zum Erhalt der Wettbewerbsfähigkeit fordert, ein. Diese Veränderungen können aber unter Umständen nicht von den Ausbildungsinhalten abgedeckt werden. Insbesondere der Dienstleistungssektor ist von einer sich schnell ändernden Nachfrage nach Qualifikationen gekennzeichnet. 


\section{Abbildung 6: Zusammenhang zwischen Ausbildungsbereitschaft und Hu- mankapital}

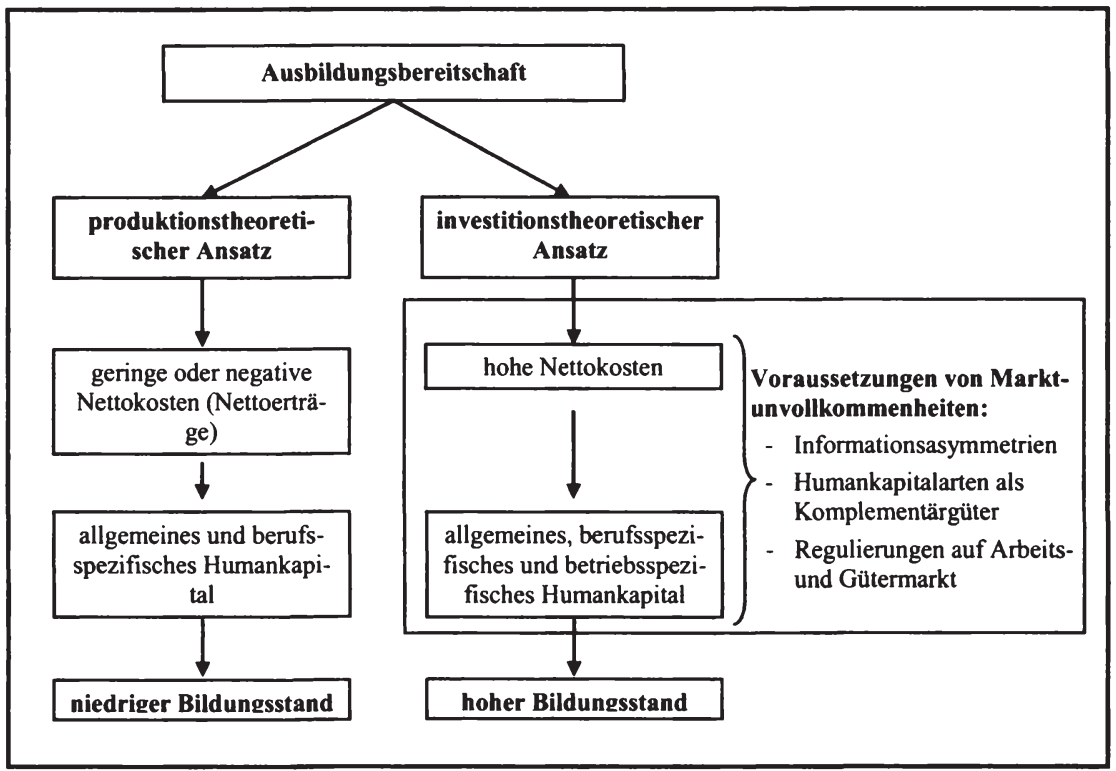

Quelle: eigene Darstellung

bei einer Weiterbeschäftigung entstehen Bildungserträge (Walden 2007), die vor allem durch das Wissen entstehen, das während des Zusammenarbeitens mit anderen Fachkräften innerhalb des Ausbildungsbetriebes weitergegeben wird; Inhalte in den Ausbildungsordnungen geben lediglich Rahmenbedingungen für Mindeststandards hinsichtlich der Qualifikation (Ryan 1998: 321). Dadurch wird die ökonomische Rentabilität gewährleistet, welche die Grundvoraussetzung der betrieblichen Ausbildung bildet (Dietrich et al. 2004). Dies setzt einen langfristigen Zeithorizont voraus, sodass Betriebe nur Personen ausbilden, wenn bei einem Angebot für eine Weiterbeschäftigung durch die bestehenden Rahmenbedingungen der freiwillige Verbleib im Betrieb gewährleistet wird (Adnett et al. 2003: 50). Daher sind Annahmen zu treffen, welche die Theorien um Mechanismen erweitern, die die Mobilität der Ausbildungsabsolventen einschränken. Grundidee ist hierbei eine Aufteilung der Finanzierung der Humankapitalinvestitionen auf die beteiligten Akteure. Dies kann in Form von entstehenden Austrittskosten sein, die bei einem Betriebswechsel entstehen. Die Motive für die unterschiedliche Ausbildungsbereitschaft werden durch die Struktur der Berufsbildung sowie die Rahmenbedingungen auf dem Arbeits- sowie Gütermarkt bestimmt, welche die Art und Höhe des Humankapitals vorgeben und somit auf die Ausbildungskosten rückwirken. Einerseits erfolgt dies durch entstehende 
Informationsasymmetrien und die fehlende Möglichkeit, die vollständige Produktivität, die sich aus formellem und informellem Wissen zusammensetzt, dem potenziellen neuen Arbeitgeber zu signalisieren. Andererseits sind Ausbildungsabsolventen bei einem Wechsel weniger produktiv, weil der betriebsspezifische Teil der Ausbildung nicht mehr angewendet werden kann. Diese beiden Aspekte dienen als Mechanismus für den Verbleib im Ausbildungsbetrieb. Darüber hinaus können Institutionen, wie gewerkschaftliche Vereinbarungen, und Transaktionskosten, wie Kündigungs- oder Einstellungskosten, die Mobilität reduzieren (Adnett et al. 2003). Danach kann die Existenz dieser Institutionen die Bindung zwischen Ausbildungsbetrieb und Ausbildungsabsolventen verstärken. ${ }^{41}$ Im System der dualen Berufsausbildung ist die Finanzierung auf Betrieb und Ausbildungsabsolvent verteilt, daher entstehen bei einer Trennung auf beiden Seiten Kosten. Unternehmen realisieren nach einer Trennung keine Erträge durch die ausgebildete Arbeitskraft. ${ }^{42}$ Auf Seiten der Ausbildungsabsolventen entstehen Kosten durch eine niedrigere Entlohnung, weil sie bei einem Wechsel ihr Wissen nicht mehr vollständig einsetzen können. Schließlich kann die Mobilität auf Seiten der Ausbildungsabsolventen reduziert werden, indem entsprechend hohe Löhne ausgehandelt werden, die über dem eines externen Unternehmens liegen (Malcomson et al. 2003). Auf Grundlage der theoretischen Erklärungsansätze für die betriebliche Ausbildungsbereitschaft ist davon auszugehen, dass die Ausbildungsbetriebe im Allgemeinen Bildungsinvestitionen vor dem Hintergrund eines langfristigen Beschäftigungsverhältnisses tätigen.

Für die Erklärung werden hierbei aber lediglich betriebliche Voraussetzungen sowie institutionelle Rahmenbedingungen für die Ausbildungsbereitschaft zugrunde gelegt. Auf den wirtschaftlichen Einfluss wird nicht eingegangen. Weiterhin gelten Annahmen, die lediglich auf die Einschränkung der Mobilität auf Seiten der Ausbildungsabsolventen abzielen bzw. es wird von einer Weiterbeschäftigung ausgegangen. Eine weitergehende Analyse, welche den Übergang an der zweiten Schwelle beschreibt, wird nicht gegeben. Diese ist aber notwendig, denn auch auf Seiten der Ausbildungsbetriebe kommt es zu Lösungen der Beschäftigungsverhältnisse, welche in den letzten Jahren zunehmen, wie die rückläufigen Übernahmequoten bescheinigen. Im folgenden Kapitel werden Bestimmungsgründe für den Verbleib nach der dualen Berufsausbildung erörtert, welche sich aus den theoretischen Ansätzen für die Ausbildungsbereitschaft ableiten lassen. Den Verbleib an der zweiten Schwelle beeinflussen jedoch neben den betrieblichen Gründen auch gesamtwirtschaftliche Einflussfaktoren, die im Folgenden ebenso zu betrachten sind.

${ }^{41}$ Nach Adnett et al. (2003) kann dagegen die Existenz von Kündigungskosten, wenn die Auszubildenden die Finanzierung der Humankapitalinvestitionen vollständig übernehmen, Anreiz für eine betriebliche Übernahme sein.

42 Allerdings fallen bei einer Trennung direkt nach Beendigung der dualen Berufsausbildung keine Kündigungskosten an, da das Ausbildungsverhältnis nur für die Dauer der Ausbildungszeit gilt. 
4 Bedeutung der unterschiedlichen Ausbildungsbereitschaft für den Verbleib nach dem Abschluss

Anders wie im letzten Kapitel erörtert wurde, wirken zum einen die Mechanismen zur Einschränkung der Mobilität auf Seiten der Auszubildenden nicht unter allen Voraussetzungen, weil weiche Faktoren, wie das Arbeitsklima, Ausbildungsabsolventen veranlassen können, den Ausbildungsbetrieb zu verlassen. Zum anderen wird die Mobilität nach der dualen Berufsausbildung auch von betrieblicher Seite angetrieben, weil Unternehmen sich trotz Nettokosten und der Möglichkeit bei einer Weiterbeschäftigung Bildungserträge zu realisieren, aus unterschiedlichen Gründen gegen eine Übernahme entscheiden. Die Betriebe sind rationale Entscheidungsträger mit einem Erfolgsziel (Blossfeld et al. 2005: 4) Allerdings basiert die Übernahmeentscheidung auf Unsicherheiten. Diese bestehen einerseits darin, dass bereits bei der Einstellung eines Schulabsolventen ein Informationsdefizit hinsichtlich seines wahren Verhaltens bzw. seiner tatsächlicher Produktivität existiert. Andererseits liegt die Unsicherheit für Unternehmen auch darin, dass sie die freiwerdende Stelle, sofern die Ausbildungsabsolventen nicht weiterbeschäftigt werden, mit den Fachkräften auf dem externen Arbeitsmarkt nicht adäquat besetzen können. So weicht die Entscheidungsgrundlage einer Ausbildung an der ersten Schwelle vom Ziel eines längerfristigen Beschäftigungsverhältnisses ab, weil Informationen über die Auszubildenden während der Ausbildungsphase gesammelt werden. Daher ist es notwendig, die Ursachen für den Entschluss gegen eine Weiterbeschäftigung auf mikro- und makroökonomischer Ebene zu analysieren. Darüber hinaus werden Gründe erläutert, weshalb auch die Mechanismen, welche die Mobilität auf Seiten der Ausbildungsabsolventen einschränken sollen, nicht immer wirken. Es werden Bestimmungsgründe dargelegt, die übernommene Ausbildungsabsolventen dazu bewegen, ihren Ausbildungsbetrieb zu verlassen. Die Wirkung der unterschiedlichen Determinanten auf die Abgangsrate aus dem Ausbildungsbetrieb und auf die Wahrscheinlichkeit, arbeitslos zu werden, wird in den nächsten Abschnitten dargestellt.

\subsection{Mikroökonomische Determinanten und der Übergang in den Ar- beitsmarkt}

Die Entscheidungsgrundlage an der ersten Schwelle für eine Ausbildung differiert zwischen den einzelnen Betrieben. Dabei ist zwischen Ausbildungsbetrieben zu unterscheiden, die bereits zu Beginn der Ausbildung keine Übernahme anstreben und denjenigen, welche die selbst ausgebildeten Ausbildungsabsolventen übernehmen wollen, sich dann aber gegen eine Weiterbeschäftigung entscheiden. Entsprechend sind die Ausbildungsinhalte auf die Entscheidungsgrundlage an der ersten Schwelle ausgerichtet. Deshalb muss analysiert werden, welche Faktoren die Ausbildungsbetriebe veranlassen, die selbst ausgebildeten Fachkräfte nicht zu übernehmen. 


\subsubsection{Produktionstheoretischer Ansatz und Verbleib nach der Ausbildung}

Ausbildungsbetriebe, die nach dem produktionstheoretischen Ansatz ausbilden, werden bereits zu Beginn der Ausbildung mit geringer Wahrscheinlichkeit an einer Übernahme der Ausbildungsabsolventen interessiert sein, weil die Bildungserträge ohnehin während der Ausbildung entstehen, aber auch entstehen müssen. Zudem lassen betriebliche Rahmenbedingungen eine Weiterbeschäftigung zu kostenintensiv erscheinen. ${ }^{43}$ Aufgrund der Realisation von Bildungserträgen ist das Unternehmen bereit, sogar ,über Bedarf" auszubilden (Neubäumer 1999), was bedeutet, dass der Ausbildungsbetrieb ein größeres Ausbildungsstellenangebot aufweist als langfristig für die Deckung seines Fachkräftebedarfs erforderlich ist. Demzufolge kann der Ausbildungsbetrieb nicht allen seinen Absolventen ein Übernahmeangebot unterbreiten, was eine hohe Fluktuation am Ende der Ausbildungsperiode bedeutet. Diese Rahmenbedingungen wirken sich sowohl auf die Art der Ausbildungsberufe als auch auf die Zusammensetzung des Wissens und der Fähigkeiten aus. Da die Ausbildungsordnungen die Lehrinhalte der Ausbildungsberufe der dualen Berufsausbildung vorgeben, werden die Ausbildungsbetriebe sich an diese Mindeststandards halten. ${ }^{44}$ Wissen und Fähigkeiten, welche darüber hinausgehen, werden nicht angeboten. Denn dies lässt die Ausbildungskosten steigen, welche bei der Entscheidung, nicht weiterzubeschäftigen, bestehen bleiben würden. Darüber hinaus sind oftmals die finanziellen Ressourcen für eine zusätzliche Wissensvermittlung nicht vorhanden. So werden auch die Externalitäten, die nicht ausbildenden Betrieben zugute kommen, weitestgehend reduziert. Die Ausbildungsqualität wird aufgrund der begrenzten Humankapitalinvestitionen gering sein, ebenso wie die Wahrscheinlichkeit einer Weiterbeschäftigung (Casey 1986: 68). Den Ausbildungsabsolventen wird daher nur in Ausnahmefällen ein weiterer Arbeitsvertrag angeboten.

Von dieser betrieblichen Entscheidung sind zumeist Schulabgänger betroffen, die einen geringen formalen Bildungsstand aufweisen. Es handelt sich häufig um Ausbildungsberufe, deren berufliche Tätigkeiten auch keinen hohen Bildungsstand erfordern. Daher finden sich Auszubildende, die bereits an der ersten Schwelle schlechte Arbeitsmarktchancen haben, in Ausbildungsbereichen mit geringen Nettokosten wieder, die auch mittelfristig (nach der dualen Berufsausbildung) schlechte Beschäftigungsaussichten aufweisen. Unternehmen, die aus produktionstheoretischer Motivation ausbilden, sind nur dann bereit, die Ausbildungsabsolventen weiterzubeschäftigen, wenn sie Fachkräftebedarf haben oder wenn Ausbildungsabsolventen einen Lohn in Höhe der Entlohnung akzeptieren,

${ }^{43}$ Gründe für die Ausbildungsbereitschaft dieser Betriebe liegen meist in der Tradition des Ausbildungsbereiches. Auch Reputationsgründe veranlassen Betriebe, sich an der Ausbildung zu beteiligen (Niederalt 2004; Sadowski 1980).

${ }_{44}$ Dies stimmt auch mit der Humankapitaltheorie von Becker (1962) überein. Danach werden Ausbildungsabsolventen nur dann nicht übernommen, wenn exogene Nachfrageschocks auftreten. In diesem Falle werden dann zunächst die Auszubildenden entlassen, die allgemeines Humankapital erlernt haben, da die Betriebe für dieses nicht aufgekommen sind. 
die ein Auszubildender bzw. ein un- oder angelernter Arbeitnehmer erhält. Da dies nicht der Fall sein wird, findet eine Weiterbeschäftigung der Ausbildungsabsolventen nicht statt (McLaughlin 1991: 4f.). Diese Ausbildungsabsolventen sind auf dem Gesamtarbeitsmarkt zwar durch einen geringeren Humankapitalstand gekennzeichnet, aber andererseits sind sie innerhalb ihrer Berufsgruppe, entgegen Gibbons und Katz (1991), nicht die weniger fähigen. Sie werden vielmehr durchschnittliche oder sogar auch überdurchschnittliche Fähigkeiten innerhalb ihrer Berufsgruppe aufweisen.

Insgesamt kann jedoch gesagt werden, dass die Wahrscheinlichkeit einer Weiterbeschäftigung bei Ausbildungsabsolventen, die in einem Betrieb mit geringen bis sogar negativen Nettokosten ausgebildet werden, geringer ist als bei Ausbildungsbetrieben mit hohen Ausbildungskosten, die in der Regel noch einen gewissen Anteil der Ausbildungsabsolventen halten. Demnach beeinflussen die Nettoausbildungskosten für die Ausbildungsbereitschaft die Wahrscheinlichkeit einer Weiterbeschäftigung positiv. Jedoch gibt es Umstände, die sich negativ auf den Verbleib im Ausbildungsbetrieb auswirken, sodass sich Ausbildungsabsolventen in Betrieben mit Nettokosten ebenfalls der Situation einer Nichtverlängerung des Arbeitsverhältnisses gegenübersehen.

\subsubsection{Investitionstheoretische Ansätze und Verbleib nach der Ausbildung}

Ausbildungsbetriebe, die nach dem investitionstheoretischen Ansatz handeln, werden vor dem Hintergrund eines längerfristigen Beschäftigungsinteresses Ausbildung anbieten, das heißt, sie bilden aus, mit dem Ziel, die eigenen Ausbildungsabsolventen zu übernehmen. Da diese Ausbildungsbetriebe vor allem ihren künftigen Fachkräftebedarf durch die eigene Ausbildung decken wollen, wird die Organisation der betrieblichen Ausbildung auf diese Unternehmensziele ausgerichtet sein, weshalb die Humankapitalakkumulation im Mittelpunkt steht (Euwals/Winkelmann 2003: 4). Die betrieblichen Bildungsinvestitionen werden hoch sein, erfordern daher eine Weiterbeschäftigung und führen somit $\mathrm{zu}$ einer Amortisation der Ausbildungskosten. Ausbildungsbetriebe, die ihre künftigen Ausbildungsabsolventen halten wollen, werden bei der Einstellung von Schulabgängern auf dieser Grundlage entscheiden. In diesem Fall wird meist jungen Erwachsenen mit hohem formalem Bildungsstand ein Ausbildungsangebot unterbreitet. An der zweiten Schwelle wird dann in der Regel eine Weiterbeschäftigung erfolgen, da Ausbildungsbetriebe mit Nettokosten ein langfristiges Beschäftigungsinteresse haben und eine Beschäftigungswahrscheinlichkeit hoch ist (McCausland/Theodossiou 2004: 68; Holm et al. 2001). Jedoch ist zunehmend zu beobachten, dass auch die Übernahmequoten in Ausbildungsbereichen, die nach investitionstheoretischem Grundsatz ausbilden, zurückgehen, weil auch in diesen Bereichen eine verstärkte Ausbildung ,über Bedarf“ durchgeführt wird. Die duale Berufsausbildung wird hierbei als ScreeningProzess betrachtet, aus dem sich die künftigen Fachkräfte herauskristallisieren (Gangl 2003: 75), welche die Wettbewerbsfähigkeit des Unternehmens gewähr- 
leisten können. So entscheiden sich auch diese Ausbildungsbetriebe bereits zu Beginn, nur einen Teil ihrer Ausbildungsabsolventen weiterzubeschäftigen, und zwar die „Besten der Besten“. Ausbildungsbetriebe mit hohen Ausbildungsinvestitionen und entsprechenden Nettoausbildungskosten bilden zunehmend , $\ddot{\mathrm{u}}$ ber Bedarf" aus. Dies wird auch als häufigster Grund für eine Nichtübernahme angegeben (Bellmann et al. 2006: 76).

Diese Selektion ist aber erst nach der dualen Berufsausbildung möglich, weil während des Ausbildungszeitraums der Ausbildungsbetrieb Informationsasymmetrien hinsichtlich der Fähigkeiten und damit der Produktivität abbaut (Acemoglu/Pischke 1998). Erst dann können die fähigeren von den weniger fähigeren Ausbildungsabsolventen unterschieden werden. Somit hängt das Übernahmeangebot von den Fähigkeiten der einzelnen Ausbildungsabsolventen ab. Dabei wird angenommen, dass auch innerhalb eines Unternehmens die Auszubildenden sich voneinander unterscheiden, die Auszubildenden demnach nicht mehr homogen sind. Grundlage der Übernahmeentscheidung ist dabei folgende Bedingung (Franz/Soskice 1995):

$$
q \cdot\left(1-q_{j}^{s c} \sum_{t=n+1}^{k} q^{s c}\left(f(\tau, s)_{t}^{g u}-f(\tau, s)_{t}^{s c}>\sum_{t=1}^{n} E_{t}-R_{t},\right.\right.
$$

Die Weiterbeschäftigung im Ausbildungsbetrieb wird durch die Realisation der Bildungserträge, die durch die Produktivität der Ausbildungsabsolventen entstehen, bestimmt. Betrachtet wird der Zeitpunkt $n+1$. Der Produktionsbeitrag der guten Ausbildungsabsolventen zu diesem Zeitpunkt beträgt $f(\tau, s)_{t}^{g u}$, der der schlechten $f(\tau, s)_{t}^{s c}$. Entscheidungsgrundlage sind die betrieblichen Mindestanforderungen, welche die Rahmenbedingungen auf dem Arbeitsmarkt und auf dem Gütermarkt, in dem das Unternehmen tätig ist, berücksichtigen (Garloff/Kuckulenz 2006; Franz/Soskice 1995). Die Differenz der Wertgrenzprodukte wird mit dem Anteil der schlechten Ausbildungsabsolventen im externen Absolventenpool $\left(q^{s c}\right)$, also mit der Wahrscheinlichkeit, einen schlechten Ausbildungsabsolventen extern rekrutieren zu können, verringert. Dieser Term wird wiederum mit der Wahrscheinlichkeit, gute betriebseigene Ausbildungsabsolventen zu erhalten, multipliziert. Demnach stellt $q_{j}^{s c}$ den Anteil der schlechten Ausbildungsabsolventen des betreffenden Ausbildungsbetriebes $j$ an allen Absolventen des Betriebes dar. $q$ stellt den Anteil der im Ausbildungsbetrieb verbleibenden Absolventen dar. Eine Übernahme findet dann statt, wenn die Produktivität der Ausbildungsabsolventen unter Berücksichtigung des externen Arbeitsmarktes die Nettokosten $\left(\sum_{t=1}^{n} E_{t}-R_{t}\right)$ übersteigt. Andernfalls wird das Beschäftigungsverhältnis nicht verlängert. Entscheidungsgrundlage ist der gegenwärtige Zeitpunkt bei Beendigung der Ausbildung. 
Die Identifizierung der Ausbildungsabsolventen, welche die Mindestanforderungen nicht erreichen, kann nur der Ausbildungsbetrieb durchführen, weil er sowohl einen Informationsvorsprung hinsichtlich der eigenen Ausbildungsabsolventen als auch Kenntnis über die durchschnittlichen (formalen) Fähigkeiten der Arbeitskräfte auf dem externen Arbeitsmarkt besitzt. Wenn der Ausbildungsabsolventenpool auf dem externen Arbeitsmarkt im Durchschnitt einen höheren Bildungsstand aufweist als die weniger fähigen Absolventen, wird den eigenen schlechteren Ausbildungsabsolventen kein Angebot für eine Verlängerung des Beschäftigungsverhältnisses unterbreitet (Casey 1986: 72; Greenwald 1986: 325). Denn auf dem externen Arbeitsmarkt besteht eine höhere Chance, eine fähige Fachkraft einzustellen. Entsprechend wird keine Verlängerung des Vertrages ausgehandelt, wodurch sich die Transaktionskosten reduzieren, weil Kündigungskosten nicht anfallen (Leuven/Oosterbeek 2001: 342). Auch nach Acemoglu und Pischke (1998) ist es für den Ausbildungsbetrieb von Vorteil, wenn diejenigen Ausbildungsabsolventen nicht übernommen werden, die unterhalb eines bestimmten Niveaus an Fähigkeiten, welches durch das allgemeine Humankapital bestimmt wird, liegen (Acemoglu/Pischke 1998), denn es gilt für alle Ausbildungsabsolventen desselben formellen Bildungsstandes der gleiche Lohn. Demzufolge kann der Ausbildungsbetrieb durch die Weiterbeschäftigung der weniger fähigen (hinsichtlich des informellen Bildungsstandes) Bildungserträge nicht in dem Maße erreichen wie er sie durch die fähigen realisiert. Zudem weist nach Mortensen und Pissarides (1994) ein bestehender Job Produktivitätsanforderungen auf, die aufgrund der zu erfüllenden Aufgaben nicht verändert werden können. Da die Produktivität der weniger fähigen Ausbildungsabsolventen aber bei einer Weiterbeschäftigung unter deren Entlohnung liegt (Leuven/Oosterbeek 2001: 343; Hashimoto 1981: 476), ist die Stelle folglich mit einer Arbeitskraft mit entsprechender Produktivität zu besetzen. Diese erfolgt bei Vorliegen eines hohen Bildungsniveaus auf dem allgemeinen Arbeitsmarkt daher durch die Rekrutierung externer Arbeitskräfte, mit denen der Ausbildungsbetrieb zugleich auch die Grenzproduktivität steigern wird.

Dieses Kapitel hat gezeigt, dass auch in investitionsorientierten Ausbildungsbetrieben die Wahrscheinlichkeit einer Nichtübernahme vorliegt, falls die Mindestanforderungen nicht erfüllt werden. In diesem Fall zeichnen sich die nicht übernommenen Ausbildungsabsolventen durch einen im gesamtwirtschaftlichen Kontext relativ hohen Humankapitalstand aus, weil Ausbildungsbetriebe hohe Humankapitalinvestitionen tätigen. Deshalb stellen diese Unternehmen Schulabsolventen mit hohem (allgemeinem) Humankapital ein. Dieser Zusammenhang zwischen Schul- und Berufsausbildung wirkt sich auf die Ausbildungsqualität der Auszubildenden aus. So findet wegen des hohen allgemeinen Bildungsstandes eine Vermittlung von berufs- und betriebsspezifischem Humankapital vor dem Hintergrund einer Übernahme statt. Einige Ausbildungsabsolventen weisen jedoch innerhalb ihrer Berufsgruppe bzw. dem Wirtschaftszweig, in dem sie die Ausbildung absolviert haben, unterdurchschnittliche Fähigkeiten auf. Nach 
Franz und Zimmermann (2001) sind dies nur ungefähr 9\% aller Ausbildungsabsolventen, bei denen deshalb keine Übernahme erfolgt. Auch neuere Studien ergaben, dass dieser Grund gegen eine Weiterbeschäftigung der Ausbildungsabsolventen im Jahr 2005 am wenigstens häufig genannt wird (Bellmann et al. 2006: 76).

\subsection{Makroökonomische Determinanten und der Übergang in den Ar- beitsmarkt}

Der auf Seiten der Betriebe induzierte Wechsel nach der dualen Berufsausbildung wird neben der firmenspezifischen Organisation der dualen Berufsausbildung auch durch die institutionellen Rahmenbedingungen bestimmt, die alle Arten von Unternehmen und Ausbildungen treffen. Entsprechend sind die Ausbildungsintensität und der Verbleib nach der Ausbildung auch sehr stark von der makroökonomischen Situation abhängig. Im Folgenden werden daher diese Begründungen der zunehmenden (unfreiwilligen) Abwanderung aus den Ausbildungsbetrieben nach der dualen Berufsausbildung genauer erörtert.

\subsubsection{Institutionelle Rahmenbedingungen an der zweiten Schwelle}

Der Einfluss der Gewerkschaften beschränkt sich nicht nur auf die Ausbildungsbereitschaft (siehe Kap. 3.2.2.3.1), sondern muss auf die zweite Schwelle ausgeweitet werden. In diesem Fall ist die Lohnstauchung zu betrachten, deren Höhe sich auf die Übernahmeentscheidung der Betriebe auswirkt. Je nach Koordinationsgrad und Macht der Gewerkschaften (Fitzenberger/Kohn 2005) wird der ausgehandelte Lohn unterschiedlich hoch sein und die Differenz zwischen Produktivität und Entlohnung entsprechend bestimmen. Dies wirkt sich wiederum auf die Amortisationsdauer der Bildungsinvestitionen aus. Zentralisierte Tarifverhandlungen werden daher die Lohnstauchung fördern (Fitzenberger/Kohn 2005; OECD 2004; Aidt/Tzannatos 2002; Flanagan 1999; Gerlach/Meyer 1995). ${ }^{45}$ Dies impliziert entsprechend höhere Löhne (Ausbildungsvergütungen) im unteren Lohnbereich und wirkt sich nach Booth und Zoega (2001) sowie Acemoglu und Pischke (1999a; 1999b; 1998) positiv auf die Höhe des Humankapitalstandes aus. Durch eine zunehmende Humankapitalinvestition wird die Lohnkompression maximiert. Daher weisen tariflich gebundene Betriebe eine hohe Übernahmewahrscheinlichkeit auf. Dies bedeutet zugleich, dass die von gewerkschaftlich organisierten Betrieben nicht übernommenen Ausbildungsabsolventen über eine gute Ausbildung verfügen, die sich durch hohes Humankapital auszeichnet. Diese Humankapitalstruktur ist gekennzeichnet von allgemeinem und betriebsspezifischem Humankapital. Dezentrale Tariflöhne dagegen sind niedriger, da die Tarifpartner eine geringere Macht haben als bei zentraler

${ }^{45}$ Allgemein berücksichtigen zentralisierte Gewerkschaften die gesamtwirtschaftlichen Rahmenbedingungen, was zu moderaten Lohnabschlüssen führt. Dies wirkt einer zu starken Lohnstauchung entgegen. 
Koordination. Sie berücksichtigen die entsprechende Situation des gewerkschaftlich organisierten Betriebes, weshalb die Löhne im unteren Bereich relativ niedrig sind. Infolgedessen bedarf es geringerer Humankapitalinvestitionen, um die Differenz zwischen Produktivität und Entlohnung zu vergrößern. Aufgrund dessen werden die Ausbildungsbetriebe nicht in dem Maße gezwungen sein, die Ausbildungsabsolventen zu übernehmen. Die Wahrscheinlichkeit einer Übernahme liegt unter derjenigen von tariflich gebundenen Unternehmen.

Sobald konjunkturelle Schwankungen auftreten, können negative makroökonomische Einflussfaktoren die mikroökonomischen Anreize für eine Weiterbeschäftigung überlagern. In diesem Falle wirken tariflich ausgehandelte Löhne hemmend auf die Flexibilität der Unternehmen, weil bei Produktions- bzw. Nachfrageschwankungen die erforderliche Anpassung über die Löhne nicht möglich ist. Deshalb können Schwankungen nur über Veränderungen in der Arbeitskräftenachfrage kompensiert werden, wovon insbesondere die Ausbildungsabsolventen betroffen sind, da deren Verträge auslaufen und somit keine weiteren Kündigungs(schutz)kosten entstehen (Neubäumer 2006). ${ }^{46}$ Folglich beeinflussen auch Kündigungsschutzregelungen den Verbleib an der zweiten Schwelle bei Verschlechterung der wirtschaftlichen Situation negativ. Explizit wirkte sich dies auf die Übernahmequote in Deutschland aus, die aufgrund der nachteiligen wirtschaftlichen Lage, wie zu Beginn der 1990er Jahre, und den geltenden institutionellen Rahmenbedingungen rückläufig war (Bispinck et al. 2002: 215). Seither wirken Arbeitnehmer- und Arbeitgebervertreter diesem Trend mit entsprechenden tariflichen Vereinbarungen entgegen, indem sie den Fokus neben der Ausbildungsförderung verstärkt auf die Übernahme nach der dualen Berufsausbildung legen. Deshalb wurden in Deutschland vor allem tarifliche Vereinbarungen hinsichtlich einer Weiterbeschäftigung getroffen. Regelungen sind in Kraft getreten, die sich insbesondere durch den Aspekt einer befristeten oder unbefristeten Übernahme bzw. einer Übernahme mit Voll- oder Teilzeitbeschäftigung unterscheiden. Bei der befristeten Übernahme handelt es sich meist um einen Zeitraum von bis zu 12 Monaten, in dem Ausbildungsabsolventen im Betrieb nach der Ausbildung weiterbeschäftigt werden. Die Übernahmeregelungen werden zwar vertraglich festgehalten, sie sind aber für die Ausbildungsbetriebe nicht bindend, sondern vielmehr als Empfehlung zu sehen (Bispinck et al. 2002). Das heißt, es erlaubt Betrieben unter bestimmten personen- oder wirtschaftlich bedingten Voraussetzungen, von den Abmachungen abzuweichen (Bispinck 2002). Trotzdem halten sich die meisten Ausbildungsbetriebe hinsichtlich einer (befristeten) Übernahme an die tariflichen Vereinbarungen. Nach der Befristung ist es den Unternehmen jedoch immer noch offen, das Arbeitsverhältnis fortzuführen oder sich ohne zusätzliche Kündigungskosten

\footnotetext{
${ }^{46}$ Dem positiven Einfluss der Übernahme durch die Gewerkschaften entgegen wirkt aber der Entlassungseffekt (Lechthaler/Snower 2008). Dadurch, so die Argumentation, verringern sich die Bildungsinvestitionen, weil durch die kürzere Beschäftigungsdauer die Bildungserträge nicht vollständig realisiert werden können.
} 
endgültig zu lösen. Gelten darüber hinaus noch niedrigere tarifliche Einstiegseinkommen, wie es für einen bestimmten Zeitraum in gewissen Industriebereichen in Deutschland während der 1990er Jahre der Fall war, können die Ausbildungsbetriebe zu einem früheren Zeitpunkt die Ausbildungskosten amortisieren (Bispinck 2002: 214), weil die Lohnkompression vergrößert wird, was wiederum zu steigenden Bildungserträgen führt. Dies schafft für Unternehmen, welche aus wirtschaftlichen Gründen zu einer Nichtübernahme tendieren, Anreize zumindest für einen gewissen Zeitraum, die Ausbildungsabsolventen zu übernehmen. Während dieser Zeit wird zugleich auch den Ausbildungsabsolventen ermöglicht, Berufserfahrung zu sammeln und so das berufsspezifische $\mathrm{Hu}-$ mankapital zu erweitern.

Im Allgemeinen werden (dezentral bzw. nicht gewerkschaftlich organisierte) Unternehmen, in denen die Differenz zwischen Ausbildungsvergütung und Produktionsbeitrag größer ausfällt, Ausbildungsabsolventen eher über Bedarf ausbilden (Fitzenberger/Kohn 2005). Entsprechend sinkt nach der dualen Berufsausbildung die Wahrscheinlichkeit einer Übernahme, da die Kosten bereits während der Ausbildungsphase amortisiert werden können. Ausbildungsabsolventen dieser Art von Unternehmen werden daher wahrscheinlicher von einer Nichtübernahme betroffen sein als Absolventen, die in einem Betrieb eine Ausbildung absolviert haben, der tariflich gebunden ist, weil diese eher aus normativen Gründen einer Übernahme nachkommen werden. So haben nicht an tarifliche Vereinbarungen gebundene Unternehmen keinen Anreiz, Ausbildungsabsolventen zu übernehmen. Entsprechend reduziert sich ebenfalls das Interesse an einer Weiterbeschäftigung.

\subsubsection{Wirtschaftliche Rahmenbedingungen bei Abschluss der Ausbildung}

Neben der jeweiligen Organisation der dualen Berufsausbildung innerhalb eines Ausbildungsbetriebes sind insbesondere die wirtschaftlichen Rahmenbedingungen zu nennen, welche die Fluktuation nach der Ausbildung determinieren. Je nach wirtschaftlicher Lage, kann dies einen Ausbildungsbetrieb zu einer Revision seiner Entscheidung für eine Weiterbeschäftigung, die er zu Beginn der Ausbildung getroffen hat, zwingen. Hierbei sind die langfristigen Rahmenbedingungen, wie der Strukturwandel oder kurzfristige, konjunkturelle Veränderungen, getrennt zu betrachten. Ein Strukturwandel ist von stetig und kontinuierlich andauernden Veränderungen in der Arbeitsnachfrage gekennzeichnet, verursacht insbesondere durch technischen Fortschritt, verbunden mit Produktionsverlagerungen ins Ausland und internationalem Handel ${ }^{47}$ (Kletzer 1998: 131). In diesem Fall wird die Nachfrage nach ausgebildeten Fachkräften durch intersektorale Produktions- bzw. Produktivitätsverlagerungen bestimmt. Vom Strukturwandel betroffene Unternehmen werden dann aber bereits zu Beginn der Ausbildung

\footnotetext{
${ }^{47}$ Vor allem bei wettbewerbsfähiger werdenden Importen werden mehr Leute entlassen (Addison et al. 1995; Kletzer 1995; Haveman 1994).
} 
mit höherer Wahrscheinlichkeit die Ausbildungsabsolventen nicht übernehmen und die Ausbildung entsprechend anpassen (siehe Kap. 4.1.1).

Eine Revision dieser Entscheidung kann erfolgen, wenn Änderungen in der konjunkturellen Situation zum Zeitpunkt des Abschlusses der dualen Berufsausbildung den Einfluss des Strukturwandels überlagern. So können konjunkturelle Veränderungen Unternehmen dazu veranlassen, Auszubildende nicht zu übernehmen, obwohl sie zu Beginn der Ausbildung ein langfristiges Beschäftigungsinteresse haben oder umgekehrt (McCausland/Theodossiou 2004). Das Übernahmeangebot für einen Ausbildungsabsolventen hängt hierbei von der Arbeitskräftenachfrage und vom -angebot ab. Beides wird wiederum von der Güternachfrage determiniert. Mit Rückgang der Nachfrage (auch nach Gütern) erhöhen die Unternehmen ihre Anforderungen an den Humankapitalstand der Ausbildungsabsolventen. Infolgedessen können nur die gut ausgebildeten Fachkräfte gehalten werden (Franz/Zimmermann 2001: 4). Nach Franz und Zimmermann (2001) ist der mangelnde Bedarf an qualifizierten Arbeitskräften im Ausbildungsbetrieb der Hauptgrund, weshalb Ausbildungsabsolventen nicht übernommen werden (38\%). Daher ist die mikroökonomische mit der makroökonomischen Sichtweise zur Erklärung des Übernahmeverhaltens über die konjunkturellen Schwankungen eng verbunden. Denn auf konjunkturelle Schwankungen können Ausbildungsbetriebe kurzfristig nur über die Anpassung ihres Übernahmeverhaltens reagieren. ${ }^{48}$ So wirkt sich eine Verschlechterung (oder auch eine Verbesserung) der wirtschaftlichen Lage für die im Ausbildungsprozess befindlichen Auszubildenden in einer sinkenden (steigenden) Wahrscheinlichkeit einer Weiterbeschäftigung aus. Weil die Entscheidung an der zweiten Schwelle für den Betrieb selbst langfristige Auswirkungen hat, werden die wirtschaftlichen Indikatoren verstärkt die Situation nach der dualen Berufsausbildung beeinflussen. ${ }^{49}$ Jedoch sind nicht alle Ausbildungsabsolventen in gleichem Maße von einer Auflösung des Arbeitsverhältnisses bei Änderungen der wirtschaftlichen Rahmenbedingungen betroffen (Buchholz 2008: 55). Insbesondere Betriebe, die stark von konjunkturellen Schwankungen betroffen sind, können nicht auf einen Auftragsrückgang mit einem flexiblen Arbeitseinsatz innerhalb des eigenen Unternehmens reagieren. Einerseits verfügen sie nicht über finanzielle Ressourcen zur Finanzierung von bspw. Kurzarbeit. Andererseits weisen sie kein vielfältiges Arbeitsplatzangebot auf, um die Ausbildungsabsolventen in den Bereichen einzusetzen, in denen Arbeitskräfte benötigt werden. Doeringer und Piore (1971)

48 Langfristig passen sie ihre Ausbildungsintensität an und reagieren somit an der ersten Schwelle. Wie Dietrich und Gerner (2008) zeigen, ist vor allem die Ausbildungsbereitschaft von konjunkturellen Rahmenbedingungen, wie die Geschäftserwartungen, stark beeinflusst. Insbesondere reagieren die Betriebe vor allem mit einem Rückgang der Ausbildung, sobald sich die Geschäftserwartungen verschlechtern.

${ }^{49}$ Vielmehr sind langfristig gesamtwirtschaftliche Folgen durch den Rückgang der Ausbildungsbereitschaft in einem Fachkräftemangel zu erkennen. Denn bei mangelndem Fachkräftenachwuchs wird die Wettbewerbsposition einer Volkswirtschaft geschwächt (Reinberg/Hummel 2005). 
und später Sengenberger (1987) begründen, dass insbesondere Betriebe im externen Segment, das insbesondere von Tätigkeiten gekennzeichnet ist, die geringe Anforderungen an die Humankapitalhöhe stellen, von konjunkturellen Schwankungen betroffen sind. Dabei erweitern sie den Kreis der Betriebsarten, denn im externen Segment bzw. auf externen Arbeitsmärkten befinden sich auch Randbelegschaften großer Betriebe.

Daraus folgt eine Ungleichverteilung des Risikos zwischen den verschiedenen Bildungsgruppen, nicht weiterbeschäftigt zu werden. Tätigkeiten in konjunkturell abhängigen Bereichen werden häufig von Fachkräften mit hohem Anteil an allgemeinem bzw. berufsspezifischem Wissen und niedrigem Bildungsstand ausgeübt (Büchel/Neubäumer 2001; Neubäumer 1999; Sengenberger 1987). In diesem Falle sind keine hohen Bildungsinvestitionen erforderlich, woraus nach der dualen Berufsausbildung geringe oder sogar negative Nettoausbildungskosten (Nettoerträge) resultieren. Dies ist notwendig, da Unsicherheit hinsichtlich der wirtschaftlichen Situation in diesem Tätigkeitsbereich besteht. Daher werden die Ausbildungsabsolventen mit einer relativ geringen Ausbildungsqualität bei einer Verschlechterung der konjunkturellen Situation nicht übernommen. Üblicherweise besitzen Ausbildungsabsolventen dieser Betriebe allgemeines und berufsspezifisches Humankapital, weshalb sie Arbeitsplätze ohne größere Qualifikationsanforderungen besetzen. ${ }^{50}$ Infolgedessen können die ausgeübten Tätigkeiten in Aufschwungphasen von externen rekrutieren Arbeitskräften ohne längere Einarbeitungsphase erledigt werden. Dagegen beschäftigen Betriebe in der Regel Fachkräfte, die zusätzlich einen bestimmten Anteil an betriebsspezifischer Ausbildung erhalten, bei einem Nachfragerückgang weiter (Becker 1962), weil aus dieser Ausbildung entstehende Kosten amortisiert werden müssen. ${ }^{51}$ Daraus ergibt sich für die Ausbildungsabsolventen ein unterschiedlich hohes Risiko einer Nichtübernahme. Ändern sich die wirtschaftlichen Gegebenheiten, werden Betriebe die Arbeitskräfte, welche die geringsten Kündigungskosten verursachen, nicht übernehmen. Um die Entlassungskosten gering zu halten, investieren stark von der Konjunktur abhängige Ausbildungsbetriebe nur wenig in die Ausbildung, weil deren Übernahmewahrscheinlichkeit wegen der ungünstigen Rahmenbedingungen von vornherein gering ist. Entsprechend werden besser ausgebildete Absolventen, übernommen, da sie Aufgaben erfüllen, die neben allgemeinem und berufsspezifischem vor allem auch betriebsspezifisches Humankapital erfordern. Über letztere Komponente verfügen extern rekrutierte Arbeitskräfte nicht, weswegen sich die Einlernphase verlängert. Diese unterschiedlichen Beschäftigungsinteressen werden bereits bei der Entscheidung über die

\footnotetext{
${ }^{50}$ Zudem spielt auch der soziale Hintergrund eine Rolle, da Ausbildungsabsolventen meist noch keinen familiären Verpflichtungen nachgehen müssen.

${ }^{51}$ Nach Becker (1962) gilt diese Differenzierung der Ausbildungsabsolventen nach der Übernahmewahrscheinlichkeit nicht mehr bei länger andauernden wirtschaftlichen Veränderungen. In diesem Falle ist es dann kostengünstiger, sich auch von gut ausgebildeten Arbeitskräften zu trennen.
} 
Ausbildungsintensität berücksichtigt (Díaz-Vázquez/Snower 2002). Insgesamt bleibt festzustellen, dass bei konjunkturellen Veränderungen schlechter qualifizierte Ausbildungsabsolventen eher den Ausbildungsbetrieb verlassen müssen als die besser qualifizierten. Dauert eine konjunkturelle schlechte Phase länger an, wird auch der Vertrag der gut ausgebildeten Fachkraft nicht verlängert (Nakamura 2008: 55). In diesem Fall sind alle Ausbildungsabsolventen, gleich welcher Ausbildung, in selbem Maße betroffen.

\subsection{Mobilität seitens der Ausbildungsabsolventen}

In den voran gehenden Abschnitten wird der Betriebswechsel vor allem durch die Betriebe veranlasst. Hierbei trennen sich die Ausbildungsbetriebe in der Regel von den schlechteren Ausbildungsabsolventen bzw. die Trennung erfolgt aufgrund von ungünstigen Rahmenbedingungen. Jedoch kann die Fluktuation auch auf freiwilliger Basis und demnach auf Wunsch der Ausbildungsabsolventen selbst erfolgen. Freiwillige Abgänge aus dem Ausbildungsbetrieb werden meist von überdurchschnittlich guten Ausbildungsabsolventen durchgeführt, die sich ihrer vorteilhaften Situation bewusst sind. Daher werden sie zunächst gegenüber ihrem Ausbildungsbetrieb einen höheren Lohn fordern, als der Betrieb ihnen bieten kann bzw. möchte (McLaughlin 1991). Denn zunächst ist auch für den Ausbildungsabsolventen selbst der Verbleib im Ausbildungsbetrieb von Vorteil, weil er aufgrund des Einsatzes seiner vollständigen Produktivität eine höhere Entlohnung als den Marktlohn erhält. Trotzdem wird er sich auf dem externen Arbeitsmarkt nach einem neuen Arbeitgeber umsehen, da es weitere Gründe gibt, welche die durch die theoretischen Annahmen bestehenden finanziellen Mechanismen zur Reduzierung der Mobilität außer Kraft setzen. Dies trifft dann zu, weil sie hinsichtlich der Präferenzen der Ausbildungsabsolventen zu kurz greifen. Denn der Ausbildungsabsolvent bezieht in seine Entscheidung ebenfalls weiche Faktoren ein, unter anderem Karrierechancen oder soziale Bindungen. Daher ist der Beginn des Erwerbslebens von häufigen Arbeitsplatzwechseln geprägt, wodurch die Erwerbssituation, vor allem zu Beginn, von kurzer Beschäftigungsdauer gekennzeichnet ist. In der Literatur wird dies auch als „Job-Hopping“ oder „Job-Shopping“ bezeichnet (Jovanovic 1979; Johnson 1978). Begründet wird dieses Verhalten der jungen Arbeitskräfte durch ihre geringe Berufserfahrung und die damit verbundenen Informationsasymmetrien hinsichtlich ihrer eigenen Produktivität. Vor allem die Ausbildungsabsolventen selbst wollen diese abbauen, indem sie zu Beginn ihrer Erwerbskarriere möglichst viele verschiedene Arbeitsplätze kennen lernen. Gleichzeitig haben die jungen Arbeitskräfte auch die Möglichkeit, die für sie optimale Arbeitsstelle zu finden, welche den größten Nutzen stiftet (Johnson 1978).

Es werden demzufolge Ausbildungsabsolventen mit einer guten betrieblichen Ausbildungsqualität den Ausbildungsbetrieb freiwillig verlassen. Sie werden in der Regel sowohl in der gesamten Volkswirtschaft wie auch innerhalb ihrer Berufsgruppe einen überdurchschnittlichen Bildungsstand aufweisen. Daher schät- 
zen sie ihre Optionen auf dem externen Arbeitsmarkt gut ein (Kock 2008: 17). Andernfalls würden sie das Übernahmeangebot annehmen. Da sie häufig auch einen relativ hohen Anteil an betriebsspezifischem Humankapital aufweisen, wird die berufliche Mobilität ${ }^{52}$ gering sein. Zudem suchen sie auch Arbeitsplätze in Unternehmen, die dem Ausbildungsbetrieb gleichen, um den Produktivitätsverlust zu verringern (Elliot/Lindley 2006; Jacoby 1983).

\subsection{Heterogene Humankapitalstruktur der Ausbildungsabsolventen}

Die unterschiedlichen Faktoren, welche die Fluktuation nach der dualen Berufsausbildung determinieren, implizieren ein heterogenes Arbeitsangebot von Ausbildungsabsolventen. Diese Heterogenität nimmt in konjunkturell schlechten Zeiten zu (Nakamura 2008: 58). Das Arbeitsangebot setzt sich sowohl aus guten als auch aus schlechten Ausbildungsabsolventen zusammen (von Wachter/Bender 2004), welche zum Teil um dieselben Arbeitsplätze konkurrieren. Hierbei ist davon auszugehen, dass Ausbildungsabsolventen aus unterschiedlichen Gründen nicht übernommen wurden bzw. nicht in ihrem Ausbildungsbetrieb verbleiben. Die Gründe unterscheiden sich zwischen den einzelnen Betrieben, weshalb eine Nichtübernahme über die Bereiche ungleich verteilt ist (Kletzer 1998), weil sich innerhalb eines Ausbildungsbereiches sowohl Betriebe, welche nach dem produktionstheoretischen Ansatz als auch Betriebe, die nach dem investitionstheoretischen Ansatz ausbilden, befinden. ${ }^{53}$ Dadurch wird eine unterschiedliche Art und Struktur von Humankapital impliziert, wodurch sich die Übernahmewahrscheinlichkeit der Ausbildungsabsolventen determiniert. Dies führt dazu, dass Ausbildungsabsolventen aus einem Betrieb mit einem niedrigen Humankapitalstand aufgrund geringer oder keiner Nettoausbildungskosten aufgrund von starker Abhängigkeit von konjunkturellen Schwankungen geringe Chancen einer Weiterbeschäftigung haben. Allerdings sind auch Ausbildungsabsolventen von einer Nichtübernahme betroffen, die in einem Betrieb mit Nettokosten ihren Beruf erlernt haben, obwohl wegen hoher Investitionen der Bildungsstand und die Ausbildungsqualität hoch ist. Dies ist dann der Fall, wenn ihre Fähigkeiten nicht den betrieblichen Qualifikationsanforderungen entsprechen bzw. konjunkturell schlechte Phasen lang andauern. So sind die Ausbildungsabsolventen, die eine Ausbildung mit höherer Qualität genossen haben, in der entsprechenden Berufsgruppe eher die unterdurchschnittlich fähigen. Schließlich sind die Ausbildungsabsolventen zu berücksichtigen, die freiwillig den Ausbildungsbetrieb verlassen, und die eher überdurchschnittlich qualifiziert sind und daher ihre Chancen auf dem Arbeitsmarkt vorteilhaft einschätzen. Infolgedessen ist innerhalb eines Wirtschaftsbereiches bzw. einer Berufsgruppe

\footnotetext{
${ }^{52}$ Darunter versteht man einen Berufswechsel.

${ }^{53}$ Daher kann man sagen, dass eine mögliche, auf die Übernahme folgende Arbeitslosigkeit (bzw. Einkommenseinbußen bei Wiederbeschäftigung), die Merkmale des Sektors, des Ausbildungsberufes aber auch der geografischen Lage widerspiegelt.
} 
davon auszugehen, dass sich Ausbildungsabsolventen unterschiedlicher Fähigkeiten um dieselben Arbeitsplätze bemühen.

Insgesamt sind jedoch nach Greenwald (1986) die schlechteren nicht übernommenen Ausbildungsabsolventen überproportional im Ausbildungsabsolventenpool vertreten. Dies liegt darin begründet, dass ein Ausbildungsbetrieb in der Regel den besten Ausbildungsabsolventen ein Übernahmeangebot unterbreitet, während der Jugendliche mit schlechteren Fähigkeiten nicht übernommen wird. Dies macht Sinn, da die Unternehmen mit qualifizierten und begabten Arbeitskräften konkurrenzfähig bleiben und im Wettbewerb um Qualität bestehen können (Bellmann/Neubäumer 2001: 190ff.).

Entsprechend resultiert daraus ein unterschiedliches Niveau an Fähigkeiten bzw. an Humankapital zwischen den nicht übernommenen Ausbildungsabsolventen und den übernommenen Ausbildungsabsolventen, die freiwillig den Ausbildungsbetrieb verlassen. Vor allem diese beiden Gruppen von Ausbildungsabsolventen bewerben sich auf dieselben Arbeitsplätze, sofern sie demselben Ausbildungsbereich angehören. Dagegen konkurrieren die nicht übernommenen Ausbildungsabsolventen unterschiedlicher Berufsgruppen nur um dieselben Stellen, wenn es sich um Hilfsarbeitertätigkeiten handelt, zu deren Ausübung die abgeschlossene Berufsausbildung nicht erforderlich ist.

Aus dem heterogenen Arbeitsangebot müssen die Unternehmen auf dem externen Arbeitsmarkt gute Ausbildungsabsolventen von schlechten Ausbildungsabsolventen unterscheiden können. Aufgrund dieses heterogenen Angebots an Ausbildungsabsolventen resultiert (Such-)Arbeitslosigkeit, welche in der Regel eine längere Phase für gute (freiwillig wechselnde) junge Erwachsene darstellt und solange anhält, bis das Matching zwischen Unternehmen und den Ausbildungsabsolventen erfolgreich ist. Da aber die bestehenden Informationsasymmetrien bei der Identifikation von adäquaten Arbeitskräften nicht vollständig abgebaut werden können, werden die Unternehmen bis zur Stellenbesetzung Unsicherheit hinsichtlich der Fähigkeiten der auf dem Arbeitsmarkt befindlichen Ausbildungsabsolventen haben. Um dem Risiko der Einstellung eines schlechteren Ausbildungsabsolventen, einer ,lemon' (Akerlof 1970) vorzubeugen, wird die Stellenbesetzung der verschiedenen Arbeitsplätze unterschiedlich lang andauern. Dies führt auf Seiten der Ausbildungsabsolventen zu Arbeitslosigkeit, deren Voraussetzungen, diese zu verlassen, differieren. Deshalb werden im Folgenden der Verbleib in und Implikationen für die Dauer der Arbeitslosigkeit genauer analysiert. 
Gabriele Somaggio - 978-3-631-74988-3

Downloaded from PubFactory at 01/11/2019 09:32:31AM

via free access 

ren Dauer

Eine Studie des BiBB zeigt im Rahmen einer Untersuchung von Erwerbsverläufen bis drei Jahre nach Abschluss der dualen Berufsausbildung das hohe Arbeitslosigkeitsrisiko der Absolventen auf. Knapp 32\% der jungen Erwachsenen der Ausbildungsabschlusskohorten von 1998 bis 2001 waren mit einer durchschnittlichen Dauer von fünf Monaten innerhalb des Betrachtungszeitraumes arbeitslos. Die Arbeitslosigkeit direkt nach Abschluss der dualen Berufsausbildung betrug 11\%, fiel aber nach dem ersten Jahr auf 3,7\% (Dorau et al. 2006: 6ff.). Die Mehrheit der Ausbildungsabsolventen bleibt nur kurze Zeit arbeitslos, was auf eine hohe Sucharbeitslosigkeit hindeutet, deren Anteil 2005 unter den Ausbildungsabsolventen knapp $25 \%$ betrug (Baethge et al. 2007). Daraus lässt sich schließen, dass nicht alle Ausbildungsabsolventen, die den Betrieb wechseln, gleichermaßen von Erwerbslosigkeit betroffen sind. Eine Arbeitslosigkeitsperiode für Ausbildungsabsolventen wird zum Teil von anderen Faktoren bestimmt als eine für Arbeitnehmer mit Berufserfahrung. Da sich die Phase der Erwerbslosigkeit am Übergang der zweiten Schwelle befindet, sind Rahmenbedingungen während der Ausbildung mit denen auf dem Arbeitsmarkt zu verknüpfen. Somit muss eine Verbindung zwischen Berufsbildungssystem und Arbeitsmarkt hergestellt werden. Im folgenden Kapitel wird zunächst auf die Situation der Ausbildungsabsolventen auf dem Gesamtarbeitsmarkt eingegangen. Mit der Einschränkung der Betrachtung auf die Ausbildungsabsolventen wird das Suchverhalten auf betrieblicher und individueller Seite getrennt erklärt, um die genaueren Wirkungen der Informationsasymmetrien auf die Arbeitslosigkeit zu beschreiben, welche sich durch die Ausbildung bestimmen. Diese lässt sich dann an Hand zweier unterschiedlicher Aspekte beleuchten. Hauptsächlich erklären die suchtheoretischen Grundlagen die unterschiedliche Dauer von Arbeitslosigkeit der Ausbildungsabsolventen. Rückschlüsse auf die Häufigkeit der Eintritte in Arbeitslosigkeit der unterschiedlichen Absolventengruppen können ebenfalls gezogen werden.

\subsection{Situation der Ausbildungsabsolventen auf dem Gesamtarbeitsmarkt}

Da Ausbildungsabsolventen, wenn sie den Ausbildungsbetrieb verlassen, auf dem externen Arbeitsmarkt auch mit Arbeitskräften, die bereits über eine längere Zeit im Erwerbsleben stehen, konkurrieren (Kock 2008: 17), wird in diesem Kapitel kurz eine Gegenüberstellung der Arbeitslosigkeitsrisiken der unterschiedlichen Gruppen von Arbeitssuchenden unternommen.

Im Allgemeinen lässt sich ein höheres Arbeitslosigkeitsrisiko von Ausbildungsabsolventen ableiten (Shimer 2001; Pissarides 2000; Mortensen/ Pissarides 1994). Gegenüber den länger am Erwerbsleben teilnehmenden Arbeitskräften weisen Ausbildungsabsolventen einen geringeren Humankapitalbestand auf, weil ihnen aufgrund der kürzeren Betriebszugehörigkeit Berufserfahrung fehlt, 
welche insbesondere die betriebs- bzw. technologiespezifische Humankapitalkomponente erhöht (Alda 2006: 56; Beckmann 2004: 40). Dies steigert zugleich die Produktivität und reduziert aber auch die Entlassungswahrscheinlichkeit bei exogenen Schocks. Aufgrund der Komplementarität von allgemeinem und betriebsspezifischem Humankapital, auch über die Ausbildung hinaus, wird impliziert, dass Unternehmen neue Arbeitskräfte mit zunehmender Berufserfahrung besser und schneller einlernen können. Wird daher ausschließlich die Produktivität betrachtet, nehmen die Ausbildungsabsolventen eine schlechtere Position gegenüber den Arbeitskräften mit Berufserfahrung ein. Wegen ihrer geringeren Produktivität weisen die Ausbildungsabsolventen schlechtere Chancen auf als Arbeitnehmer mit Berufserfahrung, wenn es um die Besetzung von vakanten Stellen geht (Burdett/Mortensen 1980: 657).

Dem erhöhten Arbeitslosigkeitsrisiko der Ausbildungsabsolventen steht aber ebenso die erhöhte Chance einer schnellen Wiederaufnahme einer Beschäftigung gegenüber, da das neu erworbene Humankapital noch nicht veraltet ist und auf den aktuellen technologischen Stand der Produktion angewendet werden kann. Dadurch profitieren Unternehmen bei der Einstellung von dem neuen Wissen der nicht im Ausbildungsbetrieb verbliebenen Absolventen. Dies bringt gegenüber den älteren Arbeitnehmern einen Wettbewerbsvorteil, da diese selbst bei ununterbrochener Beschäftigung eine Abwertung ihres Humankapitals erfahren (Ludwig/Pfeiffer 2005). Darüber hinaus legen die Ausbildungsabsolventen den Lohn, ab dem sie bereit sind, Arbeit anzunehmen, wegen der geringeren Produktivität auch niedriger fest. Schließlich ist davon auszugehen, dass jüngere Erwerbstätige geografisch flexibler sind als erfahrene Arbeitnehmer, da sie in der Regel nicht familiär gebunden sind. Allerdings zeigen Studien (Seifert 2007; Haas 2002) auch unter den Ausbildungsabsolventen eine geringe Mobilität. Schließlich haben sie gegenüber Arbeitssuchenden ohne Berufsabschluss Vorteile, weil der Abschluss der dualen Berufsausbildung soziale Kompetenzen signalisiert, die nur während einer Beschäftigung erworben werden können (Casey 1986: 73).

Die Situation bei der Betrachtung des gesamten Arbeitsangebots ist daher nicht eindeutig zu identifizieren. Jedoch deuten die Arbeitslosenzahlen darauf hin (siehe Tabelle 14 im Anhang), dass die Arbeitnehmer mit geringer Berufserfahrung, worunter auch die Ausbildungsabsolventen zu subsumieren sind, eine weniger günstige Ausgangsposition bei den Stellenbewerbungen haben. Aber auch unter den Ausbildungsabsolventen ist das Arbeitslosigkeitsrisiko unterschiedlich verteilt, wie in den folgenden Kapiteln erläutert wird. 


\subsection{Theoretische Grundlagen für Arbeitslosigkeit nach der dualen Be- rufsausbildung und deren Dauer}

\subsubsection{Suchtheoretische Ansätze}

Gerade Arbeitslosigkeitsphasen für Ausbildungsabsolventen sind in Verbindung mit den Gründen für das unterschiedliche Übernahmeverhalten zu sehen. Darüber hinaus haben die Unternehmen keine Kenntnis, ob das Verlassen des Betriebes freiwilliger oder unfreiwilliger Natur ist, was die Vollständigkeit der Informationen einschränkt. Unternehmen sehen sich daher einem heterogenen Arbeitsangebot von Ausbildungsabsolventen gegenüber, die sich nach der dualen Berufsausbildung hinsichtlich des Bildungsstandes unterscheiden. Auf Seiten der Ausbildungsabsolventen bestehen ebenfalls unvollständige Informationen gegenüber den Unternehmen (Mortensen 1986: 852). Daher spielen existierende Informationsasymmetrien eine wichtige Rolle zur Erklärung von Arbeitslosigkeit (Garloff 2003; Yashiv 2006; Mortensen/Pissarides 1994; Burdett/Mortensen 1980), weshalb die Stellenbesetzung bzw. -suche nicht ohne Unterbrechung erfolgen kann. Auch bestimmen die unterschiedlichen Rahmenbedingungen während der Ausbildung die qualifikatorischen Voraussetzungen der nicht übernommenen ${ }^{54}$ Ausbildungsabsolventen und somit deren Arbeitslosigkeitsdauer. Im Folgenden werden daher zunächst die Arbeitgeberseite und dann die Arbeitnehmerseite unter suchtheoretischen Aspekten betrachtet. Suchmodelle stellen dabei die Ströme von der Erwerbstätigkeit in die Arbeitslosigkeit sowie von der Arbeitslosigkeit in die Erwerbstätigkeit dar, wobei der Dynamik des Arbeitsmarktes Rechnung getragen wird (Maidorn 2002: 2). In der Literatur werden diese Ströme zunächst als Entscheidung der Arbeitgeber dargestellt, die versuchen den Nutzen eines Jobs durch eine adäquate Stellenbesetzung zu erhöhen (Pissarides 1994; Mortensen/Pissarides 1994; Maidorn 2002). Diese werden um den Aspekt der Stellensuche auf Arbeitnehmerseite erweitert (Wasmer 2006; Pissarides 1979). Im Folgenden werden diese Ansätze dargestellt mit einem besonderen Fokus auf das während der Ausbildung vermittelte Humankapital und dessen Folgen für die Arbeitslosigkeitsphase.

\subsubsection{Arbeitgeberseite: Adverse Selektion bei der Stellenbesetzung}

Unternehmen, die Fachkräfte auf dem externen Arbeitsmarkt einstellen wollen, müssen aus dem heterogenen Arbeitsangebot (siehe Kap. 4.4) die entsprechenden Arbeitskräfte identifizieren, die zu den zu besetzenden Stellen passen. Das Arbeitsangebot besteht vor allem aus Ausbildungsabsolventen, die ihren Ausbildungsbetrieb freiwillig verlassen haben. Weil die unfreiwilligen Wechsler nicht von den freiwilligen Wechslern unterschieden werden können, sind nach Gibbons und Katz (1991) sowie Greenwald (1986) die freiwillig in Arbeitssuche

\footnotetext{
${ }^{54}$ Im Folgenden steht der Ausdruck, nicht übernommen' auch für Ausbildungsabsolventen, die freiwillig den Betrieb wechseln.
} 
befindlichen und damit eher guten Absolventen besonders benachteiligt. Ein Wechsel bedeutet grundsätzlich ein negatives Signalling gegenüber potenziellen neuen Arbeitgebern. Um daher die freiwilligen von den unfreiwilligen, das heißt die guten von den schlechten, Absolventen unterscheiden zu können, müssen bestehende Informationsasymmetrien hinsichtlich der Fähigkeiten der Ausbildungsabsolventen zunächst abgebaut werden. Dies geschieht bereits durch die Modalitäten bei der Stellenbesetzung selbst, vor allem aber während einer Beschäftigung. Bei der Einstellung bzw. am Ende der Suchphase herrscht weiterhin Unsicherheit hinsichtlich der Art und Höhe der Fähigkeiten der Ausbildungsabsolventen, was sich auf die Qualität des Job-Matchings auswirkt (Mortensen 1986: 856). Bei der Suche nach Arbeitskräften mit ausreichenden Qualifikationen für die zu besetzenden Stellen verhalten sich die Unternehmen aber risikoneutral. Daher erfolgt die Allokation der Arbeitskräfte nur mit Zeitverzögerung.

Aufgrund dieser Unsicherheiten bieten die Unternehmen verschiedene Arten von Arbeitsplätzen an, die unterschiedliche Qualifikationsanforderungen an die Arbeitnehmer stellen. Da keine Annahmen über die Art und Struktur des Humankapitals getroffen werden, ist die Höhe der angeeigneten Bildung für die Besetzung der Stellen entscheidend. Daher wird vereinfachend angenommen, dass die Betriebe über gute und schlechte Jobs verfügen (Franz 2006: 218). Gute Jobs sind dabei durch eine höhere Produktivität als die schlechten Jobs gekennzeichnet, wobei diese höhere Produktivität durch ein höheres Bildungsniveau $\tau$ erreicht wird. Entsprechend teurer sind sie für die Unternehmen bei und nach der Besetzung (Pissarides 1994: 460), weil sie aufgrund des höheren Produktionsbeitrags $f=a(\tau) \eta$ eine höhere Entlohnung versprechen. $\eta$ stellt die informellen Fähigkeiten dar, welche für Unternehmen auf dem externen Arbeitsmarkt nicht beobachtbar sind Deshalb sind die Unternehmen auch bereit, höhere Kosten $\left(c_{g u, t+3}>c_{s c, t+3}\right)$ für die adäquate Besetzung der Arbeitsplätze einzusetzen. Dabei spiegeln $c_{g u, t+3}$ die Kosten für die Besetzung der guten Jobs und $c_{s c, t+3}$ die Kosten der schlechten Jobs wider. Unternehmen wenden Kosten zur Rekrutierung von ausgebildeten Arbeitskräften, so genannte Einstellungskosten, für die Werbung, Stellenanzeigen sowie Einstellungsverfahren auf, um Informationen über potenzielle Mitarbeiter zu vervollständigen (Bloemen 2005: 233; Pissarides 1979: 819 Yashiv 2006: 4). Da die Jobs mit den unterschiedlichen Qualifikationsanforderungen unterschiedliche Einarbeitungszeiten erfordern, sind diese Kosten ebenfalls zu berücksichtigen. Die guten Jobs verursachen aufgrund der höheren Qualifikationsanforderungen höhere Einarbeitungskosten, weil sie entsprechend auch höher entlohnt werden als die schlechten Jobs. Um einen Arbeitsplatz mit hohen Produktivitätsanforderungen mit guten Ausbildungsabsolventen zu besetzen, ist bei der Einstellung ein höherer Informationsasymmetrieabbau notwendig. Daher fallen die Kosten zur Besetzung eines schlechteren Arbeitsplatzes $c_{s c}$ geringer aus als diejenigen zur Besetzung eines guten Jobs $c_{g u, t+3}$ 
(Pissarides 1994: 460f.) ${ }^{55}$. Diese Kosten fallen zum Zeitpunkt der Stellenbesetzung an, während die Erträge und die Arbeitsplatzkosten, wie Löhne, über die gesamte Beschäftigungszeit $(t)$ anfallen. Letztere müssen daher auf den Zeitpunkt der Stellenbesetzung abgezinst werden. Formal gilt dann die Bedingung

$$
\frac{f a(\tau)-\omega_{g, t+3}}{(1+i)^{t+3}}-c_{g u}>\frac{f a(\tau) y-\omega_{s c, t+3}}{(1+i)^{\prime+3}}-c_{s c},
$$

wobei $a(\tau) f$ den Produktionsbeitrag aus einem guten und $a(\tau) y f$, mit $0<y<1$, den aus einem schlechten darstellt. Von diesen unterschiedlichen Produktionsbeiträgen wird die Entlohnung, die für Absolventen unterschiedlichen Bildungsniveaus gilt, abgezogen. Daraus ergibt sich das betriebliche Optimierungsproblem, welches durch den Trade-off gekennzeichnet ist: Die guten Stellen erfordern ein höheres Mindestniveau an Humankapital, das einen höheren Produktionsbeitrag erbringt, aber auch eine höhere Entlohnung bedeutet $\left(\omega_{g, t+3}>\omega_{s c, t+3}\right)$. Je höher diese Anforderungen aber sind, desto geringer ist die Anzahl der Absolventen, diese zu erfüllen. Die Wahrscheinlichkeit, die offenen Stellen auch adäquat zu besetzen, sinkt (Franz 2006: 218).

Die Unsicherheit über die Einstellung eines schlechten Ausbildungsabsolventen aus dem gesamten Angebot der Ausbildungsabsolventen, eines so genannten ,lemons' (Akerlof 1970) bzw. die Unsicherheit über ein schlechtes Matching, kann durch unterschiedliche Mechanismen abgebaut werden. So wird, um möglichst gute Bewerber auf die offenen Stellen zu erhalten, die Art des Arbeitsplatzes dem potenziellen Arbeitnehmer erst bei dessen Interesse an diesem Job signalisiert, sodass hier Informationsunvollständigkeiten gegenüber den arbeitssuchenden Personen bewusst in Kauf genommen werden. Dabei gehen Unternehmen davon aus, dass die individuelle Suchintensität der Ausbildungsabsolventen auch mit dem Bildungsstand steigt. Bspw. können die registrierten Arbeitslosen die Suchintensität erhöhen, indem über die Unterstützung bei der Suche durch das Arbeitsamt hinaus Informationen durch Anzeigen über freie Stellen eingeholt werden. Dies wiederum hat positive Auswirkungen auf das Jobangebot der Unternehmen. Diesen Bewerbern werden dann die guten Jobs angeboten. Auf Seiten der Unternehmen kann im Gegenzug die Suchintensität ebenfalls über eine Job-Matching-Methode verbessert werden (Pissarides 1979: 819).

Ein Unternehmen muss aus den Bewerbern auswählen, welche zufällig Interesse an einer Arbeitsstelle signalisieren, weil letztere die Qualität dieser Jobs nicht kennen. Wenn ein Unternehmen auf jeden Fall die freien unproduktiven Arbeitsplätze besetzen will, bevorzugt es daher auch arbeitslose Personen (Pissarides 1994: 462). Entsprechend werden die schlechten Jobs relativ zügig besetzt, was gleichzeitig auch eine relative kurze Arbeitslosigkeitsdauer der schlechteren (arbeitslosen) Personen impliziert. Ein Prozess der adversen Selek-

\footnotetext{
${ }^{55}$ Auch sind die neu geschaffenen Arbeitsplätze produktiver als die bestehenden und damit unter die guten Stellen zu subsumieren (Mortensen/Pissarides 1994: 398).
} 
tion setzt sich in Gang, weil wegen des heterogenen Arbeitsangebotes an Ausbildungsabsolventen der Marktlohn an der Produktivität der schlechteren Ausbildungsabsolventen ausgerichtet wird (Greenwald 1986: 325). Daher werden die Ausbildungsabsolventen, die ihren Ausbildungsbetrieb verlassen (müssen), bei der neuen Arbeitsstelle einen niedrigeren Lohn erhalten, weil zwar symmetrische, aber unvollständige Informationen angenommen werden (Garloff 2003: 2; Gibbons/Katz 1991: 354). Daraufhin werden sich die besseren Ausbildungsabsolventen nicht auf die Stellen bewerben, die unter Umständen inhaltlich ihrer Ausbildung und tatsächlichen Produktivität entsprechen, aber mit dem Marktlohn entlohnt werden. Insbesondere gute Ausbildungsabsolventen, die freiwillig den Betrieb verlassen und entsprechend überdurchschnittliche Fähigkeiten innerhalb ihrer Berufsgruppe aufweisen, werden daher solange suchen, bis sie einen für sie adäquaten Job gefunden haben. Infolgedessen verlängert sich die Suchphase, da sich die Auswahl von Stellenangeboten verringert und sich daher schwieriger gestaltet. Dagegen bewerben sich nur die schwächeren auf Stellen mit gegebenen Produktivitätsanforderungen, was für die entsprechenden Unternehmen ein schlechtes Matching bedeutet. Deshalb kann die Unsicherheit hinsichtlich der Besetzung der Arbeitsplätze mit „lemons“ reduziert sowie den asymmetrischen Informationen Rechnung getragen werden, indem bspw. befristete Verträge abgeschlossen (sie auch Kap. 5.4) oder Effizienzlöhne gezahlt werden. Bei letzterer Möglichkeit bieten die Unternehmen einen Lohn an, der den Marktlohn der jeweiligen Berufsgruppen übersteigt. Infolgedessen kann die durchschnittliche Produktivität des Betriebes erhöht werden (Yellen 1984: 200). Auf diese Weise erhoffen sich die Unternehmen, dass sich die Durchschnittsproduktivität bereits bei den Arbeitsplatzbewerbern erhöht. Aufgrund dieses positiven Zusammenhangs zwischen Arbeitsproduktivität und Lohnhöhe werden sich gute Arbeitsplatzbewerber melden. Da der Effizienzlohn aber den Markt räumenden Lohn übersteigt, werden die Unternehmen aus dem Bewerberangebot entsprechend die besten selektieren (Sesselmeier/Blauermel 1998: 161f.).

Infolgedessen werden die guten Absolventen erst nach einiger Zeit ein Arbeitsangebot erhalten, während schlechte Absolventen relativ schnell ein Arbeitsangebot bekommen. Das liegt insbesondere daran, dass die guten Jobs langfristig besetzt werden müssen und daher auch viel in die Besetzung investiert wird, damit die Unternehmen ihre Markt- und Wettbewerbsposition halten und verbessern können (Neubäumer 1997). Langfristig verschlechtert sich jedoch die Arbeitsmarktsituation der schlechteren Ausbildungsabsolventen bzw. derjenigen mit weniger guten Ausbildungsvoraussetzungen weiter, da bei dem Besetzungsprozess der Arbeitsstellen die besseren Ausbildungsabsolventen zum Zug kommen. Die Ausbildungsabsolventen mit einem niedrigen Bildungsstand und einer schlechteren Ausbildungsqualität weisen ein höheres Arbeitslosigkeitsrisiko auf. Dies bedeutet zugleich, dass auch die Vakanz der Arbeitsstellen mit steigenden Qualifikationsanforderungen länger bestehen bleibt als bei Jobs mit geringer Produktivität. Daher gilt es den Aufwand der Stellenbesetzung für die an- 
spruchsvolleren Stellen möglichst gering zu halten. Daher werden diese Arbeitsplätze auf längere Sicht besetzt.

\subsubsection{Arbeitnehmerseite: Individuelles Suchverhalten}

Die Phase der Arbeitslosigkeit hängt jedoch nicht nur von der Unternehmensseite, sondern auch von der Angebotsseite und somit von dem Ausbildungsabsolventen selbst ab. Hierbei stehen das Suchverhalten der Arbeitnehmer und deren Interaktion mit der Unternehmensseite im Mittelpunkt der Betrachtung. Mit dem suchtheoretischen Ansatz kann sowohl die Situation der nicht übernommenen, arbeitslosen, sowie der befristetet übernommenen Ausbildungsabsolventen dargestellt werden (Bloemen 2005: 233; Mortensen 1986: 869ff.).

Einerseits sind die nach einem Arbeitsplatz suchenden jungen Erwachsenen von unvollständigen Informationen betroffen, da sie im Voraus nicht wissen, um welche Art von Job es sich handelt. Andererseits können sie ihre tatsächliche Produktivität nicht einschätzen. Da sie ebenfalls, wie die Unternehmen risikoneutral handeln, müssen auch sie Informationen vervollständigen. Dieser Prozess beansprucht Zeit. Ferner verhalten sich die Akteure nutzenmaximierend, das heißt, Arbeitnehmer wägen zwischen dem Nutzen der Arbeitslosigkeit bzw. dem Freizeitwert und dem Nutzen der Erwerbstätigkeit ab. Das Entscheidungsproblem eines arbeitslosen Ausbildungsabsolventen reduziert sich darauf, ob er ein Stellenangebot akzeptiert oder ablehnt und weiter arbeitslos bleibt. Die Suchphase der Arbeitssuchenden bestimmt sich daher durch den Zeitpunkt, bei welchem die Entscheidung fällt, ein Arbeitsangebot anzunehmen oder noch auszuschlagen. Durch die Verlängerung der Suchphase erhöht sich die Chance, ein nutzenmaximierendes Angebot zu erhalten. Dabei ist die Suchphase zunächst von der Ermittlung nach entsprechenden Jobangeboten und dem darauf folgenden Prozess zur Gewinnung von Kenntnissen über den zukünftigen Arbeitsplatz gekennzeichnet. Schließlich findet eine Entscheidung darüber statt, ob die Suchphase fortgesetzt oder durch die Annahme eines Jobangebots beendet wird. Diesem Entscheidungsprozess liegt auch das so genannte Stopping-Problem zugrunde (Mortensen/Pissarides 1999: $2571 \mathrm{ff}$.), was den Zeitpunkt impliziert, zu dem ein Arbeitssuchender ein Jobangebot akzeptiert.

Die Entscheidungsgröße ist das Einkommen. Es wird zwischen dem Einkommen aus einer Erwerbstätigkeit $\omega$ und dem Wert der Freizeit $b$ unterschieden. Die Freizeit wird im Allgemeinen durch die Lohnersatzleistung aus der Arbeitslosenversicherung $b>0$ quantifiziert (Franz 2005: 219; Rogerson et al. 2004: 4; Pissarides 1979: 821). Die Suchphase wird dann gestoppt und das Jobangebot akzeptiert, wenn der angebotene Lohn $\omega$ über dem Wert der Freizeit $b$ liegt. Die Arbeitsstellensuche wird demnach beendet, sobald der Nutzen, den der arbeitssuchende Absolvent im nächsten Schritt erzielen würde, die Suchkosten 
unterschreitet. ${ }^{56}$ Mit dem Freizweitwert steigen aber auch die Suchkosten. Die Annahme des Jobangebots erfolgt, wenn die Entlohnung mindestens der Höhe des Freizeitwertes entspricht. Andere Faktoren, welche den Nutzen einer Arbeitskraft erhöhen, müssen berücksichtigt werden. Bspw. sind die Vorstellungen von einem Arbeitsplatz, wie Ort oder auch Ansehen des Jobs, zu beachten (Rogersen et al. 2004: 4). Es wird auch von nicht monetärem Einkommen gesprochen. Schließlich herrscht immer auch eine Wahrscheinlichkeit, wieder entlassen zu werden. Deshalb zieht der Arbeitssuchende ebenfalls die Fluktuationsrate in seine Entscheidungsgrundlage mit ein (Burdett/Mortensen 1980: 653). Daraus lässt sich der Reservationslohn $w_{R}$ ableiten, der den Schwellenwert beschreibt, ab dem ein Jobangebot akzeptiert wird. Dieser lautet somit:

$$
w_{R}=T\left(w_{R}\right) \equiv(1-\beta) b+\beta \int_{0}^{\infty} \max \left\{\omega, w_{R}\right\} d F(\omega) .
$$

Dabei stellt $\beta$ mit $0<\beta<1$ den Gewichtungsfaktor dar, mit dem die Entscheidung zu einem bestimmten Zeitpunkt für oder gegen einen Wiedereintritt in die Erwerbstätigkeit bewertet wird. Die Funktion $T\left(w_{R}\right)$ setzt sich aus der Summe der Gegenwahrscheinlichkeit $(1-\beta)$, dass die Suche fortgesetzt und die Arbeitslosenunterstützung geleistet wird, addiert mit der Wahrscheinlichkeit $(\beta)$, dass ein Job zum Zeitpunkt $t$ angenommen wird, zusammen. Die Entlohnung für die Freizeit erhält er demnach nur mit einer Wahrscheinlichkeit von $(1-\beta)$, während er die Entlohnung eines Jobs mit einer Wahrscheinlichkeit von $\beta$ erhält. Das Integral stellt die Verteilung dieser Wahrscheinlichkeiten dar, die sich für jeden Absolventen während dessen Suchphase ändern kann. $\beta$ wird mit zunehmender Arbeitslosigkeitsdauer steigen. Dies bedeutet, dass sich der arbeitslose junge Erwachsene für ein Jobangebot entscheidet, wenn die Entlohnung $\omega$ den Reservationslohn $w_{R}$ als Summe des Freizeitwertes und des nicht monetären Einkommens übersteigt, demnach $\omega>w_{R}$ gilt.

Für arbeitslose Ausbildungsabsolventen wird der Reservationslohn vor allem von den Rahmenbedingungen während der Ausbildung beeinflusst, weil diese Gruppe ansonsten nicht ausreichende Erfahrungen auf dem Arbeitsmarkt sammeln konnte. Daher wird das (Aus-)Bildungsniveau die Höhe des Reservationslohnes bestimmen. Ausbildungsabsolventen, deren Ausbildung in einem produktionstheoretisch orientierten Betrieb stattgefunden hat, weisen einen niedrigeren Reservationslohn auf. Dies liegt darin begründet, dass die Ausbildungsvergütungen als Grundlage für die Bestimmung des Reservationslohnes in Verbindung mit dem zu erwartenden künftigen Lohn innerhalb der Branche herangezogen werden, welche für diesen Bereich niedrig liegen. Beeinflusst wird diese Höhe zudem von dem geringen Bildungsstand und der relativ geringen Ausbildungsqualität. Entsprechend sind die Anforderungen der Absolventengruppe an

${ }^{56}$ Das heißt, dass der nächste Suchschritt teurer ist als der zu erwartete Lohn beim nächsten Stellenangebot. 
den künftigen Arbeitsplatz nicht sehr hoch. Anders stellen sich die Ansprüche der Ausbildungsabsolventen dar, deren Ausbildungsbetrieb hohe Investitionen in Bildung getätigt hat. Sie besitzen einen höheren Bildungsstand, der entsprechend den jeweiligen Reservationslohn determiniert. Weiterhin steigt der Reservationslohn durch Faktoren, wie höhere Anforderungen an Karrierechancen und dergleichen, was ebenfalls eine hohe Einschätzung der persönlichen Chancen impliziert. Diese steigen bei Absolvieren einer Ausbildung mit guter Bildungsqualität. Gleichermaßen erhöht sich auch der Reservationslohn (Franz 2005). Begründet liegt dies in einer positiven Zeitpräferenz: Je niedriger der Reservationslohn ist, desto schneller möchte der nicht übernommene Ausbildungsabsolvent wieder in Arbeit kommen, aber desto niedriger ist auch sein Humankapital bzw. die Einschätzung seiner Chancen auf dem Arbeitsmarkt. Daher fördert auch die Seite der Arbeitssuchenden die adverse Selektion. Da Unternehmen den angebotenen Lohn an den jungen Erwachsenen mit niedrigem Humankapital ausrichten, werden sie auch entsprechend aus dieser Gruppe Bewerber auswählen müssen. Denn Ausbildungsabsolventen, welche sich als gut einschätzen, weil sie eine Ausbildung mit hoher Qualität genossen haben, werden das Arbeitsangebot zu dem angebotenen Lohn nicht akzeptieren und entsprechend weitersuchen. Der Nutzen einer weiteren Suchperiode ist höher als der Nutzen aus der Annahme des Stellenangebots. Unternehmen können dann auch die Arbeitsplätze mit den hohen Qualifikationsanforderungen nicht sofort mit guten Ausbildungsabsolventen besetzen, da nur die jungen Erwachsenen mit niedrigerem Humankapitalniveau das Angebot zu dem entsprechenden Marktlohn akzeptieren werden. Infolgedessen verlängert sich die Suchphase bzw. die Arbeitslosigkeitsdauer der Ausbildungsabsolventen mit höherem Humankapitalniveau, weil sie solange suchen werden, bis sie das Jobangebot mit dem höchsten individuellen Nutzen erhalten. Daher ist es sowohl für das Unternehmen wie auch für die Ausbildungsabsolventen lohnend, wenn statt des Marktlohnes höhere Effizienzlöhne gelten.

Der suchtheoretische Ansatz reduziert sich bei der Diskussion um die Bestimmungsgründe der Arbeitslosigkeitsdauer auf die Beschreibung der unterschiedlichen Höhe des Freizeitwertes. Dabei wird lediglich die Heterogenität des Arbeitskräfteangebots auf dem Arbeitsmarkt angedeutet, indem die Reservationslöhne unterschiedlich hoch sind bzw. die Suchphase unterschiedlich lang, was vor allem durch Institutionen, wie die Ausgestaltung der Arbeitslosenunterstützung, erklärt wird. Die Unterschiede der Arbeitssuchenden hinsichtlich individueller Merkmale sowie der Struktur des Humankapitalstandes werden nicht konkretisiert. Auf die Wirkung der Bildung wird nur indirekt über die Größen, wie Suchdauer und Reservationslohn, eingegangen. Schließlich kann die Häufigkeit, mit der eine Person wieder arbeitslos wird, nachdem sie Beschäftigung gefunden hat, nicht abgeleitet werden. Jedoch geht Pissarides (2000) auf die OnThe-Job-Arbeitssuche ein. So sind befristet übernommene Ausbildungsabsolventen gegenüber Erwerbslosen im Vorteil. Sie erhalten in der Regel ein besse- 
res Stellenangebot auf dem externen Arbeitsmarkt, da die Unternehmen in der Lage sind, zwischen arbeitslosen und erwerbstätigen Arbeitssuchenden zu unterscheiden. Darüber hinaus gewinnen sie während der befristeten Weiterbeschäftigung Berufserfahrung und erlangen zunehmend Kenntnis über ihre wahre Produktivität (Jovanovic 1979).

\subsubsection{Erweiterungen des Suchmodells um humankapitaltheoretische Aspekte}

Der suchtheoretische Ansatz berücksichtigt lediglich Produktivitätsniveaus, die unterschiedliche Bildungsniveaus implizieren. Allerdings muss die Betrachtung des Einflusses der Humankapitalhöhe um die verschiedenen Humankapitalarten erweitert werden. Denn diese beeinflussen über die Höhe der Bildung den Reservationslohn. Die Einbettung der Humankapitalkomponenten in die Untersuchung ist aber notwendig, um das unterschiedliche Auftreten von Arbeitslosigkeit in der Gruppe der Ausbildungsabsolventen begründen zu können. Denn gerade das Bildungsniveau und die Art der Ausbildung, hinsichtlich der Gesamtheit der erlernten Fähigkeiten innerhalb eines Ausbildungsberufes, sind bei der Erklärung der Arbeitslosigkeit nach der dualen Berufsausbildung heranzuziehen. Humankapitaltheoretische Aspekte werden daher mit den suchtheoretischen Gesichtspunkten in Zusammenhang gebracht und die Implikationen dieses Zusammenhangs erörtert.

Hierbei ist die Entscheidung für die Art des Humankapitals auf Seiten des Ausbildungsabsolventen selbst, die er an der ersten Schwelle trifft, zu beachten. So verlagern sich die unterschiedlichen Wirkungen der Entscheidung an der ersten Schwelle über den Verbleib nach der Ausbildung hinaus auf die Arbeitslosigkeitsperiode. Denn zu Beginn der Ausbildung bestimmt sich, ob eine Tätigkeit mit vorwiegend allgemeinem oder vorwiegend betriebsspezifischem $\mathrm{Hu}-$ mankapital ausgeübt bzw. erlernt wird. Dabei sieht sich der Arbeitnehmer vor ein intertemporales Entscheidungsproblem gestellt, weil die Art des Humankapitals den Verbleib oder einen Unternehmenswechsel bestimmt. Dieses Entscheidungsproblem kann das Individuum aber durch Antizipation der Lohnhöhe und der Dauer von Beschäftigungsperioden lösen (Wasmer 2006: 813). Der Auszubildende kennt die Produktivität innerhalb des Unternehmens, antizipiert den Lohn und die Beschäftigungsdauer in dem betreffenden Betrieb und entscheidet sich auf dieser Grundlage für eine Investition in eine Humankapitalart. Die jeweilige Entscheidung wird von den Unternehmen unterstützt, wenn die Kosten für die gewählte Humankapitalart gering sind (Miyamito/Shirai 2006). Die Ausbildungsentscheidung muss nun in Bezug zu der Flexibilität des Arbeitsmarktes gesetzt werden, um den Einfluss der Humankapitalart auf die Arbeitslosigkeit zu erörtern.

Entgegen der bisherigen Annahme einer Komplementarität zwischen den Humankapitalarten (Kap. 3) wird davon ausgegangen, dass die beiden Arten von Humankapital substitutiv zueinander oder zumindest unvollständige Substitute 
sind (Wasmer 2006: 812). Da auch hier die Akteure risikoneutral handeln, entscheidet sich der Auszubildende zu Beginn der Ausbildung, in allgemeines Humankapital zu investieren, wenn die Wahrscheinlichkeit, einen neuen Job bei Arbeitslosigkeit auf dem externen Arbeitsmarkt zu finden, hoch ist. Die Ausbildung findet dann unter den Rahmenbedingungen eines flexiblen Arbeitsmarktes statt, der durch eine hohe Fluktuationsrate gekennzeichnet ist. Durch die Investition in allgemeines Humankapital wird eine effiziente Allokation ermöglicht, die aufgrund von technischem Fortschritt oder Veränderungen in der Güternachfrage notwendig wird (Caparos/Navarro 2005: 42). Auch die Untersuchungen von Elliott und Lindley (2001) ergaben, dass die Ausbildungsabsolventen mit einem hohen Anteil an allgemeinem Humankapital beruflich mobil sind und daher die Allokation vorantreiben. Die Unternehmen werden in einem flexiblen Arbeitsmarkt bereit sein, in allgemeines Humankapital zu investieren und dadurch die individuelle Entscheidung zu unterstützen. Die Unternehmen werden aber aufgrund der hohen Fluktuationsrate die Produktivitätsschwelle, ab der Ausbildung stattfindet, senken, um weiterhin ein Ausbildungsangebot ermöglichen zu können (Booth/Zoega 1999). ${ }^{57}$ Durch die geringe Produktivität sinken auch die Qualifikationsanforderungen. Dafür nimmt die Zahl der Auszubildenden zu (Booth/Zoega 1999: 383). Die Produktivität ist deshalb so niedrig, weil die Tätigkeiten innerhalb des Ausbildungsberufes sowie die wenig komplexen Arbeitsstrukturen im Ausbildungsbetrieb dies zulassen, weshalb externe Arbeitskräfte ohne größere Kosten eingestellt werden können. Aus betrieblicher Sicht können die vakanten Stellen daher schnell wieder besetzt werden, weshalb die Unternehmen trotz der hohen Abgangsrate von einer allgemeinen Humankapitalinvestition profitieren. Aufgrund der hohen vorherrschenden Abgangsraten aus den Ausbildungsbetrieben werden Personen, welche allgemeines Humankapital besitzen, häufig in Arbeitslosigkeitsphasen eintreten. Die Möglichkeit, weiterhin die vollständige Produktivität bei einem Wechsel beibehalten zu können und die geringe Einarbeitungszeit in eine neue Tätigkeit verkürzt aber die Dauer der Arbeitssuche.

Ist der Arbeitsmarkt dagegen inflexibel und infolgedessen die Arbeitskräfteallokation weniger effizient, investieren Betriebe und Auszubildende nur in betriebsspezifisches Humankapital. Die Bereitschaft zu dieser Art von Humankapitalinvestition wird durch die Existenz von institutionellen Regelungen, wie bspw. Kündigungsschutz gefördert. Dies verbessert die Erwerbsposition der eher betriebsspezifisch ausgebildeten Arbeitnehmer aufgrund einer hohen Beschäftigungssicherheit und fördert die Akkumulation betriebsspezifischen Humankapitals. Die Fluktuationsrate sinkt, weil einerseits die Vernichtung von Arbeitsplätzen gering ist, wodurch die Arbeitslosenrate zurückgeht. Andererseits verringert sich aber auch die Schaffung neuer Jobs, was die Arbeitslosigkeit verfestigt (Miyamito/Shirai 2006; Davis/Haltiwanger 1990). Unter diesen Voraussetzungen ist die Beschäftigung auf Dauer ausgerichtet und die Wahrscheinlichkeit des

${ }^{57}$ Sie nehmen hierbei auch einen unvollkommenen Markt an. 
Eintritts in Arbeitslosigkeit sehr gering. Wenn aber durch exogene Schocks die Wahrscheinlichkeit einer Arbeitslosigkeit zunimmt, ist diese von langer Dauer, weil die Ausbildungsabsolventen unter den gegebenen Annahmen in einem anderen Betrieb eine Produktivität von null aufweisen. Sie können somit nur als nicht qualifizierte Arbeitskräfte eingesetzt werden (Wasmer 2006: 815). Daher werden sich die Individuen nur dann für eine Investition in betriebsspezifisches Humankapital entscheiden, wenn die Abgangsraten aufgrund der institutionellen Rahmenbedingungen, bspw. aufgrund eines strikten Kündigungsschutzes oder niedrigen Lohnersatzleistungen, sehr gering sind (Wasmer 2006: 816).

Da die Unternehmen aber insbesondere von der Investition in betriebsspezifisches Humankapital profitieren, werden sie ihre Arbeitskräfte auch entsprechend entlohnen. Dies wiederum wirkt sich auf den Freizeitwert bzw. den Wert der Arbeitslosigkeitsphase aus, der im Vergleich zu den Ausbildungsabsolventen, die lediglich in allgemeines Humankapital investieren, höher liegt. Infolgedessen übersteigt auch der Reservationslohn der Auszubildenden mit betriebsspezifischem Humankapital denjenigen der Personen mit allgemeinem Humankapital, obwohl deren Produktivität bei einem Betriebswechsel unterhalb derer des allgemeinen Humankapitals liegt (Kletzer 1998: 127). Daher wird der Freizeitwert über dem angebotenen Marktlohn, der sich über die Produktivität bestimmt, sein. Wie aus Kap. 5.2.1.2 hervorgeht verlängert ein höherer Reservationslohn die Arbeitslosigkeitsperiode.

Zusammenfassend lässt sich sagen, dass Arbeitskräfte mit spezifischem $\mathrm{Hu}$ mankapital eine geringere Wahrscheinlichkeit aufweisen, nach der Aus-bildung arbeitslos zu werden als Arbeitskräfte mit allgemeinem Humankapital, weil sie aufgrund der spezifischen Komponente stärker an das Unternehmen gebunden sind. Werden die Ausbildungsabsolventen mit vorwiegend spezi-fischem $\mathrm{Hu}-$ mankapital dennoch arbeitslos, sinkt die Produktivität, weil sie nicht ihr vollständiges Wissen in den anderen Betrieben einsetzen können. Um den Produktivitätsverlust minimal zu halten, sind sie daher länger arbeitslos, weil die Wahl nach Arbeitsangeboten präzisiert werden muss. Zudem wirkt sich betriebsspezifisches Humankapital, sofern es in der alten Tätigkeit eine bedeu-tende Rolle gespielt hat, negativ auf die Lohnhöhe bei der Arbeitsstelle nach der Arbeitslosigkeit aus (Kriechel/Pfann 2005: 235). Darüber hinaus implizieren die Modellbeschreibungen ebenfalls, dass die Dauer der Arbeitslosigkeit für Personen mit allgemeinem Humankapital kürzer ist, da diese flexibel auf einer Vielzahl an Stellen eingesetzt werden können. Somit dauert diese Übergangs-phase oder Arbeitslosigkeit bei betriebsspezifisch ausgebildeten Individuen unter Umständen länger an als bei allgemein ausgebildeten, deren Wissen die Produktivität auf dem gesamten Arbeitsmarkt steigert und somit auch die Pro-duktivität der einstellenden anderen Unternehmen. Deshalb haben Personen mit niedrigerem Humankapital geringere Lohnansprüche, sodass sie bei hoher Lohnersatzleistung wenig Anreiz sehen, ein Arbeitsangebot anzunehmen (Winterhager 2006: 16), was die Arbeitslosigkeitsdauer verlängert. Das bedeutet auch, 
dass Unternehmen versuchen, bei gegebenem Marktlohn die Stellen mit Arbeitskräften zu besetzen, die über die höchste Produktivität verfügen, also den höchsten allgemeinen Humankapitalstand. Neben den Humankapitalkomponenten und Institutionen, die direkt den Reservationslohn bestimmen, beeinflussen indirekte Wirkungen anderer Institutionen die Arbeitslosigkeitsphase. Hierbei spielen wiederum Gewerkschaften eine bedeutende Rolle.

\subsubsection{Insider-Outsider-Theorie}

Bei den suchtheoretischen Ansätzen (Kap. 5.2.1) wird die Arbeitssuche vom Ausbildungsabsolventen ebenso wie von der Unternehmensseite beeinflusst. Allerdings ist die Dauer der Arbeitslosigkeitsphase immer auch von dem Verhalten der anderen Arbeitnehmer, die sich in Beschäftigung befinden, abhängig. Neben Informationsasymmetrien beeinflussen auch Arbeitsmarktinstitutionen die Übergänge zwischen dem Bildungssystem und dem Erwerbsleben. Bspw. fördert die Existenz von Gewerkschaften einerseits die betriebliche Ausbildungsbereitschaft bei allgemeinem Humankapital und trägt zur Erhöhung des Bildungsstandes bei. Andererseits verstärkt sie die Arbeitslosigkeit, weil durch tarifliche Verhandlungen die Löhne der Beschäftigten ausgehandelt werden ohne Berücksichtigung der Arbeitssuchenden.

Zur Erklärung des Einflusses der Gewerkschaften auf die Arbeitslosigkeit wird häufig der Insider-Outsider-Ansatz von Lindbeck und Snower (1988) herangezogen. Hierbei sind die Arbeitslosen, auch „Outsider“ genannt, auf das Verhalten der Beschäftigten, der so genannten „Insider“ und „Entrants“ angewiesen. Nach Blossfeld et al. (2005) sind Ausbildungsabsolventen als „Outsider" gekennzeichnet, weil sie gegenüber den „Insidern“ eine schwache Position haben. Dies liegt in der Tatsache begründet, dass sie nicht in ausreichendem Maße Berufserfahrung sammeln konnten. Ebenso ist die Bindung zu einer Arbeitnehmervertretung wenig ausgeprägt. Ferner liegt der Unterschied zwischen den Beschäftigtengruppen darin, dass sich „Insider" bereits seit längerem in einem Beschäftigungsverhältnis befinden, während die „Entrants“ zwar ebenfalls in einem Arbeitsverhältnis stehen, dieses aber noch von einer Einarbeitungsphase gekennzeichnet ist. Gewerkschaften als Vertreter der „Insider" treten in den Lohnverhandlungen auf, in denen die Entlohnung entsprechend der Produktivität der jeweiligen Beschäftigtengruppen festgesetzt wird. Bildet ein Betrieb aus, weisen dessen selbst ausgebildete Mitarbeiter eine höhere Produktivität auf, was sich auf die Löhne niederschlägt (Díaz-Vazquez/Snower 2002). Eine bessere Ausbildung bedeutet daher einen höheren ausgehandelten Tariflohn. Zudem werden in den Lohnverhandlungen weitere Kosten berücksichtigt, welche die „Insider“ gegenüber den „Entrants“ und den „Outsidern“ bereits verursacht haben oder noch verursachen werden. Hierbei ist der Zusammenhang zwischen Einarbeitungs- und Entlassungskosten zu nennen. Sind die Einarbeitungszeiten aufgrund von komplexen Aufgabengebieten höher, wirkt sich dies ebenfalls steigernd auf die Entlassungskosten aus. Deshalb kann der Lohn auch entspre- 
chend hoch festgelegt werden. „Outsider" haben in diesem Fall nur eine geringe Chance auf Einstellung, auch wenn sie zu einem niedrigeren Lohn bereit wären zu arbeiten.

Erhöht sich nun die Arbeitsnachfrage, hängt die Einstellung von „Outsidern“ von dem Verhalten der „Insider" bzw. der Gewerkschaften bei Lohnverhandlungen ab. Berücksichtigen die „Insider“ die Situation der „Outsider“, werden sie bei positiven Nachfrageschocks die Lohnforderungen nicht erhöhen. ${ }^{58}$ Jedoch können die „Insider“ auch die günstige Arbeitsmarktsituation nutzen, um ihre eigene Erwerbsposition zu verbessern, indem sie höhere Löhne fordern. Es werden daher weniger „Outsider“ eingestellt werden können (Sesselmeier/Blauermel 1998: 175ff.), da Arbeitssuchende wie die Beschäftigten entlohnt werden müssen. Jedoch besteht Unsicherheit hinsichtlich des Produktionsbeitrages der „Outsider“. ${ }^{59}$ Daher wird der Einstiegslohn zunächst unterhalb des Lohnes der anderen Fachkräfte liegen, der sich aber im Zeitablauf an den Lohn der „Insider“ anpasst. Nach Díaz-Vázquez und Snower (2003) ermöglicht der niedrigere Lohn der „Entrants“ auch die Kompensation des relativ höheren Lohnes der „Insider“. Dies führt dann zu einer relativ schnelleren Wiederbeschäftigung der „Outsider“ innerhalb der jeweiligen Berufsgruppen, unabhängig von deren Bildungsniveau und -qualität. Diese Annahme ist aufgrund der unvollständigen Informationen, die hinsichtlich der vorhandenen Produktivität der arbeitssuchenden Personen bestehen, durchaus sinnvoll. Die Produktivität steigt dabei mit zunehmender Beschäftigungsdauer. Der Beschäftigungseffekt hängt dann maßgeblich vom Verhalten der „Insider“ ab. Setzen letztere hohe Lohnforderungen durch, wirkt sich dies verlängernd auf die Arbeitslosigkeitsphase aus. Dieselbe Wirkung ergibt sich bei der Betrachtung des Arbeitslosigkeitsrisikos. Gelten hohe Löhne, werden „Insider“ bei Verschlechterung der wirtschaftlichen Lage entlassen. Handelt es sich um selbst ausgebildete Arbeitskräfte, ist das Unternehmen im Aufschwung gezwungen, eine höhere Anzahl der weniger produktiven „Entrants“ anzustellen (Díaz-Vazquez/Snower 2002), um dieselbe Produktivität wie vor der Rezession zu erreichen. Existieren weitere hohe Regulierungen, bspw. Kündigungskosten, erhöhen die Unternehmen die Anforderungen an die Produktivität, was neben der Weiterbeschäftigung der Ausbildungsabsolventen auch die Arbeitslosigkeit der „Outsider“ beeinflusst. Hierbei wird wieder die schwächere Position der Ausbildungsabsolventen gegenüber älteren arbeitssu-

${ }^{58}$ Dies ist vor allem dann vorstellbar, wenn ein zuvor eingetretener negativer Nachfrageschock kurzfristiger Natur ist.

${ }^{59}$ Um die Unsicherheit zu reduzieren, können Unternehmen bspw. auf die Lohnvorstellungen der einzelnen Bewerber achten. Wenn daher arbeitssuchende Ausbildungsabsolventen ihre Arbeitskraft unterhalb des Lohnes anbieten, den ein Unternehmen bereit ist, zu zahlen, signalisiert dies eine geringere Produktivität. Daher werden diese Ausbildungsabsolventen eine geringere Wahrscheinlichkeit haben, schnell einen Job zu finden. Dagegen werden Ausbildungsabsolventen mit hoher Produktivität auch von Unternehmen, die hoch entlohnen, eingestellt. Diese Einstellung ist aber sowohl auf Seiten der Unternehmen als auch auf Seiten der Arbeitssuchenden mit hohen Transaktionskosten verbunden. 
chenden Personen deutlich, die über Berufserfahrung verfügen (Müller/Gangl 2003).

Kritisch anzumerken bleibt, dass sich der Arbeitsmarkt in der Theorie zwar aus heterogenen Unternehmen zusammensetzt, welche aber alle an gewerkschaftliche Vereinbarungen gebunden sind. Es hindern aber nicht nur tariflich ausgehandelte Löhne die Arbeitslosen an einer Beschäftigungsaufnahme, sondern auch ein schlechtes Matching hinsichtlich Qualifikationen und geografischen Gegebenheiten. Vor allem die Übereinstimmung der Qualifikationen wird im Folgenden im Kontext der dualen Berufsausbildung erörtert. Da die Schlussfolgerungen des Kapitels 5.2. zu allgemein sind, als dass sich Implikationen für die konkrete Situation der von Arbeitslosigkeit betroffenen Ausbildungsabsolventen ableiten lassen können, ist es nun Ziel, die theoretischen Wirkungszusammenhänge auf den Übergang von der dualen Berufsausbildung in das Erwerbsleben zu übertragen und zu beleuchten, wie sich das Arbeitslosigkeitsrisiko bzw. die Dauer der Arbeitslosigkeit auf die Ausbildungsabsolventen verteilt.

\subsection{Implikationen der heterogenen Humankapitalstruktur der Absolven- ten für die Arbeitslosigkeit}

In den Arbeitslosigkeitstheorien wird die Dauer der Arbeitssuche durch den Reservationslohn definiert, dessen Höhe sich durch Anforderungen an den künftigen Arbeitsplatz sowie durch den zu erwartenden Lohn bestimmt. Zur Beschreibung der Arbeitslosigkeit nach der dualen Berufsausbildung muss der Höhe des Bildungsstandes Beachtung geschenkt werden. Da jedoch die Abschlüsse der dualen Berufsausbildung formal dieselbe Höhe aufweisen, greift diese Betrachtung zur Beschreibung der unterschiedlichen Arbeitslosigkeitsdauer zu kurz. Daher müssen die Art und Struktur des innerhalb der Berufsausbildung vermittelten Humankapitals sowie die daraus resultierenden unterschiedlichen Ausbildungsqualitäten als Determinanten des individuellen und betrieblichen Suchverhaltens und folglich des Arbeitslosigkeitsrisikos und der Dauer der Arbeitslosigkeit in Betracht gezogen werden (Winkelmann 1996; Steedman 1993). Damit die unterschiedliche Verteilung der Arbeitslosigkeitsrisiken von Ausbildungsabsolventen, die den Ausbildungsbetrieb verlassen, analysiert werden kann, muss die bis dahin allgemeine Betrachtung der Vermittlung des Humankapitals aufgegeben und auf die Besonderheiten der Wissensvermittlung innerhalb des dualen Berufsausbildungssystems übertragen werden. Hierbei bildet auch die Komplementarität zwischen den Humankapitalarten eine Erklärungsgrundlage. Denn Personen, die unfreiwillig wechseln, unterscheiden sich untereinander, aber auch von denjenigen, die freiwillig wechseln. Diese Unterscheidung ist erforderlich, da der Anteil der freiwilligen Wechsler in den letzten Jahren stark zugenommen hat, weshalb die Sucharbeitslosigkeit zur Verbesserung der Erwerbsposition eine immer bedeutendere Stellung einnimmt (Baethge et al. 2007; Bellmann et al. 2006). 


\subsubsection{Bedeutung von Informationsasymmetrien}

Bereits während der dualen Berufsausbildung werden Informationsasymmetrien hinsichtlich der informellen Fähigkeiten abgebaut. Ausbildungsbetriebe besitzen am Ende der Ausbildung Kenntnis über die Produktivität der einzelnen Ausbildungsabsolventen. Diejenigen, die den Betrieb verlassen müssen, weil sie den Anforderungen nicht gerecht werden, sind dem Risiko der Arbeitslosigkeit ausgesetzt. Ebenso haben Ausbildungsabsolventen eine erhöhte Wahrscheinlichkeit arbeitslos zu werden, wenn sie in ihrem Betrieb nicht weiterbeschäftigt werden können, weil dieser über seinen Fachkräftebedarf ausgebildet hat. Insgesamt weisen die nicht im Ausbildungsbetrieb weiter beschäftigten Ausbildungsabsolventen, die nach dem produktionstheoretischen Ansatz ausgebildet werden, ein höheres Arbeitslosigkeitsrisiko auf als Absolventen mit einer Ausbildung nach investitionstheoretischen Kriterien. Wenn die Absolventen letzterer Gruppe dann tatsächlich nicht weiterbeschäftigt werden, übersteigt ihre Arbeitslosigkeitsdauer die der ersteren Gruppe, weil sie einen höheren Anteil an betriebsspezifischem Wissen haben und dadurch einen höheren Produktivitätsverlust erleiden.

Arbeitslosigkeit entsteht deswegen, weil bestehende Informationsasymmetrien abgebaut werden müssen. Diese werden innerhalb der dualen Berufsausbildung zwar relativ gering gehalten, weil die Ausbildungsinhalte durch die Ausbildungsordnungen standardisiert sind. Dennoch sind Unterschiede in der Höhe der Informationsasymmetrien zu erkennen. Entsprechend tritt eine Ungleichverteilung der Informationen auf, weil, wegen der Beteiligung einer Vielzahl an unterschiedlichen Betrieben, immer auch zusätzliches Humankapital vermittelt wird. So gibt es Ausbildungsbetriebe, die lediglich Bildung in Höhe der Mindestanforderungen anbieten können. Andere aber wiederum bieten zusätzliches Humankapital an. Daher rückt bei der Übertragung der hergeleiteten Annahmen über das Risiko der Arbeitslosigkeit und deren Dauer nun die gemeinsame Vermittlung der Humankapitalarten in den Mittelpunkt der Betrachtung. Dabei verringert vor allem die Zusammensetzung der einzelnen Wissenskomponenten die Transparenz.

Kaum Informationsunvollständigkeiten existieren für Ausbildungsabsolventen, deren Ausbildung vor allem auf berufsspezifischem bzw. allgemeinem $\mathrm{Hu}-$ mankapital beruht. Dies fördert die Transparenz der Ausbildung, weil das erworbene Wissen fast vollständig entweder durch Abschlusszeugnisse oder durch die jeweiligen Ausbildungsordnungen signalisiert und auch innerhalb der Berufsgruppe uneingeschränkt eingesetzt werden kann. Informationsasymmetrien bestehen lediglich bezüglich persönlicher Charaktereigenschaften. ${ }^{60}$ Daher sind diese Ausbildungsabsolventen in der Lage, ihre tatsächliche Produktivität dem

${ }^{60}$ Vor allem kleine Betriebe verfügen über geringe materielle Ressourcen, weshalb sich die Ausbildung auf die durch die Ausbildungsordnungen vorgegeben Anforderungen beschränken. Auch die handwerklichen bzw. manuellen Berufe sind so ausgerichtet, dass selten über die Mindestanforderungen hinaus Humankapital vermittelt wird. 
künftigen Arbeitgeber zu zeigen. Aufgrund dessen besteht nahezu vollständige Kenntnis über die fachliche Humankapitalkomponente, was sich in ähnlichen Lohnvorstellungen zwischen den Akteuren widerspiegelt. Der Reservationslohn des Arbeitssuchenden weicht nicht sehr stark von dem Lohnangebot des Unternehmens ab. Die Arbeitslosigkeitsperiode ist in diesem Fall relativ kurz.

Trotz der Standardisierung der dualen Berufsausbildung und entsprechend der Gewährleistung der Transparenz bestehen Informationsasymmetrien, da die Ausbildungsberechtigung nicht an betriebliche Merkmale, wie Umsatz, Wettbewerbsfähigkeit, Betriebsgröße etc. gekoppelt ist. So beteiligen sich unterschiedliche Betriebe und Individuen an der dualen Berufsausbildung, wodurch die Struktur des Humankapitals, über die Ausbildungsordnungen und Zeugnisse hinausgehend, beeinflusst wird. Das allgemeine und betriebsspezifische Humankapital wird dementsprechend in unterschiedlicher Weise und Intensität vermittelt. Bspw. werden in einem Ausbildungsberuf mehr Tage an der Berufsschule gefordert als in einem anderen. Dies steigert das allgemeine Humankapital, was teilweise durch die Ausbildungsordnungen signalisiert wird. Weiterhin kann das allgemeine Wissen in einem Unternehmen eine bedeutende Stellung einnehmen, in dem der Kontakt zu Kunden und Mitarbeitern äußerst wichtig ist, sodass ein ausreichendes $\mathrm{Ma} ß$ an soft skills, wie Umgänglichkeit, Teamwork und Zuverlässigkeit erforderlich ist. Ebenso können komplexe Prozesse oder bestimmte Unternehmensphilosophien, die für die Wettbewerbsfähigkeit notwendig sind, den Wert des betriebsspezifischen Humankapitals erhöhen. Letztere Humankapitalkomponenten, welche über die Mindestanforderungen hinaus vermittelt werden, sind informeller Natur, woraus Informationsunvollständigkeiten innerhalb des dualen Berufsausbildungssystems entstehen. Dieses informelle Wissen kann, sofern keine Möglichkeit zur Zertifizierung besteht, nicht beobachtet werden. Dies ist vor allem in Betrieben der Fall, in denen die Bildungsinvestitionen hoch ausfallen. Entsprechend wird den Ausbildungsabsolventen Wissen weit über die in den Ausbildungsordnungen geforderten Mindeststandards vermittelt. ${ }^{61}$ Dieses zusätzliche Wissen kann nur dann signalisiert werden, wenn es zertifiziert werden kann oder der Ausbildungsbetrieb einen entsprechenden Ruf hinsichtlich der Qualität der Ausbildung hat (Acemoglu/Pischke 2000). Deshalb ist ein Abbau bestehender (hoher) Informationsasymmetrien notwendig (Yashiv 2006: 5; Pissarides 2000: 4), welche sich mit der (informellen) Bildung über die geregelten Ausbildungsinhalte erhöhen. Demnach korrelieren informelle Fähigkeiten positiv mit dem Humankapitalstand, was sich ebenfalls positiv auf den gesamten Bildungsstand auswirkt. Deshalb werden sich diese Ausbildungsabsolventen bei einem Betriebswechsel auf Stellen bewerben, die entsprechend ihrer Qualifikation hohe Anforderungen stellen. Weil sich aber die Produktivität bei einer bestehenden Stelle nicht ändert (Mortensen/Pissarides

${ }^{61}$ Dabei handelt es sich vor allem um Unternehmen, die im Hoch-Qualitätssegment tätig sind (Neubäumer 1999). Um wettbewerbsfähig zu bleiben, benötigen sie gut ausgebildete Fachkräfte. 
1994: 397), steigt die Suchdauer für ein erfolgreiches Matching mit den Anforderungen an den Job. Denn je höher die Anforderungen sind, desto geringer ist der Anteil derjenigen, die diesen gerecht werden. Dies wirkt sich wiederum auf die Dauer der Arbeitslosigkeit aus. So hat der Grad der existierenden Informationsasymmetrien Einfluss auf die Länge der Phase der Erwerbslosigkeit. Weiteren positiven Einfluss auf die Länge hat die Tatsache, dass sich die Ausbildungsabsolventen ihrer hohen Produktivität bewusst sind. Entsprechend hoch ist der Reservationslohn, ab dem sie eine neue Stelle akzeptieren werden. Jedoch werden die Unternehmen ihnen nur einen Lohn anbieten, welcher der Produktivität entspricht, die aufgrund des Einsatzes von berufsspezifischem Humankapital besteht. Dieser Lohn liegt unter den individuellen Lohnforderungen, weil bei einem Betriebswechsel ein Produktivitätsverlust, aufgrund des hohen Anteils an betriebsspezifischem Humankapital, vorliegt. Diese Ausbildungsabsolventen weisen bei einem Betriebswechsel einen höheren Produktivitätsverlust auf als die Ausbildungsabsolventen mit einem hohen Anteil an berufsspezifischem bzw. allgemeinem Humankapital, weil die betriebsspezifische Humankapitalkomponente im neuen Unternehmen nicht eingesetzt werden kann und deshalb abgeschrieben werden muss (Ludwig/Pfeiffer 2005). Zudem macht die Arbeitslosigkeit an sich bereits eine Humankapitalentwertung notwendig, was wiederum die Ausgangsposition der arbeitssuchenden Ausbildungsabsolventen verschlechtert (Ryan 2001: S. 47). Daher werden zwar die besseren Absolventen schnell anhand des formalen Bildungsstandes identifiziert, jedoch verlängert sich der Suchprozess aufgrund des notwendigen Abbaus von Informationsasymmetrien hinsichtlich des informellen Humankapitals und aufgrund der notwendigen Annäherung von betrieblichen und individuellen Lohnforderungen.

Die Arbeitslosigkeitsdauer bei der hier betrachteten Gruppe wird auch deshalb länger sein, weil sie aufgrund des formal besseren Abschlusses entsprechend höhere Ansprüche an die neue Arbeitsstelle hat bspw. in Bezug auf die Beschäftigungsdauer oder Arbeitsinhalte, Karrierechancen sowie soziale Bindungen, die nicht in allen Unternehmen gleichermaßen angeboten werden. Diese Ausbildungsabsolventen werden ein größeres Interesse an guten Jobs haben (Pissarides 1994; Pissarides/Mortensen 1994). Da diese Arbeitsplätze eine höhere Produktivität erfordern, müssen zu deren Besetzung höhere Kosten aufgewendet werden. Entsprechend ist auch die Auswahl geeigneter Arbeitskräfte auf Seiten der Unternehmen zeitintensiv.

Tabelle 3 gibt noch einmal einen zusammenfassenden Überblick über die Auswirkungen der unterschiedlichen Humankapitalarten und -höhen auf das Übernahmeverhalten sowie auf die Arbeitslosigkeitssituation. 
Tabelle 3: Auswirkungen des Humankapitals auf die zweite Schwelle

\begin{tabular}{|c|c|c|c|}
\hline & $\begin{array}{c}\text { Übernahme- } \\
\text { wahrscheinlichkeit }\end{array}$ & $\begin{array}{c}\text { Arbeitslosigkeits- } \\
\text { risiko }\end{array}$ & $\begin{array}{c}\text { Arbeitslosigkeits- } \\
\text { dauer }\end{array}$ \\
\hline \multicolumn{4}{|c|}{ Humankapitalniveau } \\
\hline niedrig & gering & hoch & kurz \\
\hline hoch & hoch & gering & lang \\
\hline \multicolumn{4}{|c|}{ Humankapitalart } \\
\hline allgemein & gering & hoch & kurz \\
\hline spezifisch & hoch & gering & lang \\
\hline
\end{tabular}

Quelle: eigene Darstellung

\subsubsection{Einfluss eines veränderten Humankapitalbedarfs der Betriebe}

Die Arbeitslosigkeit hängt jedoch nicht nur vom Suchverhalten der Absolventen und deren Möglichkeiten, künftige Arbeitgeber über ihr tatsächliches Wissen zu informieren, ab, sondern auch von der betrieblichen Nachfrage nach Fähigkeiten. Ausbildungsabsolventen, deren Humankapitalstruktur auf dem Arbeitsmarkt nachgefragt wird, weisen in diesem Fall eine kürzere Arbeitslosigkeitsperiode auf, als Ausbildungsabsolventen, deren Fähigkeiten nicht verlangt werden (Backes-Gellner/Mure 2008). Jedoch kann sich die Arbeitsnachfrage auch verschieben, sodass bestimmte Kombinationen von Humankapitalkomponenten nicht mehr nachgefragt und diese Arbeitsplätze im Extremfall abgebaut werden. Daher deutet eine Arbeitslosigkeit, die nach Ausbildungsberufen stark differiert, auch auf Passungsprobleme zwischen Ausbildungsangebot und Nachfrage nach auf dem Arbeitsmarkt erforderlichen Qualifikationen ab (DIPF 2008: 182). Die Nachfrage verändert sich aufgrund von Anpassungen in der Produktionsstruktur. Vor allem Ausbildungsabsolventen mit einem hohen Anteil an betriebsspezifischem Humankapital sind von Arbeitslosigkeit bei Nachfrageverschiebungen betroffen. Da der Arbeitsmarkt aufgrund der institutionellen Regelungen als rigide gilt, kann der Lohn nicht als Anpassungsmechanismus dienen (Howell/Hübler 2005: 3; Siebert 1997: 45). Daher wird auf Veränderungen der Arbeitsnachfrage je nach Art und Struktur der Ausbildung über Arbeitslosigkeit oder über einen flexiblen Arbeitskräfteeinsatz reagiert.

Die Anpassung erfolgt vor allem in einem rigiden Arbeitsmarkt über die Dauer der Arbeitslosigkeit. Nach Delacroix (2003) tritt die Anpassungsproblematik zwischen Arbeitsangebot und -nachfrage vorwiegend in einem stark regulierten Arbeitsmarkt auch häufiger auf, weil der Freizeitwert ${ }^{62}$ höher ist als in einem weniger regulierten Markt (Delacroix 2003: 662). Der Organisationsgrad, als Indikator für die Macht der Gewerkschaften, spielt hierbei eine Rolle. Sie sind insbesondere in Bereichen tätig, in denen Unternehmen gut qualifizierte Absolventen mit einem relativ hohen Anteil an betriebsspezifischem Humankapital

${ }^{62}$ Der Freizeitwert kann in einem regulierten Arbeitsmarkt insbesondere durch staatliche Lohnersatzleistungen getrieben sein. 
beschäftigen. Daher haben die „Insider“ in gewerkschaftlich organisierten Ausbildungsbereichen eine starke Position inne, weswegen die Fluktuation äußerst gering ist. So stehen weniger Stellen für die arbeitslosen Absolventen („Outsider") zur Verfügung, was die Arbeitslosigkeitsdauer verlängert. Allerdings bedeutet dies aber auch, bei Erhalt und Annahme eines Arbeitsangebots, eine gröBere Beschäftigungssicherheit und -dauer als „Insider“ sowie ein geringeres Arbeitslosigkeitsrisiko, was sich auch in dem flexiblen Einsatz von Absolventen mit hohem Bildungsstand innerhalb der Unternehmen zeigt. Darüber hinaus wird ein nutzenmaximierendes Individuum solange einen neuen Arbeitsplatz suchen und ihn erst dann akzeptieren, wenn dessen Entlohnung mindestens gleich dem Reservationslohn ist und die Kosten einer weiteren Suche darüber liegen. Dieser orientiert sich dabei an der eigenen Produktivität. Ausbildungsabsolventen, die nicht übernommen werden, aber zugleich einen hohen Anteil an betriebsspezifischem Humankapital aufweisen, werden zunächst die gesamte individuelle Produktivität betrachten und danach den Reservationslohn ausrichten. Aber da in einem regulierten Arbeitsmarkt die Arbeitgeber weniger flexibel auf Produktionsschwankungen reagieren können (Rae/Sollie 2007; Bassanini/Duval 2006; Enste/Hardege 2006; Botero et al. 2004), müssen die arbeitssuchenden Ausbildungsabsolventen bei der Entscheidung für die Akzeptanz eines Jobangebots einen niedrigeren Lohn akzeptieren (Delacroix 2003: 661). ${ }^{63}$ Dieser steigt dann gemäß des Insider-Outsider-Ansatzes während der Beschäftigungsphase an, sofern die „Insider“ in einer starken Position sind und hohe Lohnforderungen durchsetzen. Darüber hinaus wird der Anreiz für Unternehmen hoch sein, ,gute“ Jobs anzubieten, da bei diesen der Ertrag hoch ist. Um diesen hohen Ertrag aber zu erhalten, muss dieser auch mit gut qualifizierten Ausbildungsabsolventen besetzt werden, was wiederum dafür spricht, dass gerade diese Personen eine niedrige Arbeitslosigkeitsdauer aufweisen (Delacroix 2003: 662).

Während die Anpassungen der Arbeitsnachfrage bei betriebsspezifischer und damit besser ausgebildeten Absolventen über die Dauer der Arbeitslosigkeit geschieht, erfolgt die Anpassung bei Tätigkeiten mit geringen Qualifikationsanforderungen durch eine inadäquate Stellenbesetzung (Büchel/Neubäumer 2001; Neubäumer 1999) ${ }^{64}$ Dies ist möglich, weil Absolventen mit einem hohen Anteil an allgemeinem Humankapital innerhalb des Ausbildungsbereiches und der Volkswirtschaft flexibel einsetzbar sind. In einem rigiden Arbeitsmarkt bzw. im internen Segment üben Ausbildungsabsolventen mit höherer Wahrscheinlichkeit

\footnotetext{
${ }^{63}$ Nach der suchtheoretischen Grundlage sollten Ausbildungsabsolventen mit einer Anpassung ihres Reservationslohnes auf die gesunkene Produktivität reagieren. Da sie sich aber der guten Qualität ihrer Ausbildung bewusst sind, findet keine Anpassung statt, woraus eine längere Suchdauer resultiert, was letztlich, je nach Suchintensität, zu einem erfolgreichen Matching führt.

${ }^{64}$ Werwatz (2002) reduziert die ausbildungsinadäquate Beschäftigung auf die Effizienzfrage. Denn aufgrund des hohen Anteils an Ausbildungsabsolventen, deren Erwerbsberuf nicht ihrem Ausbildungsberuf entspricht, liegt ein Problem einer geringen Anpassungsflexibilität vor, weil die Ausbildungsinhalte nicht von den Betrieben nachgefragt werden.
} 
eine ausbildungsadäquate Beschäftigung aus als Ausbildungsabsolventen im externen Segment. Zudem haben Ausbildungsabsolventen, die freiwillig den Betrieb verlassen, eine niedrigere Wahrscheinlichkeit, arbeitslos zu werden als die unfreiwilligen Wechsler (Hassink/Broersma 2003: 1911f.), weil sie in der Regel bei einem Wechsel ein Arbeitsangebot vorliegen haben.

\subsection{Auswirkungen einer Arbeitslosigkeit auf weiteres Erwerbsleben}

Nun bedeutet aber eine Ausbildung, die vorwiegend berufsspezifisches Humankapital beinhaltet, nicht immer Vorteile bei der Arbeitssuche. Ebenso wenig signalisieren (betriebs- oder technologie-)spezifische Ausbildungsinhalte nicht nur Nachteile. Daher sind die im Kap. 5.3.1 gezogenen Schlussfolgerungen zum Teil zu relativieren. Die Häufigkeit, Arbeitslosigkeitsphasen zu durchleben, wird von besser qualifizierten Ausbildungsabsolventen geringer sein als von Ausbildungsabsolventen mit geringerem Bildungsstand.

Dies ist auf den Unterschied der betrieblichen Ausbildungsqualität zurückzuführen (Winkelmann 1996; Steedman 1993), der wiederum mit der Ausbildungsbereitschaft in Zusammenhang steht. Ausbildungen in Betrieben mit einer Knappheit an finanziellen Ressourcen sind durch einfach strukturierte Arbeitsprozesse gekennzeichnet und wirken sich auf die Befähigung des Ausbildungsabsolventen aus. Trotz des geringen Humankapitalverlustes bei einem Wechsel weisen diese Ausbildungsabsolventen in aller Regel ein geringeres gesamtes Humankapitalniveau, und damit verbunden, eine geringere Ausbildungsqualität auf als Ausbildungsabsolventen, die eine Ausbildung mit höherer Qualität besitzen. Daher finden Ausbildungsabsolventen mit niedrigem Humankapitalniveau zwar einfacher eine Arbeit, weil sie nicht in dem Maße Humankapitalverlust erleiden wie Ausbildungsabsolventen, die betriebs- oder technologiespezifisches Wissen erwerben (Wasmer 2006; Ludwig/Pfeiffer 2005; Stevens 1994), die aber durch eine relativ geringe Beschäftigungsstabilität gekennzeichnet ist.

Absolventen mit hohem Bildungsstand aufgrund eines guten Berufs- und allgemein bildenden Schulabschlusses erhalten meist eine Ausbildung in einem Betrieb mit komplexen Arbeitsprozessen, hoher Wettbewerbsfähigkeit auf dem Produktmarkt oder mit einem Einsatz an hohem Technischen Fortschritt sowie einem internen Arbeitsmarkt (Euwals/Winkelmann 2003: 5). Dies erhöht den Anteil an betriebs- oder technologiespezifischem Humankapital, mit dessen Vermittlung die Ausbildungsqualität steigt. Entsprechend erleiden diese Ausbildungsabsolventen bei einem Betriebswechsel einen höheren Produktivitätsverlust. Da diese Personen aber insgesamt einen höheren Anteil der allgemeinen Humankapitalkomponente aufweisen, liegt der gesamte Produktionsbeitrag ebenfalls höher als bei der zuvor genannten Personengruppe, was einen Vorteil bei der Arbeitsstellensuche mit sich bringt (Pissarides 1994: 461). Allerdings ist zu berücksichtigen, dass gerade die Absolventen mit höherem Bildungsniveau ihre Ausbildung in der Regel in Unternehmen machen, die eine gute Reputation hinsichtlich der Ausbildung genießen. Dies wiederum führt zu Vorteilen bei der 
Arbeitsplatzsuche. In diesem Fall lohnt es sich nach einem Selektionsprozess hohe Einarbeitungskosten und -zeiten in Kauf zu nehmen, da der Produktionsbeitrag aufgrund des hohen bereits vorhandenen Humankapitals schnell ansteigt. In diesem Zusammenhang sind nach Breen (1997) auch die unterschiedlich hohen Kontrollkosten zu berücksichtigen. Daher werden Arbeitsaufgaben, welche weniger kontrolliert werden können, den höher qualifizierten Arbeitskräften zugeteilt. Zunächst wird dies die Arbeitslosigkeitsdauer verlängern, aber aufgrund der komplexen Arbeitsaufgabengebiete wird der Arbeitgeber ein Interesse daran haben, den neu eingestellten Arbeitnehmer länger zu beschäftigen und damit Erwerbsstabilität und -sicherheit zu geben. Dies bedeutet in der Regel ein Ausbleiben von weiteren Arbeitslosigkeitsperioden. Infolgedessen wird die Position weiter auf dem Arbeitsmarkt gestärkt.

Allerdings ist in jüngerer Zeit eine Zunahme an befristeten Arbeitsverhältnissen als arbeitsmarktpolitisches Instrument gerade für die Gruppe der Berufsanfänger nach der dualen Berufsausbildung zu erkennen (Hillmert 2006: 15). Denn Beschäftigungsverhältnisse können ohne höhere Entlassungskosten bei Verschlechterung der konjunkturellen Situation gelöst werden. Darüber hinaus können Unsicherheiten bei der Einstellung durch befristete Verträge reduziert werden. Die Betroffenheit der Absolventen wird bei der Betrachtung der Veränderung des Anteils an befristeten Beschäftigungsverträgen über die Zeit deutlich. So sind Mitglieder jüngerer Geburtskohorten eher befristet beschäftigt als Berufsanfänger älterer Geburtskohorten. Im Jahr 2007 waren 10\% der Absolventen einer beruflichen Ausbildung befristetet beschäftigt (Fuchs/Ebert 2008: 12) Zudem können befristete Verträge den Screening- und Signalling-Prozess ${ }^{65}$ unterstützen und somit die Reduzierung der Informationsasymmetrien herbeiführen (Blossfeld et al. 2005: 6).

Allerdings deuten Studien (Giesecke/Groß 2003; Kurz/Steinhage 2001) darauf hin, dass befristete Beschäftigungsverhältnisse direkt nach der dualen Berufsausbildung eine signifikant höhere Arbeitslosigkeitswahrscheinlichkeit aufweisen als ein Start mit unbefristetem Beschäftigungsverhältnis. Daher sind befristete Arbeitsverträge für Berufsanfänger vielmehr als Beginn einer Folge von weiteren Befristungen als eine Integration in den Arbeitsmarkt zu sehen (McGinnity et al. 2005: 259; McGinnity/Mertens 2004: 126) und bedeuten damit steigende Unsicherheit (Ryan 2001: S. 67). Daraus folgen weitere Humankapitalabschreibungen, die aufgrund eines Verlustes an Berufserfahrung erforderlich sind (Eriksson 2006: 265f.; Heckman/Borjas 1980: 247). Von befristeten Arbeitsverträgen nach der dualen Berufsausbildung betroffen sind vor allem Ausbildungsabsolventen, die in einem regulierten bzw. koordinierten Teilsegment (bspw. die Existenz eines hohen Kündigungsschutzes) ihre Ausbildung

\footnotetext{
${ }^{65}$ Innerhalb der Signalling-Theorie (Spence 1973; 1974) wählen Betriebe Kriterien aus, die für den Arbeitsprozess relevant erscheinen und gleichzeitig leicht zu beobachten sind. Während des Screening Prozess werden die ausgewählten Kriterien als Vergleichsbasis für die Fähigkeit des Ausbildungsabsolventen herangezogen.
} 
absolvieren und dort auch eine weitere Beschäftigung anstreben. Eine genauere Differenzierung ergibt zudem, dass für nicht übernommene Ausbildungsabsolventen eine höhere Wahrscheinlichkeit, ein befristetes Beschäftigungsverhältnis zu beginnen, besteht als für übernommene Ausbildungsabsolventen. Darüber hinaus beginnen Berufsanfänger mit Migrationshintergrund häufiger das Erwerbsleben mit einem befristeten Vertrag (McGinnity et al. 2005). Mit den geringeren Übernahmequoten verlängert sich auch die Phase bis zur ersten Festanstellung bei der Gruppe der nicht übernommenen Ausbildungsabsolventen, sodass eine Diskontinuität der Erwerbsverläufe besteht und weiter zunimmt (Schaeper et al. 2000; Konietzka/Seibert 2001). Allerdings muss auch berücksichtigt werden, dass befristete Arbeitsverträge, gleich ob es sich um eine befristete Übernahme oder um eine Befristung in einem neuen Betrieb handelt, eine Steigerung der Berufserfahrung fördern. Denn schließlich hat auch für die Aufnahme einer weiteren (befristeten) Beschäftigung die Berufserfahrung einen positiv signifikanten Einfluss (Dietrich/Kleinert 2005: 199). Befristete Beschäftigungsverhältnisse oder befristete Übernahmeregelungen dienen zur Gewinnung von Berufserfahrung und können zugleich die Arbeitsmarktchancen verbessern. Die Arbeitslosigkeitsphasen werden reduziert. Jedoch kann der Übergangsprozess von der Ausbildung bis zur Festanstellung verzögert werden, wobei zwischen den Ausbildungsbereichen zu differenzieren ist. In Ausbildungsbranchen mit guten Beschäftigungsaussichten sind befristete Verträge eher als Brücke zu einer sicheren Beschäftigung zu verstehen. Meist finden diese Ausbildungsabsolventen durch befristete Arbeitsverhältnisse eine entsprechend gute Arbeit. Diese Funktion nehmen befristete Verträge aber eher bei der Besetzung von Stellen mit hohen Qualifikationsanforderungen ein. Dagegen führen für Ausbildungsabsolventen aus Bereichen mit weniger guten Beschäftigungsaussichten weniger erfolgreiche Eintritte in den Arbeitsmarkt mit höherer Wahrscheinlichkeit zu Arbeitslosigkeit, wodurch die Outsider-Position verstärkt wird (Boockmann/Hagen 2006; Bernardi/Nazio 2005; Layte et al. 2005). 
Gabriele Somaggio - 978-3-631-74988-3

Downloaded from PubFactory at 01/11/2019 09:32:31AM

via free access 

thesenbildung

Im theoretischen Teil liegt der Fokus auf der Verbindung zwischen dem Beginn einer Ausbildung innerhalb des dualen Systems und dem Übergang bzw. dem erfolgreichen Eintritt in das Erwerbsleben. Der betrachtete Teilarbeitsmarkt stellt einen Spezialfall dar, weil einerseits die einzige Erfahrung der Ausbildungsabsolventen auf dem Arbeitsmarkt die duale Berufsausbildung ist, andererseits bei der Arbeitssuche aber die Mechanismen des Gesamtarbeitsmarktes gelten. Daher sind die Bereitschaft zur betrieblichen Ausbildung als Bindeglied zwischen erster und zweiter Schwelle zu betrachten und mit den Arbeitslosigkeitstheorien zu verknüpfen. Obwohl es keine ökonomische Theorie gibt, die speziell die Investitionen in die duale Berufsausbildung erklärt, können die mikroökonomischen Theorien mit den Erweiterungen als Erklärungsgrundlage herangezogen werden (Wolter 2008: 91). Ebenso wenig geben diese theoretischen Ansätze Aufschluss über den Verbleib der Ausbildungsabsolventen an der zweiten Schwelle. Vielmehr wird von einem langfristigen Beschäftigungsverhältnis ausgegangen, sodass die Ausbildungsbereitschaft durch die Einschränkung der individuellen Mobilität nach der Ausbildung begründet werden muss. Deshalb werden aus den Ausbildungstheorien in Verbindung mit den suchtheoretischen Ansätzen Schlussfolgerungen hinsichtlich der Erwerbsituation nach der dualen Berufsausbildung abgeleitet.

Innerhalb des Theorieteils wurde herausgearbeitet, wie bereits an der ersten Schwelle Schulabsolventen mit bestimmten Merkmalen, insbesondere dem formalen Schulabschluss, von Unternehmen ausgesucht werden. So beginnt der Selektionsprozess bereits bei der Auswahl geeigneter Auszubildender für die angebotenen Ausbildungsstellen, sodass Personen mit geringer allgemeiner Bildung die Stellen mit schlechteren Beschäftigungsaussichten besetzen (Büchel 2002). Daher hat die Auswahl an der ersten Schwelle einen Einfluss auf das Übernahmeverhalten und das Arbeitslosigkeitsrisiko nach der Berufsausbildung. Damit ist diese Selektion prägend für den Verbleib an der zweiten Schwelle. Das Bildungsniveau stellt sich somit als ein bedeutendes Merkmal für den Eintritt in das Erwerbsleben dar (Isengard 2002), denn die Vergabe eines Ausbildungsplatzes richtet sich nach dem allgemein bildenden Schulabschluss, da dies beim Übergang an der ersten Schwelle als einziges Merkmal hinsichtlich der Fähigkeiten signalisiert werden kann. Dabei werden die Auszubildenden von den Betrieben unter Berücksichtigung des betrieblichen Beschäftigungshorizontes ausgewählt. Die Gründe (produktions- vs. investitionstheoretischer Ansatz) für die Ausbildungsbereitschaft determinieren daher die unterschiedlichen Beschäftigungsaussichten innerhalb des Ausbildungsbetriebes über die Ausbildung hinaus. Den mikroökonomischen Ansätzen zur Erklärung der betrieblichen Ausbildungsbereitschaft in Deutschland ist gemein, dass Unternehmen bei der Annahme vollkommenen Wettbewerbs nicht bereit sind, in allgemeines Humankapital, worunter auch die duale Berufsausbildung zu subsumieren ist, zu investie- 
ren. Nur unter bestimmten Voraussetzungen erfolgt die Finanzierung in die Ausbildung durch die Betriebe bei vollkommenem Wettbewerb. Hierbei müssen Annahmen von unvollkommenen Märkten und über die Kosten-Nutzenstruktur einer betrieblichen Ausbildung getroffen werden, die sich auf die Länge des Zeithorizontes, innerhalb dessen die Bildungserträge der Humankapitalinvestition realisiert werden können, auswirkt.

Einen eher kurzfristigen Zeithorizont weisen Betriebe auf, welche Bildungserträge bereits während bzw. nach der Ausbildung erzielen und somit nach dem produktionstheoretischen Ansatz in Humankapital investieren. Erträge übersteigen schon frühzeitig im Beschäftigungsverhältnis die Kosten. Dies liegt einerseits an dem hohen Beitrag zur Produktion des Ausbildungsabsolventen begründet, weil er als ,billige' Arbeitskraft eingesetzt wird. Andererseits betreiben die Betriebe selbst nicht viele Humankapitalaufwendungen, weswegen auch die Kosten niedrig gehalten werden können. In der Regel werden von diesen Betrieben, die nach dem produktionstheoretischen Ansatz ausbilden, Auszubildende mit einem niedrigen allgemeinen Schulabschluss ausgewählt. Die Ausbildung ist meist gekennzeichnet von manuellen bzw. einfachen Tätigkeiten, deren Output quantifiziert und damit kontrolliert werden kann. Daher liegen hier auch entsprechend wenig Informationsasymmetrien vor. Weil bereits die Bildungsinvestitionen amortisiert sind, müssen diese Betriebe nicht notwendigerweise den Ausbildungsabsolventen weiterbeschäftigen, um Ausbildungserträge zu realisieren. Daher sind die Unternehmen bereit, nur einen bestimmten Lohn zu bezahlen. Meist liegt dieser unter den Anforderungen eines Ausgebildeten, sodass die erneute Einstellung eines Auszubildenden oder die Einstellung eines Un- bzw. Angelernten günstiger ist als die Weiterbeschäftigung des selbst ausgebildeten Arbeitnehmers. Ein kurzfristiges Beschäftigungsinteresse liegt vor, was sich in der Qualität und der Höhe des Humankapitals niederschlägt. Werden Auszubildende an der ersten Schwelle in solche Betriebe selektiert, weisen diese nach Beendigung der Ausbildung eine geringe Übernahmewahrscheinlichkeit auf.

Dies spiegelt sich zugleich durch ein geringes (Weiter-) Beschäftigungsinteresse wider, da diese Art der Ausbildung häufig im externen Segment anzutreffen ist, das stark von der konjunkturellen Situation abhängt. Jedoch ist die Situation nach einer Nichtübernahme differenziert zu betrachten. So finden Absolventen innerhalb des berufsspezifischen Segments meist eine Anstellung, weil sie wegen des hohen Anteils an allgemeinem bzw. berufsspezifischem Humankapital flexibel auf dem Arbeitsmarkt einsetzbar sind, was eine relativ kurze Dauer von Erwerbslosigkeit impliziert. Diese Ausbildungsabsolventen üben meist eine andere als die erlernte Tätigkeit aus (Büchel/Neubäumer 2001; Neubäumer 1999). Dies liegt insbesondere an dem allgemeinen bzw. berufsspezifischen Humankapital, das bei einem Wechsel nicht verfällt und zu nahezu keinem Produktivitätsverlust führt. Die Dauer von Arbeitslosigkeit ist daher, insgesamt betrachtet, relativ kurz. Jedoch treten aufgrund des vorwiegenden Anteils an allgemeinem und berufsspezifischem Humankapital häufig Phasen der Er- 
werbslosigkeit auf, weil Einstellungs-, Einarbeitungs-, aber auch Entlassungskosten verhältnismäßig gering sind. Zudem ist diese Absolventengruppe von wirtschaftlichen Veränderungen mehr als andere betroffen. Der geringe gewerkschaftliche Organisationsgrad innerhalb dieser Berufsgruppen trägt zu der Beschäftigungsinstabilität weiter bei.

Wie Kostenstudien der betrieblichen Ausbildung belegen, liegen jedoch häufig Nettokosten nach Beendigung einer dualen Berufsausbildung vor (Beicht et al. 2004; Bardeleben et al 1995). Um die Amortisation der Bildungsinvestitionen zu ermöglichen, verlängert sich der Zeithorizont bei diesen Unternehmen, die nach investitionstheoretischen Gesichtspunkten ausbilden. Damit die Ausbildungsbereitschaft gewährleistet werden kann, müssen die Annahmen des vollkommenen Marktes modifiziert werden. Erst bei Existenz von Marktunvollkommenheiten und unvollkommenem Wettbewerb finden betriebliche Investitionen in Humankapital statt. Marktunvollkommenheiten sind Informationsasymmetrien, Gewerkschaften und andere Regulierungen auf dem Arbeits- und Gütermarkt sowie die Annahme einer bestehenden Komplementarität zwischen betriebsspezifischem und allgemeinem Humankapital.

Entsprechend determiniert dies die Qualität der Ausbildung und die Höhe des Humankapitalstandes sowie die Zusammensetzung der Humankapitalarten. Ausbildungsbetriebe, die einen größeren Zeithorizont aufweisen, werden Schulabsolventen mit bereits hohem Humankapital einstellen. Allerdings ist dies auch notwendig, da die Betriebe meist einen internen Arbeitsmarkt aufweisen und dessen Strukturen und Arbeitsprozesse komplex sind. In diesem Falle wird neben dem berufsspezifischen Wissen auch betriebsspezifisches Humankapital vermittelt, welches bei einem Betriebswechsel verfällt und einen Produktivitätsverlust zur Folge hat. Da die Unternehmen Humankapitalinvestitionen tätigen, um künftige Fachkräfte für den Erhalt der Wettbewerbsfähigkeit auszubilden, ist davon auszugehen, dass diese Unternehmen hohe Humankapitalinvestitionen tätigen, welche auf die Qualität der Ausbildung rückwirken. Zudem ist die Ausbildung als Informationsgewinnungsprozess zu verstehen, innerhalb dessen die Betriebe Kenntnisse über die Auszubildenden erlangen. Eine Ausbildung ist in diesem Fall kostengünstig gegenüber einer Rekrutierung von extern ausgebildeten Fachkräften, da die Arbeitsplätze hohe Einarbeits-, aber auch hohe Kontrollkosten verursachen. Zudem bestehen große Unsicherheiten bezüglich der Qualität der extern rekrutierten Arbeitskräfte, die bei einer Weiterbeschäftigung von selbst ausgebildeten Fachkräften vermieden werden. So werden während der Ausbildungsphase sowohl Einarbeitungskosten eingespart als auch Informationsasymmetrien abgebaut, was die Kontrollbedingungen beeinflusst. Daher sind diese Betriebe, die zumeist auch nach dem investitionstheoretischen Ansatz ausbilden, langfristig an einer Beschäftigung der Ausbildungsabsolventen interessiert. Dies reicht aber als Begründung für eine betriebliche Ausbildung bei existierenden Nettoausbildungskosten nicht aus. Vielmehr treten aufgrund der be- 
stehenden Marktunvollkommenheiten Mechanismen in Kraft, welche die Mobilität auf Seiten der Ausbildungsabsolventen reduzieren.

Neben der Amortisation der Ausbildungskosten, die bei einer Weiterbeschäftigung erfolgt, sind Betriebe daran interessiert, den zukünftigen Fachkräftebedarf zu decken, der notwendig ist, um wettbewerbsfähig zu bleiben. Daher werden Ausbildungsabsolventen weiterbeschäftigt, sofern sie die betrieblichen Mindestanforderungen erfüllen (Kessler/Lülfesmann 2002; Acemoglu/Pischke 1999a; 1999b; 1998). Andere, welche diese Mindestanforderungen nicht erfüllen, sind einem weniger erfolgreichen Start ins Erwerbsleben ausgesetzt. Dabei legt ein Teil der Betriebe bereits zu Beginn der Ausbildung fest, welchen Anteil eines Jahrgangs sie am Ende übernehmen. Somit bilden Betriebe mit Nettoausbildungskosten ebenfalls ,über Bedarf“ aus. Andere Betriebe entscheiden sich während der Ausbildung für oder gegen einen Ausbildungsabsolventen, den sie ursprünglich übernehmen wollten. Dadurch nutzen sie die Funktion des Informationsgewinnungsprozesses aus. Entsprechend ist die Übernahme von Ausbildungsabsolventen in den nach investitionstheoretischen Ansätzen ausbildenden Betrieben nicht sicher. Trotz des hohen Humankapitalniveaus können auch sie von Arbeitslosigkeit betroffen sein, was wiederum die Dauer der Arbeitslosigkeit verlängern kann, da der betriebsspezifische Anteil entsprechend hoch ist, der diesen Absolventen erschwert, einen ausbildungsadäquaten Arbeitsplatz ohne hohen Humankapitalverlust zu finden. Darüber hinaus sind sie sich ihrer qualitativ guten Ausbildung bewusst und setzen die Anforderungen, die ein künftiger Job erfüllen muss, entsprechend hoch. Dies drückt sich in einem höheren Reservationslohn aus. Allerdings ist auch zu berücksichtigen, dass diese Ausbildungsabsolventen sich vor allem auf Arbeitsstellen bewerben, die hohe Einstellungs-, Kontroll- und Kündigungskosten haben und bei denen daher die Unternehmen auf dem externen Arbeitsmarkt selbst einen längeren Suchprozess vornehmen, um die Stellen adäquat zu besetzen. So sind trotz der unter Umständen längeren Arbeitslosigkeitsphase die Ausbildungsabsolventen mit höherem Bildungsstand und höherer Ausbildungsqualität weniger häufig von Arbeitslosigkeit betroffen, weil die Arbeitsstellen eine höhere Beschäftigungssicherheit aufweisen. In der Regel sind diese Tätigkeits- und analog hierzu die Ausbildungsbereiche nicht in dem Maße von wirtschaftlichen Faktoren beeinflusst. Aber auch seitens der Ausbildungsabsolventen verlängert sich die Arbeitslosigkeitsdauer, weil sie hohe Ansprüche hinsichtlich des Lohnes, der Karrierechancen etc. an den Arbeitsplatz stellen. Jedoch muss diese Aussage relativiert werden, da es sich bei diesen Ausbildungsabsolventen um gut qualifizierte Arbeitskräfte handelt. Dieses höhere Ausbildungsniveau reduziert entsprechend die kumulierte Dauer der Arbeitslosigkeit. Abbildung 7 gibt einen Überblick über die $\mathrm{Zu}$ sammenhänge der einzelnen Komponenten: 
bbildung 7: Zusammenhang zwischen Ausbildungsbereitschaft und Übergang ins Erwerbsleben

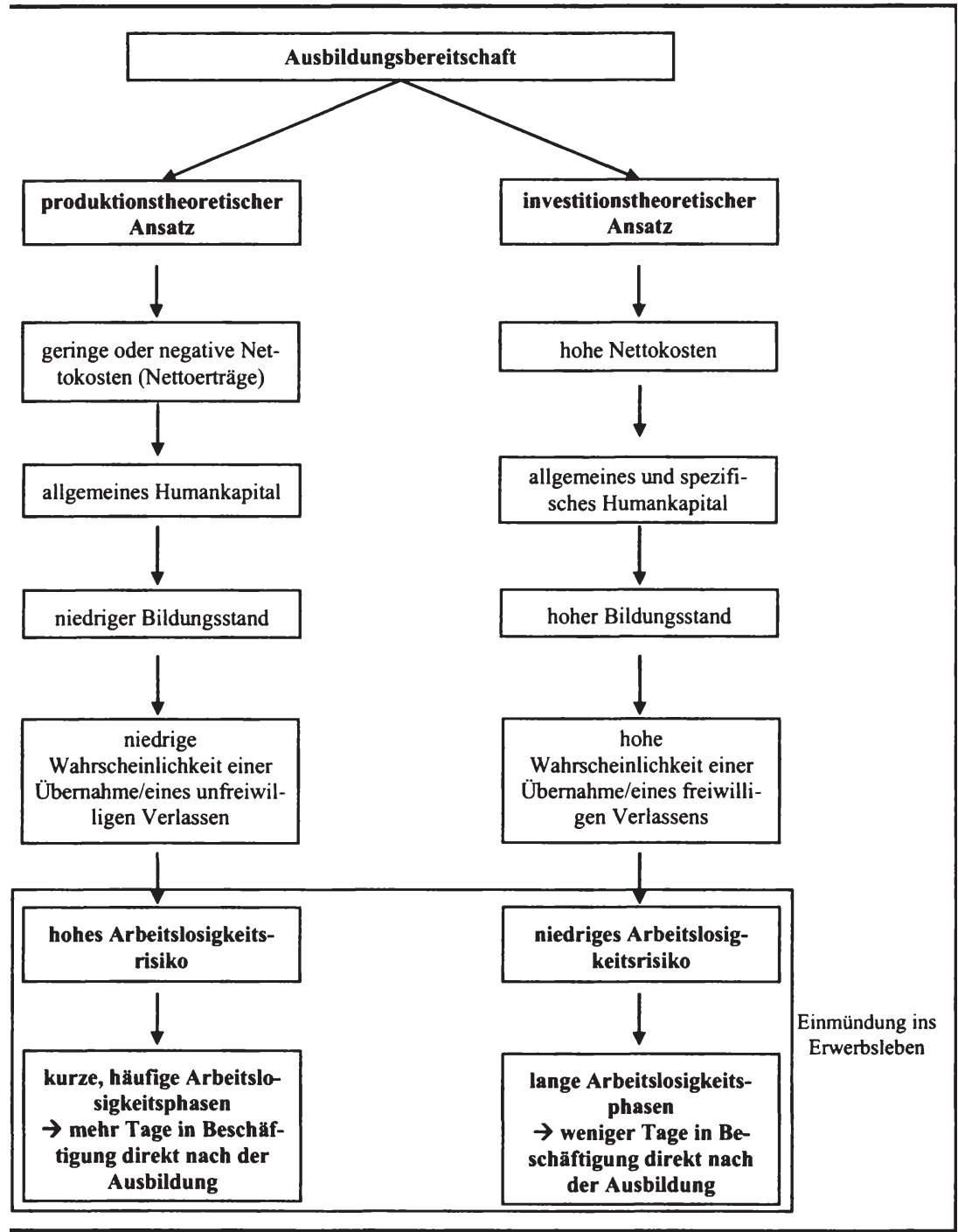

luelle: eigene Darstellung

s wird ersichtlich, dass das Bildungsniveau zu Beginn der Ausbildung über die st der Ausbildung entscheidet und die Art der Ausbildung die Qualität und das 
Bildungsniveau beeinflusst. Startet ein Jugendlicher bereits mit einem niedrigen Bildungsniveau in die Ausbildung, wird dieses nur um die Mindestanforderungen erhöht. Aufgrund der Organisation der Ausbildung sowie der geringen Ressourcen (für Bildung) setzt sich die schlechte Ausgangsposition der Auszubildenden an der zweiten Schwelle im Vergleich zu den anderen Ausbildungsabsolventen fort. So verbessert sich die Arbeitsmarktssituation nach der Ausbildung nicht wesentlich, da sie im Vergleich zu anderen nicht übernommenen Ausbildungsabsolventen immer noch ein niedriges Bildungsniveau aufweisen. Absolventen, welche eine Ausbildung nach produktionstheoretischem Ansatz absolvieren, sind daher immer noch stark von diskontinuierlichen Erwerbsverläufen betroffen (Schaeper et al. 2000). Jedoch konkurrieren nicht alle übernommenen Ausbildungsabsolventen in verschiedenem Maße miteinander. Ein Wettbewerb entsteht bei Bewerbungen um dieselben Stellen. Dies betrifft Personen, welche denselben Ausbildungsberuf oder einen Beruf derselben Berufsgruppe erlernt haben. Daher müssen auch die übernommenen Ausbildungsabsolventen einbezogen werden, die freiwillig den Betrieb verlassen. Bei dieser Betrachtung stehen absolut gesehen Ausbildungsabsolventen im Wettbewerb zueinander, die ungefähr das gleiche Bildungsniveau aufweisen. Sie unterscheiden sich lediglich darin, dass die nicht übernommenen entweder den betrieblichen Anforderungen nicht gerecht werden und daher die schlechteren Absolventen sind oder dass die Ausbildungsbetriebe sie aufgrund von gesamtwirtschaftlichen Verschlechterungen nicht weiterbeschäftigen können. Gleich welche Dimension betrachtet wird, sind die schlechteren Ausbildungsabsolventen eher davon betroffen, nicht übernommen zu werden als die guten Ausbildungsabsolventen. Infolgedessen ist das Arbeitslosigkeitsrisiko höher. Entsprechend weisen auch die Absolventen mit einem höheren gesamten Bildungsstand ein geringeres Arbeitslosigkeitsrisiko auf als junge Erwachsene mit niedrigerem Bildungsstand. Werden diese jedoch arbeitslos dauert deren Phase von Erwerbslosigkeit länger an. Die erste Zeit nach der dualen Berufsausbildung ist daher in der Regel von einer kürzeren Beschäftigungsdauer gekennzeichnet. Jedoch führt der Abgang aus Arbeitslosigkeit dann aber in ein langfristiges Beschäftigungsverhältnis.

Aus diesen theoretischen Zusammenhängen werden Hypothesen abgeleitet, die den ökonometrischen Schätzungen zugrunde liegen. Die erste Hypothese zielt auf die Implikationen des produktions- und investitionstheoretischen Ansatzes, der um die humankapitaltheoretischen Aspekte erweitert wurde. So werden Ausbildungsabsolventen mit einer Ausbildung nach produktionstheoretischen Grundsätzen weniger wahrscheinlich übernommen. Der Anteil an allgemeinem und berufsspezifischem Humankapital ist sehr hoch, woraus ein relativ niedriger Humankapitalbestand resultiert. 


\section{Hypothese}

a) Ausbildungsabsolventen mit niedrigem allgemeinem Humankapitalstand verlassen mit hoher Wahrscheinlichkeit den Ausbildungsbetrieb.

b) Bei Ausbildungsabsolventen mit niedrigem allgemeinem Humankapitalstand steigt die Wahrscheinlichkeit einer Arbeitslosigkeit.

Hypothese 1 beruht auf der Annahme, dass Ausbildungsabsolventen, die eine Ausbildung in Betrieben mit wenig finanziellen Ressourcen sowie mit mangelnder betrieblicher Ausstattung, wie eigens für die Ausbildung eingesetztes Personal, weniger Möglichkeiten zur Verfügung stehen, sich Fähigkeiten über die Mindeststandards hinaus anzueignen. Der Wechsel ist daher eher unfreiwilliger Natur. Entsprechend ist bei den wechselnden Ausbildungsabsolventen ein höheres Arbeitslosigkeitsrisiko anzunehmen (Casey 1986. 73). Begründet liegt dies darin, dass sich eine Off-the-Job-Suche, also eine Arbeitssuche außerhalb einer Beschäftigung, schwieriger gestaltet als eine On-the-Job-Suche. Selbst befristet übernommene Ausbildungsabsolventen haben einen Vorteil bei der Arbeitssuche, da sie während der befristeten Beschäftigung suchen können und zudem noch Berufserfahrung sammeln können. Weiterhin muss auf die Unterschiede zwischen den Ausbildungsabsolventen eingegangen werden. Da der Pool der Ausbildungsabsolventen, der sich nach der dualen Berufsausbildung auf dem Arbeitsmarkt befindet, heterogen ist und sich entsprechend aus unterschiedlichen Humankapitalniveaus zusammensetzt, liegen neben den unterschiedlichen Arbeitslosigkeitsrisiken ebenfalls unterschiedliche Verbleibdauern in Arbeitslosigkeit vor. Diesem Aspekt trägt die zweite Hypothese Rechnung:

\section{Hypothese}

Die Arbeitslosigkeitsdauer der Ausbildungsabsolventen steigt mit dem Humankapitalstand.

Begründet werden kann dies in dem höheren Anteil an betriebsspezifischem Humankapital und dem daraus resultieren höheren Produktivitätsverlust bei einem Wechsel. Schließlich wird ein Ausblick für Absolventen gegeben, die zu Beginn des Erwerbslebens eine Arbeitslosigkeitsphase realisieren. Hierbei wird angenommen, dass die besseren Ausbildungsabsolventen zwar länger in Arbeitslosigkeit verweilen, aber die Akzeptanz einer Beschäftigung zu einem langfristigen Arbeitsverhältnis führt. Innerhalb des ersten Jahres weisen diese zunächst wegen der längeren Arbeitslosigkeitsdauer eine kürzere Zeit in Erwerbstätigkeit auf. Dagegen sind schlechtere Ausbildungsabsolventen, trotz der kürzeren Arbeitslosigkeit häufiger arbeitslos, was sich aber bei einem kurzen Betrachtungszeitraum nicht negativ auswirkt. Aus diesem Zusammenhang resultiert die dritte Hypothese: 


\section{Hypothese:}

Die Beschäftigungsdauer im ersten Berufsjahr nach der dualen Berufsausbildung reduziert sich mit steigendem Humankapitalstand für Absolventen mit anfänglicher Arbeitslosigkeit.

Es wurden bereits einige Studien durchgeführt, die explizit die Wirkungen der Ausbildungsentscheidungen an der ersten Schwelle auf den Übergang an der zweiten Schwelle untersuchen (Büchel 2002; Hillmert 2001). Vor dem Hintergrund der wachsenden Anforderungen des Arbeitsmarktes an die Ausbildungsabsolventen und die negative Selektion der Auszubildenden an der ersten Schwelle stellt sich die Frage, inwieweit der Arbeitsmarkteintritt erschwert ist. Dabei wird im Folgenden von der Untersuchung der Erwerbsverläufe nach der dualen Berufsausbildung sowie den Wirkungen einer Arbeitslosigkeit zu Beginn der Erwerbskarriere auf den weiteren Berufsverlauf abgesehen. Vielmehr werden, vor dem Hintergrund einer zunehmenden Arbeitslosigkeit, die Ursachen des Arbeitslosigkeitsrisikos nach einer dualen Berufsausbildung empirisch untersucht. Allerdings beruhen die vorgestellten theoretischen Ansätze auf allgemeinen Auswirkungen hinsichtlich der betrieblichen Bildungsinvestitionen auf den Humankapitalstand und die Art des Humankapitals, sowie deren Einfluss auf einen Betriebswechsel und einen möglichen Eintritt in die Arbeitslosigkeit. $\mathrm{Da}$ sich aber an der dualen Berufsausbildung Betriebe beteiligen, die unterschiedliche firmenspezifische Merkmale aufweisen, müssen diese ebenfalls identifiziert werden. Denn gerade diese unterschiedlichen Eigenschaften machen die duale Berufsausbildung aus und beeinflussen die Ausbildungsqualität sowie die Ausbildungshöhe. Deshalb müssen aus diesen betrieblichen Rahmenbedingungen, wie Betriebsgröße, Wirtschaftszweig, Lohnsumme, etc. die Ursachen einer Arbeitslosigkeit abgeleitet werden. Daher ist es Ziel des Empirieteils, die theoretischen Aspekte mit den Charakteristika des dualen Berufsbildungssystems zu verknüpfen, an Hand derer die Hypothesen überprüft werden. 


\section{Empirieteil}

\section{Einflussfaktoren auf die Arbeitslosigkeit nach der dualen Berufsaus- bildung}

Da sich die Theorien bei der Erklärung der Ausbildungsbereitschaft und der Arbeitslosigkeit auf die verschiedenen Arten von Humankapital beziehen, bedarf es zur Umsetzung der aus der Theorie abgeleiteten Hypothesen (siehe Kap. 6) einiger Modifikationen. Einerseits ist dies notwendig, weil sich die Untersuchungen lediglich auf den dualen Berufsbildungsabschluss konzentrieren. ${ }^{66}$ Formal erlangen die jungen Erwachsenen dasselbe Berufsbildungsniveau mit ähnlichen Humankapitalarten. Diese bestimmen aber den Übergang vom dualen Berufsbildungssystem in den Arbeitsmarkt. So weisen Ausbildungsabsolventen unterschiedlicher Bereiche innerhalb des Berufsbildungssystems eine Vielzahl von Fähigkeiten und Arten von Wissen auf, die sich zudem in ihrer Höhe unterscheiden. Andererseits sind die Humankapitalarten nicht unmittelbar empirisch zu erfassen. Sie müssen durch Hilfsvariable in Indikatoren übersetzt werden, mit Hilfe derer sich Schätzungen durchführen lassen. Hierfür sind die jeweiligen Rahmenbedingungen während der dualen Berufsausbildung besonders geeignet, weil sie das Humankapital direkt determinieren.

\subsection{Empirische Umsetzung zur Messung des Humankapitals am Über- gang nach der Ausbildung}

Zur empirischen Analyse muss der Fokus auf die betrieblichen Rahmenbedingungen, die individuellen Merkmale, aber auch auf konjunkturelle Faktoren gelegt werden. Gerade diese Rahmenbedingungen führen zu Unterschieden im Qualifikationsniveau und verstärken zugleich auch die Unterschiede in dem Bildungsniveau. Studien (Schøne 2006; Frazis et al. 2000; Lynch/Black 1998; Oosterbeek 1998) ergaben, dass gewerkschaftlich gebundene Unternehmen, Unternehmen mit einem hohen Stand an technologischem Fortschritt sowie Unternehmen mit hoch qualifizierten Arbeitskräften viel in die Ausbildung ihrer Arbeitskräfte investieren. Daher wird eine differenzierte Betrachtung dieser Einflussfaktoren erforderlich. Dies spiegelt zugleich auch die Struktur des Systems der dualen Berufsausbildung wider. Diese Informationen können zur Beschreibung der Art des vermittelten Humankapitals innerhalb verschiedener Betriebsstrukturen herangezogen werden. Die folgende Abbildung gibt einen Überblick über die verschiedenen Einflussfaktoren, die innerhalb des dualen Berufsbildungssystems wirken.

\footnotetext{
${ }^{66}$ Die Untersuchung von verschiedenen Formen von Berufsbildungsabschlüssen, wie bspw. die Unterscheidung zwischen schulischer, betrieblicher oder universitärer Berufsbildung würde die Bestimmung des vorwiegend vermittelten Humankapitals aus der institutionellen Organisation heraus erleichtern.
} 


\section{Abbildung 8: Einflussfaktoren auf Höhe und Struktur des Humankapitals}

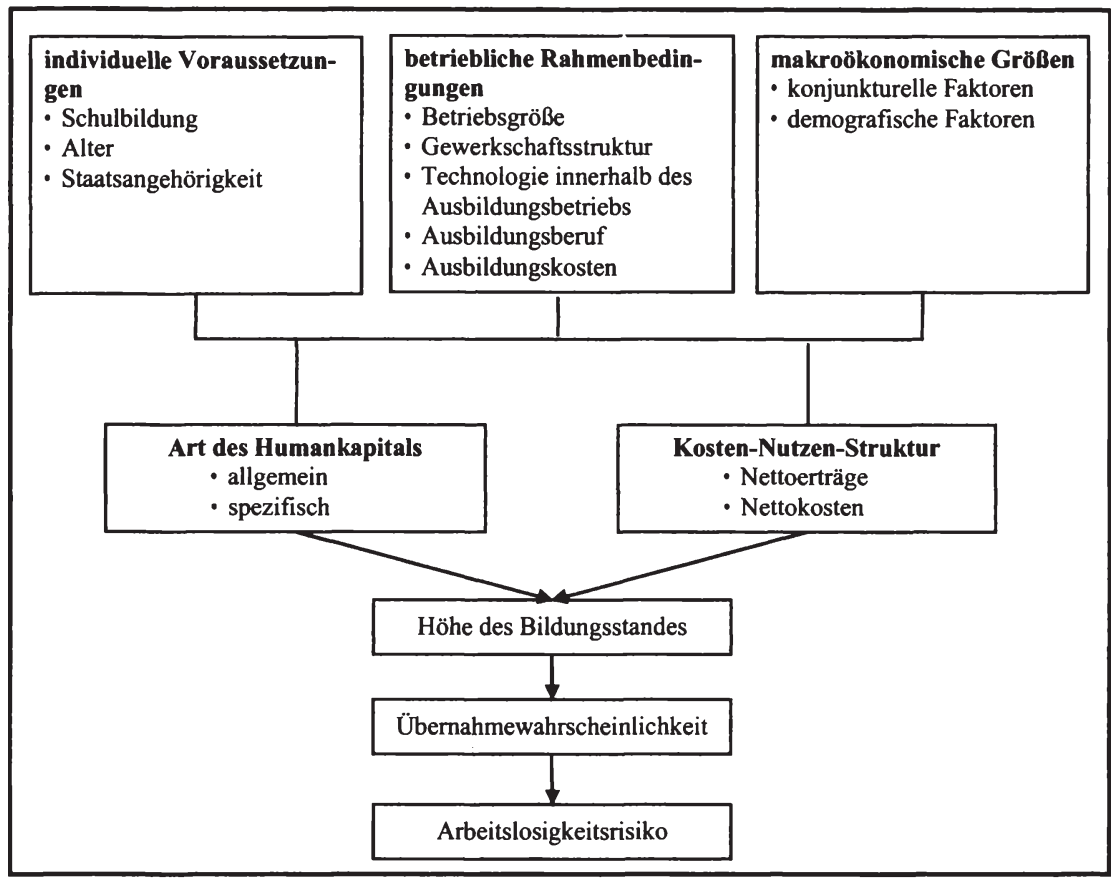

Quelle: eigene Darstellung

Es ist zu erkennen, dass individuelle Merkmale, wie Schulbildung und Staatsangehörigkeit, Einfluss auf die betriebliche Ausbildung nehmen. Wie aus Kap. 2.2 hervorgeht, beeinflusst der Schulabschluss die Bildung verschiedener Humankapitalarten. Ebenso fördern die Betriebsgröße, die Ausbildungsbereiche, die Technologie innerhalb des Unternehmens, die Ausbildungskosten sowie die gesamtwirtschaftlichen Rahmenbedingungen die Bildung der Humankapitaltypen unterschiedlich. Es sind jedoch Zusammenhänge zwischen und innerhalb der verschiedenen Ebenen (individuelle Voraussetzungen, Betriebliche Rahmenbedingungen, Makrogrößen) zu berücksichtigen, die über die Humankapitalart und den Bildungsstand die Wahrscheinlichkeit einer Arbeitslosigkeit beeinflussen. Daher eröffnen sich für Absolventen aller Schultypen nicht die gleichen Chancen, in alle Ausbildungsbereiche zu gelangen (Kap. 2.2). So sind Jugendliche mit Hauptschulabschluss vorwiegend als Auszubildende in handwerklichen Berufen zu finden, während Abiturienten hauptsächlich im Finanzdienstleistungsbereich ihre Ausbildung absolvieren. Beide genannten Bereiche weisen nach der Ausbildung unterschiedliche Chancen für den weiteren Erwerbsverlauf auf. Weiterhin sind die Zusammenhänge zwischen der Vermittlung von Humankapitalarten und der Kosten-Nutzen-Struktur eines Ausbildungsbetriebes zu beach- 
ten. Einerseits steigen die Kosten eines Ausbildungsbetriebs, wenn dieser aufgrund seiner Produktionsprozesse viel betriebs- und/oder technologiespezifisches Humankapital erfordert. Andererseits liegen die Ausbildungskosten bei Betrieben auch höher, gerade weil sie die betriebliche Ausbildung als Humankapitalinvestition sehen. Über die Identifikation des Zusammenhangs zwischen den Determinanten, die die duale Berufsausbildung kennzeichnen, aber auch strukturieren, lassen sich Rückschlüsse auf den Verbleib an der zweiten Schwelle und den Übergang in das Erwerbsleben ziehen. Zur Beschreibung dieses $\mathrm{Zu}$ sammenhangs werden bereits vorhandene Studien als Grundlage herangezogen. Die Art und insbesondere die Richtung, wie die Faktoren auf den Verbleib an der zweiten Schwelle und auf die Arbeitslosigkeit nach Abschluss der dualen Berufsausbildung wirken, sind nun zu identifizieren. Im Folgenden werden daher die Einflussfaktoren anhand der empirischen Literatur einzeln untersucht und die Richtung der Zusammenhänge zwischen ihnen und der Vermittlung der Humankapitalart sowie das Arbeitslosigkeitsrisiko nach der dualen Berufsausbildung abgeleitet.

\subsubsection{Bedeutung der individuellen Merkmale}

Wenn es um Erklärungsansätze der Arbeitslosigkeit geht, werden in den bisherigen Studien verstärkt individuelle Merkmale betrachtet. Untersuchungsgegenstand hierbei ist aber zumeist die Qualität des Abgangs aus Arbeitslosigkeit. Ob Jugendliche nach der Arbeitslosigkeit eine Beschäftigung aufnehmen oder eine Bildungsmaßnahme besuchen, hängt dabei unter anderem von der Dauer der Arbeitslosigkeit selbst ab. Ergebnis der Studie von Falk et al. (2000) ist, dass sich eine Phase der Arbeitslosigkeit bei längerer Dauer negativ auf den weiteren Erwerbsverlauf auswirkt. Erwerbslosigkeit wird als negatives Signal von künftigen Arbeitgebern gewertet, weil während der Arbeitslosigkeit das erworbene Humankapital nicht genutzt und daher obsolet wird (Ludwig/Pfeiffer 2005). Es wird angenommen, dass die erste Präferenz von arbeitslosen Jugendlichen die Aufnahme einer regulären Beschäftigung ist. Erst nach erfolgloser Jobsuche fällt die Wahl vermehrt auf eine erneute Ausbildung, die von der Einschätzung einer möglichen Wiederbeschäftigung beeinflusst wird (Dietrich/Kleinert 2006; Dietrich/Kleinert 2005; Jacob 2004; Konietzka/Seibert 2001). So ist der Übergang von der Arbeitslosigkeit in eine weitere Ausbildung bei einer Arbeitslosigkeitsdauer zwischen drei und 24 Monaten am höchsten. Zugleich weisen arbeitslose Jugendliche mit einem Hauptschulabschluss eine geringe Wahrscheinlichkeit auf, eine weitere Ausbildung aufzunehmen. Dagegen finden die meisten Übergänge von Arbeitslosigkeit in die Beschäftigung zwischen drei und sechs Monaten statt (Dietrich/Kleinert 2005). Eine arbeitsmarktpolitische Bildungsmaßnahme nehmen vor allem diejenigen auf, die länger als sechs Monate arbeitslos sind sowie arbeitslose Jugendliche in den neuen Ländern. Ferner finden sich Jugendliche, die länger als sechs Monate arbeitslos sind, auch eher in einer Niedriglohnbeschäftigung wieder als Absolventen, die weniger lang in Arbeitslosig- 
keit verbleiben. Die Vermittlungsintensität und damit die Dauer der Arbeitslosigkeit wird aber, sofern der junge Erwachsene arbeitsmarktpolitische Bildungsmaßnahmen besucht, nicht signifikant durch formale Ausbildungsmerkmale, wie Schulabschluss und Berufsausbildungsabschluss beeinflusst (Winterhager 2006: 21f.). ${ }^{67}$ Darüber hinaus verfügen Ausbildungsabsolventen mit Migrationshintergrund über eine höhere Wahrscheinlichkeit, nach der dualen Berufsausbildung arbeitslos zu werden (Burkert/Seibert 2007: 22), oder den Arbeitsmarkt gänzlich zu verlassen bzw. eine längere Phase in Arbeitslosigkeit zu verbleiben (Dietrich/Kleinert 2005). Danach sendet eine ausländische Staatsangehörigkeit eines Ausbildungsabsolventen ein negatives Signal auf die Produktivitätserwartungen der künftigen potenziellen Arbeitgeber aus (Burkert/Seibert 2007: 14, Damelang/Haas 2005: 26). Trotz der Vielzahl an verschiedenen Abgängen aus Arbeitslosigkeit nehmen aber dennoch die meisten eine reguläre $\mathrm{Be}-$ schäftigung auf.

Weiterhin ergeben Studien, dass Abiturienten am seltensten Arbeitslosigkeitsphasen mit darauf folgenden Betriebs- oder Berufswechseln realisieren (Konietzka 2002). Dietrich und Kleinert $(2005)^{68}$ kommen zu dem Ergebnis, dass die meisten der arbeitslosen Ausbildungsabsolventen (49\%) einen Realschulabschluss vorweisen, während $38 \%$ mit einem Hauptschulabschluss nach der dualen Berufsausbildung arbeitslos werden. ${ }^{69}$ So sehen sich Jugendliche mit niedrigerem (allgemeinem) Bildungsstand relativ schlechteren Beschäftigungsaussichten gegenüber. Sie realisieren häufiger Arbeitslosigkeitsphasen nach der dualen Berufsausbildung als Absolventen mit Realschulabschluss bzw. Abitur (Büchel 2002: 10ff.). Die Übernahmewahrscheinlichkeit hängt positiv mit dem Niveau des Schulabschlusses zusammen. Infolgedessen steigt das Arbeitslosigkeitsrisiko bei Ausbildungsabsolventen mit niedrigerem Schulabschluss. Dieser Zusammenhang ist nach Hillmert (2001) signifikant. Die Bedeutung des Bildungsstandes auf die Dauer der Arbeitslosigkeit ist nicht eindeutig. Dietrich und Kleinert (2005) kommen bei der Untersuchung der Arbeitslosigkeitsdauer nach der dualen Berufsausbildung zu dem Ergebnis, dass bei einem höheren Bildungsstand sich die Phase der Arbeitslosigkeit verkürzt. Dagegen führt nach Falk et al. (2000) ein höherer Bildungsabschluss zu keinem schnelleren Eintritt in eine Beschäftigung, was eine längere Suchdauer nach einem Arbeitsplatz bedeutet. In den meisten Studien nimmt aber die Bildung bzw. das Bildungssystem dennoch eine bedeutende Rolle für den Verbleib an der zweiten Schwelle ein ( $u$.

${ }^{67}$ In der empirischen Analyse wird aber vielmehr der Einfluss der Merkmale, welche die Ausbildung bestimmen, auf den Abgang aus Arbeitslosigkeit untersucht.

${ }^{68}$ In dieser Studie wurde auch die Auswirkung der Schichtzugehörigkeit auf den Eintritt in Arbeitslosigkeit nach der dualen Berufsausbildung untersucht. Schließlich kommen die meisten arbeitslosen Jugendlichen aus der Mittelschicht (63\%), während die zweitgrößte Gruppe hinsichtlich dieses Merkmals der Unterschicht entstammt, 10\% dagegen nur aus der Oberschicht (Dietrich/Kleinert 2005).

${ }^{69}$ Bei diesen Ergebnissen ist zu berücksichtigen, dass auch die Mehrheit der Absolventen des dualen Berufsausbildungssystems die Schule mit mittlerer Reife beendet hat. 
a. Albert 2007; Konietzka 2002). Auch für Reinberg und Hummel (2007) sowie Schönig und Farhauer (2004) sind die Qualifikationen für die Arbeitsmarktposition signifikant.

Bereits bei der Besetzung der Lehrstellen zu Beginn der Ausbildung ist der Schulabschluss von Bedeutung, denn er bestimmt über die Art der Ausbildung bzw. die Ausbildungsbetriebsart die Beschäftigungsaussichten eines Ausbildungsabsolventen im Ausbildungsbetrieb (Büchel/Neubäumer 2001; Schaeper et al. 2000; Schaeper 1999), dessen direkter Einfluss aus den theoretischen Herleitungen ersichtlich und durch Studien bestätigt wurde. Der Effekt der Schulbildung setzt sich aber auch indirekt in der Wirkung der Ausbildungsbereiche auf den Übergang ins Erwerbsleben fort, weil formal zwar alle Ausbildungsbereiche für jeden Jugendlichen offen sind, aber aufgrund der Anforderungen der Betriebe die verschiedenen Ausbildungsberufe nicht in selbem Maße für Schulabsolventen geeignet sind. So sind die Chancen von Jugendlichen mit niedriger Schulbildung geringer als für Jugendliche mit einer höheren Schulbildung, bereits an der ersten Schwelle einen Ausbildungsberuf mit guten Berufsaussichten zu erlernen, (Büchel 2002; Büchel/ Neubäumer 2001). Abiturienten weisen demnach die beste Ausgangsposition zu Beginn der Ausbildung wie auch nach der Ausbildung auf. Ausbildungsabsolventen, die bereits einen höheren Schulabschluss vorweisen, erlernen einen Beruf, der ebenfalls hohes Humankapital erfordert. Dies wiederum erhöht den gesamten Bildungsstand am Ende der Ausbildung stark. Dagegen können die Ausbildungsabsolventen mit einem niedrigen oder keinem Schulabschluss ihre Bildung nur um die berufsspezifische Humankapitalkomponente steigern. Aber auch innerhalb der Ausbildungsbereiche verbleiben Absolventen mit höherem Schulabschluss wahrscheinlicher im Ausbildungsbetrieb. Infolgedessen weisen sie ein geringeres Arbeitslosigkeitsrisiko als Absolventen mit niedrigem Schulabschluss im selben Ausbildungsbereich auf. Insgesamt verfügen Hauptschulabsolventen dann weiterhin über einen geringeren Bildungsstand.

Für die empirischen Ergebnisse ist daher zu erwarten, dass ein Hauptschulabschluss positiv auf die Wahrscheinlichkeit wirkt, den Betrieb zu wechseln. Ebenso werden Ausbildungsabsolventen mit Hauptschulabschluss eher arbeitslos als Ausbildungsabsolventen mit Realschulabschluss und Abitur. Einerseits ist anzunehmen, dass die Dauer der Arbeitslosigkeit mit steigender Bildung sinkt. Das heißt, dass gut ausgebildete Ausbildungsabsolventen bereits aufgrund ihres höheren formalen Bildungsstandes, der sich aus der Schulbildung aber auch durch einen bestimmten Ausbildungsbereich oder durch ein bestimmtes Unternehmen zusammensetzt, schneller eine neue Beschäftigung finden. Andererseits bewerben sich diese zumeist auf gute Stellen, die einem strengen Selektionsverfahren unterliegen. Zudem steigen die Ansprüche der guten Ausbildungsabsolventen an die Stellen. Infolgedessen wirkt sich die Stellensuche zunächst verlängernd auf die Arbeitslosigkeit aus. Personen mit Abitur weisen eine längere Arbeitslosigkeitsdauer auf, da sie in Ausbildungsbereichen sind, die einen hohen 
Anteil an betriebsspezifischem Humankapital vermitteln. Das Angebot eines ausbildungsadäquaten Arbeitsplatzes beschränkt sich daher. Infolgedessen verlängert sich die Arbeitslosigkeitsperiode.

Ein weiterer Einflussfaktor auf die Dauer der Arbeitslosigkeit stellt auch das Alter des betreffenden Ausbildungsabsolventen dar, das sich in der Studie von Dietrich und Kleinert (2005) für arbeitslose Jugendliche mit einer dualen Berufsausbildung als signifikant erweist. Je älter demnach die betroffene Person ist, desto weniger wahrscheinlich ist ein Übergang in die Beschäftigung. Das Alter übt zudem einen direkten Einfluss auf die Arbeitslosigkeitsdauer aus. Ein höheres Alter bei der Bewerbung wirkt sich dann negativ auf die Lehrstellenbewerbung aus, sofern sich diese durch ein Berufgrundbildungsjahr bzw. Berufsvorbereitungsjahr oder einen verspäteten Schulabschluss verzögert. Der Zusammenhang zwischen der ersten und zweiten Schwelle wird auch hier deutlich, da die älteren Jugendlichen mit niedrigerem Schulabschluss Ausbildungsberufe mit geringen Beschäftigungsaussichten erhalten. Daher beeinflusst in diesem Kontext das Alter die Arbeitslosigkeitsdauer.

Schließlich spielt die Staatsangehörigkeit ebenfalls eine Rolle beim Bildungsprozess. Ausländische bzw. Jugendliche mit Migrationshintergrund erlernen Ausbildungsberufe, in denen vorwiegend berufsspezifisches Humankapital vermittelt wird. Daher verfügen sie nach der Ausbildung über den Bildungsstand, der für die Ausübung des erlernten Berufes gerade notwendig ist. Die Höhe des Bildungsniveaus spiegelt demnach die durch die Ausbildungsordnungen vorgegebenen Mindeststandards wider. Eine nicht-deutsche Staatsangehörigkeit führt zu einer erhöhten Wahrscheinlichkeit, den Ausbildungsbetrieb zu verlassen. Demnach ist ein positiver Zusammenhang zwischen nicht-deutscher Staatsangehörigkeit und Betriebswechsel zu erwarten. Ebenso wird eine ausländische Staatsbürgerschaft die Wahrscheinlichkeit erhöhen, in Arbeitslosigkeit einzutreten. Die Dauer bis zur weiteren Beschäftigungsaufnahme wird in der Regel relativ kurz sein.

\subsubsection{Betriebliche Einflussfaktoren}

Mehr als bei anderen Berufsbildungsabschlüssen spielen betriebliche Faktoren während der dualen Ausbildung für den Verbleib an der zweiten Schwelle eine Rolle. Durch die Teilnahme unterschiedlicher Ausbildungsbetriebe an der dualen Berufsausbildung, welche die Vermittlung der Mindestanforderungen an Qualifikationen erfüllen müssen, ist die Berufsausbildung von einer Vielzahl an Ausprägungen der Einflussfaktoren auf betrieblicher Ebene gekennzeichnet. Dadurch werden verschiedene Anforderungen an die Qualifikationen der Auszubildenden sowie entsprechend an die zukünftigen Fachkräfte gestellt, weil sie unterschiedliche Ausbildungskosten haben, unterschiedliche Technologien in den Produktionsprozess einsetzen oder sich auch in der Wettbewerbsposition auf dem Gütermarkt unterscheiden (Elliott/Lindley 2006: 390). Diese Qualifikationsanforderungen und -arten differieren bspw. zwischen den handwerklichen 
und den kaufmännischen oder technischen Ausbildungsbereichen. So benötigt ein Auszubildender in einem kaufmännischen Beruf vor allem analytisches und logisches Denkvermögen, während in technischen Berufen neben analytischem Denken vorwiegend auch Verständnis für technische Zusammenhänge gefordert wird. Ausbildungsberufe verlangen daher meist bereits an der ersten Schwelle eine unterschiedliche Höhe an Humankapital. Auf dieser Grundlage kann während der Ausbildung unterschiedlich viel Bildung bzw. auch eine Vermittlung von betriebsspezifischem und allgemeinem Humankapital in unterschiedlicher Zusammensetzung erfolgen. Daraus resultiert wiederum am Ende der Ausbildung ein Humankapitalstand, der zwischen den unterschiedlich erlernten Berufen divergiert. Einerseits ist die berufliche Qualifikationsstruktur durch die Ausbildungsordnungen selbst gegeben, die sich zwischen Ausbildungsberufen unterscheiden. So ist zwar das berufspezifische Humankapital darin verankert, welches die Mobilität innerhalb der unterschiedlichen Berufsgruppen ohne gröBere Humankapitalverluste ermöglicht (Kambourov/Manovskii 2008). Andererseits entsteht weiteres Humankapital durch die Beteiligung des Auszubildenden am Produktionsprozess. Je nach Art der Produktion und der herzustellenden Produkte fällt dieses zusätzliche Humankapital unterschiedlich hoch aus. Daher beeinflussen die verschiedenen betrieblichen Rahmenbedingungen die Zusammensetzung des jeweiligen Qualifikationsniveaus. Infolgedessen divergiert die Höhe der Mobilität in und zwischen den einzelnen Berufsgruppen. So haben die Ausbildungsabsolventen der jeweiligen Ausbildungsbereiche am Ende der Ausbildung unterschiedliche Beschäftigungschancen. Daher müssen zur Überprüfung der Hypothesen zunächst die betrieblichen Determinanten identifiziert werden, die innerhalb der dualen Berufsausbildung die Struktur der Humankapitalarten und infolgedessen die Höhe des Bildungsniveaus festlegen. Danach können Implikationen für den Verbleib an der zweiten Schwelle getroffen werden.

In bisherigen Studien wurde insbesondere die Struktur der Übergänge in das Erwerbsleben innerhalb der unterschiedlichen Ausbildungsbereiche untersucht, die sich als bedeutend hinsichtlich der verschiedenen Eintrittsmöglichkeiten erwiesen. ${ }^{70}$ Vor allem Kfz-Mechaniker sind nach einer Arbeitslosigkeitsphase gezwungen, den Beruf zu wechseln (10,1\% aller Kfz-Mechaniker) (Konietzka 2002: 658). Als signifikant erweist sich weiterhin der Einfluss einer Ausbildung, einen nicht ausbildungsadäquaten Job zu besetzen, im Bereich Fahrzeugbau/reparatur Elektrotechnik, Nahrungs- und Genussmittel, Holz-/Textil und Verkehr/Nachrichten sowie Handel. Ausgebildete Handelskaufleute haben die höchste Wahrscheinlichkeit, den Beruf bzw. den Betrieb zu wechseln (Konietzka 2002). Das Arbeitslosigkeitsrisiko ist hoch. Jedoch weisen Tischler $(6,1 \%$ aller Tischler) die höchste Arbeitslosigkeit unter den Ausbildungsabsolventen

\footnotetext{
${ }^{70}$ Eine Studie, die auf der Erwerbstätigenbefragung des IAB und BiBB von 1991/92 basiert, ergab zudem, dass die erreichte Berufsstellung für 39\% der Lehrabsolventen als bildungsadäquat zu bezeichnen ist (Feller 2001: 123).
} 
auf (Konietzka 2002). Dagegen verbleiben Bankkaufleute (sowohl Männer als auch Frauen) mit der höchsten Wahrscheinlichkeit $(83,1 \%$ bzw. $82,0 \%$ aller Bankfachleute) im Ausbildungsbetrieb, gefolgt von den Maurern (80,8\% aller Maurer).

Gerade die Ausbildungsbereiche, in denen die meisten Ausbildungsabsolventen nach Abschluss der Berufsausbildung einen Wechsel realisieren, gehören den Bereichen an, die relativ geringe Nettoausbildungskosten ${ }^{71}$ aufweisen. Der handwerkliche Ausbildungsbereich sowie eine Ausbildung in den freien Berufen sehen sich geringen Ausbildungskosten gegenüber (Fougère/Schwerdt 2002: 330f.) und bilden somit nach dem produktionstheoretischen Ansatz aus. Nach Mohrenweiser und Backes-Gellner (2008: S. 10) verfolgen auch Ausbildungsbetriebe im (einfachen) Dienstleistungsbereich eine Substitutionsstrategie.

Hierbei ist der Zusammenhang mit einem weiteren betrieblichen Merkmal, der Betriebsgröße, zu berücksichtigen. Unternehmen, die nach dem produktionstheoretischen Ansatz ausbilden, sind zumeist kleine Betriebe (Beicht et al. 2004; Büchel/Neubäumer 2001; Franz/Zimmermann 2001; Neubäumer 1999; Bardeleben et al. 1995), deren Arbeitsprozesse übersichtlich sind, sodass die Auszubildenden nach einer kurzen Einarbeitungsphase als vollwertige Arbeitskräfte eingesetzt werden können (Neubäumer 1999). Dies trifft vor allem auf die Handwerksbetriebe und Betriebe der freien Berufe zu. Bei letzteren handelt es sich insbesondere um Arztpraxen oder Anwaltsbüros. Allgemeines bzw. berufsspezifisches Humankapital, das gerade den in den Ausbildungsordnungen vorgegebenen Mindestqualifikationen entspricht, reicht daher aus, weil die geringe Anzahl an Mitarbeitern und die einfachen Arbeitsprozesse nicht mehr verlangen. Zudem verfügen diese Betriebe in dem Ausbildungsbereich über einen geringen Grad an Technologisierung, weshalb sich auch die Qualifikation der restlichen Belegschaft auf berufsspezifische Komponenten beschränkt. Ausbildungsabsolventen, die vorwiegend manuelle bzw. handwerkliche sowie bestimmte dienstleistende Berufe erlernt haben, verfügen deshalb vorwiegend über allgemeines bzw. berufsspezifisches Humankapital. Durch die kurze Einarbeitungsphase ist der bewertete Produktionsausfall der hauptamtlichen Mitarbeiter geringer. Zudem leisten die Auszubildenden einen höheren Produktionsbeitrag, der die Differenz zwischen Kosten und Erträgen verringert. So kann bspw. ein Malerlehrling nach kurzer Einarbeitungszeit, ein Zimmer selbst anstreichen oder Auszubildende im Einzelhandel können selbst Kunden bedienen. Daher decken die Bildungserträge bereits während der Ausbildung die Kosten. Daraus ergibt sich ein Überschussangebot an Ausbildungsabsolventen (Euwals/Winkelmann 2003; Bellmann/Neubäumer 2001). Ausbildungsbetriebe in diesem Bereich haben daher wenig Anreiz, die Ausbildungsabsolventen zu übernehmen (Büchel/Neubäumer

\footnotetext{
${ }^{71}$ Im Folgenden werden weiterhin Ausbildungskosten verwendet, die über die Ausbildungsvergütungen hinausgehen. Es ist aber zu berücksichtigen, dass sich auf die in Kap. 7.2 hergeleiteten Zusammenhänge zwischen Ausbildungsvergütungen und Ausbildungskosten in Kap. 8 wieder bezogen wird.
} 
2001; Franz/Zimmermann 2001; Neubäumer 1999). Sie weisen daher ein hohes Arbeitslosigkeitsrisiko auf. Studien (Büchel/Neubäumer 2001; Neubäumer 1999; Franz/Zimmermann 1999) bestätigen dies für den Handwerkssektor. Dort finden sich vor allem junge Erwachsene, die nach Abschluss der Ausbildung über Humankapitalkomponenten verfügen, die aufgrund des geringen Anteils an betriebs- und technologiespezifischem Humankapital nahezu in allen Tätigkeitsbereichen eingesetzt werden können. Somit liegt die Wahrscheinlichkeit des Arbeitslosigkeitsrisikos für Ausbildungsabsolventen von Unternehmen mit geringen bis keinen Nettokosten bzw. Unternehmen im Handwerksbereich, im Bereich der einfachen Dienstleistungstätigkeiten etc. höher, gleichzeitig steigt aber auch die Wahrscheinlichkeit, kürzer in Arbeitslosigkeit zu verbleiben. Dies liegt daran, dass die Beschäftigung nach der Arbeitslosigkeit genau die Produktivität erfordert, die der betroffene Absolvent während der Ausbildung als allgemeines bzw. berufsspezifisches Humankapital erlernt hat. Häufig müssen diese Absolventen aber auch Stellen akzeptieren, die nicht ihrem Bildungsniveau entsprechen und die auch eine geringe Beschäftigungssicherheit aufweisen. Insbesondere weisen Ausbildungsabsolventen in kleinen Betrieben eine geringe Übernahmewahrscheinlichkeit und damit ein erhöhtes Risiko auf, arbeitslos zu werden. Die wenigsten Ausbildungsabsolventen verbleiben nach der Ausbildung im Betrieb, wenn dieser nur einen Beschäftigten hat (Euwals/Winkelmann 2002: 257).

Betriebe in Ausbildungsbereichen, die nach dem investitionstheoretischen Ansatz ausbilden, wie der Bankensektor, Energie/Bergbau sowie der Bereich Chemie und Kunststoff, werden die Ausbildungsabsolventen nach der dualen Berufsausbildung mit hoher Wahrscheinlichkeit weiterbeschäftigen. Diese weisen auch die höchste Wahrscheinlichkeit auf, eine der Ausbildung entsprechende Tätigkeit auszuüben (Büchel/Neubäumer 2001; Neubäumer 1999; Franz/ Zimmermann 2001). Gleichzeitig gehören diese Betriebe den Ausbildungsbereichen an, in denen, den Studien zu Folge, häufig ein reibungsloser Übergang gelingt. Wegen der hohen Ausbildungskosten werden Fachkräfte lediglich nach dem zukünftigen Bedarf ausgebildet. Die Nettoausbildungskosten sind deshalb hoch, weil zum einen in diesen Bereichen insbesondere Großunternehmen vorzufinden sind. Deren Arbeitsprozesse sind komplex, wodurch sich die Einarbeitungs- und Einlernphase verlängert. Ebenso wird mehr betriebsspezifisches Wissen generiert, das wiederum ein bestimmtes Niveau an allgemeinem Humankapital erfordert. Infolgedessen spielt die Betriebsgröße für die Situation nach Abschluss der dualen Berufsausbildung eine entscheidende Rolle. Dies wird bereits bei der Betrachtung der Übernahmequoten, welche zwischen den verschiedenen Betriebsgrößen differieren, deutlich. So nimmt die Weiterbeschäftigungswahrscheinlichkeit für Ausbildungsabsolventen mit der Größe des jeweiligen Ausbildungsbetriebes zu (siehe hierzu u. a. BMBF 2006). Zum anderen verwenden diese Unternehmen, unabhängig von der Betriebsgröß $\mathrm{e}^{72}$, aber auch den neuesten techno-

72 Vor allem in der Informations- und Kommunikationstechnologiebranche oder anderen Technologiezweigen gibt es kleinere Betriebe, die einen hohen technologischen Stand aufwei- 
logischen Stand bei der Produktion, weshalb der Anteil an technologiespezifischem Humankapital zunimmt, um die erreichte Wettbewerbsposition zu halten. Zudem erfordert eine komplexe Technologie Lehrwerkstätten, in denen das betriebsspezifische Humankapital vermittelt werden kann. So entstehen Nettoausbildungskosten einerseits für den Lehrwerkstättenbetrieb und andererseits, weil die Auszubildenden keinen Produktionsbeitrag während dieser Zeit leisten. Da die Ausbildungsordnungen nicht in dem Tempo angepasst werden wie technischer Fortschritt stattfindet, wird in diesen Ausbildungsbereichen und Betrieben Humankapital über die gesetzlich vorgegebenen Mindeststandards vermittelt. Die Geschwindigkeit des technologischen Fortschritts fördert zudem die Anpassungsfähigkeit und Flexibilität der Arbeitskräfte, da sich die Produktionsprozesse entsprechend der eingesetzten Technologie verändern. Hierzu sind hoch qualifizierte Arbeitskräfte notwendig, weshalb davon ausgegangen werden kann, dass das Qualifikationsniveau innerhalb dieser Betriebe hoch ist und sich die Qualifikationsstruktur zugunsten von gut ausgebildeten Fachkräften verteilt. Daher benötigen Auszubildende als künftige Fachkräfte betriebsspezifisches Humankapital, das insbesondere dann vermittelt werden kann, wenn ein hoher allgemeiner Humankapitalstand vorliegt, sowohl beim Auszubildenden selbst als auch innerhalb des Unternehmens. So weisen Ausbildungsabsolventen von groBen Unternehmen neben betriebsspezifischem auch allgemeines Humankapital auf. Insgesamt ergibt sich daraus ein hohes Bildungsniveau. Daher weisen technische und kaufmännische Berufe einen höheren Anteil an betriebs- bzw. technologiespezifischem Wissen auf, weil die Auszubildenden meist einen Überblick über die Produktionsprozesse erhalten, die zwischen den Unternehmen differieren. Diese Humankapitalkomponente verfällt dann bei einem Wechsel.

Daraus folgt, dass hohe Ausbildungskosten negativ mit dem Arbeitslosigkeitsrisiko korrelieren, da die Unternehmen einerseits die Ausbildungsabsolventen weiterbeschäftigen werden. Andererseits finden junge ausgebildete Fachkräfte, die nicht im Ausbildungsbetrieb weiterbeschäftigt werden, aufgrund ihrer guten Ausbildung eine stabile Beschäftigung. Ebenso ist davon auszugehen, dass Betriebe, die über einen hohen technologischen Stand verfügen, auch gut qualifizierte Fachkräfte beschäftigen. Demzufolge ist die Ausbildung in derartigen Ausbildungsbetrieben innerhalb der jeweiligen Branche überdurchschnittlich, was ebenfalls die Wahrscheinlichkeit für einen Betriebswechsel und das

sen. Der Anteil an Auszubildenden ist in dieser Branche relativ gering, da es sich meist um junge Unternehmen ohne Ausbildungstradition handelt, die entweder über keine Ausbildungsberechtigung oder nicht über die entsprechenden Ressourcen verfügen. Diese Unternehmen fragen außerdem häufig Arbeitskräfte mit Hochschulabschluss nach (Falk 2002). Neuere Studien ergaben jedoch, dass Unternehmen in diesen Branchen Ausbildung vor der Rekrutierung bevorzugen, weil selbst ausgebildete Fachkräfte flexibler einsetzbar sind (Steedman et al. 2006; Steedman/Wagner 2007). Dies führt auch bei Unternehmen, die in aufstrebenden Industriezweigen agieren, dazu, dass deren Ausbildungsabsolventen über eine hohe Qualifikation verfügen. 


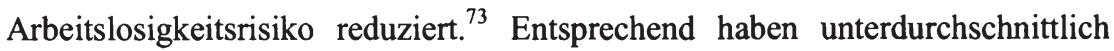
ausgebildete Ausbildungsabsolventen eine hohe Wahrscheinlichkeit, den Betrieb zu verlassen und sind zudem eher von Arbeitslosigkeit betroffen. Aufgrund des hohen Bildungsniveaus stellen die Ausbildungsabsolventen, welche den Ausbildungsbetrieb verlassen, aber hohe Ansprüche an die zukünftige Beschäftigung. Zudem sind Informationsasymmetrien vorhanden, weil die Ausbildung über die Vermittlung der Lehrinhalte, die in den Ausbildungsordnungen verankert sind, hinausgeht. Dies verlängert die Arbeitslosigkeitsdauer für Ausbildungsabsolventen in diesen Bereichen.

Aber auch aus Sicht der Auszubildenden scheinen Unternehmen in Ausbildungsbereichen der Finanzdienstleistungen, Verwaltungsberufe sowie produktionsnahen Dienstleistungen attraktiver zu sein als Unternehmen in Bereichen des Handwerks bzw. des Handels, sodass Ausbildungsabsolventen eher im Ausbildungsbetrieb verbleiben. Hierbei wird davon ausgegangen, dass insbesondere die Betriebsgröße die Entscheidung des Ausbildungsabsolventen selbst beeinflusst, ob er im Ausbildungsbetrieb verbleibt oder nicht. Euwals und Winkelmann (2002) untersuchen diesen Aspekt in ihrer Studie. Danach verbleiben über die Hälfte der übernommenen Absolventen in ihrem Ausbildungsbetrieb. Allerdings schwanken die Raten nach Unternehmensgröße. So übernehmen größere Ausbildungsbetriebe tendenziell mehr ihrer ehemaligen Auszubildenden. Die höchste Verbleibsrate weisen Unternehmen über 1000 Beschäftigte auf (Euwals/Winkelmann 2002: 257). In den letzten Jahren finden die meisten Übernahmen jedoch in Betrieben zwischen 10 und 49 Beschäftigten statt (u. a. BMBF 2008). Franz und Zimmermann (2001) ermitteln hierbei einen signifikanten Einfluss. ${ }^{74}$ Begründet werden kann die höhere Verbleibsquote in einem gröBeren Betrieb durch die besseren Karrierechancen und durch die besseren Möglichkeiten, sich beruflich weiterzuentwickeln. Ausbildungsabsolventen in einem großen Ausbildungsunternehmen treten am seltensten in Arbeitslosigkeit ein und sind auch am seltensten nach einer Arbeitslosigkeitsphase von Berufswechseln betroffen (Konietzka 2002). Dies kann wiederum auch durch den Zusammenhang zwischen Ausbildungskosten und der Betriebsgröße erklärt werden.

\subsubsection{Wirtschaftliche und demografische Einflussgrößen}

Neben betrieblichen und individuellen Faktoren bestimmen makroökonomische Größen, wie die konjunkturelle Situation, die demografischen Rahmenbedingungen sowie der regionale Arbeitsmarkt, den Verbleib im Ausbildungsbetrieb bzw. das Arbeitslosigkeitsrisiko nach der dualen Berufsausbildung. Demografi-

\footnotetext{
${ }^{73}$ Das Arbeitslosigkeitsrisiko hängt nach von Wachter/Bender (2004) auch von der Fluktuationsrate eines Betriebes ab. Ist diese hoch, erhöht sich auch das Arbeitslosigkeitsrisiko.

${ }^{74}$ Die Situation der Ausbildungsabsolventen aus kleinen Betrieben war nicht immer derart eindeutig. So haben sich die Beschäftigungsaussichten für Ausbildungsabsolventen in kleinen Betrieben aus langfristiger Sicht sogar verschlechtert.
} 
sche Rahmenbedingungen drücken sich vor allem in der Stärke der verschiedenen Jahrgänge aus. Studien über die Erwerbsmuster an den Übergängen (Mroz/Savage 2006; Dietrich/Kleinert 2005; Hillmert/Mayer 2004; Büchel 2002; Hillmert 2001; Franz/Zimmermann 2001; Konietzka/Seibert 2001; Neubäumer 1999) wurden insbesondere mit Hilfe eines Vergleichs am Beispiel der Geburtskohorten von 1964 und 1971 durchgeführt. Diese eignen sich besonders für die Untersuchung des Kohorteneffekts und die Wirkungen der wirtschaftlichen Rahmenbedingungen beim Übergang an der zweiten Schwelle (Dietrich 2001b; Hillmert 2001). Danach haben die Ausbildungskohorten, die einem strikteren Ausleseprozess an der ersten Schwelle unterzogen werden, bspw. weil der Jahrgang stärker besetzt ist, bessere Beschäftigungsaussichten an der zweiten Schwelle als andere Kohorten mit einem weniger strikten Auswahlverfahren bei der Vergabe von Ausbildungsplätzen (Hillmert 2001: 11f.). Dies ist deswegen der Fall, da sich unter den strikteren Bedingungen die besseren durchsetzen, weil das Stellenangebot geringer und die Konkurrenz entsprechend größer ist. Die Ausbildung in einem Bereich mit guten Beschäftigungsaussichten ist daher in stärkeren Jahrgängen vorrangig den besseren Schulabsolventen vorbehalten. Die Wettbewerbsposition von schlechteren Schulabsolventen verbessert sich in konjunkturellen Aufschwungphasen oder in schwach besetzten Jahrgängen. Nach Beendigung der dualen Berufsausbildung haben aber junge Erwachsene mit Hauptschulabschluss, die denselben Ausbildungsberuf erlernen wie Abiturienten, eine höhere Wahrscheinlichkeit, keinen erfolgreichen Übergang zu schaffen. Insgesamt implizieren die Studien damit aber einen Trade-off der Selektion zwischen erster und zweiter Schwelle. Dieser ist dadurch gekennzeichnet, dass junge Erwachsene, die unter wirtschaftlich schwierigen Rahmenbedingungen eine betriebliche Ausbildung beginnen, weniger Hindernisse am Übergang ins Erwerbsleben aufweisen bzw. umgekehrt, Personen, die gute Voraussetzungen bei der Aufnahme einer Lehrstelle haben, dann weniger erfolgreich ins Erwerbsleben starten. Die Art des Einstiegs setzt sich für den weiteren Berufsverlauf fort. Junge Erwachsene, die bereits an der ersten Schwelle Schwierigkeiten mit dem Arbeitseinstieg haben, weisen auch im weiteren Erwerbsleben wahrscheinlicher diskontinuierliche Erwerbsverläufe auf (Hillmert 2001; Lauterbach/Sacher 2001). Diese Zusammenhänge bedeuten, dass das Arbeitslosigkeitsrisiko mit der Kohortengröße steigt (Hillmert 2001). Unter guten Voraussetzungen hinsichtlich der demografischen Bedingungen aufgrund einer relativ schwachen Besetzung ihrer Jahrgänge traten insbesondere Jugendliche nach 2000 ins Erwerbsleben ein. Zugleich relativierte ein schlechtes Verhältnis zwischen Ausbildungsstellenangebot und -nachfrage, das eine stärke Selektion zu Beginn der Ausbildung hervorrief, diese Vorteile (siehe Tabelle 14 im Anhang). Danach wurden die positiv wirkenden demografischen Effekte von den gesamtwirtschaftlichen Effekten überlagert, was wiederum einen reibungslosen Übergang von der dualen Berufsausbildung in das Erwerbsleben förderte. 
Verschiedene Studien (u. a. McGinnity et al. 2005; Konietzka 2002) ergaben ebenfalls, dass ein erfolgreicher Eintritt in das Erwerbsleben vom Jahr, in dem der Berufsabschluss erfolgt und somit von der gesamtwirtschaftlichen Situation, signifikant beeinflusst wird. Der signifikante Einfluss der gesamtwirtschaftlichen Situation wird bei der Betrachtung der Kohorte von 1985 und 1995 ebenfalls deutlich. Die Abschlusskohorte von 1995 hatte einen wesentlich längeren Einstieg bis zur ersten regulären Beschäftigung zu verzeichnen als die Abschlusskohorten von 1985 und 1990 (Falk et al. 2000: 15). Auch Konietzka (2002) stellt fest, dass gerade ein Bildungsabschluss in den Jahren 1994 und 1995 vermehrt zu Arbeitslosigkeit bzw. zu einem Betriebswechsel führt. Wirtschaftlich schlechte Jahre erhöhen demnach die Wahrscheinlichkeit, arbeitslos zu werden. Hierbei entwickelt sich die Arbeitslosigkeit nach Abschluss der Berufsausbildung zyklisch zu der Gesamtarbeitslosigkeit. Die starke Abhängigkeit der Weiterbeschäftigung von Ausbildungsabsolventen ist auf das Auslaufen der Arbeitsverträge mit dem Ende der Ausbildung zurückzuführen (siehe auch Kap. 4). Konietzka und Seibert (2001) ziehen die Arbeitslosenrate als gesamtwirtschaftlichen Indikator heran, die auch die Arbeitslosigkeit der Ausbildungsabsolventen positiv beeinflusst. Daraus resultiert, dass unfreiwillige Betriebswechsel in wirtschaftlich schlechteren Rahmenbedingungen zunehmen, wohingegen die freiwilligen Betriebswechsel abnehmen. Ausbildungsabsolventen, die unter anderen Bedingungen wechseln würden, ziehen dann die Beschäftigung und damit die Sicherheit innerhalb des Ausbildungsbetriebes vor.

Jedoch beeinflusst die wirtschaftliche Situation die Zusammensetzung von freiwilligen und unfreiwilligen Wechseln und folglich auch die Qualität des Erwerbseinstiegs. Eine schlechte wirtschaftliche Ausgangslage erhöht den Anteil an unfreiwilligen Wechseln, wohingegen eine wirtschaftlich gute Situation Anreize für freiwillige Wechsel setzt. Beide Aspekte implizieren eine kurze Beschäftigungsdauer nach einem Betriebswechsel. Ausbildungsabsolventen, die den Betrieb verlassen (mussten), verweilen in ihrem neuen Betrieb kürzer als Ausbildungsabsolventen, die nicht wechseln. Bei unfreiwilligen Wechseln deutet dies einerseits auf ein erhöhtes Arbeitslosigkeitsrisiko und eine längere Verweildauer in Arbeitslosigkeit hin. Andererseits, sofern der Wechsel freiwilliger Natur ist, bedeutet dies vielmehr ein Job-Shopping bzw. Job-Hopping (Jovanovic 1979) im Sinne einer Verbesserung der Erwerbsposition. Einen erfolgreichen Start in das Erwerbsleben verzeichnen insbesondere Ausbildungsabsolventen, die nach der dualen Berufsausbildung im Ausbildungsbetrieb verbleiben. Sie besetzen zudem am häufigsten einen qualifikationsadäquaten Arbeitsplatz als Facharbeiter. Bereits ein Betriebswechsel kann zu einem Einstieg unterhalb einer Facharbeiterposition führen. Die Wahrscheinlichkeit nimmt zu, wenn sich der Betriebswechsel zusammen mit einem Berufswechsel vollzieht (Konietzka 2002: 665f.). Jedoch ergaben andere Studien (von Wachter/Bender 2004; Schwerdt/Bender 2003; Dustmann et al. 1997), dass Ausbildungsabsolventen, welche freiwillig den Betrieb wechseln, höhere Löhne realisieren können als 
Ausbildungsabsolventen, die im Ausbildungsbetrieb verbleiben. Dagegen beeinflusst ein unfreiwilliger Wechsel, dem eine Arbeitslosigkeitsphase folgt, signifikant das weitere Erwerbsleben bzw. den Eintritt in den Arbeitsmarkt. Diese Komponenten, welche meist ein niedriges Einkommen bei Wiederaufnahme einer Beschäftigung nach sich ziehen, spielen aber langfristig keine Rolle mehr, weil sich die Löhne angleichen (Manning 2003; Farber 1993; Blanchflower/Oswald 1994; Topel/Ward 1992; Burdett 1978). Bereits eine Arbeitslosigkeitsperiode vor der dualen Berufsausbildung, die von jungen Erwachsenen mit niedrigem Bildungsstand durchlaufen wird, beeinflusst nach Mroz und Savage (2006), den Erwerbsverlauf langfristig. So erhalten die Jugendlichen einige Zeit nach der Arbeitslosigkeitsperiode immer noch einen geringeren Lohn als Jugendliche ohne Arbeitslosigkeitserfahrung (Mroz/Savage 2006: 290).

Gesamtwirtschaftliche Faktoren können zudem zwischen den regionalen Arbeitsmärkten divergieren. Ausbildungsabsolventen in strukturschwachen Gebieten sind daher häufiger mit einem unfreiwilligen Betriebswechsel mit anschlieBender Arbeitslosigkeit konfrontiert als Ausbildungsabsolventen in strukturstarken Gebieten. Von ersteren wird daher auch eine hohe geografische Mobilität verlangt, um die Wahrscheinlichkeit einer (lang andauernden) Arbeitslosigkeit zu verringern (Haas 2002). Jedoch sind Ausbildungsabsolventen mit unterschiedlichem Bildungsniveau nicht in gleichem Maße von konjunkturellen Entwicklungen betroffen. Untersuchungen der Zusammenhänge von gesamtwirtschaftlicher Arbeitslosigkeit mit dem Bildungsniveau ergaben zwar negative Effekte für alle Absolventengruppen, jedoch verstärkte sich die Wirkung bei sinkendem Bildungsabschluss (Gangl 2003: 85).

Des Weiteren können sich die Effekte der gesamtwirtschaftlichen und demografischen Faktoren auf den Übergang an der zweiten Schwelle verstärken, aber auch aufheben. Bspw. ergaben Studien, dass im Jahr 1986/87 und Anfang der 1990er gesamtwirtschaftliche Faktoren die Wirkungen der demografischen Faktoren überlagerten. Mitte der $1980 \mathrm{er}$ beendete bspw. ein starker Jahrgang die Ausbildung, wies aber im Verhältnis eine geringe Arbeitslosigkeit auf. Dies war vor allem auf die guten wirtschaftlichen Rahmenbedingungen zurückzuführen (Bellmann/Bender 1997). Allerdings kann auch die Kohortenstärke selbst gesamtwirtschaftliche Größen beeinflussen und die Wirkungskette somit in die entgegen gesetzte Richtung führen. Diesen Zusammenhang hat Shimer (1998) untersucht, der in einer Studie zwischen zwei Effekten, die durch den Zustrom einer stärkeren Kohorte entstehen, unterscheidet. Der direkte Effekt wirkt sich auf die gesamtwirtschaftliche Arbeitslosenquote aus, während der indirekte Effekt die alterspezifischen Arbeitslosenquoten beeinflusst. Dieser Effekt ist hierbei auf die Alterskohorte der zwischen 15- und 24-jährigen, und demnach auf die Alterskohorte, der die Ausbildungsabsolventen der Regel angehören, am stärksten (Shimer 2001: 1002). Dieser Kohorteneffekt beeinflusst die Arbeitslosigkeit nach der dualen Berufsausbildung negativ (Hillmert 2001). Allerdings ergaben Untersuchungen von Hillmert (2001) weniger signifikante Einflüsse der 
wirtschaftlichen Situation als vielmehr die Signifikanz der individuellen Einflussfaktoren. Dagegen traf zu Beginn der 1990er Jahre ein schwacher Jahrgang auf eine schlechte wirtschaftliche Ausgangslage, die hohe Arbeitslosigkeit mit sich brachte (Konietzka/Seibert 2001: 77). 1995 betrug diese 15\% unter den Ausbildungsabsolventen (Konietzka/Seibert 2001: 85f.). Hierbei wird die theoretische Annahme bestätigt, dass der Übergang von der dualen Berufsausbildung in Arbeitslosigkeit mit steigender gesamtwirtschaftlicher Arbeitslosigkeit zunimmt.

Darüber hinaus beeinflusst die wirtschaftliche Situation nicht nur den Eintritt in das Erwerbsleben bzw. Arbeitslosigkeit, sondern auch die Verweildauer in Arbeitslosigkeit positiv. Dabei unterscheidet sich die Dauer der Arbeitslosigkeit nach Abschluss einer Berufsausbildung für Ost- und Westdeutschland. Während die Mehrheit der von Arbeitslosigkeit betroffenen Personengruppe in Ostdeutschland mittelfristiger Natur (zwischen 6 und 12 Monate) ist, weisen Ausbildungsabsolventen in Westdeutschland eher eine Arbeitslosigkeitsdauer von kurzfristiger Natur (unter drei Monate) auf. Wenn die wirtschaftliche Situation positiv verläuft, finden die nicht übernommenen Ausbildungsabsolventen schneller eine Beschäftigung als Ausbildungsabsolventen in einer schlechten wirtschaftlichen Situation (vgl. hierzu Caliendo 2006: 147). Dabei wird der gesamtwirtschaftliche Effekt durch die Bildungskomponente verstärkt. Vor allem Abiturienten mit dualer Berufsausbildung sind bei hoher gesamtwirtschaftlicher Arbeitslosigkeit vor einem Eintritt in Arbeitslosigkeit geschützt. Verbessert sich die wirtschaftliche Situation, werden diese mit hoher Wahrscheinlichkeit auch wieder eingestellt (Hillmert 2001: 22), das heißt, dass Abiturienten unter guten wirtschaftlichen Voraussetzungen seltener ein Studium beginnen werden. ${ }^{75}$ Bspw. hatte die Geburtskohorte 1964 relativ schlechte Chancen, einen guten Einstieg in das Erwerbsleben zu realisieren, da die betrachtete Kohorte relativ groß war und die aktuellen Arbeitsmarktbedingungen bei Ausbildungsabschluss unvorteilhaft waren. Jedoch verlief der Übergang von der Ausbildung in das Erwerbsleben für Personen mit Abitur günstig.

Auch die Art der Humankapitalbildung wird durch die wirtschaftlichen Rahmenbedingungen determiniert. Um die Wirkungen erklären zu können, sind jedoch mehr die langfristigen als die kurzfristigen Voraussetzungen zu berücksichtigen. Hierbei ist die Ebene der Ausbildungsbereiche zu betrachten. Bereiche, die auf Dauer starken wirtschaftlichen Schwankungen unterworfen sind, wie bspw. das Handwerk oder der Handel, reagieren darauf entsprechend mit Änderungen der Arbeitsnachfrage nach Fachkräften. So werden Betriebe, welche stark von der Konjunktur abhängig sind, weniger in Humankapital investie-

\footnotetext{
${ }^{75}$ Nach Behrens et al. (2008) besteht jedoch eine große Unsicherheit hinsichtlich des Übergangs von Abiturienten an der zweiten Schwelle. Die Entscheidung, ob ein Studium nach der dualen Berufsausbildung aufgenommen wird oder nicht hängt dabei von sozialen Faktoren, wie dem Einfluss der Eltern, dem generellen sozialen Status sowie von den Zukunftsplänen ab.
} 
ren. Häufige Betriebswechsel sind die Folge. Entsprechend muss diese Absolventengruppe meist un- bzw. angelernte Tätigkeiten in Sparten ausüben, die wiederum gegenüber Nachfrageschwankungen relativ instabil sind. Deshalb ist das Arbeitslosigkeitsrisiko (im weiteren Erwerbsverlauf) zwar hoch, aber die Arbeitslosigkeitsdauer wird im Verhältnis kurz sein, da die Stellenbesetzung keinen großen Screening-Prozess erfordert. Dagegen haben Betriebe, die flexibel auf wirtschaftliche Veränderungen reagieren können, mehr Anreiz, höhere finanzielle Aufwendungen für die Ausbildung zu betreiben (Sengenberger 1987; Doeringer/Piore 1975; Neubäumer 1999). So werden Betriebe dieser Ausbildungsarten auch gerade wegen der hohen Humankapitalinvestitionen und infolgedessen wegen des hohen Humankapitalniveaus flexibler reagieren können. Ausbildungsabsolventen aus diesen Bereichen sind dadurch innerhalb der Betriebe gegenüber neuen Aufgaben anpassungsfähig. Im Falle einer Lösung des Vertrages verlängert die recht betriebsspezifische Ausbildung die Arbeitslosigkeit, weil die gut ausgebildeten Absolventen auch produktive Arbeitsplätze besetzen sollen. Für deren Besetzungen werden aufwendige und kostenintensive Einstellungsverfahren praktiziert, die zudem zeitintensiv sind.

Zusammenfassend ist festzustellen, dass Ausbildungsabsolventen von den demografischen Entwicklungen unterschiedlich betroffen sind. Starke Absolventenjahrgänge weisen eine höhere Wahrscheinlichkeit auf, nach der Ausbildung arbeitslos zu werden als schwächere Jahrgänge. Ebenso verlängert sich die Verbleibdauer in Arbeitslosigkeit. Schließlich beeinflussen Ausbildungsbereiche, die starken wirtschaftlichen Schwankungen ausgeliefert sind, den Übergang der Ausbildungsabsolventen unterschiedlich stark. Ausbildungsabsolventen in Branchen, in denen nach der dualen Berufsausbildung häufige Betriebswechsel bzw. Phasen von Arbeitslosigkeit folgen, sehen sich einem höheren Risiko für das Verlassen des Ausbildungsbetriebs gegenübergestellt, wenn der Abschluss in einem wirtschaftlich schlechteren Jahr erfolgt. Dagegen verringert sich der Einfluss der Ausbildungsbereiche, die insgesamt bessere Beschäftigungsaussichten im Ausbildungsbetrieb aufweisen. Auch für die Bildungsvariable ist anzunehmen, dass die Effekte sich in weniger vorteilhaften Jahren für Ausbildungsabsolventen mit niedrigem Humankapital weiter ungünstig entwickeln, während dieser negative Einfluss in besseren Jahren geringer wird. Da diese Aspekte immer auch gleichzeitig wirken, können sich die Wirkungen gegenseitig verstärken oder abschwächen.

\section{2 Überblick über die Wirkungszusammenhänge}

Einflussfaktoren für einen weniger erfolgreichen Eintritt in das Erwerbsleben liegen neben der individuellen Ebene innerhalb des dualen Berufsausbildungssystems vor allem auf der betrieblichen Ebene, welche sich wiederum auf die Humankapitalarten und -höhe auswirken. Durch den Wirkungskanal des Humankapitals können die Ursachen für die Arbeitslosigkeit identifiziert werden und infolgedessen ebenfalls die Bestimmungsgründe für die Arbeitslosigkeits- 
dauer. Im Folgenden werden die Wirkungsrichtungen der einzelnen Einflussfaktoren auf die verschiedenen Übergänge nach der dualen Berufsausbildung in der untenstehenden Tabelle zusammengefasst. Die angegebenen Vorzeichen der Einflüsse sind aus den theoretischen Ansätzen und aus bisherigen Studien abgeleitet worden, sodass sie als Ergebnisse der empirischen Untersuchungen erwartet werden. Die Einflüsse sind in Relation zu den anderen Ausbildungsbereichen bzw. Einflussfaktoren zu sehen. Des Weiteren bezieht sich das Arbeitslosigkeitsrisiko auf alle Absolventen innerhalb eines Ausbildungsbereiches, während die Arbeitslosigkeitsdauer im Verhältnis innerhalb der Gruppe der arbeitslosen Absolventen zu verstehen ist. Auch die Richtungen der Einflussfaktoren auf diese beiden zu erklärenden Aspekte müssen im Vergleich zu den anderen Ausbildungsbereichen gesehen werden.

Tabelle 4 verdeutlicht die Faktoren, mit denen das Humankapital empirisch umgesetzt wird. So stehen die Ausbildungsbereiche für berufsspezifische, die betrieblichen Rahmenbedingungen für die betriebsspezifische und die Schulbildung für die allgemeine Humankapitalkomponente (vgl. auch Kriechel/Pfann 2005). Es ist festzustellen, dass vor allem in den Ausbildungsbereichen mit einfachen Tätigkeiten das Verlassen des Betriebes am höchsten zu sein scheint, während die kaufmännischen und technischen Ausbildungsbereiche gute Beschäftigungsaussichten innerhalb des Ausbildungsbetriebes bieten. Diese unterschiedlichen Einflüsse verdeutlichen sich auch bei der Betrachtung der Wahrscheinlichkeit, arbeitslos zu werden. Hier weisen sie ein hohes Arbeitslosigkeitsrisiko auf. Die Arbeitslosigkeitsdauer liegt aber bei Ausbildungsabsolventen mit einem Berufsbildungsabschluss in den Bereichen mit den höheren Qualifikationsanforderungen gegenüber den Personen mit einer Ausbildung im handwerklichen bzw. einfachen Dienstleistungsberufe höher. Mit dem Bildungsstand, welcher durch die Schulbildung und das betriebliche Qualifikationsniveau entsteht, reduziert sich das Risiko, den Betrieb wechseln zu müssen, und infolgedessen auch die Eintrittswahrscheinlichkeit in Arbeitslosigkeit. Konjunkturelle Schwankungen wirken sich dagegen negativ auf die Bildung von Humankapital aus und machen einen Betriebswechsel wahrscheinlicher. Die Arbeitslosigkeit tritt in der Regel häufiger und länger ein.

Diese Zusammenhänge dienen als Grundlage zur Verdeutlichung der empirischen Evidenz für die Gründe der Arbeitslosigkeit nach der dualen Berufsausbildung und deren Auswirkungen auf die Arbeitslosigkeitsdauer. Damit soll die Forschungslücke geschlossen werden, die insbesondere noch hinsichtlich der Ursachenforschung der verschiedenen Übergänge aus wirtschaftswissenschaftlicher Perspektive bestehen. Denn bisherige Studien, so wurde in den vorangehenden Kapiteln deutlich, betrachten die Übergänge vor allem vor dem Hintergrund soziologischer Fragestellungen, wie die „Erosion der Übergänge von der dualen Berufsausbildung ins Erwerbsleben“ (Konietzka/Seibert 2001: 87). In diesem Zusammenhang wird zumeist die Qualität der Übergänge von der Ar- 
Tabelle 4: Übersicht über Wirkungszusammenhänge zwischen Einflussfaktoren auf das Humankapital und dem Verbleib nach der Ausbildung

\begin{tabular}{|c|c|c|c|c|c|c|}
\hline & $\begin{array}{c}\text { allgemeines/be- } \\
\text { rufsspezifisches } \mathrm{Hu} \text { - } \\
\text { mankapital }\end{array}$ & $\begin{array}{c}\text { betriebs- } \\
\text { spezifisches } \mathrm{Hu}- \\
\text { mankapital }\end{array}$ & Bildungsstand & $\begin{array}{l}\text { Übernahme- } \\
\text { wahrscheinlich- } \\
\text { keit }\end{array}$ & $\begin{array}{l}\text { Arbeitslosig- } \\
\text { keitsrisiko }\end{array}$ & $\begin{array}{c}\text { Arbeitslosig- } \\
\text { keitsdauer }\end{array}$ \\
\hline $\begin{array}{l}\text { handwerkliche/landwirtschaftliche } \\
\text { Ausbildungsberufe }\end{array}$ & + & - & - & - & + & - \\
\hline manuelle Industrieberufe & ++ & + & - & - & + & - \\
\hline technische Industrieberufe & + & + & + & + & - & + \\
\hline kaufmännische Berufe & ++ & + & + & + & - & + \\
\hline einfache Dienstleistungsberufe & + & - & - & - & + & - \\
\hline Ausbildungskosten & + & + & + & + & - & + \\
\hline Betriebsgröße & + & + & + & + & - & + \\
\hline Qualifikationsniveau & + & ++ & + & + & - & + \\
\hline Schulbildung & + & ++ & + & + & - & + \\
\hline Qualifikationsniveau & + & + & + & + & - & - \\
\hline \multicolumn{7}{|l|}{ makroökonomische Größen } \\
\hline Abhängigkeit von Konjunktur & + & - & - & + & - & - \\
\hline Stärke der Abschlusskohorte & 1 & I & I & - & + & + \\
\hline
\end{tabular}

Quelle: eigene Darstellung 
beitslosigkeit in die Erwerbstätigkeit, in eine weitere Ausbildung oder in eine arbeitsmarktpolitische Bildungsmaßnahme etc. untersucht. So werden die Folgewirkungen des nicht erfolgreichen Eintritts in das Erwerbsleben auf den weiteren Erwerbverlauf ermittelt, der in diesem Fall zunehmend durch Arbeitslosigkeitsphasen und atypische Beschäftigungsverhältnisse für jüngere $\mathrm{Be}$ rufseinsteiger gekennzeichnet ist (Buchholz 2008; Bukodi et al. 2006; Dietrich 2001). In Kapitel 8 wird daher gegenüber den bisherigen Studien an der vorangehenden Stufe, nämlich dem Eintritt in die Arbeitslosigkeit nach der dualen Berufsausbildung, angesetzt. Deshalb werden die Wirkungen der betrieblichen Rahmenbedingungen im Zusammenhang mit den individuellen Faktoren auf diesen Teil des Übergangs untersucht, um die Ursachenbekämpfung der Arbeitslosigkeit erfolgreich gestalten zu können. Die abgeleiteten Zusammenhänge zwischen den Merkmalen, die erfasst werden können, und der Art und Höhe des Humankapitals sind für die Überprüfung der Hypothesen vor dem Hintergrund der Datengrundlage notwendig.

\subsection{Beschreibung der Datengrundlage}

Die empirischen Schätzungen werden auf Grundlage der schwach anonymisierten Version der IAB-Beschäftigten-Stichprobe 1975-2004 (im Folgenden: IABS 1975-2004) durchgeführt. ${ }^{76}$ Es handelt sich um Prozessdaten der Bundesagentur für Arbeit. Der Datensatz enthält Informationen aus der Beschäftigtenund Leistungsempfängerhistorik, sodass genaue Kenntnisse über den Erwerbsverlauf und eventuelle Unterbrechungen durch Arbeitslosigkeit erfasst werden. Sie sind in der Regel lückenlos, ${ }^{77}$ da zum einen Arbeitgeber im Rahmen des Meldeverfahrens zur Sozialversicherung Beschäftigungsinformationen über die Arbeitnehmer mitteilen müssen. Im Zuge dessen werden Beschäftigungsinformationen, mit denen die Datenbasis der Beschäftigtenhistorik gespeist wird, über die Krankenversichertennummer an die Agentur für Arbeit weitergegeben. Zum anderen werden diese Beschäftigungsinformationen um Verwaltungsdaten der Bundesagentur für Arbeit mit Kenntnissen über den Leistungsbezug aus der Arbeitslosenversicherung ergänzt. Mit der Umstellung des Meldeverfahrens zum 1. Januar 1999 werden ebenfalls Daten über geringfügig Beschäftigte erhoben. Insgesamt beinhaltet die Beschäftigtenstichprobe tagesgenaue Angaben von den Arbeitnehmern und erfasst somit die Erwerbsverläufe, aber auch Ar-

\footnotetext{
${ }^{76}$ Der Datenzugang erfolgte über einen Gastaufenthalt am Forschungsdatenzentrum der Bundesagentur für Arbeit im Institut für Arbeitsmarkt- und Berufsforschung (FDZ) und anschließend mittels kontrollierter Datenfernverarbeitung beim FDZ.

${ }^{77}$ Lücken im Datensatz entstehen nur dann, wenn die Beschäftigten nicht mehr sozialversicherungspflichtig sind, weil sie ein Studium aufgenommen haben. Darüber hinaus sind Personen dann nicht gemeldet, wenn sie als erwerbslose Personen nicht bei der Bundesagentur als arbeitslos registriert sind oder wenn sie sich in Arbeitsbeschaffungsmaßnahmen befinden.
} 
beitslosigkeitsphasen bzw. Phasen atypischer Beschäftigungen ${ }^{78}$. Sie bildet verschiedene Übergänge im Erwerbsverlauf ab, insbesondere auch den Übergang an der zweiten Schwelle. Um dies technisch abbilden zu können, enthält der Datensatz so genannte Spells: Pro Veränderung im Erwerbsstatus wird eine neue Zeile für die entsprechende Person gebildet. Erfolgen innerhalb eines Jahres keine Änderungen im Erwerbsstatus einer Person, werden dennoch zu Beginn und am Ende eines Jahres so genannte Jahresmeldungen abgegeben. Damit liegen in der IABS 1975-2004 über 21 Millionen Spells bzw. Datenzeilen vor, welche sich auf knapp 1,3 Millionen sozialversicherungspflichtig Beschäftigte verteilen. Davon sind ungefähr 1106691 Personen dem Erhebungsgebiet der alten Bundesländer zuzuordnen und 187128 Personen dem Erhebungsgebiet der neuen Bundesländer. Dies entspricht 2\% der Gesamtheit aller Arbeitnehmer, die innerhalb des erhobenen Zeitraumes mindestens einmal sozialversicherungspflichtig beschäftigt waren. Da die Grundlage eines Ausbildungsverhältnisses ein Vertrag zwischen Betrieb und Auszubildenden selbst darstellt, sind die Ausbildungsbetriebe dazu verpflichtet, Sozialversicherungsbeiträge zu leisten. Dadurch werden die Auszubildenden ebenfalls als sozialversicherungspflichtige Beschäftigte erfasst, wodurch Kenntnisse während der betrieblichen Ausbildung existieren. Eine weitere Variable in der Datenbasis enthält Informationen über die genaue Stellung innerhalb eines Berufes. Mit dieser Auskunft können Auszubildende von anderen Beschäftigten unterschieden werden (siehe auch Schimpl-Neimanns 2006; Drews et al. 2006).

In der IABS 1975-2004 sind die Berufe nicht in Dimensionen der Ausbildungsberufe erfasst, sondern als Erwerbsberufe. Dies ist nicht dieselbe Schnittmenge, da nicht alle Erwerbsberufe staatlich anerkannte Ausbildungsberufe darstellen. Darunter fallen bspw. Steinbrecher, Schweißer etc., die lediglich als Erwerbsberufe aufgezählt werden. Darüber hinaus gibt es auch Erwerbsberufe, die einem Ausbildungsberuf entsprechen, dieser zwar staatlich anerkannt ist, sich aber nicht dem System der dualen Berufsausbildung zuordnen lässt. Als Beispiel ist der Beruf der/des Krankenschwester/-pflegers zu nennen. Daher müssen zunächst die ausgeübten Tätigkeiten identifiziert werden, die einem innerhalb des dualen Berufsausbildungssystems anerkannten Ausbildungsberuf entsprechen. Aufgrund der Vielzahl an Ausbildungsberufen wird eine Zusammenfassung notwendig. Diese erfolgt anhand einer Systematik, die ähnliche Berufe zusammenschließt. Die Zusammenfassung erfolgt nach Parmentier et al. (1993) und Erlinghagen (2003), die sich in Untergruppen der produzierenden sowie der dienstleistenden Tätigkeit aufteilt. Die Zuordnung

\footnotetext{
${ }^{78}$ Die Stichprobe wird durch das Verfahren der geschichteten Zufallstichprobe gezogen. Die vier aus der Schichtenbildung resultierenden „Untergruppen“ sind nach der Staatsanghörigkeit (Deutsch/Ausländer) und nach West- und Ostdeutschland proportional geordnet (Schimpl-Neimanns 2006: 6).
} 
erfolgt nach der Häufigkeit der jeweiligen Tätigkeitsmerkmale. ${ }^{79}$ Die Untergruppen der Tätigkeitsbereiche zeichnen sich durch die Anwendung bzw. Bearbeitung eines gemeinsamen Materials aus, welche die Berufsbezeichnung selbst widerspiegelt (Parmentier et al. 1993: 103). Die Untergruppen der Dienstleistungstätigkeiten lassen sich durch eine Nachfrageorientierung und die Art der Dienstleistung bestimmen. ${ }^{80}$ Die Einteilung der Ausbildungsberufe zu den entsprechenden Tätigkeitsbereichen befindet sich in Tabelle 13 im Anhang.

Des Weiteren werden nur Auszubildende berücksichtigt, die in Westdeutschland ihre Ausbildung zwischen 1975-2004 absolviert haben. Der Fokus liegt auf Westdeutschland, da in Ostdeutschland die duale Berufsausbildung zur Zeiten der DDR wenig Tradition hatte. Diese Situation hat sich nach der Wiedervereinigung zwar angepasst, das Angebot an Ausbildungsplätzen ist aber immer noch relativ gering (Fitzenberger/Spitz 2004; Beckmann 2002b).

Da der Schwerpunkt der Arbeit in der Ursachenforschung der Arbeitslosigkeit nach der ersten abgeschlossenen dualen Berufsausbildung liegt, werden junge Erwachsene im Alter von 16 bis 24 Jahren untersucht, die innerhalb des Betrachtungszeitraumes die duale Berufsausbildung abgeschlossen haben. Einerseits werden diejenigen berücksichtigt, die unmittelbar nach dem Hauptschulabschluss einen Ausbildungsplatz finden und diesen auch regulär abschließen. Dies erfolgt im Normalfall mit 18 Jahren. Andererseits wird Jugendarbeitslosigkeit bis zu einem Alter von 25 Jahren definiert, sodass die Absolventen bei der Betrachtung bis zu einem Jahr nach Abschluss der dualen Berufsausbildung unter diese Definition fallen. Es wird nur die erste Ausbildung betrachtet, die für den Großteil der Auszubildenden mit einem Alter von 24 Jahren abgeschlossen ist, wenn man davon ausgeht, dass das Durchschnittalter der Ausbildungsanfänger ungefähr 19 Jahre beträgt (BMBF 2007: 108). ${ }^{81}$ Die Einschränkung der betrachteten Altersgruppen ergibt sich zudem aus der Berücksichtigung der Ausbildungsabsolventen mit einer Ausbildungsdauer von mehr als 450 Tagen. Zudem werden Personen erfasst, deren äußerst kurze Ausbildungszeiten dennoch zu einem erfolgreichen Abschluss führen. Diese Auszubildenden werden aufgrund der Kürze ihrer Ausbildung nicht weiter berück-

\footnotetext{
${ }^{79}$ Parmentier et al. (1993) unterteilt die Tätigkeitsmerkmale in Produktionstätigkeiten, Infrastrukturtätigkeiten, Vertriebs-, Verwaltungs- und Planungstätigkeiten sowie Dienstleistungstätigkeiten. Daten des Mikrozensus geben Auskunft über die Tätigkeitsschwerpunkte der einzelnen Ausbildungsberufe, nach denen die Zuordnung für die gesamte Berufsgruppe erfolgt. Schwieriger gestaltet sich die Zuordnung zu den Infrastrukturtätigkeiten, die sowohl produktions- als auch dienstleistungsorientierte Aufgabenbereiche enthalten.

${ }^{80}$ Unter Nachfrageorientierung ist hierbei die Erbringung von Aufgaben innerhalb einer Unternehmung bzw. innerhalb eines Haushaltes zu verstehen.

${ }^{81}$ Dieses hohe Durchschnittsalter der Auszubildenden ist vor allem auf die mit der Bildungsexpansion einhergehende Verlängerung des Schulbesuchs, auf die steigende Beteiligung von Abiturienten an der dualen Berufsausbildung sowie auf die Zunahme des Besuchs von Berufsvorbereitungs- und Berufsgrundbildungsjahren zurückzuführen (BMBF 2007: 108).
} 
sichtigt, da es sich unter Umständen um eine Fort- oder Weiterbildung handelt. Darüber hinaus sind in der IABS 1975-2004 Anlernzeiten zusammen mit den Ausbildungszeiten aggregiert, sodass diese durch die Vorgabe einer Mindestausbildungsdauer identifiziert werden und somit bei den folgenden Analysen, sofern sie diese Mindestausbildungsdauer nicht erreichen, vernachlässigt werden können. Schließlich werden lediglich die männlichen Ausbildungsabsolventen untersucht, da sich die Frauen bzw. Mädchen in der Wahl ihrer Ausbildungsberufe auf eine geringere Anzahl konzentrieren und sich zudem weniger an der dualen Berufsausbildung beteiligen (Hartung/Janik 2006). Daher werden weniger Ausbildungsberufe berücksichtigt, woraus ebenfalls verzerrte Schätzungen resultieren können.

Um die interessierende Grundgesamtheit zu erhalten, müssen vor allem Personen identifiziert werden, die eine duale Berufsausbildung abgeschlossen haben. Dies geschieht, indem nur die Einheiten beibehalten werden, für die ein Wechsel in den Variablen "Ausbildung" und "Stellung im Beruf" erfolgt ist. Kriterien sind hierfür, dass der höchst erreichte Bildungsabschluss in einen höheren Bildungsabschluss wechselt. So werden Personen als Ausbildungsabsolventen identifiziert, die einen „Haupt-/Realschulabschluss ohne Berufsausbildung“ bzw. „Abitur ohne Berufsausbildung“ haben und dann einen „Haupt/Realschulabschluss mit Berufsausbildung“ bzw. „Abitur mit Berufsausbildung“. Zudem darf die Variable „Stellung im Beruf“ im darauf folgenden Spell nicht mehr die Ausprägung ,in Ausbildung“ anzeigen (vgl. Schimpl-Neimanns 2006).

Zur Beantwortung der Fragestellung und zur Feststellung der Ursachen für die Arbeitslosigkeit nach der dualen Berufsausbildung werden sowohl betriebliche als auch individuelle Merkmale benötigt. Hierbei ist es zunächst notwendig, die arbeitslosen von den erwerbstätigen Absolventen zu unterscheiden. Die IABS 1975-2004 enthält keine Angaben darüber, ob ein Betriebswechsel freiwilliger oder unfreiwilliger Natur ist. Aus diesem Grund können übernommene Ausbildungsabsolventen, die den Betrieb freiwillig verlassen, nicht von den nicht übernommenen Ausbildungsabsolventen unterschieden werden. Aber gerade die nicht übernommenen Ausbildungsabsolventen gilt es zu identifizieren und die Ursachen für die Nichtübernahme zu untersuchen. Denn auch freiwillig wechselnde junge Erwachsene können für kurze Zeit arbeitslos sein. Diese Sucharbeitslosigkeit dient dazu, einen qualifikationsadäquaten Job zu finden, bei dem die ursprüngliche Position auf dem Arbeitsmarkt verbessert wird (siehe Kap. 5.2). In der Regel nimmt diese Sucharbeitslosigkeit keine sehr lange Zeit in Anspruch, weshalb nur die Ausbildungsabsolventen als arbeitslos angesehen werden, die länger als 30 Tage ohne Beschäftigung waren. So wird davon ausgegangen, dass die freiwillig wechselnden Absolventen innerhalb eines Monates wieder in Beschäftigung kommen. Dagegen befinden sich die Absolventen, die nicht freiwillig wechseln, länger in Arbeitslosigkeit (von Wach- 
ter/Bender 2004: 14). Die verbleibenden Fälle, die untersucht werden, sind demnach mit großer Wahrscheinlichkeit die interessierenden Einheiten.

Darüber hinaus haben die fehlenden Informationen zur Übernahme die Folge, dass auch befristet übernommene Ausbildungsabsolventen nicht identifiziert werden können. ${ }^{82}$ Diese werden zu den Ausbildungsabsolventen hinzugezählt, die keinen Betriebswechsel aufweisen und entweder sozialversicherungspflichtig oder geringfügig beschäftigt sind. Begründet wird dies durch die Annahme, dass während der befristeten Weiterbeschäftigung Berufserfahrung gesammelt wird, was wiederum die Arbeitsmarktposition der betroffenen Ausbildungsabsolventen verbessert. Eine befristete Übernahme deutet zumeist auf eine gewerkschaftliche Bindung des Ausbildungsbetriebes hin. In diesen Betrieben ist das Bildungsniveau von Ausbildungsabsolventen wegen des Interesses an Humankapitalinvestitionen relativ hoch (Kap. 3.3.2.3.1 und Kap.4.2.2). Deshalb kann davon ausgegangen werden, dass es sich um gut ausgebildete Ausbildungsabsolventen handelt.

Zur Schätzung des Einflusses auf die Arbeitslosigkeit sind Informationen über die individuellen Merkmale der Personen, wie Geschlecht, Geburtsjahr, Alter, Familienstand, Kinderzahl, Staatsangehörigkeit sowie Schul- und Berufsausbildung relevant, welche die IABS 1975-2004 enthält.

Für die empirische Evidenz ist vor allem die Bildungsvariable von besonderer Relevanz. Allerdings weist sie zwei Nachteile auf. Erstens enthält die IABS 1975-2004 keine vollständigen Informationen über die Bildung der Untersuchungseinheiten, da keine Meldepflicht besteht und es sich dementsprechend nur um eine statistische Angabe handelt (Drews 2006: 4). Dadurch ist das Bildungsmerkmal nicht sehr zuverlässig. Um es zuverlässiger zu machen, wurden verschiedene Imputationsmethoden, u. a. von Fitzenberger et al. (2005) und Drews (2006) erarbeitet, wovon die erste Imputationsalternative für die Schätzungen in der vorliegenden Arbeit verwendet wird. Die Lücken werden mit der Information des höchst erreichten Bildungsstandes der jeweiligen Person gefüllt. Hierbei werden die Bildungsinformationen mit den „,bildungsgradspezifischen Altergrenzen“" berücksichtigt (Drews 2006: 6), sodass bspw. eine 17- jährige Person keinen Hochschulabschluss vorweisen kann. Zweitens ist die Bildungsvariable eine Kombination aus Informationen der Schulbildung und der Berufausbildung, welche den höchsten erreichten (Aus-)Bildungsabschluss angibt. Die Schulbildungsvariable ist ebenfalls vergröbert, da sie „Haupt- und Realschulabschluss“ sowie „keinen Schulabschluss“ in einer Merkmalsausprägung zusammenfasst. Daher können Personen mit einem Hauptschulabschluss nicht von Personen mit Realschulabschluss oder keinem Schulabschluss unterschieden werden. Um dennoch Aussagen über den Einfluss der Bildung auf den Übergang an der zweiten Schwelle sowie das Arbeitslosigkeitsrisiko treffen zu

${ }^{82}$ Seit Mitte der 1990er Jahre gewinnen tarifliche Übernahmeregelungen immer mehr an Bedeutung. Jedoch erst ab dem Jahr 2000 wurde die Übernahmefrist in vielen Branchen auf bis zu 12 Monate verlängert. 
können, kann der Schulabschluss der Personen über das Alter identifiziert werden. ${ }^{83}$ Dadurch wird der näherungsweise Anteil der Personen erhalten, der über einen Haupt- bzw. über einen Realschulabschluss verfügt. Diese Disaggregation der Bildungsvariablen mit Hilfe des Alters ist in jüngerer Zeit jedoch kritisch zu betrachten, da der Anteil der Schulabsolventen, die an berufsvorbereitenden Maßnahmen teilnehmen, zunimmt. Teilnehmer sind insbesondere Hauptschulabsolventen oder Jugendliche, die ohne Abschluss die Schule verlassen. Aufgrund dessen erhöht sich das Durchschnittsalter dieser Personengruppe bei Aufnahme einer Ausbildung und entspricht somit nahezu dem Durchschnittsalter von Realschulabsolventen. ${ }^{84}$

Darüber hinaus gibt die IABS 1975-2004 Auskunft über die betrieblichen Rahmenbedingungen während eines Beschäftigungsspells. Erfasst werden die Wirtschaftszweige, innerhalb derer der entsprechende Betrieb agiert, sowie die Qualifikationsstruktur der Belegschaft. Hierbei existieren Informationen über die Anzahl der im Betrieb Beschäftigten jeweils ,ohne Berufsausbildung', ,mit dualer Berufsausbildung', ,mit Hochschulabschluss' sowie ,geringfügig Beschäftigte' (Drews et al 2006). Diese Variable kann Aufschluss über die betriebliche Nachfrage nach qualifizierten Arbeitskräften geben, mit der die Gründe für die Ausbildungsbereitschaft und für den Verbleib an der zweiten Schwelle identifiziert werden können. Darüber hinaus ist die Betriebsgröße erfasst und das Betriebsentgelt, welches das arithmetische Mittel der Entgelte aller im Betrieb Beschäftigter, angibt. Insgesamt ermöglichen diese Informationen, Wirkungen der im Ausbildungsbetrieb bestehenden Rahmenbedingungen auf das weitere Erwerbsleben zu ermitteln.

Als betriebliches Merkmal stellen die Ausbildungskosten in den theoretischen Modellen eine wichtige Erklärungsgrundlage für die Ausbildungsbereitschaft dar. Sie werden jedoch innerhalb der IABS 1975-2004 nicht erfasst. Um diesen Einfluss dennoch näherungsweise abbilden zu können, werden die Ausbildungsvergütungen herangezogen, die zu einem sehr großen Teil die Kosten verursachen. Zur Bestimmung des Anteils der Ausbildungsvergütungen an den Ausbildungskosten liegen als Basis in der Literatur (Beicht et al. 2004) nur die Bruttokosten vor. Bei deren Betrachtung wird deutlich, dass sich die Zusammensetzung der Kosten zwischen den Ausbildungsbereichen unterscheidet. Entsprechend entfallen im Öffentlichen Dienst $84 \%$ der Gesamtkosten auf die Ausbildungsvergütungen bzw. 78\% in Industrie und Handel. Dieser Anteil der

\footnotetext{
${ }^{83}$ Jugendliche, die nach Abschluss der Berufsausbildung jünger als 19 Jahre alt waren, wurden den Absolventen mit Hauptschulabschluss zugeordnet, während bei den Absolventen über 19 Jahren angenommen wird, dass sie einen Realschulabschluss haben.

${ }^{84}$ In diesem Fall ist die Interpretation der Wahrscheinlichkeit eines Eintritts in Arbeitslosigkeit mit der durch das Alter disaggregierten Variablen für jüngere Daten nicht mehr möglich. In der vorliegenden Arbeit tritt dieses Problem jedoch erst am aktuellsten Rand auf, sodass das Alter und die Höhe des Bildungsabschlusses nicht mehr in positivem Zusammenhang stehen.
} 
Ausbildungsvergütung ist verhältnismäßig gering, da in den Freien Berufen $91 \%$ der Gesamtkosten für Ausbildungsvergütungen aufgewendet werden müssen. ${ }^{85}$ Im Handwerk sind es $84 \%$ der Gesamtkosten. Der hohe Anteil der Ausbildungsvergütungen an den Bruttoausbildungskosten impliziert zugleich einen hohen Produktionsbeitrag, sodass geringe Nettoausbildungskosten nach der Ausbildung verbleiben. Dies ist dann möglich, wenn der Arbeitsprozess so gestaltet ist, dass die Auszubildenden ohne größere Einlernphase eingesetzt werden können. Zudem entfällt ein geringerer Anteil auf die anderen Kostenpositionen, wie Löhne der hauptberuflichen Ausbilder bzw. Löhne der Mitarbeiter, die die Lehre als zusätzliche Aufgabe erledigen, sowie Ausbildungsmaterial. In diesem Fall ist der Humankapitalstand, im Vergleich zu Ausbildungsbereichen mit einem geringen Anteil der Ausbildungsvergütungen an den Ausbildungskosten, niedrig und vor allem durch die allgemeine bzw. berufsspezifische Komponente gekennzeichnet. Daraus resultieren ebenfalls niedrige Nettoausbildungskosten, die neben den geringen Kosten durch die hohen Produktionsbeiträge klein gehalten werden. Dagegen sind die Nettokosten in Betrieben neben den hohen absoluten Ausbildungsvergütungen auch auf andere Ausbildungskosten zurückzuführen. Diese sind aufgrund des höheren Wertes am Produktionsausfall durch das Einführen und Lehren in Großunternehmen hoch. Erträge während der Ausbildung sind daher geringer. Ausbildungsvergütungen werden auch deshalb als Annäherung an die gesamten Ausbildungskosten herangezogen, da in der IABS 1975-2004 letztere nicht direkt erfasst werden. Zur Integration der Ausbildungskosten werden die absoluten Ausbildungsvergütungen als Indikator verwendet. Um Rückschlüsse ziehen zu können, ob ein Ausbildungsbetrieb nach dem produktionstheoretischen oder investitionstheoretischen Ansatz ausbildet, kann davon ausgegangen werden, dass bei höheren Ausbildungsvergütungen auch höhere Ausbildungskosten vorliegen, welche folglich für einen investitionstheoretischen Ansatz sprechen. Bspw. liegt im Handwerksbereich zwar der Anteil der Ausbildungsvergütung an den Gesamtkosten verhältnismäßig hoch, aber die absolute Höhe der Entlohnung für die Auszubildenden ist am niedrigsten. Dagegen liegt der absolute Wert der Ausbildungsvergütungen im Öffentlichen Dienst durchschnittlich wesentlich höher (ca. 3000€) bei gleichem Anteil an der Gesamtkostenstruktur. Dies deutet wiederum auf höhere Nettoausbildungskosten hin, da absolut hohe Kosten bei relativ geringer Produktivität vorliegen. Diese höhere Entlohnung spiegelt jedoch nicht die tatsächliche Produktivität wider. Denn diese höhere Produktivität kommt erst nach der Ausbildung zum Tragen, wodurch der Ausbildungsabsolvent auch einen höheren Bildungsstand aufweist, der von einem (unter Umständen relativ hohen) Anteil an betriebsspezifischem Humankapital gekenn-

\footnotetext{
${ }^{85}$ Kosten des Ausbildungspersonals sind in den Freien Berufen am höchsten, da es sich dort meist um den Chef der Praxis selbst handelt, der als Ausbilder fungiert. Ferner ist dessen Lohn relativ gesehen höher als der eines Landwirts oder einer Führungskraft in einem Betrieb.
} 
zeichnet ist. ${ }^{86}$ Da die Ausbildungsvergütungen den größten Teil der Ausbildungskosten darstellen, kann davon ausgegangen werden, dass diese negativ mit dem Arbeitslosigkeitsrisiko und positiv mit dem Verbleib im Ausbildungsbetrieb korrelieren. Hohe Ausbildungsvergütungen führen zu einer höheren Wahrscheinlichkeit, übernommen zu werden. Größere Unternehmen können meist auch wirtschaftliche Schwankungen durch flexibleren Arbeitskräfteeinsatz besser ausgleichen. Daher ist es ihnen möglich, mehr ihrer Ausbildungsabsolventen (befristet) weiterzubeschäftigen.

Auf Grundlage der in der IABS 1975-2004 enthaltenen Variablen werden die empirische Evidenz der abgeleiteten Zusammenhänge zwischen Humankapital und der Einflussfaktoren im nächsten Kapitel überprüft und Implikationen für die zweite Schwelle herausgearbeitet.

${ }^{86}$ Der Ertrag hängt nach Zwick (2007) dagegen von mehreren Faktoren ab, unter anderem von der Wettbewerbsposition und der Qualifikationsstruktur innerhalb des Betriebs. Diese Faktoren wirken jedoch dem Lohnsummeneffekt entgegen und überlagern diesen im Idealfall. Daher handelt es sich meist um Unternehmen, die nach dem investitionstheoretischen Ansatz ausbilden. Die Ausbildung erfolgt mit dem Ziel des Erhalts der Wettbewerbsposition und der Deckung des Fachkräftebedarfs. Folglich sind die Unternehmen bereit, die produktiven Arbeitsplätze hoch zu entlohnen. 


\section{8 Ökonometrische Verfahren und empirische Evidenz}

Die empirische Untersuchung konzentriert sich auf die Bestimmung der Ursachen für den Eintritt in Arbeitslosigkeit nach der dualen Berufsausbildung und die Dauer dieser ersten Arbeitslosigkeitsphase und knüpft dabei an die abgeleiteten Einflussfaktoren an. Dazu werden im ersten Schritt die verschiedenen Fälle, die sich beim Eintritt in das Erwerbsleben nach der dualen Berufsausbildung ergeben, identifiziert mit einem multinomialen Logitmodell geschätzt. Daraus lassen sich die Einflussfaktoren auf die Eintrittswahrscheinlichkeit dieser verschiedenen Fälle ableiten. Im zweiten Schritt wird geschätzt, welche Einflussgrößen die Dauer der ersten Arbeitslosigkeit der Ausbildungsabsolventen determinieren. Die betrachteten Einheiten bilden arbeitslose junge Erwachsene. Um die Wirkung der ersten Arbeitslosigkeit auf das erste Berufsjahr zu identifizieren, wird die kumulierte Beschäftigungsdauer innerhalb des ersten Jahres nach der dualen Berufsausbildung ermittelt. Denn eine kurze Arbeitslosigkeitsphase direkt nach der dualen Berufsausbildung muss nicht unbedingt $\mathrm{zu}$ einer höheren Beschäftigungsdauer innerhalb des Betrachtungszeitraums führen, weil unter Umständen eine kurzfristige Beschäftigung mit häufigen Unterbrechungen besteht. Dieser Effekt wird mit der Matching-Methode ermittelt.

\subsection{Bestimmungsgründe für den Verbleib nach der dualen Berufsaus- bildung}

In diesem Abschnitt wird der Eintritt ins Erwerbsleben unmittelbar nach der dualen Berufsausbildung untersucht. Hierbei stehen die Einflussfaktoren für einen Betriebswechsel im Mittelpunkt der Betrachtung. Der Fokus liegt aber nicht auf der Qualität des Arbeitsmarkteinstiegs, da davon ausgegangen wird, dass eine Beschäftigung, gleich welcher Art, positiv auf die Berufserfahrung wirkt, obwohl sich ein vom Normalarbeitsverhältnis abweichender Eintritt ins Erwerbsleben negativ, zumindest auf den Beginn des Berufslebens, auswirkt (siehe Kap. 7.2). Vielmehr steht die Analyse der ersten Arbeitslosigkeitsphase im Mittelpunkt der Betrachtung. Darüber hinaus können befristete Arbeitsverhältnisse nicht identifiziert werden. Geringfügige Beschäftigung hatte bis 2004 als sozialversicherungspflichtige Erwerbstätigkeit nicht die Relevanz, weil diese Beschäftigungsart erst nach dem Betrachtungszeitraum von 2003 an Bedeutung gewonnen hat (Fuchs/Ebert 2008; Schreyer 2001). Daher ist die Anzahl an Fällen unter Ausbildungsabsolventen für diesen Betrachtungszeitraum recht gering. Zudem ist anzunehmen, dass vor allem niedrig qualifizierte Personen geringfügig beschäftigt sind. Dieser Gruppe können die Absolventen jedoch aufgrund des erfolgreichen Berufsabschlusses nicht zugeordnet werden. 


\subsubsection{Methodisches Vorgehen zur Bestimmung des Arbeitsmarkteintritts}

Für die Untersuchung des Eintritts in den Arbeitsmarkt nach der dualen Berufsausbildung werden die verschiedenen Alternativen einander gegenübergestellt und mit deren Realisationswahrscheinlichkeiten bewertet. Auf diese Weise werden die ersten beiden Hypothesen untersucht: Zunächst wird erörtert, ob Ausbildungsabsolventen mit niedrigem Bildungsstand eine höhere Wahrscheinlichkeit haben, den Ausbildungsbetrieb zu verlassen (Hypothese 1a). In diesem Zusammenhang wird gleichzeitig die Hypothese $1 \mathrm{~b}$ überprüft, inwieweit Personen mit niedrigem Humankapitalstand eine höhere Wahrscheinlichkeit haben, arbeitslos zu werden. Zur Überprüfung der Hypothesen sind daher entsprechende Alternativen für den Arbeitsmarkteintritt zu wählen, die in Abbildung 9 dargestellt sind.

Abbildung 9: Darstellung der Verbleibsalternativen nach der dualen Berufsausbildung

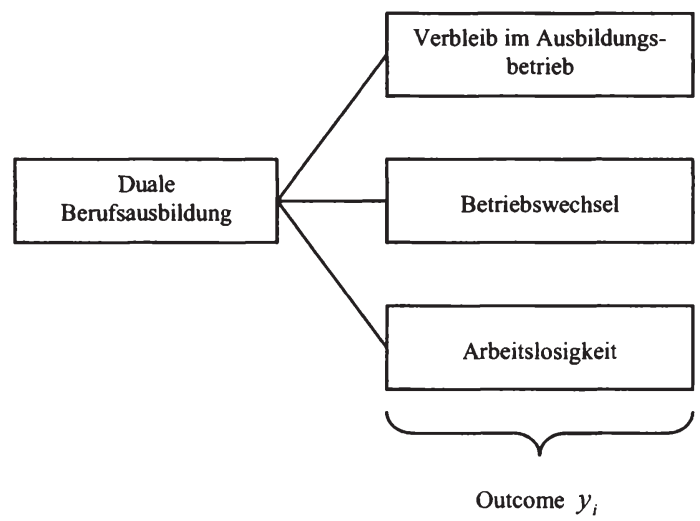

Quelle: eigene Darstellung

Wie aus der Abbildung hervorgeht, ergeben sich vor allem drei verschiedene Outcomes, die für die weiteren Untersuchungen von besonderer Bedeutung sind. Ausbildungsabsolventen können nach Abschluss der dualen Berufsausbildung im Betrieb verbleiben. In diesem Fall bleibt nur die Möglichkeit einer Beschäftigung entweder in regulärer Erwerbstätigkeit oder in einer geringfügigen Beschäftigung. Verlassen Ausbildungsabsolventen dagegen den Ausbildungsbetrieb, findet ein Teil der Ausbildungsabsolventen eine Beschäftigung. Der andere Teil tritt in Arbeitslosigkeit. Da der Ausbildungsbildungsabsolvent nur einen dieser Zustände bei Eintritt in den Arbeitsmarkt realisieren kann, wird auch von konkurrierenden Risiken gesprochen, die zugleich Unabhängigkeit 
voneinander implizieren. Diese Annahme bildet gleichzeitig die grundlegende Voraussetzung für die Schätzung der Signifikanz des Einflusses auf die verschiedenen Zustände nach Abschluss der dualen Berufsausbildung. Hierzu wird das multinomiale Logitmodell verwendet, welches eine Verallgemeinerung des binären Logitmodells (Moosmüller 2004: 199; Greene 2003: 720ff.; Ronning 1991: 38) darstellt, weil dieser Ansatz nicht nur zwei Ausprägungen einer abhängigen Variablen schätzen kann, sondern die Möglichkeit bietet, $j$ diskrete Ausprägungen einer zu erklärenden Variablen simultan zu schätzen.

Darüber hinaus eignet sich das Logitmodell, weil die abhängige Variable ein nominales Merkmal ist. Dies erfordert die Ermittlung der Wahrscheinlichkeit $p$, mit der eine bestimmte Auswahlkategorie $j$ realisiert wird. Die Auswahlkategorie entspricht den Verbleibsalternativen. $\beta$ gibt die Stärke der Einflussfaktoren $\left(x^{\prime}\right)$, die sich für die einzelnen Kategorien der abhängigen Variablen unterscheidet, wieder. Daher stellt sich das Logitmodell formal wie folgt dar:

$$
p\left\{y_{i}=j\right\}=\frac{\exp \left\{x_{i j}^{\prime} \beta\right\}}{\exp \left\{x_{i 1}^{\prime} \beta\right\}+\exp \left\{x_{i 2}^{\prime} \beta\right\}+\exp \left\{x_{i 3}^{\prime} \beta\right\}}, \quad j=1,2,3
$$

Ebenso wie das binäre Logitmodell setzt auch das multinomiale Logitmodell voraus, dass die Störterme unabhängig voneinander sind. Damit diese Voraussetzung erfüllt ist, muss die Unabhängigkeit der Alternativen gewährleistet sein, indem sie sich möglichst voneinander unterscheiden. Das heißt, ähnliche Alternativen (Outcomes) können nicht in Betracht gezogen werden. Mc Fadden (1974) nennt dies die „Independence of irrelevant alternatives“ (IIA). Zwischen regulärer Erwerbstätigkeit und Teilzeitarbeit wird daher nicht unterschieden, da es sich meist um identische Tätigkeitsbereiche handelt, nur mit unterschiedlichen Wochenarbeitsstunden. Geringfügige Beschäftigung betrifft dagegen meist andere Tätigkeitsbereiche. In dem vorliegenden Datensatz wird sie aber erst seit 1999 erfasst und ist bis 2004 in der Gruppe der Ausbildungsabsolventen anteilig noch sehr wenig verbreitet. Mit diesem Modell kann der Einfluss auf die geringfügige Beschäftigung daher nicht robust geschätzt werden. Daher wird sie unter die Alternative „Beschäftigung“ subsumiert. Aufgrund der Annahme, dass die Störterme voneinander unabhängig sind, muss deren Erwartungswert gleich null sein. Zudem weisen die Outcomes unterschiedliche Verteilungen auf, sodass diese jeweils durch die Einflussfaktoren vollständig erklärt werden können. Infolgedessen tritt eine mögliche Korrelation von unbeobachtbaren Faktoren mit beobachtbaren Faktoren nicht auf. Um die Unabhängigkeit der verschiedenen Outcomes zu überprüfen, wird das Pseudo $R^{2}$ herangezogen, das die Differenz zwischen den logistischen Werten der Outcomes bildet und diese als Prüfwerte verwendet. ${ }^{87}$ Je größer die Differenz zwischen

${ }^{87}$ Formal bedeutet dies pseudo $R^{2}=1-\frac{1}{1+2\left(\log L_{1}-\log L_{0} / N\right.}$. 
den Prüfwerten ist, desto kleiner ist das Pseudo $R^{2}$, wonach in diesem Fall die Annahmen der Unabhängigkeit eingehalten werden (Verbeek 2008).

Als beobachtbare erklärende Einflussfaktoren $\left(x^{\prime}\right)$ werden die betrieblichen Rahmenbedingungen während der Ausbildung herangezogen, da diese einen Betriebswechsel iniziieren bzw. einen Eintritt in Arbeitslosigkeit bewirken können. Da zwischen der Variable Wirtschaftszweig und Ausbildungsbereich ein systematischer Zusammenhang (Multikollinearität) besteht (Hill et al. 2001), werden in den Regressionen lediglich die Ausbildungsbereiche berücksichtigt. Begründet liegt dies in der Tatsache, dass ein Ausbildungsberuf meist nur in einem bestimmten Wirtschaftszweig zu finden ist. Daher korrelieren diese beiden Merkmale sehr stark miteinander. Allerdings gibt es auch Ausbildungsberufe, die über mehrere Wirtschaftszweige hinweg vertreten sind, wie bspw. Bürokaufleute. Diese bilden aber die Minderheit, weshalb von einem systematischen Zusammenhang ausgegangen werden kann. Somit würde die Aufnahme beider Variablen in das Modell über den Verbleib an der zweiten Schwelle keinen zusätzlichen Informationsbeitrag zur Erklärung der abhängigen Variablen bringen.

Ferner verlangen Ausbildungsberufe aufgrund der vorgegebenen Lehrinhalte bereits eine Reihe von Fähigkeiten, wodurch Schulabgänger verschiedener Schularten für bestimmte Ausbildungsbereiche einen leichteren Zutritt erlangen als für andere. Das heißt, Ausbildungsabsolventen mit einem niedrigen Schulabschluss haben meist auch nur die Möglichkeit einen Ausbildungsberuf mit schlechten Beschäftigungsaussichten zu erlernen. Gegenüber Ausbildungsabsolventen mit höherer Schulbildung, die auch entsprechend bessere Chancen auf einen Ausbildungsberuf mit guten Aussichten haben, verschlechtert sich die Situation weiter. Entsprechende Ausprägungen der genannten Einflussvariablen verstärken dann den Selektionseffekt. Deshalb wird eine Interaktionvariable, welche sich aus dem Produkt der Ausprägungen des Schulabschlusses und des jeweiligen Ausbildungsbereiches zusammensetzt, in das Regressionsmodell eingeführt. Diese gibt die Wirkung eines bestimmten Schulabschlusses innerhalb eines bestimmten Ausbildungsbereiches auf den Verbleib an der zweiten Schwelle an. Damit kann eine Aussage getroffen werden, welche Rolle die Bildung innerhalb der entsprechenden Ausbildungsbereiche einnimmt. Bei einem negativen Einfluss weisen Absolventen mit einem höheren Schulabschluss eine geringere Wahrscheinlichkeit auf, den Betrieb zu wechseln oder arbeitslos zu werden.

Darüber hinaus wird die Differenz zwischen dem Qualifikationsniveau, das der Ausbildungsabsolvent während der Ausbildung innerhalb des Betriebs aufgrund der Qualifikationen der Belegschaft erlangen kann, und dem Qualifikationsniveau, das innerhalb des entsprechenden Wirtschaftszweiges vorherrscht, gebildet (von Wachter/Bender 2004). Ist die Differenz negativ, kann das Unternehmen besser qualifizierte Fachkräfte derselben Branche extern einstellen. Ferner deutet dies auf eine Ausbildung hin, die durch den produktionstheoreti- 
schen Ansatz begründet werden kann, weil aufgrund des geringen Qualifikationsniveaus der Belegschaft vorwiegend berufsspezifisches Humankapital vermittelt wird. Der Auszubildende wird als produktive Arbeitskraft eingesetzt und ist entsprechend günstig. Deshalb wird in diesem Fall eine Arbeitslosigkeit wahrscheinlich.

Schließlich wird die durchschnittliche betriebliche Lohnsumme als weiterer Einflussfaktor verwendet. Diese erfasst alle Gehälter und Löhne aller Beschäftigten innerhalb des Ausbildungsbetriebes. Bei steigender Lohnsumme kann davon ausgegangen werden, dass es sich um ein Unternehmen handelt, das Ausbildung zur Deckung des Fachkräftebedarfs tätigt. Diese beiden Größen hängen positiv miteinander zusammen, denn je höher die Ausbildungssummen sind, desto höher ist auch die Lohnsumme. Hierbei wird ebenfalls die Übernahmewahrscheinlichkeit steigen und das Arbeitslosigkeitsrisiko sinken.

Darüber hinaus beeinflussen individuelle Merkmale, wie Schulbildung und Staatsangehörigkeit, die verschiedenen Wahrscheinlichkeiten einer j-ten Verbleibsalternative $\mathrm{zu}$ realisieren. Zudem werden regionale Unterschiede betrachtet. Hierbei werden die einzelnen Bundesländer als weitere erklärende Variable herangezogen, wodurch auf die nicht direkt beobachtbare unterschiedliche wirtschaftliche Situation und die Industriestruktur Rückschlüsse gezogen werden können.

Da mit der IABS 1975-2004 Verlaufsdaten über einen langen Zeitraum vorliegen, erscheint ein gepooltes logistisches Regressionsmodell wenig sinnvoll. Es ist anzunehmen, dass die Stärke der Einflussfaktoren, das heißt, deren Wirkung auf die abhängige Variable sich im Zeitablauf ändert. Bspw. veränderte sich die Wirkung einer Ausbildung in einem kleinen Betrieb für den Verbleib an der zweiten Schwelle in den letzten Jahren im Vergleich zum Beginn der 1980 er Jahre. Um dieser Entwicklung Rechnung zu tragen, werden die einzelnen Abschlussjahrgänge zu Kohorten zusammengefasst. So bilden die Jahrgänge von 1975-1980, 1981-1986, 1987-1991, 1992-1995, 1996-1999 sowie 20002003 jeweils eine Abschlusskohorte, wobei sich die Untersuchungen auf die Kohorten zwischen 1992 und 2003 konzentrieren. Anhand dieser Kohorten werden getrennte Schätzungen durchgeführt. Bspw. sind die Rahmenbedingungen von Absolventen, die zwischen 2000 und 2003 den Abschluss ihrer Ausbildung erworben haben, im Vergleich zu anderen Jahrgängen von einer geringen Arbeitslosigkeit der entsprechenden Altersklasse gekennzeichnet. Teilweise liegt diese sogar unter der gesamtwirtschaftlichen Arbeitslosenrate. Gerade diese Abschlusskohorte hatte aber zu Beginn der Ausbildung ungünstige Voraussetzungen, da wesentlich weniger Ausbildungsstellen angeboten wurden als Bewerber vorhanden waren. Die Selektion der Betriebe war entsprechend zu Gunsten der besseren Schulabsolventen ausgerichtet. Wie in Kapitel 7.1.3 bereits dargestellt, wird auch hier der negative Zusammenhang zwischen den Voraussetzungen vor und nach der Ausbildung deutlich. Die Indikatoren finden sich Tabelle im Anhang wieder. Die Abschlusskohorten stehen jeweils unter- 
schiedlichen Rahmenbedingungen sowohl vor als auch nach Abschluss der Ausbildung gegenüber, die sich jeweils auf den Übergang in das Erwerbsleben und auf die Dauer einer Arbeitslosigkeit auswirken. Im Folgenden werden die weiteren Einflussfaktoren empirisch überprüft.

\subsubsection{Empirische Evidenz des Arbeitsmarkteintritts}

Im folgenden Kapitel wird nun die empirische Evidenz für die verschiedenen Übergangsalternativen überprüft. Junge Erwachsene, die zwischen 2000 und 2003 ihre duale Berufsausbildung abgeschlossen haben, realisierten die in $\mathrm{Ab}$ bildung 10 beschriebenen Outcomes.

\section{Abbildung 10: Verteilung der Verbleibsalternativen der Abschlusskohor- ten 2000-2003}

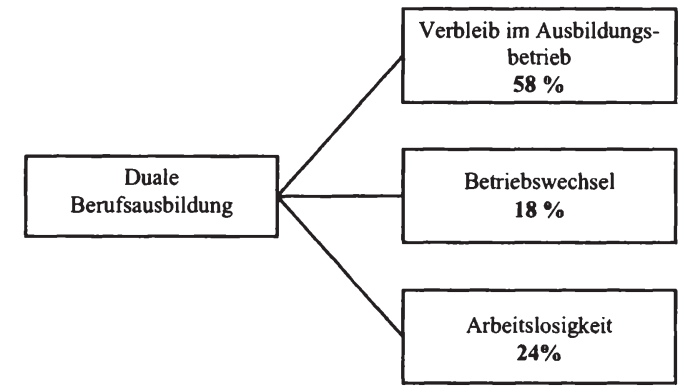

Quelle: IABS 1975-2004, eigene Berechnungen

$58 \%$ der betrachteten Ausbildungsabsolventen verblieben in ihrem Ausbildungsbetrieb. 18\% der Ausbildungsabsolventen wechselten den Betrieb, fanden aber im Anschluss daran wieder eine Beschäftigung. Dagegen waren $24 \%$ nach der dualen Berufsausbildung arbeitslos. Dies entspricht auch den Berechnungen des BiBB und der Europäischen Kommission ${ }^{88}$ (BMBF 2007 und 2006; EC 2007; Kap.2.2). Der Anteil der im Ausbildungsbetrieb verbleibenden Absolventen nahm im Vergleich zu den vorangehenden Abschlusskohorten ab, während gleichzeitig die Arbeitslosenrate zugenommen hat (siehe Tabelle 14 im Anhang). Werden Änderungen der Wechsel in einen anderen Betrieb und der Wechsel in Arbeitslosigkeit nach der dualen Berufsausbildung gemeinsam betrachtet, ist eine gegenläufige Entwicklung der Verteilung festzustellen. Dies lässt sich durch die unterschiedlichen wirtschaftlichen Rahmenbedingungen

\footnotetext{
${ }^{88}$ Die ermittelten Arbeitslosenraten der Europäischen Kommission liegen unter denen der offiziellen Statistik in Deutschland. Dies ist auf unterschiedliche Definition und Abgrenzungen der Arbeitslosigkeit zurückzuführen.
} 
erklären. Ein relativ geringer Anteil an Betriebswechseln, die in eine Beschäftigung mündeten und ein gleichzeitig hoher Anteil an Arbeitslosigkeit nach der dualen Berufsausbildung deuten auf schlechte gesamtwirtschaftliche Voraussetzungen hin. Entsprechend sind auch die Übergänge weniger erfolgreich. Ausbildungsabsolventen der Abschlusskohorten 1981-1986 waren mit einer entsprechenden Situation konfrontiert. In dieser Zeit war auch der Anteil der im Ausbildungsbetrieb Verbliebenen noch relativ hoch. Freiwillige Wechsel waren für diese Kohorten vermutlich eher unwahrscheinlich. Allerdings ist davon auszugehen, dass Ausbildungsabsolventen einer Abschlusskohorte nicht in gleichem Maße die verschiedenen Outcomes realisieren. Wie Studien zeigen (vgl. hierzu Kap. 7.1.1), spielt der Schulbildungsabschluss auch beim Übergang an der zweiten Schwelle eine entscheidende Rolle.

\section{Abbildung 11: Verbleib nach Schulabschluss für die Abschlusskohorten 2000-2003 (in \%)}

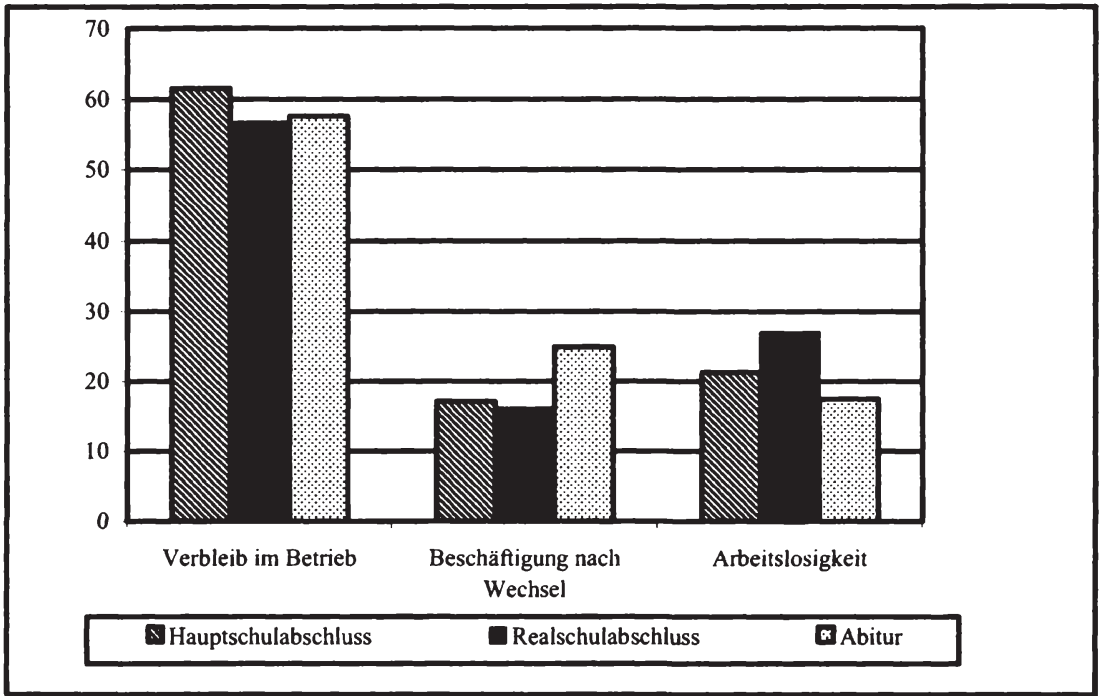

Quelle: IABS 1975-2004, eigene Berechnungen

Aus Abbildung 11 wird ersichtlich, dass sich die Anteile der im Ausbildungsbetrieb verbleibenden Ausbildungsabsolventen nicht wesentlich unterscheiden. So sind $62 \%$ aller Absolventen mit Hauptabschluss weiter in ihrem Betrieb beschäftigt gegenüber $57 \%$ aller Personen mit Realschulabschluss, deren Verbleibsquote unter allen Bildungsgruppen am geringsten ist. Absolventen mit Abitur wechseln am häufigsten den Betrieb (25\% aller Absolventen mit Abitur). Dieser hohe Anteil ergibt sich aus der Aufnahme eines Studiums, währenddessen sie aber noch erwerbstätig sind bzw. jobben. Junge Erwachsene mit 
Realschulabschluss und einer dualen Berufsausbildung realisierten am häufigsten den Eintritt in Arbeitslosigkeit (27\%). Der Schulabschluss determiniert demnach den Übergang von der dualen Berufsausbildung in das Erwerbsleben. Tabelle 5 stellt die Arbeitslosenquote im Zeitverlauf der Abschlusskohorten von 1975 bis 2003 dar.

Tabelle 5: Arbeitslosenquote nach Schulabschluss für die Ausbildungsabsolventen zwischen 1975-2003 (in \%)

\begin{tabular}{|l|c|c|c|c|}
\hline & Hauptschulabschluss & Realschulabschluss & Abitur & Gesamt \\
\hline $\mathbf{1 9 7 5 - 1 9 8 0}$ & 6,8 & 14,3 & 7,4 & 8,5 \\
\hline $\mathbf{1 9 8 1 - 1 9 8 6}$ & 15,9 & 32,0 & 11,2 & 21,0 \\
\hline $\mathbf{1 9 8 7 - 1 9 9 1}$ & 10,3 & 23,5 & 11,8 & 16,6 \\
\hline $\mathbf{1 9 9 2 - 1 9 9 5}$ & 20,6 & 23,3 & 15,5 & 21,8 \\
\hline $\mathbf{1 9 9 6 - 1 9 9 9}$ & 24,3 & 27,0 & 13,8 & 24,9 \\
\hline $\mathbf{2 0 0 0 - 2 0 0 3}$ & 21,3 & 27,0 & 17,5 & 24,4 \\
\hline
\end{tabular}

Quelle: IABS 1975-2004, eigene Berechnungen

Für die letzten 30 Jahre ist eine Zunahme der Arbeitslosigkeit auch unter den Ausbildungsabsolventen zu erkennen, wobei die Absolventen mit Realschulabschluss über alle Abschlusskohorten am meisten von Arbeitslosigkeit betroffen waren. Dieses Ergebnis ist vor allem durch die hohe Beteiligung der Realschulabsolventen an der dualen Berufsausbildung gekennzeichnet (Realschulabsolventen stellen die größte Gruppe innerhalb der dualen Berufsausbildung). Ausbildungsabsolventen mit Abitur wiesen über die Jahre insgesamt die geringsten Arbeitslosigkeitsquoten auf. Der Abstand zu den Arbeitslosenraten der anderen Bildungsabschlüsse vergrößerte sich. Allerdings zeigt sich für die Absolventen mit Abitur in der ältesten Abschlusskohorte keine geringere Arbeitslosenquote gegenüber den Hauptschulabsolventen. In den Jahrgängen zwischen 1987 bis 1991 waren Absolventen mit dem niedrigsten Schulabschluss sogar am wenigsten von Arbeitslosigkeit betroffen. Insgesamt kann diese geringe Wahrscheinlichkeit der Absolventen mit Hauptschulabschluss durch eine ausbildungsinadäquate Anstellung, bspw. als Hilfsarbeiter erklärt werden.

Weitere Studien (v.a. Konietzka 2002; Kap. 2.2) belegten zudem unterschiedliche Wahrscheinlichkeiten beim Übergang in den Arbeitsmarkt zwischen den einzelnen Ausbildungsbereichen. Die Übergänge der Abschlusskohorten zwischen 2000 und 2003 zeigt Tabelle 6. 
Tabelle 6: Verbleib nach Abschlussbereichen für die Abschlusskohorten 2000-2003 (in \%)

\begin{tabular}{|l|c|c|c|}
\hline & $\begin{array}{c}\text { im Ausbildungs- } \\
\text { betrieb }\end{array}$ & Betriebswechsel & Arbeitslosigkeit \\
\hline Land-/Forstwirtschaftsberufe & 45,3 & 27,0 & 27,7 \\
\hline Chemie-, holzverarbeitende Berufe & 55,0 & 12,8 & 32,2 \\
\hline Metallberufe & 66,1 & 12,9 & 21,0 \\
\hline Textilberufe & 53,3 & 16,7 & 30,0 \\
\hline Berufe im Nahrungsmittelbereich & 58,6 & 18,2 & 22,7 \\
\hline Elektroberufe & 60,2 & 20,0 & 19,8 \\
\hline Bauberufe & 54,9 & 12,9 & 32,2 \\
\hline $\begin{array}{l}\text { Infrastruktur-/ Transportdienstleis- } \\
\text { tungsberufe }\end{array}$ & 55,9 & 24,4 & 19,6 \\
\hline Finanzdienstleistungserufe & 56,0 & 23,9 & 20,1 \\
\hline Verwaltungsberufe & 60,2 & 21,1 & 18,7 \\
\hline $\begin{array}{l}\text { produktionsnahe Dienstleistungsbe- } \\
\text { rufe }\end{array}$ & 61,3 & 17,6 & 21,1 \\
\hline $\begin{array}{l}\text { haushalts-/personenbezogene Dienst- } \\
\text { leistungsberufe }\end{array}$ & 48,5 & 19,1 & 32,4 \\
\hline Gesamt & 57,6 & 18,0 & 24,4 \\
\hline
\end{tabular}

Quelle: IABS 1975-2004; eigene Berechnungen

Auch bei der Betrachtung des Übergangs vom Berufsbildungssystem in das Erwerbsleben nach Ausbildungsbereichen ${ }^{89}$ werden Unterschiede ersichtlich. Vor allem Ausbildungsabsolventen der manuellen Ausbildungsbereiche (Berufe der Land- und Forstwirtschaft, Chemieberufe, Bauberufe) und der einfachen Dienstleistungstätigkeiten (haushalts- und personenbezogene Dienstleistungsberufe) waren überdurchschnittlich von Arbeitslosigkeit betroffen. Gleichzeitig verblieben vor allem in den land- und forstwirtschaftlichen Ausbildungsberufen und in den haushalts- und personenbezogenen Dienstleistungsberufen die wenigsten Ausbildungsabsolventen im Ausbildungsbetrieb. Ausbildungsbereiche, wie bspw. Elektroberufe, Infrastruktur- und Transportdienstleistungsberufe sowie Verwaltungsberufe entließen ihre Ausbildungsabsolventen dagegen selten in Arbeitslosigkeit. Dies sind zumeist Berufe, die eine hohe Beschäftigungssicherheit aufweisen, wobei die Infrastruktur- und Transportdienstleistungsberufe zugleich auch unterdurchschnittliche Verbleibsquoten im Ausbildungsbe-

${ }^{89}$ Die Ausbildungsbereiche können weiterhin in unterschiedliche Gruppen unterteilt werden, welche die Ausbildungsqualität und den Bildungsstand widerspiegeln. So gehören die Bereiche Land- und Forstwirtschaft, Chemie und Holzverarbeitung, Metallverarbeitung, Textil-, Nahrungsmittel-, Elektro- und Baubranche dem Handwerk an, welches einen hohen Anteil an berufsspezifischem Humankapital aufweist. Im Allgemeinen haben diese Bereiche weniger gute Beschäftigungsaussichten. Absolventen der Infrastruktur- und Transportdienstleistungen sowie haushalts- und personenbezogene Dienstleistungen sind den einfachen Dienstleistungstätigkeiten zuzuordnen, während in den Bereichen Finanzdienstleistungen und produktionsnahe Dienstleistungen Berufe mit unterschiedlich hohen Anforderungen vertreten sind. Schließlich weisen Absolventen in den Verwaltungsberufen einen hohen Bildungsstand auf. 
trieb realisierten. ${ }^{90}$ Ein relativ hoher Anteil an Ausbildungsabsolventen verlie $\beta$ den Betrieb, fand aber auch in einem anderen Betrieb wieder eine Beschäftigung. Ausbildungsabsolventen der metallverarbeitenden Berufe haben hingegen die höchste Wahrscheinlichkeit, im Ausbildungsbetrieb weiter beschäftigt $\mathrm{zu}$ werden. In diesem Bereich scheint aus langfristigem Interesse ausgebildet zu werden, obwohl sich innerhalb dieses Bereiches unterschiedliche Arten von Betrieben an der Ausbildung beteiligen. So finden sich Betriebe der Autoindustrie genauso wie kleine Handwerksbetriebe wieder. In letzteren ist die betriebliche Mobilität recht hoch, weil innerhalb dieses Bereiches, aufgrund der Zugehörigkeit zum berufsspezifischen Teilarbeitsmarkt, Betriebswechsel ohne größeren Humankapitalverlust vollzogen werden können.

Die multinomialen logistischen Regressionen bestätigen die Tendenzen der deskriptiven Auswertungen, welche diese hinsichtlich der Überprüfung der Hypothesen geben. Tabelle 7 bis Tabelle 9 stellen die marginalen Effekte der Einflussfaktoren der Abschlusskohorten von 1992-1995, 1996-1999 sowie 2000-2003 den Verbleib an der zweiten Schwelle dar. Um die Veränderungen der Einflüsse aufzeigen zu können, wurden getrennte Schätzungen für die jeweiligen Abschlusskohorten durchgeführt. Zwei Modelle wurden geschätzt, wovon in Modell 2 Interaktionseffekte einbezogen werden, die den Zusammenhang zwischen Ausbildungsberufen und Schulbildungsvariablen berücksichtigt. Der Referenz-Outcome stellt den Verbleib im Ausbildungsbetrieb dar. Die Hypothesen 1a und $1 \mathrm{~b}$ werden im Folgenden gemeinsam betrachtet.

Ausbildungsabsolventen, deren Ausbildung mit dem Ziel eines langfristigen Beschäftigungsverhältnisses stattfindet, nehmen tendenziell weniger wahrscheinlich eine Tätigkeit in einem anderen Betrieb auf bzw. werden weniger wahrscheinlich nach der dualen Berufsausbildung arbeitslos. Diese Aussagen bestätigen vor allem die betrieblichen Faktoren, die den Verbleib an der zweiten Schwelle signifikant beeinflussen. Höhere Ausbildungsvergütungen reduzieren die Wahrscheinlichkeit, eine Beschäftigung außerhalb des Ausbildungsbetriebes aufzunehmen. Das heißt aber auch, dass Ausbildungsabsolventen, die den Betrieb wechseln, geringere Ausbildungsvergütungen haben als Ausbildungsabsolventen, die nicht wechseln. Die Wahrscheinlichkeit, eine Beschäftigung in einem anderen Betrieb aufzunehmen, ist bei einer marginalen Erhöhung der Ausbildungsvergütung ${ }^{91}$ um über 120 Prozentpunkte niedriger. Diese

\footnotetext{
${ }^{90}$ Diesem Bereich ist vor allem die Deutsche Bahn zuzuordnen, die in der Regel nur einen geringen Anteil an Ausbildungsabsolventen übernimmt. Sie bietet ihnen aber über eine Zeitarbeitsfirma die Möglichkeit zur Weiterbeschäftigung. Dadurch ist es möglich, die Arbeitskräfte flexibel auf überregionaler Ebene einzusetzen. Daher wird auch nur ein geringer Anteil an Ausbildungsabsolventen arbeitslos.

91 Die Ausbildungsvergütungen werden in logarithmierter Form dargestellt. Aus diesem Grund sind die marginalen Einflüsse auf die abhängige Variable hoch.
} 
Tabelle 7: Einflussfaktoren auf den Verbleib nach der dualen Berufsausbildung für die Abschlusskohorten 2000-2003 - Schätzungen eines multi-nomialen Logitmodells

\begin{tabular}{|c|c|c|c|c|}
\hline \multirow[b]{2}{*}{$\begin{array}{l}\text { (Referenz: Verbleib im Ausbil- } \\
\text { dungsbetrieb) }\end{array}$} & \multicolumn{2}{|c|}{$\begin{array}{c}\text { Modell 1: } \\
\text { ohne Interaktionseffekte }\end{array}$} & \multicolumn{2}{|c|}{$\begin{array}{c}\text { Modell 2: } \\
\text { mit Interaktionseffekten }\end{array}$} \\
\hline & $\begin{array}{l}\text { Beschäftigung } \\
\text { nach Wechsel }\end{array}$ & Arbeitslosigkeit & $\begin{array}{l}\text { Beschäftigung } \\
\text { nach Wechsel }\end{array}$ & Arbeitslosigkeit \\
\hline Ausbildungsvergütungen (log) & $\begin{array}{l}-1,248^{* * *} \\
(0,106)\end{array}$ & $\begin{array}{l}-1,932^{* * *} \\
(0,100)\end{array}$ & $\begin{array}{l}-1,253^{* * *} \\
(0,106)\end{array}$ & $\begin{array}{l}-1,933^{* * *} \\
(0,101)\end{array}$ \\
\hline $\begin{array}{l}\text { durchschnittliche betriebliche } \\
\text { Lohnsumme (log) }\end{array}$ & $\begin{array}{l}0,129^{* * *} \\
(0,028)\end{array}$ & $\begin{array}{l}0,096^{* * *} \\
(0,027)\end{array}$ & $\begin{array}{l}0,125^{* * *} \\
(0,028)\end{array}$ & $\begin{array}{l}0,087^{* * *} \\
(0,027)\end{array}$ \\
\hline Ausbildungsdauer (log) & $\begin{array}{l}-0,287^{* *} \\
(0,135)\end{array}$ & $\begin{array}{l}-0,659^{* * *} \\
(0,124)\end{array}$ & $\begin{array}{l}-0,267^{* *} \\
(0,136) \\
\end{array}$ & $\begin{array}{l}-0,626^{* * *} \\
(0,124)\end{array}$ \\
\hline \multicolumn{5}{|l|}{$\begin{array}{l}\text { Betriebsgröße } \\
\text { (Referenz: 1-9 Mitarbeiter) }\end{array}$} \\
\hline 10-49 Mitarbeiter & $\begin{array}{l}-0,308^{* * *} \\
(0,091) \\
\end{array}$ & $\begin{array}{l}-0,337^{* * *} \\
(0,077) \\
\end{array}$ & $\begin{array}{l}-0,308^{* * *} \\
(0,092) \\
\end{array}$ & $\begin{array}{l}-0,318^{* * *} \\
(0,078) \\
\end{array}$ \\
\hline 50-499 Mitarbeiter & $\begin{array}{l}-0,394^{* * *} \\
(0,126)\end{array}$ & $\begin{array}{l}-0,662^{* * *} \\
(0,117)\end{array}$ & $\begin{array}{l}-0,398^{* * *} \\
(0,127)\end{array}$ & $\begin{array}{l}-0,651^{* * *} \\
(0,117)\end{array}$ \\
\hline > 500 Mitarbeiter & $\begin{array}{l}-0,877^{* * *} \\
(0,196)\end{array}$ & $\begin{array}{l}-1,422^{* * *} \\
(0,191) \\
\end{array}$ & $\begin{array}{l}-0,876^{* * *} \\
(0,196)\end{array}$ & $\begin{array}{l}-1,413^{* * *} \\
(0,192) \\
\end{array}$ \\
\hline \multicolumn{5}{|l|}{$\begin{array}{l}\text { Ausbildungsberufe (Referenz: } \\
\text { Land-/ Forstwirtschaftsberufe) }\end{array}$} \\
\hline Chemieberufe & $\begin{array}{l}-0,052 \\
(0,193)\end{array}$ & $\begin{array}{l}-0,231 \\
(0,172)\end{array}$ & $\begin{array}{l}-0,625 \\
(0,607)\end{array}$ & $\begin{array}{l}-0,337 \\
(0,571)\end{array}$ \\
\hline Metallberufe & $\begin{array}{l}-0,575^{* * *} \\
(0,135)\end{array}$ & $\begin{array}{l}-0,133 \\
(0,106) \\
\end{array}$ & $\begin{array}{c}-0,661 \\
(0,435) \\
\end{array}$ & $\begin{array}{c}0,135 \\
(0,371) \\
\end{array}$ \\
\hline Textilberufe & $\begin{array}{l}-0,598^{* * *} \\
(0,100)\end{array}$ & $\begin{array}{l}-0,626^{* * *} \\
(0,087)\end{array}$ & $\begin{array}{l}-0,177 \\
(0,385)\end{array}$ & $\begin{array}{c}-0,220 \\
(0,388)\end{array}$ \\
\hline $\begin{array}{l}\text { Berufe im Nahrungsmittelbe- } \\
\text { reich"2 }\end{array}$ & ja & ja & ja & ja \\
\hline Elektroberufe & $\begin{array}{l}-0,312 \\
(0,209)\end{array}$ & $\begin{array}{l}-0,735^{* * *} \\
(0,195)\end{array}$ & $\begin{array}{c}-0,546 \\
(0,636)\end{array}$ & $\begin{array}{c}-0,065 \\
(0,631)\end{array}$ \\
\hline Bauberufe & $\begin{array}{l}-0,023 \\
(0,104) \\
\end{array}$ & $\begin{array}{l}-0,484^{* * *} \\
(0,100)\end{array}$ & $\begin{array}{c}-0,195 \\
(0,438) \\
\end{array}$ & $\begin{array}{c}0,198 \\
(0,485)\end{array}$ \\
\hline $\begin{array}{l}\text { Infrastruktur-/Transport- } \\
\text { dienstleistungsberufe }\end{array}$ & $\begin{array}{l}-0,328^{* * *} \\
(0,119)\end{array}$ & $\begin{array}{c}0,103 \\
(0,096) \\
\end{array}$ & $\begin{array}{l}-0,290 \\
(0,379)\end{array}$ & $\begin{array}{c}-0,084 \\
(0,341)\end{array}$ \\
\hline Finanzdienstleistungsberufe & $\begin{array}{c}0,056 \\
(0,160) \\
\end{array}$ & $\begin{array}{l}-0,508^{* * *} \\
(0,170)\end{array}$ & $\begin{array}{l}1,370^{* * *} \\
(0,514)\end{array}$ & $\begin{array}{c}0,823 \\
(0,585) \\
\end{array}$ \\
\hline Verwaltungsberufe & $\begin{array}{c}0,047 \\
(0,104) \\
\end{array}$ & $\begin{array}{l}-0,250^{* *} \\
(0,101)\end{array}$ & $\begin{array}{c}0,253 \\
(0,345) \\
\end{array}$ & $\begin{array}{l}-0,040 \\
(0,363) \\
\end{array}$ \\
\hline $\begin{array}{l}\text { produktionsnahe Dienstleistungs- } \\
\text { berufe }\end{array}$ & $\begin{array}{l}-0,170 \\
(0,106) \\
\end{array}$ & $\begin{array}{l}-0,503^{* * *} \\
(0,103)\end{array}$ & $\begin{array}{c}0,235 \\
(0,372) \\
\end{array}$ & $\begin{array}{l}-0,084 \\
(0,395)\end{array}$ \\
\hline $\begin{array}{l}\text { haushalts-/personenbezogene } \\
\text { Dienstleistungsberufe }\end{array}$ & $\begin{array}{l}-0,392^{* *} \\
(0,157) \\
\end{array}$ & $\begin{array}{l}-0,606^{* * *} \\
(0,147) \\
\end{array}$ & $\begin{array}{l}-0,248 \\
(0,570) \\
\end{array}$ & $\begin{array}{c}0,367 \\
(0,575) \\
\end{array}$ \\
\hline \multicolumn{5}{|l|}{ Qualifikation } \\
\hline $\begin{array}{l}\text { Durchschnitt zum Wirtschafts- } \\
\text { zweig }\end{array}$ & $\begin{array}{l}0,507^{* * *} \\
(0,060)\end{array}$ & $\begin{array}{l}0,157^{* * *} \\
(0,058) \\
\end{array}$ & $\begin{array}{l}0,515^{* * *} \\
(0,060)\end{array}$ & $\begin{array}{l}0,165^{* * *} \\
(0,058)\end{array}$ \\
\hline
\end{tabular}

\footnotetext{
${ }^{92}$ Im Nahrungsmittelbereich sind nur wenige Fälle enthalten.
} 


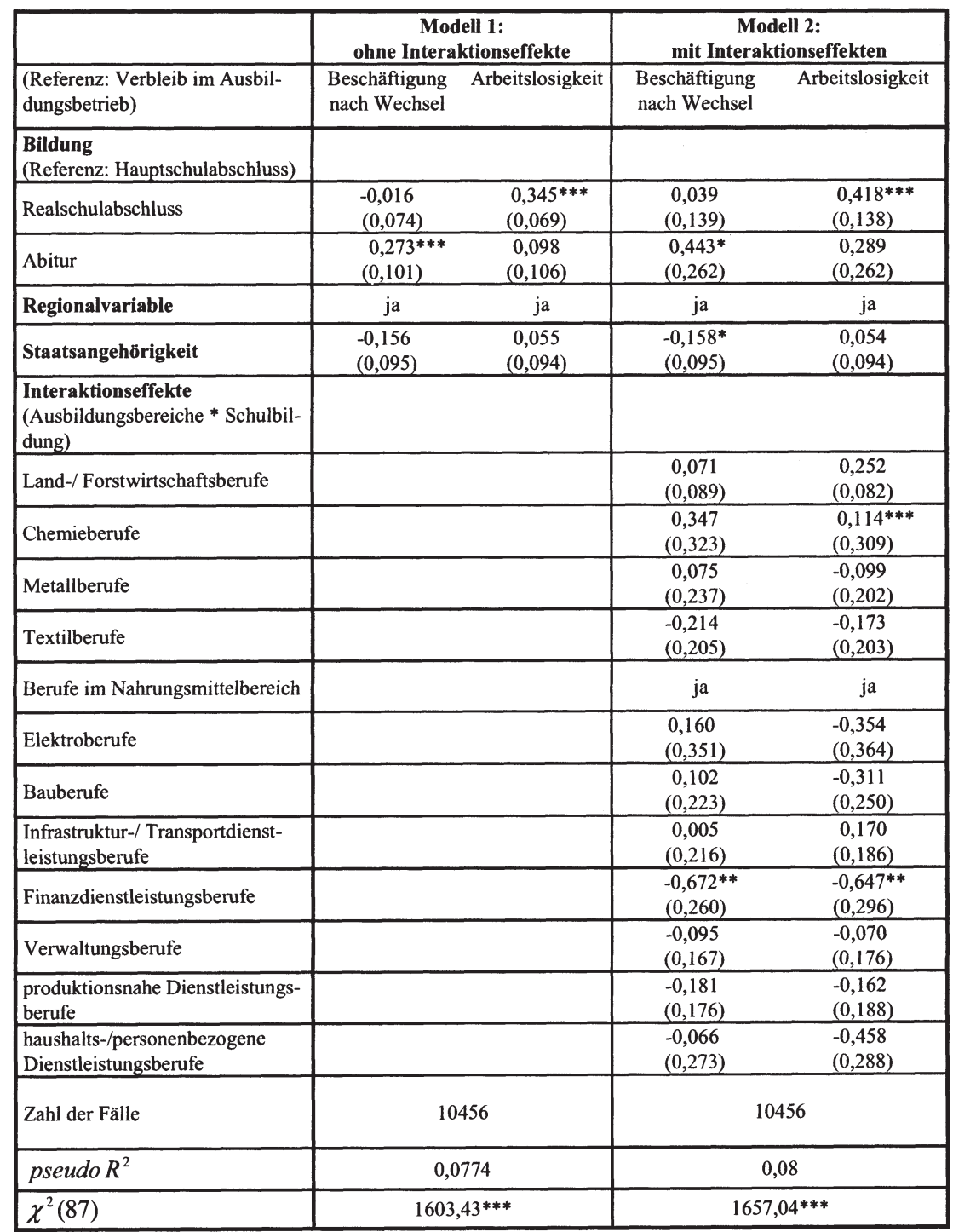

\section{Quelle: IABS 1975-2004, eigene Berechnungen}

*** $1 \%$-Signifikanzniveau; ** 5\%-Signifikanzniveau; * 10\%-Signifikanzniveau Werte in Klammern bezeichnen die Standardfehler

Bedeutung ist signifikant. Der Effekt der Ausbildungsvergütung verstärkt sich bei der Betrachtung der Wahrscheinlichkeit, nach der Ausbildung in Arbeitslo- 
sigkeit einzutreten. Aufgrund des hohen Anteils der Ausbildungsvergütungen an den gesamten Ausbildungskosten deutet dieser Einfluss darauf hin, dass die hohen durch die Ausbildung verursachten Kosten tendenziell zu besseren Aussichten auf einen Verbleib im Betrieb führen. Tätigt ein Ausbildungsbetrieb höhere Ausbildungskosten, spricht dies für eine entsprechend höhere Humankapitalinvestition. Eine Ausbildung bei hohen Ausbildungsvergütungen findet dann zur Deckung des eigenen Fachkräftebedarfs statt.

Ausbildungsvergütungen bilden gemeinsam mit den Gehältern der Fachkräfte und der Hilfsarbeiter eines Betriebes die Lohnsumme, deren Höhe von zwei Aspekten beeinflusst wird. Einerseits kommt eine hohe Lohnsumme zustande, wenn es sich um einen relativ kleinen Betrieb handelt, der aber hoch qualifizierte Arbeitskräfte beschäftigt. Dies sind insbesondere humankapitalintensive und daher produktive Unternehmen, weshalb die Gehälter auch entsprechend angepasst sind. ${ }^{93}$ Die durchschnittliche Lohnsumme ist relativ hoch. Die Ausbildung findet daher vor dem Hintergrund eines langfristigen Beschäftigungsverhältnisses statt, weshalb in diesem Fall ein negativer Einfluss auf die Wahrscheinlichkeit eines Betriebswechsels und einer Arbeitslosigkeit vorliegen müsste. Andererseits kann eine hohe Lohnsumme insbesondere bei einem großen Betrieb auf viele Beschäftigte zurückzuführen sein, die durchschnittliche (oder sogar unterdurchschnittliche) Löhne und Gehälter erhalten. Entsprechend ist die durchschnittliche Lohnsumme relativ gering. Hier sind die Auszubildenden als billige Arbeitskräfte zu betrachten. Infolgedessen sind die Beschäftigungsaussichten innerhalb des Ausbildungsbetriebes gering; die Wahrscheinlichkeit für einen Betriebswechsel steigt. Daher deutet der positiv signifikante Einfluss auf die Wahrscheinlichkeit, in einem anderen Betrieb tätig zu sein, auf einen produktionstheoretischen Zusammenhang hin. Bei einer marginalen Erhöhung der durchschnittlichen Lohnsumme steigt auch die Wahrscheinlichkeit um 13 Prozentpunkte, den Betrieb zu wechseln gegenüber der Wahrscheinlichkeit im Betrieb zu verbleiben. Dieser Effekt ist bei der Betrachtung des Arbeitslosigkeitsrisikos ebenfalls positiv und signifikant, der marginale Effekt ist aber geringer. Somit ist anzunehmen, dass vor allem berufsspezifisches $\mathrm{Hu}$ mankapital vermittelt wird und die Lohnsumme vorwiegend durch viele Arbeitskräfte zustande kommt, deren Lohn unter der einer ausgebildeten Fachkraft liegt. Da eine hohe Lohnsumme die Erträge des Unternehmens (Zwick 2007) schmälert, beschäftigen Ausbildungsbetriebe die Ausbildungsabsolventen nur dann weiter, wenn sie Fachkräfte benötigen.

Die Ausbildungsdauer hat lediglich bei der Wahrscheinlichkeit, nach der dualen Berufsausbildung arbeitslos zu werden, einen signifikanten Einfluss, welcher negativ, aber sehr gering ist. Einerseits spricht eine höhere Ausbildungs-

${ }^{93}$ Diese hohe durchschnittliche Lohnsumme der Betriebe kann auf eine Tarifbindung hindeuten, welche die Produktivität positiv beeinflusst. Auch dies bestätigt den investitionstheoretischen Ansatz, der einen negativen Effekt auf die Wahrscheinlichkeit für einen Betriebswechsel haben würde. 
dauer für eine Ausbildung, in der mehr Humankapital erworben werden kann. Die Ausbildung ist hierbei als Humankapitalinvestition zu sehen. Andererseits werden die Erträge höher bzw. die Nettokosten geringer (siehe auch Mühlemann et al. 2005), welche gegen Ende der Ausbildung entstehen bzw. vollständig gedeckt werden und sich in Bildungserträge wandeln. Eine Weiterbeschäftigung ist in diesem Fall erwünscht. So wirkt sich auch hier das erworbene Humankapital negativ auf die Wahrscheinlichkeit aus, den Betrieb zu wechseln, ebenso auf das Arbeitslosigkeitsrisiko. Denn je länger die Ausbildung dauert, desto besser sind die Bedingungen, Bildung zu erwerben, welche die Ausbildungsbetriebe nach Möglichkeit selbst nutzen. Zudem reduziert sich das Arbeitslosigkeitsrisiko bei zunehmender Ausbildungsdauer. Dieser Effekt ist auf dem 1\%-Niveau signifikant. Die Ausbildungsabsolventen mit einer längeren Ausbildung verfügen auch über einen höheren Bildungsstand. ${ }^{94}$

Auch die Betriebsgröße beeinflusst das Verlassen des Ausbildungsbetriebes signifikant. Die marginalen Effekte auf die abhängige Variable nehmen mit der Betriebsklassengröße zu. So wechseln Ausbildungsabsolventen, die in einem Betrieb mit über 500 Beschäftigten ausgebildet werden, weniger wahrscheinlich (-88 Prozentpunkte) den Ausbildungsbetrieb als Absolventen in einem Ausbildungsbetrieb mit bis zu neun Mitarbeitern. Bei der Betrachtung des Outcomes der Arbeitslosigkeit ist dieselbe Struktur der Wirkungen der Faktoren zu erkennen. Allerdings ist der marginale Effekt der Betriebsgröße auf das Arbeitslosigkeitsrisiko größer als auf die Wahrscheinlichkeit eines Betriebswechsels. Ausbildungsabsolventen aus einem größeren Ausbildungsbetrieb haben daher eine geringere Wahrscheinlichkeit, in Arbeitslosigkeit einzutreten, als Ausbildungsabsolventen aus einem Ausbildungsbetrieb, welcher der Referenzgruppe angehört. Schließlich bestätigen sie ebenfalls die Annahmen über den Einfluss der Betriebsgröße auf das Arbeitslosigkeitsrisiko. Ausbildungsabsolventen weisen ein höheres Niveau an Fähigkeiten auf, je größer der Ausbildungsbetrieb ist. Der Anteil an betriebsspezifischem Humankapital steigt mit der Größe des Unternehmens, was sich zudem auf das gesamte Bildungsniveau positiv auswirkt. Dies wiederum erklärt die gegenüber den Ausbildungsabsolventen eines kleinen Betriebs geringere Wahrscheinlichkeit für einen Betriebswechsel. Größere Betriebe bilden daher aus langfristigem Beschäftigungsinteresse aus, weshalb Ausbildungsabsolventen nach Möglichkeit weiterbeschäftigt werden, während kleinere Betriebe nach der Substitutionsstrategie ausbilden. Zudem kann eine geringe Anzahl an freiwilligen Wechseln angenommen werden, da die Möglichkeiten hinsichtlich des weiteren Erwerbslebens mit der Betriebsgröße steigen.

94 Der geringe marginale Effekt kann durch die Möglichkeit für gute Auszubildende, die Ausbildungszeit zu verkürzen, erklärt werden. Allerdings wurden in den letzten Jahren neue Ausbildungsberufe geschaffen, die eine kurze Ausbildungsdauer haben. So dauert eine Ausbildung bspw. zum/zur Änderungsschneider/in oder Metallschleifer/in nur 2 Jahre. Ein Zusammenhang zwischen Schulabschluss und Ausbildungsdauer ist daher nicht anzunehmen. 
Die Ausbildungsberufe beeinflussen ebenfalls die Wahrscheinlichkeit eines Betriebswechsels und das Arbeitslosigkeitsrisiko (Seibert 2007: 3). Die Referenz bei den Schätzungen bilden die land- und forstwirtschaftlichen Ausbildungsberufe. Gegenüber der Referenzgruppe haben andere Ausbildungsbereiche meist einen negativen marginalen Effekt auf die Wahrscheinlichkeit für einen Betriebswechsel. Ausbildungsabsolventen, die einen Beruf im Metallgewerbe erlernt haben, weisen eine signifikant niedrigere Wahrscheinlichkeit für einen Betriebswechsel auf als Ausbildungsabsolventen in land- und forstwirtschaftlichen Ausbildungsberufen. Der marginale Effekt dieses Ausbildungsbereiches ist zudem mit 58 Prozentpunkten relativ hoch. Ferner weisen die Ausbildungsabsolventen mit einem erlernten metallverarbeitenden Beruf ein geringeres Arbeitslosigkeitsrisiko gegenüber der Referenzgruppe auf. Hierbei handelt es sich zwar vor allem um produzierende handwerkliche Berufe, die sowohl in kleineren Betrieben als auch in größeren Industriebetrieben eingesetzt werden. Dennoch beschränkt sich das Humankapital nicht nur auf die berufsspezifische Komponente. Bei Verlassen des Ausbildungsbetriebes findet dieses aber gegenüber den land- und forstwirtschaftlichen Berufen einen größeren Anwendungsbereich. Mit einem Textilberuf reduziert sich gegenüber der Referenzgruppe die Wahrscheinlichkeit für einen Betriebswechsel signifikant um 60 Prozentpunkte. Der marginale Effekt ist am stärksten. Darüber hinaus weisen junge Erwachsene in diesem Bereich ebenfalls eine signifikant geringere Wahrscheinlichkeit (63 Prozentpunkte) gegenüber der Referenzgruppe auf, arbeitslos zu werden. Diese Effekte entsprechen jedoch nicht den Erwartungen, da es sich um eine Branche handelt, die stark vom Strukturwandel betroffen ist. Gerade deswegen ist der Anteil an Ausbildungsabsolventen im Textilbereich auch sehr gering, sodass die wenigen Absolventen scheinbar gute Berufsaussichten aufweisen. Auch junge Erwachsene, die ihre Ausbildung in Infrastruktur- und Transportdienstleistungsberufen abschließen, wechseln weniger wahrscheinlich als Personen in land- und forstwirtschaftlichen Berufen. Der marginale Effekt ist aber wesentlich geringer als der der Textil- und metallverarbeitenden Ausbildungsberufe. Jedoch erhöht sich das Arbeitslosigkeitsrisiko gegenüber der Referenzgruppe. Diese Ausbildungsabsolventen sehen sich einem erhöhten Arbeitslosigkeitsrisiko deshalb gegenüber, weil der Einfluss des Ausbildungsbereiches negativ auf die Verbleibswahrscheinlichkeit wirkt und die Deskription zugleich auch eine unterdurchschnittliche Verbleibsquote im Ausbildungsbetrieb angibt. Infrastruktur- und Transportdienstleistungsberufe sind zumeist von großen Unternehmen, wie der Deutschen Bahn oder städtischen Verkehrsunternehmen, gekennzeichnet, die das entsprechende Teilsegment nahezu vollständig abdecken. Daraus kann geschlossen werden, dass Fachkräfte mit dem Ziel einer Weiterbeschäftigung ausgebildet werden. Daher sind Ausbildungsbetriebe in diesem Bereich bestrebt, ihre Ausbildungsabsolventen zu übernehmen. Wenn diese aber nicht weiterbeschäftigt werden können, verringern sich die Chancen dieser jungen Erwachsenen auf dem Arbeitsmarkt relativ 
zu der Referenzgruppe. Eine Weiterbeschäftigung erfolgt dann nicht, sobald die wirtschaftliche Situation es nicht zulässt, alle Absolventen zu übernehmen oder die Fähigkeiten unter den Qualifikationsanforderungen des Ausbildungsbetriebes liegen. In diesem Fall bedeutet das Verlassen des Betriebes ein negatives „Signalling“ auf dem Arbeitsmarkt, welches anderen Unternehmen zeigt, dass der Ausbildungsabsolvent unterdurchschnittlich ist. Darüber hinaus ist die Ausbildung sehr betriebsspezifisch ${ }^{95}$, weshalb eine Beschäftigung vorwiegend in dieser Branche zu finden ist, diese für einen Wechsel aber aufgrund des Oligopols bzw. Monopols wenig Alternativen bietet. Daher handelt es sich in der Regel um gut ausgebildete Fachkräfte, die nach Möglichkeit wieder eine qualifikationsadäquate Tätigkeit aufnehmen wollen. Die in den Deskriptionen beschriebenen positiven Aspekte dieses Ausbildungsbereiches kehren sich demnach in nachteilige Beschäftigungsaussichten für diejenigen um, die nicht im Betrieb verbleiben können. Der kleine Einsatzbereich sowie die durch das betriebsspezifische Humankapital geringe Anzahl an Beschäftigungsalternativen erhöhen die Wahrscheinlichkeit, arbeitslos zu werden. Jedoch ist dieser Effekt auf das Arbeitslosigkeitsrisiko nicht signifikant. Schließlich beginnen Ausbildungsabsolventen, die einen haushalts- bzw. personenbezogenen Dienstleistungsberuf haben, nach der dualen Berufsausbildung weniger wahrscheinlich eine neue Tätigkeit in einem anderen Betrieb gegenüber der Referenzgruppe. Zudem weisen diese auch ein signifikant geringeres Arbeitslosigkeitsrisiko auf. Bei den beschriebenen Bereichen handelt es sich um Berufsgruppen, die bessere Beschäftigungssaussichten in ihren jeweiligen Bereichen und Betrieben bieten, allerdings mit einigen Ausnahmen von Berufen im haushalts- und personenbezogenen Dienstleistungsbereich (bspw. Kellner). Aufgrund des hohen Anteils an allgemeinem Humankapital sind die Ausbildungsabsolventen aber flexibel -wenn auch teilweise außerhalb des Ausbildungsbereiches als angelernte Arbeitskraft oder Hilfskraft- einsetzbar. Gegenüber der Referenzgruppe liegen hier die Ausbildungsqualität und das vermittelte Humankapital höher.

Einen signifikanten negativen Einfluss auf die Eintrittswahrscheinlichkeit in Arbeitslosigkeit haben darüber hinaus vor allem Ausbildungsabsolventen der Bereiche für Elektro- und Bauberufe, für Finanzdienstleistungen, für Verwaltungsberufe sowie für produktionsnahe Dienstleistungsberufe. Die Verwaltungsberufe üben hierbei einen signifikanten Effekt auf das Arbeitslosigkeitsrisiko nur auf dem 5\%-Niveau aus. Eine Ausbildung in diesen Tätigkeitsbereichen deutet auf gute Voraussetzungen für das weitere Erwerbsleben hin. Allerdings sind die negativen Vorzeichen für die jeweiligen Bereiche unterschiedlich zu bewerten. Für Ausbildungsabsolventen in Bauberufen ergibt sich eine Vielzahl von Gründen für das geringe Arbeitslosigkeitsrisiko gegenüber den Ausbildungsberufen der Land- und Forstwirtschaft. Zum einen fällt der Abschluss der dualen Berufsausbildung meist in das Sommerhalbjahr, in dem den

${ }^{95} \mathrm{Zu}$ diesem Bereich sind Berufe, wie u. a. Schienenfahrzeugführer, Eisenbahnbetriebsregler, -schaffner oder Postverteiler zu zählen. 
Unternehmen der Baubranche tendenziell die meisten Aufträge vorliegen, zu deren Durchführung sie Arbeitskräfte benötigen. Zum anderen liegt die Tradition der dualen Berufsausbildung in dieser Branche, weswegen das berufsspezifische Teilsegment stark ausgeprägt ist. Eine hohe Mobilität zwischen den einzelnen Betrieben dieser Branchen kennzeichnet dies, was den Ausbildungsabsolventen viele Beschäftigungsmöglichkeiten eröffnet. Schließlich aber finden Personen mit einer Ausbildung in diesem Bereich häufig auch Arbeit als angelernte Arbeitskräfte bzw. Hilfsarbeiter in Produktionsbereichen großer Unternehmen, was gute Voraussetzungen für eine unmittelbare Fortsetzung der Erwerbstätigkeit liefert. Die geringere Arbeitslosigkeitswahrscheinlichkeit in den Finanzdienstleistungsberufen, in den Verwaltungsberufen sowie in Berufen der produktionsnahen Dienstleistungen ist auf die gute Ausbildung und auf das hohe Bildungsniveau zurückzuführen. Ausbildungsabsolventen, die bereits eine gute Schulausbildung haben, erlernen einen Beruf in diesem Bereich. Zudem bietet auch diese Branche eine Vielzahl an Beschäftigungsmöglichkeiten für junge Erwachsene, die ihren Ausbildungsbetrieb verlassen mussten.

Neben den betrieblichen Faktoren tragen auch die individuellen Faktoren zur Erklärung eines Betriebswechsels sowie des Arbeitslosigkeitsrisikos nach der dualen Berufsausbildung bei. Absolventen, die innerhalb des Wirtschaftsbereiches ein unterdurchschnittliches Qualifikationsniveau durch die betriebliche Ausbildung aufweisen, haben einen signifikanten Einfluss auf die Wechselund Arbeitslosigkeitswahrscheinlichkeit. Dieser Indikator ist eine Dummyvariable und setzt sich aus der Qualifikationsstruktur zusammen, die innerhalb des jeweiligen Ausbildungsbetriebes gegeben ist und wird dann dem Qualifikationsniveau des Wirtschaftszweiges, in dem der Ausbildungsbetrieb agiert, gegenübergestellt (siehe auch Kapitel 8.1.1). Ausbildungsabsolventen erhalten nur geringes zusätzliches informelles Humankapital von den anderen Mitarbeitern innerhalb des Ausbildungsbetriebs vermittelt, wenn deren Bildungsniveau niedrig ist. Die Beschäftigten weisen dann ebenfalls kein sehr hohes Humankapital auf, was auf einen produktionstheoretischen Ausbildungsbetrieb schließen lässt (vgl. Asplund 2004; Blundell et al. 1999; Kap. 3.2.2.2.1). Ausbildungsabsolventen, die in solchen Betrieben ausgebildet werden ${ }^{96}$, haben eine um 51 Prozentpunkte höhere Wahrscheinlichkeit, eine neue Tätigkeit in einem anderen Unternehmen aufzunehmen und demnach im Ausbildungsbetrieb nicht weiterbeschäftigt zu werden. Dieser Effekt ist signifikant. Sie weisen ebenfalls ein höheres Arbeitslosigkeitsrisiko als diejenigen auf, die in einem Betrieb mit überdurchschnittlichem Niveau an Bildung ihre Ausbildung absolvieren.

Die Effekte der Schulbildung auf die Wahrscheinlichkeit, den Betrieb zu wechseln, sind nicht eindeutig. So wechseln Ausbildungsabsolventen mit Realschulabschluss weniger wahrscheinlich den Betrieb. Personen mit mittlerer

\footnotetext{
96 Absolventen eines Betriebes, der innerhalb des entsprechenden Wirtschaftszweiges ein unterdurchschnittliches Qualifikationsniveau aufweist, werden mit eins kodiert, die anderen mit null.
} 
Reife haben höhere Beschäftigungsaussichten im Betrieb, was die geringere Wechselwahrscheinlichkeit erklärt. Der Einfluss des Schulniveaus ist aber nicht signifikant. Dafür weisen sie ein signifikant höheres Arbeitslosigkeitsrisiko gegenüber den Absolventen mit Hauptschulabschluss auf. Das erhöhte Arbeitslosigkeitsrisiko, das bereits aus den deskriptiven Auswertungen hervorgeht, wird durch die Schätzungen bestätigt. Im Falle des Verlassens des Ausbildungsbetriebs beachten Ausbildungsabsolventen aufgrund des höheren und betriebsspezifischeren Humankapitals lediglich ein beschränktes Stellenangebot. Dagegen beeinflusst der höchste Schulabschluss signifikant die Betriebswechselwahrscheinlichkeit. Absolventen mit Abitur wechseln häufiger den Betrieb zur Verbesserung ihrer Arbeitsmarktsituation, aber auch wegen der Aufnahme eines Studiums. Nach den Regressionsschätzungen weisen die Ausbildungsabsolventen mit Abitur gegenüber denjenigen mit einem Hauptschulabschluss eine höhere Wahrscheinlichkeit auf, arbeitslos zu werden. Dieser Einfluss ist aber nicht signifikant. Winkelmann (1994) ermittelt sogar für beide Schulabschlüsse keinen signifikanten Einfluss auf die erste Arbeitslosigkeitsphase nach der dualen Berufsausbildung. Diese Ergebnisse stehen im Widerspruch zu der Theorie, wonach Ausbildungsabsolventen mit geringerem Humankapitalbestand eher in Arbeitslosigkeit eintreten als Personen mit hohem Humankapitalbestand, werden aber von einer Studie (Dietrich/Kleinert 2005) des IAB bestätigt. Danach wiesen junge Erwachsene mit Realschulabschluss das höchste Arbeitslosigkeitsrisiko auf.

Vor allem der Vergleich zwischen Hauptschul- und Realschulabsolventen verdeutlicht, dass ein höherer Schulabschluss und damit ein höheres Bildungsniveau nicht vor einem Arbeitsmarkteintritt, der von einem üblichen Eintritt ins Erwerbsleben nach der dualen Berufsausbildung (Weiterbeschäftigung im Ausbildungsbetrieb) abweicht, schützt. Dieser Zusammenhang entspricht nicht den Ergebnissen bisheriger Studien, wonach Realschulabsolventen gute Beschäftigungsaussichten haben (Büchel 2002; Büchel/Neubäumer 2001; Kap. 7.1.1). Dies steht ebenso im Widerspruch zu den Hypothesen, dass eine höhere Bildung, welche sich vor allem durch den Schulabschluss bestimmt, die Wahrscheinlichkeit zu wechseln bzw. arbeitslos zu werden, verringert. ${ }^{97}$ Es ist daher zu vermuten, dass die Wirkung der Schulbildung in den Hintergrund der Wirkung des Berufsausbildungsabschlusses tritt. Für einen künftigen Arbeitgeber ist daher der Berufsbildungsabschluss oder auch der Ruf des Ausbildungsbetriebs bei einer Einstellung ausschlaggebend (vgl. Niederalt 2004). Aufgrund der Erfassungsmodalitäten der Bildungsvariablen sind die Ergebnisse des Einflusses vorsichtig zu interpretieren.

Unter Berücksichtigung von Interaktionseffekten (siehe Kapitel 8.1.1) ergeben sich vor allem Änderungen bei dem Effekt der Bildungsvariablen und der

\footnotetext{
${ }^{97}$ Dieser Widerspruch zwischen Annahmen und empirischer Evidenz kommt erst in jüngerer Zeit zum Vorschein. Für die älteren Kohorten (siehe Tabellen 8 und 9) können die in den Hypothesen die angenommenen negativen Zusammenhänge nicht bestätigt werden.
} 
Ausbildungsbereiche. Absolventen mit Realschulabschluss wechseln gegenüber Personen mit Hauptschulabschluss weniger wahrscheinlich, dieser Effekt ist aber nicht signifikant. Der Effekt für Absolventen mit Abitur schwächt sich ab.

Der signifikante Einfluss der Ausbildungsberufe im ersten Modell verdeutlicht sowohl die Wahrscheinlichkeit eines Betriebswechsels als auch das Arbeitslosigkeitsrisiko. Im Modell zwei wird der Einfluss der Ausbildungsberufe insignifikant. Dies kann auf die Unterschiede in der Schulbildung zurückgeführt werden, anhand derer die Betriebe Auszubildende aus den Schulabgängern auswählen. Infolgedessen differiert auch die Bedeutung von Schulabschlüssen in den jeweiligen Ausbildungsbereichen. Denkbar ist bspw., dass im Bau praktische Fähigkeiten von Vorteil sind, weswegen vorwiegend Hauptschulabsolventen einen Beruf in dieser Branche erlernen. Dagegen werden in Finanzdienstleistungsberufen vorwiegend mathematische Fähigkeiten vorausgesetzt, über die Abiturienten aufgrund der längeren Schulzeit eher verfügen, weshalb diese in diesem Bereich häufig anzutreffen sind. Um die Bedeutung der Schulbildung innerhalb der Ausbildungsbereiche überprüfen zu können, dienen die Interaktionseffekte im zweiten Modell daher zur Kontrolle. Der Einfluss der Ausbildungsbereiche ist nicht mehr signifikant, das heißt, die Unterschiede zwischen den Bereichen sind tatsächlich auf die Schulbildung zurückzuführen. Jedoch sind die Interaktionseffekte selbst auch nicht signifikant. Daher spielen Unterschiede innerhalb der Ausbildungsbereiche, welche durch die Schulbildung hervorgerufen werden, für den Verbleib nach der dualen Berufsausbildung ebenfalls keine bedeutende Rolle. So haben Absolventen mit Abitur dieselben Chancen wie Absolventen mit Hauptschulabschluss innerhalb der jeweiligen Ausbildungsbereiche. Diese insignifikanten Ergebnisse hängen mit der systematischen Zuordnung der Schulabsolventen zu den einzelnen Ausbildungsbereichen zusammen. Entsprechend ist die Streuung innerhalb der Ausbildungsbereiche hinsichtlich der Schulbildung gering, was zu insignifikanten Einflüssen der Interaktionsvariablen führt. Wenn daher nicht über diese Variable kontrolliert wird, liegt eine hohe Streuung zwischen den Ausbildungsbereichen vor und damit sind die Unterschiede (im ersten Modell) auch signifikant.

Dennoch wirkt sich eine Ausbildung in Finanzdienstleistungsberufen signifikant positiv auf eine Beschäftigung in einem anderen Betrieb gegenüber den Referenzausbildungsberufen der Land- und Forstwirtschaft aus. Das Arbeitslosigkeitsrisiko erhöht sich gegenüber der Referenzgruppe ebenfalls, ist aber nicht signifikant. Der Schulabschluss hat aber für den Verbleib einen signifikanten Einfluss. Ein höheres Bildungsniveau mit einem Ausbildungsberuf im Finanzdienstleistungsbereich schützt vor einem Betriebswechsel sowie vor einem Arbeitslosigkeitsrisiko. In diesem Bereich sind Berufe zugeordnet, welche unterschiedliche Anforderungen an das Humankapital stellen und die junge Erwachsene mit unterschiedlichem Bildungshintergrund erlernen. Sowohl Ausbildungsberufe, die einen hohen Qualifikationsstand (Bankfachleute, Groß- 
und Einzelhandelskaufleute) als auch Berufe, die weniger hohe Qualifikationen (Verkäufer) fordern, sind enthalten. Entsprechend sinkt die Wahrscheinlichkeit für Absolventen eines Finanzdienstleistungsberufes mit höherer Schulbildung, den Betrieb zu wechseln (-67 Prozentpunkte). Bildung schützt in diesem Fall insbesondere vor unfreiwilligen Betriebswechseln. In diesem Bereich bilden die Ausbildungsbetriebe daher mit hoher Wahrscheinlichkeit aus langfristigem Beschäftigungsinteresse aus, wenn es sich um Ausbildungsberufe handelt, die hohe Qualifikationsanforderungen an die Ausbildungsabsolventen stellen. Dies führt meist auch zu einer guten Ausbildungsqualität. Vor allem Branchen, die vorwiegend dem tertiären Sektor zuzuordnen sind, weisen negative Effekte auf. Das heißt, dass sich die Wahrscheinlichkeit verringert, nach der dualen Berufsausbildung eine Tätigkeit in einem anderen Betrieb aufzunehmen je höher der Bildungsabschluss der Ausbildungsabsolventen ist. Diese Einflüsse sind aber meist nicht signifikant. Ferner ist für die Finanzdienstleistungsberufe ein die Bildung überlagernder Effekt anzunehmen, da insbesondere die Variable dieses Ausbildungsbereiches weiterhin signifikant ist. Schließlich übt die Bildung einen signifikant positiven Einfluss innerhalb der Gruppe der Chemieund holzverarbeitenden Ausbildungsberufe auf die Wahrscheinlichkeit aus, nach der dualen Berufsausbildung arbeitslos zu werden. Bessere Ausbildungsabsolventen in diesem Bereich werden wahrscheinlicher arbeitslos als Absolventen mit einer niedrigeren Schulbildung. Aufgrund dieses positiven Zusammenhangs kann auf Strukturänderungen geschlossen werden, die den Effekt der Schulbildung überlagern.

Die Tabelle 8 und Tabelle 9 zeigen Wahrscheinlichkeiten eines Betriebswechsels bzw. einer Arbeitslosigkeit der Ausbildungskohorten zwischen 19961999 sowie zwischen 1992-1995. Diese logistischen Schätzungen wurden durchgeführt, um zu überprüfen, ob die marginalen Effekte und deren Signifikanz auf die verschiedenen Outcomes zwischen den Abschlusskohorten und unter verschiedenen wirtschaftlichen Rahmenbedingungen variieren. Im Folgenden werden aber nur die Veränderungen aufgeführt, die für die Fragestellung von Bedeutung sind.

Bei der Gegenüberstellung der Abschlusskohorten ist zu erkennen, dass sich die Wirkung der Einflussfaktoren auf die Wahrscheinlichkeit eines Betriebswechsels bzw. einer Arbeitslosigkeit nicht wesentlich verändert. So bleiben über die betrachteten Abschlusskohorten Wirkungen der Ausbildungsvergütungen auf einen Betriebswechsel mit anschließender Beschäftigung und auf Arbeitslosigkeit signifikant. Während dieses Betrachtungszeitraumes nimmt aber die Bedeutung dieser Einflussvariablen zu. Außerdem verstärkt sich der Einfluss der betrieblichen Lohnsumme, während die Bedeutung der Ausbildungsdauer über die Kohorten zwar signifikant bleibt, aber geringer wird. Auch die haben Absolventen eines Ausbildungsbetriebes mit mehr als 500 Beschäftigten im Zeitablauf eine geringere Wahrscheinlichkeit für einen Betriebswechsel. 
Tabelle 8: Einflussfaktoren auf den Verbleib nach der dualen Berufsausbildung für die Abschlusskohorten 1996-1999 - Schätzungen eines multi-nomialen Logitmodells

\begin{tabular}{|c|c|c|c|c|}
\hline \multirow[b]{2}{*}{$\begin{array}{l}\text { (Referenz: Verbleib im Ausbil- } \\
\text { dungsbetrieb) }\end{array}$} & \multicolumn{2}{|c|}{$\begin{array}{c}\text { Modell 1: } \\
\text { ohne Interaktionseffekte }\end{array}$} & \multicolumn{2}{|c|}{$\begin{array}{c}\text { Modell 2: } \\
\text { mit Interaktionseffekten }\end{array}$} \\
\hline & $\begin{array}{l}\text { Beschäftigung } \\
\text { nach Wechsel }\end{array}$ & Arbeitslosigkeit & $\begin{array}{l}\text { Beschäftigung } \\
\text { nach Wechsel }\end{array}$ & Arbeitslosigkeit \\
\hline Ausbildungsvergütungen (log) & $\begin{array}{l}-1,152^{* * *} \\
(0,101) \\
\end{array}$ & $\begin{array}{l}-1,484^{* * *} \\
(0,094)\end{array}$ & $\begin{array}{l}-1,152^{* * *} \\
(0,102)\end{array}$ & $\begin{array}{l}-1,490^{* * *} \\
(0,094)\end{array}$ \\
\hline betriebliche Lohnsumme (log) & $\begin{array}{l}0,090^{* * *} \\
(0,025)\end{array}$ & $\begin{array}{l}0,087^{* * *} \\
(0,024)\end{array}$ & $\begin{array}{l}0,093^{* * *} \\
(0,025)\end{array}$ & $\begin{array}{l}0,086^{* * *} \\
(0,024)\end{array}$ \\
\hline Ausbildungsdauer (log) & $\begin{array}{l}-0,510^{* * *} \\
(0,121)\end{array}$ & $\begin{array}{l}-0,215 \\
(0,113)\end{array}$ & $\begin{array}{l}-0,507^{* * *} \\
(0,122)\end{array}$ & $\begin{array}{l}-0,200^{*} \\
(0,114)\end{array}$ \\
\hline \multicolumn{5}{|l|}{$\begin{array}{l}\text { Betriebsgröße } \\
\text { (Referenz: 1-9 Mitarbeiter) }\end{array}$} \\
\hline 10-49 Mitarbeiter & $\begin{array}{l}-0,127 \\
(0,082)\end{array}$ & $\begin{array}{l}-0,113 \\
(0,071)\end{array}$ & $\begin{array}{l}-0,132 \\
(0,082)\end{array}$ & $\begin{array}{l}-0,109 \\
(0,071)\end{array}$ \\
\hline 50-499 Mitarbeiter & $\begin{array}{l}-0,334^{* * *} \\
(0,113)\end{array}$ & $\begin{array}{l}-0,470^{* * *} \\
(0,104)\end{array}$ & $\begin{array}{l}-0,334^{*} \\
(0,113) \\
\end{array}$ & $\begin{array}{l}-0,465^{* * *} \\
(0,104) \\
\end{array}$ \\
\hline$>500$ Mitarbeiter & $\begin{array}{l}-0,684^{* * *} \\
(0,171)\end{array}$ & $\begin{array}{l}-1,530^{* * *} \\
(0,171)\end{array}$ & $\begin{array}{l}-0,677^{* * *} \\
(0,171) \\
\end{array}$ & $\begin{array}{l}-1,536^{* * *} \\
(0,171) \\
\end{array}$ \\
\hline \multicolumn{5}{|l|}{$\begin{array}{l}\text { Ausbildungsberufe (Referenz: } \\
\text { Land-/ Forstwirtschaftsberufe) }\end{array}$} \\
\hline Chemieberufe & $\begin{array}{c}0,080 \\
(0,201) \\
\end{array}$ & $\begin{array}{c}0,078 \\
(0,184)\end{array}$ & $\begin{array}{l}-0,228 \\
(0,627)\end{array}$ & $\begin{array}{c}0,079 \\
(0,595) \\
\end{array}$ \\
\hline Metallberufe & $\begin{array}{l}-0,387^{* * *} \\
(0,116)\end{array}$ & $\begin{array}{l}-0,176^{*} \\
(0,101) \\
\end{array}$ & $\begin{array}{l}-0,242 \\
(0,379) \\
\end{array}$ & $\begin{array}{l}-0,016 \\
(0,356) \\
\end{array}$ \\
\hline Textilberufe & $\begin{array}{l}-0,511^{* * *} \\
(0,092)\end{array}$ & $\begin{array}{l}-0,469^{* * *} \\
(0,082)\end{array}$ & $\begin{array}{l}-0,033 \\
(0,371) \\
\end{array}$ & $\begin{array}{c}0,008 \\
(0,362) \\
\end{array}$ \\
\hline $\begin{array}{l}\text { Berufe im Nahrungsmittelbe- } \\
\text { reich }^{98}\end{array}$ & ja & ja & ja & ja \\
\hline Elektroberufe & $\begin{array}{l}-0,303 \\
(0,212) \\
\end{array}$ & $\begin{array}{l}-0,474 \\
(0,193) \\
\end{array}$ & $\begin{array}{l}-0,177 \\
(0,648) \\
\end{array}$ & $\begin{array}{l}-0,145 \\
(0,632) \\
\end{array}$ \\
\hline Bauberufe & $\begin{array}{l}-0,150 \\
(0,097)\end{array}$ & $\begin{array}{l}-0,326^{* * *} \\
(0,091)\end{array}$ & $\begin{array}{l}-0,377 \\
(0,414)\end{array}$ & $\begin{array}{c}0,071 \\
(0,415)\end{array}$ \\
\hline $\begin{array}{l}\text { Infrastruktur-/Transport- } \\
\text { dienstleistungsberufe }\end{array}$ & $\begin{array}{l}-0,332^{* * *} \\
(0,107)\end{array}$ & $\begin{array}{c}0,179 \\
(0,089)\end{array}$ & $\begin{array}{l}-0,239 \\
(0,344)\end{array}$ & $\begin{array}{c}0,135 \\
(0,311)\end{array}$ \\
\hline Finanzdienstleistungsberufe & $\begin{array}{l}0,478^{* * *} \\
(0,151)\end{array}$ & $\begin{array}{l}-0,635^{* * *} \\
(0,190)\end{array}$ & $\begin{array}{l}0,876^{* *} \\
(0,502)\end{array}$ & $\begin{array}{l}-0,198 \\
(0,679)\end{array}$ \\
\hline Verwaltungsberufe & $\begin{array}{l}-0,438^{* * *} \\
(0,103)\end{array}$ & $\begin{array}{l}-0,463^{* * *} \\
(0,098)\end{array}$ & $\begin{array}{l}-0,095 \\
(0,342) \\
\end{array}$ & $\begin{array}{l}-0,430 \\
(0,353) \\
\end{array}$ \\
\hline $\begin{array}{l}\text { produktionsnahe Dienstleistungs- } \\
\text { berufe }\end{array}$ & $\begin{array}{l}-0,528^{* * *} \\
(0,111)\end{array}$ & $\begin{array}{l}-0,463^{* * *} \\
(0,106)\end{array}$ & $\begin{array}{l}-0,565 \\
(0,390)\end{array}$ & $\begin{array}{l}-0,476 \\
(0,388)\end{array}$ \\
\hline $\begin{array}{l}\text { haushalts-/personenbezogene } \\
\text { Dienstleistungsberufe }\end{array}$ & $\begin{array}{l}-0,607^{* * *} \\
(0,144)\end{array}$ & $\begin{array}{l}-0,478^{* * *} \\
(0,131)\end{array}$ & $\begin{array}{l}-0,278 \\
(0,479)\end{array}$ & $\begin{array}{l}-0,680 \\
(0,486)\end{array}$ \\
\hline \multicolumn{5}{|l|}{ Qualifikation } \\
\hline $\begin{array}{l}\text { Durchschnitt zum Wirtschafts- } \\
\text { zweig }\end{array}$ & $\begin{array}{l}0,592^{* * *} \\
(0,055)\end{array}$ & $\begin{array}{l}0,551^{* * *} \\
(0,051)\end{array}$ & $\begin{array}{l}0,596^{* * *} \\
0,055)\end{array}$ & $\begin{array}{l}0,554^{* * *} \\
(0,071)\end{array}$ \\
\hline Fortsetzung Tabelle 8 auf der näch & ten Seite & & & \\
\hline
\end{tabular}

${ }^{98}$ Im Nahrungsmittelbereich sind nur wenige Fälle enthalten. 


\begin{tabular}{|c|c|c|c|c|}
\hline \multirow[b]{2}{*}{$\begin{array}{l}\text { (Referenz: Verbleib im Ausbil- } \\
\text { dungsbetrieb) }\end{array}$} & \multicolumn{2}{|c|}{$\begin{array}{c}\text { Modell 1: } \\
\text { ohne Interaktionseffekte }\end{array}$} & \multicolumn{2}{|c|}{$\begin{array}{c}\text { Modell 2: } \\
\text { mit Interaktionseffekten }\end{array}$} \\
\hline & $\begin{array}{l}\text { Beschäftigung } \\
\text { nach Wechsel }\end{array}$ & Arbeitslosigkeit & $\begin{array}{l}\text { Beschäftigung } \\
\text { nach Wechsel }\end{array}$ & Arbeitslosigkeit \\
\hline \multicolumn{5}{|l|}{$\begin{array}{l}\text { Bildung } \\
\text { (Referenz: Hauptschulabschluss) }\end{array}$} \\
\hline Realschulabschluss & $\begin{array}{c}0,029 \\
(0,068)\end{array}$ & $\begin{array}{c}0,074 \\
(0,061)\end{array}$ & $\begin{array}{c}0,117 \\
(0,138)\end{array}$ & $\begin{array}{c}0,131 \\
(0,104)\end{array}$ \\
\hline Abitur & $\begin{array}{c}0,508 \\
(0,098) \\
\end{array}$ & $\begin{array}{l}-0,407 * * * \\
(0,108)\end{array}$ & $\begin{array}{l}0,694^{* * *} \\
(0,267)\end{array}$ & $\begin{array}{l}-0,339 \\
(0,171) \\
\end{array}$ \\
\hline Regionalvariable & ja & ja & ja & $\mathrm{ja}$ \\
\hline Staatsangehörigkeit & $\begin{array}{l}-0,107 \\
(0,083)\end{array}$ & $\begin{array}{l}-0,298^{* * *} \\
(0,074)\end{array}$ & $\begin{array}{l}-0,104 \\
(0,084)\end{array}$ & $\begin{array}{l}-0,295^{* * *} \\
(0,074)\end{array}$ \\
\hline \multicolumn{5}{|l|}{$\begin{array}{l}\text { Interaktionseffekte } \\
\text { (Ausbildungsbereiche * Schulbil- } \\
\text { dung) }\end{array}$} \\
\hline Land-/ Forstwirtschaftsberufe & & & $\begin{array}{l}-0,144 \\
(0,106) \\
\end{array}$ & $\begin{array}{c}0,092 \\
(0,104) \\
\end{array}$ \\
\hline Chemieberufe & & & $\begin{array}{c}0,160 \\
(0,330)\end{array}$ & $\begin{array}{c}0,013 \\
(0,329)\end{array}$ \\
\hline Metallberufe & & & $\begin{array}{l}-0,095 \\
(0,204)\end{array}$ & $\begin{array}{l}-0,081 \\
(0,195)\end{array}$ \\
\hline Textilberufe & & & $\begin{array}{l}-0,277 \\
(0,195)\end{array}$ & $\begin{array}{l}-0,252 \\
(0,192) \\
\end{array}$ \\
\hline Berufe im Nahrungsmittelbereich & & & ja & $\mathrm{ja}$ \\
\hline Elektroberufe & & & $\begin{array}{l}-0,081 \\
(0,366)\end{array}$ & $\begin{array}{l}-0,190 \\
(0,369)\end{array}$ \\
\hline Bauberufe & & & $\begin{array}{c}0,098 \\
(0,213)\end{array}$ & $\begin{array}{l}-0,208 \\
(0,219)\end{array}$ \\
\hline $\begin{array}{l}\text { Infrastruktur-/ Transportdienst- } \\
\text { leistungsberufe }\end{array}$ & & & $\begin{array}{l}-0,061 \\
(0,188)\end{array}$ & $\begin{array}{c}0,043 \\
(0,170)\end{array}$ \\
\hline Finanzdienstleistungsberufe & & & $\begin{array}{l}-0,230 \\
(0,253)\end{array}$ & $\begin{array}{l}-0,226 \\
(0,353)\end{array}$ \\
\hline Verwaltungsberufe & & & $\begin{array}{l}-0,183 \\
(0,166)\end{array}$ & $\begin{array}{l}-0,009 \\
(0,178)\end{array}$ \\
\hline $\begin{array}{l}\text { produktionsnahe Dienstleistungs- } \\
\text { berufe }\end{array}$ & & & $\begin{array}{l}-0,017 \\
(0,183)\end{array}$ & $\begin{array}{c}0,010 \\
(0,192)\end{array}$ \\
\hline $\begin{array}{l}\text { haushalts-/personenbezogene } \\
\text { Dienstleistungsberufe }\end{array}$ & & & $\begin{array}{l}-0,190 \\
(0,240) \\
\end{array}$ & $\begin{array}{c}0,114 \\
(0,248) \\
\end{array}$ \\
\hline Zahl der Fälle & & 694 & & \\
\hline pseudo $R^{2}$ & & 692 & & \\
\hline$\chi^{2}(87)$ & & $81^{* * *}$ & 162 & $1 * * *$ \\
\hline
\end{tabular}

Quelle: IABS 1975-2004, eigene Berechnungen

*** 1\%-Signifikanzniveau; ** 5\%-Signifikanzniveau; * 10\%-Signifikanzniveau

Werte in Klammern bezeichnen die Standardfehler 
Tabelle 9: Einflussfaktoren auf den Verbleib nach der dualen Berufsausbildung für die Abschlusskohorten 1992-1995 - Schätzungen eines multi-nomialen Logitmodells

\begin{tabular}{|c|c|c|c|c|}
\hline \multirow[b]{2}{*}{$\begin{array}{l}\text { (Referenz: Verbleib im Ausbil- } \\
\text { dungsbetrieb) }\end{array}$} & \multicolumn{2}{|c|}{$\begin{array}{c}\text { Modell 1: } \\
\text { ohne Interaktionseffekte }\end{array}$} & \multicolumn{2}{|c|}{$\begin{array}{c}\text { Modell 2: } \\
\text { mit Interaktionseffekten }\end{array}$} \\
\hline & $\begin{array}{l}\text { Beschäftigung } \\
\text { nach Wechsel }\end{array}$ & Arbeitslosigkeit & $\begin{array}{l}\text { Beschäftigung } \\
\text { nach Wechsel }\end{array}$ & Arbeitslosigkeit \\
\hline Ausbildungsvergütungen (log) & $\begin{array}{l}-0,387^{* * *} \\
(0,093) \\
\end{array}$ & $\begin{array}{l}-0,561^{* * *} \\
(0,090) \\
\end{array}$ & $\begin{array}{l}-0,386^{* * *} \\
(0,093) \\
\end{array}$ & $\begin{array}{l}-0,558^{* * *} \\
(0,090) \\
\end{array}$ \\
\hline betriebliche Lohnsumme (log) & $\begin{array}{r}0,040^{*} \\
(0,024) \\
\end{array}$ & $\begin{array}{c}0,033 \\
(0,024) \\
\end{array}$ & $\begin{array}{c}0,039 \\
(0,024) \\
\end{array}$ & $\begin{array}{c}0,033 \\
(0,024) \\
\end{array}$ \\
\hline Ausbildungsdauer (log) & $\begin{array}{l}-0,780^{* * *} \\
(0,110)\end{array}$ & $\begin{array}{l}-0,651^{* * *} \\
(0,108)\end{array}$ & $\begin{array}{l}-0,788^{* * *} \\
(0,110)\end{array}$ & $\begin{array}{l}-0,660^{* * *} \\
(0,108)\end{array}$ \\
\hline \multicolumn{5}{|l|}{$\begin{array}{l}\text { Betriebsgröße } \\
\text { (Referenz: 1-9 Mitarbeiter) }\end{array}$} \\
\hline 10-49 Mitarbeiter & $\begin{array}{l}-0,262^{* * *} \\
(0,079) \\
\end{array}$ & $\begin{array}{l}-0,118 \\
(0,075) \\
\end{array}$ & $\begin{array}{l}-0,260^{* * *} \\
(0,079)\end{array}$ & $\begin{array}{l}-0,121 \\
(0,075) \\
\end{array}$ \\
\hline 50-499 Mitarbeiter & $\begin{array}{l}-0,345^{* * *} \\
(0,108)\end{array}$ & $\begin{array}{l}-0,201^{*} \\
(0,106) \\
\end{array}$ & $\begin{array}{l}-0,337^{* * *} \\
(0,109)\end{array}$ & $\begin{array}{l}-0,198^{*} \\
(0,106) \\
\end{array}$ \\
\hline > 500 Mitarbeiter & $\begin{array}{l}-0,593^{* * *} \\
(0,164) \\
\end{array}$ & $\begin{array}{l}-0,869^{* * *} \\
(0,166) \\
\end{array}$ & $\begin{array}{l}-0,592^{* * *} \\
(0,164) \\
\end{array}$ & $\begin{array}{l}-0,868^{* * *} \\
(0,166) \\
\end{array}$ \\
\hline \multicolumn{5}{|l|}{$\begin{array}{l}\text { Ausbildungsberufe (Referenz: } \\
\text { Land-/Forstwirtschaftsberufe) }\end{array}$} \\
\hline Chemieberufe & $\begin{array}{c}0,254 \\
(0,174) \\
\end{array}$ & $\begin{array}{c}0,025 \\
(0,175) \\
\end{array}$ & $\begin{array}{l}-0,317 \\
(0,531) \\
\end{array}$ & $\begin{array}{l}-0,720 \\
(0,547)\end{array}$ \\
\hline Metallberufe & $\begin{array}{l}-0,542 \\
(0,110) \\
\end{array}$ & $\begin{array}{l}-0,406^{* * *} \\
(0,099) \\
\end{array}$ & $\begin{array}{l}-0,504 \\
(0,345) \\
\end{array}$ & $\begin{array}{l}-0,695^{* *} \\
(0,331) \\
\end{array}$ \\
\hline Textilberufe & $\begin{array}{l}-0,470 \\
(0,086) \\
\end{array}$ & $\begin{array}{l}-0,451^{* * *} \\
(0,078) \\
\end{array}$ & $\begin{array}{l}-0,338 \\
(0,338) \\
\end{array}$ & $\begin{array}{l}-0,513 \\
(0,329) \\
\end{array}$ \\
\hline $\begin{array}{l}\text { Berufe im Nahrungsmittelbe- } \\
\text { reich }^{99}\end{array}$ & ja & ja & ja & ja \\
\hline Elektroberufe & $\begin{array}{c}0,012 \\
(0,164) \\
\end{array}$ & $\begin{array}{l}-0,584^{* * *} \\
(0,179)\end{array}$ & $\begin{array}{c}0,119 \\
(0,508) \\
\end{array}$ & $\begin{array}{l}-0,905 \\
(0,589)\end{array}$ \\
\hline Bauberufe & $\begin{array}{l}-0,251^{* * *} \\
(0,091) \\
\end{array}$ & $\begin{array}{l}-0,493 * * * \\
(0,088)\end{array}$ & $\begin{array}{l}-0,107 \\
(0,395)\end{array}$ & $\begin{array}{l}-0,268 \\
(0,412) \\
\end{array}$ \\
\hline $\begin{array}{l}\text { Infrastruktur-/Transport- } \\
\text { dienstleistungsberufe }\end{array}$ & $\begin{array}{l}-0,487^{* * *} \\
(0,111) \\
\end{array}$ & $\begin{array}{l}-0,600^{* * *} \\
(0,104) \\
\end{array}$ & $\begin{array}{l}-0,781^{* *} \\
(0,354) \\
\end{array}$ & $\begin{array}{l}-0,927^{* * *} \\
(0,346) \\
\end{array}$ \\
\hline Finanzdienstleistungsberufe & $\begin{array}{c}0,012 \\
(0,155) \\
\end{array}$ & $\begin{array}{l}-0,942^{* * *} \\
(0,186) \\
\end{array}$ & $\begin{array}{l}-0,066 \\
(0,456) \\
\end{array}$ & $\begin{array}{l}-1,660^{* * *} \\
(0,579) \\
\end{array}$ \\
\hline Verwaltungsberufe & $\begin{array}{l}-0,560^{* * *} \\
(0,096) \\
\end{array}$ & $\begin{array}{l}-1,034^{* * *} \\
(0,098) \\
\end{array}$ & $\begin{array}{l}-0,251 \\
(0,313) \\
\end{array}$ & $\begin{array}{l}-0,447 \\
(0,325) \\
\end{array}$ \\
\hline $\begin{array}{l}\text { produktionsnahe Dienstleis- } \\
\text { tungsberufe }\end{array}$ & $\begin{array}{l}-0,683^{* * *} \\
(0,107) \\
\end{array}$ & $\begin{array}{l}-0,590^{* * *} \\
(0,100) \\
\end{array}$ & $\begin{array}{l}-1,193 * * * \\
(0,379) \\
\end{array}$ & $\begin{array}{l}-1,024^{* * *} \\
(0,360) \\
\end{array}$ \\
\hline $\begin{array}{l}\text { haushalts-/personenbezogene } \\
\text { Dienstleistungsberufe }\end{array}$ & $\begin{array}{l}-0,617^{* * *} \\
(0,135) \\
\end{array}$ & $\begin{array}{l}-0,757^{* * *} \\
(0,133)\end{array}$ & $\begin{array}{l}-0,973 * * \\
(0,446) \\
\end{array}$ & $\begin{array}{l}-1,320^{* * *} \\
(0,467)\end{array}$ \\
\hline \multicolumn{5}{|l|}{ Qualifikation } \\
\hline $\begin{array}{l}\text { Durchschnitt zum Wirtschafts- } \\
\text { zweig }\end{array}$ & $\begin{array}{c}0,490^{* * *} \\
(0,051)\end{array}$ & $\begin{array}{l}0,493^{* * *} \\
(0,050)\end{array}$ & $\begin{array}{l}0,498^{* * *} \\
(0,051)\end{array}$ & $\begin{array}{l}0,501^{* * *} \\
(0,050)\end{array}$ \\
\hline \multicolumn{5}{|c|}{ Fortsetzung Tabelle 9 auf der nächsten Seite } \\
\hline
\end{tabular}

\footnotetext{
${ }^{99}$ Im Nahrungsmittelbereich sind nur wenige Fälle enthalten.
} 


\begin{tabular}{|c|c|c|c|c|}
\hline \multirow{2}{*}{$\begin{array}{l}\text { (Referenz: Verbleib im Ausbil- } \\
\text { dungsbetrieb) }\end{array}$} & \multicolumn{2}{|c|}{$\begin{array}{c}\text { Modell 1: } \\
\text { ohne Interaktionseffekte }\end{array}$} & \multicolumn{2}{|c|}{$\begin{array}{c}\text { Modell 2: } \\
\text { mit Interaktionseffekten }\end{array}$} \\
\hline & $\begin{array}{l}\text { Beschäftigung } \\
\text { nach Wechsel }\end{array}$ & Arbeitslosigkeit & $\begin{array}{l}\text { Beschäftigung } \\
\text { nach Wechsel }\end{array}$ & Arbeitslosigkeit \\
\hline \multicolumn{5}{|l|}{$\begin{array}{l}\text { Bildung } \\
\text { (Referenz: Hauptschulabschluss) }\end{array}$} \\
\hline Realschulabschluss & $\begin{array}{c}0,065 \\
(0,064)\end{array}$ & $\begin{array}{l}0,129 * * \\
(0,063)\end{array}$ & $\begin{array}{c}0,044 \\
(0,130)\end{array}$ & $\begin{array}{c}0,061 \\
(0,125)\end{array}$ \\
\hline Abitur & $\begin{array}{l}0,299 * * * \\
(0,092)\end{array}$ & $\begin{array}{l}-0,218^{* *} \\
(0,101)\end{array}$ & $\begin{array}{c}0,266 \\
(0,252)\end{array}$ & $\begin{array}{l}-0,291 \\
(0,250)\end{array}$ \\
\hline Regionalvariable & $\mathrm{ja}$ & $\mathrm{ja}$ & $\mathrm{ja}$ & $\mathrm{ja}$ \\
\hline Staatsangehörigkeit & $\begin{array}{l}-0,336^{* * * *} \\
(0,081) \\
\end{array}$ & $\begin{array}{l}-0,558^{* * *} \\
(0,076) \\
\end{array}$ & $\begin{array}{l}-0,331^{* * *} \\
(0,081)\end{array}$ & $\begin{array}{l}-0,549^{* * *} \\
(0,076) \\
\end{array}$ \\
\hline \multicolumn{5}{|l|}{$\begin{array}{l}\text { Interaktionseffekte } \\
\text { (Ausbildungsbereiche * Schul- } \\
\text { bildung) }\end{array}$} \\
\hline Land-/ Forstwirtschaftsberufe & & & $\begin{array}{c}0,035 \\
(0,093)\end{array}$ & $\begin{array}{c}0,008 \\
(0,098)\end{array}$ \\
\hline Chemieberufe & & & $\begin{array}{c}0,338 \\
(0,288)\end{array}$ & $\begin{array}{c}0,430 \\
(0,299) \\
\end{array}$ \\
\hline Metallberufe & & & $\begin{array}{l}-0,021 \\
(0,189)\end{array}$ & $\begin{array}{c}0,165 \\
(0,181)\end{array}$ \\
\hline Textilberufe & & & $\begin{array}{l}-0,067 \\
(0,180)\end{array}$ & $\begin{array}{c}0,036 \\
(0,175)\end{array}$ \\
\hline $\begin{array}{l}\text { Berufe im Nahrungsmittelbe- } \\
\text { reich }\end{array}$ & & & ja & ja \\
\hline Elektroberufe & & & $\begin{array}{l}-0,064 \\
(0,290)\end{array}$ & $\begin{array}{c}0,186 \\
(0,332)\end{array}$ \\
\hline Bauberufe & & & $\begin{array}{l}-0,068 \\
(0,203)\end{array}$ & $\begin{array}{l}-0,113 \\
(0,213)\end{array}$ \\
\hline $\begin{array}{l}\text { Infrastruktur- und Transport- } \\
\text { dienstleistungsberufe }\end{array}$ & & & $\begin{array}{c}0,185 \\
(0,197)\end{array}$ & $\begin{array}{c}0,195 \\
(0,193)\end{array}$ \\
\hline Finanzdienstleistungsberufe & & & $\begin{array}{c}0,046 \\
(0,235) \\
\end{array}$ & $\begin{array}{c}0,383 \\
(0,291) \\
\end{array}$ \\
\hline Verwaltungsberufe & & & $\begin{array}{l}-0,134 \\
(0,154)\end{array}$ & $\begin{array}{l}-0,279^{*} \\
(0,163) \\
\end{array}$ \\
\hline $\begin{array}{l}\text { produktionsnahe Dienstleis- } \\
\text { tungsberufe }\end{array}$ & & & $\begin{array}{c}0,233 \\
(0,178) \\
\end{array}$ & $\begin{array}{c}0,205 \\
(0,175) \\
\end{array}$ \\
\hline $\begin{array}{l}\text { haushalts-/ personenbezogene } \\
\text { Dienstleistungsberufe }\end{array}$ & & & $\begin{array}{c}0,192 \\
(0,225) \\
\end{array}$ & $\begin{array}{c}0,299 \\
(0,239) \\
\end{array}$ \\
\hline Zahl der Fälle & & & & \\
\hline pseudd $R^{2}$ & & & & \\
\hline$\chi^{2}(87)$ & & & 1060 & $2^{* * *}$ \\
\hline
\end{tabular}

Quelle: IABS 1975-2004, eigene Berechnungen

*** 1\%-Signifikanzniveau; ** 5\%-Signifikanzniveau; * 10\%-Signifikanzniveau Werte in Klammern bezeichnen die Standardfehler

Jüngere Abschlusskohorten weisen demnach nach der Ausbildung gegenüber Ausbildungsabsolventen in einem Betrieb mit bis zu neun Beschäftigten eine bessere Ausgangsposition auf dem Arbeitsmarkt auf als die älteren Jahrgänge. 
Ferner ist die Bedeutung einer Ausbildung in einem Betrieb zwischen 10 und 49 Mitarbeitern für die Abschlusskohorten 1996-1999 gegenüber der Vorgängerabschlusskohorte nicht signifikant für das Arbeitslosigkeitsrisiko. Dies bedeutet, dass eine Ausbildung in dieser Betriebsgrößenklasse gegenüber der Referenzgruppe nicht vor Arbeitslosigkeit schützt.

Jedoch sind insbesondere Veränderungen der Einflüsse der Ausbildungsbereiche zwischen den einzelnen Abschlusskohorten zu erkennen. Dies verdeutlicht die unterschiedlichen Entwicklungen der Arbeitsnachfrage innerhalb der einzelnen Branchen. Ausbildungsabsolventen der Abschlusskohorten 19921995 haben in den meisten Ausbildungsberufen gegenüber der Referenzgruppe gute Startbedingungen in das Erwerbsleben. Diese positiven Wirkungen auf den Übergang setzen sich für die Abschlusskohorten 1996-1999 fort. Jedoch ist eine Abschwächung der signifikant negativen Einflüsse auf eine Wahrscheinlichkeit für einen Betriebswechsel vor allem für die handwerklichen Ausbildungsbereiche zu erkennen (Nahrungsmittelberufe, Elektroberufe sowie Berufe im Bau). Auffallend ist, dass junge Erwachsene der Ausbildungsbereiche eine signifikant geringere Wahrscheinlichkeit für einen Betriebswechsel bzw. für Arbeitslosigkeit gegenüber der Referenzgruppe in wirtschaftlich schlechten Jahren, wie 1992-1995 und 1981-1986 (siehe Tabelle 16 bis Tabelle 18 im Anhang) aufweisen.

Daher betreffen die wirtschaftlichen Schwankungen insbesondere die Ausbildungsabsolventen, die über einen geringen Bildungsstand verfügen sowie in Ausbildungsbereichen lernen, die konjunkturabhängig sind. Jedoch sind unter den wirtschaftlichen Bedingungen auch deren langfristige Auswirkungen zu betrachten. So sind die gegenüber dem land- und forstwirtschaftlichen Bereich signifikanten Einflüsse unter Umständen auch auf die schlechter werdende wirtschaftliche Lage der Landwirtschaft selbst zurückzuführen. Es fand in diesen Bereichen zwar noch Ausbildung statt, die Arbeitsnachfrage war aber rückläufig, was durch eine Steigerung der Produktivität, durch Auslagerungen der Produktionsstätten ins Ausland oder auch durch die Deckung des Arbeitsbedarfes mit ausländischen Arbeitskräften erklärt werden kann. Daraus lässt sich auf geringe Chancen der Ausbildungsabsolventen bereits zu Beginn der Ausbildung schließen, in ihrem Ausbildungsberuf bzw. Ausbildungsbetrieb tätig zu sein.

Schließlich verbessert sich die Situation auf dem Arbeitsmarkt nach der dualen Berufsausbildung für Ausbildungsabsolventen, die unterdurchschnittliche Fähigkeiten aufweisen. Sie weisen eine geringere Wahrscheinlichkeit auf, eine Beschäftigung in einem anderen Betrieb aufzunehmen sowie arbeitslos zu werden. Ferner besitzen Abiturienten der jüngeren Abschlusskohorten ein signifikant geringeres Arbeitslosigkeitsrisiko gegenüber den Hauptschulabsolventen der entsprechenden Abschlusskohorte. Sie sind besser vor konjunkturellen Schwankungen geschützt (vgl. Konietzka 2002; Kap.7.1.3). Absolventen mit Realschulabschluss haben über alle Kohorten ein signifikant höheres Arbeitslo- 
sigkeitsrisiko. Eine deutsche Staatsangehörigkeit beeinflusst vor allem den Betriebswechsel der jüngeren Abschlusskohorten negativ. Deutsche Ausbildungsabsolventen wechseln weniger häufig den Ausbildungsbetrieb als Ausbildungsabsolventen mit Migrationshintergrund.

Im zweiten Modell ergeben sich vor allem für die Abschlusskohorten zwischen 1992 und 1995 weiterhin signifikante Einflüsse der Ausbildungsbereiche. Bei einer Kontrolle der Bildungsvariablen über die Interaktionseffekte bleiben die Unterschiede zwischen den Ausbildungsbereichen weiterhin bestehen. In diesen Jahren haben andere Effekte, insbesondere sich zwischen den Bereichen unterscheidende konjunkturelle Rahmenbedingungen die unterschiedlichen Chancen für Ausbildungsabsolventen nach der dualen Berufsausbildung hervorgerufen. Diese überlagernden Effekte schwächten sich aber zwischen 1975 und $2003 \mathrm{ab}$ (siehe hierzu weiter Tabelle 15 bis Tabelle 17 im Anhang). Für die Interaktionseffekte selbst ergeben sich vorwiegend insignifikante Einflüsse. In den älteren Abschlusskohorten spielte die Bildung für einen Betriebswechsel, wie auch für die jüngste Kohorte, keine große Bedeutung. Allerdings nahm die Bedeutung der Bildung für den Eintritt in den Arbeitsmarkt über den Zeitverlauf gesehen zu, sofern ein Ausbildungsabsolvent im Bereich der Finanzdienstleistungen ausgebildet wurde. Diese Entwicklung ist auf die verschiedenen Anforderungen in den Ausbildungsberufen zurückzuführen. So liegt die Wahrscheinlichkeit für Bankfachleute höher, im Ausbildungsbetrieb weiterbeschäftigt zu werden als für Verkäufer, was durch den negativen Effekt impliziert wird. War dieser Ausbildungsbereich in der Abschlusskohorte 1992-1995 noch von einem, wenn auch nicht signifikant, höheren Arbeitslosigkeitsrisiko in diesem Ausbildungsbereich betroffen, kehrt sich das Vorzeichen für die jüngeren Abschlusskohorten um. Das Arbeitslosigkeitsrisiko sinkt, was auf ein hohes Bildungsniveau und eine gute Ausbildungsqualität schließen lässt. Allerdings ist letzterer Einfluss nicht signifikant. Ausbildungsabsolventen, die ihren Abschluss in der Verwaltungs-, Organisations- und Kommunikationsbranche zwischen 1992 und 1995 machten, hatten mit einem höheren Schulabschluss ein auf dem 10\% Signifikanzniveau niedrigeres Arbeitslosigkeitsrisiko als die jüngeren Kohorten. Dieser Effekt schwächt sich aber für die jüngeren Kohorten ab. Für die in diesem Kapitel betrachteten Hypothesen ergeben sich folgende Ergebnisse:

Die Hypothese 1a, dass schlechtere Ausbildungsabsolventen den Betrieb wechseln, kann bestätigt werden. Insbesondere betriebliche Rahmenbedingungen, wie die Ausbildungsvergütungen, die Ausbildungsdauer sowie die Betriebsgröße, beeinflussen den Humankapitalstand positiv und verringern die Wahrscheinlichkeit eines Betriebswechsels. Darüber hinaus nimmt der negative Einfluss dieser Faktoren im Zeitverlauf zu, was zugleich bedeutet, dass auch die Art des Humankapitals und der Bildungsstand für den Verbleib nach der dualen Berufsausbildung an Bedeutung gewinnen. Entsprechend scheinen für die jüngeren Abschlusskohorten die betrieblichen Rahmenbedingungen von 
größerer Relevanz zu sein als für die älteren Abschlusskohorten. Gut ausgebildete Absolventen werden somit eher in ihrem Ausbildungsbetrieb weiterbeschäftigt als Ausbildungsabsolventen, deren Rahmenbedingungen während der Ausbildung weniger vorteilhaft für eine Humankapitalbildung sind. Daher stellt die Art des Betriebs eine Voraussetzung für den Einstieg ins Erwerbsleben und den weiteren Erwerbsverlauf dar. Die Einflüsse unterscheiden sich bei sich ändernden wirtschaftlichen Rahmenbedingungen, wie die getrennten Schätzungen nach Abschlusskohorten aufzeigen. Vor allem in der jüngsten Kohorte, die eher schlechte Rahmenbedingungen hatte, zeigt sich ein besonders starker negativer Effekt. Besser ausgebildete Absolventen, die meist einen geringen Anteil an allgemeinem bzw. berufsspezifischem Humankapital haben, wechseln gegenüber den anderen Kohorten weniger wahrscheinlich den Ausbildungsbetrieb. Die Einflüsse der Ausbildungsbereiche sind vorwiegend negativ und signifikant. Dies deutet auf ein langfristiges Beschäftigungsinteresse der Ausbildungsbetriebe hin. Im Allgemeinen weisen Ausbildungsbereiche, die scheinbar einen höheren Humankapitalstand erfordern, wie z.B. Finanzdienstleistungsberufe, Verwaltungsberufe, produktionsnahe oder haushalts- und personenbezogene Dienstleistungsberufe, eine geringe Wahrscheinlichkeit auf, den Betrieb zu wechseln. Im Zeitverlauf ändern sich auch hier die Einflüsse, denn für die jüngste Kohorte weisen zwar die handwerklichen Betriebe günstige Voraussetzungen auf, in ihrem Ausbildungsbetrieb zu verbleiben, während Absolventen in Verwaltungs- und Finanzdienstleistungsberufen sogar eine positive, wenn auch nicht signifikante, Wahrscheinlichkeit besitzen, den Betrieb zu wechseln. Durch die Interaktionseffekte wird aber deutlich, dass insbesondere im Finanzdienstleistungsbereich ein hoher Bildungsabschluss die Wechselwahrscheinlichkeit negativ beeinflusst. Daher kann festgehalten werden, dass schlechtere bzw. weniger gut ausgebildete Absolventen eine geringere Wahrscheinlichkeit haben, den Betrieb zu wechseln, als besser ausgebildete Personen.

Hypothese $1 \mathrm{~b}$, dass schlechtere Ausbildungsabsolventen ein höheres Arbeitslosigkeitsrisiko haben, kann ebenfalls bestätigt werden. Insbesondere die betrieblichen Merkmale zeigen, dass gut ausgebildete Absolventen ein geringeres Eintrittsrisiko in Arbeitslosigkeit aufweisen als die schlechter ausgebildeten jungen Erwachsenen. Ausbildungsabsolventen, die innerhalb ihres Wirtschaftsbereiches unterdurchschnittliche betriebliche Qualifikationen aufweisen, realisieren eher Arbeitslosigkeitsphasen als Ausbildungsabsolventen mit überdurchschnittlichen Qualifikationen. Bei Berücksichtigung der Ausbildungsbereiche ist keine eindeutige Tendenz hinsichtlich des Arbeitslosigkeitsrisikos zu erkennen, da bis auf wenige Branchen die Einflüsse nicht signifikant sind. Vor allem Ausbildungsbereiche, die gut ausgebildete Arbeitskräfte, wie die Infrastrukturund Transportdienstleistungs-, Finanzdienstleistungs- und Verwaltungsberufe haben, weisen jedoch für alle Abschlusskohorten ein signifikant geringeres Arbeitslosigkeitsrisiko auf. Deren Einfluss hat aber für die jüngere Kohorte abgenommen. Die Interaktionseffekte lassen jedoch auf eine verminderte Eintritts- 
wahrscheinlichkeit in Arbeitslosigkeit schließen, je höher der Bildungsabschluss ist. Das Arbeitslosigkeitsrisiko liegt somit bei Ausbildungsabsolventen, die in einem Betrieb mit guten Voraussetzungen für einen hohen Bildungsstand ihre Ausbildung absolviert haben, niedriger als bei jungen Erwachsenen mit weniger guten betrieblichen Rahmenbedingungen während der Ausbildung.

Zusammenfassend bleibt zu sagen, dass trotz der Ergebnisse eine Ausbildung mit einer hohen Bildungsqualität und einem hohen Bildungsstand, die Wahrscheinlichkeit eines Betriebswechsels und das Arbeitslosigkeitsrisiko reduziert. Schließlich ist die Schulbildung zwar für die Wahl einer Ausbildung entscheidend, für den Verbleib nach der dualen Berufsausbildung scheinen aber betriebliche Rahmenbedingungen und die Ausbildungsberufe einen unmittelbaren Einfluss auszuüben, während die Schulbildung über die betrieblichen Merkmale indirekt wirkt. Dennoch können Ausbildungsabsolventen, die scheinbar gute Voraussetzungen während der Ausbildung haben, arbeitslos werden, weil die konjunkturelle Situation den Betrieben eine Weiterbeschäftigung der Ausbildungsabsolventen nicht zulässt. Diese treten dann in Konkurrenz um Stellen mit anderen Ausbildungsabsolventen. Zudem verändert sich die Bedeutung der betrieblichen Rahmenbedingungen und infolgedessen auch des Humankapitals aufgrund von langfristigen wirtschaftlichen Veränderungen. Wie sich die Einflussfaktoren auf die Dauer der Arbeitslosigkeit auswirken, wird im Folgenden untersucht.

\subsection{Dauer der Arbeitslosigkeit}

\subsubsection{Ansatz der Verlaufsdatenanalyse}

Zur Überprüfung der Hypothese 2, dass Ausbildungsabsolventen mit höherem Humankapital länger arbeitslos sind, muss die Querschnittsperspektive verlassen werden.

Es wird nun ein Zeitpunkt betrachtet, der mit dem Abschluss der dualen Berufsausbildung und dem Eintritt in Arbeitslosigkeit beginnt. Dieser Betrachtungszeitraum beginnt jedoch nicht für alle Personen zur gleichen Zeit, weshalb für jede Betrachtungseinheit unterschiedliche Zeitpunkte für den Beginn der Arbeitslosigkeit resultieren. Daher beginnt der Betrachtungszeitraum mit dem Eintritt der Arbeitslosigkeit. Weil sich der Untersuchungszeitraum über mehrere Perioden erstreckt, können Personen vor Eintritt des Ereignisses aus dem Datensatz fallen, wodurch die Informationen der interessierenden Personengruppe nicht vollständig sind (Ruud 2000). Dieses Problem der zensierten Daten tritt im Falle von Verlaufsdaten häufig auf. Sowohl den Aspekt der unterschiedlichen individuellen Verweildauern als auch den Aspekt der zensierten Daten berücksichtigen die Methoden der Verlaufsanalyse, weshalb diese im Kontext der Fragestellung angebrachter erscheint als eine OLS-Regression.

Zur Schätzung der Dauer der Arbeitslosigkeit wird ein Hazardraten-Modell verwendet. Hierbei wird nicht die Länge der Dauer, sondern über die Abgangs- 
rate (Hazardrate) aus dem betrachteten Zustand gemessen. Es wird daher die Wahrscheinlichkeit geschätzt, wie lange die betreffende Person durchschnittlich den interessierenden Zustand überlebt, unter der Bedingung, dass sie zum Zeitpunkt $t$ weiterhin in dem Zustand ist. Der Betrachtungszeitraum wird in Zeitintervalle eingeteilt, wobei zu jedem dieser Zeitintervalle $T$ geschätzt wird, wie viele Ausbildungsabsolventen die Arbeitslosigkeit verlassen haben bzw. wie hoch die Wahrscheinlichkeit ist, dass die in Arbeitslosigkeit verbleibenden Ausbildungsabsolventen das nächste Zeitintervall $t+\Delta t$ erleben bzw. aus dem Zustand abgehen. Die Hazardrate $\lambda(t)$ bestimmt sich durch die Bedingung

$$
\text { (10) } \lambda(t)=\lim _{\Delta \rightarrow 0+} \frac{P(t \leq T \leq t+\Delta t \mid T \geq t)}{\Delta t} \Rightarrow f(t)=\lim _{\Delta t \rightarrow 0+} \frac{P(t \leq T \leq t+\Delta t)}{\Delta t} \Rightarrow \lambda(t) \frac{f(t)}{S(t)} \text {. }
$$

Der rechte Term gibt Auskunft über die Wahrscheinlichkeit, wie lange es dauert, bis der arbeitslose Ausbildungsabsolvent wieder in Beschäftigung kommt. Daraus ergibt sich die Überlebensfunktion bzw. Survival-Funktion $S(t)$ :

$$
S(t)=e^{-\int_{0}^{\prime} \lambda(t) d t}
$$

Zur Schätzung der Dauer der Arbeitslosigkeit als abhängige Variable wird zunächst die nicht parametrische Methode von Kaplan und Meier (1958) verwendet. Sie beschreibt die Überlebensfunktion der betrachteten Untersuchungseinheiten in Arbeitslosigkeit. Hierbei wird die Arbeitslosigkeitsdauer ihrer Länge nach geordnet und dann die durchschnittliche Verbleibsrate nach einem Zeitpunkt ermittelt. Die zensierten Daten von Personen, die zwar aus der Arbeitslosigkeit abgehen, aber deren Zielzustand unklar ist, werden bei den Berechnungen nicht berücksichtigt. Entsprechend wird die Grundgesamtheit um deren Anzahl zum jeweiligen Betrachtungszeitpunkt reduziert (Ronning 1991).

Für die Analyse des Einflusses von Kovariaten wird die Dauer der Arbeitslosigkeit mit einem semi-parametrischen Modell der Überlebensdaueranalyse geschätzt, indem zeitabhängige Variablen aufgenommen werden (Ronning 1991: 185f.). Es wird davon ausgegangen, dass die erste Arbeitslosigkeitsphase, welche hier betrachtet wird, relativ kurz ist und sich daher die Einflussfaktoren während dieser Zeit nicht ändern. Darüber hinaus beginnt das interessierende Zeitintervall nach dem Ende der dualen Berufsausbildung. Daher variiert $x$ hinsichtlich der Arbeitslosigkeitsdauer nicht. Jedoch kann sich die Hazardrate über den Betrachtungszeitraum dennoch verändern, sodass nicht von einer konstanten Hazardrate zu jedem Zeitpunkt ausgegangen werden kann. Hierzu schlägt Cox (1972) die Einführung einer Grundhazardrate $\lambda_{0}$ vor, die das Modell der proportionalen Hazardrate in eine Komponente zerlegt, welche die für alle betrachteten Individuen geltenden Hazardrate $\lambda_{v}$ angibt. Die zweite Komponente bildet die individuelle Heterogenität, $e^{\beta_{x}}$. Die zweite Komponente beinhaltet eine Funktion in Abhängigkeit der Einflussvariablen, welche die gesamte Hazardrate determiniert. 
Darüber hinaus verlangt dieses Modell keine Annahme über die Verteilung. Die Variablen $x$ unterscheiden sich ebenfalls für die einzelnen Individuen. Die Schätzung des Einflusses ist aufgrund der individuellen Grundhazardrate nicht möglich, weshalb die verschiedenen Hazardraten ins Verhältnis gesetzt werden, mit der sich eine Schätzung der Grundhazardrate erübrigt. Daraus ergibt sich die zu schätzende Gleichung:

$$
\frac{\lambda\left(t, x_{1}\right)}{\lambda\left(t, x_{2}\right)}=e^{\chi^{\prime}\left(x_{1}-x_{2}\right)}
$$

Die Koeffizienten werden durch die partielle Likelihood-Methode nach Cox (1972) geschätzt. Sie beschreibt die Wahrscheinlichkeit, zu der ein Ausbildungsabsolvent, der zum betrachteten Rand des Zeitintervalls noch arbeitslos ist, diesen Zustand verlässt, unter der Bedingung, dass nur eine Untersuchungseinheit aus Arbeitslosigkeit in $t$ tritt. Daraus ergibt sich die Wahrscheinlichkeit, mit der ein Individuum $i$ die Arbeitslosigkeit verlässt:

$$
P\left(\left(t_{i}=T_{K} \mid R\left(t_{i}\right)\right) \mid \text { ein Abgang aus } R\left(t_{i}\right)\right)=\frac{e^{\beta x^{\prime} x_{i}}}{\sum_{j \in R\left(t_{i}\right)} e^{\beta^{\prime} x_{j}}},
$$

wobei $R\left(t_{i}\right)$ die Anzahl aller Personen darstellt, die zum Zeitpunkt $t_{i}$ noch in Arbeitslosigkeit verweilen.

Für die Analyse des Einflusses auf die durchschnittliche Abgangsrate werden wiederum die betrieblichen und gesamtwirtschaftlichen Rahmenbedingungen sowie individuelle Voraussetzungen herangezogen.

\subsubsection{Einflussfaktoren auf die Dauer der Arbeitslosigkeit}

In Kapitel 8.1.2 wurde untersucht, welche Einflussfaktoren den Eintritt in das Erwerbsleben determinieren. Eine höhere Schulbildung scheint hierbei vor Arbeitslosigkeit nicht zu schützen. Auch bei der Betrachtung der Abgangsraten aus Arbeitslosigkeit sind kaum Unterschiede sowohl zwischen den jeweiligen Bildungsgruppen als auch zwischen den Abschlusskohorten zu erkennen. Aus den Abbildungen 12 bis 14 wird zunächst deutlich, dass die Verbleibsdauern der Ausbildungsabsolventen nach Bildungsabschlüssen für die Abschlusskohorten 1992-1995, 1996-1999 sowie 2000-2003 leicht stiegen. Die durchschnittliche Arbeitslosigkeitsdauer betrug für die Abschlusskohorten 20002003 für Absolventen mit Abitur bzw. Hauptschulabschluss ungefähr 20 Wochen, während der Durchschnitt der Abschlusskohorten von 1992-1995 und von 1996-1999 jeweils für Personen mit Abitur bei ungefähr 17 Wochen lag. Jedoch konnten bereits in der Abschlusskohorte 1981-1986 lange Verbleibsdauern in Arbeitslosigkeit beobachtet werden (siehe Abbildung 15 im Anhang). 
Diese Veränderungen der Arbeitslosigkeitsdauer zwischen den Abschlusskohorten bedeutet einen signifikanten Einfluss der Abschlussjahre, wie ein Logrank-Test ergibt. ${ }^{100}$

\section{Abbildung 12: Abgang aus Arbeitslosigkeit der Abschlusskohorten 2000- 2003 nach Schulabschluss - Schätzung eines Kaplan-Meier- Modells}

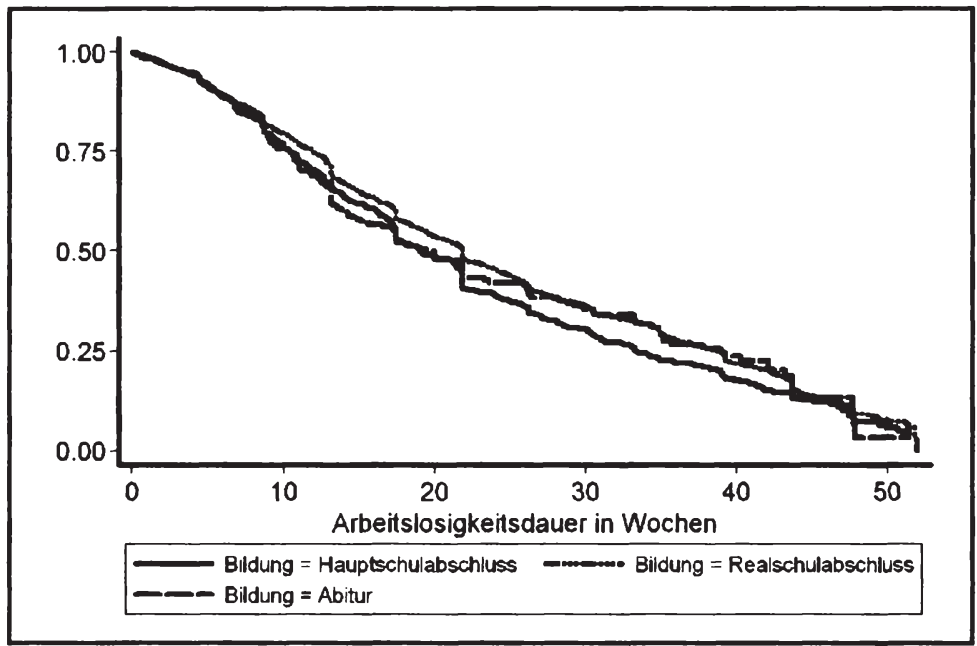

Quelle: IABS 1975-2004, eigene Berechnungen

Der Verbleib in Arbeitslosigkeit nach der dualen Berufsausbildung unterschied sich ebenfalls bei den Bildungsabschlüssen innerhalb der betrachteten Ausbildungskohorten. Dieser Unterschied nahm im Zeitverlauf ab. So wiesen die Abiturienten der Abschlusskohorten 2000-2003 nahezu dieselbe Hazardrate auf wie Ausbildungsabsolventen mit Haupt- bzw. Realschulabschluss. Diese verringerte sich aber nach ungefähr einem halben Jahr in Arbeitslosigkeit. Der Schulbildungsabschluss scheint auch bei Betrachtung der Hazardraten nicht vor einer längeren Arbeitslosigkeitsdauer zu schützen, denn Abiturienten der jüngsten Abschlusskohorte konnten die Phase der Erwerbslosigkeit nicht schneller verlassen als Hauptschulabsolventen mit einem Berufsabschluss. Im Gegenteil, Hauptschulabsolventen gingen nach ungefähr der 20. Woche am schnellsten in Beschäftigung über.

${ }^{100}$ Dieser überprüft die Hypothese, ob die Abgangsraten der betrachteten Abschlusskohorten einander entsprechen. Die Hypothese muss verworfen werden. 
Abbildung 13: Abgang aus der Arbeitslosigkeit der Abschlusskohorten 1996-1999 nach Schulabschluss - Schätzung eines KaplanMeier-Modells

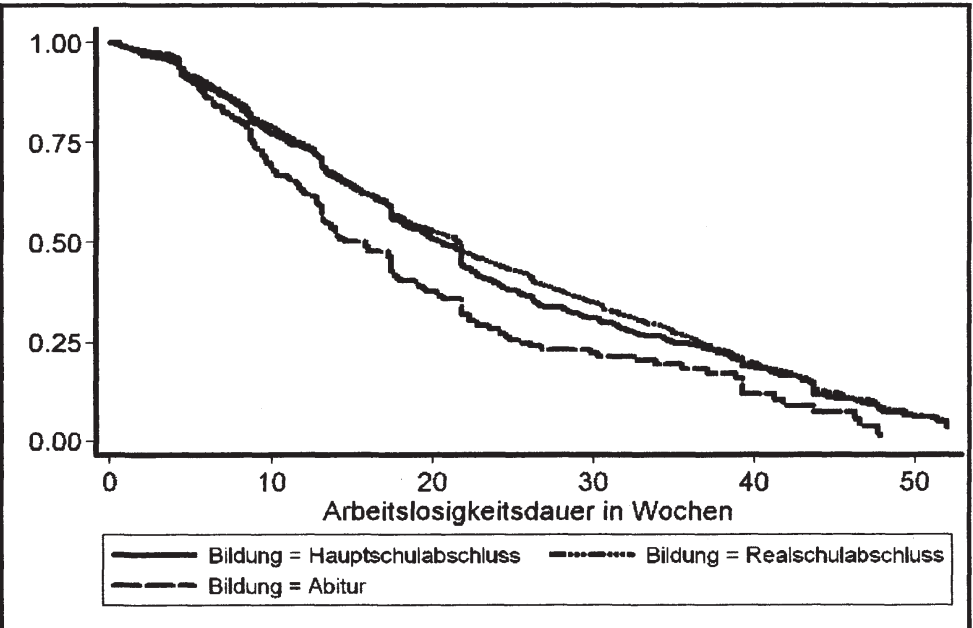

Quelle: IABS 1975-2004, eigene Berechnungen.

Abbildung 14: Abgang aus der Arbeitslosigkeit der Abschlusskohorten 1992-1995 nach Schulabschluss - Schätzung eines KaplanMeier-Modells

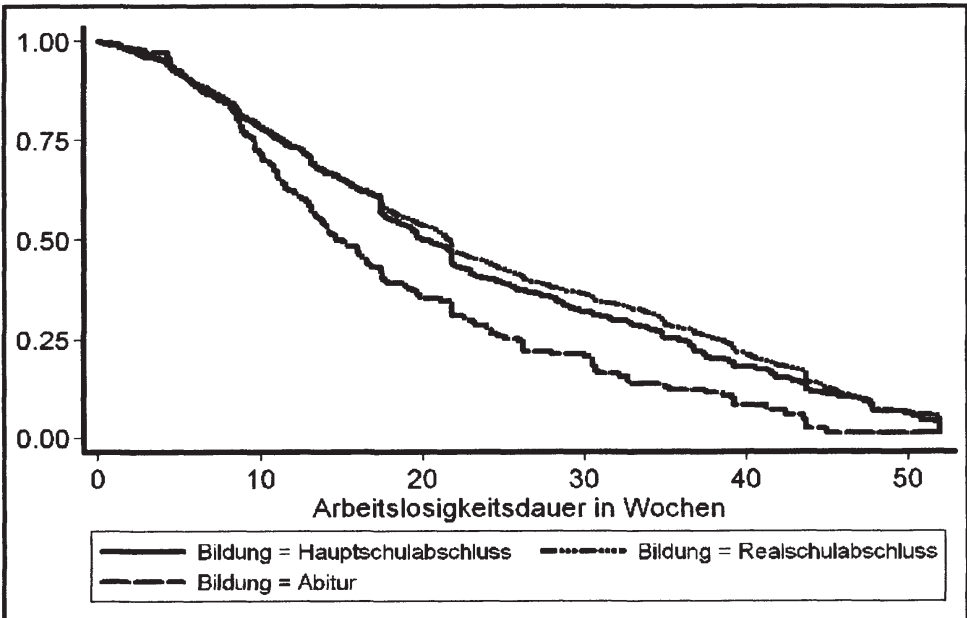

Quelle: IABS 1975-2004, eigene Berechnungen

Signifikant bei $\alpha<0,05$ 
Für die Abschlusskohorten 1996-1999 sind nach einigen Wochen in Arbeitslosigkeit Bildungsunterschiede deutlich erkennbar. Die Hazardraten der Bildungsgruppen sind zu Beginn der Phase der Erwerbslosigkeit nahezu identisch, nach fünf Wochen gehen jedoch Absolventen mit Abitur am ehesten in eine Erwerbstätigkeit über. Ab der 20. Woche reduzierte sich die Abgangsrate für die Ausbildungsabsolventen mit Realschulabschluss leicht. Sie verblieben aber ebenso wie junge Erwachsene mit Hauptschulabschluss am längsten in Arbeitslosigkeit. Die niedrigere Abgangsrate der Absolventen mit Abitur zu Beginn der Arbeitslosigkeitsperiode ist auf die Suche nach einer ausbildungsadäquaten Tätigkeit zurückzuführen, die ihre Zeit dauert.

In den Abschlusskohorten 1992-1995 werden Unterschiede erst ab der 10. Woche deutlich. Zuvor verblieben die jeweiligen Bildungsgruppen ungefähr gleich lang in Arbeitslosigkeit. Nach diesem Zeitpunkt realisieren dann aber die Absolventen mit Abitur die höchste Abgangsrate aus Arbeitslosigkeit. Auch in der ältesten Kohorte verblieben die jungen Erwachsenen mit Realschulabschluss am längsten in Arbeitslosigkeit. Der Logrank-Test ergibt, dass die Schulbildung die Abgangsraten aus Arbeitslosigkeit signifikant beeinflusst.

Bedeutende Unterschiede in den Abgangsraten sind über die gesamten Abschlusskohorten auch bei der Betrachtung der Ausbildungsbereiche zu erkennen (siehe Abbildung 16 im Anhang). Absolventen mit einer Ausbildung in Chemieberufen, metallverarbeitenden Berufen, in Elektroberufen sowie in Bauberufen wiesen relativ homogene Hazardraten zwischen den Bildungsgruppen auf. Hier spielten Schulabschlüsse scheinbar eine untergeordnete Rolle beim Abgang aus der Arbeitslosigkeit. Darüber hinaus hatten Absolventen der Bauberufe sowie der Elektroberufe eine relativ niedrige Hazardrate.

In den Ausbildungsberufen der Infrastruktur- und Transportdienstleistungen sowie der produktionsnahen Dienstleistungen sind dagegen Unterschiede zwischen den Bildungsgruppen beim Abgang aus der Arbeitslosigkeit zu erkennen. In diesen Ausbildungsbranchen wichen die Abgangsraten der unterschiedlichen Bildungsabschlüsse zu Beginn der Arbeitslosigkeitsperiode kaum voneinander ab. Bei einer längeren Dauer nahm die Bedeutung der Bildung aber zu. So waren in den Infrastruktur- und Transportdienstleistungen 50\% der Absolventen mit Haupt- und Realschulabschluss ungefähr 21 Wochen arbeitslos, während derselbe Anteil mit Abitur ungefähr 14 Wochen ohne Erwerbstätigkeit war. Bessere Ausbildungsabsolventen hatten eine kürzere Arbeitslosigkeitsdauer. Im Bereich der produktionsnahen Dienstleistungsberufe lag die durchschnittliche Verweildauer in Arbeitslosigkeit bei Absolventen mit Abitur bei 14 Wochen, der durchschnittliche Dauer ohne Erwerbstätigkeit bei Absolventen mit Hauptschulabschluss belief sich auf 20 Wochen. Ebenso wie im Infrastruktur- und Transportdienstleistungsbereich wiesen junge Fachkräfte mit Realschulabschluss nach einer längeren Periode ohne Arbeit die niedrigste Abgangsrate aus Arbeitslosigkeit auf. 
Die deskriptiven Auswirkungen deuten auf unterschiedliche Verweildauern der einzelnen Bildungsgruppen sowohl zwischen den betrachteten Ausbildungskohorten wie auch in den jeweiligen Ausbildungsbereichen hin. Tabelle 10 stellt die Bedeutung der Einflüsse auf die Höhe der Abgangsrate dar. Aus diesem Zusammenhang ist es möglich auf die Bestimmungsgründe der Arbeitslosigkeitsdauern zu schließen. Die Koeffizienten zur Beschreibung des Einflusses auf die Hazardraten stellen die Hazardratios dar. Diese beschreiben den Effekt einer unabhängigen Variablen auf den Verbleib in Arbeitslosigkeit im Verhältnis zum unveränderten Effekt der jeweiligen Variablen.

Tabelle 10: Einflussfaktoren auf die Abgangsrate aus Arbeitslosigkeit Schätzungen eines Cox-Modells für die Abschlusskohorten zwischen 1992 und 2003 (Hazardratios)

\begin{tabular}{|c|c|c|c|c|c|c|}
\hline & \multicolumn{2}{|c|}{$\begin{array}{l}\text { Abschlusskohorte } \\
2000-2003\end{array}$} & \multicolumn{2}{|c|}{$\begin{array}{c}\text { Abschlusskohorte } \\
\text { 1996-1999 }\end{array}$} & \multicolumn{2}{|c|}{$\begin{array}{c}\text { Abschlusskohorte } \\
\text { 1992-1995 }\end{array}$} \\
\hline & Modell 1" & Modell 2 & Modell 1" & Modell 2 & Modell 1" & Modell $2^{\text {"W" }}$ \\
\hline & $\begin{array}{l}\text { Abgangs- } \\
\text { rate }\end{array}$ & $\begin{array}{l}\text { Abgangs- } \\
\text { rate }\end{array}$ & $\begin{array}{l}\text { Abgangs- } \\
\text { rate }\end{array}$ & $\begin{array}{l}\text { Abgangs- } \\
\text { rate }\end{array}$ & $\begin{array}{l}\text { Abgangs- } \\
\text { rate }\end{array}$ & $\begin{array}{l}\text { Abgangs- } \\
\text { rate }\end{array}$ \\
\hline $\begin{array}{l}\text { Ausbildungsvergütung } \\
\text { (log) }\end{array}$ & $\begin{array}{c}1,080 \\
(0,078) \\
\end{array}$ & $\begin{array}{c}1,080 \\
(0,079) \\
\end{array}$ & $\begin{array}{r}1,072 \\
(0,073) \\
\end{array}$ & $\begin{array}{c}1,074 \\
(0,074) \\
\end{array}$ & $\begin{array}{r}1,089 \\
(0,075) \\
\end{array}$ & $\begin{array}{c}1,101 \\
(0,076) \\
\end{array}$ \\
\hline $\begin{array}{l}\text { betriebliche Lohnsumme } \\
\text { (log) }\end{array}$ & $\begin{array}{l}0,953^{* *} \\
(0,020)\end{array}$ & $\begin{array}{l}0,957^{* *} \\
(0,021)\end{array}$ & $\begin{array}{c}1,017 \\
(0,018) \\
\end{array}$ & $\begin{array}{r}1,019 \\
(0,018) \\
\end{array}$ & $\begin{array}{c}1,000 \\
(0,019)\end{array}$ & $\begin{array}{c}0,997 \\
(0,019) \\
\end{array}$ \\
\hline Ausbildungsdauer (log) & $\begin{array}{c}0,886 \\
(0,081) \\
\end{array}$ & $\begin{array}{c}0,877 \\
(0,081) \\
\end{array}$ & $\begin{array}{l}0,810^{* *} \\
(0,070)\end{array}$ & $\begin{array}{l}0,801^{* *} \\
(0,069)\end{array}$ & $\begin{array}{l}0,816^{* *} \\
(0,069) \\
\end{array}$ & $\begin{array}{l}0,829^{* *} \\
(0,071) \\
\end{array}$ \\
\hline \multicolumn{7}{|l|}{$\begin{array}{l}\text { Betriebsgröße (Referenz: } \\
\text { 1- } 9 \text { Mitarbeiter) }\end{array}$} \\
\hline 10-49 Mitarbeiter & $\begin{array}{c}1,053 \\
(0,066)\end{array}$ & $\begin{array}{c}1,037 \\
(0,066)\end{array}$ & $\begin{array}{l}0,877^{* *} \\
(0,047)\end{array}$ & $\begin{array}{l}0,874 * * \\
(0,047)\end{array}$ & $\begin{array}{c}0,898 \\
(0,054)\end{array}$ & $\begin{array}{r}0,906^{*} \\
(0,055)\end{array}$ \\
\hline 50-499 Mitarbeiter & $\begin{array}{c}1,070 \\
(0,100) \\
\end{array}$ & $\begin{array}{c}1,057 \\
(0,099) \\
\end{array}$ & $\begin{array}{c}0,885 \\
(0,068) \\
\end{array}$ & $\begin{array}{r}0,882 \\
(0,068) \\
\end{array}$ & $\begin{array}{c}0,926 \\
(0,077) \\
\end{array}$ & $\begin{array}{c}0,935 \\
(0,078) \\
\end{array}$ \\
\hline > 500 Mitarbeiter & $\begin{array}{l}1,363^{* *} \\
(0,215)\end{array}$ & $\begin{array}{l}1,337^{*} \\
(0,211)\end{array}$ & $\begin{array}{c}0,860 \\
(0,112)\end{array}$ & $\begin{array}{c}0,861 \\
(0,112)\end{array}$ & $\begin{array}{c}0,997 \\
(0,129)\end{array}$ & $\begin{array}{c}1,002 \\
(0,130)\end{array}$ \\
\hline \multicolumn{7}{|l|}{$\begin{array}{l}\text { Ausbildungsbereiche } \\
\text { (Referenz: Land-/ Forst- } \\
\text { wirtschaftsberufe) }\end{array}$} \\
\hline Chemieberufe & $\begin{array}{c}0,906 \\
(0,120)\end{array}$ & $\begin{array}{c}2,009 \\
(1,017)\end{array}$ & $\begin{array}{c}0,919 \\
(0,126)\end{array}$ & $\begin{array}{c}1,647 \\
(0,810)\end{array}$ & $\begin{array}{l}0,787^{*} \\
(0,105)\end{array}$ & $\begin{array}{c}0,560 \\
(0,226)\end{array}$ \\
\hline Metallberufe & $\begin{array}{c}1,104 \\
(0,092)\end{array}$ & $\begin{array}{c}1,143 \\
(0,335)\end{array}$ & $\begin{array}{c}0,910 \\
(0,071)\end{array}$ & $\begin{array}{c}1,055 \\
(0,294)\end{array}$ & $\begin{array}{c}0,917 \\
(0,073)\end{array}$ & $\begin{array}{c}0,758 \\
(0,205)\end{array}$ \\
\hline Textilberufe & $\begin{array}{c}1,004 \\
(0,070) \\
\end{array}$ & $\begin{array}{c}1,087 \\
(0,356) \\
\end{array}$ & $\begin{array}{c}0,972 \\
(0,061) \\
\end{array}$ & $\begin{array}{c}1,111 \\
(0,329) \\
\end{array}$ & $\begin{array}{c}0,907 \\
(0,057) \\
\end{array}$ & $\begin{array}{c}0,813 \\
(0,214) \\
\end{array}$ \\
\hline $\begin{array}{l}\text { Berufe im Nahrungsmit- } \\
\text { telbereich }\end{array}$ & ja & ja & ja & ja & ja & ja \\
\hline Elektroberufe & $\begin{array}{c}1,312 \\
(0,218)\end{array}$ & $\begin{array}{l}4,931^{* * *} \\
(2,836)\end{array}$ & $\begin{array}{r}1,292 \\
(0,198) \\
\end{array}$ & $\begin{array}{c}1,808 \\
(0,966)\end{array}$ & $\begin{array}{c}1,073 \\
(0,160) \\
\end{array}$ & $\begin{array}{c}1,641 \\
(0,873)\end{array}$ \\
\hline Bauberufe & $\begin{array}{c}0,981 \\
(0,083) \\
\end{array}$ & $\begin{array}{c}1,374 \\
(0,664) \\
\end{array}$ & $\begin{array}{c}0,972 \\
(0,069) \\
\end{array}$ & $\begin{array}{r}1,209 \\
(0,419) \\
\end{array}$ & $\begin{array}{l}0,861^{* *} \\
(0,062)\end{array}$ & $\begin{array}{r}1,040 \\
(0,339) \\
\end{array}$ \\
\hline $\begin{array}{l}\text { Infrastruktur-/Transport- } \\
\text { dienstleistungsberufe }\end{array}$ & $\begin{array}{l}0,866^{* *} \\
(0,065)\end{array}$ & $\begin{array}{r}0,738 \\
(0,219) \\
\end{array}$ & $\begin{array}{c}0,968 \\
(0,064) \\
\end{array}$ & $\begin{array}{c}1,589 \\
(0,388) \\
\end{array}$ & $\begin{array}{l}0,779^{* * *} \\
(0,067)\end{array}$ & $\begin{array}{c}0,732 \\
(0,206) \\
\end{array}$ \\
\hline
\end{tabular}

Fortsetzung Tabelle 10 auf der nächsten Seite 


\begin{tabular}{|c|c|c|c|c|c|c|}
\hline & \multicolumn{2}{|c|}{$\begin{array}{c}\text { Abschlusskohorte } \\
2000-2003\end{array}$} & \multicolumn{2}{|c|}{$\begin{array}{c}\text { Abschlusskohorte } \\
1996-1999\end{array}$} & \multicolumn{2}{|c|}{$\begin{array}{c}\text { Abschlusskohorte } \\
\text { 1992-1995 }\end{array}$} \\
\hline & Modell $1^{\#}$ & Modell $2^{\text {\# }}$ & Modell $1^{\#}$ & Modell 2 & Modell 1" & Modell 2" \\
\hline & $\begin{array}{l}\text { Abgangs- } \\
\text { rate }\end{array}$ & $\begin{array}{l}\text { Abgangs- } \\
\text { rate }\end{array}$ & $\begin{array}{l}\text { Abgangs- } \\
\text { rate }\end{array}$ & $\begin{array}{l}\text { Abgangs- } \\
\text { rate }\end{array}$ & $\begin{array}{l}\text { Abgangs- } \\
\text { rate }\end{array}$ & $\begin{array}{l}\text { Abgangs- } \\
\text { rate }\end{array}$ \\
\hline $\begin{array}{l}\text { Finanzdienstleistungs- } \\
\text { berufe }\end{array}$ & $\begin{array}{c}1,170 \\
(0,167)\end{array}$ & $\begin{array}{c}1,097 \\
(0,495) \\
\end{array}$ & $\begin{array}{c}0,888 \\
(0,143) \\
\end{array}$ & $\begin{array}{c}0,630 \\
(0,435) \\
\end{array}$ & $\begin{array}{c}1,013 \\
(0,164) \\
\end{array}$ & $\begin{array}{c}0,502 \\
(0,295) \\
\end{array}$ \\
\hline Verwaltungsberufe & $\begin{array}{c}0,887 \\
(0,077) \\
\end{array}$ & $\begin{array}{c}0,975 \\
(0,298) \\
\end{array}$ & $\begin{array}{c}1,058 \\
(0,085) \\
\end{array}$ & $\begin{array}{c}1,210 \\
(0,358) \\
\end{array}$ & $\begin{array}{l}0,784^{* * *} \\
(0,065)\end{array}$ & $\begin{array}{c}0,788 \\
(0,212) \\
\end{array}$ \\
\hline $\begin{array}{l}\text { produktionsnahe Dienst- } \\
\text { leistungsberufe }\end{array}$ & $\begin{array}{l}0,806^{* *} \\
(0,071) \\
\end{array}$ & $\begin{array}{c}0,966 \\
(0,348) \\
\end{array}$ & $\begin{array}{c}0,968 \\
(0,084) \\
\end{array}$ & $\begin{array}{c}1,452 \\
(0,473) \\
\end{array}$ & $\begin{array}{l}0,790^{* * *} \\
(0,066) \\
\end{array}$ & $\begin{array}{c}1,235 \\
(0,374) \\
\end{array}$ \\
\hline $\begin{array}{l}\text { haushalts- und personen- } \\
\text { bezogene Dienstleistungs- } \\
\text { berufe }\end{array}$ & $\begin{array}{c}0,975 \\
(0,121)\end{array}$ & $\begin{array}{c}1,174 \\
(0,704)\end{array}$ & $\begin{array}{c}1,171 \\
(0,124)\end{array}$ & $\begin{array}{c}0,802 \\
(0,338)\end{array}$ & $\begin{array}{c}0,967 \\
(0,109)\end{array}$ & $\begin{array}{l}0,866 \\
(0,427)\end{array}$ \\
\hline \multicolumn{7}{|l|}{ Qualifikation } \\
\hline $\begin{array}{l}\text { Durchschnitt zum Wirt- } \\
\text { schaftszweig }\end{array}$ & $\begin{array}{c}1,098 \\
(0,053) \\
\end{array}$ & $\begin{array}{l}1,101^{* *} \\
(0,053) \\
\end{array}$ & $\begin{array}{l}1,111^{* * *} \\
(0,045)\end{array}$ & $\begin{array}{l}1,108^{* *} \\
(0,045) \\
\end{array}$ & $\begin{array}{l}1,134^{* * *} \\
(0,047)\end{array}$ & $\begin{array}{l}1,137^{* * *} \\
(0,047) \\
\end{array}$ \\
\hline \multicolumn{7}{|l|}{$\begin{array}{l}\text { Fortsetzung Tabelle } 10 \text { auf } \\
\text { der nächsten Seite }\end{array}$} \\
\hline \multicolumn{7}{|l|}{$\begin{array}{l}\text { Bildung (Referenz: Haupt- } \\
\text { schulabschluss) }\end{array}$} \\
\hline Realschulabschluss & $\begin{array}{c}0,916 \\
(0,054)\end{array}$ & $\begin{array}{c}0,974 \\
(0,114) \\
\end{array}$ & $\begin{array}{l}1,010 \\
(0,051)\end{array}$ & $\begin{array}{c}1,144 \\
(0,123)\end{array}$ & $\begin{array}{c}0,962 \\
(0,052)\end{array}$ & $\begin{array}{c}0,929 \\
(0,091)\end{array}$ \\
\hline Abitur & $\begin{array}{c}1,057 \\
(0,101) \\
\end{array}$ & $\begin{array}{c}1,219 \\
(0,272) \\
\end{array}$ & $\begin{array}{l}1,252^{* *} \\
(0,119)\end{array}$ & $\begin{array}{c}1,604^{* *} \\
(0,359) \\
\end{array}$ & $\begin{array}{l}1,438^{* * *} \\
(0,128) \\
\end{array}$ & $\begin{array}{r}1,442^{*} \\
(0,284) \\
\end{array}$ \\
\hline Regionalvariable & ja & ja & ja & $\mathrm{ja}$ & ja & $\mathrm{ja}$ \\
\hline Staatsangehörigkeit & $\begin{array}{c}0,923 \\
(0,071) \\
\end{array}$ & $\begin{array}{c}0,916 \\
(0,071) \\
\end{array}$ & $\begin{array}{l}1,164^{* * *} \\
(0,067)\end{array}$ & $\begin{array}{l}1,165^{* * *} \\
(0,067)\end{array}$ & $\begin{array}{c}1,080 \\
(0,064) \\
\end{array}$ & $\begin{array}{c}1,078 \\
(0,064) \\
\end{array}$ \\
\hline \multicolumn{7}{|l|}{$\begin{array}{l}\text { Interaktionseffekte (Aus- } \\
\text { bildungsbereiche * Schul- } \\
\text { bildung) }\end{array}$} \\
\hline $\begin{array}{l}\text { Land-/ Forstwirtschaftsbe- } \\
\text { rufe }\end{array}$ & & $\begin{array}{c}0,919 \\
(0,057) \\
\end{array}$ & & $\begin{array}{c}0,933 \\
(0,077) \\
\end{array}$ & & $\begin{array}{l}1,187^{* *} \\
(0,097) \\
\end{array}$ \\
\hline Chemieberufe & & $\begin{array}{c}0,640 \\
(0,174) \\
\end{array}$ & & $\begin{array}{c}0,714 \\
(0,196) \\
\end{array}$ & & $\begin{array}{c}1,229 \\
(0,263) \\
\end{array}$ \\
\hline Metallberufe & & $\begin{array}{c}0,969 \\
(0,153) \\
\end{array}$ & & $\begin{array}{c}0,920 \\
(0,140) \\
\end{array}$ & & $\begin{array}{c}1,130 \\
(0,166) \\
\end{array}$ \\
\hline Textilberufe & & $\begin{array}{c}0,945 \\
(0,162) \\
\end{array}$ & & $\begin{array}{c}0,925 \\
(0,146) \\
\end{array}$ & & $\begin{array}{c}1,077 \\
(0,151) \\
\end{array}$ \\
\hline $\begin{array}{l}\text { Berufe im Nahrungsmit- } \\
\text { telbereich }\end{array}$ & & $\mathrm{ja}$ & & ja & & ja \\
\hline Elektroberufe & & $\begin{array}{c}0,447^{* *} \\
(0,153) \\
\end{array}$ & & $\begin{array}{c}0,822 \\
(0,259) \\
\end{array}$ & & $\begin{array}{c}0,790 \\
(0,238) \\
\end{array}$ \\
\hline Bauberufe & & $\begin{array}{c}0,826 \\
(0,207) \\
\end{array}$ & & $\begin{array}{c}0,883 \\
(0,162) \\
\end{array}$ & & $\begin{array}{c}0,919 \\
(0,155) \\
\end{array}$ \\
\hline $\begin{array}{l}\text { Infrastruktur-/ Transport- } \\
\text { dienstleistungs-berufe }\end{array}$ & & $\begin{array}{c}1,088 \\
(0,175) \\
\end{array}$ & & $\begin{array}{l}0,748^{* *} \\
(0,100)\end{array}$ & & $\begin{array}{c}1,048 \\
(0,164)\end{array}$ \\
\hline $\begin{array}{l}\text { Finanzdienstleistungs- } \\
\text { berufe }\end{array}$ & & $\begin{array}{c}1,016 \\
(0,233) \\
\end{array}$ & & $\begin{array}{c}1,201 \\
(0,438) \\
\end{array}$ & & $\begin{array}{c}1,474 \\
(0,435) \\
\end{array}$ \\
\hline Verwaltungsberufe & & $\begin{array}{c}0,934 \\
(0,140) \\
\end{array}$ & & $\begin{array}{c}0,919 \\
(0,137) \\
\end{array}$ & & $\begin{array}{c}1,005 \\
(0,136) \\
\end{array}$ \\
\hline $\begin{array}{l}\text { produktionsnahe Dienst- } \\
\text { leistungsberufe }\end{array}$ & & $\begin{array}{c}0,898 \\
(0,153) \\
\end{array}$ & & $\begin{array}{c}0,805 \\
(0,131) \\
\end{array}$ & & $\begin{array}{c}0,822 \\
(0,121) \\
\end{array}$ \\
\hline \multicolumn{7}{|c|}{ Fortsetzung Tabelle 10 auf der nächsten Seite } \\
\hline
\end{tabular}




\begin{tabular}{|c|c|c|c|c|c|c|}
\hline & \multicolumn{2}{|c|}{$\begin{array}{c}\text { Abschlusskohorte } \\
2000-2003\end{array}$} & \multicolumn{2}{|c|}{$\begin{array}{c}\text { Abschlusskohorte } \\
1996-1999\end{array}$} & \multicolumn{2}{|c|}{$\begin{array}{c}\text { Abschlusskohorte } \\
1992-1995\end{array}$} \\
\hline & Modell 1" & Modell 2 & Modell $1^{n}$ & Modell 2 & Modell 1 & Modell 2 \\
\hline & $\begin{array}{l}\text { Abgangs- } \\
\text { rate }\end{array}$ & $\begin{array}{l}\text { Abgangs- } \\
\text { rate }\end{array}$ & $\begin{array}{l}\text { Abgangs- } \\
\text { rate }\end{array}$ & $\begin{array}{l}\text { Abgangs- } \\
\text { rate }\end{array}$ & $\begin{array}{l}\text { Abgangs- } \\
\text { rate }\end{array}$ & $\begin{array}{l}\text { Abgangs- } \\
\text { rate }\end{array}$ \\
\hline $\begin{array}{l}\text { haushalts-/ personenbezo- } \\
\text { gene Dienstleistungsberufe }\end{array}$ & & $\begin{array}{c}0,894 \\
(0,274)\end{array}$ & & $\begin{array}{c}1,220 \\
(0,266)\end{array}$ & & $\begin{array}{c}1,074 \\
(0,271)\end{array}$ \\
\hline Zahl der Fälle n & 5427 & 5427 & 5907 & 5907 & 5460 & 5460 \\
\hline$\chi^{2}(29)$ & $46,43^{* *}$ & & $70,11^{* * *}$ & & $84,13^{* * *}$ & \\
\hline$\chi^{2}(41)$ & & $59,94 * *$ & & $81,70^{* * *}$ & & $101,91^{* * *}$ \\
\hline
\end{tabular}

Quelle: IABS 1975-2004, eigene Berechnungen

\# ohne Interaktionseffekte; \#\# mit Interaktionseffekten

*** 1\%-Signifikanzniveau; ** 5\%-Signifikanzniveau; * 10\%-Signifikanzniveau

Werte in Klammern bezeichnen die Standardfehler

Die Bedeutung der Einflussvariablen auf die Abgangsrate aus Arbeitslosigkeit verändert sich im Vergleich zu der Betrachtung der verschiedenen Outcomes an der zweiten Schwelle. Sind für die Abschlusskohorten 2000-2003 die Ausbildungsvergütungen und auch die Ausbildungsdauer für den Verbleib im Betrieb von besonderer Relevanz, wirken sie sich zwar auch im Falle der Arbeitslosigkeitsdauer immer noch positiv auf die Hazardrate aus, diese Einflüsse sind aber nicht signifikant. Dagegen wirkt sich die Ausbildungsdauer negativ auf die Hazardrate und dementsprechend positiv auf die Verweildauer in Arbeitslosigkeit aus. Dieser Zusammenhang ist nicht zu erwarten, da hier ebenfalls angenommen wird, dass durch die längere Ausbildungsdauer ein höherer Bildungsstand vorliegt, der sich dann positiv auf die Abgangsrate auswirkt. Allerdings kann gerade dieser höhere Bildungsstand zu einer längeren Arbeitslosigkeitsdauer führen, weil die Suche nach einem ausbildungsadäquaten Arbeitsplatz zeitintensiver ist. ${ }^{101}$ Dagegen ist die logarithmierte Lohnsumme des Ausbildungsbetriebs für die Höhe der Abgangsrate von Bedeutung. Der Effekt ist negativ. Eine marginale Erhöhung der Lohnsumme des Ausbildungsbetriebes ergibt eine Hazardrate der arbeitslosen Ausbildungsabsolventen von nur ungefähr 95\% der Hazardrate der Absolventen in einem Ausbildungsbetrieb mit geringerer Lohnsumme. Personen mit einer Ausbildung in einem Ausbildungsbetrieb, dessen Löhne und Gehälter für die ausgebildeten Fachkräfte hoch sind, fordern entsprechende Löhne bei der Jobsuche. Dies wirkt über den Reservationslohn, ab dem ein Ausbildungsabsolvent bereit ist, einen Arbeitsplatz anzu-

${ }^{101}$ Zudem besteht die Möglichkeit für Ausbildungsabsolventen, die während der Ausbildung überdurchschnittliche Leistungen erbringen, die Ausbildungsdauer um ein Jahr zu verkürzen. So weisen Ausbildungsabsolventen mit einer kurzen Ausbildungsphase dennoch einen hohen Bildungsstand auf. Dies wirkt sich ebenfalls verkürzend auf die Arbeitslosigkeitsdauer aus. 
nehmen. Da aber Informationsasymmetrien hinsichtlich der tatsächlichen Produktivität sowie der Gründe für das Verlassen des Ausbildungsbetriebes existieren, werden künftige Unternehmen bei der Bewerberauswahl selektiv vorgehen und ihnen nach Möglichkeit einen Lohn entsprechend der Produktivitätshöhe, über die sie Kenntnis besitzen, anbieten. Die Stellenbesetzung bzw. der Abgang aus Arbeitslosigkeit wird dann erst erfolgen, wenn die unterschiedlichen Lohnvorstellungen ausgeglichen sind. Daher spricht dies für die Bestätigung der zweiten Hypothese, dass Ausbildungsabsolventen, die nach der dualen Berufsausbildung arbeitslos werden, länger in Arbeitslosigkeit verweilen, wenn der Bildungsstand entsprechend hoch ist.

Beeinflusst die Größe des Ausbildungsbetriebes den Verbleib an der zweiten Schwelle, insbesondere den Betriebswechsel signifikant, schwächt sich dieser bei der Betrachtung der Arbeitslosigkeitsdauer ab. Lediglich Ausbildungsabsolventen, deren Ausbildung in einem Betrieb mit mehr als 500 Beschäftigten stattfindet, haben signifikante Vorteile, die Arbeitslosigkeit schnell wieder zu verlassen. So liegt die Hazardrate $36 \%$ über derjenigen für Ausbildungsabsolventen mit einer Ausbildung in einem Betrieb bis zu neun Beschäftigten. Dieser Effekt ist auf dem 5\%- Niveau signifikant. Unter der Annahme, dass in Großunternehmen die Bildung und die Ausbildungsqualität höher liegen als in einem Betrieb mit bis zu neun Beschäftigten bedeutet dies eine Verkürzung der Arbeitslosigkeitsdauer. Die zu überprüfende Hypothese kann durch diesen Einflussfaktor nicht bestätigt werden. Danach müssten Ausbildungsabsolventen der großen Betriebe aufgrund ihres anzunehmenden höheren Humankapitals länger nach einer neuen Arbeitsstelle suchen. Das Gegenteil ist der Fall. Eine bessere und demnach investitionstheoretisch ausgerichtete Ausbildung bringt arbeitslose Ausbildungsabsolventen schneller wieder in Erwerbstätigkeit.

Die meisten Ausbildungsbereiche üben keinen signifikanten Einfluss auf die Abgangsrate aus Arbeitslosigkeit auf. Lediglich die Infrastruktur- und Transportdienstleistungsberufe sowie die produktionsnahen Dienstleistungsberufe beeinflussen die Arbeitslosigkeitsdauer ihrer Absolventen entscheidend. Aus beiden Bereichen weisen Ausbildungsabsolventen gegenüber der Referenzgruppe eine niedrigere Abgangsrate aus Arbeitslosigkeit auf. Die Hazardrate für Absolventen mit einer Ausbildung im produktionsnahen Bereich liegt bei $80 \%$ der Abgangsrate für Absolventen mit einem Ausbildungsberuf in der Land- und Forstwirtschaft. Dieser Effekt ist auf dem 5\%-Niveau signifikant. Auch dieser Einfluss ist zu erwarten, denn dem produktionsnahen Dienstleistungsbereich lassen sich insbesondere technische Ausbildungsberufe zuordnen, die in der Regel hohes technologiespezifisches Wissen vorweisen, was wiederum einen hohen allgemeinen Bildungsstand erfordert. Auch dies stellt Ansprüche an den zukünftigen Arbeitsplatz hinsichtlich der Vermeidung eines zu hohen Produktivitätsverlustes. In diesem Tätigkeitsbereich erfordern insbesondere die an den Arbeitsplätzen zu erledigenden Aufgaben hohe Anforderungen, weswegen auch die qualifikationsadäquate Besetzung der Stellen auf Seiten der 
Unternehmen eine gewisse Zeit beansprucht. Dies wirkt sich für Ausbildungsabsolventen, die eine Arbeitsstelle suchen, positiv auf die Arbeitslosigkeitsdauer aus. Ausbildungsabsolventen mit einer Ausbildung im Infrastruktur- und Transportbereich haben eine höhere Hazardrate, sie beträgt aber nur $86 \%$ der Abgangsrate von Absolventen des Bereiches der Land- und Forstwirtschaft. Ebenso wie das erhöhte Arbeitslosigkeitsrisiko dieser Absolventen lässt sich auch deren längere Arbeitslosigkeit durch die relativ geringen Beschäftigungsalternativen dieses Teilmarktes und des hohen Anteils an betriebsspezifischem Humankapital erklären (vgl. hierzu auch Kap. 8.1.2).

Die Qualifikationsvariablen lassen keinen eindeutigen Effekt auf die Länge der Arbeitslosigkeitsperiode erkennen. So realisieren Ausbildungsabsolventen, deren Fähigkeiten unterhalb des durchschnittlichen Niveaus innerhalb des Wirtschaftszweiges liegen, eine höhere Abgangsrate als Personen mit überdurchschnittlichen Fähigkeiten. Hier liegt die Abgangsrate 10\% über der Abgangsrate derjenigen, die innerhalb des Wirtschaftszweiges überdurchschnittliche Fähigkeiten aufweisen. Dieser Effekt ist auf dem 10\%-Niveau signifikant. Daneben zeigen die Schätzungen, dass Absolventen mit höherem Bildungsabschluss eine kürzere Arbeitslosigkeitsdauer als Absolventen mit niedrigerem Schulabschluss haben. Geht ein geringerer Teil der Ausbildungsabsolventen mit Realschulabschluss gegenüber der Referenzgruppe in Beschäftigung über, ist die Abgangsrate der Personen mit Abitur um 6\% höher als diejenige der Hauptschulabsolventen. Absolventen mit einem Realschulabschluss weisen aber nur $91 \%$ der Hazardrate von Hauptschulabsolventen auf. Die niedrige Hazardrate der Personen mit mittlerem Schulabschluss lässt sich anhand verschiedener Aspekte erklären. Absolventen mit Realschulabschluss erlernen Berufe sowohl im handwerklichen als auch kaufmännischen Bereich, weshalb entsprechend stark auch die erlernten Ausbildungsberufe innerhalb dieser Absolventengruppe streuen. So besetzen sie einerseits Berufe, die auch von Hauptschulabsolventen besetzt werden und die unter Umständen schlechte Beschäftigungsaussichten bieten. Da die jungen Erwachsenen mit Realschulabschluss gegenüber denjenigen mit Hauptschulabschluss eine bessere Ausgangsposition haben, zielt die Suche vorwiegend auf einen qualifikationsadäquaten Arbeitsplatz ab. Dagegen werden Hauptschulabsolventen häufig Stellen besetzen, die nicht ihrer Ausbildung entsprechen. Andererseits erlernen Ausbildungsabsolventen mit mittlerer Reife jedoch auch Berufe, in denen Abiturienten anzutreffen sind, wobei letztere die besseren Bildungsvoraussetzungen mitbringen. Dies beeinflusst die Wahrscheinlichkeit, arbeitslos zu werden, positiv.

Im zweiten Modell, unter Berücksichtigung der Interaktionseffekte, lassen sich ähnliche Einflüsse erkennen. Erwähnenswert sind jedoch nur die Einflüsse hinsichtlich der Ausbildungsberufe. So weisen Absolventen mit einer Ausbildung in den Elektroberufen eine signifikant höhere Abgangsrate aus Arbeitslosigkeit auf als Absolventen der Referenzgruppe. Innerhalb dieses Ausbildungsbereiches sinkt jedoch die Abgangsrate mit steigender Schulbildung. Die Ab- 
gangsrate der Absolventen mit Abitur liegt nur bei 45\% gegenüber der Hazardrate von Absolventen mit mittlerer Reife. Der Effekt ist auf dem 5\%Niveau signifikant. Hierbei ist davon auszugehen, dass Ausbildungsabsolventen mit hohem Bildungsstand betriebsspezifischere Ausbildungsberufe erlernen als Personen mit einer geringeren Schulbildung. Infolgedessen verlängert sich entsprechend der theoretischen Annahmen die Dauer der Arbeitslosigkeit. Dagegen spielen Schulabschlüsse für Ausbildungsberufe der Infrastruktur- und Transportdienstleistungen und der produktionsnahen Dienstleistungen im zweiten Modell keine signifikante Rolle bei der Erklärung von Arbeitslosigkeitsdauer.

Für die beiden älteren Abschlusskohorten lassen sich einige Unterschiede erkennen, die unter dem Aspekt verschiedener gesamtwirtschaftlicher Rahmenbedingungen $\mathrm{zu}$ betrachten sind. So hat die Ausbildungsdauer für die Abschlusskohorte 1996-1999 zwar auch einen negativen Effekt. Dieser ist aber signifikant. Je länger die Ausbildungsdauer, desto länger ist auch die Arbeitslosigkeitsdauer, denn die Steigerung der Ausbildungsdauer weist eine 19\%Punkte niedrigere Abgangsrate (Hazardratio $=0,81$ ) auf. Signifikant negative Effekte auf die Abgangsrate aus Arbeitslosigkeit in eine Beschäftigung sind neben den Chemie- und holzverarbeitenden Berufen auch für metallverarbeitende Berufe, Infrastruktur- und Transportdienstleistungsberufe sowie produktionsnahe Dienstleistungsberufe zu konstatieren. Diese haben gegenüber der Referenzgruppe (land- und forstwirtschaftliche Ausbildungsberufe) eine niedrigere Abgangsrate, obwohl diese Ausbildungsberufe einen höheren Humankapitalstand benötigen und fördern. Allerdings sind diese Einflussfaktoren nicht signifikant.

Ebenso wie die jüngere Abschlusskohorte haben Ausbildungsabsolventen 1996-1999 mit einem überdurchschnittlichen Ausbildungsniveau innerhalb eines Wirtschaftszweiges eine niedrigere Abgangsrate. Diese hat für die Abschlusskohorten 1996-1999 einen signifikanten Einfluss. Weiterhin gilt auch für Abiturienten dieser Abschlusskohorte gegenüber Hauptschulabsolventen mit dualer Berufsausbildung eine signifikant höhere Abgangsrate aus Arbeitslosigkeit.

Unter Berücksichtigung der Interaktionseffekte bleiben die Einflüsse der erklärenden Variablen unverändert. Nur für Ausbildungsberufe der Infrastrukturund Transportdienstleistungen übt der Bildungsstand einen signifikanten Einfluss auf die Verweildauer in Arbeitslosigkeit aus. In diesem Bereich ist die Abgangsrate von Ausbildungsabsolventen mit einem höheren Bildungsstand geringer als von jungen Erwachsenen mit niedrigerem Bildungsstand. Dieser Einfluss ist auf eine gute Ausbildung zurückzuführen, die jedoch arbeitslosen Absolventen geringe (adäquate) Beschäftigungsmöglichkeiten innerhalb des Bereiches bietet (siehe Kap. 8.1.2).

Für die Abschlusskohorten 1992-1995 hat die Betriebsgrößenklasse keinen signifikanten Effekt. Lediglich im zweiten Modell weist eine Ausbildung in 
einem Betrieb zwischen 10 und 49 Mitarbeitern einen negativen Effekt auf dem 10\%- Signifikanzniveau auf. In diesem Fall liegt der Hazardratio bei 91\% gegenüber derjenigen für Ausbildungsabsolventen von Kleinstbetrieben, den $\mathrm{Zu}-$ stand der Arbeitslosigkeit zu dem betrachteten Zeitintervall zu verlassen. Die Bedeutung dieser Betriebsgröße nimmt für die Abschlusskohorten 1996-1999 zu. Im gesamten Zeitraum von 1975 und 2003 wirkt sich die Größe des Betriebs, innerhalb dessen die Ausbildung durchgeführt wird, für die Arbeitslosigkeitsdauer nicht signifikant aus (siehe Tabelle 18 im Anhang).

Weiterhin weisen für die Abschlusskohorten 1992-1995 insbesondere die Ausbildungsberufe des Baus, der Infrastruktur- und Transportdienstleistungen, der Verwaltung sowie der produktionsnahen Dienstleistungen signifikant negative Effekte auf die Abgangsrate aus Arbeitslosigkeit auf. So haben die Absolventen in den Bauberufen eine höhere Wahrscheinlichkeit $(86 \%$ gemessen am Hazardratio für die Referenzgruppe) zu einem betrachteten Zeitintervall aus Arbeitslosigkeit abzugehen als die Absolventen in Infrastruktur- und Transportdienstleistungsberufen (78\% gemessen am Hazradratio für die Referenzgruppe), jedoch ist letzterer Effekt auf 1\%- Niveau signifikant. Bei der Betrachtung der Berufe mit signifikanten Einflüssen ist festzustellen, dass die Absolventen der Verwaltungsberufe und der produktionsnahen Dienstleistungsberufe tendenziell ein höheres Ausbildungsniveau gegenüber der Referenzgruppe aufzeigen und auch die geringste Abgangsrate aus Arbeitslosigkeit aufgrund der Suche nach einer ausbildungsadäquaten Beschäftigung aufweisen. Bei der Berücksichtigung der Interaktionseffekte ändern sich die Wirkungen der verschiedenen Ausbildungsberufe. So haben die Chemieberufe signifikant positive Auswirkungen auf die Hazardrate. Diese Ausbildungsabsolventen weisen eine längere Arbeitslosigkeit auf. Ferner haben über den gesamten Betrachtungszeitraum die Bauberufe die größten Veränderungen im Einfluss auf die Arbeitslosigkeitsdauer aufgewiesen (siehe Tabelle 18 im Anhang). Dies zeigt die relativ große Abhängigkeit von konjunkturellen Entwicklungen dieses Bereiches.

In den Abschlusskohorten 1992-1995 sowie 1996-1999 sind die Bildungsvariablen, mit Ausnahme des Realschulabschlusses, signifikant. Auch hier scheint die Variable, welche die unterdurchschnittlichen formalen Qualifikationen der Ausbildungsabsolventen widerspiegelt, die Hypothese zu bestätigen. Denn der signifikante Hazardratio wirkt positiv auf die Abgangsrate aus Arbeitslosigkeit, die bei einem unterdurchschnittlich ausgebildeten gegenüber einem überdurchschnittlich ausgebildeten jungen Erwachsenen um 13\% höher liegt. Dagegen scheint sich bei der Betrachtung der Schulbildungsvariablen die Hypothese nicht bestätigen zu lassen, denn die Absolventen mit Abitur haben eine um 44\% höhere Abgangsrate zum Betrachtungszeitpunkt als die Absolventen mit Hauptschulabschluss. Die Ergebnisse der Kaplan-Meier Schätzung werden nicht bestätigt. Im Allgemeinen ist ein hohes Bildungsniveau bei der Stellensuche von Vorteil. 
Schließlich schwächt sich die Stärke der Einflussfaktoren der Bauberufe, des Bereiches der Infrastruktur- und Transportdienstleistungsberufe, der Verwaltungsberufe sowie der produktionsnahen Dienstleistungsberufe ab. Dagegen wirkt sich eine Ausbildung im Nahrungsmittelbereich gegenüber einer Ausbildung im Land- und Forstwirtschaftsbereich signifikant und positiv auf die Abgangsrate aus Arbeitslosigkeit aus. Die Schulbildung war in dieser Abschlusskohorte insbesondere in Land- und Forstwirtschaftsberufen sowie in Elektroberufen von besonderer Relevanz, allerdings mit unterschiedlichen Wirkungsrichtungen. Während Absolventen mit höherer Bildung in Land- und Forstwirtschaftsberufen schneller aus Arbeitslosigkeit abgingen, verblieben diese mit einer Ausbildung in einem Elektroberuf länger in Arbeitslosigkeit. Vor allem im ersten Bereich gibt es eine Vielzahl von Möglichkeiten, wie die Selbständigkeit, aber auch eine ausbildungsinadäquate Beschäftigung, um den Zustand der Arbeitslosigkeit zu beenden.

Hypothese 2, dass Ausbildungsabsolventen mit einem höheren Abschluss eine längere Arbeitslosigkeitsperiode realisieren, kann nicht eindeutig verworfen bzw. bestätigt werden: Betriebliche Faktoren, wie Ausbildungsvergütung und Betriebsgröße, und die Schulbildungsvariable implizieren eine höhere Abgangsrate aus Arbeitslosigkeit für junge Erwachsene mit einem höheren Bildungsabschluss und einer höheren Ausbildungsqualität. Dagegen haben Ausbildungsabsolventen, die unterdurchschnittliche Fähigkeiten innerhalb ihres Wirtschaftszweiges besitzen, eine höhere Abgangsrate als diejenigen mit überdurchschnittlichen Fähigkeiten. Auch einige Ausbildungsbereiche, denen zugeschrieben wird, dass sie eine gute Ausbildungsqualität liefern, haben einen negativen Einfluss auf die Abgangsrate, was ebenfalls impliziert, dass die besser ausgebildeten Absolventen eine längere Arbeitslosigkeitsdauer aufweisen, insbesondere in den Ausbildungsbereichen der Verwaltungsberufe und den produktionsnahen sowie haushalts- und personenbezogenen Dienstleistungen. Diese geringere Hazardrate steigt im Zeitverlauf aber gegenüber der Referenzgruppe, sodass vor allem Ausbildungsbereiche mit einem höheren Bildungsstand auch höhere Abgangsraten haben, wodurch sich auch in diesen Bereichen die Arbeitslosigkeitsdauer mit höherem (Aus-)Bildungsniveau verkürzt. Umgekehrt liegen insbesondere die Abgangsraten der handwerklichen Ausbildungsbereiche über der Abgangsrate der Referenzgruppe. Dies deutet auch darauf hin, dass Absolventen in Berufen mit niedrigerem Bildungsstand schneller aus Arbeitslosigkeit abgehen. Auch die Interaktionseffekte weisen keine klare Einflussstruktur auf die Arbeitslosigkeitsdauer aus. So gilt nur für wenige Ausbildungsberufe bei höherer Bildung eine geringere Abgangsrate aus Arbeitslosigkeit. Eine bessere Schul- bzw. Berufsausbildung schützt demnach in den meisten Fällen vor einer längeren Arbeitslosigkeit. Allerdings sind die Einflüsse nicht signifikant, weshalb keine klare Aussage hinsichtlich der Bestätigung oder Verwerfung der Hypothese gemacht werden kann. Betrachtet man aber die Abschlusskohorten getrennt voneinander, so lassen sich Tendenzen einer 
längeren Arbeitslosigkeitsdauer für besser ausgebildete Absolventen 19921995 erkennen. Viele der Ausbildungsbereiche, denen eine hohe Ausbildungsqualität zuzuschreiben ist, weisen negative Hazardratios auf. Ebenso ist der Einfluss der Betriebsgröße gegenüber der Referenzgröße negativ, aber insignifikant.

\subsection{Bedeutung der Arbeitslosigkeit für die Beschäftigungsdauer zu Be- ginn des Erwerbslebens}

Bisher lag der Fokus auf dem Verbleib der Ausbildungsabsolventen an der zweiten Schwelle. Dabei wurden betriebliche und individuelle Einflussfaktoren auf die Wahrscheinlichkeit einer Weiterbeschäftigung im Ausbildungsbetrieb, eines Betriebswechsels, einer Arbeitslosigkeit sowie auf die Arbeitslosigkeitsdauer untersucht. Im Folgenden wird die empirische Untersuchung um den Aspekt des Einflusses der Arbeitslosigkeit zu Beginn des Erwerbslebens auf den Erwerbsverlauf innerhalb des ersten Jahres nach der dualen Berufsausbildung erweitert. ${ }^{102}$ Es wird die Beschäftigungsdauer der direkt nach der dualen Berufsausbildung arbeitslosen mit derjenigen der erwerbstätigen Absolventen verglichen. Ziel ist es daher zu analysieren, ob die Arbeitslosigkeit zu Beginn des Erwerbslebens für bestimmte Absolventengruppen einen größeren Nachteil bringt als für andere. Daher wird die dritte Hypothese, dass besser ausgebildete Absolventen, die direkt nach der dualen Berufsausbildung, arbeitslos sind, eine kürzere kumulierte Beschäftigungsdauer aufweisen als Absolventen, die direkt nach Beendigung der Ausbildung erwerbstätig sind, untersucht. Hierbei wird die Wirkung der Arbeitslosigkeit auf die Anzahl der Tage in Beschäftigung innerhalb des ersten Jahres nach Abschluss der dualen Berufsausbildung analysiert.

\subsubsection{Beschreibung der Methode}

Das Matching-Verfahren wird zur Beschreibung eines kausalen Effekts auf ein nachgelagertes Ereignis verwendet. Dabei wird der Einfluss eines bestimmt eintretenden Ereignisses auf ein später eintretendes Ereignis untersucht. Die Wirkungen anderer Einflussfaktoren werden dadurch ausgeschaltet. Üblicherweise wird das Matching-Verfahren zur Beurteilung von arbeitsmarktpolitischen Maßnahmen verwendet. Hierbei lässt sich die Fragestellung behandeln, inwieweit die Teilnahme an einer solchen Maßnahme künftige Beschäftigungsaussichten verbessert. Vor diesem Hintergrund ist denkbar, dass Arbeitslosigkeit direkt nach der dualen Berufsausbildung die kumulierte Beschäftigungsdauer nach einem Jahr beeinflusst. Deshalb ist es möglich, das MatchingVerfahren ebenfalls auf die hier behandelte Fragestellung anzuwenden.

${ }^{102}$ Der Zeitraum des ersten Jahres nach der dualen Berufsausbildung wurde bei der technischen Umsetzung um zwei Monate verschoben. Das heißt, der hier betrachtete Zeitraum erstreckt sich vom zweiten bis zum 14. Monat nach Abschluss der dualen Berufsausbildung. 
Der Bestimmung des kausalen Effekts liegt die Idee zugrunde, dass ein $\mathrm{Zu}$ stand eintritt, wenn diesem ein bestimmtes Ereignis vorausgeht. Dies basiert auf der Vorstellung des Eintrittes potenzieller Ereignisse Y, der in Abhängigkeit von dem kausal wirksamen Faktor $D$ (Arbeitslosigkeit direkt nach der dualen Berufsausbildung) steht (Gangl/DiPrete 2004: 4). Im Kontext der zu behandelnden Fragestellung ist das potenzielle Ereignis die Dauer der Beschäftigung in Tagen innerhalb des ersten Jahres nach der dualen Berufsausbildung. Um den Effekt der Arbeitslosigkeit analysieren zu können, muss die hypothetische Frage gestellt werden: „Wie hoch wäre die Beschäftigungsdauer innerhalb des ersten Jahres nach Abschluss der Ausbildung, wenn der Ausbildungsabsolvent weiterbeschäftigt gewesen wäre?". Dies erfordert eine Betrachtung der Ausbildungsabsolventen sowohl im tatsächlich realisierten wie auch im hypothetischen Zustand. Da der Ausbildungsabsolvent jedoch nicht in zwei unterschiedlichen Zuständen betrachtet werden kann, wird auch oftmals von einem Problem fehlender Daten und in diesem Zusammenhang von einem fundamentalen Evaluierungsproblem gesprochen (Imbens 2004: 5). ${ }^{103}$ Der direkte Vergleich einer Arbeitslosigkeitsperiode und einer Beschäftigungsperiode direkt nach der dualen Berufsausbildung für ein und dieselbe Person ist nicht möglich. Der Ausbildungsabsolvent kann nur in dem tatsächlich realisierten $\mathrm{Zu}$ stand beobachtet werden, entweder, wenn er arbeitslos oder, wenn er beschäftigt ist.

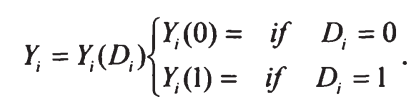

Die nach der Ausbildung beschäftigten Ausbildungsabsolventen, bei $D_{i}=0$, weisen eine kumulierte Beschäftigungsdauer in Höhe von $Y_{i}(0)$ auf. Dagegen realisieren die Ausbildungsabsolventen, die arbeitslos waren, $D_{i}=1$, die Ergebnisvariable in der Form $Y_{i}(1)$, welche die Länge der Periode in Erwerbstätigkeit dieser Absolventen angibt.

Beobachtet werden kann jedoch nur die Beschäftigungsdauer, die ein arbeitsloser Ausbildungsabsolvent innerhalb des ersten Jahres tatsächlich erreicht. Die kumulierte Länge der Periode in Erwerbstätigkeit, die er nach einem Jahr realisieren würde, wenn er direkt nach der dualen Berufsausbildung beschäftigt gewesen wäre, kann nicht beobachtet werden. Diese Beobachtung ist aber notwendig, um den Effekt der Arbeitslosigkeit auf die Beschäftigungsdau-

${ }^{103}$ Um Rückschlüsse auf die Effekte zwischen der Experimentalgruppe und der Kontrollgruppe zu ziehen, wird ein ökonometrisches Modell mit identifizierenden Annahmen betrachtet. Daher müssen wiederum Annahmen auf die hypothetische Bevölkerung, die auf der beobachteten Bevölkerung basiert, getroffen werden. Das wiederum bedeutet, dass Annahmen über die nicht realisierten Zustände der beobachteten Ausbildungsabsolventen getroffen werden (Caliendo 2006: 20). Bspw. kann keine Aussage darüber gemacht werden, ob direkt nach der Ausbildung beschäftigte Absolventen innerhalb des ersten Jahres tatsächlich arbeitslos werden und sich somit die Beschäftigungsdauer verkürzt. 
er zu ermitteln. Dies ist durch den Vergleich zweier Personen möglich, die ihre Ausbildung unter denselben Bedingungen absolvieren und auch ansonsten dieselben individuellen Merkmale aufweisen (vgl. Hujer et al. 2003: 18). Folglich unterscheiden sie sich nur im Ereignis $D$. Um einen derartigen Vergleich zu erreichen, müssen zwei Gruppen gebildet werden: die Experimentalgruppe und die Kontrollgruppe. Der Experimentalgruppe werden Fälle zugeordnet, die nach der dualen Berufsausbildung arbeitslos sind, $D=1$. Die Kontrollgruppe wird als Vergleichsbasis herangezogen. In dieser Gruppe sind alle Ausbildungsabsolventen enthalten, die nach der dualen Berufsausbildung erwerbstätig sind, somit gilt $D=0 .{ }^{104}$ Der kausale Effekt einer Arbeitslosigkeit direkt nach der dualen Berufsausbildung auf die Dauer der Beschäftigung lässt sich durch die Differenz zwischen der Anzahl der Tage, die ein arbeitsloser Absolvent in Beschäftigung verbringt, und der Anzahl der Tage, die ein direkt nach der Ausbildung erwerbstätiger Absolvent in Beschäftigung ist, bestimmen. Formal ist die Wirkung auf die Beschäftigungsperiode der Unterschied zwischen $Y_{1 i}=Y \mid D=1$ bei einem Verlassen des Ausbildungsbetriebes und $Y_{0 i}=Y \mid D=0$ bei einer Beschäftigung (im Ausbildungsbetrieb oder einem anderen Betrieb), $\left(Y_{1 i}=Y \mid D=1\right)-\left(Y_{0 i}=Y \mid D=0\right)$. Zur Bestimmung der Wirkung einer Arbeitslosigkeit nach der dualen Berufsausbildung müssen Zwillingspaare gebildet werden, wovon der eine Partner aus der Experimentalgruppe und der andere Partner aus der Kontrollgruppe stammt. Folglich werden sich die Paare nur durch das Ereignis einer Arbeitslosigkeits- bzw. Beschäftigungsperiode unterscheiden. Die Zuordnung der einzelnen Partner erfolgt nach den beobachtbaren Kovariaten $X$, welche betriebliche Rahmenbedingungen und individuelle Merkmale enthalten. Je mehr Einflussfaktoren jedoch bei der Bildung von MatchingPartnern berücksichtigt werden müssen, desto geringer ist die Wahrscheinlichkeit ein Paar mit derselben Merkmalsstruktur zu finden (Dehejia/Wahba 2002: 152). Um dieses so genannte Dimensionalitätsproblem zu lösen (D'Agostino 1998: 2267; Hujer et al. 2001: 178), wird meist der Propensity Score als Identifikationsvariable herangezogen. Nach Rosenbaum und Rubin (1983) reicht es aus, wenn Untersuchungseinheiten mit einer ähnlich hohen Eintrittswahrscheinlichkeit in Arbeitslosigkeit einander zugeordnet werden.

Der Ermittlung des kausalen Effekts von Arbeitslosigkeit auf die Beschäftigungsdauer innerhalb des ersten Jahres nach Abschluss der Ausbildung geht die Schätzung eines Regressionsmodells voraus. Dieses bestimmt den Einfluss der Kovariaten auf das Ereignis $\mathrm{Y}$ und dementsprechend auf den Eintritt der Arbeitslosigkeit

$$
Y_{i}=\alpha+X_{i}^{\prime} \beta_{2}+\varepsilon_{i t}
$$

${ }^{104}$ Die Kontrollgruppe wird mit Hilfe des Cross-Section-Estimators gebildet, wozu Querschnittsinformationen ausreichen. Dies ist von Vorteil, da aufgrund der relativ kurzen Arbeitsmarkterfahrung von Ausbildungsabsolventen nur wenige Längsschnittdaten für die Verwendung anderer Schätzer (bspw. Difference-in-Difference-Estimator) vorliegen. 
Die Übertragung des Regressionsmodells auf die Idee des MatchingVerfahrens erfolgt nun über eine Erweiterung eines eintretenden Ereignisses. So muss dem Ergebniszustand $Y_{i}\left(D_{i}\right)$ ein Ereignis $D$ vorangehen, durch welches das Ergebnis $Y$ erklärt werden kann. Daher muss das Ereignis wiederum als erklärende Variable in dem Regressionsmodell berücksichtigt werden. Die Matching-Methode basiert daher auf folgendem Zusammenhang:

$$
Y_{i}\left(D_{i}\right)=\alpha+\tau D_{i}+X_{i}^{\prime} \beta_{2}+\varepsilon_{i t}
$$

Das Ereignis darf aber wiederum nicht von dem Ergebnis beeinflusst werden. Daher gilt $Y_{0}, Y_{1} \amalg D \mid X$, wobei $\amalg$ für Unabhängigkeit steht (Caliendo 2006:30). Dann sind gleiche Verteilungen hinsichtlich des Arbeitslosigkeitsrisikos bzw. der Wahrscheinlichkeit der Erwerbstätigkeit zwischen den beiden Gruppen gewährleistet. ${ }^{105}$ Zudem muss bei dieser Regressionsgleichung die Unabhängigkeit zwischen der Arbeitslosigkeit direkt nach der dualen Berufsausbildung $(D)$ und den Kovariaten $(X)$, den betrieblichen und individuellen Voraussetzungen, und demnach $X_{i} \amalg D_{i} \mid e\left(X_{i}\right)$ gelten (Imbens/Wooldridge 2007). Schließlich basiert die Matching- Methode auf der Annahme der Exogenität (Unconfoundedness), also muss die Unabhängigkeit zwischen $\varepsilon_{i} \amalg X_{i}$ gewährleistet sein. Das heißt, alle Merkmale, die im ersten Berufsjahr die Beschäftigungstage nach einer anfänglichen Arbeitslosigkeit beeinflussen, müssen beobachtbar sein. Es ist möglich, dass letztere Bedingung durch das Selektionsproblem verletzt wird, da Schulabgänger bspw. aufgrund ihres Abschlusses ${ }^{106}$ bestimmten Ausbildungsberufen zugeteilt werden. Daher unterscheiden sich die Mitglieder der Kontrollgruppe stark von denen der Experimentalgruppe, ${ }^{107}$ sodass keine Matching-Partner gefunden werden können bzw. daraus eine schlechte Qualität des Matchings resultiert. In diesem Fall kann der Einfluss nicht robust geschätzt werden. Das Selektionsproblem wird teilweise durch die

${ }^{105}$ Bei der Annahme der Unabhängigkeit muss die Verteilung der Merkmale innerhalb der Kontrollgruppe der Verteilung innerhalb der Experimentalgruppe entsprechen. Gilt dies, sind die Erwartungswerte des Ereignisses identisch.

${ }^{106}$ Es ist auch die unbeobachtete Heterogenität zu berücksichtigen. So können Jugendliche aufgrund informeller Fähigkeiten bestimmten Betrieben oder auch Ausbildungsbereichen zugeteilt werden. Auch in diesem Fall können nicht genügend Matching-Partner gefunden werden, bzw. die Matchingqualität ist aufgrund der zu unterschiedlichen Merkmalsstruktur nicht ausreichend. Die Schätzung kann dann durch weitere Lockerung der Annahmen hinsichtlich der Identifikation bei den Matching-Partnern oder durch Verwendung einer passenden Kontrolleinheit für mehrere arbeitslose Absolventen (,Ziehen mit Zurücklegen“) durchgeführt werden (Gensler et al 2005: 43). Allerdings ist für die Art der Ausbildung und für den Verbleib danach das formal erworbene Humankapital ausschlaggebend, selbst bei Selektion zu Beginn der Ausbildung (Adda et al. 2007).

${ }^{107}$ Bspw. kann es im Extremfall sein, dass der Kontrollgruppe nur Ausbildungsabsolventen, die über Abitur verfügen und eine Ausbildung im produktionsnahen Dienstleistungsbereich absolviert haben, zugeordnet werden, während die Experimentalgruppe ausschließlich ausgebildete Landwirte mit Hauptschulabschluss enthält. 
Matching-Methode berücksichtigt. Hierzu ist es aber notwendig, dass der Bereich des ,Common Support' entsprechend groß ist. Demnach ist dies dann gegeben, wenn eine ausreichende Anzahl von Ausbildungsabsolventen in der Kontrollgruppe eine ähnliche Merkmalsstruktur aufweist wie Ausbildungsabsolventen in der Experimentalgruppe. Nur diese Fälle werden dann einander als Matching-Partner zugewiesen und dienen so als Grundlage zur Ermittlung des kausalen Effekts. So lässt sich die systematische Zuordnung zu einer bestimmten Gruppe abschwächen (Blundell et al. 2005, Imbens 2007). In diesem Fall finden sich genügend Matching-Partner. Um die Ähnlichkeit von MatchingPartnern weiter zu gewährleisten und somit das Selektivitätsproblem zu reduzieren, werden für die vorliegende Untersuchung Subgruppen nach den Merkmalen gebildet, von denen angenommen wird, dass diese die systematische $\mathrm{Zu}$ ordnung verstärken. ${ }^{108}$

Werden die Annahmen erfüllt, kann der kausale Effekt ermittelt werden. Hierfür gibt es unterschiedliche Methoden eines Matching-Verfahrens, die in der Regel auf dem Rubin Causal Modell basieren (u.a. Rubin 1974, 1978; Holland 1986; Pratt/Schlaifer 1988; Manski 1995; Heckman et al. 1997; Rosenbaum 2002). Der Average Treatment Effect on the Treated (ATT) untersucht hierbei den durchschnittlichen Wirkungszusammenhang auf die kumulierte Beschäftigungsdauer der direkt nach der dualen Berufsausbildung arbeitslosen Ausbildungsabsolventen, die nach einem Jahr wieder arbeitslos sind (vgl. Fier et al. 2005: 6; Gangl/DiPrete 2004: 6). Der Indikator gibt daher die Erwerbstätigkeitdauer an, welche Ausbildungsabsolventen, die den Ausbildungsbetrieb verlassen, aufweisen, wenn sie eigentlich entweder im Ausbildungsbetrieb verblieben oder in einem anderen Betrieb beschäftigt worden wären. Entsprechend weisen sie eine höhere Beschäftigungsdauer auf $\left(Y_{0}\right)$ :

$$
A T T=E\left(\Delta_{i} \mid D=1\right)=E\left(Y_{1} \mid D=1\right)-E\left(Y_{0} \mid D=1\right) .
$$

Entsprechend wird hier der tatsächliche Einfluss eines zufällig ausgewählten arbeitslosen Ausbildungsabsolventen auf die Beschäftigungsdauer innerhalb des ersten Jahres nach Abschluss der dualen Berufsausbildung berechnet $(\mathrm{Ca}-$ liendo/Hujer 2005: 3; Gangl/DiPrete 2004: 8). Dies bedeutet, dass der Zustand aller Ausbildungsabsolventen aus der Experimentalgruppe ein Jahr nach der dualen Berufsausbildung betrachtet wird. Nimmt der ATT einen negativen Wert an, ist eine direkt nach der dualen Berufsausbildung arbeitslose Person weniger Tage in Beschäftigung als eine direkt nach der Ausbildung beschäftig-

\footnotetext{
${ }^{108}$ Rosenbaum und Rubin (1983) schlagen einen Momentenschätzer vor, der die Annahme der Unabhängigkeit und des ,common support“ abschwächt. Nach Heckman et al. (1998) ist die Annahme der starken Ignoranz jedoch immer noch zu strikt. Entsprechend reicht eine im Durchschnitt erwartete Unabhängigkeit aus. $E\left(Y_{0} \mid X, D=1\right)=E\left(Y_{0} \mid X, D=0\right)$ genügt daher. Schließlich reicht es aus, wenn das Ergebnis der Vergleichsgruppe unabhängig von dem Ereignis ist, $Y_{0} \amalg D \mid X$ und die Wahrscheinlichkeit kleiner $1, P(D=1 \mid X)<1$.
} 
te Person. Ist der Effekt positiv, hat die Arbeitslosigkeit nach der dualen Berufsausbildung sogar vorteilhafte Auswirkungen auf die Beschäftigungsdauer.

Der Fokus liegt darauf, inwieweit die Dauer der Beschäftigung im ersten Jahr nach der dualen Berufsausbildung durch eine anfängliche Arbeitslosigkeitsphase verkürzt wird. Untersuchungsgegenstand ist die Struktur des Einflusses der Arbeitslosigkeitsphasen zwischen den Schulabschlüssen bzw. den Ausbildungsbereichen. Hierbei werden die Unterschiede sowohl zwischen den verschiedenen Schulabschlüssen als auch zwischen den einzelnen Ausbildungsbereichen unabhängig von anderen Einflussfaktoren untersucht. Daher sind die Effekte der betrieblichen und der konjunkturellen Faktoren zu kontrollieren, indem sie als Identifikationsvariablen, welche die Matching-Partner bestimmen, dienen. Diese Variablen sind neben den Abschlusskohorten die Betriebsgröße, das Alter bei Abschluss der dualen Berufsausbildung, die Staatsangehörigkeit sowie, ob der Absolvent, innerhalb seines Wirtschaftszweiges unterdurchschnittliche Qualifikationen erlangt hat. So werden durch die Verwendung des Abschlussjahres der Ausbildung als Identifikationsvariable konjunkturelle Effekte weitestgehend aufgehoben. ${ }^{109}$

\subsubsection{Empirische Evidenz des Einflusses der Arbeitslosigkeit}

In diesem Kapitel wird die empirische Evidenz des durchschnittlichen Effekts einer Arbeitslosigkeitsphase direkt nach der dualen Berufsausbildung auf die Beschäftigungsdauer innerhalb des ersten Jahres nach der dualen Berufsausbildung untersucht. Zunächst werden die verschiedenen Bildungsgruppen getrennt voneinander betrachtet. Tabelle 11 zeigt die durchschnittliche Beschäftigungsdauer der Experimentalgruppe und somit derjenigen, die den Start ins Erwerbsleben mit einer Arbeitslosigkeitsperiode vollziehen. Daneben wird die durchschnittliche Anzahl an Arbeitstagen der Kontrollgruppe aufgezeigt. Für die Schätzungen der ATT in Tabelle 11 und 12 lässt sich eine gute Matchingqualität feststellen. Dies ist am Mean Standardised Bias (MSB) zu erkennen, der sich nach der Zuweisung der Matchingpartner zueinander jeweils deutlich reduziert hat. ${ }^{110}$ Der durchschnittliche Effekt der Arbeitslosigkeit zu Beginn des Berufslebens bildet die Differenz der ermittelten Beschäftigungsdauern. Diese Indikatoren werden zunächst für alle drei Schulabschlüsse ermittelt.

Tabelle 11 zeigt, dass ein Einstieg in den Arbeitsmarkt, der durch Arbeitslosigkeit gekennzeichnet ist, die Beschäftigung negativ beeinflusst. Demnach verbringt ein Ausbildungsabsolvent, der direkt nach Abschluss der dualen Be-

\footnotetext{
${ }^{109}$ Von einem Einfluss der Konjunktur der Wirkung der Arbeitslosigkeit auf die Beschäftigungsdauer kann nicht vollständig abgesehen werden, da sich der ATT in wirtschaftlich ungünstigen Situationen vergrößert bzw. sich in stark konjunkturell abhängigen Ausbildungsbereichen auch verstärkt auswirken kann. Nach Abschlusskohorten getrennte Schätzungen ergaben aber dieselben Muster wie bei der gepoolten Ermittlung des ATT.

${ }^{110}$ Von einer guten Matchingqualität spricht man, wenn der MSB nach dem Matching maximal zwischen 3 und $5 \%$ hat.
} 
rufsausbildung arbeitslos wird, innerhalb des ersten Berufsjahres weniger Tage in Erwerbstätigkeit als ein Ausbildungsabsolvent, der unmittelbar nach der dualen Berufsausbildung erwerbstätig gewesen ist. Die kausalen Effekte wirken sich innerhalb der Bildungsgruppen unterschiedlich aus. So ist festzustellen, dass Ausbildungsabsolventen mit Hauptschulabschluss, die direkt nach der dualen Berufsausbildung arbeitslos sind, 60 Tage weniger in Beschäftigung sind als

\section{Tabelle 11: Average Treatment Effect der Arbeitslosigkeit auf die Beschäf- tigungsdauer im ersten Berufsjahr - nach Schulbildung (in Tagen)}

\begin{tabular}{|l|c|c|c|c|c|c|c|}
\hline & \multicolumn{4}{|c|}{ Kausaler Zusammenhang } & \multicolumn{2}{c|}{$\begin{array}{c}\text { Matching } \\
\text { Qualität (Means Stan- } \\
\text { dardised Bias (in \%) }\end{array}$} \\
\hline & $\begin{array}{c}\text { arbeitslose } \\
\text { Absolventen }\end{array}{ }^{\prime \prime \prime}$ & $\begin{array}{c}\text { erwerbstätige } \\
\text { Absolventen }\end{array}$ & ATT & $\begin{array}{c}\text { Standard- } \\
\text { fehler }\end{array}$ & Fälle & $\begin{array}{c}\text { vor } \\
\text { Matching }\end{array}$ & $\begin{array}{c}\text { nach } \\
\text { Matching }\end{array}$ \\
\hline Hauptschulabschluss & 259 & 319 & -60 & 2,40 & 49366 & 10,18 & 1,90 \\
\hline Realschulabschluss & 213 & 301 & -88 & 2,29 & 45504 & 7,79 & 0,89 \\
\hline Abitur & 165 & 278 & -113 & 7,27 & 8009 & 8,29 & 2,82 \\
\hline
\end{tabular}

Quelle: IABS 1975-2004, eigene Berechnungen

Absolventen, die übernommen werden oder erfolgreich den Betrieb wechseln. Der ATT erhöht sich mit zunehmendem Bildungsstand. Je negativer demnach der ATT, desto größer ist der Effekt auf die Verringerung der Beschäftigungsperiode. So arbeiten Personen mit Realschulabschluss und einer dualen Berufsausbildung, die arbeitslos werden, knapp 88 Tage weniger als Personen mit mittlerer Reife, die ohne Unterbrechung beschäftigt gewesen sind. Abiturienten mit dualer Berufsausbildung weisen die höchste Differenz zwischen Experimental- und Kontrollgruppe auf, was den geringsten negativen ATT-Wert bedeutet. Abiturienten, die nach der Ausbildung erwerbslos sind, weisen eine um 113 Tage geringere Beschäftigungsperiode auf als Absolventen, die keine Arbeitslosigkeitsphase durchlaufen. Der höhere ATT der Absolventen mit Realschulabschluss deutet darauf hin, dass Berufserfahrung für diese Gruppe von besonderer Bedeutung ist. In diesem Fall dient vor allem eine befristete Beschäftigung als Brücke in den Arbeitsmarkt.

Eine anfängliche Arbeitslosigkeitsdauer im ersten Berufsjahr ist in der Gruppe der Absolventen mit Hauptschule weniger gravierend als in der Gruppe

${ }^{111}$ Hierunter werden alle Absolventen subsumiert, die direkt nach der Ausbildung mindestens 30 Tage arbeitslos sind.

${ }^{112}$ Ausbildungsabsolventen, die direkt nach Abschluss der Ausbildung weiter erwerbstätig sind, können im Laufe des ersten Berufsjahres auch arbeitslos werden, weil das Beschäftigungsverhältnis befristet ist. 
der Absolventen mit mittlerer Reife oder Abitur. Diese Unterschiede können auf Besonderheiten der einzelnen Ausbildungsbereiche zurückgeführt werden. Ausbildungsabsolventen mit Hauptschulabschluss sind vorwiegend in handwerklichen Ausbildungsbereichen vorzufinden, die von einem hohen Anteil an berufsspezifischem Humankapital gekennzeichnet sind. Betriebliche Mobilität verbunden mit einem flexiblen Arbeitskräfteeinsatz, der auch zwischen verschiedenen Tätigkeitsbereichen stattfinden kann, ist die Folge. Dies impliziert häufig eine Beschäftigung als angelernter Mitarbeiter, was eine Verkürzung der Arbeitslosigkeitsperiode und entsprechend eine Erhöhung der Anzahl der Arbeitstage gegenüber Absolventen mit Realschulabschluss bzw. Abitur bedeutet. So ist zu erwarten, dass der ATT in den handwerklichen Bereichen relativ gering ist. Darüber hinaus ist vor allem bei nach der dualen Berufsausbildung erwerbslosen Absolventen mit mittlerer Reife zu vermuten, dass sie unterdurchschnittliche Fähigkeiten aufweisen oder in Bereichen als Auszubildender sind, die schlechte Beschäftigungsaussichten aufweisen. Zudem verfügen Ausbildungsabsolventen mit Realschulabschluss über einen relativ höheren Anteil an betriebsspezifischerem Humankapital, was die Suchdauer verlängert. Während es für letztere Gruppe einen Einstieg in das Erwerbsleben darstellt, werden geringer Qualifizierte nach kurzen Beschäftigungsdauern wieder arbeitslos. Schließlich lässt die geringere Beschäftigungsdauer bei arbeitslosen Absolventen auf die verstärkte Suche nach einem ausbildungsadäquaten Job schließen, welche die Annahme eines Arbeitsangebotes hinauszögert. Jedoch deutet das speziell bei Abiturienten im Allgemeinen geringe Niveau an Arbeitstagen eher auf den Beginn eines Studiums hin. Diese Alternative wird unter Umständen im Falle einer Arbeitslosigkeit bevorzugt gewählt, um eine solche wieder zu verlassen.

Tabelle 12 gibt Auskunft über die kausalen Effekte der Arbeitslosigkeit direkt nach der dualen Berufsausbildung auf eine weitere Periode von Erwerbslosigkeit nach einem Jahr.

So unterscheidet sich der kausale Effekt einer Arbeitslosigkeit zu Beginn des Erwerbslebens zwischen den einzelnen Ausbildungsberufen zum Teil erheblich. Hierbei weisen die Absolventen in den Ausbildungsbereichen der Landund Forstwirtschaft und dem Bau den niedrigsten ATT auf. Dies bedeutet, dass arbeitslose Absolventen in diesen Bereich gegenüber direkt nach der Ausbildung erwerbstätigen Absolventen ungefähr 59 bzw. 61 Tage weniger in Beschäftigung sind. Zudem ist die Beschäftigungsdauer für Absolventen im Bau relativ lang, was darauf hindeutet, dass mögliche Arbeitslosigkeitsperioden relativ kurz, aber trotzdem wiederholt auftreten können. Dabei handelt es sich zwar um handwerkliche Bereiche, welche Ausbildungsabsolventen auch mit einem relativ geringen Bildungsstand in den Arbeitsmarkt entlassen, die selten in ihrem Ausbildungsbereich eine Beschäftigung finden. Dafür sind sie aber häufig in anderen Bereichen als angelernte Arbeitskräfte tätig, weshalb die Differenz zu den übernommenen Absolventen bzw. den Betriebswechslern relativ 
gering ist. Allerdings kann aber nicht für alle handwerklichen Bereiche ein niedriger ATT festgestellt werden. So ist die Anzahl der Arbeitstage für arbeitslose Absolventen mit einer Ausbildung in den Bereichen der Chemie und Holzverarbeitung, der Metallverarbeitung, der Nahrungsmittel sowie im Elektrobereich zwischen 78 und 81 Tage geringer als für Absolventen, die keine Unterbrechung nach der dualen Berufsausbildung in der Erwerbstätigkeit aufweisen.

Tabelle 12: Average Treatment Effect der Arbeitslosigkeit auf die Beschäftigungsdauer im ersten Berufsjahr - nach Ausbildungsbereichen (in Tagen)

\begin{tabular}{|l|c|c|c|c|c|c|c|}
\hline & \multicolumn{5}{|c|}{ Kausaler Zusammenhang } & \multicolumn{2}{c|}{$\begin{array}{c}\text { Matching } \\
\text { Qualiät (Means Stan- } \\
\text { dardised Bias (in \%) }\end{array}$} \\
\hline & $\begin{array}{c}\text { arbeitslose } \\
\text { Absolventen }\end{array}$ & $\begin{array}{c}\text { erwerbstätige } \\
\text { Absolventen }\end{array}$ & ATT & $\begin{array}{c}\text { Standard- } \\
\text { fehler }\end{array}$ & Fälle & $\begin{array}{c}\text { vor Mac- } \\
\text { thing }\end{array}$ & $\begin{array}{c}\text { nach } \\
\text { Matching }\end{array}$ \\
\hline $\begin{array}{l}\text { Land-und Forstwirt- } \\
\text { schaft }\end{array}$ & 225 & 284 & -59 & 9,48 & 3410 & 11,60 & 3,48 \\
\hline $\begin{array}{l}\text { Chemie-, holzverarbei- } \\
\text { tende Berufe }\end{array}$ & 234 & 314 & -79 & 5,56 & 7961 & 10,74 & 2,72 \\
\hline $\begin{array}{l}\text { Metallverarbeitende } \\
\text { Berufe }\end{array}$ & 236 & 314 & -78 & 3,66 & 20168 & 11,56 & 1,93 \\
\hline Textilberufe & 265 & 339 & -74 & 26,96 & 447 & 16,66 & 10,19 \\
\hline $\begin{array}{l}\text { Berufe im Nahrungs- } \\
\text { mittelbereich }\end{array}$ & 248 & 328 & -81 & 8,68 & 3391 & 11,96 & 4,31 \\
\hline Elektroberufe & 217 & 296 & -79 & 5,99 & 11822 & 10,30 & 2,51 \\
\hline Bauberufe & 243 & 304 & -61 & 4,38 & 11502 & 10,64 & 1,69 \\
\hline $\begin{array}{l}\text { Infrastruktur- } \\
\text { /Transport- } \\
\text { dienstleistungsberufe }\end{array}$ & 220 & 321 & -101 & 13,70 & 1934 & 8,74 & 4,87 \\
\hline $\begin{array}{l}\text { Finanzdienstleistungs- } \\
\text { berufe }\end{array}$ & 209 & 316 & -107 & 5,19 & 11984 & 9,46 & 3,21 \\
\hline Verwaltungsberufe & 201 & 302 & -101 & 6,07 & 9217 & 9,02 & 3,05 \\
\hline $\begin{array}{l}\text { unternehmensbezogene } \\
\text { Dienstleistungsberufe }\end{array}$ & 210 & 297 & -87 & 8,38 & 4535 & 10,30 & 3,55 \\
\hline $\begin{array}{l}\text { haushalts-/ personenbe- } \\
\text { zogene Dienstleistungs- } \\
\text { berufe }\end{array}$ & 227 & 310 & -83 & 3,65 & 14680 & 9,14 & 2,12 \\
\hline
\end{tabular}

Quelle: IABS 1975-2004, eigene Berechnungen

Dagegen zieht eine Arbeitslosigkeit bei Eintritt in den Arbeitsmarkt vor allem für Absolventen mit einer Ausbildung in dienstleistenden Berufen (Infrastruktur- und Transportdienstleistungsberufe, Finanzdienstleistungsberufe, Verwaltungsberufe, produktionsnahe Dienstleistungsberufe) eine kürzere Beschäftigungsdauer bzw. eine verlängerte Arbeitslosigkeitsphase nach sich. Der kausale Effekt liegt für die Infrastruktur- und Transportdienstleistungsberufe, Finanzdienstleistungsberufe, Verwaltungsberufe über 100 Tage, wobei sich Arbeitslosigkeit für Absolventen mit einer Ausbildung im Infrastruktur- und Transportdienstleistungsbereich mit einem ATT von 101 Tagen gravierend auf 
die Beschäftigung auswirkt. Ausbildungsabsolventen, die eine Ausbildung mit guten Voraussetzungen für die Beschäftigung absolvieren und dennoch arbeitslos werden, haben innerhalb des ersten Jahres zunächst Nachteile auf dem Arbeitsmarkt. So realisieren sie mit höherer Wahrscheinlichkeit eine längere Arbeitslosigkeitsperiode als nach Abschluss der Ausbildung erwerbstätige Personen, obwohl sie in der Regel über einen hohen Humankapitalstand verfügen, der ebenfalls einen hohen Anteil an betriebsspezifischem Humankapital enthält. In Ausbildungsberufen mit prinzipiell guten Beschäftigungsaussichten wiegt eine Arbeitslosigkeitsphase zu Beginn des Arbeitslebens schwerer als in Bereichen mit weniger guten Beschäftigungsaussichten. Darüber hinaus ist zu erkennen, dass eine Ausbildung in Infrastruktur- und Transportdienstleistungsberufen eine relativ lange Beschäftigungsperiode für weiterbeschäftigte Absolventen verspricht, was eine Ausbildung nach investitionstheoretischem Ansatz impliziert, diese demnach auf Dauer angelegt ist. Für Absolventen in produktionsnahen sowie haushalts- und personenbezogenen Dienstleistungsberufen ist davon auszugehen, dass Arbeitslosigkeitsphasen unter den Arbeitskräften mit geringerer Bildung häufiger sind. Daher können Unternehmen in diesen Bereichen in bestimmten Ausbildungsberufen nicht ohne weiteres auf Arbeitsplatzbewerber mit lückenlosen Erwerbsverläufen zurückgreifen. Dies impliziert einen schnellen Wiedereintritt ins Erwerbsleben und eine längere Beschäftigungsperiode. Dagegen verbleiben Absolventen von Ausbildungsberufen mit guter Ausbildungsqualität länger in Arbeitslosigkeit. Daher ist der ATT relativ gesehen weder hoch noch niedrig (siehe auch Kap. 8.2.1. und Kap. 8.2.2).

Die Hypothese 3, dass Absolventen in Ausbildungsbereichen mit hohem Humankapital, die direkt nach der dualen Berufsausbildung, arbeitslos sind, im ersten Berufsjahr eine kürzere Beschäftigungsperiode realisieren als arbeitslos werdende Absolventen in Ausbildungsbereichen mit niedrigem Humankapital, kann bestätigt werden. Werden besser ausgebildete Absolventen direkt nach der dualen Berufsausbildung arbeitslos, weisen sie sowohl gegenüber ihresgleichen als auch gegenüber weniger gut ausgebildeten arbeitslos werdenden Absolventen eine wesentlich geringere Anzahl an Tagen in Beschäftigung auf. Dies impliziert zugleich auch eine entsprechend längere Arbeitslosigkeitsphase. 
Gabriele Somaggio - 978-3-631-74988-3

Downloaded from PubFactory at 01/11/2019 09:32:31AM

via free access 


\section{Schlussbemerkung}

Ziel der Arbeit war es, die Ursachen für die Arbeitslosigkeit nach der dualen Berufsausbildung zu identifizieren. Ausgangspunkt stellte der Rückgang der Anzahl von Übernahmen nach der dualen Berufsausbildung dar, die immer häufiger in Arbeitslosigkeit mündeten. Aus diesen Entwicklungen ergab sich die Fragestellung, inwieweit die Art der betrieblichen Ausbildungsbereitschaft den Übergang von der dualen Berufsausbildung ins Erwerbsleben beeinflusst. Hierbei wurde insbesondere der Zeitpunkt direkt nach der dualen Berufsausbildung betrachtet, weil dieser einen Übergang von dem Berufsausbildungssystem in den Arbeitsmarkt darstellt. Die betrachtete Personengruppe sieht sich damit Rahmenbedingungen sowohl des Ausbildungssystems als auch des Arbeitsmarktes gegenüber, woraus Besonderheiten resultieren. Daher greifen einerseits die Erklärungsansätze der Ausbildungsbereitschaft aus Mangel an Implikationen für die Arbeitslosigkeitsphase direkt nach der dualen Berufsausbildung zu kurz. Andererseits setzen die Modelle zur Begründung von Arbeitslosigkeit erst beim Eintritt in die Erwerbslosigkeit an. Gerade bei der Betrachtung der Ausbildungsabsolventen erweist sich jedoch die Situation während der dualen Berufsausbildung für den Einstieg in das Erwerbsleben als relevant. Diese Zusammenhänge können aber in der Theorie nicht durch einen eigenen Ansatz vollständig abgebildet werden, weshalb die Modelle zur Erklärung der Ausbildungsbereitschaft um den Aspekt des Verbleibs nach der dualen Berufsausbildung erweitert werden müssen. Deshalb wurden die Kenntnisse über die betriebliche Ausbildungsbereitschaft mit den theoretischen Erklärungsansätzen der Arbeitslosigkeit verknüpft, um Informationen über die Verteilung des Arbeitslosigkeitsrisikos und die Dauer der Arbeitslosigkeit der unterschiedlichen Ausbildungsabsolventen zu erlangen. Anhand dieser beiden Theoriestränge konnte der Übergang analysiert und durch die verschiedenen Zusammensetzungen des Humankapitals, welche aus der unterschiedlichen Gestaltung der Ausbildung entstehen, erklärt werden. Der Übergang in das Erwerbsleben und die unterschiedliche Betroffenheit von Arbeitslosigkeit nach der dualen Berufsausbildung hinsichtlich des Humankapitals wurde bereits in Kapitel 6 zusammengefasst. Als Einflussfaktoren kristallisierten sich hierbei insbesondere die betrieblichen Rahmenbedingungen, die während der Ausbildung vorherrschten, heraus, die um individuelle Merkmale ergänzt wurden.

Um einen Ausblick geben und Handlungsempfehlungen ableiten zu können, muss nun die Arbeitslosigkeit unter dem Aspekt des Eintrittsrisikos sowie der Dauer für die einzelnen Gruppen von Ausbildungsabsolventen betrachtet werden. Die Gruppen werden anhand der Höhe ihres Bildungsstandes klassifiziert, wobei die Grenzen durchlässig sind. Die resultierende zweistufige Vorgehensweise zur Identifikation der Bestimmungsgründe, ausgehend von der Ausbildungsbereitschaft, scheint bei der Betrachtung des Arbeitslosigkeitsrisikos und der Dauer der Arbeitslosigkeit notwendig, weil aufgrund der theoretischen Annahmen davon auszugehen ist, dass das Arbeitslosigkeitsrisiko innerhalb einer 
Absolventengruppe eine andere Bedeutung einnimmt als die Wahrscheinlichkeit, die Arbeitslosigkeit wieder zu verlassen.

Aufgrund der Beteiligung unterschiedlicher Betriebe an der dualen Berufsausbildung ist deren Ausbildungsbereitschaft auf verschiedene Gründe zurückzuführen. Dadurch wird die Zusammensetzung des notwendigen Humankapitals und die Höhe der Ausbildungskosten bestimmt, was den Eintritt ins Erwerbsleben determiniert. Hierbei ist es erforderlich, vor allem den Humankapitalstand und dessen Struktur auf die einzelnen betrieblichen Merkmale in Verbindung mit den individuellen Eigenschaften genauer zu betrachten. Neben diesen Ebenen wirken sich die konjunkturellen Rahmenbedingungen auf den Übergang zwischen dualem Berufsbildungssystem und Arbeitsmarkt aus. Da von Änderungen der Stärke der Einflussfaktoren im Zeitablauf auszugehen ist, werden mehrere Abschlusskohorten zusammengefasst und miteinander verglichen. Die Gegenüberstellung konzentriert sich auf die Jahre zwischen 1992 und 2003. Der Fokus der empirischen Auswertungen liegt somit auf der Analyse der Arbeitslosigkeit nach der dualen Berufsausbildung, deren Eintritt im ersten Schritt sich aber nicht losgelöst von der Betrachtung anderer Einstiegsalternativen betrachten lässt. Berücksichtigung findet daher zugleich die Aufnahme einer Tätigkeit in einem anderen Betrieb. Im zweiten Schritt wird die Arbeitslosigkeitsperiode der arbeitslos werdenden Absolventen genauer betrachtet. Die zweistufige Vorgehensweise aus dem Theorieteil wird bei der Überprüfung der Hypothesen im empirischen Teil fortgesetzt.

Bei der Umsetzung ist zu berücksichtigen, dass Humankapital empirisch nicht direkt erfasst werden kann. Zur Bestimmung des Einflusses der Höhe und der Art des Humankapitals sind daher Variablen zu verwenden, die in Prozessdaten erfasst werden können und gleichzeitig Auskunft über die Bildung geben. Betriebliche Faktoren, wie Betriebsgröße, Höhe der Ausbildungsvergütungen, betriebliche Lohnsumme, erfüllen diese Kriterien ebenso wie die verschiedenen Ausbildungsberufe, die zu Ausbildungsbereichen zusammengefasst werden, und die Bildungs- und Qualifikationsvariablen.

Nach der theoretischen Argumentation werden Ausbildungsabsolventen, welche ihre Ausbildung in einem Betrieb mit kurzfristigem Beschäftigungsinteresse erworben haben, häufig den Betrieb wechseln müssen. Dies liegt in den geringen betrieblichen Bildungsinvestitionen begründet, weil Betriebe keine hohen Aufwendungen tätigen können und sie auch aufgrund der Betriebsstrukturen nicht benötigen. Entsprechend sind produktionstheoretisch orientierte Unternehmen meist kleine Betriebe, die geringe Ausbildungsvergütungen bezahlen und bei denen die Löhne und Gehälter der Beschäftigten insgesamt nicht sehr hoch sind. Eine kleine Betriebsgröße spricht somit für einfache Betriebsstrukturen und Arbeitsprozesse. Es handelt sich meist um Betriebe, die eine Ausbildung in handwerklichen Bereichen (bspw. metallverarbeitende, Elektround Baubereich), aber auch in Bereichen mit einfachen dienstleistenden Tätigkeiten (bspw. haushalts- und personenbezogenen Dienstleistungsbereich) an- 
bieten. Hier sind vor allem Hauptschulabsolventen anzutreffen. Absolventen der genannten Bereiche weisen daher ein geringes Humankapital auf, weil ihre Ausbildung in der Regel produktionstheoretisch begründet ist. Eine Weiterbeschäftigung erfolgt weniger wahrscheinlich, da dies auch mit einer Ausbildung „über Bedarf" verbunden ist und Fachkräfte nicht in dem Maße, wie die hohe Ausbildungsintensität impliziert, benötigt werden. Durch das Verlassen des Ausbildungsbetriebs werden sie den Arbeitsmarktrisiken direkt ausgesetzt sein, was wiederum das Arbeitslosigkeitsrisiko erhöht. Die Zusammensetzung des Humankapitals ermöglicht im Falle einer Arbeitslosigkeit einen schnellen Wiedereintritt in die Erwerbstätigkeit, da wegen des hohen Anteils an allgemeinem Humankapital kaum ein Produktivitätsverlust entsteht. Für einen schnellen Wiedereinstieg in die Erwerbstätigkeit ist es zudem erforderlich, dass die Arbeitsplätze keine hohen Anforderungen an betriebsspezifische Qualifikationen stellen, was entsprechend einfache Tätigkeitsbereiche kennzeichnet. Somit verkürzt sich die Einarbeitungs- und Einlernphase und die Einstellungs- aber auch die Entlassungskosten verringern sich. Diese geringen Einarbeitungs- und Entlassungskosten sind zudem aufgrund der Abhängigkeit von wirtschaftlichen Entwicklungen notwendig. Hier sind Anpassungen der Produktionsprozesse an starke konjunkturelle und strukturelle Veränderungen unerlässlich. Daher wirkt sich in dieser Gruppe ein zu Beginn des Erwerbslebens notwendiger Betriebswechsel eher negativ auf die Beschäftigungsstabilität aus. Die Beschäftigungssicherheit ist relativ gering.

Dagegen gestaltet sich der Übergang für Ausbildungsabsolventen mit hohem Bildungsstand theoretisch reibungslos. Mit zunehmender Betriebsgröße steigt die Komplexität der Arbeitsprozesse. Dies erfordert betriebsspezifisches Wissen zusätzlich zu einem höheren allgemeinen Humankapitalstand. Dadurch werden vor allem Personen zumindest mit einem Realschulabschluss eine Ausbildung in einem derartigen Unternehmen absolvieren. Größere Unternehmen zahlen, meist aus tariflichen Verpflichtungen heraus entstandene, höhere Ausbildungsvergütungen. Zudem werden Ausbildungsberufe innerhalb der Industriebranche und innerhalb des Bereiches von Dienstleistungstätigkeiten gegenüber handwerklichen Berufen und Berufe der Land- und Forstwirtschaft höhere Anforderungen an das Humankapital stellen. Die Wahrscheinlichkeit eines Betriebswechsel und der Arbeitslosigkeit ist daher gering. Dennoch verlassen bessere Ausbildungsabsolventen, die sowohl im Ausbildungsbetrieb als auch auf dem externen Arbeitsmarkt gute Beschäftigungsaussichten haben, ebenfalls den Ausbildungsbetrieb. Der Wechsel kann freiwillig sein, weil sie ihre Arbeitsmarktschancen gut einschätzen und ihre Erwerbssituation durch einen Betriebswechsel verbessern wollen. Dieser Wechsel kann unfreiwillig sein, weil auch investitionstheoretisch orientierte Betriebe teilweise mehr Fachkräfte ausbilden als sie tatsächlich benötigen und nach der Ausbildung nur den „Besten der Besten" ein Beschäftigungsangebot unterbreiten. So werden die Ausbildungsabsolventen von Betriebswechseln betroffen sein, die unterdurchschnitt- 
liche Qualifikationen innerhalb des Wirtschaftszweiges aufweisen. Dieser Einflussfaktor wird mit Hilfe der Qualifikationsstruktur der Beschäftigten im Ausbildungsbetrieb empirisch gemessen. Aus der Überprüfung der Hypothesen im empirischen Teil resultieren folgende Ergebnisse:

\section{Hohes Arbeitslosigkeitsrisiko für Ausbildungsabsolventen mit geringem $\mathrm{Hu}$ - mankapital}

Hypothese 1 wurde bestätigt. Personen mit einem hohen Humankapitalstand und einer höheren Ausbildungsqualität weisen eine geringere Wahrscheinlichkeit auf, arbeitslos zu werden, als diejenigen mit niedrigerem Humankapitalstand. Absolventen mit einer Ausbildung in einem großen Unternehmen verfügen über eine höhere Bildung mit zunehmender betriebspezifischer Komponente und werden weniger wahrscheinlich arbeitslos als Absolventen mit Ausbildung in einem kleineren Betrieb. Ebenso nehmen das Arbeitslosigkeitsrisiko und die Wahrscheinlichkeit eines Betriebswechsels mit steigenden Ausbildungsvergütungen ab, denn je geringer die Entlohnung für Auszubildende, desto geringer ist das vermittelte Humankapital. Dieser Einfluss ist aber erst in jüngerer Zeit zu erkennen.

Die Struktur der Ausbildung, welche die Ausbildungsbereiche und die Dauer der Ausbildung bestimmt sowie die berufsspezifische Humankapitalkomponente kennzeichnet, zeigt ebenfalls ein eindeutiges Ergebnis für den Verbleib nach der dualen Berufsausbildung auf. Dennoch sind die Einflüsse der Ausbildungsbereiche unterschiedlich zu deuten. Eine Ausbildung in Land- und Forstwirtschaftsberufen bietet die geringsten Aussichten, im Ausbildungsbetrieb zu verbleiben. Das Arbeitslosigkeitsrisiko liegt in diesen Berufen am höchsten, was deren struktureller Wandel als Ursache bekräftigt. Ebenso haben Auszubildende handwerklicher Berufe, wie die Elektro-, und Bauberufe, eine hohe Wahrscheinlichkeit, den Betrieb zu wechseln. Hier werden einerseits die Strukturänderungen, andererseits die kurzfristigen wirtschaftlichen Entwicklungen deutlich, welche sich auf die Humankapitalbildung und dessen Höhe auswirken. Absolventen in metallverarbeitenden Berufen sowie in den Infrastrukturund Transportdienstleistungsberufen wechseln weniger wahrscheinlich den Betrieb. Wenn sie aber den Betrieb verlassen, weisen sie eine erhöhte Eintrittswahrscheinlichkeit in Arbeitslosigkeit auf. Obwohl die Wahrscheinlichkeit für einen Betriebswechsel in Finanzdienstleistungsberufen und in Verwaltungsberufen hoch ist, ist das Arbeitslosigkeitsrisiko gering. Vor allem hinsichtlich des Bankengewerbes ist zu vermuten, dass Abiturienten in diesem Bereich häufig eine Ausbildung wählen, um anschließend ein Studium aufzunehmen. Ebenso ist auch das Risiko, nach der dualen Berufsausbildung, arbeitslos zu werden, innerhalb der einzelnen Bereiche mit einem höheren Bildungsstand geringer als das der handwerklichen Ausbildungsbereiche. Im Allgemeinen wechseln Absolventen der haushalts- und personenbezogenen Dienstleistungen weniger 
wahrscheinlich den Betrieb und haben auch ein wesentlich geringeres Arbeitslosigkeitsrisiko als Absolventen anderer Ausbildungsbereiche.

Die Qualifikationsvariablen und damit die allgemeine Humankapitalkomponente lassen keinen eindeutigen Zusammenhang zwischen Bildungshöhe und Wechselwahrscheinlichkeit erkennen. Absolventen mit Realschulabschluss beginnen am unwahrscheinlichsten eine Tätigkeit in einem anderen Betrieb, weisen aber die höchste Wahrscheinlichkeit auf, arbeitslos zu werden. Die hohe Wahrscheinlichkeit der Abiturienten, nach der dualen Berufsausbildung den Betrieb zu wechseln, lässt sich $\mathrm{u}$. a durch einen hohen Anteil an freiwilligen Betriebswechseln erklären. Sie jobben während des Studiums oder versuchen in einem anderen Betrieb ihre Erwerbssituation zu verbessern. Absolventen, welche unterdurchschnittliche Qualifikationen innerhalb des Wirtschaftszweiges aufweisen, wechseln wahrscheinlicher den Betrieb. Der Ausbildungsbetrieb kann in diesem Fall nicht für die notwendigen Ressourcen für hohe Bildungsinvestitionen aufkommen. Dies schränkt auch die Möglichkeiten einer Weiterbeschäftigung in diesem Betrieb ein. Der Betriebswechsel wird daher unfreiwilliger Natur sein. Da sie aufgrund der unterdurchschnittlichen Qualifikationen ungünstige Berufsaussichten gegenüber anderen Absolventen desselben Ausbildungsbereichs aufweisen, ist das Arbeitslosigkeitsrisiko gegenüber Absolventen mit überdurchschnittlichen Qualifikationen hoch. Insgesamt hat die Schulbildung jedoch nicht den zu erwartenden (signifikanten) Effekt auf den Verbleib an der zweiten Schwelle. Vielmehr wirkt sie sich über die Ausbildungsbereiche auf die Wahrscheinlichkeit, den Betrieb zu wechseln bzw. arbeitslos zu werden, aus. So kann ein Zusammenhang zwischen Ausbildungsbereich und Schulbildung hergestellt werden. Die Ergebnisse verdeutlichen eine Zuordnung von Absolventen in Ausbildungsberufe nach Schulabschlüssen. Lediglich in Ausbildungsberufen mit unterschiedlichen Qualifikationsanforderungen, wie im Ausbildungsbereich Finanzdienstleistungen, ist ein Zusammenhang zwischen Bildung und der Wahrscheinlichkeit eines Wechsels bzw. einer Arbeitslosigkeit zu erkennen. Ein niedriger Bildungsabschluss erhöht innerhalb der einzelnen Ausbildungsbereiche die Wahrscheinlichkeit für einen Betriebswechsel. Gleichermaßen wirkt sich ein niedriger Schulabschluss innerhalb der einzelnen Ausbildungsbereiche positiv auf das Arbeitslosigkeitsrisiko aus.

\section{Verlängerung der Dauer von Arbeitslosigkeit mit zunehmendem Humankapi- talstand}

Hypothese 2 konnte anhand der vorliegenden empirischen Schätzungen nicht eindeutig bestätigt werden. Tendenziell ist zu erkennen, dass Ausbildungsabsolventen mit niedrigem Bildungsstand länger in Arbeitslosigkeit verweilen als diejenigen mit höherer Bildung. Die Arbeitslosigkeitsdauer ist für Absolventen mit einer Ausbildung in einem größeren Betrieb kürzer als für Absolventen mit einer Ausbildung in einem kleineren Betrieb. Ebenso lässt sich ein positiver Zusammenhang zwischen der Höhe der Ausbildungsvergütungen und der Ar- 
beitslosigkeitsdauer erkennen. Allein die Höhe der durchschnittlichen Lohnsumme der Betriebe wirkt sich negativ auf die Arbeitslosigkeitsdauer aus. Je mehr die Beschäftigten im Ausbildungsbetrieb verdienen, desto kürzer sind deren Ausbildungsabsolventen, die den Betrieb verlassen, arbeitslos. Auch ist der Einfluss der Ausbildungsbereiche auf die Dauer der Arbeitslosigkeit nicht eindeutig zu bestimmen. Aufgrund des hohen allgemeinen und berufsspezifischen Humankapitals verleiben Ausbildungsabsolventen der handwerklichen Ausbildungsbereiche meist, wenn auch nicht signifikant, relativ kurz in Arbeitslosigkeit. Darüber hinaus sind diese Ausbildungsbereiche zumeist stark von konjunkturellen Schwankungen abhängig, wodurch das hohe Arbeitslosigkeitsrisiko, aber auch die kurze Verweildauer in Arbeitslosigkeit ebenfalls erklärt werden kann. Gleichzeitig weisen Absolventen von metallverarbeitenden Berufen aber auch eine kürzere Arbeitslosigkeitsdauer auf, was auf die sehr berufsspezifische Berufsausbildung in diesen Bereichen hindeutet. Verstärkt durch den hohen allgemeinen Anteil an Humankapital treten diese Ausbildungsabsolventen relativ zügig wieder in die Erwerbstätigkeit ein. Dieser zügigere Wiedereintritt ist allerdings erst in den jüngeren Abschlusskohorten zu beobachten, für welche sich scheinbar die Arbeitsmarktsituation verbessert hat. Dabei ist aber anzunehmen, dass nur diese Ausbildungsabsolventen weniger in ihren Ausbildungsberufen als vielmehr als Hilfsarbeiter tätig sind. Absolventen der produktionsnahen Dienstleistungsberufe, der Finanzdienstleistungsberufe sowie der Verwaltungsberufe verweilen gegenüber Absolventen mit einer Ausbildung im unternehmens- bzw. haushalts- und personenbezogenen Dienstleistungsbereich, aber auch gegenüber denjenigen in handwerklichen Ausbildungsbereichen relativ lang in Arbeitslosigkeitsdauer. Dem erhöhten Arbeitslosigkeitsrisiko der unterdurchschnittlich ausgebildeten Absolventen folgt im Vergleich zu den Ausbildungsabsolventen mit überdurchschnittlichen Fähigkeiten eine kürzere Arbeitslosigkeitsperiode. Dies deutet auf geringere Ansprüche in Bezug auf die künftige Stelle hin, wodurch folglich der Reservationslohn entsprechend angepasst wird. Absolventen mit Realschulabschluss bleiben länger als Absolventen mit Hauptschulabschluss ohne Beschäftigung. Der kurze Verbleib in Arbeitslosigkeit für Absolventen mit einem niedrigeren Schulabschluss ist auf die erhöhte Akzeptanz von Stellenangeboten zurückzuführen, die Hilfsarbeitertätigkeiten beinhalten. Ebenso wird eine weiterführende Ausbildung (bspw. Meisterprüfung) unter Umständen von den besseren Absolventen mit Realschulabschluss angestrebt. Die Suche nach einer Arbeitsstelle, die hierfür entsprechend ausgestaltet ist, muss aus einer begrenzten Anzahl an Angeboten gefunden werden. Dies benötigt Zeit. Darüber hinaus erlernen Absolventen mit Realschulabschluss Berufe, die gegenüber den von Hauptschulabsolventen erlernten Berufen einen höheren Anteil an betriebsspezifischem Humankapital aufweisen. Auch hier ist das Stellenangebot, das der Ausbildung entspricht, begrenzt. Zudem stehen sie zumeist auch in Konkurrenz mit den Ausbildungsabsolventen, die Abitur aufweisen und die aufgrund des daraus 
resultierenden höheren Bildungsstandes auch höhere Chancen auf ein Arbeitsangebot haben. Dies belegen auch die höheren Abgangsraten aus Arbeitslosigkeit bei höherem Bildungsstand. Entsprechend ist für Absolventen mit Realschulabschluss die Beschäftigungsperiode innerhalb des ersten Jahres nach der dualen Berufsausbildung gegenüber den Absolventen mit Hauptschulabschluss kürzer. Dementsprechend sinkt die Abgangsrate aus Arbeitslosigkeit mit zunehmender Schulbildung innerhalb der einzelnen Ausbildungsbereiche.

Anzahl der Tage in Beschäftigung der arbeitslos werdenden gegenüber erwerbstätigen Absolventen sinkt mit Humankapitalstand

Hypothese 3 konnte bestätigt werden. Je höher der Bildungsstand, desto geringer ist die Anzahl der Tage in Beschäftigung für Absolventen mit einer anfänglichen Arbeitslosigkeit gegenüber Absolventen desselben Bildungsniveaus, die nach der dualen Berufsausbildung erwerbstätig sind. So reduziert eine Arbeitslosigkeit von Absolventen mit Hauptschulabschluss gegenüber direkt nach der Ausbildung in Erwerbstätigkeit verbleibenden Absolventen derselben Schulbildung die Beschäftigungsdauer innerhalb des ersten Berufsjahres nicht wesentlich, was ebenfalls darauf hindeutet, dass die Arbeitslosigkeitsdauer relativ kurz ist. Diese Differenz nimmt mit steigender Schulbildung zu. Treten Absolventen mit Abitur zu Beginn des Erwerbslebens in Arbeitslosigkeit, hat diese Phase auf ersten Eintritt in eine Beschäftigung große Bedeutung: Sie weisen eine wesentlich kürze Beschäftigungsdauer gegenüber den Absolventen mit derselben Schulbildung auf, die zu Beginn erwerbstätig sind. Eine Erwerbstätigkeit, gleich ob im Ausbildungsbetrieb oder in einem anderen Betrieb, fördert einen erfolgreichen Einstieg in das Erwerbsleben. Berufserfahrung ist daher vor allem in metallverarbeitenden, Elektro- und Bauberufen von besonderer Relevanz. In diesen handwerklichen Bereichen führt eine Arbeitslosigkeitsphase zu einer wesentlich kürzeren Beschäftigungsdauer innerhalb des ersten Jahres nach Abschluss der dualen Berufsausbildung. Der Einstieg in den Arbeitsmarkt durch Beschäftigung liefert hier gute Voraussetzungen für das weitere Erwerbsleben. Zugleich bedeutet dies aber auch, dass die kürzere Arbeitslosigkeitsdauer mit häufigen Arbeitslosigkeitsphasen, welche die kumulierte Beschäftigungsdauer innerhalb des ersten Jahres reduziert, verbunden sein kann. Die lange Arbeitslosigkeitsphase und die relativ kurze Beschäftigungsdauer deuten in den Ausbildungsbereichen der Verwaltungsberufe, unternehmensbzw. haushalts- und personenbezogenen Dienstleistungen auf eine mittel- bis langfristig hohe Beschäftigungsstabilität bei Wiedereintritt in die Erwerbstätigkeit hin.

Insgesamt ist festzustellen, dass die betrieblichen Rahmenbedingungen vor allem die Art des Eintritts in das Erwerbsleben bestimmen. Sie wirken auf die Arbeitslosigkeitsdauer nicht mehr so stark, sodass andere Faktoren von größerer Bedeutung sind. Dies stellt einen weiteren wichtigen Forschungsgegenstand dar. Daher sind z. B. die tariflichen Regelungen bei der Betrachtung des Ein- 
flusses an der zweiten Schwelle genauer zu berücksichtigen. Ein Betriebswechsel wird bspw. durch die Übernahmevereinbarungen verzögert, ebenso der Eintritt in die Arbeitslosigkeit; letzterer wird unter Umständen auch vermieden. Jedoch wirken aber andere tarifliche oder institutionelle Rahmenbedingungen, wie bspw. Löhne oder Kündigungsschutz hemmend auf den Wiedereintritt in Beschäftigung. Aus den Ergebnissen kann aber dennoch ein Ausblick gegeben werden. Über den Zeitablauf ergaben sich für den Erwerbseinstieg vor allem dahingehend Änderungen, dass zwar immer noch bestimmte Berufsgruppen bzw. Ausbildungsabsolventen mit bestimmten Merkmalen mehr als andere Absolventen von Arbeitslosigkeit betroffen waren, jedoch scheinen sich diese Effekte in den letzten Jahren leicht abzuschwächen. Unter den jüngeren Abschlusskohorten ist somit nur noch ein schwacher Trend (zu Lasten der Absolventen mit niedrigem Bildungsstand) auszumachen, sodass die geringere Strukturierung des Übergangs auch auf wirtschaftliche Veränderungen in kurz- und langfristiger Sicht zurückzuführen ist. Neben dem wirtschaftlichen Umfeld sind aber auch demografische Entwicklungen zu berücksichtigen. In Zukunft werden mehr Arbeitskräfte aus dem Erwerbsleben aus- als eintreten, wodurch sich der Fachkräftemangel verstärkt fortsetzt (Fischer et al. 2008; Reinberg/Hummel 2004). Infolgedessen werden Betriebe auf die Ausbildungsabsolventen mittel- bis langfristig angewiesen sein. Es ist deshalb zu vermuten, dass sich die Übergangsproblematik in zukunftsträchtigen Ausbildungsbereichen bessert. Zwei Aspekte sind hier zu berücksichtigen: Erstens ist die Ausbildung zu intensivieren. Denn durch die duale Berufsausbildung werden Grundlagen geschaffen, um dem Fachkräftemangel entgegenzusteuern. Darüber hinaus bildet sie die Basis für Lebenslanges Lernen, das in einer alternden Gesellschaft an Bedeutung gewinnt und auch die Voraussetzung für die Beschäftigungsfähigkeit der älteren Arbeitnehmer bildet. Damit wird auch der Grundstein für die Fort- und Weiterbildung der Mitarbeiter gelegt, was die Wettbewerbsfähigkeit des Unternehmens im Kontext einer zunehmenden Internationalisierung erhöht. Zweitens muss der Fokus auf den Übergang von der dualen Berufsausbildung in das Erwerbsleben erweitert werden. Die Ausbildung kann nur erfolgreich sein, wenn Ausbildungsabsolventen die Chance bekommen, ihr erworbenes Humankapital adäquat einzusetzen. Um die ausbildungsadäquate Beschäftigung zu gewährleisten, muss zugleich auch die Ausbildung ,über Bedarf“ zurückgehen. Denn auch bei einer Beschäftigung in einem anderen Beruf erfolgt eine Humankapitalentwertung, weil das erlernte Wissen nicht vollständig eingesetzt werden kann (Ludwig/Pfeiffer 2005; Schönig/Farhauer 2004).

Aus diesen Folgerungen lassen sich Handlungsempfehlungen für Bereiche der Bildungspolitik sowie der Arbeitsmarktpolitik ableiten, die Rahmenbedingungen schaffen, falls ökonomische Größen für einen erfolgreichen Übergang in das Erwerbsleben nicht greifen. Diese Rahmenbedingungen müssen eine Beschäftigung nach Abschluss der Ausbildung ermöglichen, denn durch das Brachliegen von Humankapital geht der Volkswirtschaft Wissen und entspre- 
chend auch Produktivität verloren. Die tariflich ausgehandelten Übernahmeregelungen sind dabei sicherlich eine notwendige Maßnahme zum Erhalt des Humankapitals und zur Gewinnung von Berufserfahrung. Es ist jedoch zu überlegen, ob diese tarifvertraglichen Sollvorschriften nicht verpflichtend für die Betriebe sein können. Zwar erfüllen die Betriebe in der Regel die Vereinbarungen, aber unter bestimmten Bedingungen (aus persönlichen oder wirtschaftlichen Gründen) ist es möglich, von den Übernahmeregelungen abzuweichen.

Weiterhin sind Anpassungen durchzuführen, welche die bildungspolitische Ebene mit dem Arbeitsmarkt verknüpft. Es ist erforderlich, bei der Organisation der Ausbildung anzusetzen. Insbesondere ist eine Flexibilisierung des dualen Berufssystems notwendig, um auf den sozioökonomischen Wandel und die Veränderungen vor dem Hintergrund der Globalisierung, die in immer kürzer werdenden Zeitabständen auftreten, angemessen reagieren zu können. Dies erfordert aber auch eine schnellere Anpassung der Ausbildungsinhalte an die Anforderungen des Arbeitsmarktes. Damit die betriebliche Ausbildung diesen sich wandelnden Anforderungen an den Arbeitsmarkt gerecht wird, müssen die Lerninhalte entsprechend verändert werden. So ist es auf dem Weg hin zur Wissensgesellschaft notwendig, Schlüsselqualifikationen zu erlangen, wie Personalkompetenz, Sozialkompetenz, Fachkompetenz und Lernkompetenz (Weigel et al. 2007: 62ff.; vgl. auch Mulder et al. 2007: 81). Vor allem allgemeine Humankapitalkomponenten spielen daher eine immer wichtigere Rolle, die berufs- und betriebsspezifische Komponenten in den Hintergrund treten lassen. Dadurch würde ein größerer Teil des erworbenen Ausbildungswissens dem Arbeitsmarkt in einer ebenso größeren Anzahl an Arbeitsmarktbereichen zur Verfügung stehen. Diese allgemeinen Humankapitalkomponenten sind in den Ausbildungsordnungen bereits vorhanden, müssen aber verstärkt gefördert werden. Dadurch kann der Anreiz zu einer Weiterbeschäftigung nach der dualen Berufsausbildung erhöht werden, ebenso wie der Anreiz, die Fachkräfte ausbildungsadäquat einzusetzen. Durch die Fokussierung auf die Schlüsselqualifikationen werden die Einsatzbereiche der einzelnen Ausbildungsberufe erweitert und die Wahrscheinlichkeit einer qualifikationsadäquaten Beschäftigung entsprechend erhöht. Die duale Berufsausbildung lässt sich dadurch sowohl für die Absolventen als auch für die Betriebe selbst aufwerten. Schließlich wird die duale Berufsausbildung weiter eine wichtige Rolle bei der arbeitsmarktpolitischen Diskussion um die Erhöhung der Beschäftigungsfähigkeit spielen. Zwar mögen in Zukunft Bachelorabschlüsse an Bedeutung gewinnen, der Zugang bleibt aber immer noch nur den Abiturienten vorbehalten. Deshalb wird die duale Berufsausbildung weiterhin von Bedeutung zur Erlangung einer beruflichen Qualifikation, gerade für die Jugendliche ohne Hochschulzugangsberechtigung, sein, weil schwächeren Schülern dadurch der Eintritt in das Erwerbsleben ermöglicht wird. Hierzu leistet sie einen Beitrag, da, trotz der Unterschiede in den Qualifikationsanforderungen der einzelnen Berufe, dennoch gut ausgebildete Arbeitskräfte herangezogen werden, die flexibel innerhalb der Berufs- 
gruppe, aber auch innerhalb der gesamten Volkswirtschaft eingesetzt werden können (Sanders/deGrip 2004). Ferner zeugt sie vom Erwerb sozialer Fähigkeiten (Casey 1986: 73), welche in Zukunft durch die Tertiarisierung an Bedeutung gewinnen werden.

Neben den bildungspolitischen Maßnahmen ist es auf Seiten der Arbeitgeber weiterhin notwendig, Anreize für eine Weiterbeschäftigung bzw. für einen reibungslosen Übergang in einen anderen Betrieb zu schaffen. So sollten die positiven Externalitäten der Bildung auf die Volkswirtschaft ausgenutzt werden, indem die anderen Unternehmen dazu gebracht werden, Arbeitskräfte mit weniger Berufserfahrung einzustellen. So können nicht ausbildende Betriebe bei einer Erhöhung des allgemeinen Humankapitals während der Ausbildung die Produktivität der Absolventen vollständig nutzen. Als unterstützendes Instrument kann der Lohn die entsprechende Funktion ausüben, indem er flexibler auf die unteren Qualifikationsbereiche (vor allem hinsichtlich der Arbeitsmarkterfahrung) reagiert. Bei einer Arbeitslosigkeit sind Maßnahmen zu ergreifen, die auf einen schnellen Wiedereintritt in die Beschäftigung hinwirken. Allerdings sollte dies vielmehr auf Beschäftigung abzielen als auf weitere Qualifizierungsmaßnahmen, da das während der dualen Berufsausbildung erlernte Wissen gerade bei Ausbildungsabsolventen (noch) nicht überholt ist. Damit wird auch Ausbildungsabsolventen mit schlechteren Beschäftigungsaussichten ein erfolgreicher Start ins Erwerbsleben institutionell ermöglicht. Denn die Weiterbeschäftigung stellt ein Bindeglied zwischen der Ausbildung und dem Eintritt in den Arbeitsmarkt dar. 


\section{Literaturverzeichnis}

Acemoglu, D./ Pischke, J.-S. (1998): Why do firms train?. In: Quarterly Journal of Economics, Vol. 113(1), S. 79-119.

Acemoglu, D./ Pischke, J.-S. (1999a): Beyond Becker: Training in imperfect labour markets. In: The Economic Journal, Vol. 109(453), S. F112-F142.

Acemoglu, D./ Pischke, J.-S. (1999b): The structure of wages and investment in general training. In: Journal of Political Economy, Vol. 117(3), S. 539572.

Acemoglu, D./ Pischke, J.-S. (2000): Certification of training and training Outcomes. In: European Economic Review, Vol. 44(4-6), S. 917-927.

Adda, J./ Dustmann, C./ Meghir, J.-M./ Robin, J.-M. (2007): Career progression and formal versus on the job training. In: CEPR Discussion Paper Nr. 6084.

Addison, J./ Fox, D. A./ Ruhm, C. J. (1995) Trade and displacement in manufacturing. In: Monthly Labor Review, Vol. 118(4), S. 58-67.

Adnett, N./ Boughas, S./ Georgellis, Y. (2003): On the trade-off between workrelated training and labor mobility: The role of firing and exit costs. In: Journal of Economics (Zeitschrift für Nationalökonomie), Vol. 82(1), S. 49-70.

Aidt, J./ Tzannatos, Z. (2002): Unions and collective bargaining - Economic effects in a global environment. The World Bank, Washington D.C..

Akerlof, G. A. (1970): The market for "lemon": Quality, uncertainty and the market mechanism. In: Quarterly Journal of Economics, Vol. 84(3), S. 488-500.

Albert, E. (2007): Wie beeinflusst das Bildungssystem die Übergangsprozesse von der Schule in den Beruf. Soziologisches Institut der Universität Zürich. http://socio.ch/arbeit/t_albert2.pdf. Zugriff am 23.09.2007.

Alda, H. (2006): Beobachtbare und unbeobachtbare Betriebs- und Personeneffekte auf die Entlohnung. Beiträge zur Arbeitsmarkt- und Berufsforschung, Band 298.

Alewell, D./ Richter, J. (1999): Die Finanzierung der betrieblichen Berufsausbildung in der Diskussion - Ausbildungsplatzabgaben versus Rückzahlungsklauseln. In: Beer, D./Frick, B./Neubäumer, R./Sesselmeier, W. (Hrsg.): Die wirtschaftlichen Folgen von Aus- und Weiterbildung, München, S.43-69.

Allmendinger, J. (1989): Educational systems and labor market outcomes. In: European Sociological Review, Vol. 5(3), S. 28-31.

Anger, C./ Plünnecke, A./ Seyda, S./ Werber, D./ Schäfer, H. (2006): Bildungsarmut und Humankapitalschwäche in Deutschland. Gutachten des Institutes der deutschen Wirtschaft, Köln.

Asplund, R. (2004): The provision and effects of company training: A brief review of the literature. In: ETLA Discussion Paper Nr. 907. 
Autor, D. (2001): Why do temporary help firms provide free general skills training?. In: Quarterly Journal of Economics, Vol 116(4), S. 1409-1448.

Backes-Gellner, U. (2008): Zur Logik betrieblicher Qualifizierungsstrategien im internationalen Vergleich - Betriebliche Aus- und Weiterbildung als optimale Vorratshaltung. In: Leading House Working Paper Nr. 20. Überarbeitete Version der Ausgabe "Berufliche Weiterbildung in europäischer Perspektive" herausgegeben von Dieter Timmermann (1999), S. 65-92.

Backes-Gellner, U./ Mure, J. (2008): The skill-weights approach on firm specific human capital: Empirical results for Germany. Papier präsentiert auf der Tagung " International Conference on Economics of Education, 25.27. Juni 2008 in Zürich, Schweiz.

Baethge, M./ Solga, H./ Wieck, M. (2007): Berufsbildung im Umbruch, Berlin. Balmaceda, F. (2005): Firm-sponsored general training. In: Journal of Labor Economics, Vol. 23(1), S. 115-133.

Bartel, A. P./ Sichermann, N. (1998): Technological change and skill acquisition of young worker. In: Journal of Labour Economics, Vol. 16(4), S. 718-755.

Bassanini, A./ Duval, R. (2006), Employment patterns in OECD Countries: Reassessing the role of policies and institutions. In: OECD Economics Department Working Paper Nr. 486.

BBiG (2005): Novellierung des Berufsbildungsgesetzes vom 23. März 2005, Berlin.

Beck, U./ Brater, M./ Wegener, B. (1979): Berufswahl und Berufszuweisung. Ergebnisse einer empirischen Untersuchung zur sozialen Verwandtschaft von Ausbildungsberufen, Frankfurt a. M.

Becker, G. (1962): Investment in human capital. A theoretical analysis. In: The Journal of Political Economy. Vol 70(5), S. 9-49.

Beckmann, M. (2004): Betriebliche Personalpolitik im technologischen und organisatorischen Innovationsprozess, Mering.

Beckmann, M. (2002a): Lohnstrukturverzerrung und betriebliche Ausbildung. Empirische Analyse des Acemoglu-Pischke-Modells mit Daten des IABBetriebspanels. In: Mitteilungen aus der Arbeitsmarkt- und Berufsforschung, Vol. 35(2), S. 189-204.

Beckmann, M. (2002b): Wage compression and firm-sponsored training in Germany: Empiriacal evidence for the Acemoglu-Pischke-Model from a zeroinflated count data model. In: Konjunkturpolitik, Vol. 48(3-4), S. 369389.

Behrens, M./ Pilz, M./ Greuling, O. (2008): Taking a straightforward detour: Learning and labour market participation in the german apprenticeship system. In: Journal of Vocational Education \& Training, Vol. 60(1), S. 93104. 
Beicht, U./ Berger, K. (2003): Schaffung von Ausbildungsplätzen durch Vereinbarungen der Tarifparteien. In: Berufsbildung in Wissenschaft und Praxis, Vol. 32/Sonderausgabe, S. 24-27.

Beicht, U./ Walden, G./ Herget, H. (2004): Kosten und Nutzen der betrieblichen Berufsausbildung in Deutschland. Bundesinstitut für berufliche Bildung, Bertelsmann, Bielefeld.

Bellmann, L./ Bender, S. (1997): Die Analyse der Stabilität von Beschäftigungsverhältnissen bei Berufsanfängern. In: Mitteilungen aus der Arbeitsmarkt- und Berufsforschung, Vol. 30(3), S. 681-687;

Bellmann, L./ Bielenski, H./ Bilger, F./ Dahms, V./ Fischer, G./ Frei, M./ Wahse, J. (2006): Personalbewegungen und Fachkräfterekrutierung. Ergebnisse des IAB-Betriebspanels 2005. In: IAB Forschungsbericht Nr. 11.

Bellmann, L./ Janik, F. (2007): To recruit skilled workers or to train one's own? In: Zeitschrift für Arbeitsmarktforschung, Vol. 40(2-3), S. 205-221.

Bellmann, L./ Neubäumer, R. (2001): Die Übernahme betrieblich Ausgebildeter - theoretische Überlegungen und empirische Ergebnisse auf der Basis des IAB-Betriebpanels 1998. In: von Weizsäcker, R.K. (Hrsg.): Bildung und Beschäftigung, Schriften des Vereins für Socialpolitik Band 284, Berlin, S. 179-212.

Bender, S./ Dietrich, H. (2001): Unterschiedliche Startbedingungen haben langfristige Folgen. Der Einmündungsverlauf der Geburtskohorten 1964 und 1971 in Ausbildung und Beschäftigung - Befunde aus einem IAB-Projekt. In: IAB Werkstattbericht Nr. 11.

Bernardi, F./ Nazio, T. (2005): Globalization and transition to adulthood in Italy. In: Blossfeld, H.-P./ Klijzing, E./ Mills, M./ Kurz, K. (Hrsg.): Globalization, uncertainty and youth in society, London, New York, S. 375-402.

Bispinck, R. (2002): Tarifliche Ausbildungsförderung begrenzt erfolgreich eine empirische Wirkungsanalyse. In: WSI Mitteilungen Nr. 4/2002, S. 213-219.

Blanchflower, D. G./ Oswald A. J. (1994): The wage curve. Cambridge, MA, London.

Blinder, A. S. / Krueger, A. B. (1996): Labour turnover in the US and Japan: A tale of two countries. In: Pacific Economic Review, Vol. 1(1), S. 27-57.

Bloemen, H. G. (2005): Job search, search intensity and labor market transitions. In: Journal of Human Resources, Vol. 40(1), S. 231-269.

Blossfeld, H.-P. (1990): Berufsverläufe und Arbeitsmarktprozesse. Ergebnisse soziostruktureller Längsschnittuntersuchungen. In: Mayer, K. U. (Hrsg.): Lebensverläufe und sozialer Wandel. Sonderheft 31 der Kölner Zeitschrift für Soziologie und Sozialpsychologie, S. 118-145.

Blossfeld, H.-P./ Buchholz, S./ Bukodi, E./ Ebralidze, E./ Kurz, K./ Relikowski, I./ Schmelzer, P. (2005): Flexibility processes and social inequalities at labor market entry and in the early career. In: flexCAREER Working Paper Nr. 1. 
Blossfeld, H.-P./ Stockmann, R. (1999): Globalization and chances in vocational training systems in developing and advanced industrialized societies, Vol. I-III, Armonk, NY.

Blundell, R./ Dearden, L./ Meghir, C./ Sianesi, B. (1999): Human capital investment: The returns from education and training to the individualism the firm and the economy. In: Fiscal Studies, Vol. 20(1), S. 1-23.

BMBF (2003): Berufsbildungsbericht 2003, Bonn.

BMBF (2004): Berufsbildungsbericht 2004, Bonn.

BMBF (2005): Berufsbildungsbericht 2005, Bonn.

BMBF (2006): Berufsbildungsbericht 2006, Bonn.

BMBF (2007): Berufsbildungsbericht 2007, Bonn.

BMBF (2008): Berufsbildungsbericht 2008, Bonn.

Boockmann, B./ Hagen, T. (2006): Befristete Beschäftigungsverhältnisse Brücken in den Arbeitsmarkt oder Instrumente der Segmentierung. In: ZEW-Wirtschaftsanalysen, Band 80. Baden-Baden.

Booth, A. L./ Zoega, G. (2000): Why do firms invest in general training? 'Good' firms and 'bad' firms as a source of monopsony power. In: Working Paper des Institute for Labour Research of the University of Essex Nr. 058.

Booth, A. L./ Zoega, G. (2001): Is wage compression a necessary condition for firm-financed general training. In: CEPR Discussion Paper Nr. 2845.

Booth, A. L./ Zoega, G. (1999): Do quits cause under-training? In: Oxford economic Papers, Vol. 51(2), S. 374-386.

Booth, A. L./ Bryan, M. L. (2002): Who pays for general training? New evidence for british men and women. In: IZA Discussion Paper Nr. 486

Booth, A. L./ Chatterji, M. (1998): Unions and efficient training. In: Economic Journal, Vol. 108(447), S. 328-343.

Botero, J./ Djankov, S./ La Porta; R./ Lopez-de Silanes, F./ Shleifer, A (2004): The Regulation of Labor. In: Quarterly Journal of Economics, Vol. 119(4), S. 1339-1380.

Breen, R. (1997): Risk, recommodification and stratification. In: Sociology, Vol. 31(3), S. 473-489.

Brunello, G. (2001): On the complementarity between education and training in Europe. In: IZA Discussion Paper Nr. 309.

Brunello, G./ Giannini, M./ Ariga, K. (2004): The optimal timing of school tracking. In: IZA Discussion Paper Nr. 995.

Brunello, G./ Medio, A. (2001): An explanation of international differences in education and workplace training. In: European Economic Review, Vol. 45(2), S. 307-322.

Büchel, F. (2002): Successful apprenticeship-to-work transitions: On the longterm change in significance of the german school-leaving certificate. In: IZA Discussion Paper, Nr. 425. 
Büchel, F./ Neubäumer, R. (2001): Ausbildungsadäquate Beschäftigung als Folge branchenspezifischer Ausbildungsstrategien. In: Mitteilungen aus der Arbeitsmarkt- und Berufsforschung, Vol. 34(3), S. 269-285.

Buchholz, S. (2008): Die Flexibilisierung des Erwerbsverlaufs. Eine Analyse von Einstiegs- und Ausstiegsprozessen in Ost- und Westdeutschland, Wiesbaden.

Büchtemann, C. F./ Schupp, J./ Soloff, D. J. (1993): Übergänge von der Schule in den Beruf - Deutschland und USA im Vergleich. In: Mitteilungen der Arbeitsmarkt- und Berufsforschung, Vol. 26(3), S. 507-520.

Bukodi, E./ Ebralidze, E./ Schmelzer, P./ Relikowski, I. (2006): Increasing flexibility at labour market entry in the early career. In: flexCareer Working Paper Nr. 6.

Burdett, K. (1978): A theory of employee job search and quit rates. In: American Economic Review, Vol. 68(1), S. 212-220.

Burdett, K./ Mortensen, D. T. (1980): Search, layoffs and labor market equilibrium. In: Journal of Politicial Economy, Vol. 88(4), S. 652-672.

Burkert, C./ Seibert, H. (2007): Labour market outcomes after vocational training in Germany. Equal opportunities for migrants and natives?. In: IAB Discussion Paper Nr. 31.

Caliendo, M. (2006): Microeconometric evaluation of labour market policies, Heidelberg.

Caliendo, M./ Hujer, R. (2005): The Microeconometric estimation of treatment effects - An overview. In: IZA Discussion Paper Nr. 1653.

Caparros, A./ Navarro, M. L. (2005): Factors affecting quits and layoffs in Spain. In: Applied Econometrics and International Development, Vol. 5(4), S. 41-60.

Capelli, P. (2002): Why do employers pay for college?. In: NBER Working Paper Nr. 9225.

Casey, B. (1986): The dual apprenticeship system and the recruitment and retention of young person in West Germany. In: BJIR: An International Journal of Employment Relations/London School of Economics, Vol. 24(1), S. 63-81.

Clark, D. (2001): Why do german firms subsidize apprenticeship training? Tests of asymmetric information and mobility cost explanations. In: Vierteljahreshefte zur Wirtschaftsforschung, Vol. 70(1), S. 102-106.

Clark, D./ Fahr, R. (2001): The promise of workplace training for non-collegebound youth: Theory and evidence from german apprenticeship. In: IZA Discussion Paper Nr. 378.

Cox, D. (1972): Regression models and life tables. In: Journal of the Royal Statistical Society, Series B, Vol. 34(2), S. 187-220.

D'Agostino, R. (1998): Tutorial in biostatistics: Propensity score methods for bias reduction in the comparison of a treatment to a non-randomized control group. In: Statistics in Medicine, Vol. 17(19), S. 2265-2281. 
Damelang, A./ Haas, A. (2005): Arbeitsmarkteinstieg nach dualer Berufsausbildung - Migranten und Deutsche im Vergleich. In: IAB Forschungsbericht Nr. 17.

Davis, S. J./ Haltiwanger, J.C. (1990): Gross job creation and destruction. In: NBER Macroeconomic Annual 1990, Cambridge.

Dehejia, R. H./ Wahba, S. (2002): Propensity score-matching methods for nonexperimental causal studies. In: Review of Economics and Statistics, Vol. 84(1), S. 151-161.

Delacroix, A. (2003): Transitions into unemployment and the nature of firing costs. In: Review of Economic dynamics, Vol. 6(3), S. 651-671.

Deutsches Institut für Internationale Pädagogische Forschung (DIPF) (2008): Bildung in Deutschland 2008, KMK und BMBF (Hrsg.), Bonn.

Díaz-Vazques,P./ Snower, D. (2002): On-the-job-training and the effects of insider power. In: IZA Discussion Paper Nr. 586.

Díaz-Vázquez, P./ Snower, D. (2003): On-the-job-training, firing costs and employment. In: IZA Discussion Paper Nr. 910.

Dietrich, H. (2001a): Wege aus der Jugendarbeitslosigkeit - Von der Arbeitslosigkeit in die Maßnahme? In: Mitteilungen aus der Arbeitsmarkt- und Berufsforschung, Vol. 34(4), S. 461-478.

Dietrich, H. (2001b): Unterschiedliche Startbedingungen haben langfristige Folgen. Der Einmündungsverlauf der Geburtskohorten 1964 und 1971 in Ausbildung und Beschäftigung - Befunde aus einem IAB-Projekt. In: IAB-Werkstattbericht, Nr. 11.

Dietrich, H./ Abraham, M. (2005): Eintritt in den Arbeitsmarkt. In: Abraham, M./ Hinz, T. (Hrsg.), Arbeitsmarktsoziologie. Probleme, Theorien, empirische Befunde, Wiesbaden, S. 69-98.

Dietrich, H./ Dietz, M./ Stops, M./ Walwei, U. (2008): Ausbildungsmarkt: Mehr Schwung durch neue Regelungen. In: Orientierungen zur Wirtschafts- und Gesellschaftspolitik, Vol. 115(1), S. 51-56.

Dietrich, H./ Gerner, H.-D. (2008): Betriebliches Ausbildungsverhalten und Geschäftserwartungen. In: Sozialer Fortschritt, Vol. 57(4), S. 87-93.

Dietrich, H./ Kleinert, C. (2005): Reemployment - but what kind of job? Job prospects of young unemployed. In: Beiträge zur Arbeitsmarkt- und Berufsforschung, Band 295, S. 185-203.

Dietrich, H./ Kleinert, C. (2006): Der lange Arm der sozialen Herkunft. Bildungsentscheidungen arbeitsloser Jugendlicher. In: Tully, C. J. (Hrsg.): Lernen in flexibilisierten Welten. Wie sich das Lernen der Jugend verändert, Weinheim, S. 111-130.

Dietrich, H./ Koch, S./ Stops, M. (2004): Ausbildung muss sich lohnen - auch für Betriebe. In: IAB- Kurzbericht. Nr. 6.

Dionisius, R./ Mühlemann, S./ Pfeifer, H./ Walden, G./ Wenzelmann, F./ Wolter, S. C. (2008): Cost and benefit of apprenticeship training: A comparison of Germany and Switzerland. In: IZA Discussion Paper Nr. 3465. 
Doeringer, P. B./ Piore, M. J. (1971): Internal markets and manpower analysis, Lexington.

Dorau, R./ Granato, M./ Höhns, G./ Schwerin, C./ Uhly, A. (2006): Übergänge und beruflichen Entwicklungsmöglichkeiten junger Fachkräfte an der zweiten Schwelle. Abschlussbericht des Bundesinstituts für Berufsbildung, Bonn.

Drews, N. (2006): Qualitätsverbesserung der Bildungsvariablen in der IABBeschäftigten-Stichprobe 1975-2000. In: FDZ-Methodenreport Nr. $5 / 2006$.

Drews, N./ Hamann, S./ Köhler, M./ Krug, G./ Wübbeke, C. (2006): Variablen der schwach anonymisierten Version der IAB-Beschäftigten-Stichprobe 1975-2001. In: FDZ-Datenreport Nr. 01/2006.

Dundler, A./ Müller, D. (2006): Ein Leben ohne Arbeitslosigkeit - nur noch Fiktion?. In: IAB Kurzbericht Nr. 27.

Dustmann, C./ Euwals, R./ van Soest, A. (1997): Wages in the first job after apprenticeship: Mover versus stayers. In: Mitteilungen der Arbeitsmarktund Berufsforschung, Vol. 30(3), S. 671-674.

Dustmann, C./ Schönberg, U. (2002): Why do firms paying for training? Testing alternatives explanations using firm and longitudinal data, Discussion Paper, University College London.

Dustmann, C./ Schönberg, U. (2004): Training and union wages. In: IZA Discussion Paper Nr. 1435.

Eberhard, V. (2006): Das Konzept der Ausbildungsreife - ein ungeklärtes Konstrukt im Spannungsfeld unterschiedlicher Interessen: Ergebnis aus dem BiBB. In: Wissenschaftliche Diskussionspapiere Nr. 083.

EC (2007): Employment in Europe 2007, Brüssel.

Elliott, R. J. R./ Lindley, J. (2006): Skill specificity and labor mobility: occupational and sectoral dimensions. In: The Manchester School, Vol. 74(3), S. 389-413.

Enste, D. H./ Hardege, S. (2006): IW-Regulierungsindex. In: IW-Analysen, Forschungsberichte Nr. 16.

Eriksson, S. (2006): Skill loss, ranking of job applicants and the dynamics of unemployment. In: German Economic Review, Vol. 7(3), S. 265-296.

Erlinghagen, M. (2003): Die Restrukturierung des Arbeitsmarktes. Arbeitsmarktmobilität und Beschäftigungsstabilität im Zeitverlauf, Wiesbaden.

Estevez-Abe, M./ Iversen, T./ Soskice, D. (2000): Social protection and the formation of skills: A reinterpretation of the welfare state. Paper presented at Wiener inequality and Social Policy Seminar, 13. März 2000 an der Harvard University, USA.

Euwals, R./ Winkelmann, R. (2002): Mobility after apprenticeship - Evidence from register data. In: Konjunkturpolitik, Vol 48(3-4), S. 257-278. 
Euwals, R./ Winkelmann, R. (2003): Training intensity and first labor market outcomes of apprenticeship graduates. In: Working Paper Series of Socioeconomic Institute, University of Zurich Nr. 0308.

Evans, K./ Heinz, W. R. (1994): Becoming adults in England and Germany. Anglo-german foundation, London.

Falk, M. (2002): IKT-Fachkräftemangel und Anpassungsreaktionen der Unternehmen. In: Bellmann, L./Velling, H. (Hrsg.): Arbeitsmärkte für Hochqualifizierte. In: Beiträge zur Arbeitsmarkt- und Berufsforschung, Band 256, S. 37-73.

Falk, S./ Sackmann, R./ Struck, O./ Weymann, A./ Windzio, M./ Wingens, M. (2000): Gemeinsame Startbedingungen in Ost- und West? Risiken beim Berufseinstieg und deren Folgen im weiteren Erwerbsverlauf. In: Arbeitspapier des Sonderforschungsbereichs 186 der Universität Bremen Nr. 65.

Farber, H. (1993): The incidence and cost of job loss: 1982-1991. In: Brookings Papers of Economic Activity, Department of Economics, Industrial Relations Section, S. 73-132.

Feller, G. (2001): Berufseinmündung und Verbleib von Absolventen/Absolventinnen der Berufsfachschulen und des dualen Systems der Berufsausbildung. In: Feller, G. (Hrsg.): Auf dem Schulweg zum Beruf. Befunde zur Ausbildung an Berufsfachschulen als Teil des deutschen Berufsbildungssystems, Bundesinstitut für Berufliche Bildung, Der Generalsekretär, S.117-128.

Fier, A./ Heger, D./ Hussinger, K. (2005): Die Wirkungsanalyse staatlicher Förderprogramme durch den Einsatz von Matching- und Selektionsmodellen am Beispiel der Fertigungstechnik. In: ZEW Discussion Paper Nr. 0509.

Figlio, D. N./ Page, M. E. (2002): School choice and the distributional effects of ability tracking: Does separation increase inequality?. In: Journal of Urban Economics, Vol. 51(3), S. 497-514.

Fischer, G./ Dahms, V./ Bechmann, S./ Bilder, F./ Frei, M./ Wahse, J./ Möller, I. (2008): Langfristig handeln, Mangel vermeiden: Betriebliche Strategien zur Deckung des Fachkräftebedarfs. Ergebnisse des IAB-Betriebspanels 2007. In: IAB Forschungsbericht Nr. 3.

Fitzenberger, B./ Kohn, K. (2005): Gleicher Lohn für gleiche Arbeit? Zum Zusammenhang zwischen Mitgliedschaft und Lohnstruktur in Westdeutschland 1985-1997. In: ZEW Discussion Paper Nr. 05-36.

Fitzenberger, B./ Osikominu, A./ Völter, R. (2005): Imputation rules to improve the education variable in the IAB Employment Subsample. In: ZEW Discussion Paper Nr. 05-10.

Fitzenberger, B./ Spitz, A. (2004): Die Anatomie des Berufswechsels: Eine empirische Bestandsaufnahme auf der Basis der BiBB/IAB-Daten 1998/1999. In: ZEW Discussion Paper Nr 04-05. 
Flanagan, R. J. (1999): Macroeconomic performance and collective bargaining: An institutional persepctive. In: Journal of Economic Literature, Vol. 3(3), S. 1150-1175.

Fougère, D./ Schwerdt, W. (2002): Are apprentices productive? In: Konjunkturpolitik, Vol. 48(3-4), S. 317-337.

Franz, W./ Zimmermann, V. (1999): Mobilität nach der beruflichen Erstausbildung: Eine Empirische Studie für Westdeutschland. In: Jahrbücher für Nationalökonomie und Statistik, Vol. 219(1-2), S. 143-164.

Franz, W./ Soskice, D. (1995): The german apprenticeship system. In: Discussion Papier of the Center for International Labor Economics Nr.11-1994.

Franz, W./ Steiner, V./ Zimmermann, V. (2000): Die betriebliche Ausbildungsbereitschaft im technologischen und demografischen Wandel, BadenBaden.

Frazis, H./ Gittleman, M./ Joyce, M. (2000): Correlates of training: An analysis using both employer and employee characteristics. In: Industrial and Labor Relation Review, Vol. 53(2), S. 443-462.

Fuchs, S./ Schettkat, R. (2000): Germany: A regulated flexibility. In: EspingAndersen, G./ Regini, M. (Hrsg.): Why deregulate Labour Markets?, Oxford, S. 211-244.

Fuchs, T./ Ebert, A. (2008): Was ist gute Arbeit? Anforderungen an den Berufseinstieg aus Sicht der jungen Generation. Ergebnisse einer repräsentativen Befragung von Berufseinsteigern im Alter zwischen 18 und 34 Jahren mit abgeschlossener Berufsausbildung. Forschungsbericht des Internationalen Institut für empirische Sozialökonomie (Inifes), Nr. 375 und Gutachten Im Auftrag der Bundesanstalt für Arbeitsschutz und Arbeitsmedizin (BAuA) und dem Bundesministerium für Arbeit und Soziales (BMAS).

Fürstenberg, F. (2000): Berufsgesellschaft in der Krise, Berlin.

Gangl, M. (2003): Bildung und Übergangsrisiken beim Einstieg in den Beruf. Ein europäischer Vergleich zum Arbeitsmarktwert von Bildungsabschlüssen. In: Zeitschrift für Erziehungswissenschaft, Vol. 6(1), S. 72-89.

Gangl, M./ DiPrete, T. A. (2004): Kausalanalyse durch Matchingverfahren. In: Dieckmann, A. (Hrsg.) Methoden der Sozialforschung. Sonderheft 2004 der Kölner Zeitschrift für Soziologie und Sozialpsychologie. In Mimeo.

Garloff, A. (2003): Lohndispersion und Arbeitslosigkeit. Neuere Ansätze der Suchtheorie. In: ZEW Discussion Paper Nr. 03-60.

Garloff, A./ Kuckulenz, A. (2006): Training, mobility and wages: Specific versus general human capital. In: Jahrbücher für Nationalökonomie und Statistik, Vol. 226(1), S. 55-81.

Genda, Y./ Paziena, M. P./ Sognorelli, M. (2001): Labour market performance and job creation. Comparing economic systems: Italy and Japan, Hampshire. 
Gensler, S./ Skiera, B./ Böhm, M. (2005): Einsatzmöglichkeiten der Matching Methode zur Berücksichtigung von Selbstselektion. In: Journal für Betriebswirtschaftslehre, Vol. 55(1), S. 37-62.

Gerlach, K./ Meyer, W. (1995): Tarifverhandlungssysteme, Lohnhöhe und Beschäftigung. In: Mitteilungen aus der Arbeitsmarkt- und Berufsforschung, Vol. 28(3), S. 383-390.

Gerlach, K./ Stephan, G. (2005): Tarifverträge und betriebliche Entlohnungsstrukturen. In: IAB Discussion Paper Nr. 20.

Gersbach, H./ Schmutzler, A. (2001): A product market theory of worker training. In: IZA Discussion Paper Nr. 327.

Gersbach, H./ Schmutzler, A. (2006): The effects of globalization on worker training. In: IZA Discussion Paper Nr. 2403.

Gibbons, R./ Katz, L. (1991): Layoffs and lemons. In: Journal of Labor Economics, Vol. 9(4), S. 351-380.

Giesecke, J./ Groß, M. (2003): Temporary employment: Chance or risk? In: European Sociological Review, Vol. 19(2), S. 161-177.

Görg, H./ Strobl, E./ Walsh, F. (2007): Why do foreign-owned firms pay more? The role of on-the-job training. In: CEPR Discussion Paper Nr 6171.

Granato, M./ Dorau, R. (2004): Junge Fachkräfte an der zweiten Schwelle. In: Ibv, Nr. 25.

Greene, W. (2003): Econometric Analysis. 5. Auflage, Upper Saddle River.

Greenwald, B. (1986): Adverse selection in the labor market. In: The Review of Economic Studies. Vol. 53(3), S. 325-347.

Greinert, W.-D. (1998): Das „deutsche System“ der Berufsausbildung. Studien zur vergleichenden Berufspädagogik, Baden-Baden.

Großmann, S./ Meyer, H.L.(2002): Berufsausbildung im Dualen System - eine lohnende Investition?, Frankfurt a. M.

Grünewald, U./ Moraal, D. (Hrsg.) (2001): Duale Ausbildungssysteme. Institutionelle Rahmenbedingungen und Leistungsfähigkeit der dualen Ausbildung im Baugewerbe. In: Berichte zur beruflichen Bildung, Bielefeld Nr. 245.

Haas, A. (2002): Arbeitsmobilität nach Abschluss einer Dualen Berufsausbildung. Befunde aus der IAB-Historikdatei 1992-1997. In: IAB Werkstattbericht Nr. 3.

Hanushek, E. A./ Wößmann, L. (2006): Does educational tracking affect performance and inequality? Differences-in-differences evidence across countries. In: The Economic Journal, Vol. 116(510), S. C63-C76.

Harhoff, D./ Kane, T. J. (1997): Is the German Apprenticeship System a Panacea for the U.S. Labor Market? In: Journal of Population Economics, Vol. 10(2), S.171-196.

Hartung, S./ Janik, F. (2006): Seltener am Start, genauso oft am Ziel: Frauen in der betrieblichen Berufsausbildung. In: IAB Kurzbericht Nr. 15. 
Hashimoto, M. (1981): Firm-specific human capital as a shared investment. In: American Economic Review, Vol. 7(3), S. 475-482.

Hassink, W./ Broersma, L. (2003): Quits, layoffs and job destruction. In: Applied Economics, Vol. 35(18), S. 1911-1914.

Haveman, J. D. (1994): The Influence of changing trade patterns on displacement of labor. Purdue University, Krannert School of Management, Center for International Business Education and Research (CIBER).

Heckman, J. J./ Ichimura, H./ Todd, P. E. (1997): Matching as an econometric evaluation estimator: Evidence from evaluating a job training programme. In: Review of Economic Studies, Vol. 64(4), S. 605-654.

Heckman, J. J./ Ichimura, H./ Todd, P. E. (1998): Matching as an econometric evaluation estimator. In: Review of Economic Studies, Vol. 65(2), S. 261294.

Heckman, J. J./ Borjas, G. J. (1980): Does unemployment cause future unemployment? Definitions, questions and answers from a continuous time model of heterogeneity and state dependence. In: Economica, Vol. 47(187), S. 247-283.

Heinz, W. R./ Krüger, H./ Rettke, U./ Wachtveitl, E./ Witzel, A. (1987): „Hauptsache eine Lehrstelle“. Jugendliche vor den Hürden des Arbeitsmarktes, Weinheim.

Hill, R. C./ Griffith, W. E./ Judge, G. G. (2001): Undergraduate econometrics, 2.Auflage, Hoboken.

Hillmert, S. (2001): Kohortendynamik und Konkurrenz an den zwei Schwellen des dualen Ausbildungssystems. Übergänge zwischen Schule und Arbeitsmarkt im Kontext ökonomischen und demografischen Wandels. Papier aus Projekt „Ausbildungs- und Berufsverläufe der Geburtskohorten 1964 und 1971 in Westdeutschland Nr.5.

Hillmert, S. (2004): Berufseinstieg in Krisenzeiten. Ausbildungs- und Arbeitsmarktchancen in den 1980er und 1990er Jahren. In: Hillmert, S./Mayer, K. U. (Hrsg.): Geboren 1964 und 1971. Untersuchungen zu Ausbildungs- und Berufschancen, Wiesbaden, S. 23-38.

Hillmert, S. (2006): Übergänge zwischen Schule und Arbeitsmarkt: Ergebnisse der Westdeutschen Lebensverlaufstudie. In: Übergänge zwischen Schule und Beruf und darauf bezogene Hilfesysteme in Deutschland. Gesprächskreis Arbeit und Qualifizierung der Friedrich-Ebert-Stiftung und des Instituts für Arbeitsmarkt- und Berufsforschung der Bundesagentur für Arbeit, S. 10-20.

Hillmert, S. (2007): Soziale Ungleichheit im Bildungsverlauf: Zum Verhältnis von Bildungsinstitutionen und Entscheidungen. In: Becker, R./Lauterbach, W. (Hrsg.): Bildung als Privileg. Erklärungen und Befunde zu den Ursachen der Bildungsungleichheit, 2. Auflage, Wiesbaden, S. 71-99.

Hillmert, S./ Mayer, K. U. (2004): Geboren 1964 und 1971. Neuere Untersuchungen zu Ausbildungs- und Berufschancen Wiesbaden. 
Hofer, H./ Pichelmann, K. (1995): Zur Arbeitsmarktintegration von Lehrlingen in Österreich. In: Steiner, V./Bellmann, L. (Hrsg.): Mikroökonomik des Arbeitsmarktes, Beiträge zur Arbeitsmarkt- und Berufsforschung Band 192, S. 327-352.

Hofmann, A. (2001): Humankapital als Standortfaktor - volkswirtschaftliche Betrachtungsweisen, Aachen.

Holland, P. W. (1986): Statistics and causal inference. In: Journal of the American Statistical Association, Vol. 81(396), S. 945-960.

Holm, A./ Gros, N./ Olsen, T. H. (2001): Youth unemployment and opportunities in the labour market - The myth of lifelong hysteresis. In: Labour, Vol. 15(4), S. 531-554.

Howell, D. R./ Hübler, F. (2005): Wage compression and the unemployment crisis: Labor market institutions, skill, and inequality-unemployment tradeoffs. In: Howell, D. R. (Hrsg.): Fighting unemployment: The limits of free market orthodoxy, New York.

Hujer, R./ Caliendo, M./ Radic, D. (2001): Nobody knows...how different evaluation estimators perform in a simulated labour market experiment? Working Paper der J. W. Goethe-Universität Frankfurt.

Hujer, R./ Caliendo, M./ Radic, D. (2003): Methods and limitation of evaluation and impact research. Report on VET Research in Europe. In: Working Paper des CEDEFOP Project 0730.

Imbens, G. (2004): Nonparametric estimation of average treatment effects under exogeneity: A review. In: Review of Economic Statistics, Vol. 86(1), S. 4-29.

Imbens, G./ Wooldridge, J. (2007): Recent developments in the econometrics of program evaluation. Unveröffentlichtes Dokument, Department of Economics of Harvard University.

Isengard, B. (2002): Youth unemployment: Individual risk factors and institutional determinants. A case study of Germany and the United Kingdom. In: DIW Discussion Papers Nr. 284.

Jacob, M. (2004): Mehrfachausbildungen in Deutschland. Karriere, Collage, Kompensation?, Wiesbaden.

Jacoby, S. (1983): Industrial labor mobility in historical perspective. In: Industrial Relations, Vol. 22(2), S. 261-282.

Johnson, W. R. (1978): A theory of job shopping. In: Quarterly Journal of Economic, Vol. 92(2), S. 261-278.

Jovanovic, B. (1979): Job matching and the theory of turnover. In: Journal of Political Economy, Vol. 87(5), S. 972-990.

Kahn, D. (2000): Wage inequality, collective Bargains and relative employment from 1985 to 1994: Evidence from fifteen OECD countries. In: Review of Economics and Statistics, Vol. 82(4), S. 564-579. 
Kambourov, G./ Manovskii, I. (2008): Rising occupational and industry mobility in the United States: 1968-1997. In: International Economic Review, Vol. 49(1), S. 41-79.

Kaplan, E./ Meier, P. (1958): Nonparametric estimation from incomplete observations. In: Journal of the American Statistical Association, Vol. 53(282), S. 457-481.

Katz, E./ Ziderman, A. (1990): Investment in general training: The role of information and labour mobility. In: Economic Journal, Vol. 100(403), S. 1147-1158.

Katz, L. F./ Murphy, K. M. (1991): Changes in relative wages, 1963-1987: supply and demand factors. In: NBER Working Paper Nr. 3927.

Kempf, T. (1985): Theorie und Empirie betrieblicher Ausbildungsplatzangebote, Frankfurt/Main.

Kerckhoff, A. C. (2000): Transition from school to work in comparative perspective. In: Hallinan, M. T. (Hrsg.): Handbook of the Sociology of Education, S. 543-574, New York, Boston, M.A..

Kessler, A./ Lülfesmann, C. (2002): The theory of human capital revisited: On the interaction of general and specific investments. In: CESifo Working Paper Nr. 776.

Kletzer, L. G. (1998): Job displacement. In: Journal of Economic Perspectives, Vol. 12(1), S. 115-136.

Kletzer, L. G. (1995): International trade and job loss in U.S. manufacturing, 1979-91. In: Collins, S. M. (Hrsg.): Imports, exports and the american worker, Washington, D.C..

Kock, K. (2008): Auf Umwegen in den Beruf. Destandardisierte und prekäre Beschäftigung von Jugendlichen an der zweiten Schwelle - eine Auswertung empirischer Befunde. Projekt im Auftrag der Hans-Böckler-Stiftung.

Konietzka, D. (2002): Die soziale Differenzierung der Übergangsmuster in den Beruf. Die ,zweite Schwelle“ im Vergleich der Berufseinstiegskohorten 1976-1995. In: Kölner Zeitschrift für Soziologie und Sozialpsychologie, Vol. 54(4), S. 645-673.

Konietzka, D. (2007): Berufliche Bildung und der Übergang in den Arbeitsmarkt. In: Becker, R./Lauterbach, W. (Hrsg.): Bildung als Privileg. Erklärungen und Befunde zu den Ursachen der Bildungsungleichheit, 2. Auflage, Wiesbaden, S. 273-302.

Konietzka, D./ Seibert, H. (2001): Die Erosion eines Übergangsregimes? Arbeitslosigkeit nach der Dualen Berufsausbildung und ihre Folgen für den Berufseinstieg - ein Vergleich der Berufseinstiegskohorten 1976- 1995. In Berger, P. A./ Konietzka, D. (Hrsg.): Die Erwerbsgesellschaft. Neue Ungleichheiten und Unsicherheiten. In der Reihe Sozialstrukturanalyse, S. 65-95.

Kriechel, B./ Pfann, G. (2005): The role of specific and general human capital after displacement. In: Education Economics, Vol. 13(2), S. 223-236. 
Kurz, K./ Steinhage, N. (2001): Globaler Wettbewerb und Unsicherheiten beim Einstieg in den Arbeitsmarkt. Analysen für Deutschland in den 80er und 90er Jahren. In: Berliner Journal für Soziologie, Vol. 11(4), S. 513-531.

Lauterbach, W./ Sacher, M. (2001): Erwerbseinstieg und erste Erwerbsjahre. Ein Vergleich von vier westdeutschen Geburtskohorten. In: Kölner Zeitschrift für Soziologie und Sozialpsychologie, Vol.53(2), S. 258-282.

Layte, R./ O'Connell. P. J./ Fahey, T./ McCoy, S. (2005): Ireland and economic globalization: The experiences of a small open economy. In: Blossfeld, H.-P./ Klijzing, E./ Mills, M./ Kurz, K. (Hrsg.): Globalization, uncertainty and youth in society, London, New York.

Lazear, E. P. (2004): Firm-specific human capital: A skill-weights approach. In: NBER Working Paper Nr. 9679.

Lechner, M. (1998): Mikroökonomische Evaluationsstudien: Anmerkungen zu Theorie und Praxis. In: Pfeiffer, F./Pohlmeier, W. (Hrsg.): Qualifikation, Weiterbildung und Arbeitsmarkterfolg. ZEW-Wirtschaftsanalysen, Band 31, Baden-Baden, S. 13-38.

Lechthaler, W./ Snower, D. J. (2008): Mindestlöhne und Humankapital. In: IfoSchnelldienst, Sonderausgabe: Mindestlohn: Für und Wider, S. 43-44.

Lehne, H. (1991): Bestimmungsgründe der betrieblichen Ausbildungsplatzangebote in der Dualen Berufsausbildung, München.

Leuven, E. (2005): The economics of training: A survey of the literature. In: Journal of Economic Survey, Vol. 19(1), S. 91-111.

Leuven, E./ Oosterbeek, H. (2001): Firm-specific human capital as a shared investment: Comment. In: American Economic Review, Vol. 91(1), S. 342-347.

Lindbeck, A./ Snower, D. (1988): The insider-outsider theory of employment and unemployment, Cambridge/Mass.

Lindley, R. M. (1975): The demand for apprentice recruits by the engineering industry, 1951-71. In: Scottish Journal of Political Economy, Vol. 22(1), S. 1-24.

Loewenstein, M. A./ Spletzer, J. R.(1998): Dividing the costs and returns to general training. In: Journal of Labor Economics, Vol. 16(1), S. 142-171.

Loewenstein, M. A./ Spletzer, J. R.(1999): General and specific training: Evidence and implications. In: Journal of Human Resources, Vol. 34(4), S. 710-733.

Lorenz, K./ Ebert, F./ Krüger, M. (2005): Das neue Berufsbildungsgesetz Chancen und Grenzen für die berufsbildenden Schulen in Deutschland. In: Wirtschaft und Erziehung, Vol. 57(5), S. 167-174.

Ludwig, V./ Pfeiffer, F. (2005): Abschreibungsraten allgemeiner und beruflicher Ausbildungsinhalte. In: ZEW Discussion Paper Nr. 05-036.

Lynch L. M./ Black, S. E. (1998): Beyond the incidence of employer-provided training. In: Industrial and Labor Relations Review, Vol. 52(1), S. 64-81. 
Maidorn, S. (2002): Transitions in the german labour market. In: Graduate College "Allocation Theory, Economic Policy and Collective Decisions" Ruhr-University Bochum/Dortmund University.

Malcomson, J. M./ Maw, J. W./ McCormick, B. (2003): General training by firms, apprentice contracts, and public policy. In: European Economic Review, Vol. 47(2), S. 197-227.

Manning, A. (2003): Monopsy in motion: Imperfect competition in labor markets, Princeton.

Manski, C. F. (1995): Identification problems in the social sciences, Cambridge, MA.

Marini, M. M./ Singer, B. (1988): Causality in social sciences. In: Sociological Methodology, Vol. 18(1988), 347-409.

Mayer, K.-U. (2000): Arbeit und Wissen. Die Zukunft von Bildung und Beruf. In: Kocka, J./Offe, C. (Hrsg.): Geschichte und Zukunft der Arbeit, Frankfurt am M., S. 383-409.

Mayer, K.-U. (2001): Lebensverlauf. In: Schäfers, B./Zapf, W. (Hrsg.): Handwörterbuch zur Gesellschaft Deutschlands, Opladen, S. 446-460.

McCausland, W. D./ Theodossiou, I. (2004): Training and hysteresis effects on the wage inflation: Unemployment Relationship. In: Journal of PostKeynesian Economics, Vol. 27(1), S. 67-86.

McFadden, D. (1974): The measurement of urban travel demand. In: Journal of Public Economics, Vol. 3(4), S. 303-328.

McGinnity, F./ Mertens, A. (2004): Befristete Verträge und Berufseinstieg. In: Hillmert, S./ Mayer, K.-U. (Hrsg.): Geboren 1964 und 1971. Neue Untersuchungen zu Ausbildungs- und Berufschance in Westdeutschland. VS Verlag für Sozialwissenschaften, Wiesbaden, S. 115-132.

McGinnity, F./ Mertens, A./ Gundert, S. (2005): A bad Start? Fixed-term contracts and the transition from education to work in West Germany. In: European Sociological Review, Vol. 21(4), S. 359-374.

McLaughlin, K. (1991): A theory of quits and layoffs with efficient turnover. In: The Journal of Political Economy, Vol. 99(1), S. 1-29.

Merilees, W. (1983): Alternative models of apprentice recruitment: With special reference to the British Engineering Industry. In: Applied Economics, Vol. 15(1), S. 1-21.

Mertens, D./ Parmentier, K. (1982): Zwei Schwellen - acht Problembereiche. Grundzüge eines Diskussions- und Aktionsrahmens zu den Beziehungen zwischen Bildungs- und Beschäftigungssystem. In: Mertens, D. (Hrsg.): Konzepte der Arbeitsmarkt- und Berufsforschung, Beiträge zu Arbeitsmarkt- und Berufsforschung, Band 70, S. 357-396.

Miyamito, H./ Shirai, Y. (2006): Job flows and unemployment in an equilibrium unemployment model with firm-specific skill training. In: The Japanese Economic Review, Vol. 57(4), S. 547-561. 
Mohrenweiser, J./ Backes-Gellner, U. (2008): Apprenticeship training - What for? Investment in human capital or substitution of cheap labour? In: Leading House Working Paper Nr. 17.

Mohrenweiser, J./Zwick, T. (2008): Why do firms train apprentices? The net cost puzzle reconsidered. Präsentiertes Papier bei der "International Conference on Economics of Education, Firm Behaviour and Training Policy", 25-27, Juni 2008 in Zürich, Schweiz.

Moosmüller, G. (2004): Methoden der empirischen Wirtschaftsforschung, München.

Mortensen, D. T. (1986): Job search and labor market analysis. In: Ashenfelter, O./Layard, R. (Hrsg.): Handbook of Labor Economics Vol. 2, North Holland, S. 849-919.

Mortensen, D. T./Pissarides, C. A. (1994): Job creation and job destruction in the theory of unemployment. In: Review of Economic Studies. Vol. 61(3), S. 397-415.

Mortensen, D. T./Pissarides, C. (1999): New developments in models of search in the labour market. In: Ashenfelter, O./Card, D. (Hrsg.): Handbook of Labour Economics Vol. 3B, North Holland, S. 2567-2627.

Mroz, T. A./Savage, T. H. (2006): The long-term effects of youth unemployment. In: The Journal of Human Resources, Vol. 61(2), S. 259-293.

Mühlemann, S./Schweri, J./Winkelmann, R./Wolter, S. C. (2005): A structural model of demand for apprentices. In: CESifo Working Paper Nr. 1417.

Mühlenweg, A. (2007): Educational effects of early or later secondary school tracking in Germany. In: ZEW Discussion Paper Nr. 07-079.

Mulder, M./Weigel, T./Collins, K. (2007): The concept of competence in the development of vocational education and training in selected EU member states: a critical analysis. In: In: Journal of vocational education and Training, Vol. 59(1), S. 67-88.

Müller, W. (2005): Education and youth integration into european labour market. In: International Journal of Comparative Sociology, Vol. 46(5-6), S. $461-485$.

Müller, W./Gangl, M. (2003): The transition from school to work: A european perspective. In: Müller, W./Gangl, M. (Hrsg.): Transition from education to work in Europe. The integration of youth into labour markets, New York, S. 1-22.

Müller, W./Shavit, Y. (1998): The institutional embeddedness of the stratification process. In: Schavit, Y./Müller, W. (Hrsg.): From school to work. A comparative study of educational qualifications and occupational destinations, Oxford, S. 1-48.

Nakamura, E. (2008): Layoffs and lemons over business cycle. In: Economic Letters, Vol. 99(1), S. 55-58. 
Neubäumer, R (1999): Der Ausbildungsstellenmarkt in der Bundesrepublik Deutschland: Eine theoretische Analyse und empirische Analyse, Schriften des Vereins für Socialpolitik, Berlin.

Neubäumer, R. (1997): „Ausbildung über oder unter Bedarf“ und die Finanzierung der Dualen Ausbildung - Theoretische Erklärungen, empirische Ergebnisse und wirtschaftspolitische Schlussfolgerungen. Referat im bildungsökonomischen Ausschuss des Vereins für Socialpolitik am 6. Februar 1997.

Neubäumer, R. (2002): Institutionelle Rahmenbedingungen der Dualen Berufsausbildung. In: Fachinger, U./Rothgang, H./Viebrok, H. (Hrsg.): Die Konzeption sozialer Sicherung, Baden-Baden, S. 303-318.

Neubäumer, R. (2006): Der gesetzliche Kündigungsschutz aus ökonomischer Sicht. In: Sozialer Fortschritt, Vol. 55(2-3), S. 25-34.

Neubäumer, R./Somaggio, G. (2006): Die Duale Berufsausbildung aus humankapitaltheoretischer und institutioneller Sicht. In: WISU - Das Wirtschaftsstudium, Nr. 5/2006, S. 690-697.

Niederalt, M. (2004): Zur ökonomischen Analyse betrieblicher Lehrstellenangebote in der Bundesrepublik Deutschland. Frankfurt am M..

OECD (2004): Employment Outlook 2004, Paris.

OECD (2008): Employment Outlook 2008, Paris.

Oosterbeek, H. (1998): Unravelling supply and demand factors in work-related training. In: Oxford Economic Papers, Vol. 50(2), S. 266-283.

Oosterbeek, H./Sloof, R./Sonnemans, J. (2007): Who should invest in specific training? In: Journal of Population Economics- Journal of the European Society for Population Vol. 20(2), S. 329-357.

Parmentier, K./Schade H.-J./Schreyer, F. (1993): Berufsspezifische Strukturund Entwicklungsdaten 1980-1991. Erwerbsberufe im Spiegel der Statistik. 6. Auflage; Nümberg.

Pearl, J. (2000): Causality, models, reasoning and inference, Cambridge.

Pigou, A. C. (1912): Wealth and Welfare, London.

Pissarides, C. A. (1979): Job matchings with state employment agencies and random search. In: The Economic Journal, Vol. 89(12), S. 818-833.

Pissarides, C. A. (1994): Search unemployment with on-the-job-search. In: Review of Economic Studies, Vol. 61(3), S. 457-475.

Pissarides, C.A. (2000): Equilibrium unemployment theory. Cambridge, MA.

Poletaev, M./Robinson, C. (2008): Human capital skill and industry specificity: Evidence from the dictionary of occupational titles and displaced worker surveys 1984-2000. In: Journal of Labor Economics, Vol. 26(3), S. 387420.

Pratt, J. W./Schlaifer, R. (1988): On the interpretation and observations of Laws. In: Journal of Econometrics, Vol. 39(1-2), S. 23-52.

Pütz, U. (2003): Zur Finanzierung beruflicher Bildung. Ansätze aus humankapitaltheoretischer und institutionenökonomischer Perspektive, Berlin. 
Rae, D./Sollie, M. (2007): Globalisation and the European Union. Which countries are best placed to cope? In: OECD Economics Department Working Paper Nr. 586.

Rees, A. (1986): An essay on youth joblessness. In: Journal of Economic Literature, Vol. 24(2), S. 613-628.

Reichenbach, S. (2001): Die Ausbildungsentscheidung im Dualen System der Berufsausbildung. Bestimmungsgründe der Allokation von Ausbildungsinteressenten auf Lehrberufe, Mannheim.

Reinberg, A./Hummel, M. (2004): Fachkräftemangel bedroht Wettbewerbsfähigkeit der deutschen Wirtschaft. In: Aus Politik und Zeitgeschichte, Nr. 28, S. 3-10.

Reinberg, A./Hummel, M. (2005): Vertrauter Befund: Höhere Bildung schützt auch in der Krise vor Arbeitslosigkeit. In: IAB Kurzbericht Nr. 9.

Reinberg, A./Hummel, M. (2007): Qualifikationsspezifische Arbeitslosigkeit im Jahr 2005 und die Einführung der Hartz-IV-Reform. In IAB Forschungsbericht Nr. 9.

Riphan, R. T. (2002): Residential location and youth unemployment: The economic geography of school-to-work transitions. In: Journal of Population Economics, Vol. 15(1), S.115-135.

Rogerson, R./ Shimer, R./ Wright, R. (2004): Search-theoretic models of the labour market: A survey. In: NBER Working Paper Nr. 10655.

Ronning, G. (1991): Mikroökonometrie, Heidelberg.

Rosen, S. (1976): A Theory of life earnings. In: Journal of Political Economy, Vol. 84(4), S. 545-568.

Rosenbaum, P. R. (2002): Observational Studies, 2. Auflage, New York.

Rosenbaum, P. R./ Rubin, D. (1983): The central role of the propensity score in the observational studies for causal effects. In: Biometrica, Vol. 70(1), S. 41-50.

Rothe, T./ Tinter, S. (2007): Jugendliche auf dem Arbeitsmarkt - eine Analyse von Beständen und Bewegungen. In: IAB Forschungsbericht Nr. 4.

Rubin, D. (1990): Comment on "Neyman (1923) and causal inference in experiments and observational studies. In: Statistic Studies, Vol. 5(4), S. 472480.

Rubin, D. B. (1974): Estimating causal effects of treatments in randomized and nonrandomized studies. In: Journal of Educational Psychology, Vol. 66(5), S. 688-701.

Rubin, D. B. (1978): Bayesian inference for causal effects. In: Annals of Statistics, Vol. 6(1), S. 34-58.

Ruud, P. A. (2000): An introduction to a classical econometric theory. Oxford University Press, New York.

Ryan, P. (1998): Is apprenticeship better? A review of the economic evidence. In: Journal of Vocational Education and Training, Vol. 50(2), S. 289-329. 
Ryan, P. (2001): The school-to-work transition: A cross national perspective. In: Journal of Economic Literature, Vol. 39(1), S. 34- 92.

Saar, E./ Unt, M./ Kogan, I. (2008): Transition from educational system to labour market in the European Union: A comparison between new and old members. In: International Journal of Comparative Sociology, Vol. 49(1), S. 31-49.

Sachverständigenkommission Kosten und Finanzierung der beruflichen Bildung (1974): Kosten und Finanzierung der außerschulischen beruflichen Bildung (Abschlußbericht). Bielefeld.

Sackmann, R. (2001): Generationenspezifische Arbeitsmarktchancen im internationalen Vergleich. In: Berger, P.A./Konietzka, D. (Hrsg.): Die Erwerbsgesellschaft. Neue Ungleichheiten und Unsicherheiten, Opladen, S. 253-276.

Sadowski, D. (1980): Berufliche Bildung und betriebliches Bildungsbudget, Stuttgart.

Sanders, J./ de Grip, A. (2004): Training task flexibility and the employability of low-skilled workers. In: International Journal of Manpower, Vol. 25(1), S. 73-89.

Schaeper, H. (1999): Erwerbsverläufe von Ausbildungsabsolventinnen und absolventen. Eine Anwendung der Optimal-Matching-Technik. In: Arbeitspapier des Sonderforschungsbereichs 186 der Universität Bremen, Projekt: Statuspassagen und Risikolagen im Lebensverlauf, Nr. 57.

Schaeper, H./ Kühn, T./ Witzel, A. (2000): Diskontinuierliche Erwerbskarrieren und Berufswechsel in den 1990ern: Strukturmuster und biografische Umgangsweisen betrieblich ausgebildeter Fachkräfte. In: Mitteilungen aus der Arbeitsmarkt- und Berufsforschung Vol. 33(1), S. 80-100.

Schettkat, R. (1992): The labor market dynamics of economic restructuring: The United States and Germany in transition, New York.

Schimpl-Neimanns, B. (2006): Berufliche Ausbildungsverläufe bis zum Übergang ins Erwerbsleben - Analysen zur Stichprobenselektivität des Mikrozensuspanels 1996- 1999. ZUMA-Arbeitsbericht Nr. 2006/02.

Schnabel, C. (2005): Gewerkschaften und Arbeitgeberverbände: Organisationsgrade, Tarifbindung und Einflüsse auf Löhne und Beschäftigung. In: Zeitschrift für Arbeitsmarktforschung, Vol. 38(3), S. 181-196.

Schøne, P. (2006): Determinants and developments of employer provided training: Evidence from wage compresses environment. In: Journal of Vocational Education and Training, Vol. 58(2), S. 237-254.

Schönig, W./ Farhauer, O. (2004): Bildungsförderung, Verteilungspolitik und soziale Durchlässigkeit. Zur Theorie und Empirie eines vernachlässigten Handlungsfeldes. In: Aus Politik und Zeitgeschichte, Nr. 28, S. 17-23.

Schreyer, F. (2001): Unsichere Beschäftigung und berufliche Qualifikation ein Blick auf Erwerbsarbeit jenseits des Normalarbeitsverhältnisses. In: Beiträge zur Arbeitsmarkt- und Berufsforschung, Band 246, S. 147-172. 
Schwerdt, W./ Bender, S. (2003): Was tun Lehrlinge nach ihrer Ausbildung? Eine Analyse mit dem Linked Employer-Employee-Datensatz des IAB. In: Mitteilungen aus der Arbeitsmarkt- und Berufsforschung, Vol. 36(1), S. 46-59.

Schweri, J. (2006): Das Ausbildungsverhalten Schweizer Firmen: Welche Rolle spielen asymmetrische Information und firmenspezifisches Training?, Berlin.

Seibert, H. (2007): Wenn der Schuster nicht bei seinem Leisten bleibt.... In: IAB Kurzbericht Nr. 1.

Seifert, H. (2007): Frühe Flexibilisierung? Regionale Mobilität nach der Lehrausbildung in Deutschland zwischen 1977 und 2004. In: IAB Discussion Paper Nr. 9.

Sengenberger, W. (1987): Struktur und Funktionsweise von Arbeitsmärkten: Die Bundesrepublik Deutschland im internationalen Vergleich, Frankfurt am Main, New York.

Sesselmeier, W./ Blauermel, G. (1998): Arbeitsmarkttheorien. Ein Überblick, 2. Auflage, Heidelberg.

Shimer, R. (1998): Why is the U.S. employment rate so much lower?. In Bernanke, B./ Rotemberg, J. (Hrsg.): NBER Macroeconomics Annual, Vol. 13, Cambridge, MA, S. 11-61.

Shimer, R. (2001): The impact of young workers in the aggregate labour market. In: The Quarterly Journal of Economics, Vol. 116(3), S. 969-1007.

Siebert, H. (1997): Labour market rigidities: At the root of unemployment in Europe. In: Journal of Economic Perspectives, Vol. 11(3), S. 37-54.

Smits,W./ Stromback, T. (2001): The economics of the apprenticeship system, Cheltenham/Northampton.

Soskice, D. (1994): Reconciling markets and institutions: The german apprenticeship system. In: Lynch, L. (Hrsg.): Training and the private sector. International comparison, Chicago, S. 25-60.

Spence, M. (1974): Market signalling, Cambridge.

Spence, M. (1973): Job market signalling. In: Quarterly Journal of Economics, Vol. 87(3), S. 255-274.

Steedman, H. (1993): The economics of youth training in Germany. In: Economic Journal Vol. 103(420), S. 1279-1291.

Steedman, H./ Wagner, K. (2007): The impact of rational ICT qualification systems on companies' recruitment practices - an anglo-german comparison. In: Zeitschrift für Arbeitsmarktforschung, Vol. 40 (2-3), S. 235-251.

Steedman, H./ Wagner, K./ Foreman, J. (2006): Das Angebot an IKTFachkräften im Vereinigten Königreich und in Deutschland. In: Europäische Zeitschrift für Berufsbildung, Vol. 37(1), S. 9-23.

Stegmann, H./ Kraft, H. (1983): Vom Ausbildungs- zum Arbeitsvertrag. In: Mitteilungen aus der Arbeitsmarkt- und Berufsforschung Vol. 16(3), S. 235- 251 . 
Stegmann, H./ Kraft, H. (1982): Jugendliche an der Schwelle von der Berufsausbildung in die Erwerbstätigkeit: Methode und erste Ergebnisse der Wiederholungserhebung Ende 1980. In: Mitteilungen aus der Arbeitsmarkt- und Berufsforschung, Vol. 14(1), S. 20-35.

Steinmann, S. (2000): Bildung, Ausbildung und Arbeitsmarktchancen in Deutschland. Eine Studie zum Wandel der Übergänge von der Schule in das Erwerbsleben, Opladen.

Stevens, M. (1996): Transferable training and poaching externalities. In: Booth, A. L./ Snower, D. (Hrsg.): Acquiring Skills. Market Failures, their Symptoms and Policy Responses, New York, S. 19-40.

Stevens, M. (2001): Should firms be required to pay for vocational training?. In: Economic Journal, Royal Economic Society, Vol. 111(473), S. 485505.

Szydlik, M. (1996): Übereinstimmung von Ausbildung und Arbeitsplatzanforderungen in der Bundesrepublik Deutschland. In: Mitteilung der Berufsund Arbeitsmarktforschung, Vol. 29(2), S. 295-306.

Topel, R./ Ward M. (1992): Job mobility and the careers of young men. In: Quarterly Journal of Economics, Vol. 107(2), S.439-479.

Verbeek, M. (2008): A guide to modern econometrics, 3. Auflage, Hoboken.

Von Bardeleben, R./ Beicht, U./ Fehér, K. (1995): Betriebliche Kosten und Nutzen der Ausbildung. In: Berichte zur beruflichen Bildung Nr. 187, Bielefeld.

Von Wachter, T./ Bender, S. (2004): In the right place at the wrong time: The role of firms and luck in young workers' careers. In: IZA Discussion Paper Nr. 1348.

Walden, G. (2007): Short-term and long-term benefits as determinants of the training behaviour of companies. In: Zeitschrift für Arbeitsmarktforschung, Vol. 40(2-3), S. 169-193.

Wasmer, E. (2006): General versus specific skills in Labour Markets with Search frictions and firing costs. In: American Economic Review, Vol. 36(3), S. 811-831.

Weigel, T./ Mulder, M./ Collins, K. (2007): The concept of competence in the development of vocational education and training in selected EU member states. In: Journal of Vocational Education and Training, Vol. 59(1), S. 5366.

Werwatz, A. (2002): Occupational mobility after apprenticeship - How effective is the german apprenticeship system? In: Konjunkturpolitik, Vol. 48(3-4), S. 279-303.

Williamson, O. E. (1975): Markets and hierarchies: Analysis and antitrust implications, New York.

Winkelmann, R. (1994): Apprenticeship and after: Does it really matter? In: CEPR Discussion Paper Nr. 1034. 
Winkelmann, R. (1996): Employment prospects and skill acquisition of apprenticeship-trained workers in Germany. In: Industrial and Labour Relations Review, Vol. 49(4), S. 658-672.

Winterhager, H. (2006): Determinanten der Arbeitslosigkeitsdauer - Neue Erkenntnisse aus der IEB. In: ZEW Discussion Paper Nr. 06-077.

Wolter, S. C. (2008): Ausbildungskosten und -nutzen und die betriebliche Nachfrage nach Lehrlingen. In: Perspektiven der Wirtschaftspolitik, Vol. 9 (Sonderheft), S. 90-108.

Yashiv, E. (2006): Search and matching in macroeconomics. In: CEPR Discussion Paper Nr. 5459.

Yellen, J. L. (1984): Efficiency wage models of unemployment. In: American Economic Review P+P, Vol. 74(2), S. 200-205.

Zwick, T. (2007): Apprenticeship training in Germany - investment or productivity driven? In: Zeitschrift für Arbeitsmarktforschung, Vol. 40(2-3), S. 193-204. 


\section{Anhang}

Tabelle 13: Zuordnung der staatlich anerkannten Ausbildungsberufe zu den Ausbildungsbereichen

\begin{tabular}{|c|c|c|}
\hline & Ausbildungsbereiche & Ausbildungsberufe \\
\hline \multirow{7}{*}{$\begin{array}{l}\text { landwirtschaftliche } \\
\text { Berufe }\end{array}$} & \multirow[t]{7}{*}{ Landwirtschaft } & Landwirte \\
\hline & & Weinbauern \\
\hline & & Tierzüchter \\
\hline & & Tierpfleger und verwandte Berufe \\
\hline & & Gärtner, Gartenarbeiter \\
\hline & & Forstverwalter, Förster, Jäger \\
\hline & & Waldarbeiter, Waldnutzer \\
\hline \multirow[t]{28}{*}{ handwerkliche Berufe } & \multirow{28}{*}{$\begin{array}{l}\text { Chemie-, Papier-, Keramik- und holz- } \\
\text { verarbeitende Berufe }\end{array}$} & Edelsteinbrenner \\
\hline & & Formstein-, Betonhersteller \\
\hline & & Keramiker \\
\hline & & Hohlglasmacher \\
\hline & & Glasbläser \\
\hline & & Glasbearbeiter, Glasveredler \\
\hline & & Chemiebetriebswerker \\
\hline & & Vulkaniseure \\
\hline & & Kunststoffverarbeiter \\
\hline & & Papier-, Zellstoffhersteller \\
\hline & & Verpackungsmittelhersteller \\
\hline & & Buchbinderberufe \\
\hline & & Schriftsetzer \\
\hline & & Druckstockhersteller \\
\hline & & Buchdrucker (Hochdruck) \\
\hline & & Flach-, Tiefdrucker \\
\hline & & Spezialdrucker, Siebdrucker \\
\hline & & Vervielfältiger \\
\hline & & Holzaufbereiter \\
\hline & & $\begin{array}{l}\text { Holzverformer und zugehörige Beru- } \\
\text { fe }\end{array}$ \\
\hline & & Holzwarenmacher \\
\hline & & Korb-, Flechtwarenmacher \\
\hline & & Musikinstrumentenbauer \\
\hline & & Modelltischler, Formentischler \\
\hline & & Stellmacher, Böttcher \\
\hline & & Sonst. Holz-, Sportgerätebauer \\
\hline & & Warenmaler, -lackierer \\
\hline & & Holzoberflächenveredler, Furnierer \\
\hline \multirow[t]{11}{*}{ manuelle Industrieberufe } & \multirow[t]{11}{*}{ metallverarbeitende Berufe } & Eisen-, Metallerzeuger, Schmelzer \\
\hline & & Metallzieher \\
\hline & & Former, Kernmacher \\
\hline & & Formgießer \\
\hline & & Drahtverformer, -verarbeiter \\
\hline & & Dreher \\
\hline & & Fräser, \\
\hline & & Metallschleifer \\
\hline & & Metallpolierer \\
\hline & & Graveure, Ziseleure \\
\hline & & Galvaniseure, Metallfärber \\
\hline
\end{tabular}




\begin{tabular}{|c|c|c|}
\hline & & $\begin{array}{l}\text { Emaillierer, Feuerverzinker u. and. } \\
\text { Metalloberflächenveredler }\end{array}$ \\
\hline & & Stahlschmiede \\
\hline & & $\begin{array}{l}\text { Behälterbauer, Kupferschmiede und } \\
\text { verwandte Berufe }\end{array}$ \\
\hline & & Feinblechner \\
\hline & & Rohrinstallateure \\
\hline & & Rohrnetzbauer, Rohrschlosser \\
\hline & & Bauschlosser \\
\hline & & Betriebsschlosser,Reparaturschlosser \\
\hline & & Stahlbauschlosser, Eisenschiffbauer \\
\hline & & Sonstige Mechaniker \\
\hline & & Werkzeugmacher \\
\hline & & Metallfeinbauer a.n.g. \\
\hline & & Edelmetallschmiede \\
\hline & & Warenmaler, -lackierer \\
\hline & & Kerammaler \\
\hline handwerkliche Berufe & Textilberufe & Spuler, Zwirner, Seiler \\
\hline & & Weber \\
\hline & & Maschenwarenfertiger \\
\hline & & Schneider \\
\hline & & Oberbekleidungsnäher \\
\hline & & Sticker \\
\hline & & Hut-, Mützenmacher \\
\hline & & Näher a.n.g. \\
\hline & & Textilausrüster \\
\hline & & Lederhersteller, Darmsaitenmacher \\
\hline & & Schuhmacher \\
\hline & & Schuhwarenhersteller \\
\hline & & $\begin{array}{l}\text { Groblederwarenhersteller, Banda- } \\
\text { gisten }\end{array}$ \\
\hline & & Handschuhmacher \\
\hline & & Fellverarbeiter \\
\hline handwerkliche Berufe & Nahrungs- und Genussmittelerzeugen- & Konditoren \\
\hline & de Industrien & Fleischer \\
\hline & & Weinkühler \\
\hline & & Brauer, Mälzer \\
\hline & & Sonstige Getränkehersteller, Koster \\
\hline & & Milch-, Fettverarbeiter \\
\hline & & Mehl-, Nährmittelhersteller \\
\hline & & Zucker-, Süßwaren-, Speisehersteller \\
\hline handwerkliche Berufe & Bauberufe & Maurer \\
\hline & & Betonbauer \\
\hline & & Zimmerer \\
\hline & & Gerüstbauer \\
\hline & & Dachdecker \\
\hline & & Strassenbauer \\
\hline & & Gleisbauer \\
\hline & & Kultur-, Wasserbauwerker \\
\hline & & $\begin{array}{l}\text { Sonstige Tiefbauer } \\
\end{array}$ \\
\hline & & Stukkateure, Gipser, Verputzer \\
\hline & & Isolierer, Abdichter \\
\hline & & Fliesenleger \\
\hline
\end{tabular}




\begin{tabular}{|c|c|c|}
\hline & & Glaser \\
\hline & & Estrich-, Terazzoleger \\
\hline & & Maler, Lackierer (Ausbau) \\
\hline & & Baumaschinenführer \\
\hline technische Berufe & Berufe des Elektrofaches \& feinme- & Feinmechaniker \\
\hline & & Uhrmacher \\
\hline & & Elektroinstallateure, -monteure \\
\hline & & $\begin{array}{l}\text { Elektromotorenbauer, Transformato- } \\
\text { renbauer }\end{array}$ \\
\hline & & Elektrogerätebauer \\
\hline & & $\begin{array}{l}\text { Warenaufmacher, Versandfertigma- } \\
\text { cher }\end{array}$ \\
\hline & & Speditionskaufleute \\
\hline & & Schienenfahrzeugführer \\
\hline & & Eisenbahnbetriebsregler, -schaffner \\
\hline $\begin{array}{l}\text { (z. T. einfache) Dienst- } \\
\text { leistungsberufe } \\
\text { sowie }\end{array}$ & $\begin{array}{ll}\text { Infrastruktur-l } & \text { Transportdienstleis- } \\
\text { tungen } & \end{array}$ & $\begin{array}{l}\text { Sonstige Fahrbetriebsregler, Schaff- } \\
\text { ner }\end{array}$ \\
\hline kaufmännische Berufe & & Kraftfahrzeugführer \\
\hline & & Straßenwarte \\
\hline & & Postverteiler \\
\hline & & Lagerverwalter, Magaziner \\
\hline & & $\begin{array}{l}\text { Groß- und Einzelhandelskaufleute, } \\
\text { Einkäufer }\end{array}$ \\
\hline & & Verkäufer \\
\hline & & Verlagskaufleute, Buchhändler \\
\hline & & Tankwarte \\
\hline kaufmännische Berufe & ökonomische Dienstleistungen & Bankfachleute \\
\hline & (Finanzdienstleistungen) & Lebens-, Sachversicherungskaufleute \\
\hline & & Werbefachleute \\
\hline & & Datenverarbeitungsfachleute \\
\hline & & Bürofachkräfte \\
\hline & & Chemielaborwerker \\
\hline kaufmännische Berufe & Verwaltungs-, $\quad$ Organisations- und & Landmaschineninstandsetzer \\
\hline & & Flugzeugmechaniker \\
\hline & & Raumausstatter \\
\hline $\begin{array}{l}\text { vorwiegend technische } \\
\text { Berufe }\end{array}$ & produktionsnahe Dienstleistungsberufe & Polsterer, Matratzenhersteller \\
\hline & & Maschinenwärter, Maschinistenhelfer \\
\hline & & Bautechniker \\
\hline & & Vermessungstechniker \\
\hline & & $\begin{array}{l}\text { Biologisch-technische Sonderfach- } \\
\text { kräfte }\end{array}$ \\
\hline & & $\begin{array}{l}\text { Physikalisch- und mathematisch- } \\
\text { technische Sonderfachkräfte }\end{array}$ \\
\hline & & Chemielaboranten \\
\hline & & Photolaboranten \\
\hline & & Technische Zeichner \\
\hline & & $\begin{array}{l}\text { Textilreiniger, Färber und Chemisch- } \\
\text { reiniger }\end{array}$ \\
\hline & & Floristen \\
\hline & & KFZ-Instandsetzer \\
\hline & & Zahntechniker \\
\hline
\end{tabular}




\begin{tabular}{|c|c|c|}
\hline & & Augenoptiker \\
\hline \multirow[t]{25}{*}{$\begin{array}{l}\text { ( z. T. einfache) Dienst- } \\
\text { leistungsberufe }\end{array}$} & \multirow[t]{25}{*}{$\begin{array}{l}\text { haushalts-/ personenbezogene Dienst- } \\
\text { leistungsberufe }\end{array}$} & Funk-, Tongerätemechaniker \\
\hline & & Köche \\
\hline & & Drogisten \\
\hline & & Apothekenhelferinnen \\
\hline & & $\begin{array}{l}\text { Krankenversicherungskaufleute } \\
\text { (nicht Sozialversicherung) }\end{array}$ \\
\hline & & Fremdenverkehrsfachleute \\
\hline & & Sprechstundenhelfer \\
\hline & & Friseure \\
\hline & & Friseure \\
\hline & & $\begin{array}{l}\text { Gastwirte, Hoteliers, Gaststättenkauf- } \\
\text { leute }\end{array}$ \\
\hline & & Kellner, Stewards \\
\hline & & Floristen \\
\hline & & KFZ-Instandsetzer \\
\hline & & Zahntechniker \\
\hline & & Augenoptiker \\
\hline & & Funk-, Tongerätemechaniker \\
\hline & & Köche \\
\hline & & Drogisten \\
\hline & & Apothekenhelferinnen \\
\hline & & $\begin{array}{l}\text { Krankenversicherungskaufleute } \\
\text { (nicht Sozialversicherung) }\end{array}$ \\
\hline & & Fremdenverkehrsfachleute \\
\hline & & Sprechstundenhelfer \\
\hline & & Friseure \\
\hline & & $\begin{array}{l}\text { Gastwirte, Hoteliers, Gaststättenkauf- } \\
\text { leute }\end{array}$ \\
\hline & & Kellner, Stewards \\
\hline
\end{tabular}

Quelle: in Anlehnung an Parmentier et al. (1993); Erlinghagen (2003) 


\begin{tabular}{|c|c|c|c|c|c|c|c|c|c|c|c|c|}
\hline $\begin{array}{l}\text { Jahr } \\
\text { Kennzahlen }\end{array}$ & 1992 & 1993 & 1994 & 1995 & 1996 & 1997 & 1998 & 1999 & 2000 & 2001 & 2002 & 2003 \\
\hline $\begin{array}{l}\text { Erwerbslosenquote (ILO) } \\
\text { Gesamtdeutschland* }\end{array}$ & 6,9 & 8,1 & 8,0 & 8,2 & 9,0 & 9,5 & 8,7 & 7,8 & 7,4 & 7,9 & 8,9 & 9,4 \\
\hline $\begin{array}{l}\text { Erwerbslosenquote (Al- } \\
\text { tersklasse unter } 25)^{* *}\end{array}$ & 8,3 & 9,7 & 9,0 & 9,1 & 9,8 & 9,7 & 9,0 & 7,7 & 7,3 & 8,3 & 9,6 & 10,0 \\
\hline $\begin{array}{l}\text { Ausbildungsstellenange- } \\
\text { bot }\end{array}$ & - & - & - & 616.988 & 609.290 & 613.381 & 636.175 & 654.454 & 647.383 & 638.387 & 590.328 & 572.452 \\
\hline $\begin{array}{l}\text { Ausbildungsstellennach- } \\
\text { frage }\end{array}$ & - & - & - & 597.736 & 612.800 & 634.938 & 648.446 & 660.380 & 645.335 & 634.314 & 595.706 & 592.627 \\
\hline $\begin{array}{l}\text { Angebot-Nachfrage- } \\
\text { Relation }\end{array}$ & - & - & - & 103,2 & 99,4 & 96,6 & 98,1 & 99,1 & 100,3 & 100,6 & 99,1 & 96,6 \\
\hline
\end{tabular}

* Angaben für Dezember des jeweiligen Jahres; Quelle:

http://www.bundesbank.de/statistik/statistik_zeitreihen.php?lang=de\&open=ewu\&func=row\&tr=YSD329, Angaben in \%

** Angaben für Dezember des jeweiligen Jahres; Quelle: httsps: www.genesis.destatis.de/genesis/online/dWerteabruf_Page;jsessionid=DA12D4192E22B09A5D89DFBDE2F830A6.tc21, Angaben in \%

1) Quelle: Berufsbildungsberichte (BMBF)Archiv bis 1995, Angaben in TSD 
Tabelle 14: Häufigkeitsverteilung der Ausbildungsabsolventen an der zweiten Schwelle (1975 und 2003 in \%)

\begin{tabular}{|l|c|c|c|}
\hline Abschlusskohorte & $\begin{array}{c}\text { Verbleib im Ausbil- } \\
\text { dungsbetrieb }\end{array}$ & $\begin{array}{c}\text { Beschäftigung nach } \\
\text { Wechsel }\end{array}$ & Arbeitslosigkeit \\
\hline $\mathbf{1 9 7 5 - 1 9 8 0}$ & 70,9 & 20,6 & 8,5 \\
\hline $\mathbf{1 9 8 1 - 1 9 8 6}$ & 62,8 & 16,1 & 21,0 \\
\hline $\mathbf{1 9 8 7 - 1 9 9 1}$ & 58,8 & 24,5 & 16,7 \\
\hline $\mathbf{1 9 9 2 - 1 9 9 5}$ & 58,3 & 19,9 & 21,8 \\
\hline $\mathbf{1 9 9 6 - 1 9 9 9}$ & 56,7 & 18,3 & 25,0 \\
\hline $\mathbf{2 0 0 0 - 2 0 0 3}$ & 57,7 & 17,9 & 24,4 \\
\hline
\end{tabular}

Quelle: IABS 1975-2004, eigene Berechnungen

Tabelle 15: Eintritt in den Arbeitsmarkt nach der dualen Berufsausbildung für die Abschlusskohorten 1975-1980 - multinomiales Logitmodell

\begin{tabular}{|c|c|c|c|c|}
\hline \multirow[b]{2}{*}{$\begin{array}{l}\text { (Referenz: Verbleib im Ausbil- } \\
\text { dungsbetrieb') }\end{array}$} & \multicolumn{2}{|c|}{$\begin{array}{c}\text { Modell 1: } \\
\text { ohne Interaktionseffekte }\end{array}$} & \multicolumn{2}{|c|}{$\begin{array}{c}\text { Modell 2: } \\
\text { mit Interaktionseffekten }\end{array}$} \\
\hline & $\begin{array}{l}\text { Beschäftigung } \\
\text { nach Wechsel }\end{array}$ & Arbeitslosigkeit & $\begin{array}{l}\text { Beschäftigung } \\
\text { nach Wechsel }\end{array}$ & Arbeitslosigkeit \\
\hline Ausbildungsvergütungen (log) & $\begin{array}{l}-0,230^{* * *} \\
(0,041)\end{array}$ & $\begin{array}{l}-0,121^{*} \\
(0,071)\end{array}$ & $\begin{array}{l}-0,225^{* * *} \\
(0,041)\end{array}$ & $\begin{array}{l}-0,138 * * \\
(0,071)\end{array}$ \\
\hline $\begin{array}{l}\text { durchschnittliche betriebliche } \\
\text { Lohnsumme (log) }\end{array}$ & $\begin{array}{l}0,082^{* * *} \\
(0,018)\end{array}$ & $\begin{array}{l}0,172^{* * *} \\
(0,028)\end{array}$ & $\begin{array}{l}0,083^{* * *} \\
(0,018)\end{array}$ & $\begin{array}{l}0,169^{* * *} \\
(0,029)\end{array}$ \\
\hline Ausbildungsdauer (log) & $\begin{array}{l}-0,638^{* * *} \\
(0,040)\end{array}$ & $\begin{array}{l}0,222^{* * *} \\
(0,073) \\
\end{array}$ & $\begin{array}{l}-0,640^{* * *} \\
(0,041)\end{array}$ & $\begin{array}{l}0,239^{* * *} \\
(0,074)\end{array}$ \\
\hline \multicolumn{5}{|l|}{$\begin{array}{l}\text { Betriebsgröße } \\
\text { (Referenz: 1-9 Mitarbeiter) }\end{array}$} \\
\hline 10-49 Mitarbeiter & $\begin{array}{l}-0,356^{* * *} \\
(0,056)\end{array}$ & $\begin{array}{l}-0,398 * * * \\
(0,087)\end{array}$ & $\begin{array}{l}-0,357 * * * \\
(0,056)\end{array}$ & $\begin{array}{l}-0,388^{* * * *} \\
(0,087)\end{array}$ \\
\hline 50-499 Mitarbeiter & $\begin{array}{l}-0,509^{* * *} \\
(0,081)\end{array}$ & $\begin{array}{l}-0,571^{* * *} \\
(0,126)\end{array}$ & $\begin{array}{l}-0,504 * * * \\
(0,081)\end{array}$ & $\begin{array}{l}-0,565^{* * *} \\
(0,127) \\
\end{array}$ \\
\hline$>500$ Mitarbeiter & $\begin{array}{l}-0,730^{* * *} \\
(0,125)\end{array}$ & $\begin{array}{l}-0,872^{* * *} \\
(0,192)\end{array}$ & $\begin{array}{l}-0,722^{* * *} \\
(0,125)\end{array}$ & $\begin{array}{l}-0,893^{* * *} \\
(0,193)\end{array}$ \\
\hline \multicolumn{5}{|l|}{$\begin{array}{l}\text { Ausbildungsberufe (Referenz: } \\
\text { Land-/Forstwirtschaftsberufe) }\end{array}$} \\
\hline Chemieberufe & $\begin{array}{l}0,274^{* *} \\
(0,110) \\
\end{array}$ & $\begin{array}{l}-0,022 \\
(0,183) \\
\end{array}$ & $\begin{array}{l}0,644^{* *} \\
(0,289)\end{array}$ & $\begin{array}{l}-0,706 \\
(0,509) \\
\end{array}$ \\
\hline Metallberufe & $\begin{array}{l}-0,582^{* * *} \\
(0,085)\end{array}$ & $\begin{array}{l}-0,617^{* * *} \\
(0,133)\end{array}$ & $\begin{array}{l}-0,922 * * * \\
(0,232)\end{array}$ & $\begin{array}{l}-0,973^{* * *} \\
(0,369)\end{array}$ \\
\hline Textilberufe & $\begin{array}{l}-0,516^{* * *} \\
(0,061)\end{array}$ & $\begin{array}{l}-0,652^{* * *} \\
(0,095)\end{array}$ & $\begin{array}{l}-0,471^{* * * *} \\
(0,175)\end{array}$ & $\begin{array}{l}-0,457^{*} \\
(0,274) \\
\end{array}$ \\
\hline $\begin{array}{l}\text { Berufe im Nahrungsmittelbe- } \\
\text { reich }^{113}\end{array}$ & ja & ja & ja & ja \\
\hline
\end{tabular}

${ }^{113}$ Im Nahrungsmittelbereich sind nur wenige Fälle enthalten. 


\begin{tabular}{|c|c|c|c|c|}
\hline \multirow[b]{2}{*}{$\begin{array}{l}\text { (Referenz: Verbleib im Ausbil- } \\
\text { dungsbetrieb') }\end{array}$} & \multicolumn{2}{|c|}{$\begin{array}{c}\text { Modell 1: } \\
\text { ohne Interaktionseffekte } \\
\end{array}$} & \multicolumn{2}{|c|}{$\begin{array}{c}\text { Modell 2: } \\
\text { mit Interaktionseffekten }\end{array}$} \\
\hline & $\begin{array}{l}\text { Beschäftigung } \\
\text { nach Wechsel }\end{array}$ & Arbeitslosigkeit & $\begin{array}{l}\text { Beschäftigung } \\
\text { nach Wechsel }\end{array}$ & Arbeitslosigkeit \\
\hline Elektroberufe & $\begin{array}{l}0,261^{* * *} \\
(0,089)\end{array}$ & $\begin{array}{l}-0,817^{* * *} \\
(0,186)\end{array}$ & $\begin{array}{c}0,432 \\
(0,284) \\
\end{array}$ & $\begin{array}{l}-0,456 \\
(0,572) \\
\end{array}$ \\
\hline Bauberufe & $\begin{array}{l}-0,246^{* * *} \\
(0,068) \\
\end{array}$ & $\begin{array}{l}-0,097 \\
(0,101) \\
\end{array}$ & $\begin{array}{l}-0,371^{*} \\
(0,192) \\
\end{array}$ & $\begin{array}{l}-0,381 \\
(0,295) \\
\end{array}$ \\
\hline $\begin{array}{l}\text { Infrastruktur-/Transport- } \\
\text { dienstleistungsberufe }\end{array}$ & $\begin{array}{l}-0,657^{* * *} \\
(0,076) \\
\end{array}$ & $\begin{array}{l}-1,199^{* * *} \\
(0,118) \\
\end{array}$ & $\begin{array}{l}-1,145^{* * *} \\
(0,217)\end{array}$ & $\begin{array}{l}-1,984^{* * *} \\
(0,332)\end{array}$ \\
\hline Finanzdienstleistungsberufe & $\begin{array}{l}-0,024 \\
(0,148)\end{array}$ & $\begin{array}{c}0,011 \\
(0,306)\end{array}$ & $\begin{array}{l}-0,024 \\
(0,405)\end{array}$ & $\begin{array}{c}0,433 \\
(0,865)\end{array}$ \\
\hline Verwaltungsberufe & $\begin{array}{l}-0,480^{* * *} \\
(0,071)\end{array}$ & $\begin{array}{l}-0,147 \\
(0,119)\end{array}$ & $\begin{array}{l}-0,266 \\
(0,176)\end{array}$ & $\begin{array}{l}-0,216 \\
(0,312)\end{array}$ \\
\hline $\begin{array}{l}\text { produktionsnahe Dienstleistungs- } \\
\text { berufe }\end{array}$ & $\begin{array}{l}-0,620^{* * *} \\
(0,076) \\
\end{array}$ & $\begin{array}{l}-0,113 \\
(0,119) \\
\end{array}$ & $\begin{array}{l}-0,804^{* * *} \\
(0,201) \\
\end{array}$ & $\begin{array}{l}-0,600^{*} \\
(0,335)\end{array}$ \\
\hline $\begin{array}{l}\text { haushalts-/personenbezogene } \\
\text { Dienstleistungsberufe }\end{array}$ & $\begin{array}{l}-0,361^{* * *} \\
(0,091)\end{array}$ & $\begin{array}{l}-0,287^{* *} \\
(0,142) \\
\end{array}$ & $\begin{array}{l}-0,209 \\
(0,245) \\
\end{array}$ & $\begin{array}{l}-0,188 \\
(0,406) \\
\end{array}$ \\
\hline \multicolumn{5}{|l|}{ Qualifikation } \\
\hline $\begin{array}{l}\text { Durchschnitt zum Wirtschafts- } \\
\text { zweig }\end{array}$ & $\begin{array}{l}0,406^{* * *} \\
(0,038)\end{array}$ & $\begin{array}{l}0,275^{* * *} \\
(0,061)\end{array}$ & $\begin{array}{l}0,409^{* * *} \\
(0,038)\end{array}$ & $\begin{array}{l}0,275^{* * *} \\
(0,061)\end{array}$ \\
\hline \multicolumn{5}{|l|}{$\begin{array}{l}\text { Bildung } \\
\text { (Referenz: Hauptschulabschluss) }\end{array}$} \\
\hline Realschulabschluss & $\begin{array}{l}0,810^{* * *} \\
(0,044) \\
\end{array}$ & $\begin{array}{l}1,037^{* * *} \\
(0,067)\end{array}$ & $\begin{array}{l}0,803^{* * *} \\
(0,085)\end{array}$ & $\begin{array}{l}0,871^{* * *} \\
(0,129)\end{array}$ \\
\hline Abitur & $\begin{array}{l}0,560^{* * *} \\
(0,099) \\
\end{array}$ & $\begin{array}{l}0,787^{* * *} \\
(0,195) \\
\end{array}$ & $\begin{array}{l}0,611^{* * *} \\
(0,189)\end{array}$ & $\begin{array}{c}0,425 \\
(0,312) \\
\end{array}$ \\
\hline Regionalvariable & $\mathrm{ja}$ & $\mathrm{ja}$ & $\mathrm{ja}$ & ja \\
\hline Staatsangehörigkeit & $\begin{array}{l}-0,384 \\
(0,040) \\
\end{array}$ & $\begin{array}{l}-6,711 \\
(0,380) \\
\end{array}$ & $\begin{array}{l}-0,384^{* * *} \\
(0,040) \\
\end{array}$ & $\begin{array}{l}-6,722^{* * *} \\
(0,380) \\
\end{array}$ \\
\hline \multicolumn{5}{|l|}{$\begin{array}{l}\text { Interaktionseffekte } \\
\text { (Ausbildungsbereiche * Schulbil- } \\
\text { dung) }\end{array}$} \\
\hline Land-/Forstwirtschaftsberufe & & & $\begin{array}{l}-0,160 \\
(0,099)\end{array}$ & $\begin{array}{l}0,459^{* * *} \\
(0,142)\end{array}$ \\
\hline Chemieberufe & & & $\begin{array}{l}-0,300 \\
(0,211)\end{array}$ & $\begin{array}{c}0,548 \\
(0,351)\end{array}$ \\
\hline Metallberufe & & & $\begin{array}{c}0,253 \\
(0,169) \\
\end{array}$ & $\begin{array}{c}0,331 \\
(0,267) \\
\end{array}$ \\
\hline Textilberufe & & & $\begin{array}{l}-0,057 \\
(0,132)\end{array}$ & $\begin{array}{l}-0,108 \\
(0,205)\end{array}$ \\
\hline Berufe im Nahrungsmittelbereich & & & ja & ja \\
\hline Elektroberufe & & & $\begin{array}{l}-0,171 \\
(0,238)\end{array}$ & $\begin{array}{l}-0,286 \\
(0,463)\end{array}$ \\
\hline Bauberufe & & & $\begin{array}{c}0,079 \\
(0,136)\end{array}$ & $\begin{array}{c}0,265 \\
(0,209)\end{array}$ \\
\hline $\begin{array}{l}\text { Infrastruktur- und Transportdienst- } \\
\text { leistungsberufe }\end{array}$ & & & $\begin{array}{l}0,398^{* *} \\
(0,167) \\
\end{array}$ & $\begin{array}{l}0,689^{* * *} \\
(0,248)\end{array}$ \\
\hline Finanzdienstleistungsberufe & & & $\begin{array}{l}-0,017 \\
(0,241)\end{array}$ & $\begin{array}{l}-0,187 \\
(0,518)\end{array}$ \\
\hline Verwaltungsberufe & & & $\begin{array}{l}-0,151 \\
(0,115) \\
\end{array}$ & $\begin{array}{c}0,113 \\
(0,204) \\
\end{array}$ \\
\hline
\end{tabular}




\begin{tabular}{|c|c|c|c|}
\hline \multirow[b]{2}{*}{$\begin{array}{l}\text { (Referenz: Verbleib im Ausbil- } \\
\text { dungsbetrieb') }\end{array}$} & $\begin{array}{c}\text { Modell 1: } \\
\text { ohne Interaktionseffekte } \\
\end{array}$ & \multicolumn{2}{|c|}{$\begin{array}{c}\text { Modell 2: } \\
\text { mit Interaktionseffekten }\end{array}$} \\
\hline & $\begin{array}{l}\text { Beschäftigung Arbeitslosigkeit } \\
\text { nach Wechsel }\end{array}$ & $\begin{array}{l}\text { Beschäftigung } \\
\text { nach Wechsel }\end{array}$ & Arbeitslosigkeit \\
\hline $\begin{array}{l}\text { produktionsnahe Dienstleistungs- } \\
\text { berufe }\end{array}$ & & $\begin{array}{c}0,098 \\
(0,126)\end{array}$ & $\begin{array}{c}0,380^{*} \\
(0,209)\end{array}$ \\
\hline $\begin{array}{l}\text { haushalts-/personenbezogene } \\
\text { Dienstleistungsberufe }\end{array}$ & & $\begin{array}{l}-0,128 \\
(0,169)\end{array}$ & $\begin{array}{l}-0,016 \\
(0,281)\end{array}$ \\
\hline Fälle & 20718 & \multicolumn{2}{|c|}{20718} \\
\hline$\chi^{2}(87)$ & $5931,16^{* * *}$ & & \\
\hline$\chi^{2}(123):$ & & \multicolumn{2}{|c|}{$6001,02^{* * *}$} \\
\hline pseudd ${ }^{2}$ & 0,1839 & \multicolumn{2}{|c|}{0,1861} \\
\hline
\end{tabular}

Quelle: IABS 1975-2004, eigene Berechnungen

*** 1\%-Signifikanzniveau; ** 5\%-Signifikanzniveau; * 10\%-Signifikanzniveau Werte in Klammern bezeichnen die Standardfehler

\section{Tabelle 16: Eintritt in den Arbeitsmarkt nach der dualen Berufsausbildung für die Abschlusskohorten 1981-1986 - multinomiales Logit- modell}

\begin{tabular}{|c|c|c|c|c|}
\hline & \multicolumn{2}{|c|}{$\begin{array}{c}\text { Modell 1: } \\
\text { ohne Interaktionseffekte }\end{array}$} & \multicolumn{2}{|c|}{$\begin{array}{c}\text { Modell 2: } \\
\text { mit Interaktionseffekten } \\
\end{array}$} \\
\hline $\begin{array}{l}\text { (Referenz: Verbleib im Ausbil- } \\
\text { dungsbetrieb) }\end{array}$ & $\begin{array}{l}\text { Beschäftigung } \\
\text { nach Wechsel }\end{array}$ & Arbeitslosigkeit & $\begin{array}{l}\text { Beschäftigung } \\
\text { nach Wechsel }\end{array}$ & Arbeitslosigkeit \\
\hline Ausbildungsvergütungen (log) & $\begin{array}{l}-0,290 * * * \\
(0,055)\end{array}$ & $\begin{array}{l}-0,496 * * * \\
(0,053)\end{array}$ & $\begin{array}{l}-0,287 * * * \\
(0,055)\end{array}$ & $\begin{array}{l}-0,494^{* * *} \\
(0,053)\end{array}$ \\
\hline $\begin{array}{l}\text { durchschnittliche betriebliche } \\
\text { Lohnsumme }(\log )\end{array}$ & $\begin{array}{l}0,107 * * * \\
(0,019)\end{array}$ & $\begin{array}{c}0,184 * * * \\
(0,018)\end{array}$ & $\begin{array}{c}0,105 * * * \\
(0,019)\end{array}$ & $\begin{array}{l}0,181^{* * *} \\
(0,018)\end{array}$ \\
\hline Ausbildungsdauer (log) & $\begin{array}{l}0,982^{* * * *} \\
(0,069)\end{array}$ & $\begin{array}{l}1,426^{* * *} \\
(0,069)\end{array}$ & $\begin{array}{l}0,978 * * * \\
(0,069) \\
\end{array}$ & $\begin{array}{l}1,419^{* * *} \\
(0,069)\end{array}$ \\
\hline \multicolumn{5}{|l|}{$\begin{array}{l}\text { Betriebsgröße } \\
\text { (Referenz: 1-9 Mitarbeiter) }\end{array}$} \\
\hline 10-49 Mitarbeiter & $\begin{array}{l}-0,268^{* * *} \\
(0,056)\end{array}$ & $\begin{array}{l}-0,463^{* * *} \\
(0,052)\end{array}$ & $\begin{array}{l}-0,264 * * * \\
(0,056)\end{array}$ & $\begin{array}{l}-0,457^{* * *} \\
(0,052)\end{array}$ \\
\hline 50-499 Mitarbeiter & $\begin{array}{l}-0,457^{* * *} \\
(0,081)\end{array}$ & $\begin{array}{l}-0,965^{* * *} \\
(0,078)\end{array}$ & $\begin{array}{l}-0,447^{* * *} \\
(0,081)\end{array}$ & $\begin{array}{l}-0,956^{* * *} \\
(0,078)\end{array}$ \\
\hline$>500$ Mitarbeiter & $\begin{array}{l}-0,827^{* * *} \\
(0,126)\end{array}$ & $\begin{array}{l}-1,990^{* * *} \\
(0,126)\end{array}$ & $\begin{array}{l}-0,828^{* * *} \\
(0,126)\end{array}$ & $\begin{array}{l}-1,993 * * * \\
(0,127)\end{array}$ \\
\hline \multicolumn{5}{|l|}{$\begin{array}{l}\text { Ausbildungsberufe (Referenz: } \\
\text { Land-/Forstwirtschaftsberufe) }\end{array}$} \\
\hline Chemieberufe & $\begin{array}{l}0,726 * * * \\
(0,096)\end{array}$ & $\begin{array}{c}0,149 \\
(0,110)\end{array}$ & $\begin{array}{l}0,877^{* * *} \\
(0,238)\end{array}$ & $\begin{array}{l}-0,694 * * \\
(0,288)\end{array}$ \\
\hline Metallberufe & $\begin{array}{l}-0,604^{* * *} \\
(0,080)\end{array}$ & $\begin{array}{l}-0,389^{* * *} \\
(0,072)\end{array}$ & $\begin{array}{l}-0,427^{* *} \\
(0,212)\end{array}$ & $\begin{array}{l}-0,383^{*} \\
(0,201)\end{array}$ \\
\hline \multicolumn{5}{|c|}{ Fortsetzung Tabelle 17 auf der nächsten Seite } \\
\hline
\end{tabular}




\begin{tabular}{|c|c|c|c|c|}
\hline \multirow[b]{2}{*}{$\begin{array}{l}\text { (Referenz: Verbleib im Ausbil- } \\
\text { dungsbetrieb) }\end{array}$} & \multicolumn{2}{|c|}{$\begin{array}{c}\text { Modell 1: } \\
\text { ohne Interaktionseffekte }\end{array}$} & \multicolumn{2}{|c|}{$\begin{array}{c}\text { Modell 2: } \\
\text { mit Interaktionseffekten }\end{array}$} \\
\hline & $\begin{array}{l}\text { Beschäftigung } \\
\text { nach Wechsel }\end{array}$ & Arbeitslosigkeit & $\begin{array}{l}\text { Beschäftigung } \\
\text { nach Wechsel }\end{array}$ & Arbeitslosigkeit \\
\hline Textilberufe & $\begin{array}{l}-0,824^{* * * *} \\
(0,062)\end{array}$ & $\begin{array}{l}-0,661^{* * *} \\
(0,056) \\
\end{array}$ & $\begin{array}{l}-0,947^{* * *} \\
(0,174) \\
\end{array}$ & $\begin{array}{l}-0,805^{* * *} \\
(0,163) \\
\end{array}$ \\
\hline $\begin{array}{l}\text { Berufe im Nahrungsmittelbe- } \\
\text { reich }{ }^{14}\end{array}$ & ja & ja & ja & ja \\
\hline Elektroberufe & $\begin{array}{l}0,290^{* * *} \\
(0,088)\end{array}$ & $\begin{array}{l}-0,200^{* *} \\
(0,092)\end{array}$ & $\begin{array}{c}0,395 \\
(0,253)\end{array}$ & $\begin{array}{l}-0,400 \\
(0,268)\end{array}$ \\
\hline Bauberufe & $\begin{array}{l}-0,718^{* * *} \\
(0,070)\end{array}$ & $\begin{array}{l}-0,772^{* * *} \\
(0,066)\end{array}$ & $\begin{array}{l}-0,870^{* * *} \\
(0,211)\end{array}$ & $\begin{array}{l}-0,893^{* * *} \\
(0,205)\end{array}$ \\
\hline $\begin{array}{l}\text { Infrastruktur-/Transport- } \\
\text { dienstleistungsberufe }\end{array}$ & $\begin{array}{l}-0,534^{* * *} \\
(0,073)\end{array}$ & $\begin{array}{l}-0,231^{* * *} \\
(0,066)\end{array}$ & $\begin{array}{l}-0,600^{* * *} \\
(0,193)\end{array}$ & $\begin{array}{l}-0,565^{* * *} \\
(0,178)\end{array}$ \\
\hline Finanzdienstleistungsberufe & $\begin{array}{l}-0,075 \\
(0,139)\end{array}$ & $\begin{array}{l}-0,605^{* * *} \\
(0,161)\end{array}$ & $\begin{array}{l}0,676^{*} \\
(0,362)\end{array}$ & $\begin{array}{l}-0,817^{*} \\
(0,489)\end{array}$ \\
\hline Verwaltungsberufe & $\begin{array}{l}-0,368^{* * *} \\
(0,070)\end{array}$ & $\begin{array}{l}-0,620^{* * *} \\
(0,073)\end{array}$ & $\begin{array}{l}-0,037 \\
(0,174)\end{array}$ & $\begin{array}{l}-0,302 \\
(0,192)\end{array}$ \\
\hline $\begin{array}{l}\text { produktionsnahe Dienstleistungs- } \\
\text { berufe }\end{array}$ & $\begin{array}{l}-0,658^{* * *} \\
(0,081)\end{array}$ & $\begin{array}{l}-0,745^{* * *} \\
(0,083)\end{array}$ & $\begin{array}{l}-0,971^{* * *} \\
(0,226)\end{array}$ & $\begin{array}{l}-1,516^{* * *} \\
(0,250)\end{array}$ \\
\hline $\begin{array}{l}\text { haushalts-/personenbezogene } \\
\text { Dienstleistungsberufe }\end{array}$ & $\begin{array}{l}-0,825^{* * *} \\
(0,100)\end{array}$ & $\begin{array}{l}-0,583^{* * *} \\
(0,089)\end{array}$ & $\begin{array}{l}-0,609^{* *} \\
(0,271)\end{array}$ & $\begin{array}{l}-0,948^{* * *} \\
(0,260)\end{array}$ \\
\hline \multicolumn{5}{|l|}{ Qualifikation } \\
\hline $\begin{array}{l}\text { Durchschnitt zum Wirtschafts- } \\
\text { zweig }\end{array}$ & $\begin{array}{l}0,404^{* * *} \\
(0,038)\end{array}$ & $\begin{array}{l}0,355^{* * *} \\
(0,036)\end{array}$ & $\begin{array}{l}0,410^{* * *} \\
(0,038)\end{array}$ & $\begin{array}{l}0,361^{* * *} \\
(0,036)\end{array}$ \\
\hline \multicolumn{5}{|l|}{$\begin{array}{l}\text { Bildung } \\
\text { (Referenz: Hauptschulabschluss) }\end{array}$} \\
\hline Realschulabschluss & $\begin{array}{l}0,794^{* * *} \\
(0,041)\end{array}$ & $\begin{array}{l}0,958^{* * *} \\
(0,038)\end{array}$ & $\begin{array}{l}0,790^{* * *} \\
(0,078)\end{array}$ & $\begin{array}{l}0,835^{* * *} \\
(0,076)\end{array}$ \\
\hline Abitur & $\begin{array}{l}1,118^{* * *} \\
(0,073)\end{array}$ & $\begin{array}{l}0,290^{* * *} \\
(0,097)\end{array}$ & $\begin{array}{l}1,198^{* * *} \\
(0,163)\end{array}$ & $\begin{array}{c}0,043 \\
(0,177) \\
\end{array}$ \\
\hline Regionalvariable & $\mathrm{ja}$ & $\mathrm{ja}$ & $\mathrm{ja}$ & ja \\
\hline Staatsangehörigkeit & $\begin{array}{l}-0,733^{* * *} \\
(0,069)\end{array}$ & $\begin{array}{l}-1,982^{* * *} \\
(0,053)\end{array}$ & $\begin{array}{l}-0,734^{* * *} \\
(0,069)\end{array}$ & $\begin{array}{l}-1,981^{* * *} \\
(0,053)\end{array}$ \\
\hline \multicolumn{5}{|l|}{$\begin{array}{l}\text { Interaktionseffekte } \\
\text { (Ausbildungsbereiche * Schulbil- } \\
\text { dung) }\end{array}$} \\
\hline Land-/Forstwirtschaftsberufe & & & $\begin{array}{c}0,107 \\
(0,083)\end{array}$ & $\begin{array}{l}0,190^{* *} \\
(0,089)\end{array}$ \\
\hline Chemieberufe & & & $\begin{array}{l}-0,075 \\
(0,158) \\
\end{array}$ & $\begin{array}{l}0,581^{* * *} \\
(0,181) \\
\end{array}$ \\
\hline Metallberufe & & & $\begin{array}{l}-0,110 \\
(0,138)\end{array}$ & $\begin{array}{c}0,007 \\
(0,133)\end{array}$ \\
\hline Textilberufe & & & $\begin{array}{c}0,103 \\
(0,111) \\
\end{array}$ & $\begin{array}{c}0,121 \\
(0,106)\end{array}$ \\
\hline Berufe im Nahrungsmittelbereich & & & $\mathrm{ja}$ & $\mathrm{ja}$ \\
\hline Elektroberufe & & & $\begin{array}{l}-0,062 \\
(0,187) \\
\end{array}$ & $\begin{array}{c}0,154 \\
(0,195) \\
\end{array}$ \\
\hline Bauberufe & & & $\begin{array}{c}0,111 \\
(0,126)\end{array}$ & $\begin{array}{c}0,107 \\
(0,125)\end{array}$ \\
\hline
\end{tabular}

${ }^{114}$ Im Nahrungsmittelbereich sind nur wenige Fälle enthalten. 


\begin{tabular}{|c|c|c|c|}
\hline \multirow[b]{2}{*}{$\begin{array}{l}\text { (Referenz: Verbleib im Ausbil- } \\
\text { dungsbetrieb) }\end{array}$} & $\begin{array}{c}\text { Modell 1: } \\
\text { ohne Interaktionseffekte } \\
\end{array}$ & \multicolumn{2}{|c|}{$\begin{array}{c}\text { Modell 2: } \\
\text { mit Interaktionseffekten }\end{array}$} \\
\hline & $\begin{array}{l}\text { Beschäftigung Arbeitslosigkeit } \\
\text { nach Wechsel }\end{array}$ & $\begin{array}{l}\text { Beschäftigung } \\
\text { nach Wechsel }\end{array}$ & Arbeitslosigkeit \\
\hline $\begin{array}{l}\text { Infrastruktur- und Transport- } \\
\text { dienstleistungsberufe }\end{array}$ & & $\begin{array}{c}0,072 \\
(0,130) \\
\end{array}$ & $\begin{array}{c}0,257 \\
(0,121) \\
\end{array}$ \\
\hline Finanzdienstleistungsberufe & & $\begin{array}{l}-0,448^{* *} \\
(0,214)\end{array}$ & $\begin{array}{l}0,141^{* *} \\
(0,276)\end{array}$ \\
\hline Verwaltungsberufe & & $\begin{array}{l}-0,177^{*} \\
(0,100)\end{array}$ & $\begin{array}{l}-0,157 \\
(0,116)\end{array}$ \\
\hline $\begin{array}{l}\text { produktionsnahe Dienstleistungs- } \\
\text { berufe }\end{array}$ & & $\begin{array}{c}0,171 \\
(0,120)\end{array}$ & $\begin{array}{l}0,460^{* * *} \\
(0,137)\end{array}$ \\
\hline $\begin{array}{l}\text { haushalts-/personenbezogene } \\
\text { Dienstleistungsberufe }\end{array}$ & & $\begin{array}{l}-0,115 \\
(0,163)\end{array}$ & $\begin{array}{c}0,257 \\
(0,158)\end{array}$ \\
\hline Fälle & 26912 & \multicolumn{2}{|c|}{26912} \\
\hline$\chi^{2}(87)$ & $6012,65^{* * *}$ & & \\
\hline$\chi^{2}(123)$ & & \multicolumn{2}{|c|}{$6080,47^{* * *}$} \\
\hline pseudd ${ }^{2}$ & 0,1221 & \multicolumn{2}{|c|}{0,1235} \\
\hline
\end{tabular}

Quelle: IABS 1975-2004, eigene Berechnungen

*** 1\%-Signifikanzniveau; ** 5\%-Signifikanzniveau; * 10\%-Signifikanzniveau

Werte in Klammern bezeichnen die Standardfehler

\section{Tabelle 17: Eintritt in den Arbeitsmarkt nach der dualen Berufsausbildung für die Abschlusskohorten 1987-1991 - multinomiales Logit- modell}

\begin{tabular}{|c|c|c|c|c|}
\hline \multirow[b]{2}{*}{$\begin{array}{l}\text { (Referenz: Verbleib im Ausbil- } \\
\text { dungsbetrieb) }\end{array}$} & \multicolumn{2}{|c|}{$\begin{array}{c}\text { Modell 1: } \\
\text { ohne Interaktionseffekte }\end{array}$} & \multicolumn{2}{|c|}{$\begin{array}{c}\text { Modell 2: } \\
\text { mit Interaktionseffekten }\end{array}$} \\
\hline & $\begin{array}{l}\text { Beschäftigung } \\
\text { nach Wechsel }\end{array}$ & Arbeitslosigkeit & $\begin{array}{l}\text { Beschäftigung } \\
\text { nach Wechsel }\end{array}$ & Arbeitslosigkeit \\
\hline Ausbildungsvergütungen (log) & $\begin{array}{l}-0,449^{* * *} \\
(0,059)\end{array}$ & $\begin{array}{l}-1,112^{* * *} \\
(0,072)\end{array}$ & $\begin{array}{l}-0,441^{* * *} \\
(0,059) \\
\end{array}$ & $\begin{array}{l}-1,112 * * * \\
(0,072)\end{array}$ \\
\hline $\begin{array}{l}\text { durchschnittliche betriebliche } \\
\text { Lohnsumme (log) }\end{array}$ & $\begin{array}{l}0,131^{* * *} \\
(0,018)\end{array}$ & $\begin{array}{l}0,186^{* * *} \\
(0,021)\end{array}$ & $\begin{array}{l}0,133^{* * *} \\
(0,018)\end{array}$ & $\begin{array}{l}0,184^{* * *} \\
(0,022)\end{array}$ \\
\hline Ausbildungsdauer (log) & $\begin{array}{l}0,727 * * * \\
(0,072)\end{array}$ & $\begin{array}{l}0,994^{* * *} \\
(0,089)\end{array}$ & $\begin{array}{l}0,716^{* * *} \\
(0,072)\end{array}$ & $\begin{array}{l}0,982^{* * *} \\
(0,090)\end{array}$ \\
\hline \multicolumn{5}{|l|}{$\begin{array}{l}\text { Betriebsgröße } \\
\text { (Referenz: 1-9 Mitarbeiter) }\end{array}$} \\
\hline 10-49 Mitarbeiter & $\begin{array}{l}-0,502^{* * *} \\
(0,058)\end{array}$ & $\begin{array}{l}-0,643^{* *} \\
(0,068)\end{array}$ & $\begin{array}{l}-0,507^{* * *} \\
(0,059)\end{array}$ & $\begin{array}{l}-0,640^{* * *} \\
(0,068)\end{array}$ \\
\hline 50-499 Mitarbeiter & $\begin{array}{l}-0,856^{* * *} \\
(0,082)\end{array}$ & $\begin{array}{l}-1,042^{* *} \\
(0,097)\end{array}$ & $\begin{array}{l}-0,853^{* * *} \\
(0,083)\end{array}$ & $\begin{array}{l}-1,035 * * * \\
(0,097)\end{array}$ \\
\hline > 500 Mitarbeiter & $\begin{array}{l}-1,438 * * * \\
(0,127)\end{array}$ & $\begin{array}{l}-1,705^{* *} \\
(0,151)\end{array}$ & $\begin{array}{l}-1,426^{* * *} \\
(0,127)\end{array}$ & $\begin{array}{l}-1,700^{* * *} \\
(0,151) \\
\end{array}$ \\
\hline \multicolumn{5}{|l|}{$\begin{array}{l}\text { Ausbildungsberufe (Referenz: } \\
\text { Land-/Forstwirtschaftsberufe) }\end{array}$} \\
\hline Chemieberufe & $\begin{array}{l}0,381^{* * *} \\
(0,112)\end{array}$ & $\begin{array}{l}0,614^{* * *} \\
(0,126)\end{array}$ & $\begin{array}{c}0,403 \\
(0,301) \\
\end{array}$ & $\begin{array}{r}0,623^{*} \\
(0,363) \\
\end{array}$ \\
\hline
\end{tabular}




\begin{tabular}{|c|c|c|c|c|}
\hline \multirow[b]{2}{*}{$\begin{array}{l}\text { (Referenz: Verbleib im Ausbil- } \\
\text { dungsbetrieb) }\end{array}$} & \multicolumn{2}{|c|}{$\begin{array}{c}\text { Modell 1: } \\
\text { ohne Interaktionseffekte }\end{array}$} & \multicolumn{2}{|c|}{$\begin{array}{c}\text { Modell 2: } \\
\text { mit Interaktionseffekten }\end{array}$} \\
\hline & $\begin{array}{l}\text { Beschäftigung } \\
\text { nach Wechsel }\end{array}$ & Arbeitslosigkeit & $\begin{array}{l}\text { Beschäftigung } \\
\text { nach Wechsel }\end{array}$ & Arbeitslosigkeit \\
\hline Metallberufe & $\begin{array}{l}-0,410^{* * *} \\
(0,083)\end{array}$ & $\begin{array}{l}-0,251^{* * *} \\
(0,096)\end{array}$ & $\begin{array}{l}-0,264 \\
(0,234)\end{array}$ & $\begin{array}{l}-0,346 \\
(0,303)\end{array}$ \\
\hline Textilberufe & $\begin{array}{l}-0,661^{* * *} \\
(0,063)\end{array}$ & $\begin{array}{l}-0,658^{* * *} \\
(0,073)\end{array}$ & $\begin{array}{l}-0,576^{* * *} \\
(0,190)\end{array}$ & $\begin{array}{l}-0,750^{* * *} \\
(0,249)\end{array}$ \\
\hline $\begin{array}{l}\text { Berufe im Nahrungsmittelbe- } \\
\text { reich }^{115}\end{array}$ & ja & ja & ja & ja \\
\hline Elektroberufe & $\begin{array}{c}0,044 \\
(0,099) \\
\end{array}$ & $\begin{array}{l}-0,338^{* * *} \\
(0,127)\end{array}$ & $\begin{array}{c}0,286 \\
(0,276) \\
\end{array}$ & $\begin{array}{r}0,318 \\
(0,376) \\
\end{array}$ \\
\hline Bauberufe & $\begin{array}{l}-0,696^{* * *} \\
(0,073)\end{array}$ & $\begin{array}{l}-0,884^{* * *} \\
(0,086)\end{array}$ & $\begin{array}{l}-0,950^{* * *} \\
(0,245)\end{array}$ & $\begin{array}{l}-0,798^{* *} \\
(0,327)\end{array}$ \\
\hline $\begin{array}{l}\text { Infrastruktur-/Transport- } \\
\text { dienstleistungsberufe }\end{array}$ & $\begin{array}{l}-0,435^{* * *} \\
(0,082)\end{array}$ & $\begin{array}{c}0,004 \\
(0,091)\end{array}$ & $\begin{array}{l}-0,615^{* * *} \\
(0,236)\end{array}$ & $\begin{array}{l}-0,011 \\
(0,281)\end{array}$ \\
\hline Finanzdienstleistungsberufe & $\begin{array}{c}0,102 \\
(0,123)\end{array}$ & $\begin{array}{l}-0,477^{* * *} \\
(0,180)\end{array}$ & $\begin{array}{l}0,671^{* *} \\
(0,336)\end{array}$ & $\begin{array}{l}-0,185 \\
(0,576)\end{array}$ \\
\hline Verwaltungsberufe & $\begin{array}{l}-0,324^{* * *} \\
(0,074)\end{array}$ & $\begin{array}{l}-0,375^{* * *} \\
(0,092)\end{array}$ & $\begin{array}{c}0,062 \\
(0,197)\end{array}$ & $\begin{array}{l}-0,022 \\
(0,269)\end{array}$ \\
\hline $\begin{array}{l}\text { produktionsnahe Dienstleistungs- } \\
\text { berufe }\end{array}$ & $\begin{array}{l}-0,498^{* * *} \\
(0,082)\end{array}$ & $\begin{array}{l}-0,115 \\
(0,092)\end{array}$ & $\begin{array}{l}-0,640^{* *} \\
(0,252)\end{array}$ & $\begin{array}{c}0,097 \\
(0,296)\end{array}$ \\
\hline $\begin{array}{l}\text { haushalts-/personenbezogene } \\
\text { Dienstleistungsberufe }\end{array}$ & $\begin{array}{l}-0,564^{* * *} \\
(0,103)\end{array}$ & $\begin{array}{l}-0,378^{* * *} \\
(0,114)\end{array}$ & $\begin{array}{l}-0,033 \\
(0,285)\end{array}$ & $\begin{array}{l}-0,622^{*} \\
(0,361)\end{array}$ \\
\hline \multicolumn{5}{|l|}{ Qualifikation } \\
\hline $\begin{array}{l}\text { Durchschnitt zum Wirtschafts- } \\
\text { zweig }\end{array}$ & $\begin{array}{l}0,421^{* * *} \\
(0,039)\end{array}$ & $\begin{array}{l}0,574^{* * *} \\
(0,046)\end{array}$ & $\begin{array}{l}0,421^{* * *} \\
(0,040)\end{array}$ & $\begin{array}{l}0,574^{* * *} \\
(0,046)\end{array}$ \\
\hline \multicolumn{5}{|l|}{\begin{tabular}{|l|} 
Bildung \\
(Referenz: Hauptschulabschluss)
\end{tabular}} \\
\hline Realschulabschluss & $\begin{array}{l}0,760^{* * *} \\
(0,043) \\
\end{array}$ & $\begin{array}{l}1,091^{* * *} \\
(0,052) \\
\end{array}$ & $\begin{array}{l}0,826 * * * \\
(0,081) \\
\end{array}$ & $\begin{array}{l}1,120^{* * *} \\
(0,097)\end{array}$ \\
\hline Abitur & $\begin{array}{l}0,851^{* * *} \\
(0,072)\end{array}$ & $\begin{array}{l}0,543^{* * *} \\
(0,098)\end{array}$ & $\begin{array}{l}1,063^{* * *} \\
(0,166)\end{array}$ & $\begin{array}{l}0,692^{* * *} \\
(0,206)\end{array}$ \\
\hline Regionalvariable & ja & $\mathrm{ja}$ & ja & ja \\
\hline Staatsangehörigkeit & $\begin{array}{l}-0,339^{* * *} \\
(0,082)\end{array}$ & $\begin{array}{l}-0,400^{* * *} \\
(0,096)\end{array}$ & $\begin{array}{l}-0,334^{* * *} \\
(0,083)\end{array}$ & $\begin{array}{l}-0,398^{* * *} \\
(0,096)\end{array}$ \\
\hline \multicolumn{5}{|l|}{$\begin{array}{l}\text { Interaktionseffekte } \\
\text { (Ausbildungsbereiche * Schulbil- } \\
\text { dung) }\end{array}$} \\
\hline Land-/Forstwirtschaftsberufe & & & $\begin{array}{l}-0,220^{* *} \\
(0,087)\end{array}$ & $\begin{array}{c}0,013 \\
(0,094)\end{array}$ \\
\hline Chemieberufe & & & $\begin{array}{l}-0,040 \\
(0,176) \\
\end{array}$ & $\begin{array}{l}-0,011 \\
(0,208)\end{array}$ \\
\hline Metallberufe & & & $\begin{array}{l}-0,116 \\
(0,142) \\
\end{array}$ & $\begin{array}{c}0,052 \\
(0,175) \\
\end{array}$ \\
\hline Textilberufe & & & $\begin{array}{l}-0,075 \\
(0,113) \\
\end{array}$ & $\begin{array}{c}0,051 \\
(0,143) \\
\end{array}$ \\
\hline Berufe im Nahrungsmittelbereich & & & ja & $\mathrm{ja}$ \\
\hline Elektroberufe & & & $\begin{array}{l}-0,189 \\
(0,177) \\
\end{array}$ & $\begin{array}{l}-0,442^{*} \\
(0,238) \\
\end{array}$ \\
\hline
\end{tabular}

${ }^{115}$ Im Nahrungsmittelbereich sind nur wenige Fälle enthalten. 


\begin{tabular}{|c|c|c|c|}
\hline \multirow[b]{2}{*}{$\begin{array}{l}\text { (Referenz: Verbleib im Ausbil- } \\
\text { dungsbetrieb) }\end{array}$} & $\begin{array}{c}\text { Modell 1: } \\
\text { ohne Interaktionseffekte }\end{array}$ & \multicolumn{2}{|c|}{$\begin{array}{c}\text { Modell 2: } \\
\text { mit Interaktionseffekten }\end{array}$} \\
\hline & $\begin{array}{l}\text { Beschäftigung Arbeitslosigkeit } \\
\text { nach Wechsel }\end{array}$ & $\begin{array}{l}\text { Beschäftigung } \\
\text { nach Wechsel }\end{array}$ & Arbeitslosigkeit \\
\hline Bauberufe & & $\begin{array}{c}0,115 \\
(0,137) \\
\end{array}$ & $\begin{array}{l}-0,046 \\
(0,180) \\
\end{array}$ \\
\hline $\begin{array}{l}\text { Infrastruktur-/ Transportdienst- } \\
\text { leistungsberufe }\end{array}$ & & $\begin{array}{c}0,102 \\
(0,145)\end{array}$ & $\begin{array}{c}0,016 \\
(0,168)\end{array}$ \\
\hline Finanzdienstleistungsberufe & & $\begin{array}{l}-0,362^{*} \\
(0,186)\end{array}$ & $\begin{array}{l}-0,182 \\
(0,305)\end{array}$ \\
\hline Verwaltungsberufe & & $\begin{array}{l}-0,234^{* *} \\
(0,105)\end{array}$ & $\begin{array}{l}-0,195 \\
(0,142)\end{array}$ \\
\hline $\begin{array}{l}\text { produktionsnahe Dienstleistungs- } \\
\text { berufe }\end{array}$ & & $\begin{array}{c}0,015 \\
(0,124)\end{array}$ & $\begin{array}{l}-0,122 \\
(0,151)\end{array}$ \\
\hline $\begin{array}{l}\text { haushalts-/personenbezogene } \\
\text { Dienstleistungsberufe }\end{array}$ & & $\begin{array}{l}-0,334^{* *} \\
(0,159)\end{array}$ & $\begin{array}{c}0,120 \\
(0,193)\end{array}$ \\
\hline Fälle & 18357 & \multicolumn{2}{|c|}{18357} \\
\hline$\chi^{2}(87)$ & $2918,99^{* * *}$ & & \\
\hline$\chi^{2}(123)$ & & \multicolumn{2}{|c|}{$2957,82^{* * *}$} \\
\hline pseuddR ${ }^{2}$ & 0,0830 & \multicolumn{2}{|c|}{0,0841} \\
\hline
\end{tabular}

Quelle: IABS 1975-2004, eigene Berechnungen

*** 1\%-Signifikanzniveau; ** 5\%-Signifikanzniveau; * 10\%-Signifikanzniveau Werte in Klammern bezeichnen die Standardfehler 
Abbildung 15: Abgang aus der Arbeitslosigkeit der Abschlusskohorten 1975-1991 nach Schulabschluss - Schätzung eines KaplanMeier-Modells

Abschlusskohorten von 1975-1980

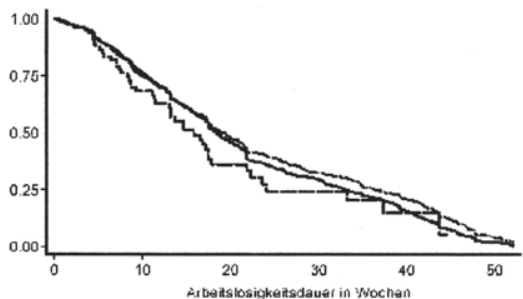

- Bildung $=$ Hauptschulabschluss - Bildung $=$ Realschulabschluss - Bildung $=$ Alitur

Abschlusskohorten von 1987-1991

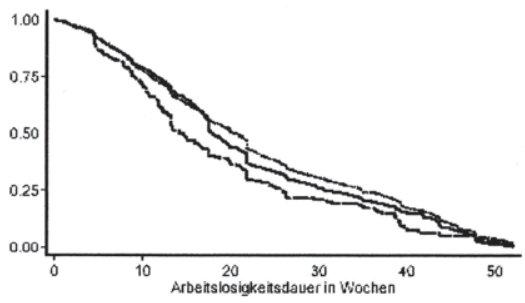

- Biddung $=$ Hauptschul abschlues -..- Bildung $=$ Realachulatsochluss - Bildung = Abilur

Quelle: IABS 1975-2004, eigene Berechnungen

Abschlusskohorten von 1981-1986

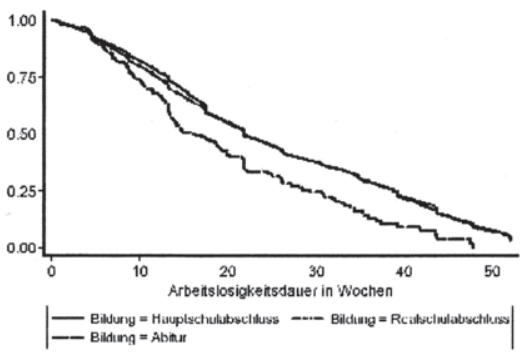




\section{Abbildung 16: Abgang aus der Arbeitslosigkeit nach Ausbildungsbereichen - Schätzung eines Kaplan-Meier-Modells}
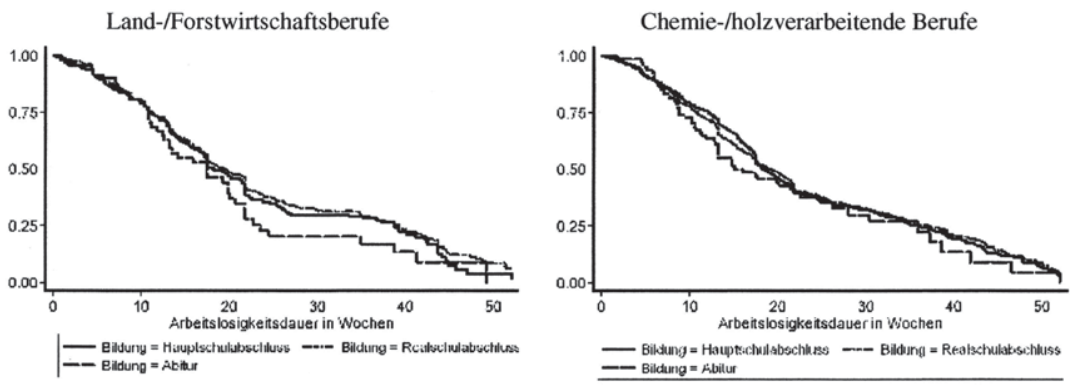

metallverarbeitende Berufe

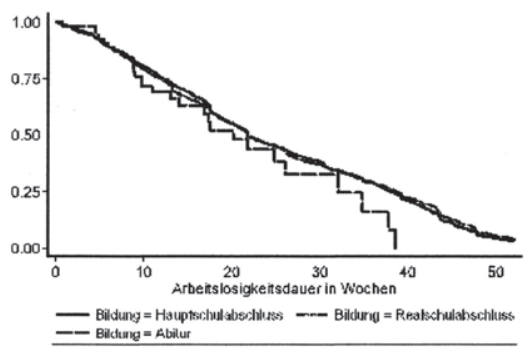

Textilberufe

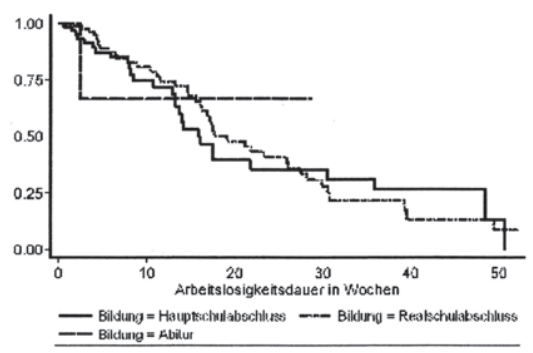

Berufe der Nahrungsmittelindustrie

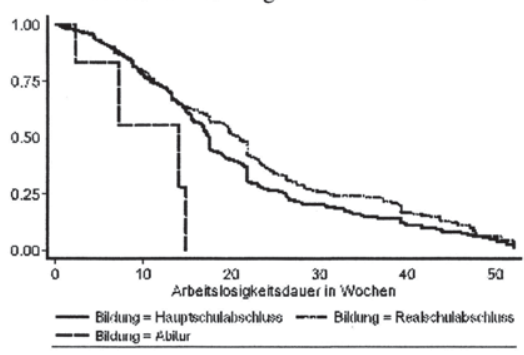

Elektroberufe

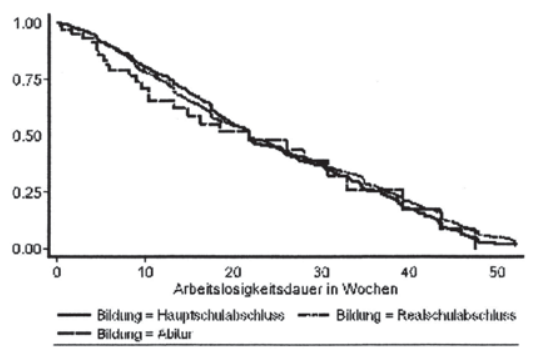

Fortsetzung Abbildung 16 auf der nächsten Seite 


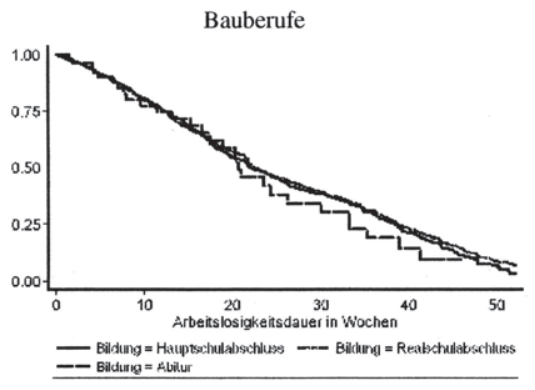

Infrastruktur-/Transportdienstleistungsberufe

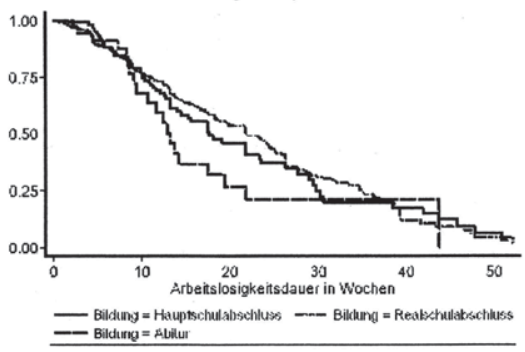

Finanzdienstleistungsberufe

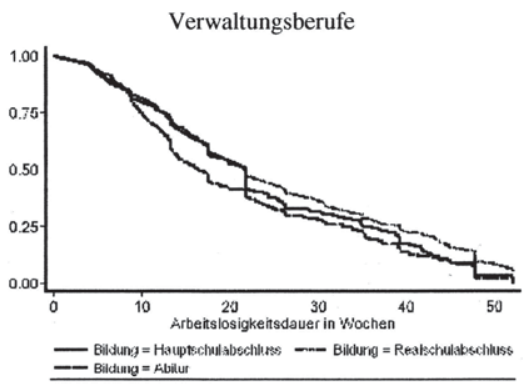

produktionsnahe Dienstleistungsberufe

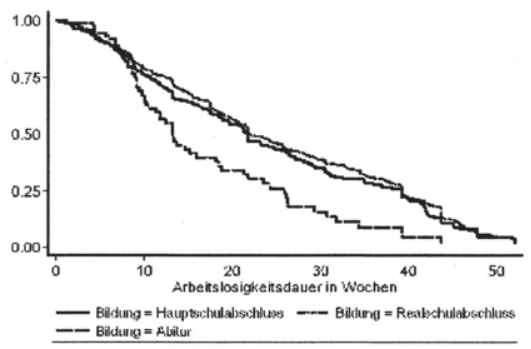

haushalts-/personenbezogene Dienstleistungsberufe

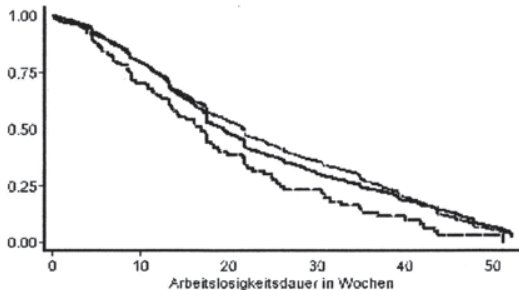

- Bildung $=$ Hauptschul abschlus $\quad-$ Bildung $=$ Realschulakschlus - Bildung $=$ suilur

\section{Quelle: IABS 1975-2004, eigene Berechnungen}


Tabelle 18: Einflussfaktoren auf die Abgangsrate aus Arbeitslosigkeit Schätzungen eines Cox-Modells für die Abschlusskohorten zwischen 1975 und 1991 (Hazardratios)

\begin{tabular}{|c|c|c|c|c|c|c|}
\hline & \multicolumn{2}{|c|}{$\begin{array}{l}\text { Abschlusskohorte } \\
1975-1980\end{array}$} & \multicolumn{2}{|c|}{$\begin{array}{l}\text { Abschlusskohorte } \\
1981-1986\end{array}$} & \multicolumn{2}{|c|}{$\begin{array}{c}\text { Abschlusskohorte } \\
\text { 1987-1991 }\end{array}$} \\
\hline & Modell $1^{\#}$ & Modell $2^{\text {"W }}$ & Modell 1" & Modell $2^{\# \#}$ & Modell $1^{\#}$ & Modell 2 \\
\hline & $\begin{array}{l}\text { Abgangs- } \\
\text { rate }\end{array}$ & $\begin{array}{l}\text { Abgangs- } \\
\text { rate }\end{array}$ & $\begin{array}{l}\text { Abgangs- } \\
\text { rate }\end{array}$ & $\begin{array}{l}\text { Abgangs- } \\
\text { rate }\end{array}$ & $\begin{array}{l}\text { Abgangs- } \\
\text { rate }\end{array}$ & $\begin{array}{l}\text { Abgangs- } \\
\text { rate }\end{array}$ \\
\hline $\begin{array}{l}\text { Ausbildungsvergütungen } \\
(\log )\end{array}$ & $\begin{array}{c}0,947 \\
(0,056)\end{array}$ & $\begin{array}{c}0,959 \\
(0,058)\end{array}$ & $\begin{array}{c}1,030 \\
(0,042)\end{array}$ & $\begin{array}{c}1,027 \\
(0,042)\end{array}$ & $\begin{array}{l}0,894^{* *} \\
(0,045)\end{array}$ & $\begin{array}{l}0,897^{* *} \\
(0,046)\end{array}$ \\
\hline $\begin{array}{l}\text { durchschnittl. betriebliche } \\
\text { Lohnsumme }(\log )\end{array}$ & $\begin{array}{c}1,013 \\
(0,022)\end{array}$ & $\begin{array}{c}1,017 \\
(0,022)\end{array}$ & $\begin{array}{c}1,014 \\
(0,014)\end{array}$ & $\begin{array}{c}1,013 \\
(0,014) \\
\end{array}$ & $\begin{array}{c}1,003 \\
(0,017) \\
\end{array}$ & $\begin{array}{c}1,003 \\
(0,018) \\
\end{array}$ \\
\hline Ausbildungsdauer (log) & $\begin{array}{c}1,013 \\
(0,055)\end{array}$ & $\begin{array}{c}1,010 \\
(0,055) \\
\end{array}$ & $\begin{array}{l}0,834^{* * *} \\
(0,044)\end{array}$ & $\begin{array}{l}0,832^{* * *} \\
(0,044) \\
\end{array}$ & $\begin{array}{l}0,752^{* * *} \\
(0,060)\end{array}$ & $\begin{array}{l}0,748^{* * *} \\
(0,060) \\
\end{array}$ \\
\hline \multicolumn{7}{|l|}{$\begin{array}{l}\text { Betriebsgröße } \\
\text { (Referenz: 1-9 Mitarbeiter) }\end{array}$} \\
\hline 10-49 Mitarbeiter & $\begin{array}{c}0,921 \\
(0,066)\end{array}$ & $\begin{array}{c}0,914 \\
(0,065) \\
\end{array}$ & $\begin{array}{c}0,982 \\
(0,039)\end{array}$ & $\begin{array}{c}0,986 \\
(0,039) \\
\end{array}$ & $\begin{array}{c}0,993 \\
(0,056)\end{array}$ & $\begin{array}{c}0,993 \\
(0,057)\end{array}$ \\
\hline 50-499 Mitarbeiter & $\begin{array}{c}0,857 \\
(0,086)\end{array}$ & $\begin{array}{l}0,837^{*} \\
(0,083)\end{array}$ & $\begin{array}{c}1,002 \\
(0,060)\end{array}$ & $\begin{array}{c}1,007 \\
(0,060)\end{array}$ & $\begin{array}{c}1,046 \\
(0,083)\end{array}$ & $\begin{array}{c}1,051 \\
(0,084)\end{array}$ \\
\hline > 500 Mitarbeiter & $\begin{array}{c}0,941 \\
(0,142)\end{array}$ & $\begin{array}{c}0,926 \\
(0,139)\end{array}$ & $\begin{array}{c}0,965 \\
(0,092)\end{array}$ & $\begin{array}{c}0,967 \\
(0,092)\end{array}$ & $\begin{array}{c}1,107 \\
(0,131)\end{array}$ & $\begin{array}{c}1,113 \\
(0,132)\end{array}$ \\
\hline \multicolumn{7}{|l|}{$\begin{array}{l}\text { Ausbildungsberufe (Refe- } \\
\text { renz: Land-/ Forstwirt- } \\
\text { schaftsberufe) }\end{array}$} \\
\hline Chemieberufe & $\begin{array}{r}1,339^{*} \\
(0,202) \\
\end{array}$ & $\begin{array}{r}1,140 \\
(0,465) \\
\end{array}$ & $\begin{array}{r}1,069 \\
(0,090) \\
\end{array}$ & $\begin{array}{l}0,774 \\
(0,187 \\
\end{array}$ & $\begin{array}{c}0,857 \\
(0,084) \\
\end{array}$ & $\begin{array}{c}1,070 \\
(0,300) \\
\end{array}$ \\
\hline Metallberufe & $\begin{array}{c}1,102 \\
(0,124) \\
\end{array}$ & $\begin{array}{c}0,695 \\
(0,200) \\
\end{array}$ & $\begin{array}{c}1,039 \\
(0,058) \\
\end{array}$ & $\begin{array}{c}0,933 \\
(0,149) \\
\end{array}$ & $\begin{array}{c}0,949 \\
(0,075) \\
\end{array}$ & $\begin{array}{c}1,063 \\
(0,272) \\
\end{array}$ \\
\hline Textilberufe & $\begin{array}{c}1,036 \\
(0,084) \\
\end{array}$ & $\begin{array}{l}0,613^{* * *} \\
(0,141) \\
\end{array}$ & $\begin{array}{c}0,923 \\
(0,039) \\
\end{array}$ & $\begin{array}{c}0,823 \\
(0,108) \\
\end{array}$ & $\begin{array}{c}0,929 \\
(0,056) \\
\end{array}$ & $\begin{array}{c}0,875 \\
(0,202) \\
\end{array}$ \\
\hline $\begin{array}{l}\text { Berufe im Nahrungsmittel- } \\
\text { bereich }\end{array}$ & ja & ja & ja & ja & ja & ja \\
\hline Elektroberufe & $\begin{array}{c}1,099 \\
(0,185)\end{array}$ & $\begin{array}{c}1,901 \\
(1,019)\end{array}$ & $\begin{array}{l}1,176^{* *} \\
(0,082)\end{array}$ & $\begin{array}{c}1,314 \\
(0,274) \\
\end{array}$ & $\begin{array}{c}1,040 \\
(0,111)\end{array}$ & $\begin{array}{c}1,012 \\
(0,330) \\
\end{array}$ \\
\hline Bauberufe & $\begin{array}{c}0,983 \\
(0,079) \\
\end{array}$ & $\begin{array}{l}0,511^{* * *} \\
(0,121)\end{array}$ & $\begin{array}{l}1,113^{* *} \\
(0,056)\end{array}$ & $\begin{array}{c}1,007 \\
(0,172) \\
\end{array}$ & $\begin{array}{l}0,848^{* *} \\
(0,062)\end{array}$ & $\begin{array}{c}0,712 \\
(0,219) \\
\end{array}$ \\
\hline $\begin{array}{l}\text { Infrastruktur-/Transport- } \\
\text { dienstleistungsberufe }\end{array}$ & $\begin{array}{l}0,758^{* * *} \\
(0,080)\end{array}$ & $\begin{array}{c}0,693 \\
(0,192) \\
\end{array}$ & $\begin{array}{c}0,959 \\
(0,049) \\
\end{array}$ & $\begin{array}{c}0,862 \\
(0,123) \\
\end{array}$ & $\begin{array}{l}0,843^{* *} \\
(0,062)\end{array}$ & $\begin{array}{c}0,711 \\
(0,181) \\
\end{array}$ \\
\hline $\begin{array}{l}\text { Finanzdienstleistungs- } \\
\text { berufe }\end{array}$ & $\begin{array}{c}0,930 \\
(0,226) \\
\end{array}$ & $\begin{array}{c}0,351 \\
(0,280) \\
\end{array}$ & $\begin{array}{c}1,084 \\
(0,144) \\
\end{array}$ & $\begin{array}{c}0,854 \\
(0,423) \\
\end{array}$ & $\begin{array}{l}0,727^{* *} \\
(0,117) \\
\end{array}$ & $\begin{array}{l}1,470 \\
(0,879) \\
\end{array}$ \\
\hline Verwaltungsberufe & $\begin{array}{c}1,043 \\
(0,099) \\
\end{array}$ & $\begin{array}{c}0,698 \\
(0,179) \\
\end{array}$ & $\begin{array}{c}0,991 \\
(0,057) \\
\end{array}$ & $\begin{array}{c}0,868 \\
(0,139) \\
\end{array}$ & $\begin{array}{c}0,888 \\
(0,069) \\
\end{array}$ & $\begin{array}{c}0,993 \\
(0,247) \\
\end{array}$ \\
\hline $\begin{array}{l}\text { produktionsnahe Dienst- } \\
\text { leistungsberufe }\end{array}$ & $\begin{array}{l}0,776^{* * *} \\
(0,072)\end{array}$ & $\begin{array}{r}0,587^{*} \\
(0,166) \\
\end{array}$ & $\begin{array}{c}0,930 \\
(0,063)\end{array}$ & $\begin{array}{c}0,712 \\
(0,162) \\
\end{array}$ & $\begin{array}{l}0,813^{* * *} \\
(0,062)\end{array}$ & $\begin{array}{c}0,774 \\
(0,212) \\
\end{array}$ \\
\hline $\begin{array}{l}\text { haushalts-/ personenbezo- } \\
\text { gene Dienstleistungsberufe }\end{array}$ & $\begin{array}{c}0,864 \\
(0,100)\end{array}$ & $\begin{array}{c}0,804 \\
(0,277)\end{array}$ & $\begin{array}{c}0,933 \\
(0,065)\end{array}$ & $\begin{array}{c}0,997 \\
(0,210)\end{array}$ & $\begin{array}{l}0,825^{* *} \\
(0,078)\end{array}$ & $\begin{array}{c}0,736 \\
(0,231)\end{array}$ \\
\hline \multicolumn{7}{|c|}{ Fortsetzung Tabelle 19 auf der nächsten Seite } \\
\hline
\end{tabular}




\begin{tabular}{|c|c|c|c|c|c|c|}
\hline & \multicolumn{2}{|c|}{$\begin{array}{c}\text { Abschlusskohorte } \\
1975-1980 \\
\end{array}$} & \multicolumn{2}{|c|}{$\begin{array}{c}\text { Abschlusskohorte } \\
\text { 1981-1986 } \\
\end{array}$} & \multicolumn{2}{|c|}{$\begin{array}{c}\text { Abschlusskohorte } \\
\text { 1987-1991 } \\
\end{array}$} \\
\hline & Modell 1 & Modell $2^{\# \#}$ & Modell $1^{\#}$ & Modell $2^{\text {\#\# }}$ & Modell $1^{*}$ & Modell $2^{\text {*n }}$ \\
\hline & $\begin{array}{l}\text { Abgangs- } \\
\text { rate }\end{array}$ & $\begin{array}{l}\text { Abgangs- } \\
\text { rate }\end{array}$ & $\begin{array}{l}\text { Abgangs- } \\
\text { rate }\end{array}$ & $\begin{array}{l}\text { Abgangs- } \\
\text { rate }\end{array}$ & $\begin{array}{l}\text { Abgangs- } \\
\text { rate }\end{array}$ & $\begin{array}{l}\text { Abgangs- } \\
\text { rate }\end{array}$ \\
\hline \multicolumn{7}{|l|}{ Qualifikation } \\
\hline $\begin{array}{l}\text { Durchschnitt zum Wirt- } \\
\text { schaftszweig }\end{array}$ & $\begin{array}{c}1,060 \\
(0,054)\end{array}$ & $\begin{array}{c}1,064 \\
(0,054)\end{array}$ & $\begin{array}{l}1,064 * * \\
(0,030)\end{array}$ & $\begin{array}{l}1,066^{* *} \\
(0,030)\end{array}$ & $\begin{array}{l}1,089^{* *} \\
(0,042)\end{array}$ & $\begin{array}{l}1,085^{* *} \\
(0,042)\end{array}$ \\
\hline \multicolumn{7}{|l|}{$\begin{array}{l}\text { Bildung } \\
\text { (Referenz: Hauptschulab- } \\
\text { schluss) }\end{array}$} \\
\hline Realschulabschluss & $\begin{array}{l}0,894^{* *} \\
(0,048) \\
\end{array}$ & $\begin{array}{l}0,746^{* * *} \\
(0,072) \\
\end{array}$ & $\begin{array}{r}1,054^{*} \\
(0,996) \\
\end{array}$ & $\begin{array}{c}0,996 \\
(0,054) \\
\end{array}$ & $\begin{array}{r}0,978 \\
(0,045) \\
\end{array}$ & $\begin{array}{c}0,982 \\
(0,076) \\
\end{array}$ \\
\hline Abitur & $\begin{array}{c}1,257 \\
(0,196) \\
\end{array}$ & $\begin{array}{c}0,902 \\
(0,208) \\
\end{array}$ & $\begin{array}{l}1,442^{* * *} \\
(1,231)\end{array}$ & $\begin{array}{c}1,231 \\
(0,172) \\
\end{array}$ & $\begin{array}{l}1,229^{* *} \\
(0,107)\end{array}$ & $\begin{array}{c}1,290 \\
(0,208) \\
\end{array}$ \\
\hline Regionalvariable & $\mathrm{ja}$ & ja & ja & ja & ja & ja \\
\hline Staatsangehörigkeit & $\begin{array}{c}0,907 \\
(0,354) \\
\end{array}$ & $\begin{array}{r}0,924 \\
(0,369) \\
\end{array}$ & $\begin{array}{l}0,870^{* * *} \\
(0,027) \\
\end{array}$ & $\begin{array}{l}0,870^{* * *} \\
(0,027) \\
\end{array}$ & $\begin{array}{r}1,132 \\
(0,087) \\
\end{array}$ & $\begin{array}{c}1,131 \\
(0,087)\end{array}$ \\
\hline \multicolumn{7}{|l|}{$\begin{array}{l}\text { Interaktionseffekte } \\
\text { (Ausbildungsbereiche * } \\
\text { Schulbildung) }\end{array}$} \\
\hline $\begin{array}{l}\text { Land-/ Forstwirtschaftsbe- } \\
\text { rufe }\end{array}$ & & $\begin{array}{c}0,864 \\
(0,083) \\
\end{array}$ & & $\begin{array}{r}1,047 \\
(0,067) \\
\end{array}$ & & $\begin{array}{c}0,919 \\
(0,068) \\
\end{array}$ \\
\hline Chemieberufe & & $\begin{array}{c}1,103 \\
(0,290) \\
\end{array}$ & & $\begin{array}{c}1,231 \\
(0,172) \\
\end{array}$ & & $\begin{array}{c}0,870 \\
(0,133) \\
\end{array}$ \\
\hline Metallberufe & & $\begin{array}{c}1,367 \\
(0,268) \\
\end{array}$ & & $\begin{array}{r}1,078 \\
(0,109) \\
\end{array}$ & & $\begin{array}{c}0,927 \\
(0,132)\end{array}$ \\
\hline Textilberufe & & $\begin{array}{l}1,454^{* *} \\
(0,242) \\
\end{array}$ & & $\begin{array}{c}1,081 \\
(0,087) \\
\end{array}$ & & $\begin{array}{r}1,026 \\
(0,131) \\
\end{array}$ \\
\hline Berufe i. Nahrungsmittelb. & & ja & & ja & & ja \\
\hline Elektroberufe & & $\begin{array}{c}0,611 \\
(0,262) \\
\end{array}$ & & $\begin{array}{c}0,922 \\
(0,130) \\
\end{array}$ & & $\begin{array}{c}1,011 \\
(0,203) \\
\end{array}$ \\
\hline Bauberufe & & $\begin{array}{l}1,563^{* * *} \\
(0,251)\end{array}$ & & $\begin{array}{r}1,070 \\
(0,107) \\
\end{array}$ & & $\begin{array}{c}1,091 \\
(0,180) \\
\end{array}$ \\
\hline $\begin{array}{l}\text { Infrastruktur-/ Transport- } \\
\text { dienstleistungsberufe }\end{array}$ & & $\begin{array}{c}1,033 \\
(0,203) \\
\end{array}$ & & $\begin{array}{r}1,078 \\
(0,098) \\
\end{array}$ & & $\begin{array}{c}1,100 \\
(0,161) \\
\end{array}$ \\
\hline $\begin{array}{l}\text { Finanzdienstleistungs- } \\
\text { berufe }\end{array}$ & & $\begin{array}{r}1,860 \\
(0,886) \\
\end{array}$ & & $\begin{array}{r}1,158 \\
(0,317) \\
\end{array}$ & & $\begin{array}{c}0,681 \\
(0,214) \\
\end{array}$ \\
\hline Verwaltungsberufe & & $\begin{array}{c}1,288 \\
(0,206) \\
\end{array}$ & & $\begin{array}{r}1,095 \\
(0,103) \\
\end{array}$ & & $\begin{array}{c}0,933 \\
(0,119) \\
\end{array}$ \\
\hline $\begin{array}{l}\text { produktionsnahe Dienst- } \\
\text { leistungsberufe }\end{array}$ & & $\begin{array}{c}1,192 \\
(0,198) \\
\end{array}$ & & $\begin{array}{r}1,171 \\
(0,141) \\
\end{array}$ & & $\begin{array}{r}1,012 \\
(0,137) \\
\end{array}$ \\
\hline $\begin{array}{l}\text { haushalts-/ personenbezo- } \\
\text { gene Dienstleistungsberufe }\end{array}$ & & $\begin{array}{c}1,034 \\
(0,239) \\
\end{array}$ & & $\begin{array}{c}0,970 \\
(0,120) \\
\end{array}$ & & $\begin{array}{c}1,054 \\
(0,172) \\
\end{array}$ \\
\hline Fälle & 3565 & 3565 & 12256 & 12256 & 6336 & 6336 \\
\hline$\chi^{2}(29)$ & $54,27 * * *$ & & $138,81^{* * *}$ & & $83,92^{* * *}$ & \\
\hline$\chi^{2}(41)$ & & $74,62^{* * *}$ & & $146,56^{* * *}$ & & $89,69 * * *$ \\
\hline
\end{tabular}

Quelle: IABS 1975-2004, eigene Berechnungen

" ohne Interaktionseffekte; ${ }^{\#}$ mit Interaktionseffekten

*** $1 \%$-Signifikanzniveau; ** 5\%-Signifikanzniveau; * 10\%-Signifikanzniveau

Werte in Klammern bezeichnen die Standardfehler 
Gabriele Somaggio - 978-3-631-74988-3

Downloaded from PubFactory at 01/11/2019 09:32:31AM

via free access 


\section{SOZIALÖKONOMISCHE SCHRIFTEN}

Herausgegeben von Professor Dr. Dr. h.c. Bert Rürup und

Professor Dr. Werner Sesselmeier

Band 1 Marietta Jass: Erfolgskontrolle des Abwasserabgabengesetzes. Ein Konzept zur Erfassung der Gesetzeswirkungen verbunden mit einer empirischen Untersuchung in der $\mathrm{Pa}$ pierindustrie. 1990.

Band 2 Frank Schulz-Nieswandt: Stationäre Altenpflege und "Pflegenotstand" in der Bundesrepublik Deutschland. 1990.

Band 3 Helmut Böhme, Alois Peressin (Hrsg.): Sozialraum Europa. Die soziale Dimension des Europäischen Binnenmarktes. 1990.

Band 4 Stephan Ruß: Telekommunikation als Standortfaktor für Klein- und Mittelbetriebe. Telekommunikative Entwicklungstendenzen und regionale Wirtschaftspolitik am Beispiel Hessen. 1991.

Band 5 Reinhard Grūnewald: Tertiärisierungsdefizite im Industrieland Bundesrepublik Deutschland. Nachweis und politische Konsequenzen. 1992.

Band 6 Bert Rürup, Uwe H. Schneider (Hrsg.): Umwelt und Technik in den Europäischen Gemeinschaften. Teil I: Die grenzüberschreitende Entsorgung von Abfällen. Bearbeitet von: Thomas Kemmler, Thomas Steinbacher. 1993.

Band 7 Mihai Nedelea: Erfordernisse und Möglichkeiten einer wachstumsorientierten Steuerpolitik in Rumänien. Dargestellt am Beispiel der Textil- und Bekleidungsindustrie. 1995.

Band 8 Andreas Schade: Ganzjăhrige Beschäftigung in der Bauwirtschaft - Eine Wirkungsanalyse. Analyse und Ansătze für eine Reform der Winterbauförderung. 1995.

Band 9 Frank Schulz-Nieswandt: Ǒkonomik der Transformation als wirtschafts- und gesellschaftspolitisches Problem. Eine Einführung aus wirtschaftsanthropologischer Sicht. 1996.

Band 10 Werner Sesselmeier, Roland Klopfleisch, Martin Setzer: Mehr Beschăftigung durch eine Negative Einkommensteuer. Zur beschäftigungspolitischen Effektivităt und Effizienz eines integrierten Steuer- und Transfersystems. 1996.

Band 11 Sylvia Liebler: Der Einfluß der Unabhängigkeit von Notenbanken auf die Stabilität des Geldwertes. 1996.

Band 12 Werner Sesselmeier: Einkommenstransfers als Instrumente der Beschăftigungspolitik. Negative Einkommensteuer und Lohnsubventionen im Lichte moderner Arbeitsmarkttheorien und der Neuen Institutionenökonomik. 1997.

Band 13 Stefan Lorenz: Der Zusammenhang von Arbeitsgestaltung und Erwerbsleben unter besonderer Berücksichtigung der Erwerbstătigkeiten von Frauen und Älteren. 1997.

Band 14 Volker Ehrlich: Arbeitslosigkeit und zweiter Arbeitsmarkt. Theoretische Grundlagen, Probleme und Erfahrungen. 1997.

Band 15 Philipp Hartmann: Grenzen der Versicherbarkeit. Private Arbeitslosenversicherung. 1998.

Band 16 Martin Setzer, Roland Klopfleisch, Werner Sesselmeier: Langzeitarbeitslose und Erster Arbeitsmarkt. Eine kombinierte Strategie zur Erhőhung der Wiederbeschăftigungschancen. 1999.

Band 17 Dorothea Wenzel: Finanzierung des Gesundheitswesens und Interpersonelle Umverteilung. Mikrosimulationsuntersuchung der Einkommenswirkung von Reformvorschlăgen zur GKV-Finanzierung. 1999.

Band 18 Ingo Schroeter: Analyse und Bewertung der intergenerativen Verteilungswirkungen einer Substitution des Umlage- durch das Kapitalstocksverfahren zur Rentenfinanzierung. 1999. 
Band 19 Roland Klopfleisch: Fiskalische Konsequenzen der Europäischen Währungsunion. Die Verånderung des Seigniorage und dessen Bedeutung für die nationalen EWU-11 Haushalte. 2000.

Band 20 Klaus Heubeck, Bert Rürup: Finanzierung der Altersversorgung des öffentlichen Dienstes. Probleme und Optionen. 2000.

Band 21 Manon Pigeau: Der Einfluß der Arbeitszeit auf die Erwerbsbeteiligung von Frauen. Empirische Befunde, mikroökonomische Modellierung und politische Konsequenzen. 2002.

Band 22 Carsten Müller: Existenzgründungshilfen als Instrument der Struktur- und Beschäftigungspolitik. 2002.

Band 23 Stefan Lewe: Wachstumseffiziente Untemehmensbesteuerung. 2003.

Band 24 Robert Coppik: Konzeption eines Transformationsansatzes zur Substitution des kameralen, inputorientierten Budgetkreislaufs der offentlichen Verwaltung in einen outputorientierten Budgetkreislauf innerhalb des New Public Management.

Band 25 Alexander Meindel: Intergenerative Verteilungswirkung beim Übergang zu einer nachgelagerten Rentenbesteuerung. 2004.

Band 26 Jochen Gunnar Jagob: Das Äquivalenzprinzip in der Alterssicherung. 2004.

Band 27 Tobias Fehr: Recht des außerbörslichen Aktienhandels vor dem Hintergrund des Rechts des börslichen Aktienhandels. Das Kapitalmarktszenario für kapitalmarktaktive Aktiengesellschaften, deren Unternehmensführungen und aktuelle und potentielle Aktionăre und für Wertpapierdienstleister. 2006.

Band 28 Stefan Fetzer: Zur nachhaltigen Finanzierung des gesetzlichen Gesundheitssystems. 2006.

Band 29 Oliver Ehrentraut: Alterung und Altersvorsorge. Das deutsche Drei-Săulen-System der Alterssicherung vor dem Hintergrund des demografischen Wandels. 2006.

Band 30 Martin Debus: Arbeitsmarkteffekte des demografischen Wandels. 2007.

Band 31 Jens Hujer: Regionalökonomische Effekte von Flughăfen. 2008.

Band 32 Zulia Gubaydullina: Nicht-monetăre Inflationsursachen in Russland. Eine empirische Analyse. 2008.

Band 33 Jasmin Häcker: Die Soziale Pflegeversicherung: Eine Generationenbilanz. 2008.

Band 34 Christina Benita Wilke: German Pension Reform. On Road Towards a Sustainable MultiPillar System. 2009.

Band 35 Stefan Pfaffenbach: Nachhaltigkeit in der Gesetzlichen Rentenversicherung - Was leistet die kinderzahlabhångige Rente. 2009.

Band 36 Anabell Kohlmeier: Die Ausweitung des Versichertenkreises der Gesetzlichen Rentenversicherung. Bestimmungsgrūnde und Verteilungswirkungen. 2009.

Band 37 Matthias Heidler: Reformen der gesetzlichen Rentenversicherung: Politisches Risiko und intergenerative Umverteilung. 2009.

Band 38 Anna Rosinus: Vermőgensdekonzentration und Mitarbeiterkapitalbeteiligungsgesetz. 2009.

Band 39 Gabriele Somaggio: Start mit Hindernissen. Eine theoretische und empirische Analyse der Ursachen von Arbeitslosigkeit nach der dualen Berufsausbildung. 2009.

www.peterlang.de 
Gabriele Somaggio - 978-3-631-74988-3

Downloaded from PubFactory at 01/11/2019 09:32:31AM

via free access 
Gabriele Somaggio - 978-3-631-74988-3

Downloaded from PubFactory at 01/11/2019 09:32:31AM

via free access 